\title{
Regioselective Synthesis of Difluorinated C-furanosides Involving a Debenzylative Cycloetherification
}

\author{
Julien A. Delbrouck, ${ }^{*}, \dagger$ Valentin N. Bochatay, ${ }^{*}, \dagger$ Abdellatif Tikad ${ }^{*}$ and Stéphane P. Vincent ${ }^{*}, \dagger$

\begin{abstract}
$\dagger$ University of Namur, Département de Chimie, Laboratoire de Chimie Bio-Organique, rue de Bruxelles 61, B-5000 Namur, Belgium;

${ }^{\ddagger}$ Laboratoire de Chimie Moléculaire et Substances Naturelles, Faculté des Sciences, Université Moulay Ismail, B.P. 1120, Zitoune, Meknès, Morocco.
\end{abstract}

\section{Materials and methods:}

Materials and methods: All reactions were carried out under an argon atmosphere. Yields refer to chromatographically and spectroscopically homogeneous materials. Reagents and chemicals were purchased from Sigma-Aldrich or Acros at ACS grade and were used without purification. All reactions were performed using purified and dried solvents: tetrahydrofuran (THF) was refluxed over sodium-benzophenone, dichloromethane $\left(\mathrm{CH}_{2} \mathrm{Cl}_{2}\right)$, triethylamine $\left(\mathrm{NEt}_{3}\right)$, and pyridine were refluxed over calcium hydride $\left(\mathrm{CaH}_{2}\right)$. All reactions were monitored by thin-layer chromatography (TLC) carried out on Merck aluminum roll silica gel 60-F254 using UV light and a phosphomolybdic acid solution as revelator. Merck silica gel (60, particle size 40$63 \mu \mathrm{m})$ was employed for flash column chromatography and preparative thin layer chromatography using technically solvent distilled prior to use as eluting solvents. NMR spectra were recorded on a JEOL ECX 400 or 500 with solvent peaks as reference. All compounds were characterized by ${ }^{1} \mathrm{H}$ and ${ }^{13} \mathrm{C}$ NMR as well as by ${ }^{1} \mathrm{H}-{ }^{1} \mathrm{H}$ and ${ }^{1} \mathrm{H}-{ }^{13} \mathrm{C}$ correlation experiments when necessary. The following abbreviations are used to describe the multiplicities: $s=$ singlet, $d=$ doublet, $\mathrm{t}=$ triplet, $\mathrm{q}=$ quartet, $\mathrm{m}=$ multiplet, $\mathrm{br}=$ broad . The numbering of the protons and carbons is illustrated in the Scheme below. Aromatic, benzyl and methyl (carbons and protons) are respectively labeled with "Arom", " $\mathrm{CH}_{2} \mathrm{Bn}$ ", quaternary carbons are indicated with a "q" superscript. Chemical shifts $(\delta)$ are reported in ppm and referenced indirectly to residual solvent signals. High-resolution mass spectra (HRMS) were performed on a Bruker maXis mass spectrometer with an accuracy tolerance of 2 ppm by the "Fédération de Recherche" ICOA/CBM (FR2708) platform. 


\section{Dithioacetal}

2,3,4,5-Tetra-O-benzyl diethyl dithioacetal D-arabinose (1a).<smiles>CCC(O)C(Br)C(Br)C(Br)C(CC)OCc1ccccc1</smiles>

1 a

An aqueous solution of concentrated hydrochloric acid (37\%, $60 \mathrm{ml}, 724 \mathrm{mmol}, 1.8$ equiv.) was added to D-arabinose $(60.1 \mathrm{~g}, 400 \mathrm{mmol})$ at room temperature. Then, ethanethiol $\left(60 \mathrm{ml}, 810 \mathrm{mmol}, 2.0\right.$ equiv.) was added dropwise at $0{ }^{\circ} \mathrm{C}$ and the product was crystallized after $10 \mathrm{~min}$ of stirring at $0{ }^{\circ} \mathrm{C}$. Then, the solid was washed with ice water and cooled diethyl ether to give diethyl dithioacetal D-arabinose as a white powder (73.5 $\mathrm{g}, 72 \%)$.

To a solution of diethyl dithioacetal D-arabinose (36.3 g, $142 \mathrm{mmol})$ in dry DMF (345 $\mathrm{ml}$ ) under argon atmosphere was added benzyl bromide (74.6 ml, $624 \mathrm{mmol}, 4.4$ equiv.) at room temperature. The solution was cooled at $0{ }^{\circ} \mathrm{C}$ and $\mathrm{NaH}(60 \%$ in mineral oil, 23.8 g, $595 \mathrm{mmol}, 4.2$ equiv.) was added portionwise and the reaction was stirred for $48 \mathrm{~h}$. Ice water was added carefully and the mixture was diluted with $\mathrm{Et}_{2} \mathrm{O}$ and the aqueous phase was extracted with $\mathrm{Et}_{2} \mathrm{O}$ (2 times). The combined organic phases were washed with saturated aqueous solution of $\mathrm{NH}_{4} \mathrm{Cl}$ (3 times) and brine (3 times), dried over $\mathrm{MgSO}_{4}$ and concentrated. Purification by chromatography on silica gel (Cy/EtOAc 95:5) gave molecule $1 \mathrm{a}(79.9 \mathrm{~g}, 91 \%)$.

$[\alpha]^{20} \mathrm{D}:+5.5\left(c\right.$ 1.0, $\left.\mathrm{CHCl}_{3}\right) .{ }^{1} \mathrm{H} \mathrm{NMR}\left(400 \mathrm{MHz}, \mathrm{CDCl}_{3}\right): \delta=7.38-7.26\left(\mathrm{~m}, 20 \mathrm{H}, \mathrm{H}^{\text {arom }}\right)$, $4.83\left(\mathrm{AB}, 1 \mathrm{H}, J_{\mathrm{A}-\mathrm{B}}=11.0 \mathrm{~Hz}, \mathrm{CH}_{2}{ }^{\mathrm{Bn}}\right), 4.78\left(\mathrm{AB}, 1 \mathrm{H}, J_{\mathrm{A}-\mathrm{B}}=11.5 \mathrm{~Hz}, \mathrm{CH}_{2}{ }^{\mathrm{Bn}}\right), 4.75(\mathrm{AB}$, $\left.1 \mathrm{H}, J_{\mathrm{A}-\mathrm{B}}=11.5 \mathrm{~Hz}, \mathrm{CH}_{2}{ }^{\mathrm{Bn}}\right), 4.73\left(\mathrm{AB}, 1 \mathrm{H}, J_{\mathrm{A}-\mathrm{B}}=11.0 \mathrm{~Hz}, \mathrm{CH}_{2}{ }^{\mathrm{Bn}}\right), 4.71\left(\mathrm{AB}, 1 \mathrm{H}, J_{\mathrm{A}-\mathrm{B}}=\right.$ $\left.11.9 \mathrm{~Hz}, \mathrm{CH}_{2}{ }^{\mathrm{Bn}}\right), 4.57\left(\mathrm{AB}, 1 \mathrm{H}, J_{\mathrm{A}-\mathrm{B}}=11.9 \mathrm{~Hz}, \mathrm{CH}_{2}{ }^{\mathrm{Bn}}\right), 4.53\left(\mathrm{AB}, 1 \mathrm{H}, J_{\mathrm{A}-\mathrm{B}}=12.4 \mathrm{~Hz}\right.$, $\left.\mathrm{CH}_{2}{ }^{\mathrm{Bn}}\right), 4.50\left(\mathrm{AB}, 1 \mathrm{H}, J_{\mathrm{A}-\mathrm{B}}=12.4 \mathrm{~Hz}, \mathrm{CH}_{2}{ }^{\mathrm{Bn}}\right), 4.26\left(\mathrm{dd}, 1 \mathrm{H}, J_{2-3}=5.7 \mathrm{~Hz}, J_{3-4}=5.0 \mathrm{~Hz}\right.$, $\mathrm{H}-3), 4.01\left(\mathrm{~d}, 1 \mathrm{H}, J_{1-2}=4.8 \mathrm{~Hz}, \mathrm{H}-1\right), 3.96\left(\mathrm{dd}, 1 \mathrm{H}, J_{1-2}=4.8 \mathrm{~Hz}, J_{2-3}=5.7 \mathrm{~Hz}, \mathrm{H}-2\right)$, 3.88-3.85 (m, 2H, H-4, H-5a), 3.69 (ABX, 1H, $\left.J_{5 \mathrm{a}-5 \mathrm{~b}}=9.9 \mathrm{~Hz}, J_{4-5 \mathrm{~b}}=4.6 \mathrm{~Hz}, \mathrm{H}-5 \mathrm{~b}\right), 2.70$ $\left(\mathrm{dq}, 2 \mathrm{H}, J_{\mathrm{CH} 2 \mathrm{CH} 3}=2.3 \mathrm{~Hz}, J_{\mathrm{CH}_{2} \mathrm{CH} 3}=7.3 \mathrm{~Hz}, \mathrm{SCH}_{2} \mathrm{CH}_{3}\right), 2.53\left(\mathrm{dq}, 2 \mathrm{H}, J_{\mathrm{CH} 2 \mathrm{CH} 3}=3.4 \mathrm{~Hz}\right.$, $\left.J_{\mathrm{CH} 2 \mathrm{CH} 3}=7.3 \mathrm{~Hz}, \mathrm{SCH}_{2} \mathrm{CH}_{3}\right), 1.19\left(\mathrm{t}, 3 \mathrm{H}, J_{\mathrm{CH} 2 \mathrm{CH} 3}=7.3 \mathrm{~Hz}, \mathrm{SCH}_{2} \mathrm{CH}_{3}\right), 1.15(\mathrm{t}, 3 \mathrm{H}$, $\left.J_{\mathrm{CH} 2 \mathrm{CH} 3}=7.3 \mathrm{~Hz}, \mathrm{SCH}_{2} \mathrm{CH}_{3}\right) \mathrm{ppm} .{ }^{13} \mathrm{C} \mathrm{NMR}\left(125 \mathrm{MHz}, \mathrm{CDCl}_{3}\right) \delta=139.0\left(\mathrm{C}_{\mathrm{q}}{ }^{\text {arom }}\right), 138.9$ $\left(\mathrm{C}_{\mathrm{q}}{ }^{\text {arom }}\right), 138.6\left(\mathrm{C}_{\mathrm{q}}{ }^{\text {arom }}\right), 138.3\left(\mathrm{C}_{\mathrm{q}}{ }^{\text {arom }}\right)$, 128.6-127.4 $\left(\mathrm{CH}^{\text {arom }}\right), 83.1(\mathrm{C}-2), 80.5(\mathrm{C}-3), 79.0$ (C-4), $75.4\left(\mathrm{CH}_{2}{ }^{\mathrm{Bn}}\right), 74.9\left(\mathrm{CH}_{2}{ }^{\mathrm{Bn}}\right), 73.5\left(\mathrm{CH}_{2}{ }^{\mathrm{Bn}}\right), 71.8\left(\mathrm{CH}_{2}{ }^{\mathrm{Bn}}\right), 69.3(\mathrm{C}-5), 54.0(\mathrm{C}-1)$, $25.4\left(\mathrm{SCH}_{2} \mathrm{CH}_{3}\right), 25.2\left(\mathrm{SCH}_{2} \mathrm{CH}_{3}\right), 14.6\left(\mathrm{SCH}_{2} \mathrm{CH}_{3}\right), 14.5\left(\mathrm{SCH}_{2} \mathrm{CH}_{3}\right)$ ppm. These analytical data correspond to published ones. ${ }^{1}$

\footnotetext{
${ }^{1}$ Delbrouck J. A., Abdellatif Tikad A., Vincent S. P. Chem. Commun. 2018, 54, 9845.
} 


\section{${ }^{1}$ H NMR}

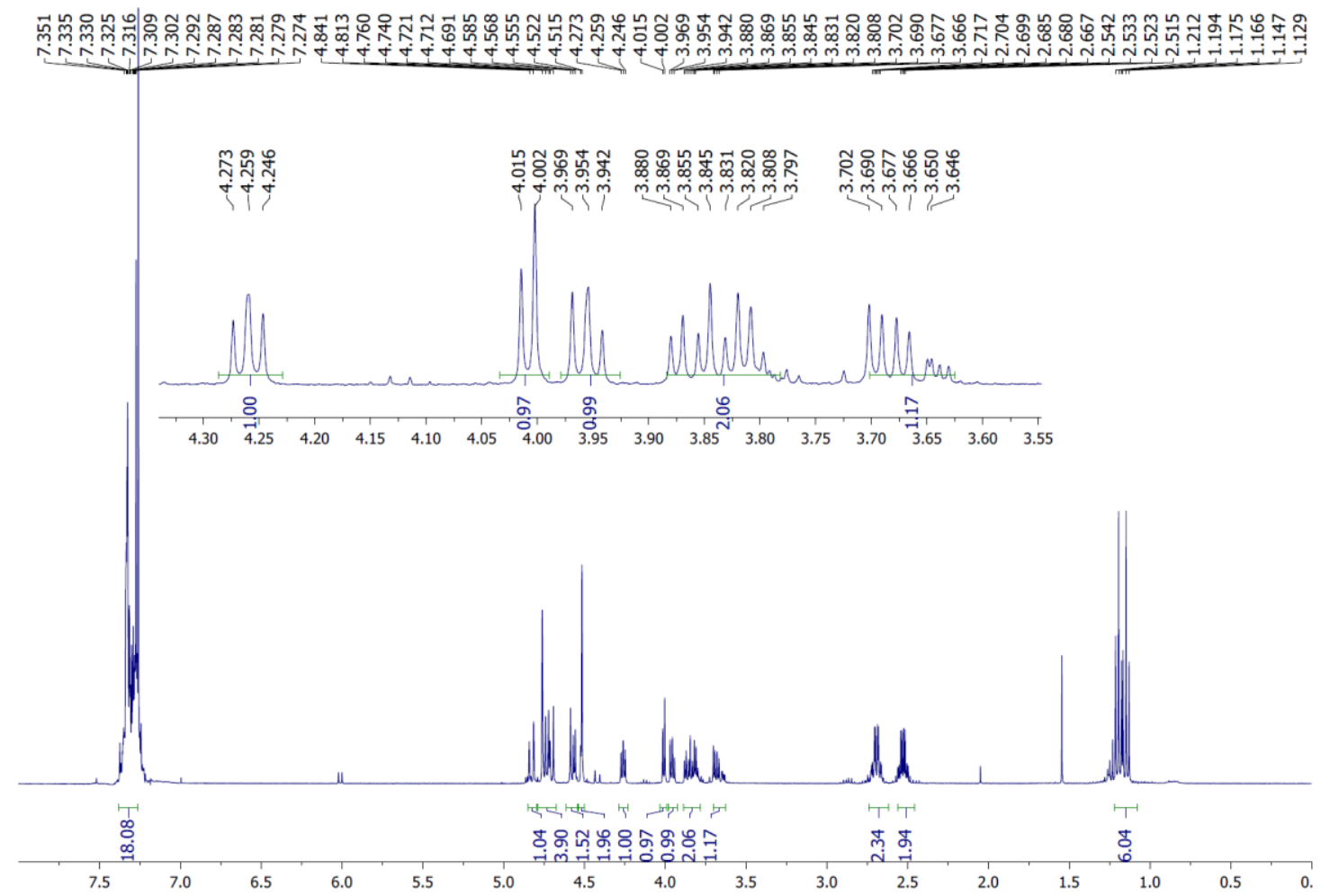

\section{${ }^{13}$ C NMR}

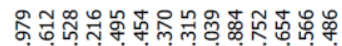

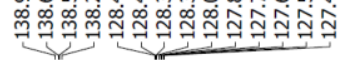

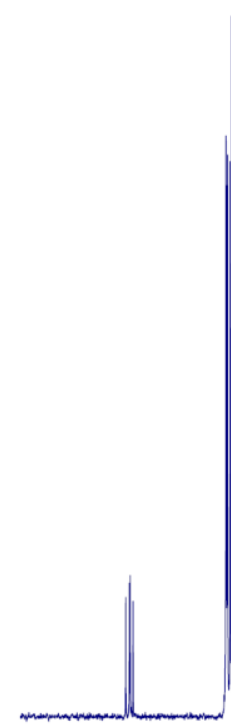

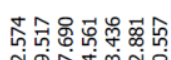

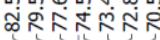

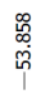

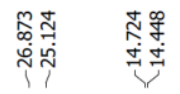

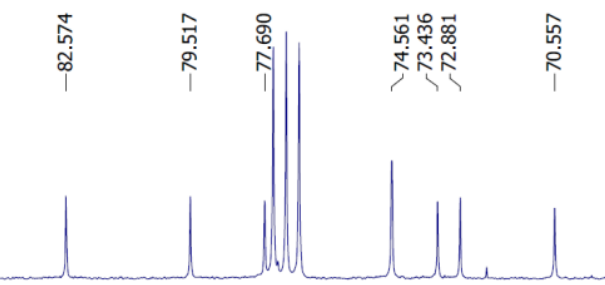

$\begin{array}{lllllllllllllllllllll}7 & 7 & 83 & 82 & 81 & 80 & 79 & 78 & 77 & 76 & 75 & 74 & 73 & 72 & 71 & 70\end{array}$

$\begin{array}{llllllllllllllllllllllllllllllll}145 & 140 & 135 & 130 & 125 & 120 & 115 & 110 & 105 & 100 & 95 & 90 & 85 & 80 & 75 & 70 & 65 & 60 & 55 & 50 & 45 & 40 & 35 & 30 & 25 & 20 & 15 & 10 & 5 & 5\end{array}$ 
<smiles>CCC(Br)C(Br)C(Br)C(Br)C(CC)Cc1ccccc1</smiles>

$1 \mathrm{~b}$

An aqueous solution of concentrated hydrochloric acid $(37 \%, 52.0 \mathrm{ml}, 627 \mathrm{mmol}, 1.8$ equiv.) was added to D-ribose $(52.6 \mathrm{~g}, 350 \mathrm{mmol})$ at $0{ }^{\circ} \mathrm{C}$ and the mixture was stirred for $10 \mathrm{~min}$ at $0{ }^{\circ} \mathrm{C}$. Then, ethanethiol $(52.0 \mathrm{ml}, 702 \mathrm{mmol}, 2.0$ equiv. $)$ was added and the solution was stirred for $20 \mathrm{~min}$ at $0{ }^{\circ} \mathrm{C}$ and $18 \mathrm{~h}$ at room temperature. Methanol $(700 \mathrm{ml})$ was added and the solution was neutralized with $\mathrm{Na}_{2} \mathrm{CO}_{3(\mathrm{~s})}$ to $\mathrm{pH}=7$. Filtration on celite ${ }^{\circledR}$, concentration of the filtrate under reduced pressure and recrystallization from hot toluene $\left(50{ }^{\circ} \mathrm{C}\right)$ afforded diethyl dithioacetal D-ribose as an orange powder $(32.3 \mathrm{~g}, 36 \%)$.

To a solution of diethyl dithioacetal D-ribose $(15.9 \mathrm{~g}, 62 \mathrm{mmol})$ in dry DMF $(210 \mathrm{ml})$ under argon atmosphere was added benzyl bromide $(32.6 \mathrm{ml}, 273 \mathrm{mmol}, 4.4$ equiv.) at room temperature. The solution was cooled at $0{ }^{\circ} \mathrm{C}$ and $\mathrm{NaH}(60 \%$ in mineral oil, $12.9 \mathrm{~g}$, $312 \mathrm{mmol}, 5.0$ equiv.) was added portionwise and the reaction was stirred for $20 \mathrm{~h}$. Ice water was added carefully and the mixture was diluted with $\mathrm{Et}_{2} \mathrm{O}$ and the aqueous phase was extracted with $\mathrm{Et}_{2} \mathrm{O}$ (2 times). The combined organic phases were washed with saturated aqueous solution of $\mathrm{NH}_{4} \mathrm{Cl}$ (3 times) and brine (3 times), dried over $\mathrm{MgSO}_{4}$ and concentrated. Purification by chromatography on silica gel (Cy/EtOAc 95:5) gave compound $\mathbf{1 b}(37.7 \mathrm{~g}, 98 \%)$.

$[\alpha]^{20} \mathrm{D}:+42.3\left(c 0.5, \mathrm{CHCl}_{3}\right) .{ }^{1} \mathrm{H} \mathrm{NMR}\left(400 \mathrm{MHz}, \mathrm{CDCl}_{3}\right): \delta=7.37-7.26\left(\mathrm{~m}, 20 \mathrm{H}, \mathrm{H}^{\text {arom }}\right)$, $4.91\left(\mathrm{AB}, 1 \mathrm{H}, J_{\mathrm{A}-\mathrm{B}}=11.0 \mathrm{~Hz}, \mathrm{CH}_{2}{ }^{\mathrm{Bn}}\right), 4.81\left(\mathrm{AB}, 1 \mathrm{H}, J_{\mathrm{A}-\mathrm{B}}=11.2 \mathrm{~Hz}, \mathrm{CH}_{2}{ }^{\mathrm{Bn}}\right), 4.73(\mathrm{AB}$, $\left.1 \mathrm{H}, J_{\mathrm{A}-\mathrm{B}}=11.9 \mathrm{~Hz}, \mathrm{CH}_{2}{ }^{\mathrm{Bn}}\right), 4.70\left(\mathrm{AB}, 1 \mathrm{H}, J_{\mathrm{A}-\mathrm{B}}=11.9 \mathrm{~Hz}, \mathrm{CH}_{2}{ }^{\mathrm{Bn}}\right), 4.64\left(\mathrm{AB}, 1 \mathrm{H}, J_{\mathrm{A}-\mathrm{B}}=\right.$ $\left.11.0 \mathrm{~Hz}, \mathrm{CH}_{2}{ }^{\mathrm{Bn}}\right), 4.61\left(\mathrm{AB}, 1 \mathrm{H}, J_{\mathrm{A}-\mathrm{B}}=11.2 \mathrm{~Hz}, \mathrm{CH}_{2}{ }^{\mathrm{Bn}}\right), 4.48\left(\mathrm{AB}, 1 \mathrm{H}, J_{\mathrm{A}-\mathrm{B}}=12.1 \mathrm{~Hz}\right.$, $\left.\mathrm{CH}_{2}{ }^{\mathrm{Bn}}\right), 4.44\left(\mathrm{AB}, 1 \mathrm{H}, J_{\mathrm{A}-\mathrm{B}}=12.1 \mathrm{~Hz}, \mathrm{CH}_{2}{ }^{\mathrm{Bn}}\right), 4.24\left(\mathrm{~d}, 1 \mathrm{H}, J_{1-2}=3.0 \mathrm{~Hz}, \mathrm{H}-1\right), 4.14(\mathrm{dd}$, $\left.1 \mathrm{H}, J_{2-3}=7.8 \mathrm{~Hz}, J_{3-4}=2.5 \mathrm{~Hz}, \mathrm{H}-3\right), 4.08(\mathrm{~m}, 1 \mathrm{H}, \mathrm{H}-4), 3.97\left(\mathrm{dd}, 1 \mathrm{H}, J_{1-2}=3.0 \mathrm{~Hz}, J_{2-3}\right.$ $=7.8 \mathrm{~Hz}, \mathrm{H}-2), 3.74\left(\mathrm{ABX}, 1 \mathrm{H}, J_{4-5 \mathrm{a}}=4.4 \mathrm{~Hz}, J_{5 \mathrm{a}-5 \mathrm{~b}}=10.5 \mathrm{~Hz}, \mathrm{H}-5 \mathrm{a}\right), 3.70(\mathrm{ABX}, 1 \mathrm{H}$, $\left.J_{4-5 \mathrm{~b}}=6.2 \mathrm{~Hz}, J_{5 \mathrm{a}-5 \mathrm{~b}}=10.5 \mathrm{~Hz}, \mathrm{H}-5 \mathrm{~b}\right), 2.67-2.55\left(\mathrm{~m}, 4 \mathrm{H}, 2 \mathrm{SCH}_{2} \mathrm{CH}_{3}\right), 1.20\left(\mathrm{q}, 6 \mathrm{H}, J_{\mathrm{CH} 2 \mathrm{CH}}\right.$ $\left.=7.6 \mathrm{~Hz}, 2 \mathrm{SCH}_{2} \mathrm{CH}_{3}\right)$ ppm. ${ }^{13} \mathrm{C} \mathrm{NMR}\left(125 \mathrm{MHz}, \mathrm{CDCl}_{3}\right): \delta=138.9\left(\mathrm{C}_{\mathrm{q}}{ }^{\text {arom }}\right), 138.6(2 \mathrm{x}$ $\left.\mathrm{C}_{\mathrm{q}}{ }^{\text {arom }}\right), 138.4\left(\mathrm{C}_{\mathrm{q}}{ }^{\text {arom }}\right), 128.6-127.4\left(\mathrm{CH}^{\text {arom }}\right), 82.3(\mathrm{C}-2), 79.9(\mathrm{C}-3), 79.2(\mathrm{C}-4), 74.7$ $\left(\mathrm{CH}_{2}{ }^{\mathrm{Bn}}\right), 73.6\left(\mathrm{CH}_{2}{ }^{\mathrm{Bn}}\right), 73.3\left(\mathrm{CH}_{2}{ }^{\mathrm{Bn}}\right), 72.6\left(\mathrm{CH}_{2}{ }^{\mathrm{Bn}}\right), 70.9(\mathrm{C}-5), 54.0(\mathrm{C}-1), 26.4$ $\left(\mathrm{SCH}_{2} \mathrm{CH}_{3}\right), 25.1\left(\mathrm{SCH}_{2} \mathrm{CH}_{3}\right), 14.6\left(2 \mathrm{SCH}_{2} \mathrm{CH}_{3}\right) \mathrm{ppm}$. These analytical data correspond to published ones. ${ }^{1}$ 


\section{${ }^{1}$ H NMR}

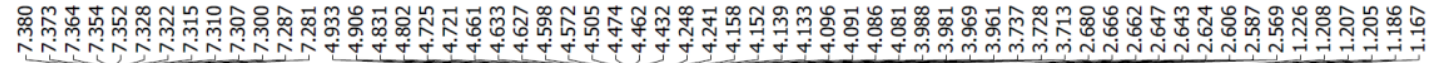

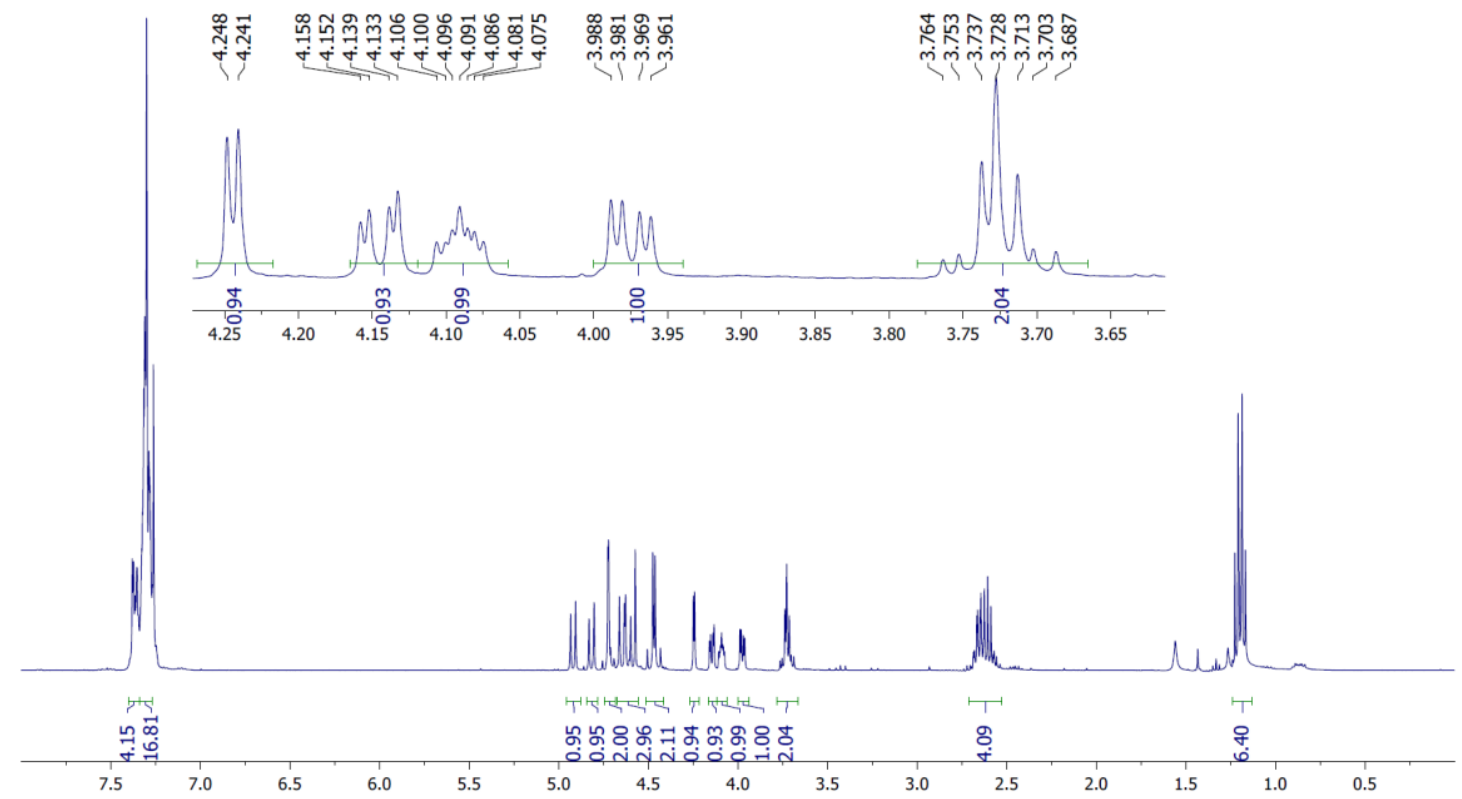

\section{${ }^{13}$ C NMR}

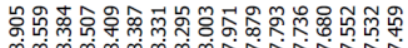

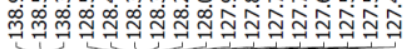

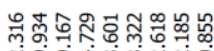

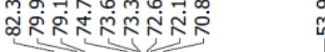

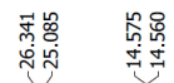
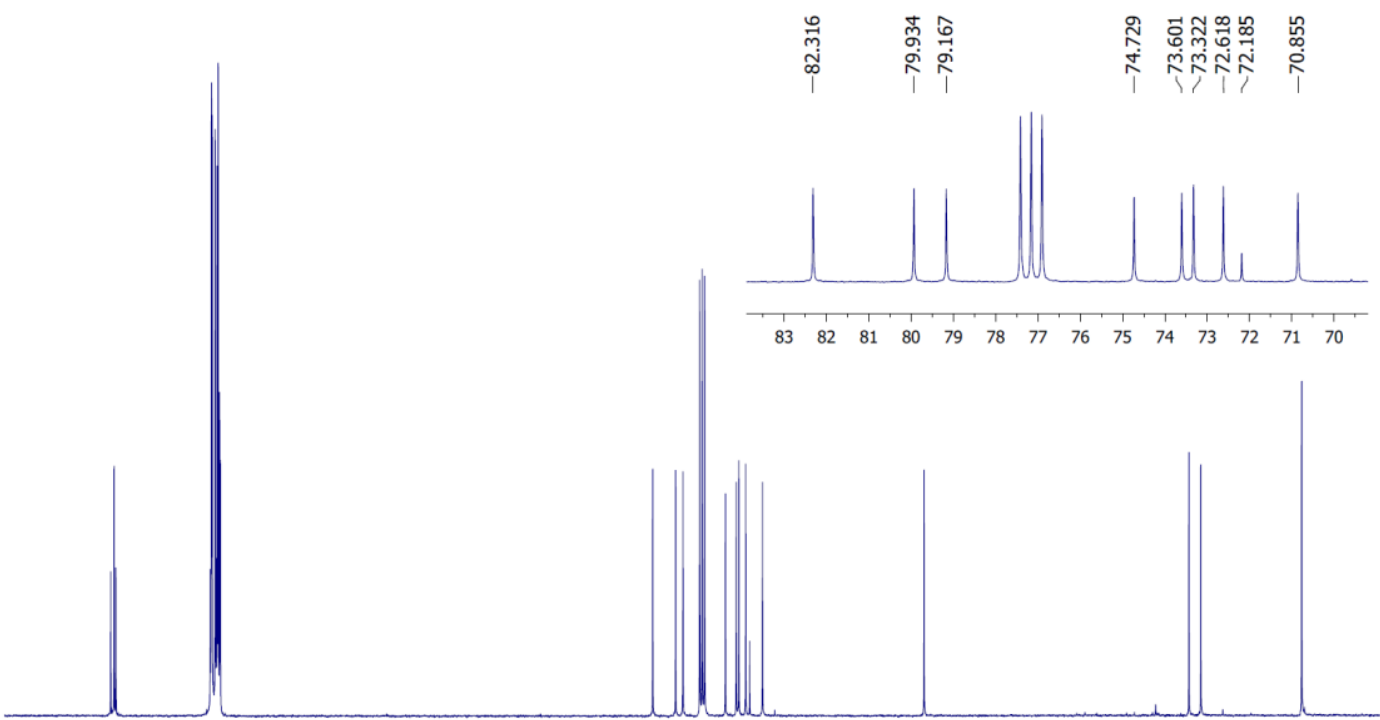

$\begin{array}{lllllllllllllllllllllllllllll}145 & 140 & 135 & 130 & 125 & 120 & 115 & 110 & 105 & 100 & 95 & 90 & 85 & 80 & 75 & 70 & 65 & 60 & 55 & 50 & 45 & 40 & 35 & 30 & 25 & 20 & 15 & 10 & 5\end{array}$ 


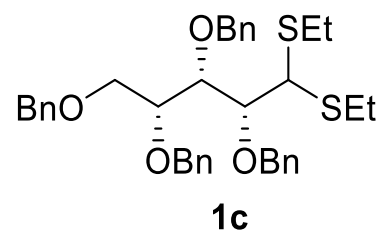

D-xylose (30.0 g, $200 \mathrm{mmol})$ was dissolved in an aqueous solution of concentrated hydrochloric acid $\left(37 \%, 25 \mathrm{ml}, 302 \mathrm{mmol}, 1.5\right.$ equiv.) at $0{ }^{\circ} \mathrm{C}$ under argon atmosphere. Then, ethanethiol ( $35 \mathrm{ml}, 472 \mathrm{mmol}, 2.4$ equiv.) was added dropwise at $0{ }^{\circ} \mathrm{C}$ over $15 \mathrm{~min}$ and the reaction was stirred for $30 \mathrm{~min}$ at r.t. The mixture was cooled again to $0{ }^{\circ} \mathrm{C}$ and neutralized with $\mathrm{NH}_{4} \mathrm{OH}$ solution $(28-30 \mathrm{wt} \%, 35 \mathrm{ml}, 14.5 \mathrm{M})$. The crude was extracted with hexane (4 times) to remove unreacted ethanethiol. The aqueous layer was concentrated to give an impure yellow oil, which was dissolved in a mixture of (acetone/ethyl acetate, 2:1, $700 \mathrm{ml}$ ), filtered through a celite ${ }^{\circledR}$ pad and concentrated. The yellow oil was recrystallized from dichloromethane/diethyl ether to yield 1c as a white solid $(42.0 \mathrm{~g}, 82 \%)$.

To a solution of diethyl dithioacetal D-xylose $(15.7 \mathrm{~g}, 61 \mathrm{mmol})$ in dry DMF $(210 \mathrm{ml})$ under argon atmosphere was added benzyl bromide $(32.3 \mathrm{ml}, 270 \mathrm{mmol}, 4.4$ equiv.) at r.t. The solution was cooled at $0{ }^{\circ} \mathrm{C}$ and $\mathrm{NaH}$ (60\% in mineral oil, $12.2 \mathrm{~g}, 306 \mathrm{mmol}, 5.0$ equiv.) was added portionwise and the reaction was stirred for $24 \mathrm{~h}$. Ice water was added carefully and the mixture was diluted with $\mathrm{Et}_{2} \mathrm{O}$ and the aqueous phase was extracted with $\mathrm{Et}_{2} \mathrm{O}$ (2 times). The combined organic phases were washed with saturated aqueous solution of $\mathrm{NH}_{4} \mathrm{Cl}$ (3 times) and brine (3 times), dried over $\mathrm{MgSO}_{4}$ and concentrated. Purification by chromatography on silica gel (Cy/EtOAc 95:5) gave molecule 1c (33.6 g, $89 \%)$.

$[\alpha]^{20} \mathrm{D}:-9.2\left(c\right.$ 1.0, $\left.\mathrm{CHCl}_{3}\right) .{ }^{1} \mathrm{H} \mathrm{NMR}\left(400 \mathrm{MHz}, \mathrm{CDCl}_{3}\right): \delta=7.38-7.26\left(\mathrm{~m}, 20 \mathrm{H}, \mathrm{H}^{\text {arom }}\right)$, $4.86\left(\mathrm{AB}, 1 \mathrm{H}, J_{\mathrm{A}-\mathrm{B}}=11.2 \mathrm{~Hz}, \mathrm{CH}_{2}{ }^{\mathrm{Bn}}\right), 4.77\left(\mathrm{AB}, 1 \mathrm{H}, J_{\mathrm{A}-\mathrm{B}}=11.2 \mathrm{~Hz}, \mathrm{CH}_{2}{ }^{\mathrm{Bn}}\right), 4.76(\mathrm{AB}$, $\left.1 \mathrm{H}, J_{\mathrm{A}-\mathrm{B}}=11.5 \mathrm{~Hz}, \mathrm{CH}_{2}{ }^{\mathrm{Bn}}\right), 4.75\left(\mathrm{AB}, 1 \mathrm{H}, J_{\mathrm{A}-\mathrm{B}}=11.9 \mathrm{~Hz}, \mathrm{CH}_{2}{ }^{\mathrm{Bn}}\right), 4.64\left(\mathrm{AB}, 1 \mathrm{H}, J_{\mathrm{A}-\mathrm{B}}=\right.$ $\left.11.5 \mathrm{~Hz}, \mathrm{CH}_{2}{ }^{\mathrm{Bn}}\right), 4.59\left(\mathrm{AB}, 1 \mathrm{H}, J_{\mathrm{A}-\mathrm{B}}=11.9 \mathrm{~Hz}, \mathrm{CH}_{2}{ }^{\mathrm{Bn}}\right), 4.46\left(\mathrm{AB}, 1 \mathrm{H}, J_{\mathrm{A}-\mathrm{B}}=11.2 \mathrm{~Hz}\right.$, $\left.\mathrm{CH}_{2}{ }^{\mathrm{Bn}}\right), 4.43\left(\mathrm{AB}, 1 \mathrm{H}, J_{\mathrm{A}-\mathrm{B}}=11.2 \mathrm{~Hz}, \mathrm{CH}_{2}{ }^{\mathrm{Bn}}\right), 4.14\left(\mathrm{dd}, 1 \mathrm{H}, J_{2-3}=6.6 \mathrm{~Hz}, J_{3-4}=4.1 \mathrm{~Hz}\right.$, $\mathrm{H}-3), 4.03\left(\mathrm{dd}, 1 \mathrm{H}, J_{1-2}=4.4 \mathrm{~Hz}, J_{2-3}=6.6 \mathrm{~Hz}, \mathrm{H}-2\right), 3.87\left(\mathrm{~d}, 1 \mathrm{H}, J_{1-2}=4.4 \mathrm{~Hz}, \mathrm{H}-1\right)$, $3.79(\mathrm{~m}, 1 \mathrm{H}, \mathrm{H}-4), 3.68\left(\mathrm{ABX}, 1 \mathrm{H}, J_{4-5 \mathrm{a}}=5.5 \mathrm{~Hz}, J_{5 \mathrm{a}-5 \mathrm{~b}}=10.3 \mathrm{~Hz}, \mathrm{H}-5 \mathrm{a}\right), 3.64$ (ABX, $\left.1 \mathrm{H}, J_{4-5 \mathrm{~b}}=4.1 \mathrm{~Hz}, J_{5 \mathrm{a}-5 \mathrm{~b}}=10.3 \mathrm{~Hz}, \mathrm{H}-5 \mathrm{~b}\right), 2.66\left(\mathrm{q}, 2 \mathrm{H}, J=7.6 \mathrm{~Hz}, \mathrm{SCH}_{2} \mathrm{CH}_{3}\right), 2.55-2.49$ $\left(\mathrm{m}, 2 \mathrm{H}, \mathrm{SCH}_{2} \mathrm{CH}_{3}\right), 1.18\left(\mathrm{t}, 3 \mathrm{H}, J_{\mathrm{CH} 2 \mathrm{CH} 3}=7.6 \mathrm{~Hz}, \mathrm{SCH}_{2} \mathrm{CH}_{3}\right), 1.15\left(\mathrm{t}, 3 \mathrm{H}, J_{\mathrm{CH} 2 \mathrm{CH} 3}=7.6\right.$ $\left.\mathrm{Hz}, \mathrm{SCH}_{2} \mathrm{CH}_{3}\right)$ ppm. ${ }^{13} \mathrm{C}$ NMR $\left(101 \mathrm{MHz}, \mathrm{CDCl}_{3}\right): \delta=138.8\left(\mathrm{C}_{\mathrm{q}}{ }^{\text {arom }}\right), 138.7\left(\mathrm{C}_{\mathrm{q}}^{\text {arom }}\right)$, $138.4\left(\mathrm{C}_{\mathrm{q}}{ }^{\text {arom }}\right), 138.3\left(\mathrm{C}_{\mathrm{q}}{ }^{\text {arom }}\right), 128.5-127.4\left(\mathrm{CH}^{\text {arom }}\right), 82.7(\mathrm{C}-2), 80.2(\mathrm{C}-3), 77.8(\mathrm{C}-4)$, $75.1\left(\mathrm{CH}_{2}{ }^{\mathrm{Bn}}\right), 75.0\left(\mathrm{CH}_{2}{ }^{\mathrm{Bn}}\right), 73.4\left(\mathrm{CH}_{2}{ }^{\mathrm{Bn}}\right), 72.4\left(\mathrm{CH}_{2}{ }^{\mathrm{Bn}}\right), 70.4(\mathrm{C}-5), 53.7(\mathrm{C}-1), 25.3$ $\left(\mathrm{SCH}_{2} \mathrm{CH}_{3}\right), 25.2\left(\mathrm{SCH}_{2} \mathrm{CH}_{3}\right), 14.5\left(2 \times \mathrm{SCH}_{2} \mathrm{CH}_{3}\right)$ ppm. These analytical data correspond to published ones. ${ }^{1}$ 


\section{${ }^{1}$ H NMR}

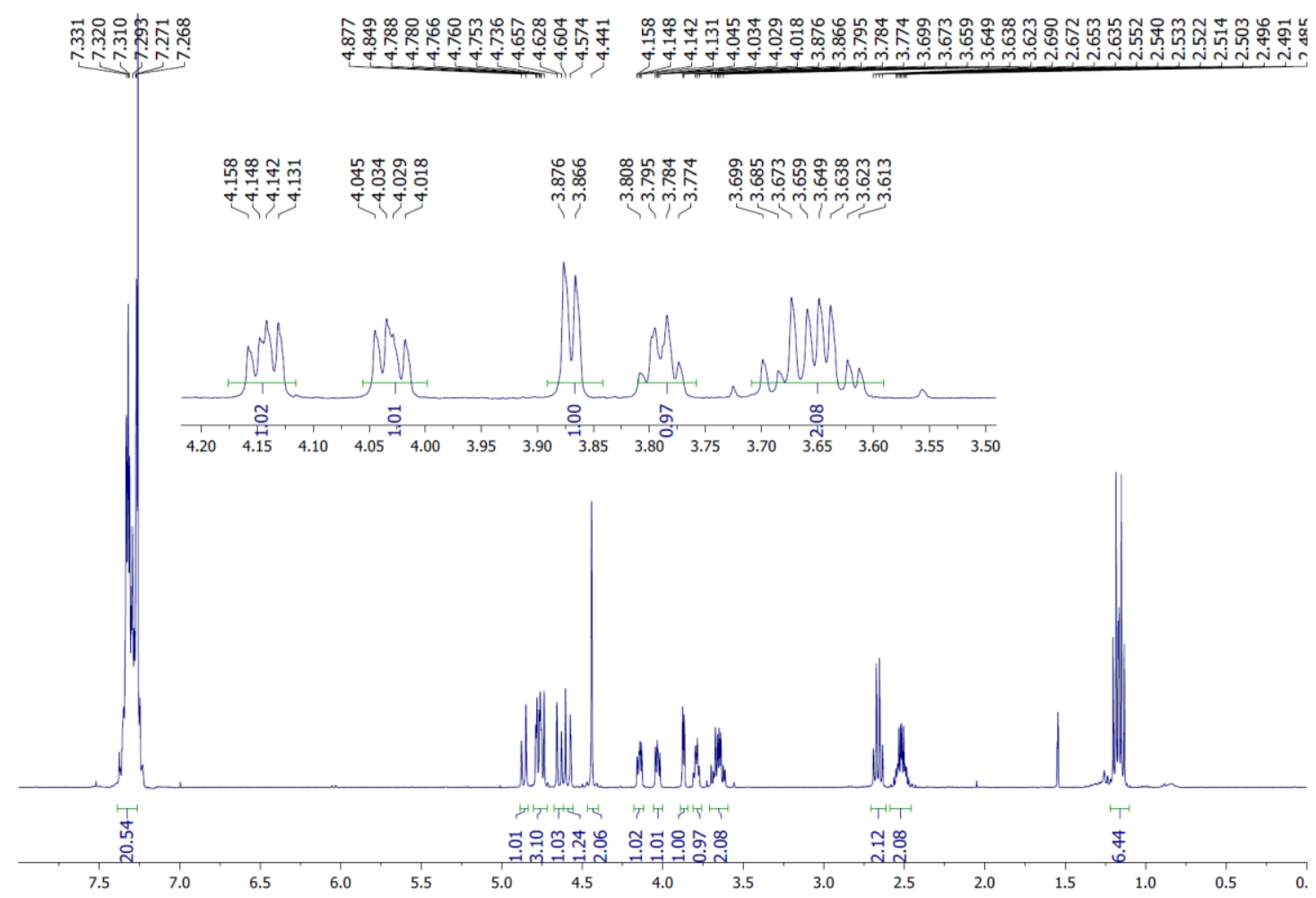

${ }^{13}$ C NMR

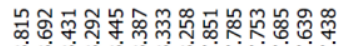

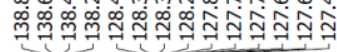

\begin{tabular}{|c|c|c|}
\hline 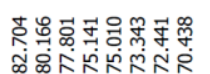 & 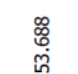 & 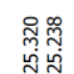 \\
\hline
\end{tabular}

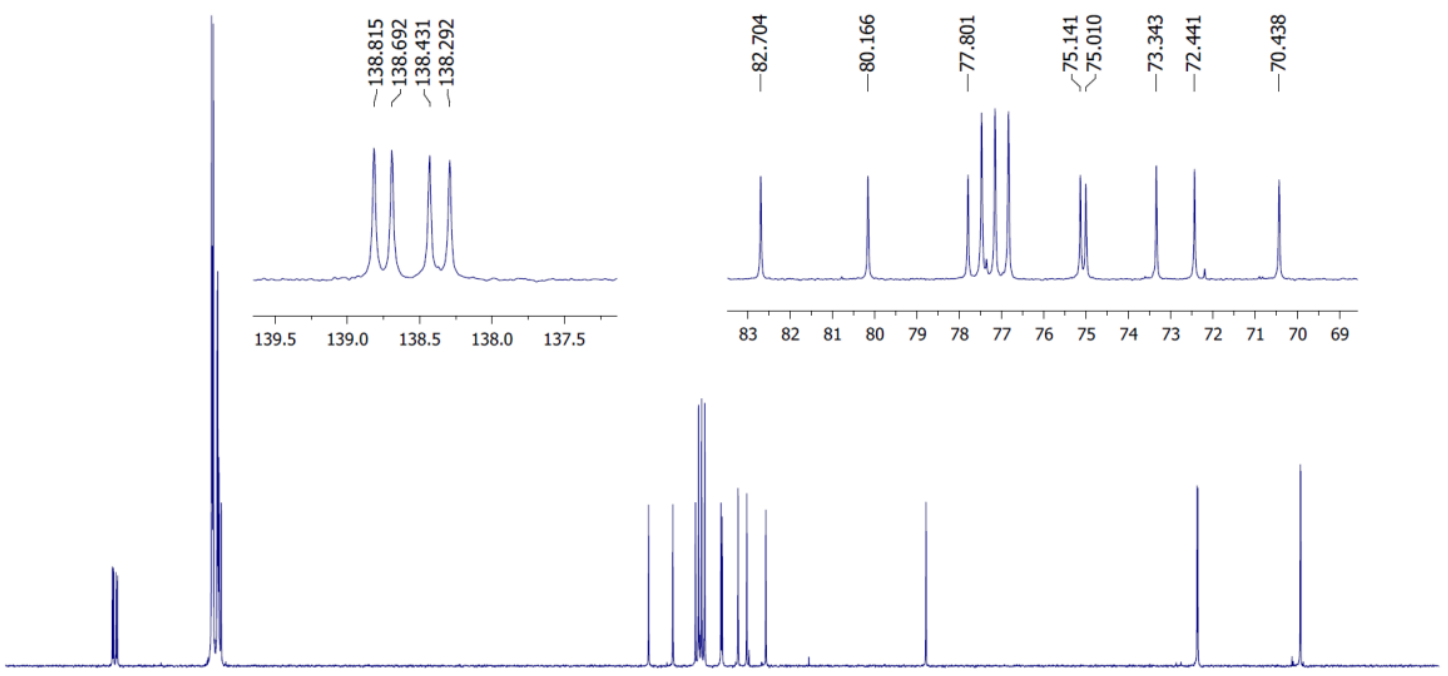

$\begin{array}{llllllllllllllllllllllllllllllllllllll}50 & 145 & 140 & 135 & 130 & 125 & 120 & 115 & 110 & 105 & 100 & 95 & 90 & 85 & 80 & 75 & 70 & 65 & 60 & 55 & 50 & 45 & 40 & 35 & 30 & 25 & 20 & 15 & 10 & 5 & 1\end{array}$ 


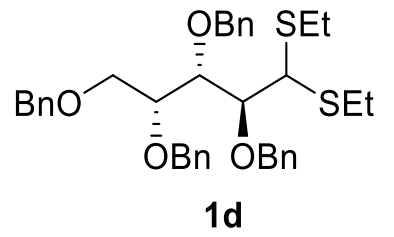

An aqueous solution of concentrated hydrochloric acid (37\%, $23.1 \mathrm{ml}, 279 \mathrm{mmol}, 1.81$ equiv.) was added to D-lyxose $(23.1 \mathrm{~g}, 154 \mathrm{mmol})$ at room temperature. Then, ethanethiol ( $23.1 \mathrm{ml}, 312 \mathrm{mmol}, 2.0$ equiv.) was added dropwise at $0{ }^{\circ} \mathrm{C}$ and the solution was stirred $2 \mathrm{~h}$ at $0{ }^{\circ} \mathrm{C}$ without solid formation. The mixture was neutralized carefully with potassium carbonate to reach $\mathrm{pH} 7$ and stirred for $16 \mathrm{~h}$ at room temperature. The white solid was filtered, washed with $\mathrm{Et}_{2} \mathrm{O}$ (3 times) and the filtrate was stirred with decolorizing carbon for $1 \mathrm{~h}$ at r.t. After filtration on celite ${ }^{\circledR}$, evaporation of solvents, the product was recrystallized in ethanol $(95 \%, 125 \mathrm{ml})$ at $0{ }^{\circ} \mathrm{C}$. Diethyl dithioacetal D-lyxose was obtained as a white powder $(12.8 \mathrm{~g}, 33 \%)$.

To a solution of diethyl dithioacetal D-lyxose $(5.3 \mathrm{~g}, 21 \mathrm{mmol})$ in dry DMF (80ml) under argon atmosphere was added benzyl bromide (10.9 ml, $91 \mathrm{mmol}, 4.4$ equiv.) at r.t. The solution was cooled at $0{ }^{\circ} \mathrm{C}$ and $\mathrm{NaH}$ (60\% in mineral oil, $4.1 \mathrm{~g}, 103 \mathrm{mmol}, 5.0$ equiv.) was added portionwise and the reaction was stirred for $24 \mathrm{~h}$. Ice water was added carefully and the mixture was diluted with $\mathrm{Et}_{2} \mathrm{O}$ and the aqueous phase was extracted with $\mathrm{Et}_{2} \mathrm{O}(2$ times). The combined organic phases were washed with saturated aqueous solution of $\mathrm{NH}_{4} \mathrm{Cl}$ (3 times) and brine (3 times), dried over $\mathrm{MgSO}_{4}$ and concentrated. Purification by chromatography on silica gel (Cy/EtOAc 95:5) gave compound 1c (11.8 g, 92\%).

$[\alpha]^{20} \mathrm{D}:+22.7\left(c 1.0, \mathrm{CHCl}_{3}\right) .{ }^{1} \mathrm{H} \mathrm{NMR}\left(400 \mathrm{MHz}, \mathrm{CDCl}_{3}\right): \delta=7.39-7.26\left(\mathrm{~m}, 20 \mathrm{H}, \mathrm{H}^{\text {arom }}\right)$, $5.03\left(\mathrm{AB}, 1 \mathrm{H}, J_{\mathrm{A}-\mathrm{B}}=11.2 \mathrm{~Hz}, \mathrm{CH}_{2}{ }^{\mathrm{Bn}}\right), 4.74-4.70\left(\mathrm{~m}, 3 \mathrm{H}, \mathrm{CH}_{2}{ }^{\mathrm{Bn}}\right), 4.56\left(\mathrm{AB}, 1 \mathrm{H}, J_{\mathrm{A}-\mathrm{B}}=\right.$ $\left.11.9 \mathrm{~Hz}, \mathrm{CH}_{2}{ }^{\mathrm{Bn}}\right), 4.50-4.44\left(\mathrm{~m}, 3 \mathrm{H}, \mathrm{CH}_{2}{ }^{\mathrm{Bn}}\right), 4.26\left(\mathrm{~d}, 1 \mathrm{H}, J_{1-2}=2.3 \mathrm{~Hz}, \mathrm{H}-1\right), 4.20$ (dd, $\left.1 \mathrm{H}, J_{2-3}=8.0 \mathrm{~Hz}, J_{3-4}=2.0 \mathrm{~Hz}, \mathrm{H}-3\right), 4.06-4.02(\mathrm{~m}, 2 \mathrm{H}, \mathrm{H}-2, \mathrm{H}-4), 3.71$ (ABX, 1H, $J_{4-5 \mathrm{a}}$ $\left.=5.7 \mathrm{~Hz}, J_{5 \mathrm{a}-5 \mathrm{~b}}=9.9 \mathrm{~Hz}, \mathrm{H}-5 \mathrm{a}\right), 3.67\left(\mathrm{ABX}, 1 \mathrm{H}, J_{4-5 \mathrm{~b}}=5.7 \mathrm{~Hz}, J_{5 \mathrm{a}-5 \mathrm{~b}}=9.9 \mathrm{~Hz}, \mathrm{H}-5 \mathrm{~b}\right)$, 2.77-2.60 (m, 4H, 2x SCH $\left.\mathrm{CH}_{3}\right), 1.24\left(\mathrm{t}, 3 \mathrm{H}, J_{\mathrm{CH} 2 \mathrm{CH} 3}=7.3 \mathrm{~Hz}, \mathrm{SCH}_{2} \mathrm{CH}_{3}\right), 1.22(\mathrm{t}, 3 \mathrm{H}$, $\left.J_{\mathrm{CH} 2 \mathrm{CH} 3}=7.3 \mathrm{~Hz}, \mathrm{SCH}_{2} \mathrm{CH}_{3}\right) \mathrm{ppm} .{ }^{13} \mathrm{C} \mathrm{NMR}\left(101 \mathrm{MHz}, \mathrm{CDCl}_{3}\right): \delta=139.0\left(\mathrm{C}_{\mathrm{q}}{ }^{\text {arom }}\right), 138.6$ $\left(\mathrm{C}_{\mathrm{q}}{ }^{\text {arom }}\right), 138.5\left(\mathrm{C}_{\mathrm{q}}{ }^{\text {arom }}\right), 138.2\left(\mathrm{C}_{\mathrm{q}}{ }^{\text {arom }}\right)$, 128.5-127.5 $\left(\mathrm{CH}^{\text {arom }}\right), 82.6(\mathrm{C}-3), 79.5(\mathrm{C}-2), 77.7$ (C-4), 74.6 (2 x C $\left.\mathrm{CH}_{2}{ }^{\mathrm{Bn}}\right), 73.4\left(\mathrm{CH}_{2}{ }^{\mathrm{Bn}}\right), 72.9\left(\mathrm{CH}_{2}{ }^{\mathrm{Bn}}\right), 70.6(\mathrm{C}-5), 53.9(\mathrm{C}-1), 26.9$ $\left(\mathrm{SCH}_{2} \mathrm{CH}_{3}\right), 25.1\left(\mathrm{SCH}_{2} \mathrm{CH}_{3}\right), 14.7\left(\mathrm{SCH}_{2} \mathrm{CH}_{3}\right), 14.5\left(\mathrm{SCH}_{2} \mathrm{CH}_{3}\right)$ ppm. These analytical data correspond to published ones. ${ }^{1}$ 


\section{${ }^{1} \mathbf{H}$ NMR}

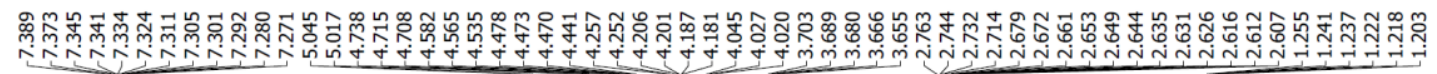

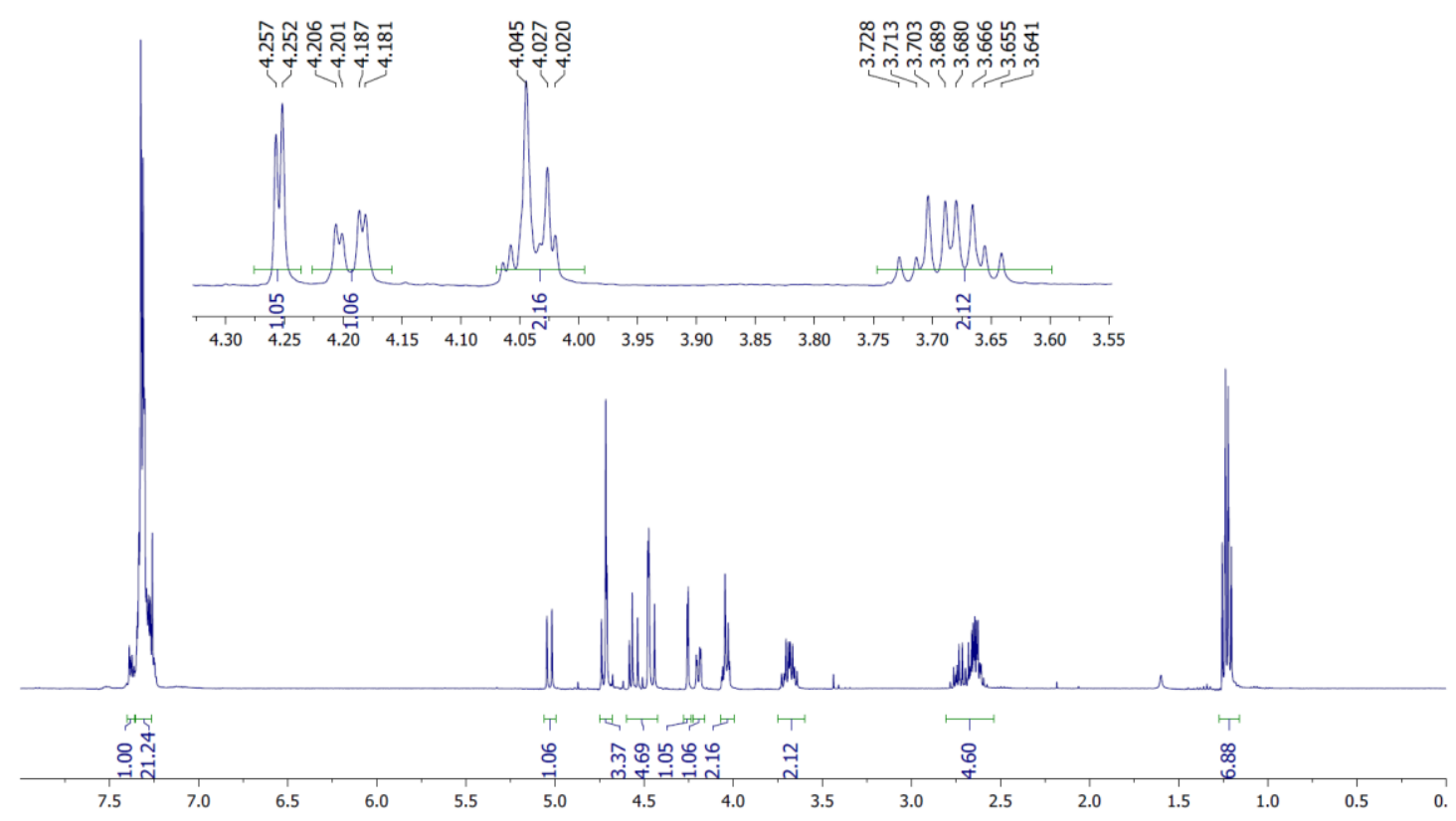

\section{${ }^{13}$ C NMR}

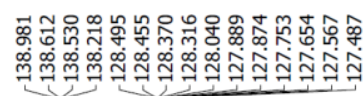

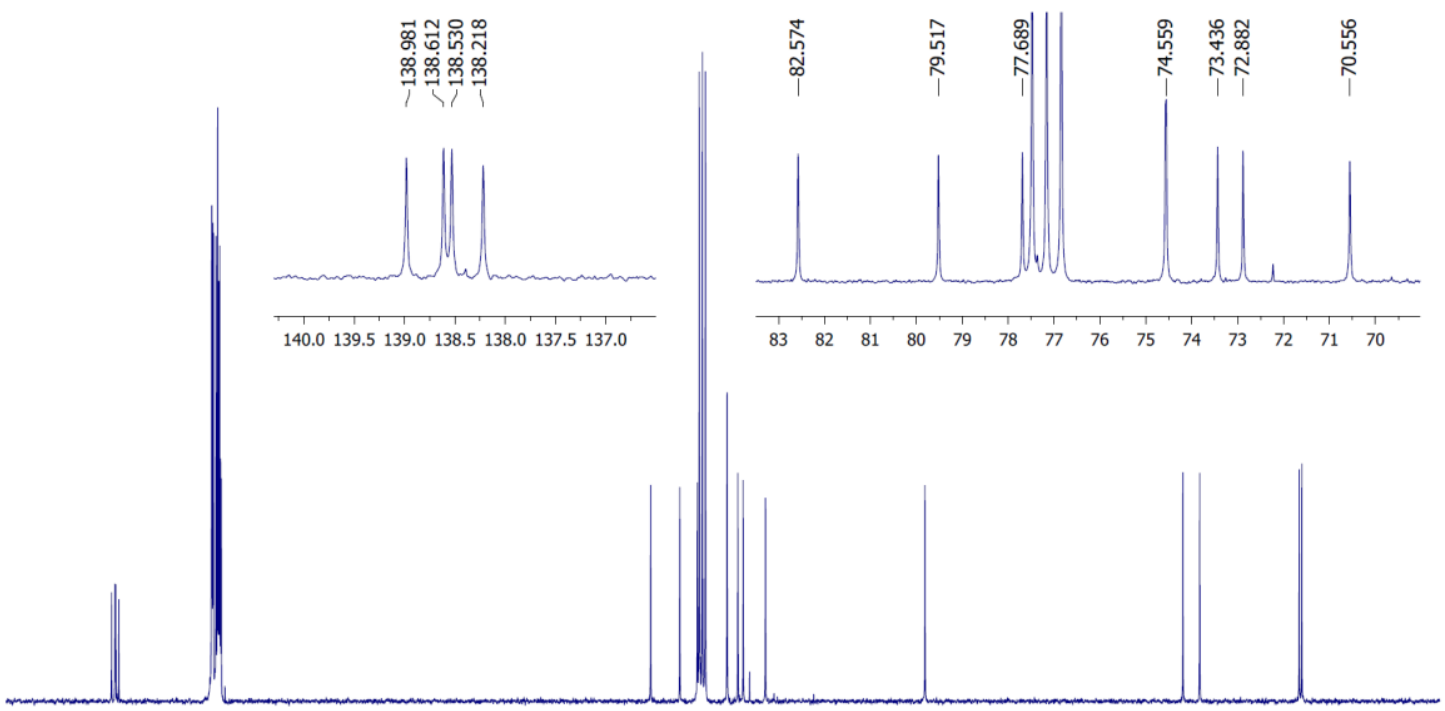

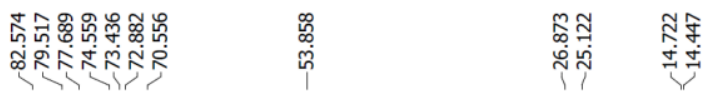

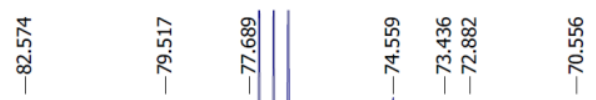

$\begin{array}{llllllllllllllllllllllllllllllllll}145 & 140 & 135 & 130 & 125 & 120 & 115 & 110 & 105 & 100 & 95 & 90 & 85 & 80 & 75 & 70 & 65 & 60 & 55 & 50 & 45 & 40 & 35 & 30 & 25 & 20 & 15 & 10 & 5 & 1\end{array}$ 
<smiles>CCC(Br)C(Br)C(Br)C(Br)C(Br)C(Br)C(C)OC</smiles>

L-Fucose (15.9 g, $97 \mathrm{mmol})$ was dissolved in an aqueous solution of concentrated hydrochloric acid (37\%, $16 \mathrm{ml}, 193 \mathrm{mmol}, 2.0$ equiv.) at r.t. under argon atmosphere. Then, ethanethiol (16 ml, $216 \mathrm{mmol}, 2.2$ equiv.) was added dropwise at r.t. and the product was crystallized after 10 min of vigorous stirring. Then, the solid was filtered, washed with ice water and cooled diethyl ether ( 2 times) and dried under reduced pressure to give diethyl dithioacetal L-fucose $(17.3 \mathrm{~g}, 64 \mathrm{mmol}, 66 \%)$ as a white powder.

To a solution of diethyl dithioacetal L-fucose $(16.0 \mathrm{~g}, 59 \mathrm{mmol})$ in dry DMF $(210 \mathrm{ml})$ under argon atmosphere was added benzyl bromide $(31.1 \mathrm{ml}, 260 \mathrm{mmol}, 4.4$ equiv.) at room temperature. The solution was cooled at $0{ }^{\circ} \mathrm{C}$ and $\mathrm{NaH}(60 \%$ in mineral oil, $11.8 \mathrm{~g}$, $296 \mathrm{mmol}, 5.0$ equiv.) was added portionwise and the reaction was stirred for $24 \mathrm{~h}$. Ice water was added carefully and the mixture was diluted with $\mathrm{Et}_{2} \mathrm{O}$ and the aqueous phase was extracted with $\mathrm{Et}_{2} \mathrm{O}$ (2 times). The combined organic phases were washed with saturated aqueous solution of $\mathrm{NH}_{4} \mathrm{Cl}$ (3 times) and brine (3 times), dried over $\mathrm{MgSO}_{4}$ and concentrated. Purification by chromatography on silica gel (Cy/EtOAc 95:5) gave molecule 1e (36.0 g, 97\%).

$[\alpha]^{20} \mathrm{D}:+7.6\left(c\right.$ 1.0, $\left.\mathrm{CHCl}_{3}\right) .{ }^{1} \mathrm{H} \mathrm{NMR}\left(400 \mathrm{MHz}, \mathrm{CDCl}_{3}\right) \delta=7.38-7.23\left(\mathrm{~m}, 20 \mathrm{H}, \mathrm{H}^{\text {arom }}\right)$, $4.83\left(\mathrm{AB}, 2 \mathrm{H}, J_{\mathrm{A}-\mathrm{B}}=11.5 \mathrm{~Hz}, \mathrm{CH}_{2}{ }^{\mathrm{Bn}}\right), 4.77-4.71\left(\mathrm{~m}, 3 \mathrm{H}, \mathrm{CH}_{2}{ }^{\mathrm{Bn}}\right), 4.66\left(\mathrm{AB}, 1 \mathrm{H}, J_{\mathrm{A}-\mathrm{B}}=\right.$ $\left.11.9 \mathrm{~Hz}, \mathrm{CH}_{2}{ }^{\mathrm{Bn}}\right), 4.65\left(\mathrm{AB}, 1 \mathrm{H}, J_{\mathrm{A}-\mathrm{B}}=11.9 \mathrm{~Hz}, \mathrm{CH}_{2}{ }^{\mathrm{Bn}}\right), 4.49\left(\mathrm{AB}, 1 \mathrm{H}, J_{\mathrm{A}-\mathrm{B}}=11.9 \mathrm{~Hz}\right.$, $\left.\mathrm{CH}_{2}{ }^{\mathrm{Bn}}\right), 4.29\left(\mathrm{t}, 1 \mathrm{H}, J_{2-3}=J_{3-4}=5.3 \mathrm{~Hz}, \mathrm{H}-3\right), 4.06(\mathrm{~m}, 2 \mathrm{H}, \mathrm{H}-1, \mathrm{H}-2), 3.84(\mathrm{~m}, 1 \mathrm{H}, \mathrm{H}-$ 5), $3.67\left(\mathrm{dd}, 1 \mathrm{H}, J_{3-4}=5.3 \mathrm{~Hz}, J_{4-5}=5.0 \mathrm{~Hz}, \mathrm{H}-4\right), 2.65\left(\mathrm{qd}, 2 \mathrm{H}, J_{\mathrm{CH} 2 \mathrm{CH} 3}=2.3 \mathrm{~Hz}, J_{\mathrm{CH} 2 \mathrm{CH} 3}\right.$ $\left.=7.3 \mathrm{~Hz}, \mathrm{SCH}_{2} \mathrm{CH}_{3}\right), 2.49\left(\mathrm{q}, 2 \mathrm{H}, J_{\mathrm{CH} 2 \mathrm{CH}}=7.3 \mathrm{~Hz}, \mathrm{SCH}_{2} \mathrm{CH}_{3}\right), 1.33\left(\mathrm{~d}, 3 \mathrm{H}, J_{5-6}=6.4\right.$ $\left.\mathrm{Hz}, \mathrm{CH}_{3}\right), 1.18\left(\mathrm{t}, 3 \mathrm{H}, J_{\mathrm{CH} 2 \mathrm{CH} 3}=7.3 \mathrm{~Hz}, \mathrm{SCH}_{2} \mathrm{CH}_{3}\right), 1.11\left(\mathrm{t}, 3 \mathrm{H}, J_{\mathrm{CH} 2 \mathrm{CH} 3}=7.3 \mathrm{~Hz}\right.$, $\left.\mathrm{SCH}_{2} \mathrm{CH}_{3}\right) \mathrm{ppm} .{ }^{13} \mathrm{C} \mathrm{NMR}\left(101 \mathrm{MHz}, \mathrm{CDCl}_{3}\right) \delta=139.1\left(2 \mathrm{C}_{\mathrm{q}}{ }^{\text {arom }}\right), 138.9\left(\mathrm{C}_{\mathrm{q}}{ }^{\text {arom }}\right), 138.7$ $\left(\mathrm{C}_{\mathrm{q}}{ }^{\text {arom }}\right), 128.5-127.4\left(\mathrm{CH}^{\text {arom }}\right), 83.5(\mathrm{C}-4), 83.1(\mathrm{C}-2), 80.8(\mathrm{C}-3), 75.4(\mathrm{C}-5), 75.0$ $\left(\mathrm{CH}_{2}{ }^{\mathrm{Bn}}\right), 74.4\left(\mathrm{CH}_{2}{ }^{\mathrm{Bn}}\right), 74.0\left(\mathrm{CH}_{2}{ }^{\mathrm{Bn}}\right), 71.2\left(\mathrm{CH}_{2}{ }^{\mathrm{Bn}}\right), 54.3(\mathrm{C}-1), 25.3\left(\mathrm{SCH}_{2} \mathrm{CH}_{3}\right), 25.2$ $\left(\mathrm{SCH}_{2} \mathrm{CH}_{3}\right), 16.8(\mathrm{C}-6), 14.6\left(\mathrm{SCH}_{2} \mathrm{CH}_{3}\right), 14.5\left(\mathrm{SCH}_{2} \mathrm{CH}_{3}\right) \mathrm{ppm}$. These analytical data correspond to published ones. ${ }^{1}$ 


\section{${ }^{1} \mathbf{H}$ NMR}

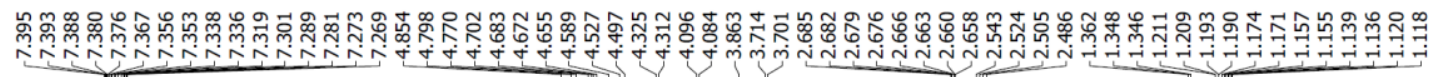

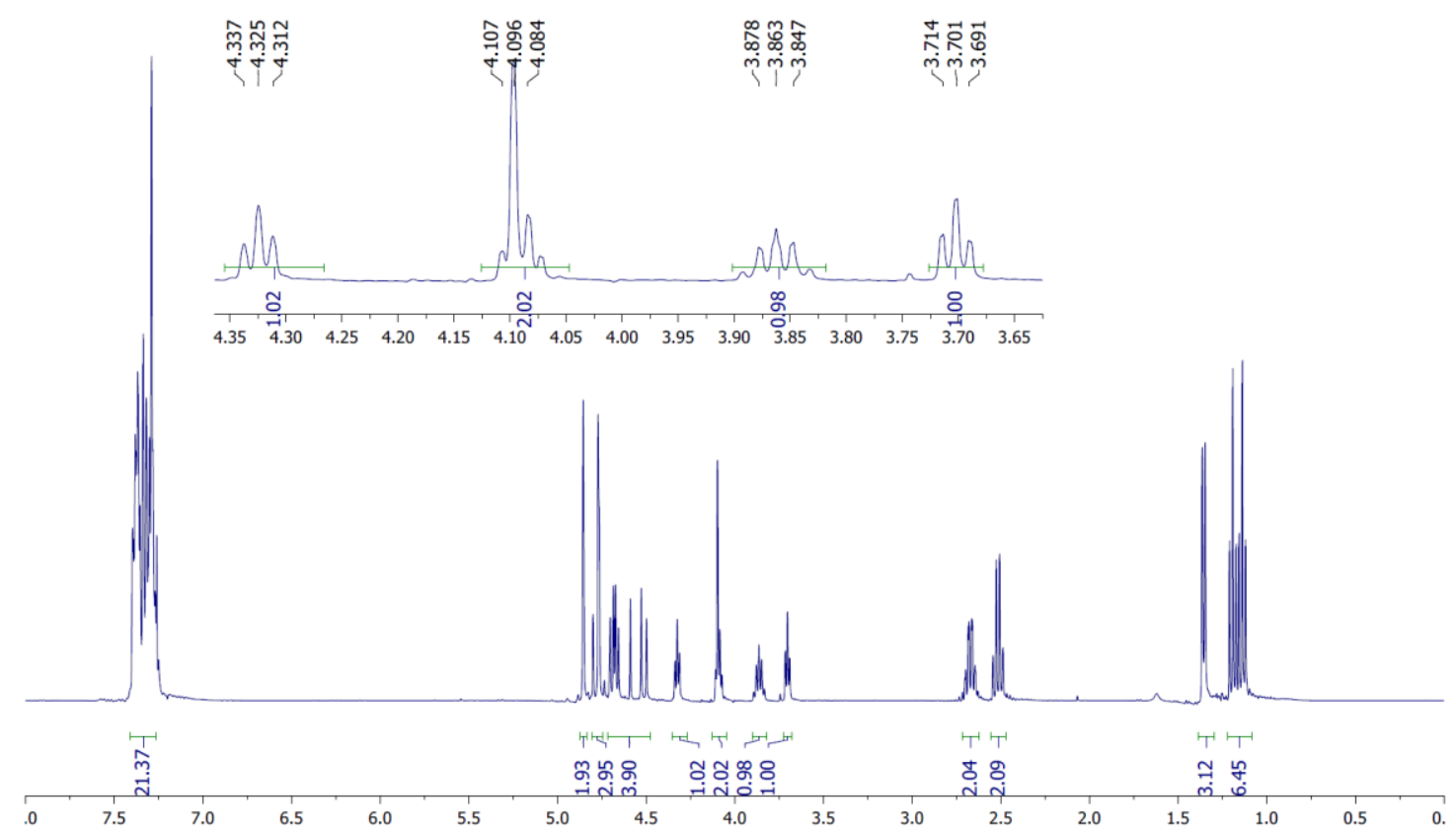

\section{${ }^{13}$ C NMR}

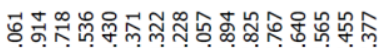



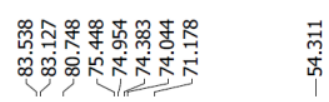

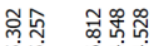

บั่

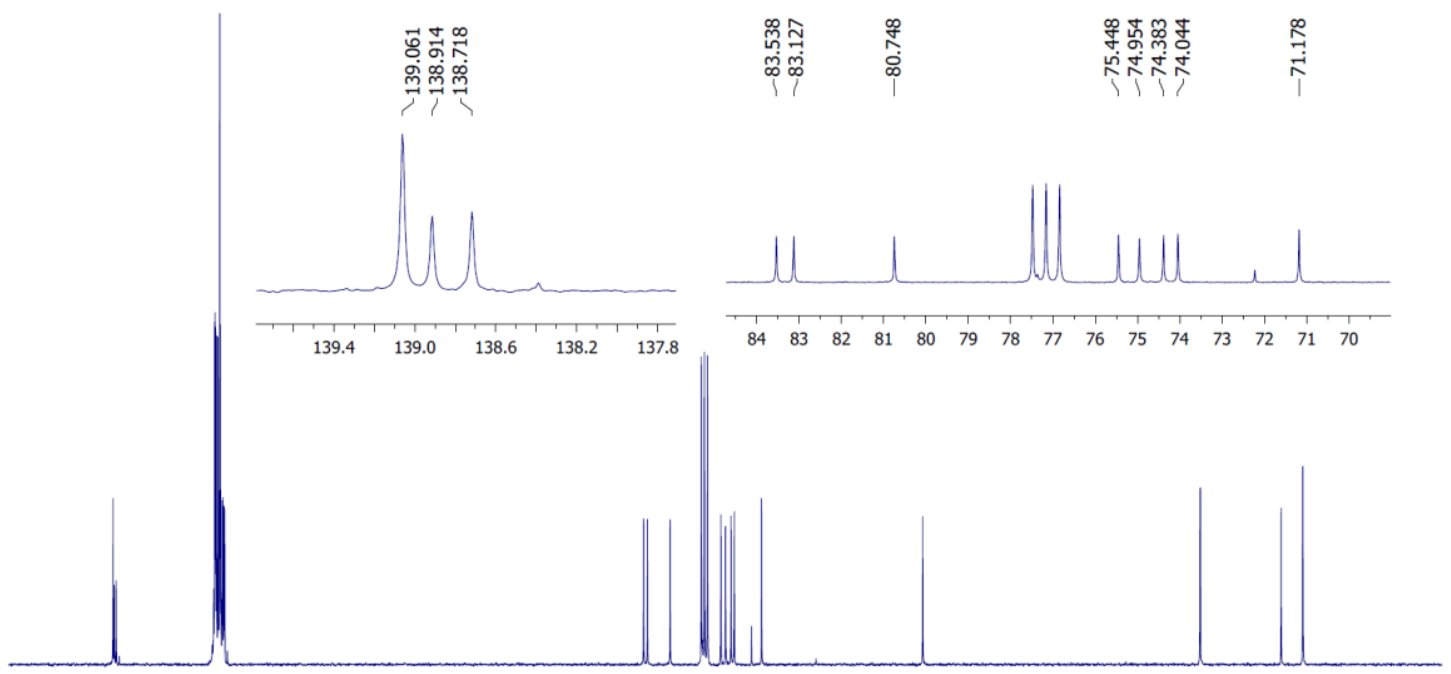

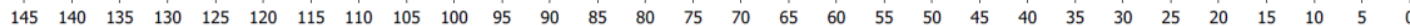




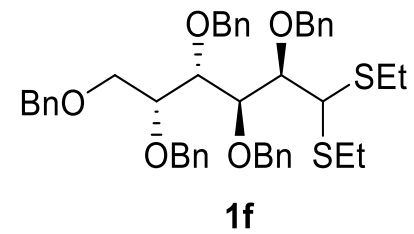

D-galactose $(50.0 \mathrm{~g}, 278 \mathrm{mmol})$ was dissolved in an aqueous solution of concentrated hydrochloric acid (37\%, $75 \mathrm{ml}, 905 \mathrm{mmol}, 3.25$ equiv.) at r.t. under argon atmosphere. Then, ethanethiol (50 ml, $675 \mathrm{mmol}, 2.4$ equiv.) was added and the mixture was stirred vigorously at r.t. while the pressure was released occasionally. After a few minutes, an increase of the temperature was observed and a small amount of ice were added, which caused almost instantaneously solidification of the reaction mixture. The solid was filtered and washed with ice water $(50 \mathrm{ml})$ and small amount of acetone to afford diethyl dithioacetal D-galactose as a white solid $(69.9 \mathrm{~g}, 88 \%)$.

To a solution of diethyl dithioacetal D-galactose $(15.5 \mathrm{~g}, 54 \mathrm{mmol})$ in dry DMF $(150 \mathrm{ml})$ under argon atmosphere was added benzyl bromide $(38.8 \mathrm{ml}, 325 \mathrm{mmol}, 6.0$ equiv.) at r.t. The solution was cooled at $0{ }^{\circ} \mathrm{C}$ and $\mathrm{NaH}$ (60\% in mineral oil, $11.9 \mathrm{~g}, 298 \mathrm{mmol}, 5.5$ equiv.) was added portionwise and the reaction was stirred for $24 \mathrm{~h}$. Ice water was added carefully and the mixture was diluted with $\mathrm{Et}_{2} \mathrm{O}$ and the aqueous phase was extracted with $\mathrm{Et}_{2} \mathrm{O}$ (2 times). The combined organic phases were washed with saturated aqueous solution of $\mathrm{NH}_{4} \mathrm{Cl}$ (3 times) and brine (3 times), dried over $\mathrm{MgSO}_{4}$ and concentrated. Purification by chromatography on silica gel (Cy/EtOAc 95:5) gave compound 1 (f 34.5 $\mathrm{g}, 86 \%)$.

$[\alpha]^{20} \mathrm{D}:+12.0\left(c 1.3, \mathrm{CHCl}_{3}\right) .{ }^{1} \mathrm{H} \mathrm{NMR}\left(500 \mathrm{MHz}, \mathrm{CDCl}_{3}\right): \delta=7.35-7.22\left(\mathrm{~m}, 25 \mathrm{H}, \mathrm{H}^{\mathrm{arom}}\right)$, $4.79\left(\mathrm{~s}, 2 \mathrm{H}, \mathrm{CH}_{2}{ }^{\mathrm{Bn}}\right), 4.76\left(\mathrm{AB}, 1 \mathrm{H}, J_{\mathrm{A}-\mathrm{B}}=12.0 \mathrm{~Hz}, \mathrm{CH}_{2}{ }^{\mathrm{Bn}}\right), 4.71\left(\mathrm{AB}, 2 \mathrm{H}, J_{\mathrm{A}-\mathrm{B}}=11.5 \mathrm{~Hz}\right.$, $\left.J_{\mathrm{A}-\mathrm{B}}=10.9 \mathrm{~Hz}, \mathrm{CH}_{2}{ }^{\mathrm{Bn}}\right), 4.70\left(\mathrm{AB}, 1 \mathrm{H}, J_{\mathrm{A}-\mathrm{B}}=10.9 \mathrm{~Hz}, \mathrm{CH}_{2}{ }^{\mathrm{Bn}}\right), 4.66\left(\mathrm{AB}, 1 \mathrm{H}, J_{\mathrm{A}-\mathrm{B}}=12.0\right.$ $\left.\mathrm{Hz}, \mathrm{CH}_{2}{ }^{\mathrm{Bn}}\right), 4.62\left(\mathrm{AB}, 1 \mathrm{H}, J_{\mathrm{A}-\mathrm{B}}=12.0 \mathrm{~Hz}, \mathrm{CH}_{2}{ }^{\mathrm{Bn}}\right), 4.49\left(\mathrm{AB}, 1 \mathrm{H}, J_{\mathrm{A}-\mathrm{B}}=11.5 \mathrm{~Hz}, \mathrm{CH}_{2}{ }^{\mathrm{Bn}}\right)$, $4.45\left(\mathrm{AB}, 1 \mathrm{H}, J_{\mathrm{A}-\mathrm{B}}=12.0 \mathrm{~Hz}, \mathrm{CH}_{2}{ }^{\mathrm{Bn}}\right), 4.31\left(\mathrm{dd}, 1 \mathrm{H}, J_{2-3}=6.3 \mathrm{~Hz}, J_{3-4}=4.6 \mathrm{~Hz}, \mathrm{H}-3\right)$, 4.05-4.00 (m, 2H, H-1, H-2), 3.93 (dd, 1H, J4-5 = 4.6 Hz, J5-6a,6b = 9.7 Hz, H-5), 3.86 (t, $\left.1 \mathrm{H}, J_{3-4}=J_{4-5}=4.6 \mathrm{~Hz}, \mathrm{H}-4\right), 3.78(\mathrm{~m}, 2 \mathrm{H}, \mathrm{H}-6 \mathrm{a}, \mathrm{H}-6 \mathrm{~b}), 2.67\left(\mathrm{q}, 2 \mathrm{H}, J_{\mathrm{CH} 2 \mathrm{CH} 3}=7.5 \mathrm{~Hz}\right.$, $\left.\mathrm{SCH}_{2} \mathrm{CH}_{3}\right), 2.44\left(\mathrm{dq}, 2 \mathrm{H}, J_{\mathrm{CH} 2 \mathrm{CH} 3}=2.3 \mathrm{~Hz}, J_{\mathrm{CH} 2 \mathrm{CH} 3}=7.5 \mathrm{~Hz}, \mathrm{SCH}_{2} \mathrm{CH}_{3}\right), 1.17(\mathrm{t}, 3 \mathrm{H}$, $\left.J_{\mathrm{CH} 2 \mathrm{CH} 3}=7.5 \mathrm{~Hz}, \mathrm{SCH}_{2} \mathrm{CH}_{3}\right), 1.09\left(\mathrm{t}, 3 \mathrm{H}, J_{\mathrm{CH} 2 \mathrm{CH} 3}=7.5 \mathrm{~Hz}, \mathrm{SCH}_{2} \mathrm{CH}_{3}\right) \mathrm{ppm} .{ }^{13} \mathrm{C} \mathrm{NMR}$ $\left(125 \mathrm{MHz}, \mathrm{CDCl}_{3}\right): \delta=139.1\left(\mathrm{C}_{\mathrm{q}}^{\text {arom }}\right), 139.0\left(\mathrm{C}_{\mathrm{q}}{ }^{\text {arom }}\right), 138.8\left(\mathrm{C}_{\mathrm{q}}{ }^{\text {arom }}\right), 138.6\left(\mathrm{C}_{\mathrm{q}}{ }^{\text {arom }}\right), 138.3$ $\left(\mathrm{C}_{\mathrm{q}}{ }^{\text {arom }}\right), 128.5-127.2\left(\mathrm{CH}^{\text {arom }}\right), 83.5(\mathrm{C}-2), 81.6(\mathrm{C}-3), 79.4(\mathrm{C}-4), 78.9(\mathrm{C}-5), 75.3$ $\left(\mathrm{CH}_{2}{ }^{\mathrm{Bn}}\right), 74.9\left(\mathrm{CH}_{2}{ }^{\mathrm{Bn}}\right), 73.4\left(\mathrm{CH}_{2}{ }^{\mathrm{Bn}}\right), 73.3\left(\mathrm{CH}_{2}{ }^{\mathrm{Bn}}\right), 73.1\left(\mathrm{CH}_{2}{ }^{\mathrm{Bn}}\right), 71.0(\mathrm{C}-6), 54.6(\mathrm{C}-1)$, $25.3\left(\mathrm{SCH}_{2} \mathrm{CH}_{3}\right), 25.0\left(\mathrm{SCH}_{2} \mathrm{CH}_{3}\right), 14.6\left(\mathrm{SCH}_{2} \mathrm{CH}_{3}\right), 14.5\left(\mathrm{SCH}_{2} \mathrm{CH}_{3}\right)$ ppm. These analytical data correspond to published ones. ${ }^{1}$ 


\section{${ }^{1} \mathbf{H}$ NMR}

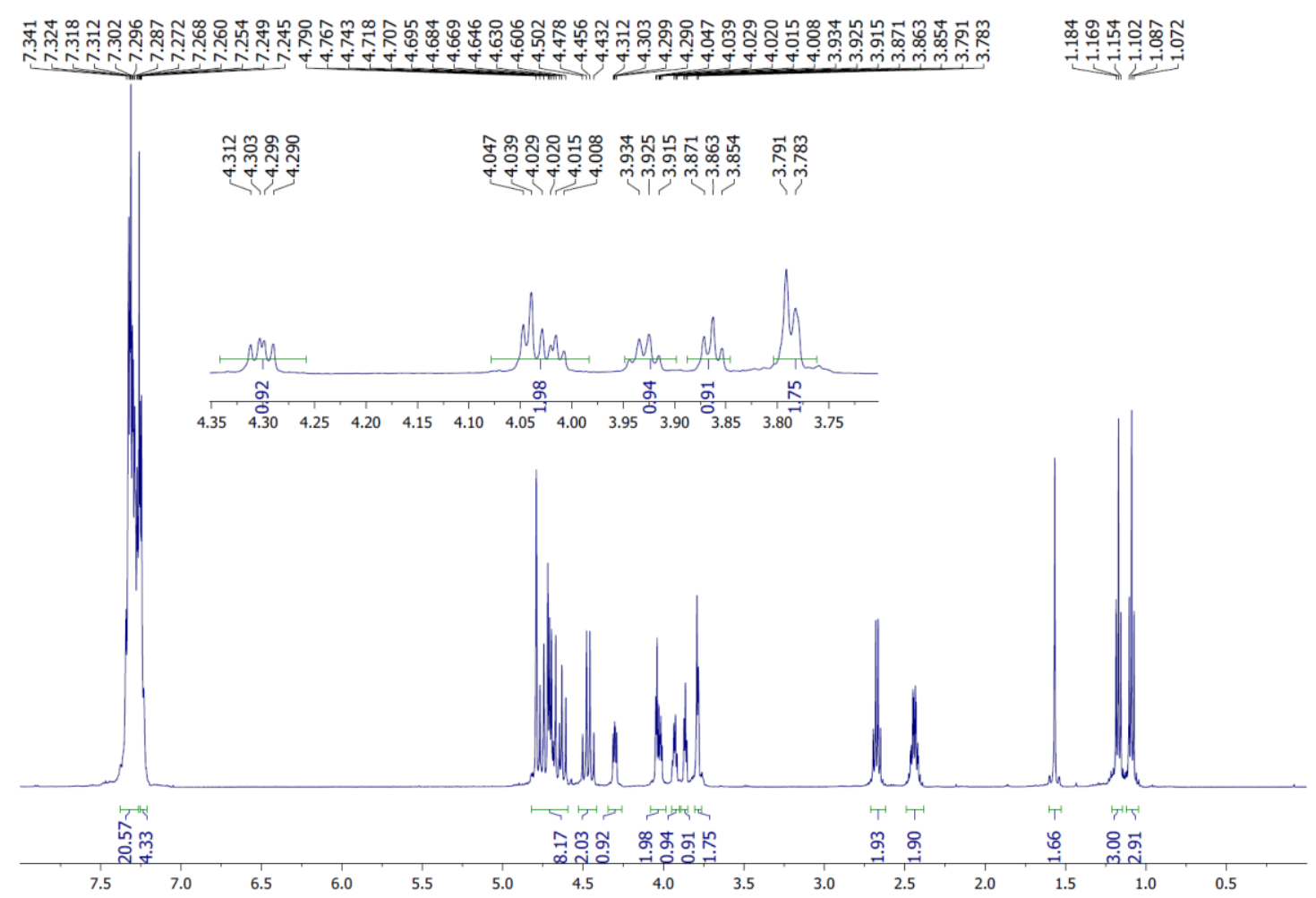

\section{${ }^{13}$ C NMR}

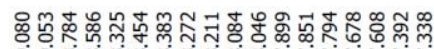

D.

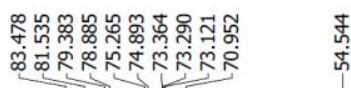

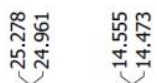
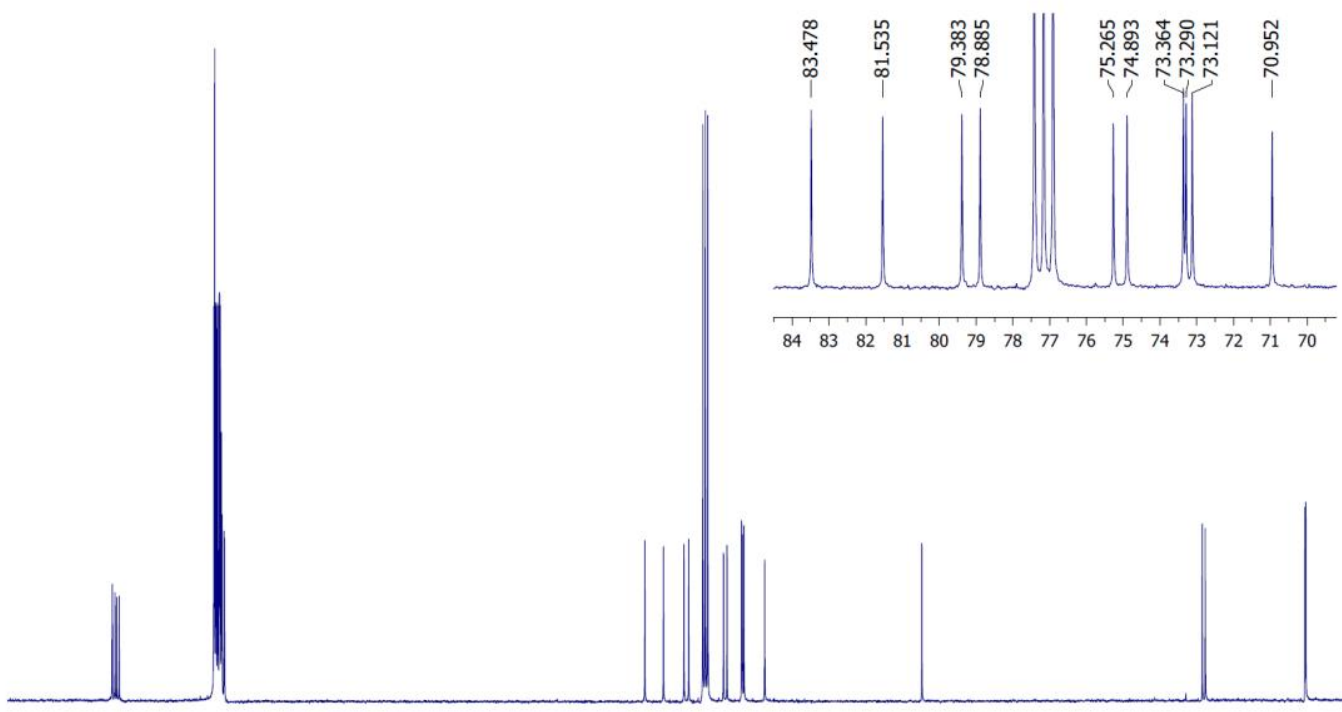

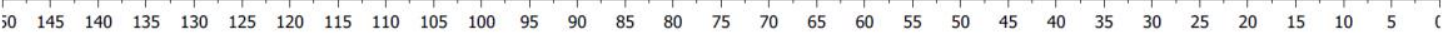




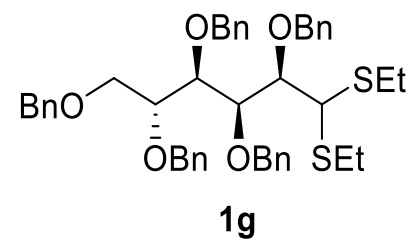

D-glucose $(60.0 \mathrm{~g}, 333 \mathrm{mmol})$ was dissolved in an aqueous solution of concentrated hydrochloric acid (37\%, $51 \mathrm{ml}, 615 \mathrm{mmol}, 1.85$ equiv.) at r.t. under argon atmosphere. Then, ethanethiol (60 ml, $810 \mathrm{mmol}, 2.4$ equiv.) was added and the mixture was stirred vigorously at r.t. for $15 \mathrm{~min}$. Temperature was maintained below $25^{\circ} \mathrm{C}$ by adding small amount of ice and the stirring was continued until crystallization occurs. Then, the mixture was cooled for $30 \mathrm{~min}$ in an ice-salt bath, the white solid was filtered, subsequently washed with cold water and diethyl ether, dried under reduced pressure to afford diethyl dithioacetal D-glucose (83.8 g, $0.293 \mathrm{~mol}, 88 \%)$.

To a solution of diethyl dithioacetal D-glucose $(21.3 \mathrm{~g}, 74 \mathrm{mmol})$ in dry DMF (250 ml) under argon atmosphere was added benzyl bromide $(48.9 \mathrm{ml}, 409 \mathrm{mmol}, 5.5$ equiv.) at room temperature. The solution was cooled at $0{ }^{\circ} \mathrm{C}$ and $\mathrm{NaH}(60 \%$ in mineral oil, $16.4 \mathrm{~g}$, $409 \mathrm{mmol}, 5.5$ equiv.) was added portionwise and the reaction was stirred for $24 \mathrm{~h}$. Ice water was added carefully and the mixture was diluted with $\mathrm{Et}_{2} \mathrm{O}$ and the aqueous phase was extracted with $\mathrm{Et}_{2} \mathrm{O}$ (2 times). The combined organic phases were washed with saturated aqueous solution of $\mathrm{NH}_{4} \mathrm{Cl}$ ( 3 times) and brine (3 times), dried over $\mathrm{MgSO}_{4}$ and concentrated. Purification by chromatography on silica gel (Cy/EtOAc 95:5) gave molecule $1 \mathrm{~g}(42.8 \mathrm{~g}, 78 \%)$.

$[\alpha]^{20}{ }_{\mathrm{D}}:-2.0\left(c \mathrm{1} .0, \mathrm{CHCl}_{3}\right) .{ }^{1} \mathrm{H} \mathrm{NMR}\left(400 \mathrm{MHz}, \mathrm{CDCl}_{3}\right): \delta=7.36-7.24\left(\mathrm{~m}, 25 \mathrm{H}, \mathrm{H}^{\text {arom }}\right)$, $4.85\left(\mathrm{AB}, 1 \mathrm{H}, J_{\mathrm{A}-\mathrm{B}}=11.2 \mathrm{~Hz}, \mathrm{CH}_{2}{ }^{\mathrm{Bn}}\right), 4.81\left(\mathrm{AB}, 1 \mathrm{H}, J_{\mathrm{A}-\mathrm{B}}=11.5 \mathrm{~Hz}, \mathrm{CH}_{2}{ }^{\mathrm{Bn}}\right), 4.79(\mathrm{AB}$, $\left.1 \mathrm{H}, J_{\mathrm{A}-\mathrm{B}}=11.2 \mathrm{~Hz}, \mathrm{CH}_{2}{ }^{\mathrm{Bn}}\right), 4.77\left(\mathrm{AB}, 1 \mathrm{H}, J_{\mathrm{A}-\mathrm{B}}=11.5 \mathrm{~Hz}, \mathrm{CH}_{2}{ }^{\mathrm{Bn}}\right), 4.69\left(\mathrm{AB}, 1 \mathrm{H}, J_{\mathrm{A}-\mathrm{B}}=\right.$ $\left.11.2 \mathrm{~Hz}, \mathrm{CH}_{2}{ }^{\mathrm{Bn}}\right), 4.68\left(\mathrm{AB}, 1 \mathrm{H}, J_{\mathrm{A}-\mathrm{B}}=12.1 \mathrm{~Hz}, \mathrm{CH}_{2}{ }^{\mathrm{Bn}}\right), 4.65\left(\mathrm{AB}, 1 \mathrm{H}, J_{\mathrm{A}-\mathrm{B}}=12.1 \mathrm{~Hz}\right.$, $\left.\mathrm{CH}_{2}{ }^{\mathrm{Bn}}\right), 4.53\left(\mathrm{AB}, 1 \mathrm{H}, J_{\mathrm{A}-\mathrm{B}}=12.4 \mathrm{~Hz}, \mathrm{CH}_{2}{ }^{\mathrm{Bn}}\right), 4.50\left(\mathrm{AB}, 1 \mathrm{H}, J_{\mathrm{A}-\mathrm{B}}=12.4 \mathrm{~Hz}, \mathrm{CH}_{2}{ }^{\mathrm{Bn}}\right), 4.46$ $\left(\mathrm{AB}, 1 \mathrm{H}, J_{\mathrm{A}-\mathrm{B}}=11.2 \mathrm{~Hz}, \mathrm{CH}_{2}{ }^{\mathrm{Bn}}\right), 4.22\left(\mathrm{dd}, 1 \mathrm{H}, J_{2-3}=4.1 \mathrm{~Hz}, J_{3-4}=6.4 \mathrm{~Hz}, \mathrm{H}-3\right), 4.03$ $\left(\mathrm{dd}, 1 \mathrm{H}, J_{3-4}=6.4 \mathrm{~Hz}, J_{4-5}=4.6 \mathrm{~Hz}, \mathrm{H}-4\right), 3.97-3.90$ (m, 4H, H-1, H-2, H-5, H-6a), 3.78$3.73(\mathrm{~m}, 1 \mathrm{H}, \mathrm{H}-6 \mathrm{~b}), 2.69\left(\mathrm{dq}, 2 \mathrm{H}, J_{\mathrm{CH} 2 \mathrm{CH} 3}=3.2 \mathrm{~Hz}, J_{\mathrm{CH} 2 \mathrm{CH} 3}=7.3 \mathrm{~Hz}, \mathrm{SCH}_{2} \mathrm{CH}_{3}\right), 2.58-$ $247\left(\mathrm{~m}, 2 \mathrm{H}, \mathrm{SCH}_{2} \mathrm{CH}_{3}\right), 1.21\left(\mathrm{t}, 3 \mathrm{H}, J_{\mathrm{CH} 2 \mathrm{CH} 3}=7.3 \mathrm{~Hz}, \mathrm{SCH}_{2} \mathrm{CH}_{3}\right), 1.15\left(\mathrm{t}, 3 \mathrm{H}, J_{\mathrm{CH} 2 \mathrm{CH} 3}=\right.$ $\left.7.3 \mathrm{~Hz}, \mathrm{SCH}_{2} \mathrm{CH}_{3}\right) \mathrm{ppm} .{ }^{13} \mathrm{C} \mathrm{NMR}\left(101 \mathrm{MHz}, \mathrm{CDCl}_{3}\right): \delta=138.9\left(\mathrm{C}_{\mathrm{q}}{ }^{\text {arom }}\right), 138.8\left(\mathrm{C}_{\mathrm{q}}{ }^{\text {arom }}\right)$, $138.7\left(\mathrm{C}_{\mathrm{q}}{ }^{\text {arom }}\right), 138.5\left(\mathrm{C}_{\mathrm{q}}{ }^{\text {arom }}\right), 138.4\left(\mathrm{C}_{\mathrm{q}}{ }^{\text {arom }}\right), 128.5-127.5\left(\mathrm{CH}^{\text {arom }}\right), 83.0(\mathrm{C}-4), 81.1(\mathrm{C}-$ 3), $79.6(\mathrm{C}-2), 78.9(\mathrm{C}-5), 75.5\left(\mathrm{CH}_{2}{ }^{\mathrm{Bn}}\right), 74.8\left(\mathrm{CH}_{2}{ }^{\mathrm{Bn}}\right), 73.7\left(\mathrm{CH}_{2}{ }^{\mathrm{Bn}}\right), 73.4\left(\mathrm{CH}_{2}{ }^{\mathrm{Bn}}\right), 72.1$ $\left(\mathrm{CH}_{2}{ }^{\mathrm{Bn}}\right), 70.2(\mathrm{C}-6), 53.7(\mathrm{C}-1), 25.1\left(\mathrm{SCH}_{2} \mathrm{CH}_{3}\right), 25.0\left(\mathrm{SCH}_{2} \mathrm{CH}_{3}\right), 14.6\left(\mathrm{SCH}_{2} \mathrm{CH}_{3}\right)$, $14.4\left(\mathrm{SCH}_{2} \mathrm{CH}_{3}\right) \mathrm{ppm}$. These analytical data correspond to published ones. ${ }^{1}$ 


\section{${ }^{1}$ H NMR}

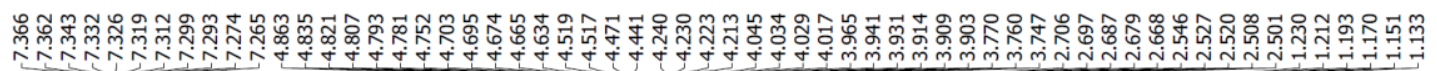

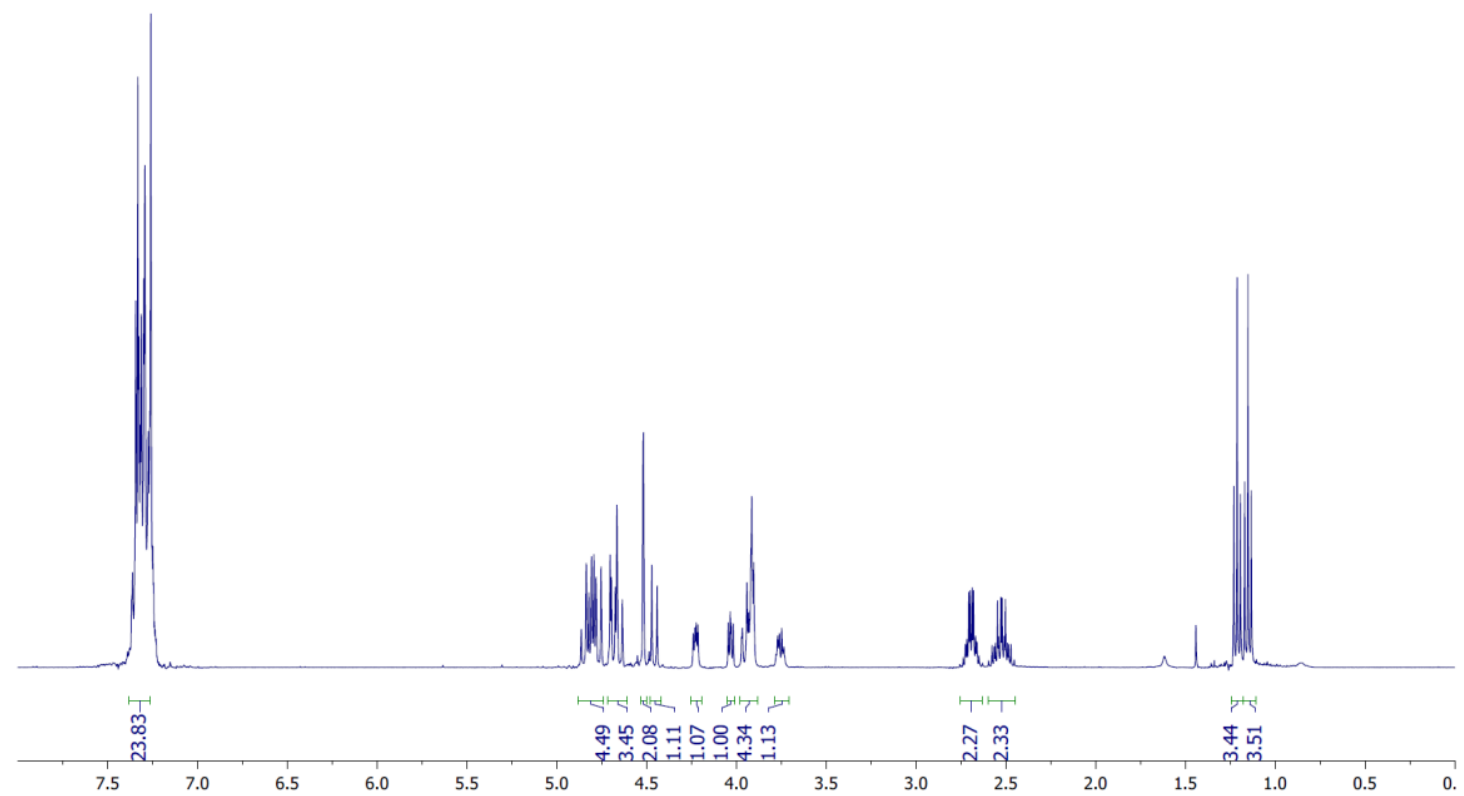

${ }^{13}$ C NMR

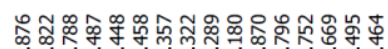

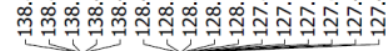

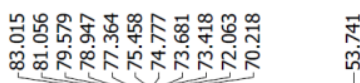

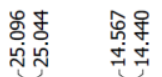

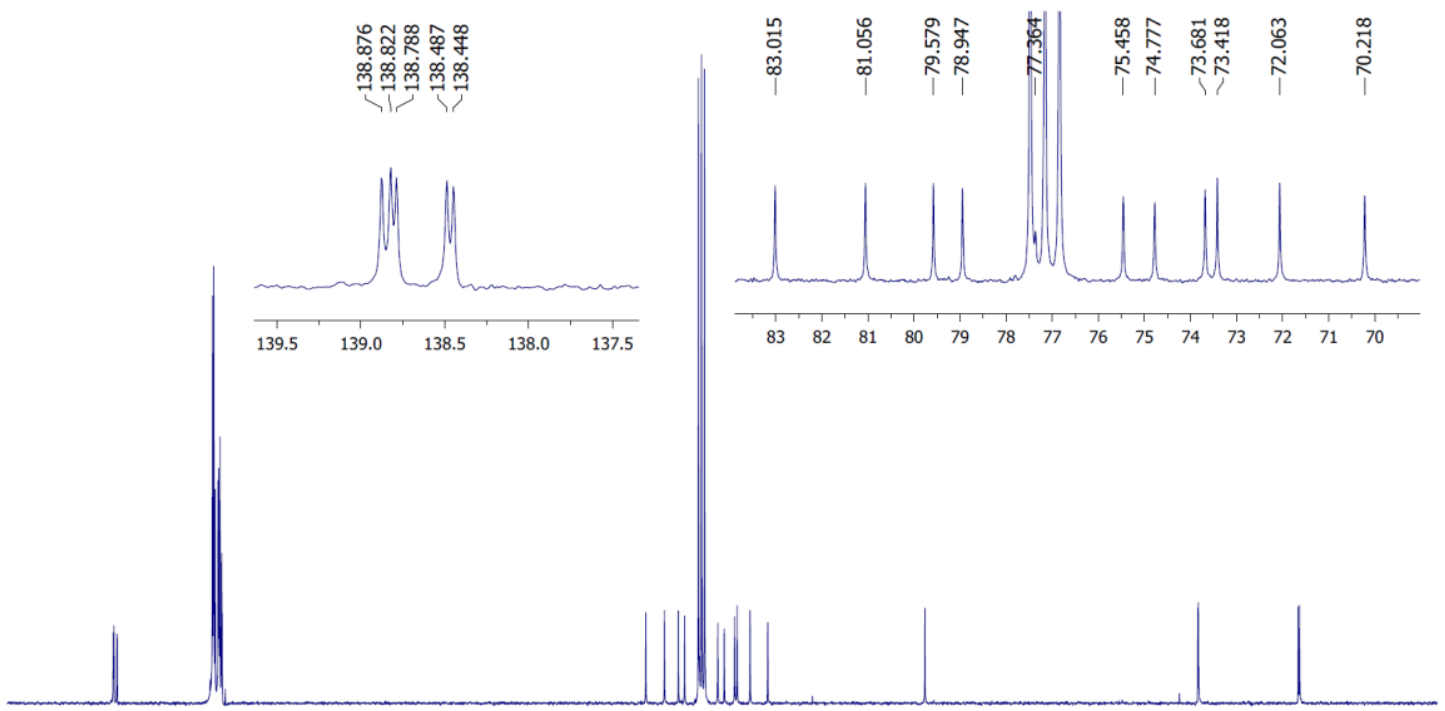

\begin{tabular}{llllllllllllllllllllllllllllllllllllll}
\hline 0 & 145 & 140 & 135 & 130 & 125 & 120 & 115 & 110 & 105 & 100 & 95 & 90 & 85 & 80 & 75 & 70 & 65 & 60 & 55 & 50 & 45 & 40 & 35 & 30 & 25 & 20 & 15 & 10 & 5 & 1
\end{tabular} 


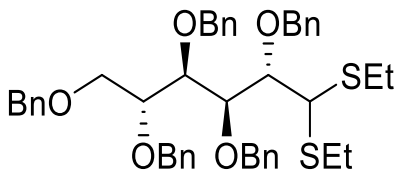

$1 \mathrm{~h}$

D-Mannose (26.1 g, $145 \mathrm{mmol}$ ) was dissolved in an aqueous solution of concentrated hydrochloric acid (37\%, $39 \mathrm{ml}, 470 \mathrm{mmol}, 3.24$ equiv.) at r.t. under argon atmosphere. Then, ethanethiol (26 ml, $351 \mathrm{mmol}, 2.4$ equiv.) was added and the mixture was stirred vigorously at r.t. for $1 \mathrm{~h}$. A small amount of ice was added, which causes almost instantaneously solidification of the reaction mixture. The solid was filtered and washed with cold water and cooled diethyl ether to give diethyl dithioacetal D-mannose as a white crystalline solid $(28.8 \mathrm{~g}, 70 \%)$.

To a solution of diethyl dithioacetal D-mannose $(14.5 \mathrm{~g}, 51 \mathrm{mmol})$ in dry DMF $(250 \mathrm{ml})$ under argon atmosphere was added benzyl bromide $(33.3 \mathrm{ml}, 278 \mathrm{mmol}, 5.5$ equiv.) at r.t. The solution was cooled at $0{ }^{\circ} \mathrm{C}$ and $\mathrm{NaH}(60 \%$ in mineral oil, $11.1 \mathrm{~g}, 278 \mathrm{mmol}, 5.5$ equiv.) was added portionwise and the reaction was stirred for $24 \mathrm{~h}$. Ice water was added carefully and the mixture was diluted with $\mathrm{Et}_{2} \mathrm{O}$ and the aqueous phase was extracted with $\mathrm{Et}_{2} \mathrm{O}$ (2 times). The combined organic phases were washed with saturated aqueous solution of $\mathrm{NH}_{4} \mathrm{Cl}$ (3 times) and brine (3 times), dried over $\mathrm{MgSO}_{4}$ and concentrated. Purification by chromatography on silica gel (Cy/EtOAc 95:5) gave compound $\mathbf{1 h}$ (26.3 g, $71 \%)$.

$[\alpha]^{20}$ D: $-8.9\left(c 1.0, \mathrm{CHCl}_{3}\right) .{ }^{1} \mathrm{H} \mathrm{NMR}\left(400 \mathrm{MHz}, \mathrm{CDCl}_{3}\right): \delta=7.38-7.26\left(\mathrm{~m}, 25 \mathrm{H}, \mathrm{H}^{\text {arom }}\right)$, $5.06\left(\mathrm{AB}, 1 \mathrm{H}, J_{\mathrm{A}-\mathrm{B}}=11.5 \mathrm{~Hz}, \mathrm{CH}_{2}{ }^{\mathrm{Bn}}\right), 4.76\left(\mathrm{AB}, 1 \mathrm{H}, J_{\mathrm{A}-\mathrm{B}}=11.7 \mathrm{~Hz}, \mathrm{CH}_{2}{ }^{\mathrm{Bn}}\right), 4.75(\mathrm{AB}$, $\left.1 \mathrm{H}, J_{\mathrm{A}-\mathrm{B}}=12.1 \mathrm{~Hz}, \mathrm{CH}_{2}{ }^{\mathrm{Bn}}\right), 4.70\left(\mathrm{AB}, 1 \mathrm{H}, J_{\mathrm{A}-\mathrm{B}}=10.0 \mathrm{~Hz}, \mathrm{CH}_{2}{ }^{\mathrm{Bn}}\right), 4.68\left(\mathrm{AB}, 1 \mathrm{H}, J_{\mathrm{A}-\mathrm{B}}=\right.$ $\left.10.0 \mathrm{~Hz}, \mathrm{CH}_{2}{ }^{\mathrm{Bn}}\right), 4.58\left(\mathrm{AB}, 1 \mathrm{H}, J_{\mathrm{A}-\mathrm{B}}=11.2 \mathrm{~Hz}, \mathrm{CH}_{2}{ }^{\mathrm{Bn}}\right), 4.56\left(\mathrm{AB}, 1 \mathrm{H}, J_{\mathrm{A}-\mathrm{B}}=11.7 \mathrm{~Hz}\right.$, $\left.\mathrm{CH}_{2}{ }^{\mathrm{Bn}}\right), 4.52\left(\mathrm{AB}, 1 \mathrm{H}, J_{\mathrm{A}-\mathrm{B}}=12.1 \mathrm{~Hz}, \mathrm{CH}_{2}{ }^{\mathrm{Bn}}\right), 4.49\left(\mathrm{AB}, 1 \mathrm{H}, J_{\mathrm{A}-\mathrm{B}}=11.2 \mathrm{~Hz}, \mathrm{CH}_{2}{ }^{\mathrm{Bn}}\right), 4.47$ $\left(\mathrm{AB}, 1 \mathrm{H}, J_{\mathrm{A}-\mathrm{B}}=11.5 \mathrm{~Hz}, \mathrm{CH}_{2}{ }^{\mathrm{Bn}}\right), 4.26\left(\mathrm{~d}, 1 \mathrm{H}, J_{1-2}=1.8 \mathrm{~Hz}, \mathrm{H}-1\right), 4.19-4.12(\mathrm{~m}, 3 \mathrm{H}, \mathrm{H}-$ $2, \mathrm{H}-3, \mathrm{H}-4), 4.00\left(\mathrm{td}, 1 \mathrm{H}, J_{4-5}=J_{5-6 \mathrm{a}}=2.5 \mathrm{~Hz}, J_{5-6 \mathrm{~b}}=5.5 \mathrm{~Hz}, \mathrm{H}-5\right), 3.95\left(\mathrm{ABX}, 1 \mathrm{H}, J_{5-}\right.$ $\left.6 \mathrm{a}=2.5 \mathrm{~Hz}, J_{6 \mathrm{a}-6 \mathrm{~b}}=10.8 \mathrm{~Hz}, \mathrm{H}-6 \mathrm{a}\right), 3.75\left(\mathrm{ABX}, 1 \mathrm{H}, J_{5-6 \mathrm{~b}}=5.5 \mathrm{~Hz}, J_{6 \mathrm{a}-6 \mathrm{~b}}=10.8 \mathrm{~Hz}, \mathrm{H}-\right.$ 6b), 2.75-2.58 (m, 4H, $\left.2 \mathrm{SCH}_{2} \mathrm{CH}_{3}\right), 1.22\left(\mathrm{t}, 3 \mathrm{H}, \mathrm{J}_{\mathrm{CH} 2 \mathrm{CH} 3}=7.3 \mathrm{~Hz}, \mathrm{SCH}_{2} \mathrm{CH}_{3}\right), 1.21(\mathrm{t}$, $\left.3 \mathrm{H}, J_{\mathrm{CH} 2 \mathrm{CH} 3}=7.3 \mathrm{~Hz}, \mathrm{SCH}_{2} \mathrm{CH}_{3}\right) \mathrm{ppm} .{ }^{13} \mathrm{C} \mathrm{NMR}\left(101 \mathrm{MHz}, \mathrm{CDCl}_{3}\right): \delta=139.0\left(\mathrm{C}_{\mathrm{q}}{ }^{\text {arom }}\right)$, $138.9\left(\mathrm{C}_{\mathrm{q}}{ }^{\text {arom }}\right), 138.8\left(\mathrm{C}_{\mathrm{q}}{ }^{\text {arom }}\right), 138.5\left(2 \mathrm{C}_{\mathrm{q}}{ }^{\text {arom }}\right), 128.5-127.5\left(\mathrm{CH}^{\text {arom }}\right), 83.2(\mathrm{C}-4), 79.6(\mathrm{C}-$ 2), $79.4(\mathrm{C}-5), 78.6(\mathrm{C}-3), 74.3\left(\mathrm{CH}_{2}{ }^{\mathrm{Bn}}\right), 74.2\left(\mathrm{CH}_{2}{ }^{\mathrm{Bn}}\right), 73.6\left(\mathrm{CH}_{2}{ }^{\mathrm{Bn}}\right), 73.4\left(\mathrm{CH}_{2}{ }^{\mathrm{Bn}}\right), 71.8$ $\left(\mathrm{CH}_{2}{ }^{\mathrm{Bn}}\right), 70.2(\mathrm{C}-6), 53.8(\mathrm{C}-1), 27.0\left(\mathrm{SCH}_{2} \mathrm{CH}_{3}\right), 25.2\left(\mathrm{SCH}_{2} \mathrm{CH}_{3}\right), 14.7\left(\mathrm{SCH}_{2} \mathrm{CH}_{3}\right)$, $14.5\left(\mathrm{SCH}_{2} \mathrm{CH}_{3}\right) \mathrm{ppm}$. These analytical data correspond to published ones. ${ }^{1}$ 


\section{${ }^{1}$ H NMR}

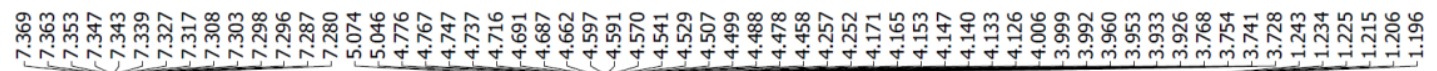

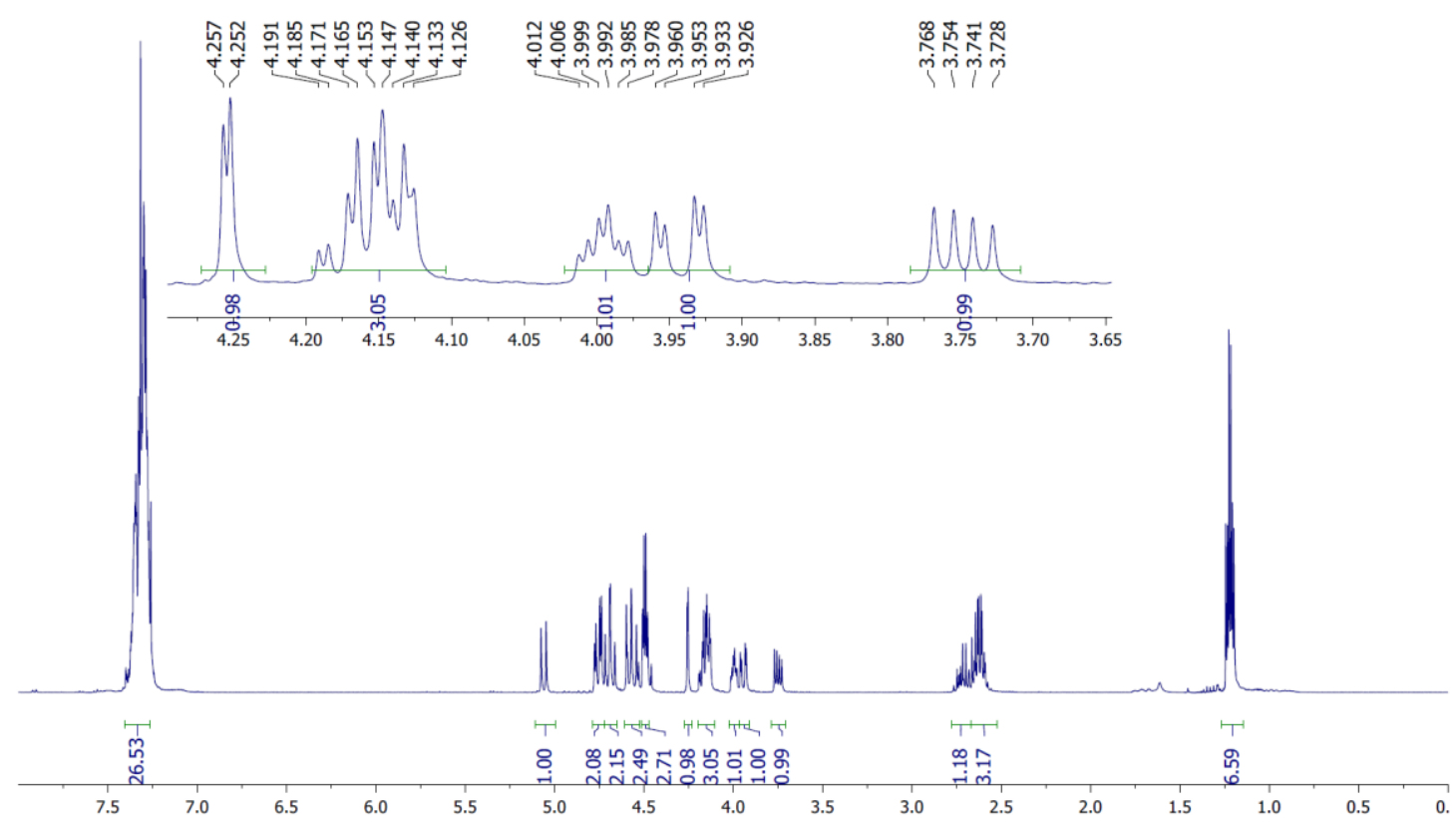

\section{${ }^{13}$ C NMR}



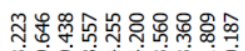

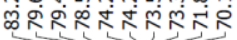

商

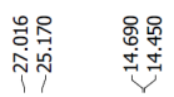

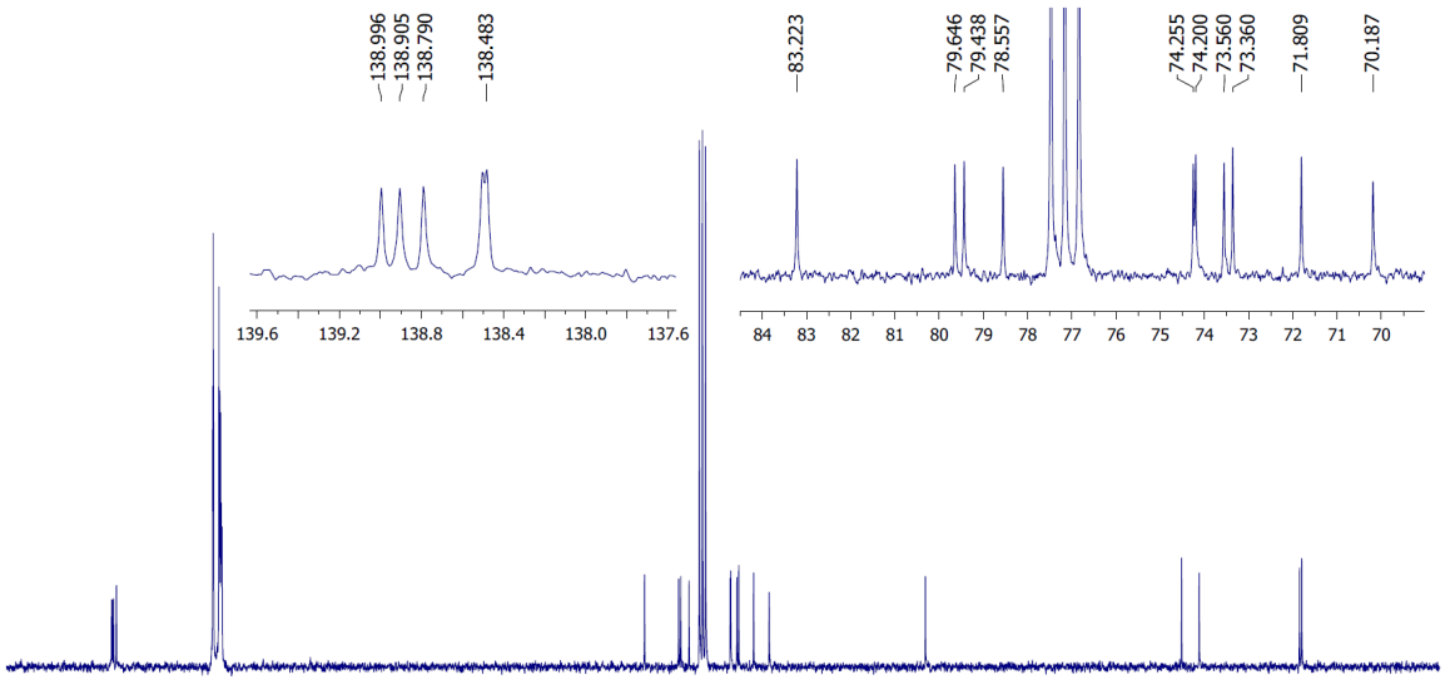

$\begin{array}{llllllllllllllllllllllllllllllllll}50 & 145 & 140 & 135 & 130 & 125 & 120 & 115 & 110 & 105 & 100 & 95 & 90 & 85 & 80 & 75 & 70 & 65 & 60 & 55 & 50 & 45 & 40 & 35 & 30 & 25 & 20 & 15 & 10 & 5 & 1\end{array}$ 


\section{Aldehydes}

2,3,4,5-Tetra-O-benzyl aldehydo D-arabinose (2a).<smiles>O=CC(Br)(Br)C(Br)C(Br)C(Cc1ccccc1)[C@H](Br)COc1ccccc1</smiles>

2a

2,3,4,5-Tetra-O-benzyl diethyl dithioacetal D-arabinose 1a (11.14 g, $43.2 \mathrm{mmol})$ was dissolved in an acetone/water mixture $(4 / 1,440 \mathrm{ml})$. Sodium bicarbonate $(8.0 \mathrm{~g}, 95 \mathrm{mmol}$, 2.2 equiv.) and iodine ( $24.1 \mathrm{~g}, 95 \mathrm{mmol}, 2.2$ equiv.) were subsequently added at r.t. The mixture was stirred vigorously for $1 \mathrm{~h}$ at room temperature. Then, saturated solution of sodium bicarbonate $(100 \mathrm{ml})$ and saturated solution of sodium thiosulfate $(100 \mathrm{ml})$ were added and acetone was removed under reduced pressure. The mixture was dissolved in $\mathrm{Et}_{2} \mathrm{O}$, saturated solution of sodium thiosulfate $(100 \mathrm{ml})$ was added and the solution was stirred for $15 \mathrm{~min}$. (until formation of a clear solution). The aqueous phase was extracted with $\mathrm{Et}_{2} \mathrm{O}$ and the combined organic layer were washed with brine (2 times), dried over $\mathrm{MgSO}_{4}$ and concentrated under reduced pressure. Purification by chromatography on silica gel (Cy/EtOAc 95:5) gave compound 2a (74.4 g, 81\%) as a colorless oil.

$[\alpha]^{20} \mathrm{D}:-2.6\left(c\right.$ 0.9, $\left.\mathrm{CHCl}_{3}\right) .{ }^{1} \mathrm{H}$ NMR $\left(400 \mathrm{MHz}, \mathrm{CDCl}_{3}\right): \delta=9.60\left(\mathrm{~d}, 1 \mathrm{H}, J_{1-2}=1.4 \mathrm{~Hz}\right.$, $\mathrm{H}-1), 7.32-7.26\left(\mathrm{~m}, 18 \mathrm{H}, \mathrm{H}^{\mathrm{arom}}\right), 7.21-7.19\left(\mathrm{~m}, 2 \mathrm{H}, \mathrm{H}^{\mathrm{arom}}\right), 4.67\left(\mathrm{AB}, 1 \mathrm{H}, J_{\mathrm{A}-\mathrm{B}}=11.7 \mathrm{~Hz}\right.$, $\left.\mathrm{CH}_{2}{ }^{\mathrm{Bn}}\right), 4.61\left(\mathrm{AB}, 1 \mathrm{H}, J_{\mathrm{A}-\mathrm{B}}=11.5 \mathrm{~Hz}, \mathrm{CH}_{2}{ }^{\mathrm{Bn}}\right), 4.53-48\left(\mathrm{~m}, 5 \mathrm{H}, \mathrm{CH}_{2}{ }^{\mathrm{Bn}}\right), 4.35(\mathrm{AB}, 1 \mathrm{H}$, $\left.J_{\mathrm{A}-\mathrm{B}}=11.5 \mathrm{~Hz}, \mathrm{CH}_{2}{ }^{\mathrm{Bn}}\right), 4.14-4.09(\mathrm{~m}, 2 \mathrm{H}, \mathrm{H}-2, \mathrm{H}-3), 3.84-3.78$ (m, 2H, H-4, H-5a), 3.67 $\left(\mathrm{ABX}, 1 \mathrm{H}, J_{4-5 \mathrm{~b}}=3.9 \mathrm{~Hz}, J_{5 \mathrm{a}-5 \mathrm{~b}}=10.5 \mathrm{~Hz}, \mathrm{H}-5 \mathrm{~b}\right) \mathrm{ppm} .{ }^{13} \mathrm{C} \mathrm{NMR}\left(125 \mathrm{MHz}, \mathrm{CDCl}_{3}\right): \delta=$ $202.3(\mathrm{C}-1), 138.2\left(2 \mathrm{C}_{\mathrm{q}}{ }^{\text {arom }}\right), 137.7\left(\mathrm{C}_{\mathrm{q}}^{\text {arom }}\right), 137.4\left(\mathrm{C}_{\mathrm{q}}^{\text {arom }}\right), 128.6-127.8\left(\mathrm{CH}^{\text {arom }}\right), 84.2$ (C-2), 78.4 (C-3), $77.5(\mathrm{C}-4), 74.2\left(\mathrm{CH}_{2}{ }^{\mathrm{Bn}}\right), 73.5\left(\mathrm{CH}_{2}{ }^{\mathrm{Bn}}\right), 73.4\left(\mathrm{CH}_{2}{ }^{\mathrm{Bn}}\right), 72.1\left(\mathrm{CH}_{2}{ }^{\mathrm{Bn}}\right)$, 68.3 (C-5) ppm. HRMS (ESI+): $\mathrm{m} / \mathrm{z}$ calculated for $\mathrm{C}_{33} \mathrm{H}_{34} \mathrm{O}_{5} \mathrm{Na}[\mathrm{M}+\mathrm{Na}]^{+}$: calc. 533.2298; found: 533.2291. 
${ }^{1}$ H NMR

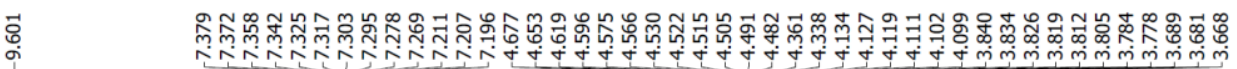

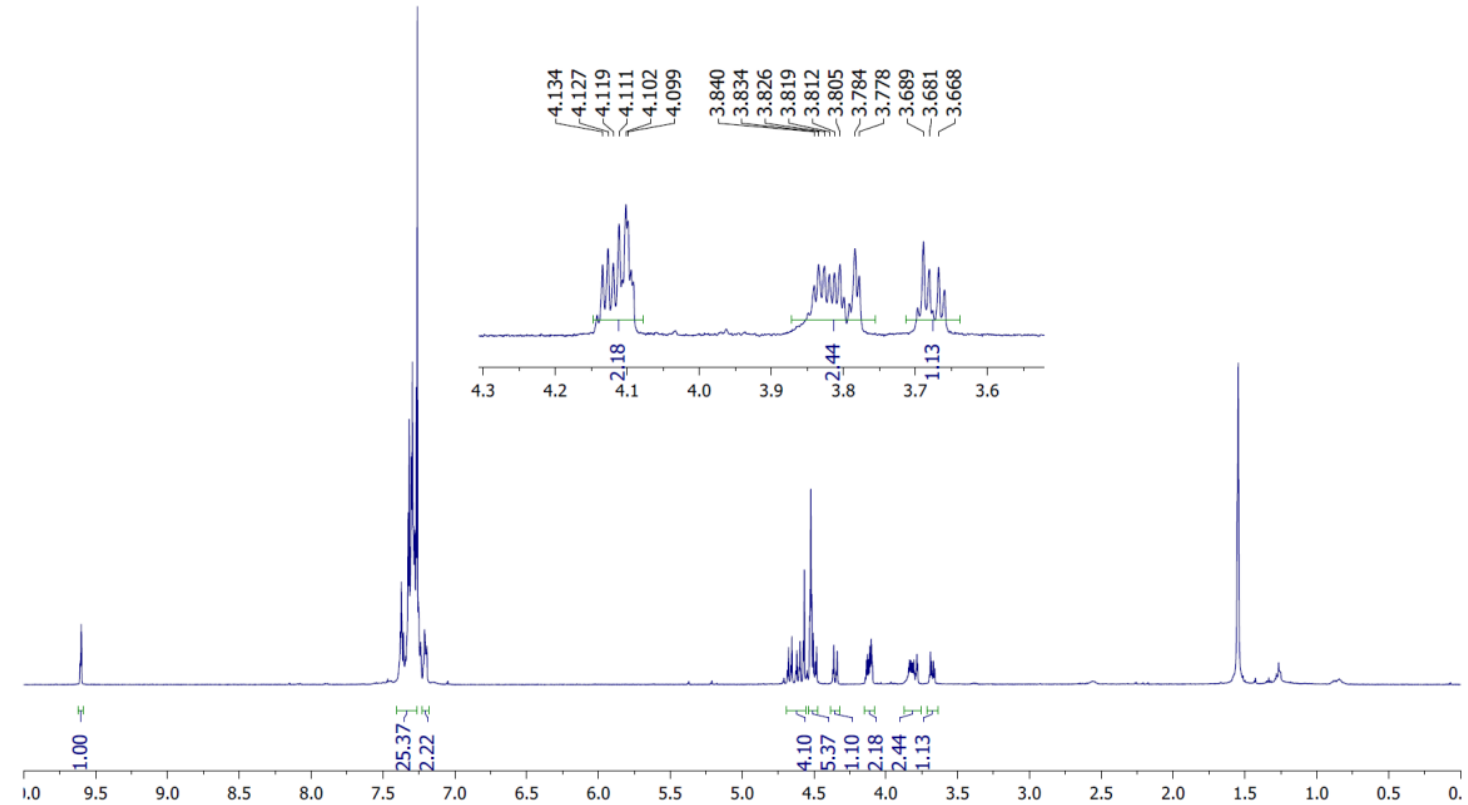

${ }^{13}$ C NMR

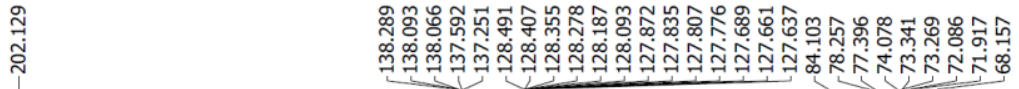

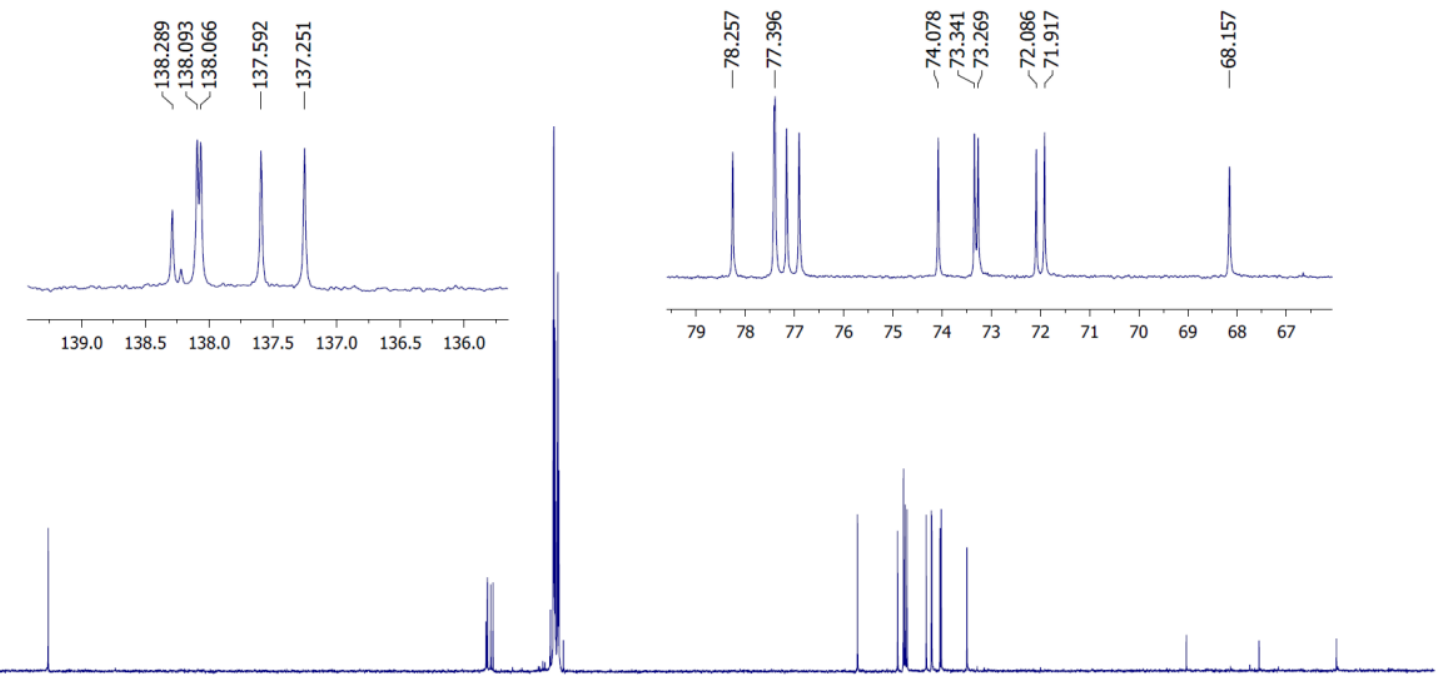

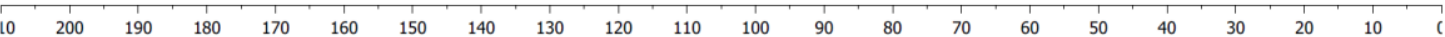


<smiles>O=CC(Br)(Br)C(Br)C(Br)C(COc1ccccc1)C(Br)Cc1ccccc1</smiles>

2b

2,3,4,5-Tetra-O-benzyl diethyl dithioacetal D-ribose $\mathbf{1 b}$ (1.7 g, $2.76 \mathrm{mmol})$ was dissolved in an acetone/water mixture (4/1, $28 \mathrm{ml})$. Sodium bicarbonate $(510 \mathrm{mg}, 6.07 \mathrm{mmol}, 2.2$ equiv.) and iodine ( $1.54 \mathrm{~g}, 6.07 \mathrm{mmol}, 2.2$ equiv.) were subsequently added at r.t. The mixture was stirred vigorously for $1 \mathrm{~h}$ at room temperature. Then, saturated solution of sodium bicarbonate $(8 \mathrm{ml})$ and saturated solution of sodium thiosulfate $(8 \mathrm{ml})$ were added and acetone was removed under reduced pressure. The mixture was dissolved in $\mathrm{Et}_{2} \mathrm{O}$, saturated solution of sodium thiosulfate $(10 \mathrm{ml})$ was added and the solution was stirred for $15 \mathrm{~min}$. (until formation of a clear solution). The aqueous phase was extracted with $\mathrm{Et}_{2} \mathrm{O}$ and the combined organic layer were washed with brine (2 times), dried over $\mathrm{MgSO}_{4}$ and concentrated under reduced pressure. Purification by chromatography on silica gel (Cy/EtOAc 95:5) gave compound $\mathbf{2 b}(1.037 \mathrm{~g}, 74 \%)$ as a colorless oil.

$[\alpha]_{20}{ }^{\mathrm{D}}:+20.4\left(\mathrm{c} 1.0, \mathrm{CHCl}_{3}\right) .{ }^{1} \mathrm{H}$ NMR $\left(400 \mathrm{MHz}, \mathrm{CDCl}_{3}\right): \delta=9.48\left(\mathrm{~d}, 1 \mathrm{H}, J_{1-2}=0.9 \mathrm{~Hz}\right.$, $\mathrm{H}-1), 7.35-7.27\left(\mathrm{~m}, 18 \mathrm{H}, \mathrm{H}^{\text {arom }}\right), 7.23-7.21\left(\mathrm{~m}, 2 \mathrm{H}, \mathrm{H}^{\text {arom }}\right), 4.74\left(\mathrm{AB}, 1 \mathrm{H}, J_{\mathrm{A}-\mathrm{B}}=12.1 \mathrm{~Hz}\right.$, $\left.\mathrm{CH}_{2}{ }^{\mathrm{Bn}}\right), 4.71\left(\mathrm{AB}, 1 \mathrm{H}, J_{\mathrm{A}-\mathrm{B}}=11.5 \mathrm{~Hz}, \mathrm{CH}_{2}{ }^{\mathrm{Bn}}\right), 4.69\left(\mathrm{AB}, 1 \mathrm{H}, J_{\mathrm{A}-\mathrm{B}}=11.9 \mathrm{~Hz}, \mathrm{CH}_{2}{ }^{\mathrm{Bn}}\right), 4.60$ (bs, $\left.1 \mathrm{H}, \mathrm{CH}_{2}{ }^{\mathrm{Bn}}\right), 4.57$ (bs, $\left.1 \mathrm{H}, \mathrm{CH}_{2}{ }^{\mathrm{Bn}}\right), 4.52\left(\mathrm{AB}, 1 \mathrm{H}, J_{\mathrm{A}-\mathrm{B}}=12.1 \mathrm{~Hz}, \mathrm{CH}_{2}{ }^{\mathrm{Bn}}\right), 4.48(\mathrm{AB}$, $\left.1 \mathrm{H}, J_{\mathrm{A}-\mathrm{B}}=11.5 \mathrm{~Hz}, \mathrm{CH}_{2}{ }^{\mathrm{Bn}}\right), 4.47\left(\mathrm{AB}, 1 \mathrm{H}, J_{\mathrm{A}-\mathrm{B}}=12.1 \mathrm{~Hz}, \mathrm{CH}_{2}{ }^{\mathrm{Bn}}\right), 4.11\left(\mathrm{dd}, 1 \mathrm{H}, J_{1-2}=\right.$ $\left.0.9 \mathrm{~Hz}, J_{2-3}=2.2 \mathrm{~Hz}, \mathrm{H}-2\right), 4.01\left(\mathrm{dd}, 1 \mathrm{H}, J_{2-3}=2.2 \mathrm{~Hz}, J_{3-4}=8.6 \mathrm{~Hz}, \mathrm{H}-3\right), 3.92-3.88$ $\left(\mathrm{ddd}, 1 \mathrm{H}, J_{3-4}=8.6 \mathrm{~Hz}, J_{4-5 \mathrm{a}}=2.5 \mathrm{~Hz}, J_{4-5 \mathrm{~b}}=4.4 \mathrm{~Hz}, \mathrm{H}-4\right), 3.70\left(\mathrm{ABX}, 1 \mathrm{H}, J_{4-5 \mathrm{a}}=2.5\right.$ $\left.\mathrm{Hz}, J_{5 \mathrm{a}-5 \mathrm{~b}}=10.5 \mathrm{~Hz}, \mathrm{H}-5 \mathrm{a}\right), 3.61\left(\mathrm{ABX}, 1 \mathrm{H}, J_{4-5 \mathrm{~b}}=4.4 \mathrm{~Hz}, J_{5 \mathrm{a}-5 \mathrm{~b}}=10.5 \mathrm{~Hz}, \mathrm{H}-5 \mathrm{~b}\right) \mathrm{ppm}$. ${ }^{13} \mathrm{C}$ NMR (100 MHz, $\left.\mathrm{CDCl}_{3}\right): \delta=201.3(\mathrm{C}-1), 138.4\left(\mathrm{C}_{\mathrm{q}}{ }^{\text {arom }}\right), 138.2\left(\mathrm{C}_{\mathrm{q}}{ }^{\text {arom }}\right) 137.8$ $\left(\mathrm{C}_{\mathrm{q}}{ }^{\text {arom }}\right), 137.6\left(\mathrm{C}_{\mathrm{q}}{ }^{\text {arom }}\right), 128.6-127.7\left(\mathrm{CH}^{\text {arom }}\right), 82.6(\mathrm{C}-2), 80.7(\mathrm{C}-3), 76.8(\mathrm{C}-4), 73.5$ $\left(\mathrm{CH}_{2}{ }^{\mathrm{Bn}}\right), 73.3\left(\mathrm{CH}_{2}{ }^{\mathrm{Bn}}\right), 73.0\left(\mathrm{CH}_{2}{ }^{\mathrm{Bn}}\right), 72.8\left(\mathrm{CH}_{2}{ }^{\mathrm{Bn}}\right), 69.3$ (C-5) ppm. HRMS (ESI+): m/z calculated for $\mathrm{C}_{33} \mathrm{H}_{34} \mathrm{O}_{5} \mathrm{~K}[\mathrm{M}+\mathrm{K}]^{+}$: calc. 549.2038; found: 549.2039 . 


\section{${ }^{1}$ H NMR}

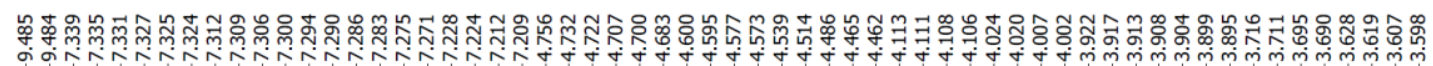

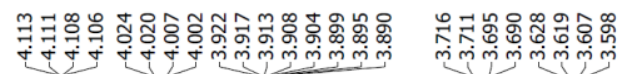

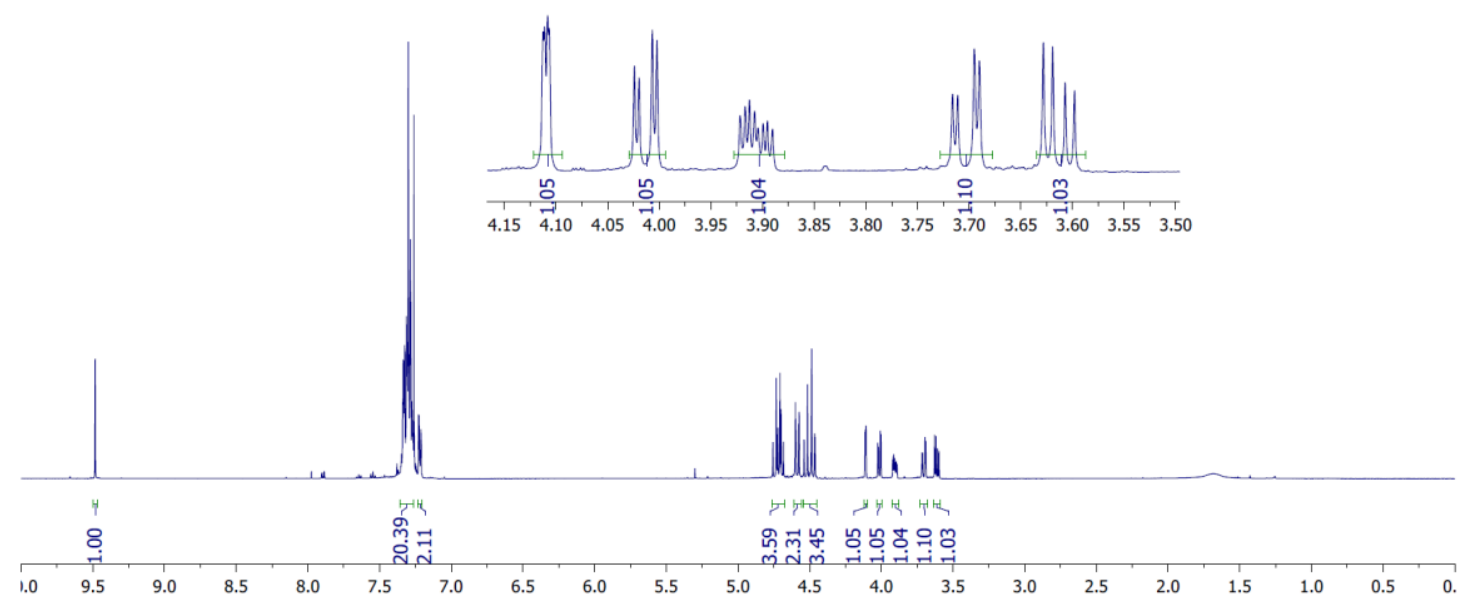

${ }^{13}$ C NMR

芦:

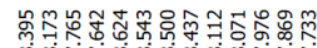

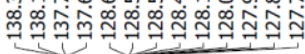

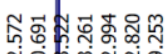

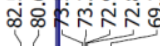

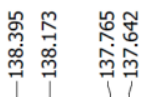
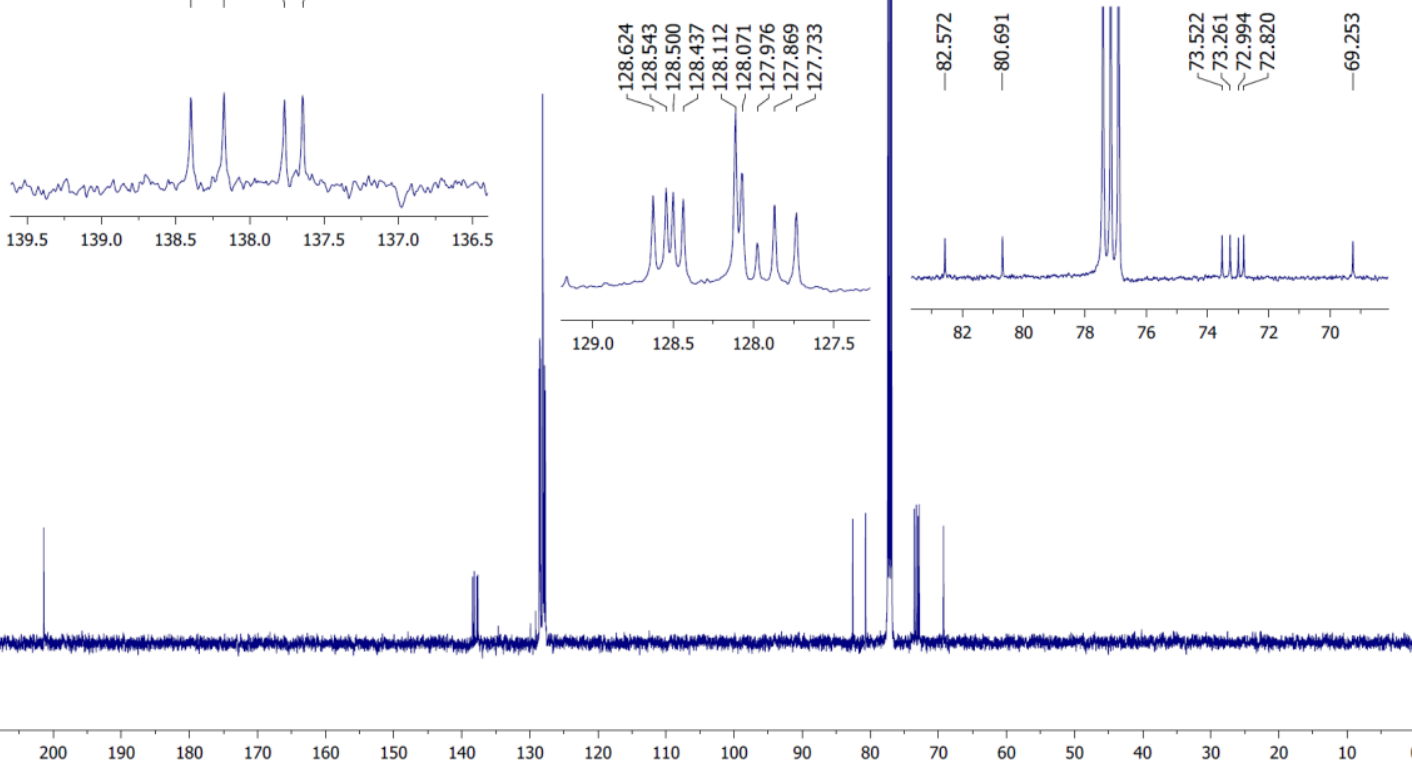
2,3,4,5-Tetra-O-benzyl aldehydo D-xylose (2c).<smiles>O=CC(Br)(Br)C(Br)C(Cc1ccccc1)[C@@H](Br)COCc1ccccc1</smiles>

2c

2,3,4,5-Tetra-O-benzyl diethyl dithioacetal D-xylose 1c (530 $\mathrm{mg}, 0.856 \mathrm{mmol})$ was dissolved in an acetone/water mixture (4/1, $15 \mathrm{ml})$. Sodium bicarbonate (158 mg, 1.884 mmol, 2.2 equiv.) and iodine ( $478 \mathrm{mg}, 1.884 \mathrm{mmol}, 2.2$ equiv.) were subsequently added at r.t. The mixture was stirred vigorously for $1 \mathrm{~h}$ at room temperature. Then, saturated solution of sodium bicarbonate $(8 \mathrm{ml})$ and saturated solution of sodium thiosulfate $(8 \mathrm{ml})$ were added and acetone was removed under reduced pressure. The mixture was dissolved in $\mathrm{Et}_{2} \mathrm{O}$, saturated solution of sodium thiosulfate $(10 \mathrm{ml})$ was added and the solution was stirred for $15 \mathrm{~min}$. (until formation of a clear solution). The aqueous phase was extracted with $\mathrm{Et}_{2} \mathrm{O}$ and the combined organic layer were washed with brine (2 times), dried over $\mathrm{MgSO}_{4}$ and concentrated under reduced pressure. Purification by chromatography on silica gel (Cy/EtOAc 95:5) gave compound $\mathbf{2 b}$ (284 mg, 65\%) as a colorless oil.

$[\alpha]^{20} \mathrm{D}:+7.7\left(\mathrm{c} 1.0, \mathrm{CHCl}_{3}\right) .{ }^{1} \mathrm{H}$ NMR $\left(400 \mathrm{MHz}, \mathrm{CDCl}_{3}\right): \delta=9.68(\mathrm{~s}, 1 \mathrm{H}, \mathrm{H}-1), 7.35-7.26$ $\left(\mathrm{m}, 18 \mathrm{H}, \mathrm{H}^{\text {arom }}\right), 7.23-7.20\left(\mathrm{~m}, 2 \mathrm{H}, \mathrm{H}^{\text {arom }}\right), 4.73\left(\mathrm{AB}, 1 \mathrm{H}, J_{\mathrm{A}-\mathrm{B}}=12.0 \mathrm{~Hz}, \mathrm{CH}_{2}{ }^{\mathrm{Bn}}\right), 4.60$ $\left(\mathrm{AB}, 1 \mathrm{H}, J_{\mathrm{A}-\mathrm{B}}=11.5 \mathrm{~Hz}, \mathrm{CH}_{2}{ }^{\mathrm{Bn}}\right), 4.56\left(\mathrm{AB}, 1 \mathrm{H}, J_{\mathrm{A}-\mathrm{B}}=11.5 \mathrm{~Hz}, \mathrm{CH}_{2}{ }^{\mathrm{Bn}}\right), 4.55(\mathrm{AB}, 1 \mathrm{H}$, $\left.J_{\mathrm{A}-\mathrm{B}}=11.5 \mathrm{~Hz}, \mathrm{CH}_{2}{ }^{\mathrm{Bn}}\right), 4.53\left(\mathrm{AB}, 1 \mathrm{H}, J_{\mathrm{A}-\mathrm{B}}=11.5 \mathrm{~Hz}, \mathrm{CH}_{2}{ }^{\mathrm{Bn}}\right), 4.46\left(\mathrm{AB}, 1 \mathrm{H}, J_{\mathrm{A}-\mathrm{B}}=12.0\right.$ $\left.\mathrm{Hz}, \mathrm{CH}_{2}{ }^{\mathrm{Bn}}\right), 4.41\left(\mathrm{AB}, 1 \mathrm{H}, J_{\mathrm{A}-\mathrm{B}}=12.6 \mathrm{~Hz}, \mathrm{CH}_{2}{ }^{\mathrm{Bn}}\right), 4.39\left(\mathrm{AB}, 1 \mathrm{H}, J_{\mathrm{A}-\mathrm{B}}=12.6 \mathrm{~Hz}, \mathrm{CH}_{2}{ }^{\mathrm{Bn}}\right)$, 3.97 (dd, $\left.1 \mathrm{H}, J_{2-3}=4.0 \mathrm{~Hz}, J_{3-4}=4.6 \mathrm{~Hz}, \mathrm{H}-3\right), 3.91-3.3 .87$ (m, 2H, H-2, H-4), 3.60 (ABX, $\left.1 \mathrm{H}, J_{4-5 \mathrm{a}}=5.7 \mathrm{~Hz}, J_{5 \mathrm{a}-5 \mathrm{~b}}=9.7 \mathrm{~Hz}, \mathrm{H}-5 \mathrm{a}\right), 3.48\left(\mathrm{ABX}, 1 \mathrm{H}, J_{4-5 \mathrm{~b}}=5.2 \mathrm{~Hz}, J_{5 \mathrm{a}-5 \mathrm{~b}}=9.7 \mathrm{~Hz}\right.$, $\mathrm{H}-5 \mathrm{~b}) \mathrm{ppm} .{ }^{13} \mathrm{C}$ NMR $\left(125 \mathrm{MHz}, \mathrm{CDCl}_{3}\right): \delta=201.2(\mathrm{C}-1), 138.2\left(\mathrm{C}_{\mathrm{q}}{ }^{\text {arom }}\right), 138.1\left(\mathrm{C}_{\mathrm{q}}{ }^{\text {arom }}\right)$ $137.7\left(\mathrm{C}_{\mathrm{q}}{ }^{\text {arom }}\right), 137.4\left(\mathrm{C}_{\mathrm{q}}{ }^{\text {arom }}\right), 128.7-127.8\left(\mathrm{CH}^{\text {arom }}\right), 81.5(\mathrm{C}-2), 79.3(\mathrm{C}-3), 77.0(\mathrm{C}-4)$, $74.2\left(\mathrm{CH}_{2}{ }^{\mathrm{Bn}}\right), 73.5\left(\mathrm{CH}_{2}{ }^{\mathrm{Bn}}\right), 73.2\left(2 \mathrm{CH}_{2}{ }^{\mathrm{Bn}}\right), 69.2$ (C-5) ppm. HRMS (ESI+): m/z calculated for $\mathrm{C}_{33} \mathrm{H}_{34} \mathrm{O}_{5} \mathrm{~K}[\mathrm{M}+\mathrm{K}]^{+}$: calc. 549.2038; found: 549.2040. 


\section{${ }^{1}$ H NMR}

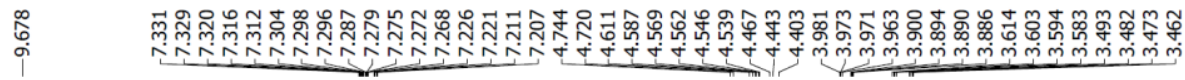

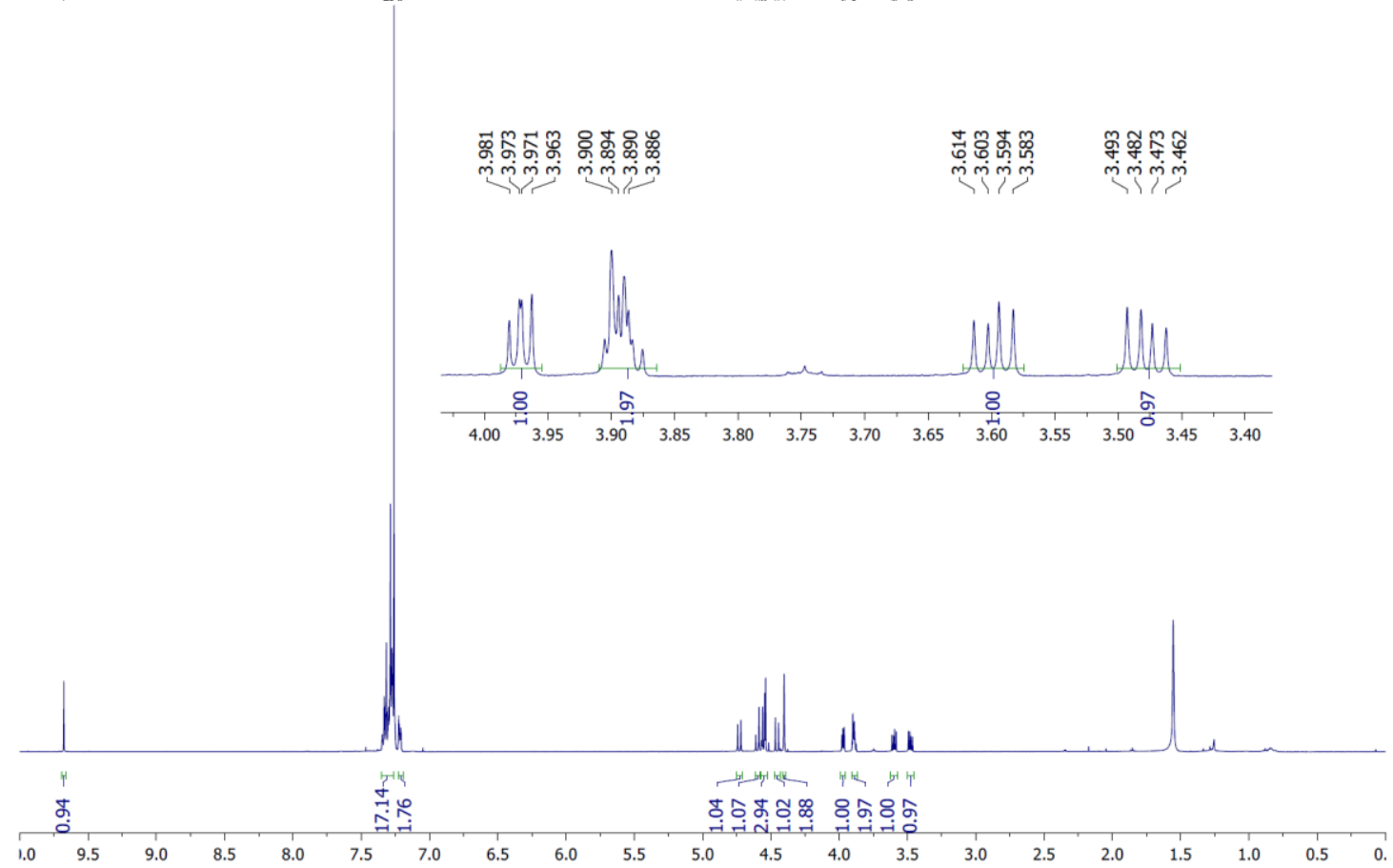

\section{${ }^{13}$ C NMR}
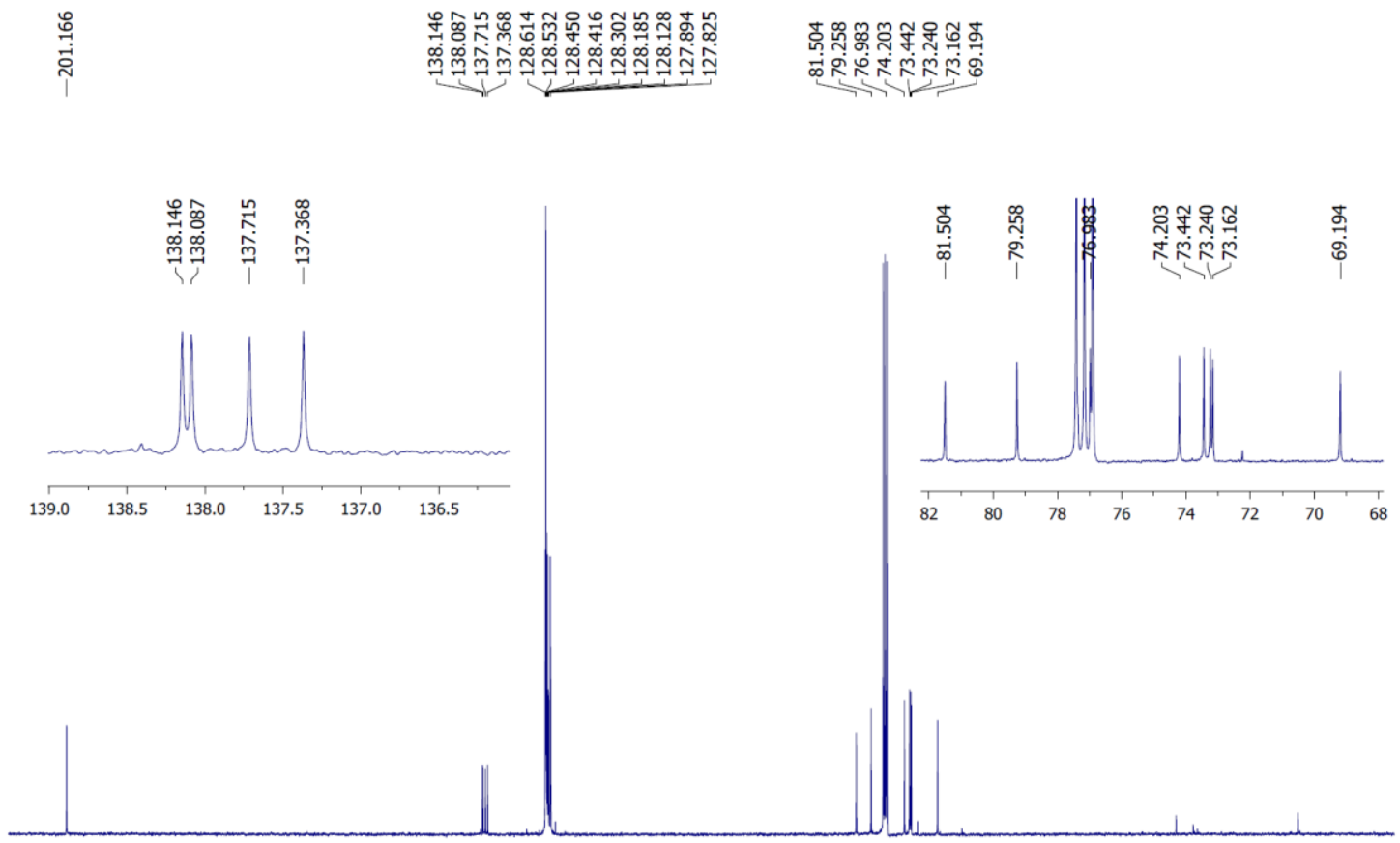

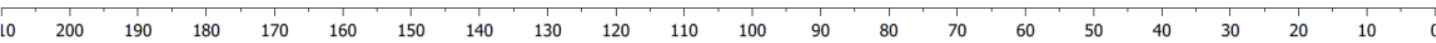


2,3,4,5-Tetra-O-benzyl aldehydo D-lyxose (2d).<smiles>O=CC(OCc1ccccc1)[C@H](Br)[C@H](Br)C(COc1ccccc1)Cc1ccccc1</smiles>

2d

2,3,4,5-Tetra-O-benzyl diethyl dithioacetal D-lyxose 1d (2.619 g, $4.25 \mathrm{mmol})$ was dissolved in an acetone/water mixture (4/1, $43 \mathrm{ml}$ ). Sodium bicarbonate (786 mg, 9.35 mmol, 2.2 equiv.) and iodine ( $2.37 \mathrm{~g}, 9.35 \mathrm{mmol}, 2.2$ equiv. $)$ were subsequently added at r.t. The mixture was stirred vigorously for $2 \mathrm{~h} 30$ at room temperature. Then, saturated solution of sodium bicarbonate $(10 \mathrm{ml})$ and saturated solution of sodium thiosulfate (10 $\mathrm{ml}$ ) were added and acetone was removed under reduced pressure. The mixture was dissolved in $\mathrm{Et}_{2} \mathrm{O}$, saturated solution of sodium thiosulfate $(10 \mathrm{ml})$ was added and the solution was stirred for $15 \mathrm{~min}$. (until formation of a clear solution). The aqueous phase was extracted with $\mathrm{Et}_{2} \mathrm{O}$ and the combined organic layer were washed with brine (2 times), dried over $\mathrm{MgSO}_{4}$ and concentrated under reduced pressure. The crude was purified by flash chromatography on silica gel (Cy/EtOAc 92:8) to provide 2,3,4,5-tetraO-benzyl-aldehydo-D-lyxose $\mathbf{2 d}$ as a colorless oil (1.492 g, 69\%).

$[\alpha]^{20} \mathrm{D}:-15.0\left(\mathrm{c} 1.0, \mathrm{CHCl}_{3}\right) .{ }^{1} \mathrm{H} \mathrm{NMR}\left(400 \mathrm{MHz}, \mathrm{CDCl}_{3}\right): \delta=9.70\left(\mathrm{~d}, J_{1-2}=1.5 \mathrm{~Hz}, 1 \mathrm{H}\right.$, $\mathrm{H}-1), 7.39-7.28\left(\mathrm{~m}, 20 \mathrm{H}, \mathrm{H}^{\text {arom }}\right), 4.69\left(\mathrm{AB}, 1 \mathrm{H}, \mathrm{J}_{\mathrm{A}-\mathrm{B}}=11.8 \mathrm{~Hz}, \mathrm{CH}_{2}{ }^{\mathrm{Bn}}\right), 4.68\left(\mathrm{AB}, 1 \mathrm{H}, J_{\mathrm{A}-}\right.$ В $\left.=11.5 \mathrm{~Hz}, \mathrm{CH}_{2}{ }^{\mathrm{Bn}}\right), 4.67\left(\mathrm{AB}, 1 \mathrm{H}, J_{\mathrm{A}-\mathrm{B}}=11.7 \mathrm{~Hz}, \mathrm{CH}_{2}{ }^{\mathrm{Bn}}\right), 4.61\left(\mathrm{AB}, 1 \mathrm{H}, J_{\mathrm{A}-\mathrm{B}}=11.6\right.$ $\left.\mathrm{Hz}, \mathrm{CH}_{2}{ }^{\mathrm{Bn}}\right), 4.57\left(\mathrm{AB}, 1 \mathrm{H}, J_{\mathrm{A}-\mathrm{B}}=11.8 \mathrm{~Hz}, \mathrm{CH}_{2}{ }^{\mathrm{Bn}}\right), 4.52\left(\mathrm{AB}, 1 \mathrm{H}, J_{\mathrm{A}-\mathrm{B}}=11.9 \mathrm{~Hz}, \mathrm{CH}_{2}{ }^{\mathrm{Bn}}\right)$, $4.50\left(\mathrm{AB}, 1 \mathrm{H}, J_{\mathrm{A}-\mathrm{B}}=11.9 \mathrm{~Hz}, \mathrm{CH}_{2}{ }^{\mathrm{Bn}}\right), 4.47\left(\mathrm{AB}, 1 \mathrm{H}, J_{\mathrm{A}-\mathrm{B}}=11.9 \mathrm{~Hz}, \mathrm{CH}_{2}{ }^{\mathrm{Bn}}\right), 4.10(\mathrm{dd}$, $\left.J_{2-3}=3.5, J_{1-2}=1.5 \mathrm{~Hz}, 1 \mathrm{H}, \mathrm{H}-2\right), 4.08\left(\mathrm{dd}, J_{3-4}=4.9, J_{2-3}=3.6 \mathrm{~Hz}, \mathrm{H}-3\right), 3.92\left(\mathrm{q}, 1 \mathrm{H}, J_{3-}\right.$ $4=5.0 \mathrm{~Hz}, \mathrm{H}-4), 3.79(\mathrm{~s}, 1 \mathrm{H}, \mathrm{H}-5), 3.78\left(\mathrm{~d}, 1 \mathrm{H}, J_{5 \mathrm{a}-5 \mathrm{~b}}=1.0 \mathrm{~Hz}, \mathrm{H}-5\right) \mathrm{ppm} .{ }^{13} \mathrm{C} \mathrm{NMR}(125$ $\left.\mathrm{MHz}, \mathrm{CDCl}_{3}\right): \delta=201.5(\mathrm{C}-1), 138.19\left(\mathrm{C}_{\mathrm{q}}^{\text {arom }}\right), 138.17\left(\mathrm{C}_{\mathrm{q}}^{\text {arom }}\right) 137.8\left(\mathrm{C}_{\mathrm{q}}^{\text {arom }}\right), 137.4$ $\left(\mathrm{C}_{\mathrm{q}}{ }^{\text {arom }}\right), 128.5\left(\mathrm{CH}^{\text {arom }}\right), 128.55\left(\mathrm{CH}^{\text {arom }}\right), 128.48\left(\mathrm{CH}^{\text {arom }}\right), 128.45\left(\mathrm{CH}^{\text {arom }}\right), 128.4$ $\left(\mathrm{CH}^{\text {arom }}\right), 128.2\left(2 \mathrm{CH}^{\text {arom }}\right), 128.04\left(\mathrm{CH}^{\text {arom }}\right), 127.97\left(\mathrm{CH}^{\text {arom }}\right), 127.9\left(\mathrm{CH}^{\text {arom }}\right), 127.82$ $\left(\mathrm{CH}^{\text {arom }}\right), 127.80\left(\mathrm{CH}^{\text {arom }}\right), 127.7\left(\mathrm{CH}^{\text {arom }}\right), 83.8(\mathrm{C}-2), 80.1(\mathrm{C}-3), 78.4(\mathrm{C}-4), 73.7$ $\left(\mathrm{CH}_{2}{ }^{\mathrm{Bn}}\right), 73.3\left(\mathrm{CH}_{2}{ }^{\mathrm{Bn}}\right), 73.1\left(\mathrm{CH}_{2}{ }^{\mathrm{Bn}}\right), 72.9\left(\mathrm{CH}_{2}{ }^{\mathrm{Bn}}\right), 70.0$ (C-5) ppm. HRMS (ESI+): m/z calculated for $\mathrm{C}_{33} \mathrm{H}_{34} \mathrm{O}_{5} \mathrm{Na}[\mathrm{M}+\mathrm{Na}]^{+}$: calc. 533.2298; found: 533.2297. 
${ }^{1}$ H NMR

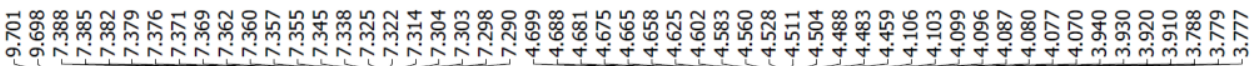

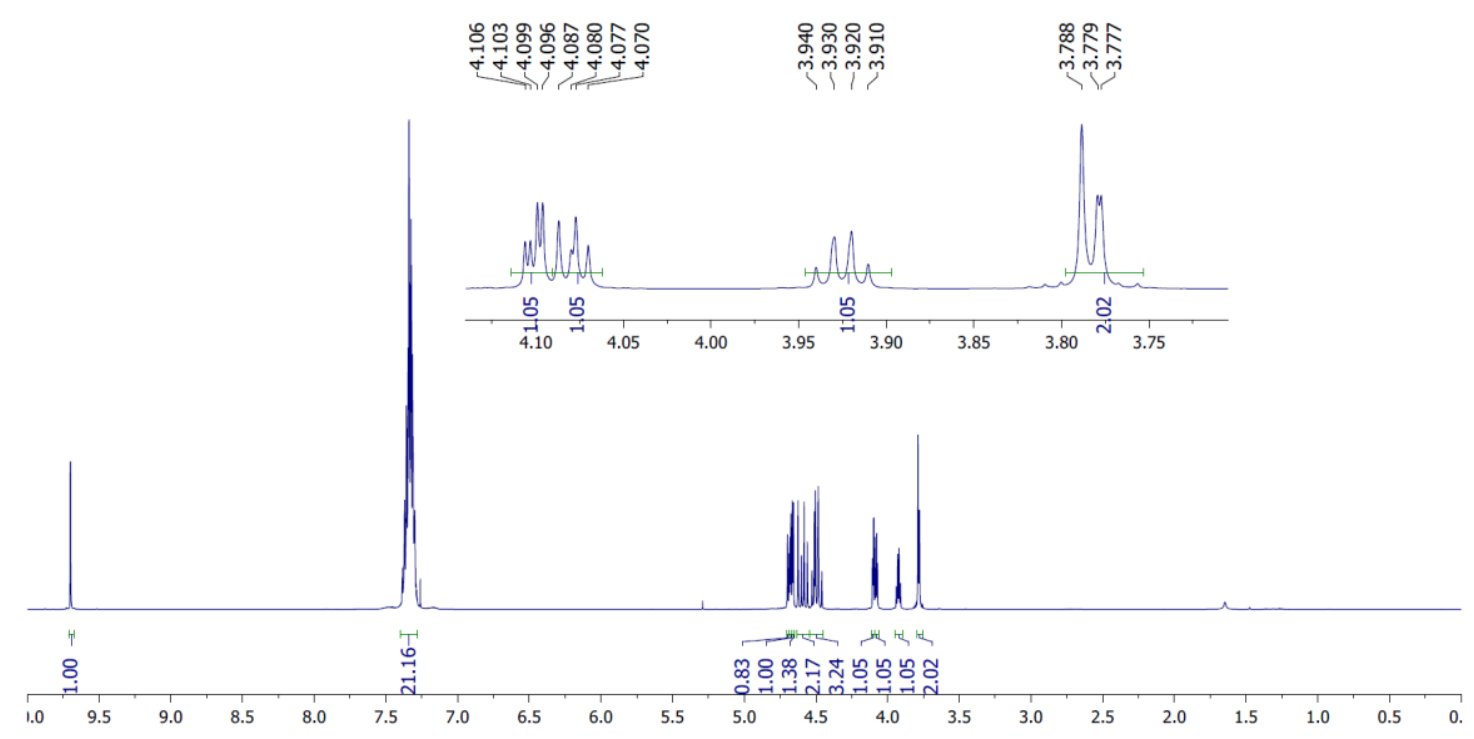

${ }^{13}$ C NMR
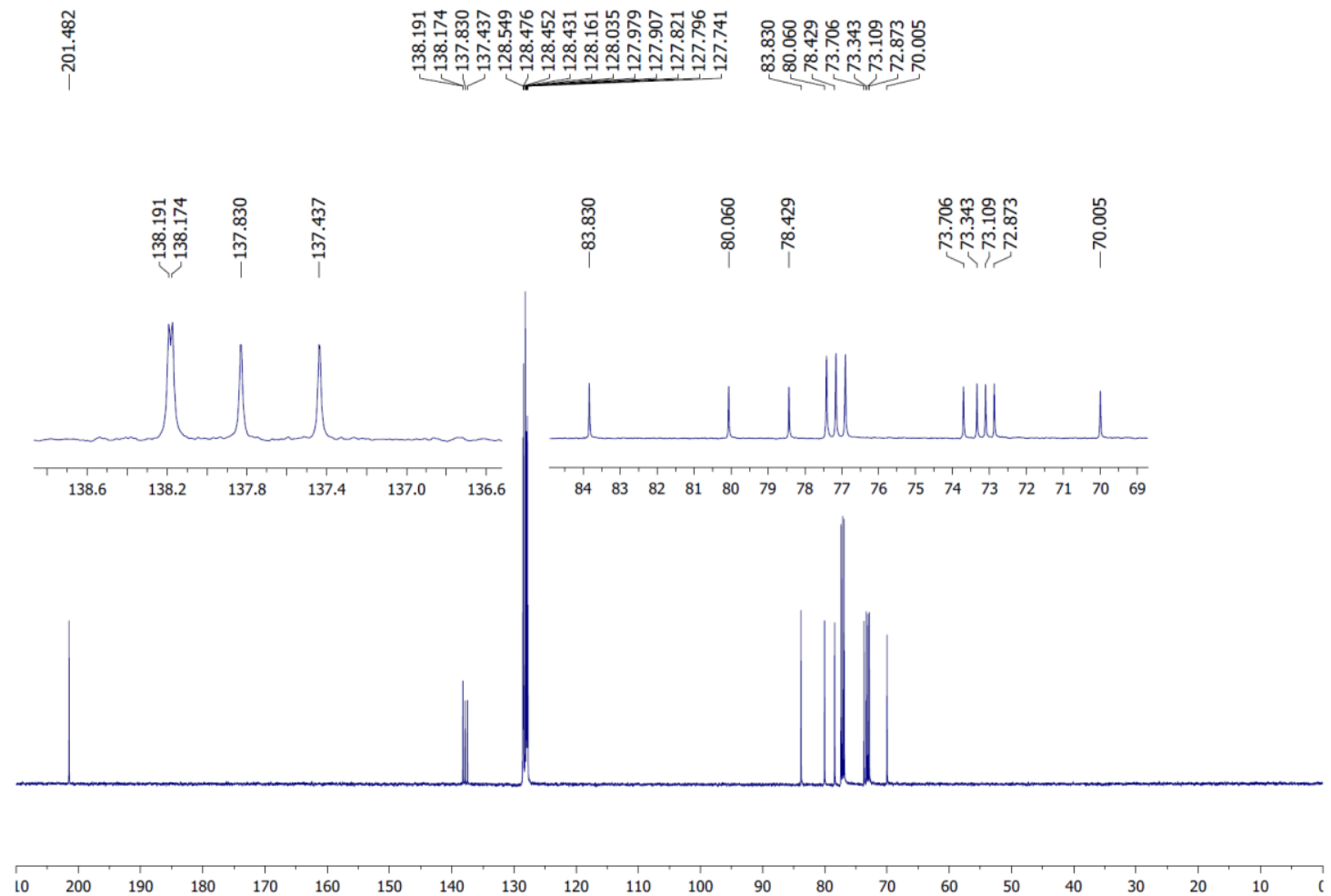
2,3,4,5-Tetra-O-benzyl aldehydo L-fucose (2e).<smiles>CC(O)[C@H](OCc1ccccc1)[C@@H](Br)C(=O)C(Cc1ccccc1)c1ccccc1</smiles>

$2 e$

2,3,4,5-Tetra-O-benzyl diethyl dithioacetal L-fucose 1e $(1.755 \mathrm{~g}, 2.78 \mathrm{mmol})$ was dissolved in an acetone/water mixture (4/1, $28 \mathrm{ml})$. Sodium bicarbonate $(514 \mathrm{mg}, 5.56$ mmol, 2.2 equiv.) and iodine ( $1.55 \mathrm{~g}, 5.56 \mathrm{mmol}, 2.2$ equiv.) were subsequently added at r.t. The mixture was stirred vigorously for $3 \mathrm{~h} 30$ at room temperature. Then, saturated solution of sodium bicarbonate $(10 \mathrm{ml})$ and saturated solution of sodium thiosulfate (10 $\mathrm{ml}$ ) were added and acetone was removed under reduced pressure. The mixture was dissolved in $\mathrm{Et}_{2} \mathrm{O}$, saturated solution of sodium thiosulfate $(10 \mathrm{ml})$ was added and the solution was stirred for $15 \mathrm{~min}$. (until formation of a clear solution). The aqueous phase was extracted with $\mathrm{Et}_{2} \mathrm{O}$ and the combined organic layer were washed with brine (2 times), dried over $\mathrm{MgSO}_{4}$ and concentrated under reduced pressure. The crude was purified by flash chromatography on silica gel (Cy/EtOAc 92:8) to provide 2,3,4,5-tetraO-benzyl-aldehydo-L-fucose $2 \mathrm{e}$ as a white solid (949 $\mathrm{mg}, 65 \%)$.

$[\alpha]_{20} \mathrm{D}:-12.1\left(\mathrm{c} 1.0, \mathrm{CHCl}_{3}\right) .{ }^{1} \mathrm{H} \mathrm{NMR}\left(400 \mathrm{MHz}, \mathrm{CDCl}_{3}\right): \delta=9.74\left(\mathrm{~d}, J_{1-2}=1.4 \mathrm{~Hz}, 1 \mathrm{H}\right.$, $\mathrm{H}-1)$, 7.37-7.28 (m, $\left.18 \mathrm{H}, \mathrm{H}^{\text {arom }}\right), 7.27-7.24\left(\mathrm{~m}, 2 \mathrm{H}, \mathrm{H}^{\text {arom }}\right), 4.76\left(\mathrm{AB}, 1 \mathrm{H}, J_{\mathrm{A}-\mathrm{B}}=11.7 \mathrm{~Hz}\right.$, $\left.\mathrm{CH}_{2}{ }^{\mathrm{Bn}}\right), 4.73\left(\mathrm{AB}, 1 \mathrm{H}, J_{\mathrm{A}-\mathrm{B}}=11.5 \mathrm{~Hz}, \mathrm{CH}_{2}{ }^{\mathrm{Bn}}\right), 4.670\left(\mathrm{AB}, 1 \mathrm{H}, J_{\mathrm{A}-\mathrm{B}}=11.7 \mathrm{~Hz}, \mathrm{CH}_{2}{ }^{\mathrm{Bn}}\right)$, $4.668\left(\mathrm{AB}, 1 \mathrm{H}, J_{\mathrm{A}-\mathrm{B}}=11.5 \mathrm{~Hz}, \mathrm{CH}_{2}{ }^{\mathrm{Bn}}\right), 4.55\left(\mathrm{AB}, 1 \mathrm{H}, J_{\mathrm{A}-\mathrm{B}}=11.7 \mathrm{~Hz}, \mathrm{CH}_{2}{ }^{\mathrm{Bn}}\right), 4.53(\mathrm{AB}$, $\left.1 \mathrm{H}, J_{\mathrm{A}-\mathrm{B}}=11.5 \mathrm{~Hz}, \mathrm{CH}_{2}{ }^{\mathrm{Bn}}\right), 4.500\left(\mathrm{AB}, 1 \mathrm{H}, J_{\mathrm{A}-\mathrm{B}}=11.6 \mathrm{~Hz}, \mathrm{CH}_{2}{ }^{\mathrm{Bn}}\right), 4.497\left(\mathrm{AB}, 1 \mathrm{H}, J_{\mathrm{A}-\mathrm{B}}\right.$ $\left.=11.7 \mathrm{~Hz}, \mathrm{CH}_{2}{ }^{\mathrm{Bn}}\right), 4.21\left(\mathrm{dd}, 1 \mathrm{H}, J=6.4, J_{2-3}=3.6 \mathrm{~Hz}, \mathrm{H}-3\right), 4.17\left(\mathrm{dd}, 1 \mathrm{H}, J_{2-3}=3.6, J_{1}\right.$. $2=1.4 \mathrm{~Hz}, \mathrm{H}-2), 3.98\left(\mathrm{qd}, 1 \mathrm{H}, J_{4-5}=6.4, J_{5-6}=4.4 \mathrm{~Hz}, \mathrm{H}-5\right), 3.76\left(\mathrm{dd}, 1 \mathrm{H}, J_{3-4}=6.4, J_{4-5}\right.$ $=4.3 \mathrm{~Hz}, \mathrm{H}-4), 1.30\left(\mathrm{~d}, 3 \mathrm{H}, J_{5-6}=6.4 \mathrm{~Hz}, \mathrm{H}-6\right) \mathrm{ppm} .{ }^{13} \mathrm{C} \mathrm{NMR}\left(125 \mathrm{MHz}, \mathrm{CDCl}_{3}\right): \delta=$ $202.2(\mathrm{C}-1), 138.8\left(\mathrm{C}_{\mathrm{q}}{ }^{\text {arom }}\right), 138.4\left(\mathrm{C}_{\mathrm{q}}{ }^{\text {arom }}\right), 137.8\left(\mathrm{C}_{\mathrm{q}}{ }^{\text {arom }}\right), 137.4\left(\mathrm{C}_{\mathrm{q}}{ }^{\text {arom }}\right), 128.6(2$ $\left.\mathrm{CH}^{\text {arom }}\right), 128.5\left(2 \mathrm{CH}^{\text {arom }}\right), 128.40\left(2 \mathrm{CH}^{\text {arom }}\right), 128.39\left(2 \mathrm{CH}^{\text {arom }}\right), 128.2\left(2 \mathrm{CH}^{\text {arom }}\right), 128.1$ $\left(\mathrm{CH}^{\text {arom }}\right), 127.90\left(2 \mathrm{CH}^{\text {arom }}\right), 127.88\left(\mathrm{CH}^{\text {arom }}\right), 127.86\left(2 \mathrm{CH}^{\text {arom }}\right), 127.8\left(2 \mathrm{CH}^{\text {arom }}\right), 127.63$ $\left(\mathrm{CH}^{\text {arom }}\right), 127.56\left(\mathrm{CH}^{\text {arom }}\right), 83.8(\mathrm{C}-2), 82.4(\mathrm{C}-4), 79.3(\mathrm{C}-3), 74.7(\mathrm{C}-5), 74.6\left(\mathrm{CH}_{2}{ }^{\mathrm{Bn}}\right)$, $73.4\left(\mathrm{CH}_{2}{ }^{\mathrm{Bn}}\right), 73.2\left(\mathrm{CH}_{2}{ }^{\mathrm{Bn}}\right), 71.2\left(\mathrm{CH}_{2}{ }^{\mathrm{Bn}}\right), 16.7$ (C-6) ppm. HRMS (ESI+): m/z calculated for $\mathrm{C}_{34} \mathrm{H}_{36} \mathrm{O}_{5} \mathrm{Na}[\mathrm{M}+\mathrm{Na}]^{+}$: calc. 547.2455; found: 547.2454. 


\section{${ }^{1}$ H NMR}

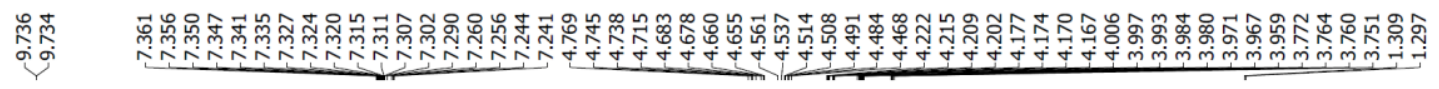

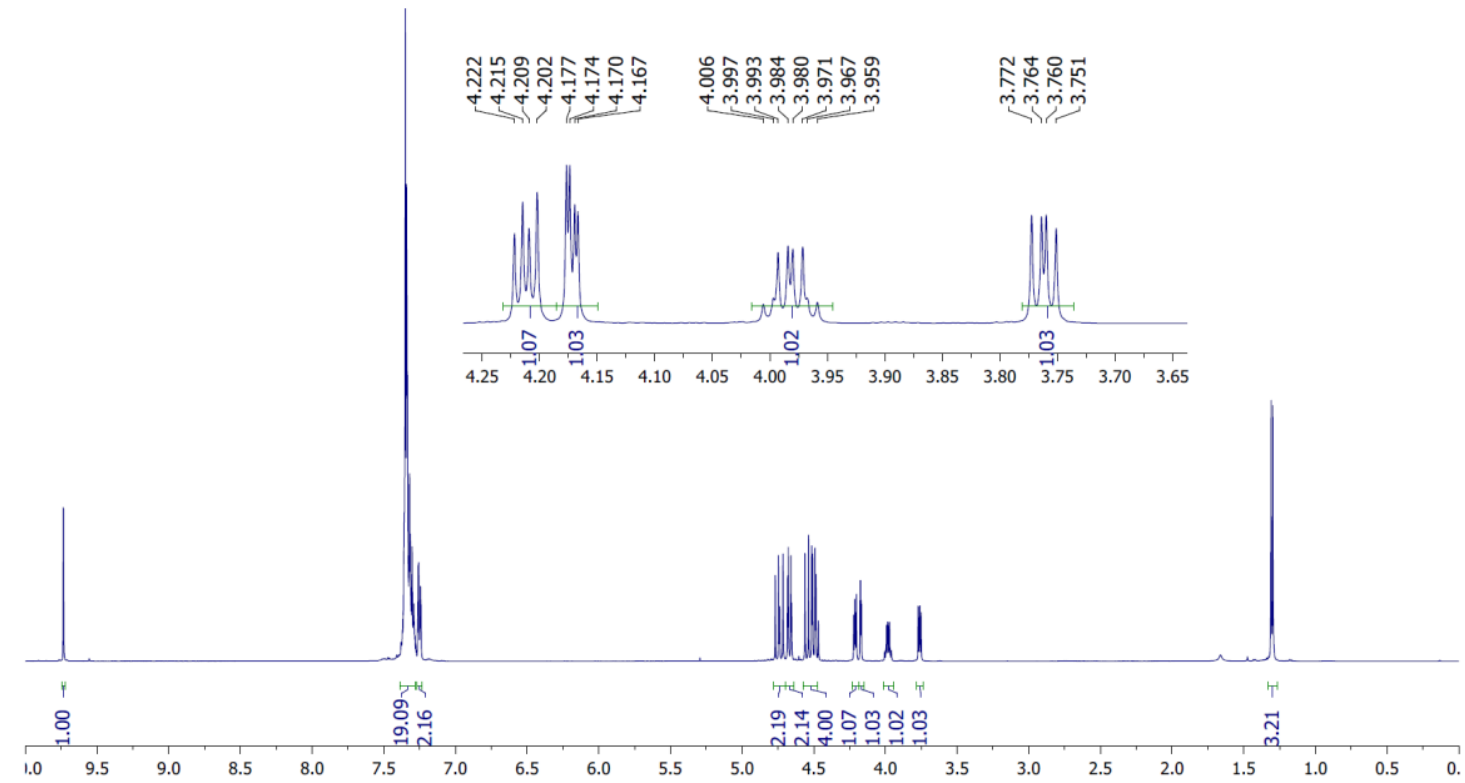

${ }^{13}$ C NMR
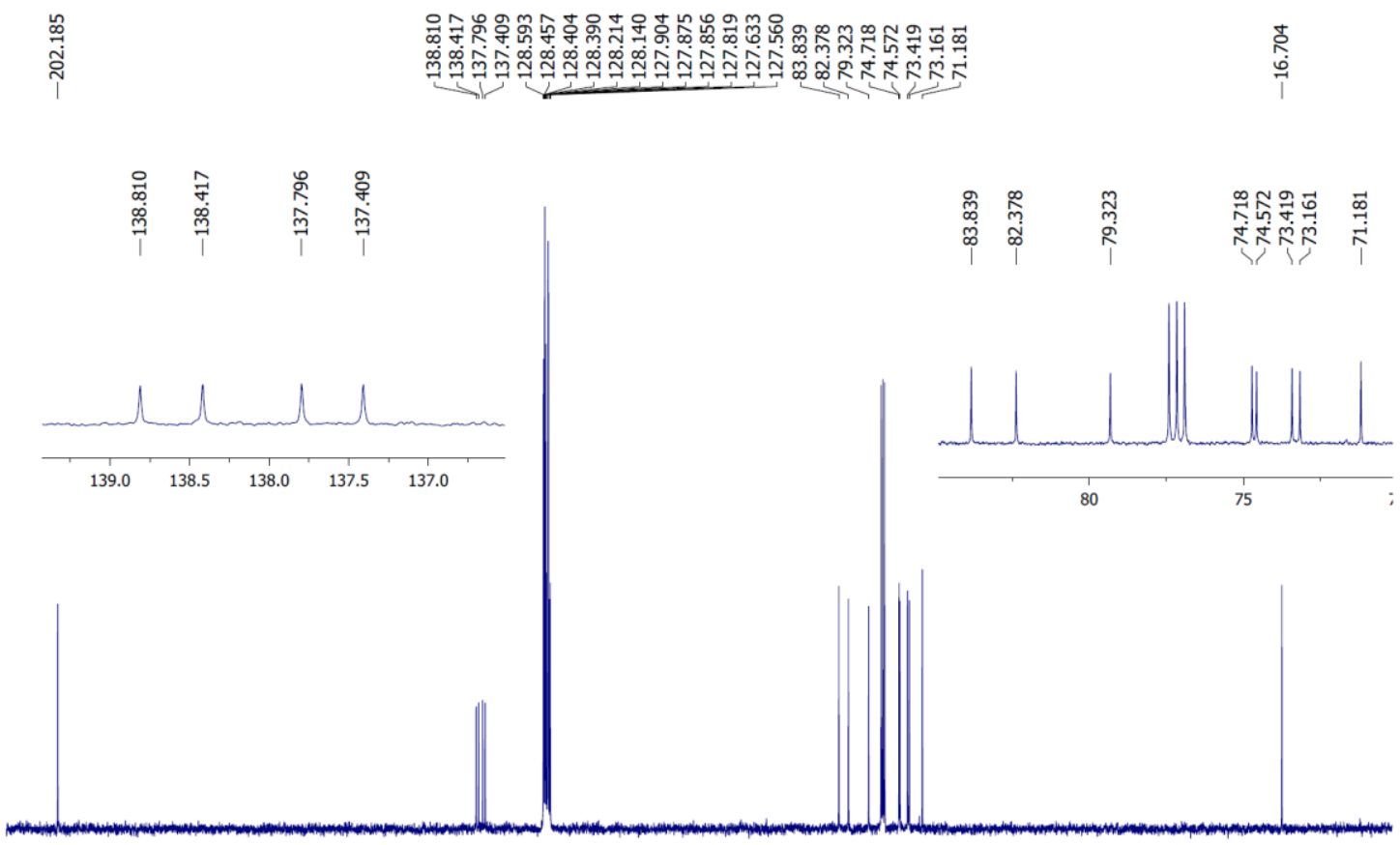

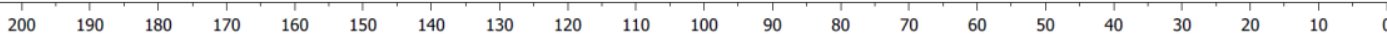


2,3,4,5,6-Penta-O-benzyl aldehydo D-galactose (2f).<smiles>O=CC(Br)(Br)C(Br)C(Br)C(Br)C(Br)COCc1ccccc1</smiles>

$2 f$

2,3,4,5,6-Penta-O-benzyl diethyl dithioacetal D-galactose $\mathbf{1 f}(1.111 \mathrm{~g}, 1.507 \mathrm{mmol})$ was dissolved in an acetone/water mixture (4/1, $25 \mathrm{ml})$. Sodium bicarbonate (279 $\mathrm{mg}, 3.32$ mmol, 2.2 equiv.) and iodine ( $843 \mathrm{mg}, 3.32 \mathrm{mmol}, 2.2$ equiv.) were subsequently added at r.t. The mixture was stirred vigorously for $1 \mathrm{~h}$ at room temperature. Then, saturated solution of sodium bicarbonate $(10 \mathrm{ml})$ and saturated solution of sodium thiosulfate $(10$ $\mathrm{ml}$ ) were added and acetone was removed under reduced pressure. The mixture was dissolved in $\mathrm{Et}_{2} \mathrm{O}$, saturated solution of sodium thiosulfate $(10 \mathrm{ml})$ was added and the solution was stirred for $15 \mathrm{~min}$. (until formation of a clear solution). The aqueous phase was extracted with $\mathrm{Et}_{2} \mathrm{O}$ and the combined organic layer were washed with brine (2 times), dried over $\mathrm{MgSO}_{4}$ and concentrated under reduced pressure. The crude was purified by flash chromatography on silica gel (Cy/EtOAc 9:1) to provide 2,3,4,5,6Penta-O-benzyl-aldehydo-D-galactose $\mathbf{2 f}$ as a yellow oil (671 $\mathrm{mg}, 71 \%)$.

$[\alpha]^{20} \mathrm{D}:-0.3\left(\mathrm{c} 1.0, \mathrm{CHCl}_{3}\right) .{ }^{1} \mathrm{H}$ NMR $\left(400 \mathrm{MHz}, \mathrm{CDCl}_{3}\right): \delta=9.69\left(\mathrm{~d}, 1 \mathrm{H}, J_{1-2}=1.2 \mathrm{~Hz}\right.$, $\mathrm{H}-1), 7.35-7.19\left(\mathrm{~m}, 25 \mathrm{H}, \mathrm{H}^{\mathrm{arom}}\right), 4.674\left(\mathrm{AB}, 1 \mathrm{H}, J_{\mathrm{A}-\mathrm{B}}=12.0 \mathrm{~Hz}, \mathrm{CH}_{2}{ }^{\mathrm{Bn}}\right), 4.672(\mathrm{AB}, 1 \mathrm{H}$, $\left.J_{\mathrm{A}-\mathrm{B}}=11.8 \mathrm{~Hz}, \mathrm{CH}_{2}{ }^{\mathrm{Bn}}\right), 4.62\left(\mathrm{AB}, 1 \mathrm{H}, J_{\mathrm{A}-\mathrm{B}}=11.6 \mathrm{~Hz}, \mathrm{CH}_{2}{ }^{\mathrm{Bn}}\right), 4.570\left(\mathrm{AB}, 1 \mathrm{H}, J_{\mathrm{A}-\mathrm{B}}=11.6\right.$ $\left.\mathrm{Hz}, \mathrm{CH}_{2}{ }^{\mathrm{Bn}}\right), 4.568\left(\mathrm{AB}, 1 \mathrm{H}, J_{\mathrm{A}-\mathrm{B}}=11.7 \mathrm{~Hz}, \mathrm{CH}_{2}{ }^{\mathrm{Bn}}\right), 4.54\left(\mathrm{AB}, 1 \mathrm{H}, J_{\mathrm{A}-\mathrm{B}}=11.5 \mathrm{~Hz}, \mathrm{CH}_{2}{ }^{\mathrm{Bn}}\right)$, $4.46\left(\mathrm{AB}, 2 \mathrm{H}, J_{\mathrm{A}-\mathrm{B}}=11.8 \mathrm{~Hz}, \mathrm{CH}_{2}{ }^{\mathrm{Bn}}\right), 4.42\left(\mathrm{AB}, 2 \mathrm{H}, J_{\mathrm{A}-\mathrm{B}}=11.7 \mathrm{~Hz}, \mathrm{CH}_{2}{ }^{\mathrm{Bn}}\right), 4.12(\mathrm{dd}$, $\left.1 \mathrm{H}, J_{3-4}=5.7, J_{2-3}=3.8 \mathrm{~Hz}, \mathrm{H}-3\right), 4.09\left(\mathrm{dd}, 1 \mathrm{H}, J_{2-3}=3.7, J_{1-2}=1.2 \mathrm{~Hz}, \mathrm{H}-2\right), 4.03(\mathrm{dd}$, $\left.1 \mathrm{H}, J_{3-4}=5.7, J_{4-5}=4.6 \mathrm{~Hz}, \mathrm{H}-4\right), 3.98\left(\mathrm{q}, 1 \mathrm{H}, J_{4-5}=4.9 \mathrm{~Hz}, \mathrm{H}-5\right), 3.62\left(\mathrm{dq}, \mathrm{H}, J_{6 \mathrm{a}-6 \mathrm{~b}}=\right.$ 10.0, $\left.J_{5-6}=5.0 \mathrm{~Hz}, \mathrm{H}-6\right) \mathrm{ppm} .{ }^{13} \mathrm{C} \mathrm{NMR}\left(125 \mathrm{MHz}, \mathrm{CDCl}_{3}\right): \delta=201.9(\mathrm{C}-1), 138.8$ $\left(\mathrm{C}_{\mathrm{q}}{ }^{\text {arom }}\right), 138.4(\mathrm{Cq}$ arom $), 138.2\left(\mathrm{C}_{\mathrm{q}}{ }^{\text {arom }}\right), 137.9\left(\mathrm{C}_{\mathrm{q}}{ }^{\text {arom }}\right) 137.4\left(\mathrm{C}_{\mathrm{q}}{ }^{\text {arom }}\right), 128.6-127.6$ $\left(\mathrm{CH}^{\text {arom }}\right), 83.7(\mathrm{C}-2), 79.4(\mathrm{C}-3), 79.2(\mathrm{C}-4), 78.2(\mathrm{C}-5), 74.6\left(\mathrm{CH}_{2}{ }^{\mathrm{Bn}}\right), 73.5\left(\mathrm{CH}_{2}{ }^{\mathrm{Bn}}\right), 73.21$ $\left(\mathrm{CH}_{2}{ }^{\mathrm{Bn}}\right), 73.17\left(\mathrm{CH}_{2}{ }^{\mathrm{Bn}}\right), 73.1\left(\mathrm{CH}_{2}{ }^{\mathrm{Bn}}\right), 70.1(\mathrm{C}-6) \mathrm{ppm}$. HRMS (ESI+): m/z calculated for $\mathrm{C}_{40} \mathrm{H}_{42} \mathrm{O}_{6} \mathrm{Na}[\mathrm{M}+\mathrm{Na}]^{+}$: calc. 653.2874 ; found : 653.2875 . 


\section{${ }^{1}$ H NMR}

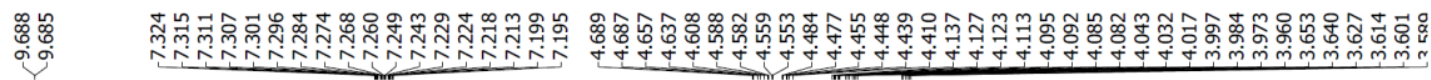

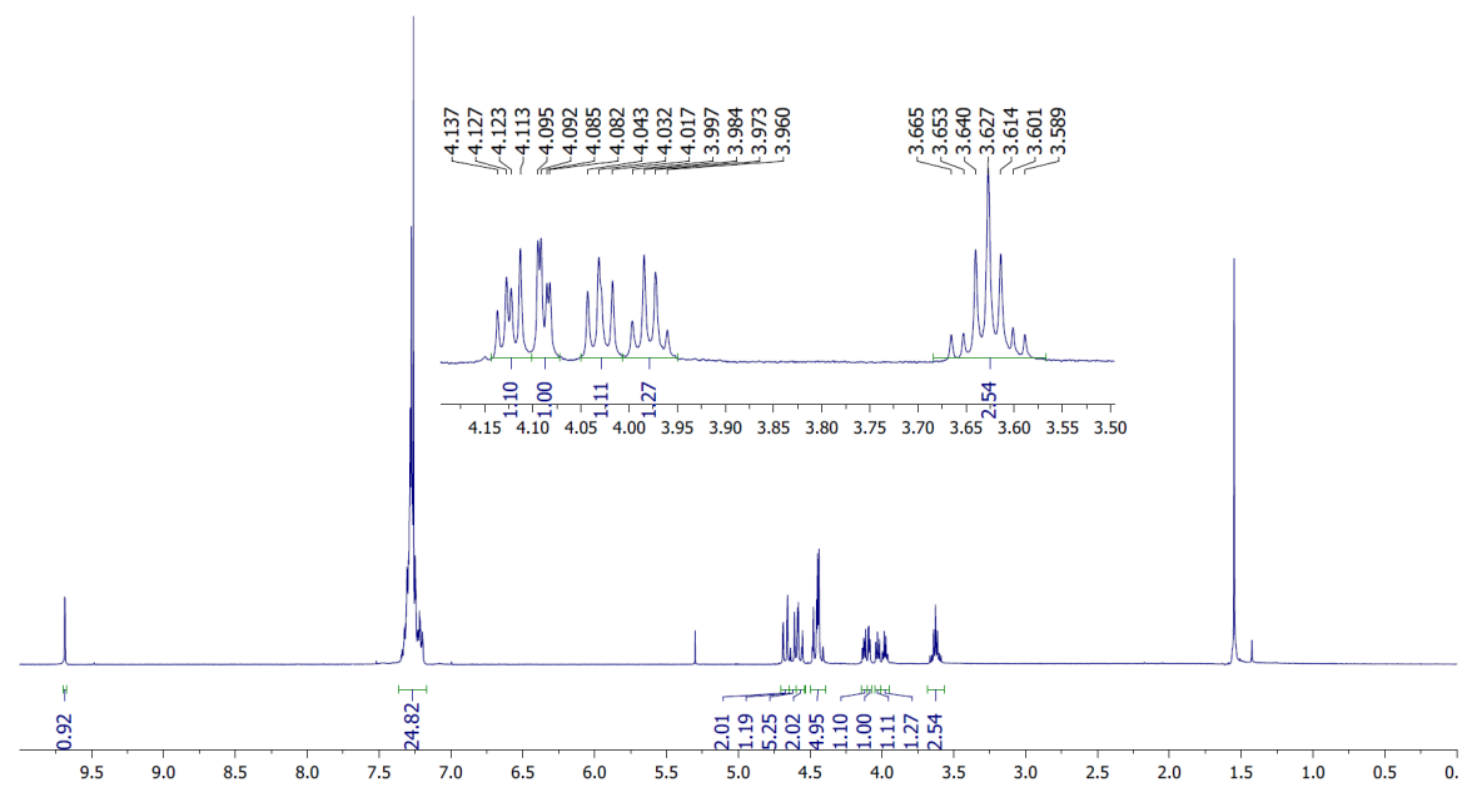

${ }^{13}$ C NMR
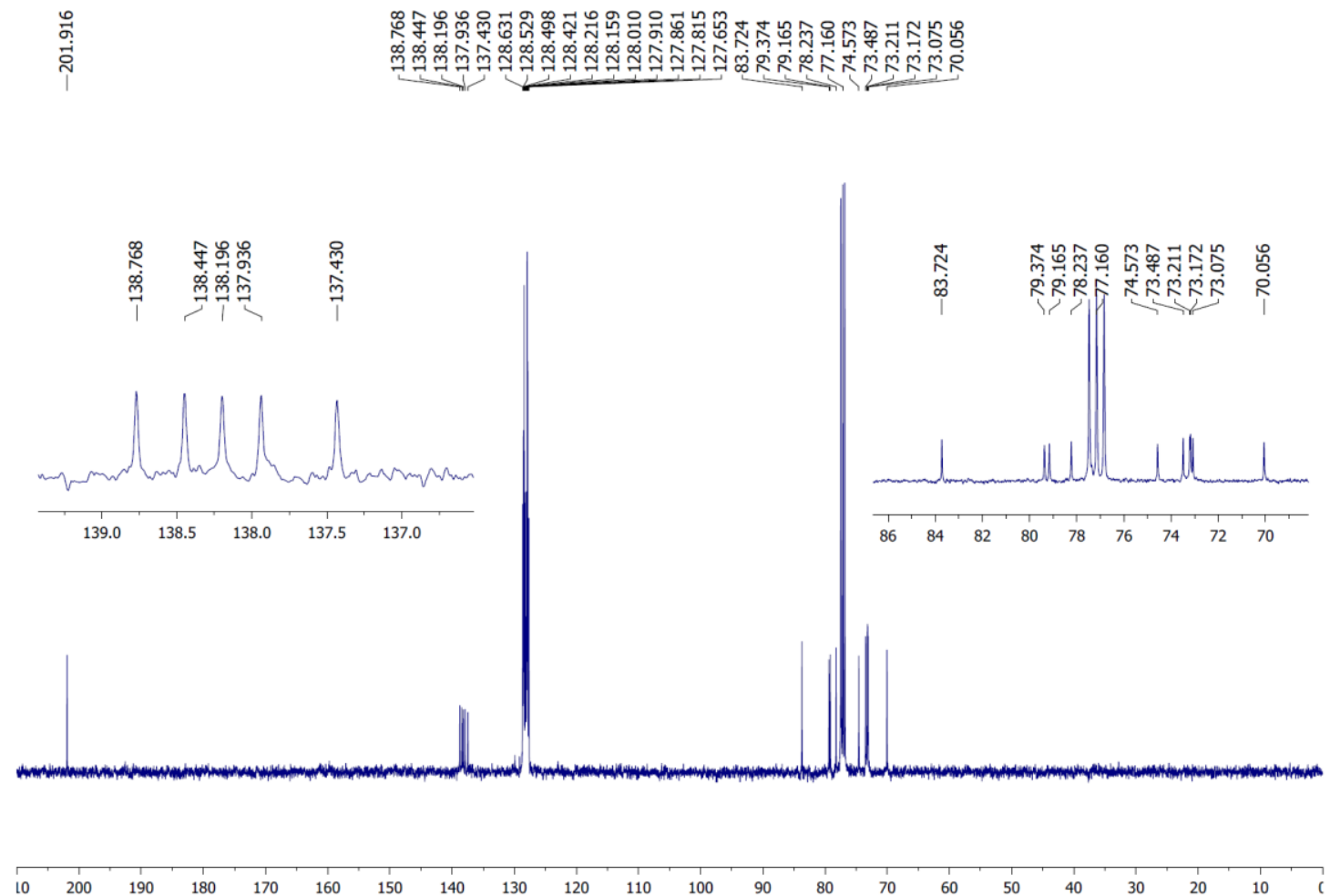
<smiles>O=CC(Br)(Br)C(Br)C(Br)C(Br)C(Br)C(Cc1ccccc1)OCc1ccccc1</smiles>

$2 g$

2,3,4,5,6-Penta-O-benzyl diethyl dithioacetal D-glucose $1 \mathrm{~g}$ (1.289 g, $1.749 \mathrm{mmol})$ was dissolved in an acetone/water mixture (4/1, $28 \mathrm{ml}$ ). Sodium bicarbonate (324 $\mathrm{mg}, 3.848$ mmol, 2.2 equiv.) and iodine (977 mg, $3.848 \mathrm{mmol}, 2.2$ equiv.) were subsequently added at r.t. The mixture was stirred vigorously for $1 \mathrm{~h}$ at room temperature. Then, saturated solution of sodium bicarbonate $(10 \mathrm{ml})$ and saturated solution of sodium thiosulfate (10 $\mathrm{ml}$ ) were added and acetone was removed under reduced pressure. The mixture was dissolved in $\mathrm{Et}_{2} \mathrm{O}$, saturated solution of sodium thiosulfate $(10 \mathrm{ml})$ was added and the solution was stirred for $15 \mathrm{~min}$. (until formation of a clear solution). The aqueous phase was extracted with $\mathrm{Et}_{2} \mathrm{O}$ and the combined organic layer were washed with brine (2 times), dried over $\mathrm{MgSO}_{4}$ and concentrated under reduced pressure. The crude was purified by flash chromatography on silica gel (Cy/EtOAc 9:1) to provide 2,3,4,5,6Penta-O-benzyl-aldehydo-D-glucose $\mathbf{2 g}$ as a yellow oil (887 $\mathrm{mg}, 80 \%)$.

$[\alpha]_{20} \mathrm{D}:+2.8\left(\mathrm{c} 1.0, \mathrm{CHCl}_{3}\right) .{ }^{1} \mathrm{H} \mathrm{NMR}\left(400 \mathrm{MHz}, \mathrm{CDCl}_{3}\right): \delta=9.71(\mathrm{~s}, 1 \mathrm{H}, \mathrm{H}-1), 7.34-7.20$ $\left(\mathrm{m}, 23 \mathrm{H}, \mathrm{H}^{\text {arom }}\right), 7.18-7.15\left(\mathrm{~m}, 2 \mathrm{H}, \mathrm{H}^{\text {arom }}\right), 4.78\left(\mathrm{AB}, 1 \mathrm{H}, J_{\mathrm{A}-\mathrm{B}}=12.0 \mathrm{~Hz}, \mathrm{CH}_{2}{ }^{\mathrm{Bn}}\right), 4.65$ $\left(\mathrm{AB}, 1 \mathrm{H}, J_{\mathrm{A}-\mathrm{B}}=11.7 \mathrm{~Hz}, \mathrm{CH}_{2}{ }^{\mathrm{Bn}}\right), 4.59-4.46\left(\mathrm{~m}, 7 \mathrm{H}, \mathrm{CH}_{2}{ }^{\mathrm{Bn}}\right), 4.41\left(\mathrm{AB}, 1 \mathrm{H}, J_{\mathrm{A}-\mathrm{B}}=11.7\right.$ $\left.\mathrm{Hz}, \mathrm{CH}_{2}{ }^{\mathrm{Bn}}\right), 4.12\left(\mathrm{dd}, 1 \mathrm{H}, J_{2-3}=5.5, J_{3-4}=3.5 \mathrm{~Hz}, \mathrm{H}-3\right), 4.03\left(\mathrm{dd}, 1 \mathrm{H}, J_{4-5}=6.8, J_{3-4}=\right.$ $3.5 \mathrm{~Hz}, \mathrm{H}-4), 3.89$ (d, $\left.1 \mathrm{H}, J_{2-3}=5.5 \mathrm{~Hz}, \mathrm{H}-2\right), 3.87-3.80$ (m, 2H, H-6), 3.68 (dd, $1 \mathrm{H}, J_{5-6}$ $\left.=10.4, J_{4-5}=4.0 \mathrm{~Hz}, \mathrm{H}-5\right) \mathrm{ppm} .{ }^{13} \mathrm{C}$ NMR $\left(125 \mathrm{MHz}, \mathrm{CDCl}_{3}\right): \delta=200.9(\mathrm{C}-1), 138.7$ $\left(\mathrm{C}_{\mathrm{q}}{ }^{\text {arom }}\right), 138.3\left(\mathrm{C}_{\mathrm{q}}{ }^{\text {arom }}\right) 138.2\left(\mathrm{C}_{\mathrm{q}}{ }^{\text {arom }}\right), 137.9\left(\mathrm{C}_{\mathrm{q}}{ }^{\text {arom }}\right), 137.5\left(\mathrm{C}_{\mathrm{q}}{ }^{\text {arom }}\right), 128.6-127.7$ $\left(\mathrm{CH}^{\text {arom }}\right), 81.0(\mathrm{C}-2), 80.2(\mathrm{C}-5), 78.3(\mathrm{C}-3), 77.4(\mathrm{C}-4), 74.2\left(\mathrm{CH}_{2}{ }^{\mathrm{Bn}}\right), 73.8\left(\mathrm{CH}_{2}{ }^{\mathrm{Bn}}\right), 73.5$ $\left(\mathrm{CH}_{2}{ }^{\mathrm{Bn}}\right), 73.2\left(\mathrm{CH}_{2}{ }^{\mathrm{Bn}}\right), 68.7$ (C-6) ppm. HRMS (ESI+): m/z calculated for $\mathrm{C}_{40} \mathrm{H}_{42} \mathrm{O}_{6} \mathrm{Na}$ $[\mathrm{M}+\mathrm{Na}]^{+}$: calc. 653.2874 ; found: 653.2872 . 


\section{${ }^{1}$ H NMR}

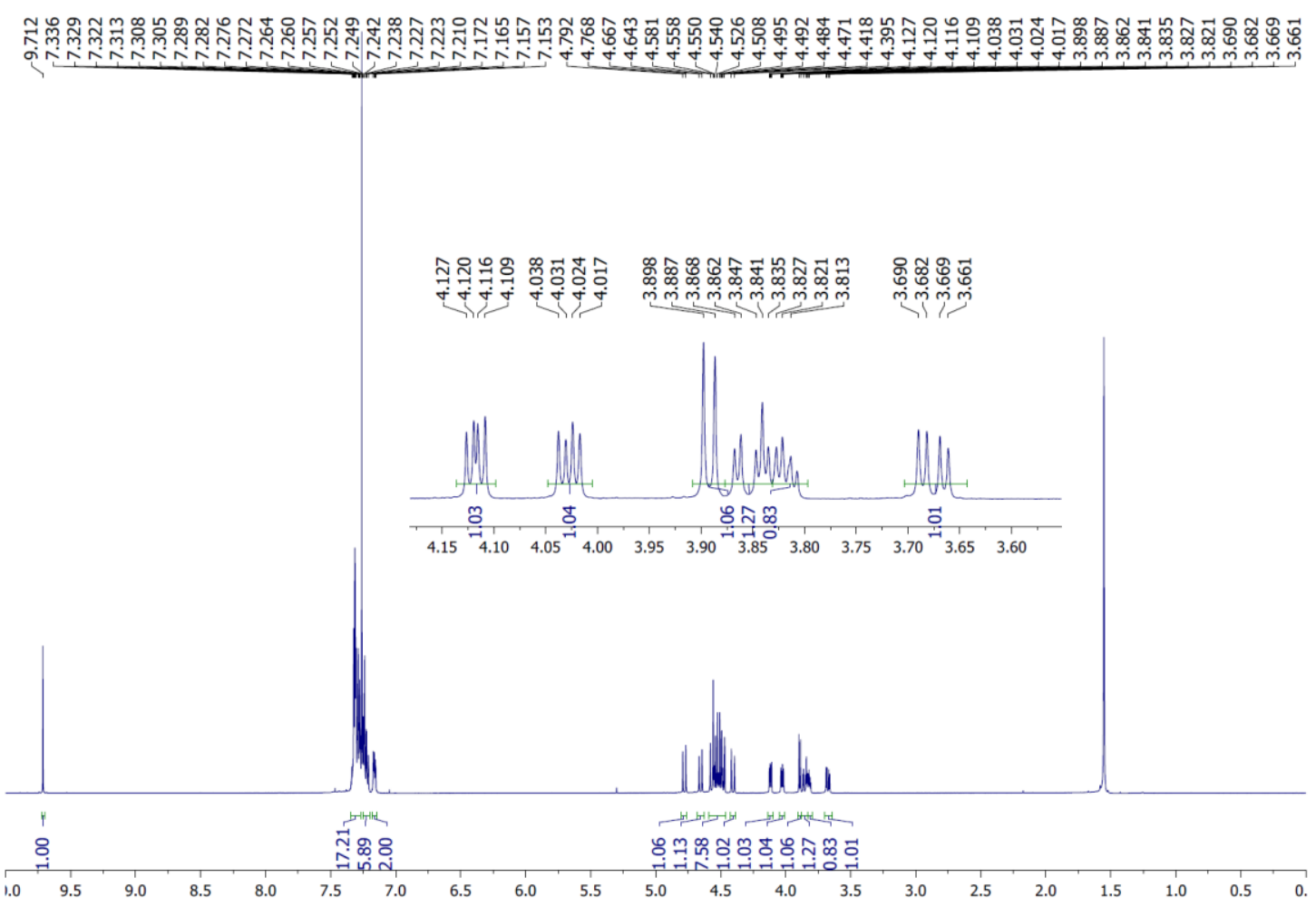

\section{${ }^{13}$ C NMR}
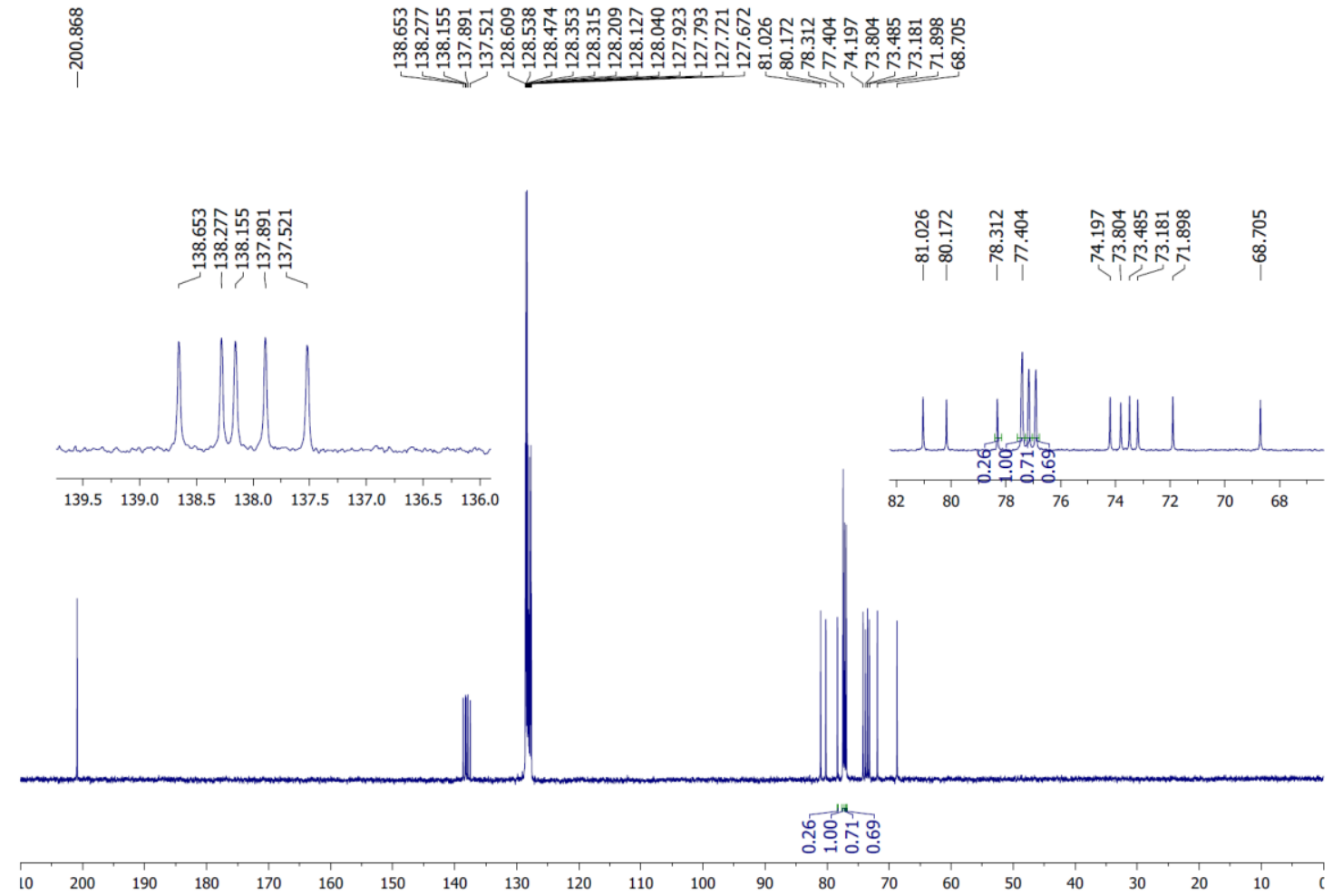


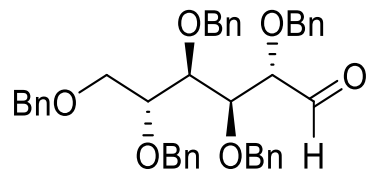

$2 \mathrm{~h}$

2,3,4,5,6-Penta-O-benzyl diethyl dithioacetal D-mannose $\mathbf{1 h}$ (1.236 g, $1.677 \mathrm{mmol})$ was dissolved in an acetone/water mixture (4/1, $28 \mathrm{ml})$. Sodium bicarbonate $(311 \mathrm{mg}, 3.689$ mmol, 2.2 equiv.) and iodine (939 mg, $3.689 \mathrm{mmol}, 2.2$ equiv.) were subsequently added at r.t. The mixture was stirred vigorously for $2 \mathrm{~h}$ at room temperature. Then, saturated solution of sodium bicarbonate $(10 \mathrm{ml})$ and saturated solution of sodium thiosulfate (10 $\mathrm{ml}$ ) were added and acetone was removed under reduced pressure. The mixture was dissolved in $\mathrm{Et}_{2} \mathrm{O}$, saturated solution of sodium thiosulfate $(10 \mathrm{ml})$ was added and the solution was stirred for $15 \mathrm{~min}$. (until formation of a clear solution). The aqueous phase was extracted with $\mathrm{Et}_{2} \mathrm{O}$ and the combined organic layer were washed with brine (2 times), dried over $\mathrm{MgSO}_{4}$ and concentrated under reduced pressure. The crude was purified by flash chromatography on silica gel (Cy/EtOAc 9:1) to provide 2,3,4,5,6Penta-O-benzyl-aldehydo-D-mannose $\mathbf{2 h}$ as a colorless oil (727 mg, 69\%).

$[\alpha]_{20}$ D: $-4.6\left(\mathrm{c} 1.0, \mathrm{CHCl}_{3}\right) .{ }^{1} \mathrm{H} \mathrm{NMR}\left(500 \mathrm{MHz}, \mathrm{CDCl}_{3}\right): \delta=9.69(\mathrm{~d}, 1 \mathrm{H}, \mathrm{J}=1.8 \mathrm{~Hz}, \mathrm{H}-$ 1), 7.33-7.26 (m, 20H, $\left.\mathrm{H}^{\text {arom }}\right), 7.25-7.21\left(\mathrm{~m}, 5 \mathrm{H}, \mathrm{H}^{\text {arom }}\right), 4.67\left(\mathrm{AB}, 1 \mathrm{H}, J_{\mathrm{A}-\mathrm{B}}=5.7 \mathrm{~Hz}\right.$, $\left.\mathrm{CH}_{2}{ }^{\mathrm{Bn}}\right), 4.65\left(\mathrm{AB}, 1 \mathrm{H}, J_{\mathrm{A}-\mathrm{B}}=5.7 \mathrm{~Hz}, \mathrm{CH}_{2}{ }^{\mathrm{Bn}}\right), 4.64\left(\mathrm{AB}, 1 \mathrm{H}, J_{\mathrm{A}-\mathrm{B}}=11.7 \mathrm{~Hz}, \mathrm{CH}_{2}{ }^{\mathrm{Bn}}\right), 4.57$ $\left(\mathrm{AB}, 1 \mathrm{H}, J_{\mathrm{A}-\mathrm{B}}=8.2 \mathrm{~Hz}, \mathrm{CH}_{2}{ }^{\mathrm{Bn}}\right), 4.54\left(\mathrm{AB}, 2 \mathrm{H}, J_{\mathrm{A}-\mathrm{B}}=8.4 \mathrm{~Hz}, \mathrm{CH}_{2}{ }^{\mathrm{Bn}}\right), 4.52\left(\mathrm{AB}, 1 \mathrm{H}, J_{\mathrm{A}-}\right.$ $\left.\mathrm{B}=11.7 \mathrm{~Hz}, \mathrm{CH}_{2}{ }^{\mathrm{Bn}}\right), 4.48\left(\mathrm{AB}, 1 \mathrm{H}, J_{\mathrm{A}-\mathrm{B}}=12.0 \mathrm{~Hz}, \mathrm{CH}_{2}{ }^{\mathrm{Bn}}\right), 4.44\left(\mathrm{AB}, 1 \mathrm{H}, J_{\mathrm{A}-\mathrm{B}}=11.7\right.$ $\mathrm{Hz}, \mathrm{CH}_{2}{ }^{\mathrm{Bn}}$ ), $4.31\left(\mathrm{AB}, 1 \mathrm{H}, J_{\mathrm{A}-\mathrm{B}}=11.7 \mathrm{~Hz}, \mathrm{CH}_{2}{ }^{\mathrm{Bn}}\right.$ ), 4.12 (t, $\left.1 \mathrm{H}, J_{2-3}=4.2 \mathrm{~Hz}, \mathrm{H}-2\right), 3.98$ $\left(\mathrm{dq}, 2 \mathrm{H}, J_{2-3}=4.3, J_{3-4}=2.4 \mathrm{~Hz}, \mathrm{H}-3\right.$ and H-4), 3.89-3.83 (m, 2H, H-5 and H-6), 3.713.67 (m, 1H, H-6) ppm. $\left.{ }^{13} \mathrm{C} \mathrm{NMR} \mathrm{(125} \mathrm{MHz,} \mathrm{CDCl}_{3}\right): \delta=201.8(\mathrm{C}-1), 138.5\left(\mathrm{C}_{\mathrm{q}}{ }^{\text {arom }}\right)$, $138.3\left(\mathrm{C}_{\mathrm{q}}{ }^{\text {arom }}\right) 138.2\left(\mathrm{C}_{\mathrm{q}}{ }^{\text {arom }}\right), 138.1\left(\mathrm{C}_{\mathrm{q}}{ }^{\text {arom }}\right), 137.4\left(\mathrm{C}_{\mathrm{q}}{ }^{\text {arom }}\right), 128.5-127.7\left(\mathrm{CH}^{\text {arom }}\right), 84.4$ (C-3 or C-4), 80.5 (C-2), $79.0\left(\mathrm{C}-3\right.$ or C-4), $78.4(\mathrm{C}-5), 74.4\left(\mathrm{CH}_{2}{ }^{\mathrm{Bn}}\right), 74.2\left(\mathrm{CH}_{2}{ }^{\mathrm{Bn}}\right), 73.5$ $\left(\mathrm{CH}_{2}{ }^{\mathrm{Bn}}\right), 72.5\left(\mathrm{CH}_{2}{ }^{\mathrm{Bn}}\right), 71.9\left(\mathrm{CH}_{2}{ }^{\mathrm{Bn}}\right), 68.9$ (C-6) ppm. HRMS (ESI+): m/z calculated for $\mathrm{C}_{40} \mathrm{H}_{42} \mathrm{O}_{6} \mathrm{Na}[\mathrm{M}+\mathrm{Na}]^{+}$: calc. 653.2874 ; found: 653.2874 . 


\section{${ }^{1}$ H NMR}

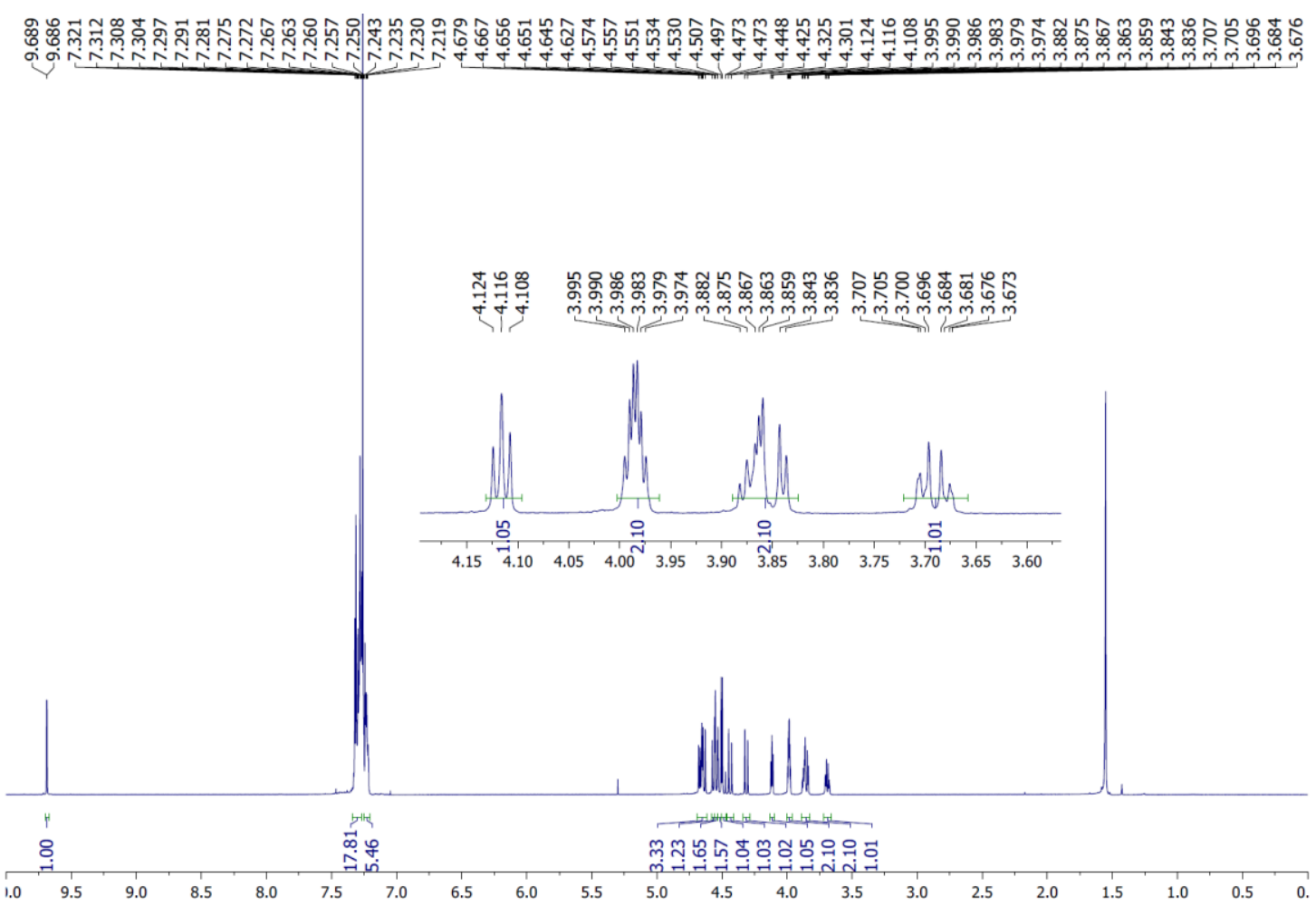

\section{${ }^{13}$ C NMR}
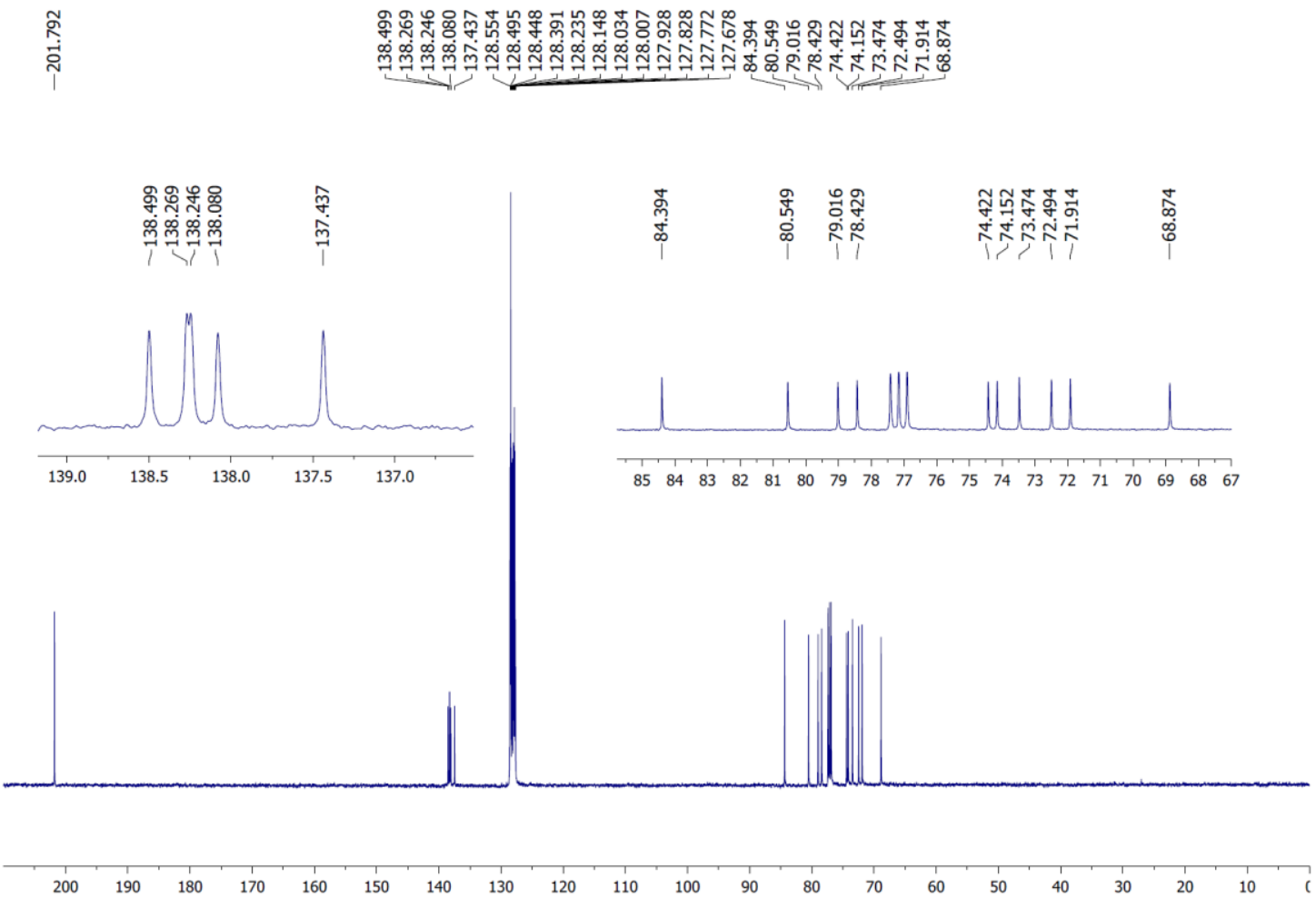


\section{Reformatsky products}

\section{General procedure Zinc activation}

To a Buchner with two filter paper $(\phi 110 \mathrm{~mm})$ was added zinc dust $(100 \mathrm{~g}, 98+\%,<10$ microns). The solid was washed abundantly with $\mathrm{HCl}(10 \%, 500 \mathrm{ml})$ and triturated under the hood. Then, the solid was subsequently washed and triturated with $\mathrm{H}_{2} \mathrm{O}(500 \mathrm{ml})$, $\mathrm{EtOH}(500 \mathrm{ml})$ and $\mathrm{Et}_{2} \mathrm{O}(500 \mathrm{ml})$. The grey powder was dried and stored in the oven at $110^{\circ} \mathrm{C}$.

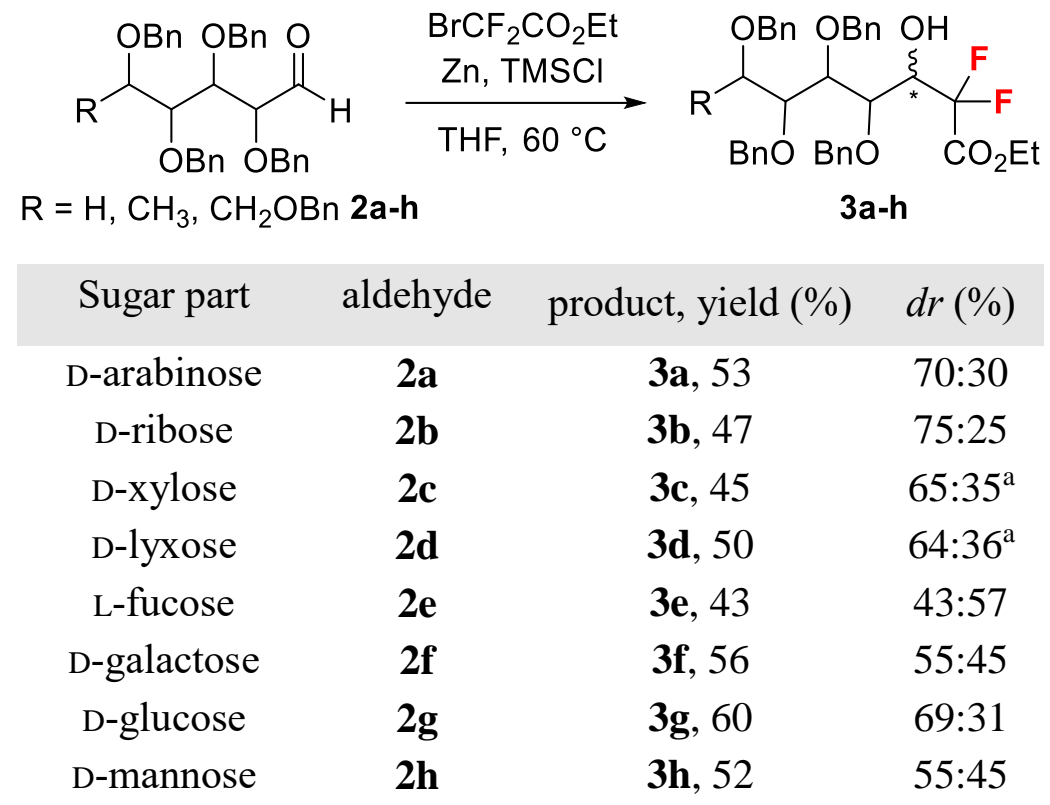

${ }^{a}$ Inseparable mixture by silica gel chromatography 


\section{D-Arabinose}

Ethyl $\quad(1 R / 1 S, 2 R, 3 R, 4 R)$-2,3,4,5-tetra-O-benzyl-1',1'-difluoro-1-hydroxyheptanoate $((R / S)-3 a)$.<smiles>O=C[C@H](OCc1ccccc1)[C@H](Br)OCc1ccccc1</smiles>

$2 \mathbf{a}$<smiles>CCOC(=O)C(F)(F)Br</smiles>

THF<smiles>CCOC(=O)C(F)(F)[C@@H](O)[C@H](OCc1ccccc1)[C@H](Br)[C@H](Br)COc1ccccc1</smiles>

(S)-3a
$53 \%$ $\mathrm{R}: \mathrm{S} d r 70: 30$<smiles>CCOC(=O)C(F)(F)[C@@H](O)[C@H](Br)[C@H](Br)[C@H](Br)[C@H](Br)COCc1ccccc1</smiles>

(R)-3a

To a dry two-necked round bottomed flask was added "activated zinc" powder (1.88 g, $28.77 \mathrm{mmol}, 5.7$ equiv.). The flask was heated up at $40^{\circ} \mathrm{C}$ and the zinc powder suspended in dry THF (15 ml) under argon atmosphere. Then, TMSCl $(0.27 \mathrm{ml}, 2.17 \mathrm{mmol}, 0.4$ equiv.) was added dropwise and the reaction was stirred at $60^{\circ} \mathrm{C}$ for 15 mins. Next, a solution of aldehyde $\mathbf{2 a}$ ( $2.57 \mathrm{~g}, 5.04 \mathrm{mmol}, 1.0$ equiv.) and ethyl bromo-difluoroacetate ( $0.77 \mathrm{ml}, 6.05 \mathrm{mmol}, 1.2$ equiv.) dissolved homogenously in dry THF (15 ml) under argon atmosphere was added dropwise at $60^{\circ} \mathrm{C}$. The reaction was stirred vigorously for $1 \mathrm{~h}$ at $60^{\circ} \mathrm{C}$ and then cooled at room temperature. Ice water $(30 \mathrm{ml})$ and $\mathrm{HCl}(1 \mathrm{M}, 30 \mathrm{ml})$ were added and the mixture was diluted with EtOAc. The organic phase was washed with $\mathrm{NaHCO}_{3}$ (sat) (2 times), brine, dried over $\mathrm{MgSO}_{4}$ and concentrated under reduced pressure. Crude NMR analysis showed the formation of two diastereoisomer $(R)-3 \mathbf{a}$ and 3a-(S) in 70:30 $d r$ ratio. Purification by silica gel chromatography (Cy/EtOAc 9:1) afforded pure diastereoisomer $(S)$-3a and an inseparable mixture of two diastereoisomers $(S)$-3a and $(R)-3 \mathbf{a}$ in 89:11 $d r$ ratio in 53\% overall yield. Noteworthy, few milligrams (10 $\mathrm{mg}$ ) of the diastereoisomer $(R)$-3a could be isolated and fully characterized. 
Ethyl (1R,2R,3R,4R)-2,3,4,5-tetra-O-benzyl-1',1'-difluoro-1-hydroxyheptanoate $\quad((R)$ $3 a)$.

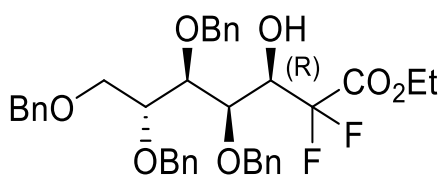

(R)-3a

$[\alpha]^{20} \mathrm{D}:-3.1\left(\mathrm{c} 1.0, \mathrm{CHCl}_{3}\right) .{ }^{1} \mathrm{H}$ NMR $\left(500 \mathrm{MHz}, \mathrm{CDCl}_{3}\right) \delta=7.35-7.27\left(\mathrm{~m}, 14 \mathrm{H}, \mathrm{H}^{\text {arom }}\right)$, 7.26-7.20 (m, 6H, $\left.\mathrm{H}^{\text {arom }}\right), 4.73\left(\mathrm{AB}, 1 \mathrm{H}, J_{\mathrm{A}-\mathrm{B}}=11.5 \mathrm{~Hz}, \mathrm{CH}_{2}{ }^{\mathrm{Bn}}\right), 4.67\left(\mathrm{AB}, 1 \mathrm{H}, J_{\mathrm{A}-\mathrm{B}}=\right.$ $\left.11.5 \mathrm{~Hz}, \mathrm{CH}_{2}{ }^{\mathrm{Bn}}\right), 4.64\left(\mathrm{AB}, 1 \mathrm{H}, J_{\mathrm{A}-\mathrm{B}}=11.5 \mathrm{~Hz}, \mathrm{CH}_{2}{ }^{\mathrm{Bn}}\right), 4.54-4.49\left(\mathrm{~m}, 4 \mathrm{H}_{,} \mathrm{CH}_{2}{ }^{\mathrm{Bn}}\right), 4.45$ $\left(\mathrm{AB}, 1 \mathrm{H}, J_{\mathrm{A}-\mathrm{B}}=10.9 \mathrm{~Hz}, \mathrm{CH}_{2}{ }^{\mathrm{Bn}}\right), 4.38(\mathrm{~m}, 1 \mathrm{H}, \mathrm{H}-1), 4.05\left(\mathrm{dd}, 1 \mathrm{H}, J_{2-3}=4.0 \mathrm{~Hz}, J_{3-4}=\right.$ $5.7 \mathrm{~Hz}, \mathrm{H}-3), 4.03-3.95$ (m, 2H, $\left.\mathrm{CH}_{2} \mathrm{CH}_{3}\right), 3.93-3.88$ (m, 2H, H-4, H-5a), 3.87 (t, $1 \mathrm{H}, J_{2-}$ $\left.3_{3}=3.4 \mathrm{~Hz}, \mathrm{H}-2\right), 3.71\left(\mathrm{ABX}, 1 \mathrm{H}, J_{4-5 \mathrm{~b}}=4.6 \mathrm{~Hz}, J_{5 \mathrm{a}-5 \mathrm{~b}}=10.3 \mathrm{~Hz}, \mathrm{H}-5 \mathrm{~b}\right), 1.14(\mathrm{t}, 3 \mathrm{H}$, $\left.J_{\mathrm{CH} 2 \mathrm{CH} 3}=7.5 \mathrm{~Hz}, \mathrm{CH}_{2} \mathrm{CH}_{3}\right)$ ppm. ${ }^{13} \mathrm{C} \mathrm{NMR}\left(125 \mathrm{MHz}, \mathrm{CDCl}_{3}\right) \delta=163.3\left(\mathrm{t}, J_{\mathrm{C}-\mathrm{F}}=31.4\right.$ $\left.\mathrm{Hz}, \mathrm{C}_{\mathrm{q}} \mathrm{O}_{2} \mathrm{Et}\right), 138.1\left(\mathrm{C}_{\mathrm{q}}{ }^{\text {arom }}\right), 138.0\left(\mathrm{C}_{\mathrm{q}}{ }^{\text {arom }}\right), 137.9\left(\mathrm{C}_{\mathrm{q}}{ }^{\text {arom }}\right), 137.7\left(\mathrm{C}_{\mathrm{q}}{ }^{\text {arom }}\right), 128.6-127.7$ $\left(\mathrm{CH}^{\text {arom }}\right), 114.8\left(\mathrm{dd}, J_{\mathrm{C}-\mathrm{F}}=252.3 \mathrm{~Hz}, J_{\mathrm{C}-\mathrm{F}}=255.9 \mathrm{~Hz}, \mathrm{CF}_{2}\right), 78.7(\mathrm{C}-4), 78.2(\mathrm{C}-3), 76.6$ (C-2), $74.1\left(\mathrm{CH}_{2}{ }^{\mathrm{Bn}}\right), 73.6\left(\mathrm{CH}_{2}{ }^{\mathrm{Bn}}\right), 73.5\left(\mathrm{CH}_{2}{ }^{\mathrm{Bn}}\right), 72.4\left(\mathrm{CH}_{2}{ }^{\mathrm{Bn}}\right), 71.6\left(\mathrm{t}, J_{\mathrm{C}-\mathrm{F}}=21.8 \mathrm{~Hz}, \mathrm{C}-\right.$ 1), $69.2(\mathrm{C}-5), 62.8\left(\mathrm{CH}_{2} \mathrm{CH}_{3}\right), 13.9\left(\mathrm{CH}_{2} \mathrm{CH}_{3}\right)$ ppm. ${ }^{19} \mathrm{~F} \mathrm{NMR}\left(471 \mathrm{MHz}, \mathrm{CDCl}_{3}\right) \delta=-$ $116.9\left(\mathrm{dd}, 1 \mathrm{~F}, J_{\mathrm{H}-\mathrm{Fa}}=9.9 \mathrm{~Hz}, J_{\mathrm{Fa}-\mathrm{Fb}}=260.9 \mathrm{~Hz}, \mathrm{Fa}\right),-120.7\left(\mathrm{dd}, 1 \mathrm{~F}, J_{\mathrm{H}-\mathrm{Fb}}=15.3 \mathrm{~Hz}, J_{\mathrm{Fa}-}\right.$ $\mathrm{Fb}=260.9 \mathrm{~Hz}, \mathrm{Fb})$ ppm. HRMS (ESI+): $\mathrm{m} / \mathrm{z}$ calculated for $\mathrm{C}_{37} \mathrm{H}_{40} \mathrm{~F}_{2} \mathrm{NaO}_{7}[\mathrm{M}+\mathrm{Na}]^{+}$: calc. 657.2634; found: 657.2638 . 


\section{${ }^{1} \mathrm{H}$ NMR}

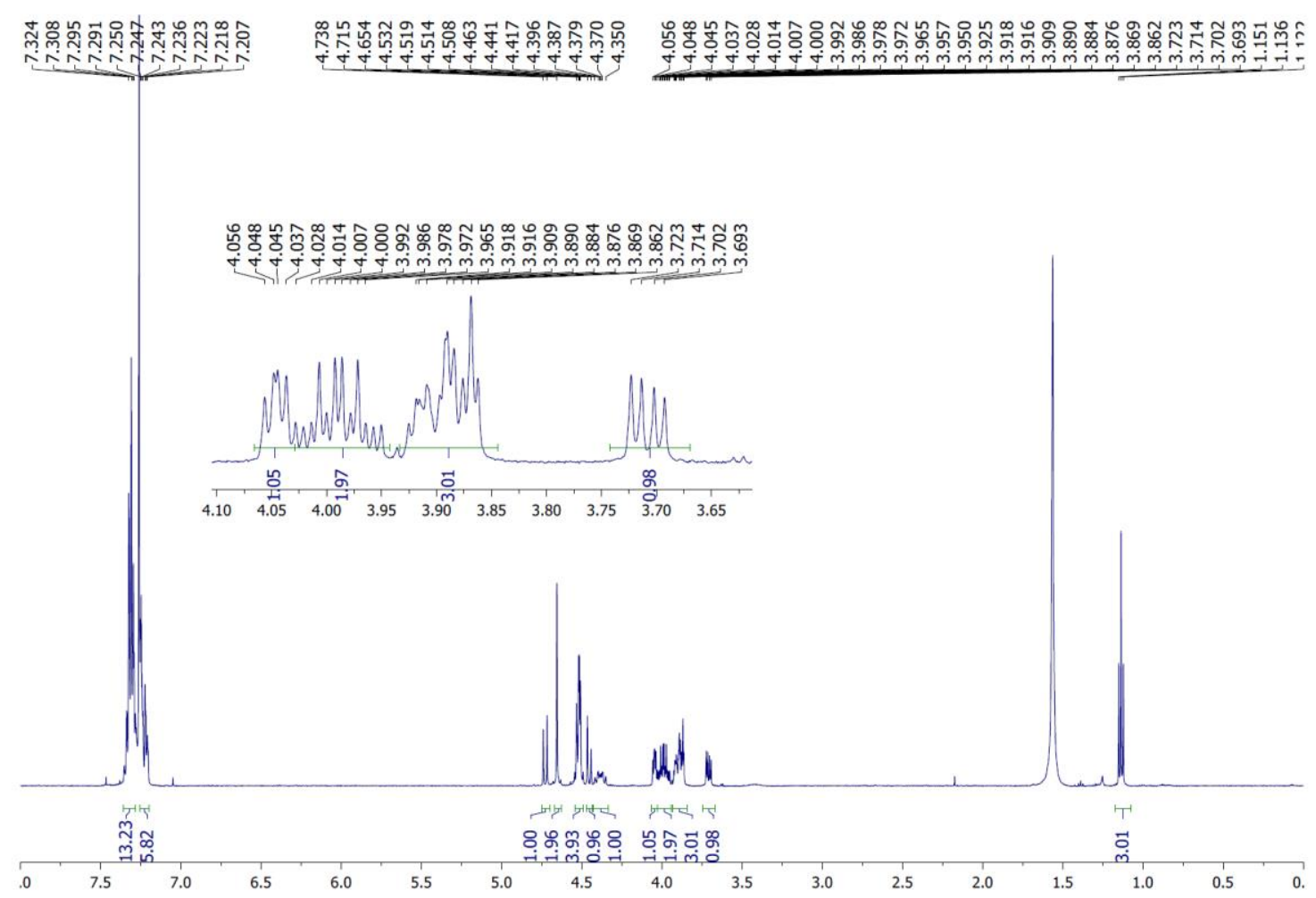

${ }^{19}$ F NMR

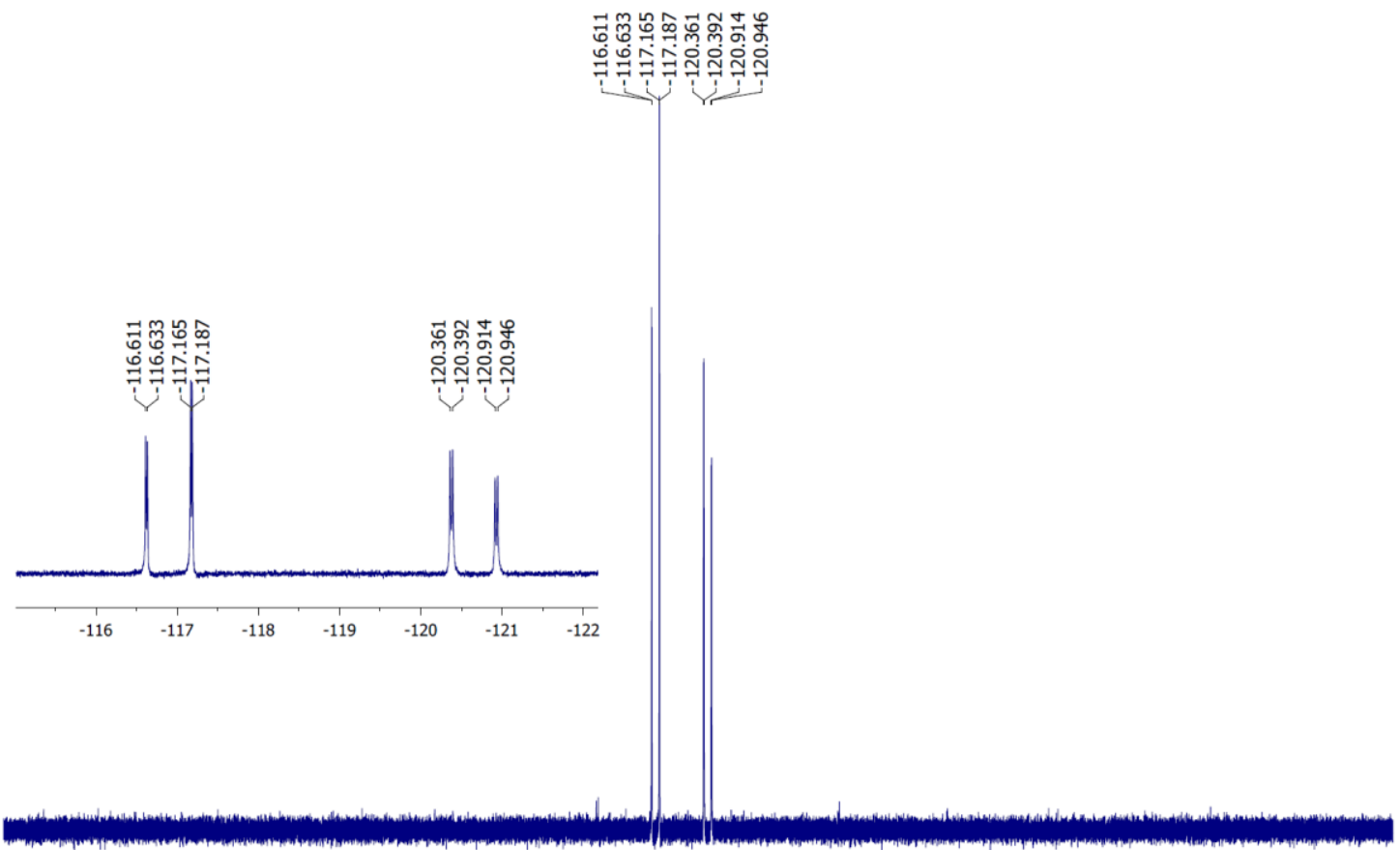

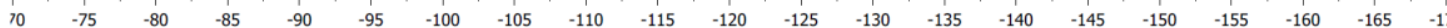


${ }^{13}$ C NMR

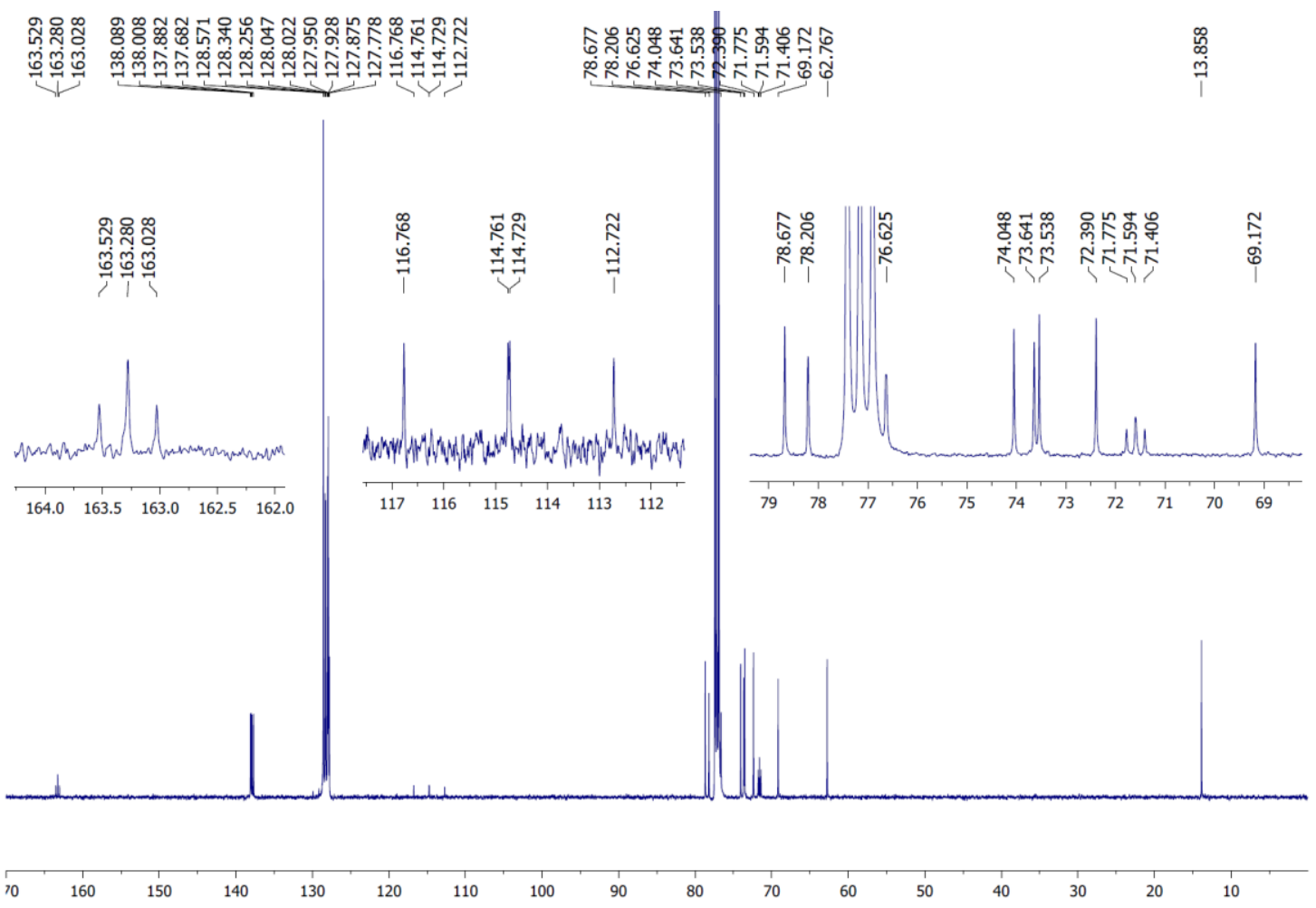


Ethyl (1S,2R,3R,4R)-2,3,4,5-tetra-O-benzyl-1',1'-difluoro-1-hydroxyheptanoate ((S)$3 a)$.<smiles>CCOC(F)(F)[C@@H](O)[C@H](Br)[C@H](Br)[C@H](Br)[C@H](Br)COCc1ccccc1</smiles>

(S)-3a

$[\alpha]^{20} \mathrm{D}:+2.1\left(\mathrm{c} 1.0, \mathrm{CHCl}_{3}\right) .{ }^{1} \mathrm{H} \mathrm{NMR}\left(500 \mathrm{MHz}, \mathrm{CDCl}_{3}\right) \delta=7.33-7.26\left(\mathrm{~m}, 16 \mathrm{H}, \mathrm{H}^{\mathrm{arom}}\right)$, 7.25-7.21 (m, $\left.4 \mathrm{H}, \mathrm{H}^{\mathrm{arom}}\right), 4.70\left(\mathrm{~m}, 2 \mathrm{H}, \mathrm{CH}_{2}{ }^{\mathrm{Bn}}\right), 4.64\left(\mathrm{AB}, 1 \mathrm{H}, J_{\mathrm{A}-\mathrm{B}}=11.5 \mathrm{~Hz}, \mathrm{CH}_{2}{ }^{\mathrm{Bn}}\right)$, $4.61\left(\mathrm{AB}, 1 \mathrm{H}, J_{\mathrm{A}-\mathrm{B}}=11.5 \mathrm{~Hz}, \mathrm{CH}_{2}{ }^{\mathrm{Bn}}\right), 4.57\left(\mathrm{AB}, 1 \mathrm{H}, J_{\mathrm{A}-\mathrm{B}}=11.5 \mathrm{~Hz}, \mathrm{CH}_{2}{ }^{\mathrm{Bn}}\right), 4.53-4.48$ $\left(\mathrm{m}, 3 \mathrm{H}, \mathrm{CH}_{2}{ }^{\mathrm{Bn}}\right), 4.28\left(\mathrm{dd}, 1 \mathrm{H}, J_{1-2}=J_{1-\mathrm{Fa}}=5.7 \mathrm{~Hz}, J_{1-\mathrm{Fb}}=18.3 \mathrm{~Hz}, \mathrm{H}-1\right), 4.16(\mathrm{q}, 2 \mathrm{H}$, $\left.J_{\mathrm{CH} 2 \mathrm{CH} 3}=7.5 \mathrm{~Hz}, \mathrm{CH}_{2} \mathrm{CH}_{3}\right), 4.01\left(\mathrm{~d}, 1 \mathrm{H}, J_{1-2}=J_{2-3}=5.7 \mathrm{~Hz}, \mathrm{H}-2\right), 3.91\left(\mathrm{t}, 1 \mathrm{H}, J_{2-3}=J_{3-4}\right.$ $=5.7 \mathrm{~Hz}, \mathrm{H}-3), 3.87(\mathrm{~m}, 1 \mathrm{H}, \mathrm{H}-4), 3.80\left(\mathrm{ABX}, 1 \mathrm{H}, J_{4-5 \mathrm{a}}=4.0 \mathrm{~Hz}, J_{5 \mathrm{a}-5 \mathrm{~b}}=10.3 \mathrm{~Hz}, \mathrm{H}-5 \mathrm{a}\right)$, $3.70\left(\mathrm{ABX}, 1 \mathrm{H}, J_{4-5 \mathrm{~b}}=5.2 \mathrm{~Hz}, J_{5 \mathrm{a}-5 \mathrm{~b}}=10.3 \mathrm{~Hz}, \mathrm{H}-5 \mathrm{~b}\right), 1.23\left(\mathrm{t}, 3 \mathrm{H}, J_{\mathrm{CH} 2 \mathrm{CH} 3}=7.5 \mathrm{~Hz}\right.$, $\left.\mathrm{CH}_{2} \mathrm{CH}_{3}\right) \mathrm{ppm} .{ }^{13} \mathrm{C} \mathrm{NMR}\left(125 \mathrm{MHz}, \mathrm{CDCl}_{3}\right) \delta=163.6\left(\mathrm{dd}, J_{\mathrm{C}-\mathrm{F}}=30.2 \mathrm{~Hz}, J_{\mathrm{C}-\mathrm{F}}=33.8\right.$ $\left.\mathrm{Hz}, \mathrm{C}_{\mathrm{q}} \mathrm{O}_{2} \mathrm{Et}\right), 138.2\left(2 \mathrm{C}_{\mathrm{q}}{ }^{\text {arom }}\right), 138.1\left(\mathrm{C}_{\mathrm{q}}{ }^{\text {arom }}\right), 137.6\left(\mathrm{C}_{\mathrm{q}}^{\text {arom }}\right), 128.5-127.7\left(\mathrm{CH}^{\text {arom }}\right), 114.5$ $\left(\mathrm{dd}, J_{\mathrm{C}-\mathrm{F}}=253.5 \mathrm{~Hz}, J_{\mathrm{C}-\mathrm{F}}=259.5 \mathrm{~Hz}, \mathrm{CF}_{2}\right), 79.0(\mathrm{C}-4), 78.6(\mathrm{C}-3), 75.2(\mathrm{C}-2), 74.4$ $\left(\mathrm{CH}_{2}{ }^{\mathrm{Bn}}\right), 74.2\left(\mathrm{CH}_{2}{ }^{\mathrm{Bn}}\right), 73.5\left(\mathrm{CH}_{2}{ }^{\mathrm{Bn}}\right), 72.5\left(\mathrm{CH}_{2}{ }^{\mathrm{Bn}}\right), 70.4\left(\mathrm{dd}, J_{\mathrm{C}-\mathrm{F}}=24.7 \mathrm{~Hz}, J_{\mathrm{C}-\mathrm{F}}=28.4\right.$ $\mathrm{Hz}, \mathrm{C}-1), 69.3(\mathrm{C}-5), 63.1\left(\mathrm{CH}_{2} \mathrm{CH}_{3}\right), 14.0\left(\mathrm{CH}_{2} \mathrm{CH}_{3}\right)$ ppm. ${ }^{19} \mathrm{~F} \mathrm{NMR}\left(471 \mathrm{MHz}, \mathrm{CDCl}_{3}\right)$ $\delta=-111.6\left(\mathrm{dd}, 1 \mathrm{~F}, J_{\mathrm{H} 1-\mathrm{Fa}}=5.7 \mathrm{~Hz}, J_{\mathrm{Fa}-\mathrm{Fb}}=260.9 \mathrm{~Hz}, \mathrm{Fa}\right),-122.4\left(\mathrm{dd}, 1 \mathrm{~F}, J_{\mathrm{H}-\mathrm{Fb}}=18.3\right.$ $\left.\mathrm{Hz}, J_{\mathrm{Fa}-\mathrm{Fb}}=260.9 \mathrm{~Hz}, \mathrm{Fb}\right)$ ppm. HRMS $(\mathrm{ESI}+): \mathrm{m} / \mathrm{z}$ calculated for $\mathrm{C}_{37} \mathrm{H}_{40} \mathrm{~F}_{2} \mathrm{NaO}_{7}$ $[\mathrm{M}+\mathrm{Na}]^{+}$: calc. 657.2634; found: 657.2640 . 


\section{${ }^{1}$ H NMR}

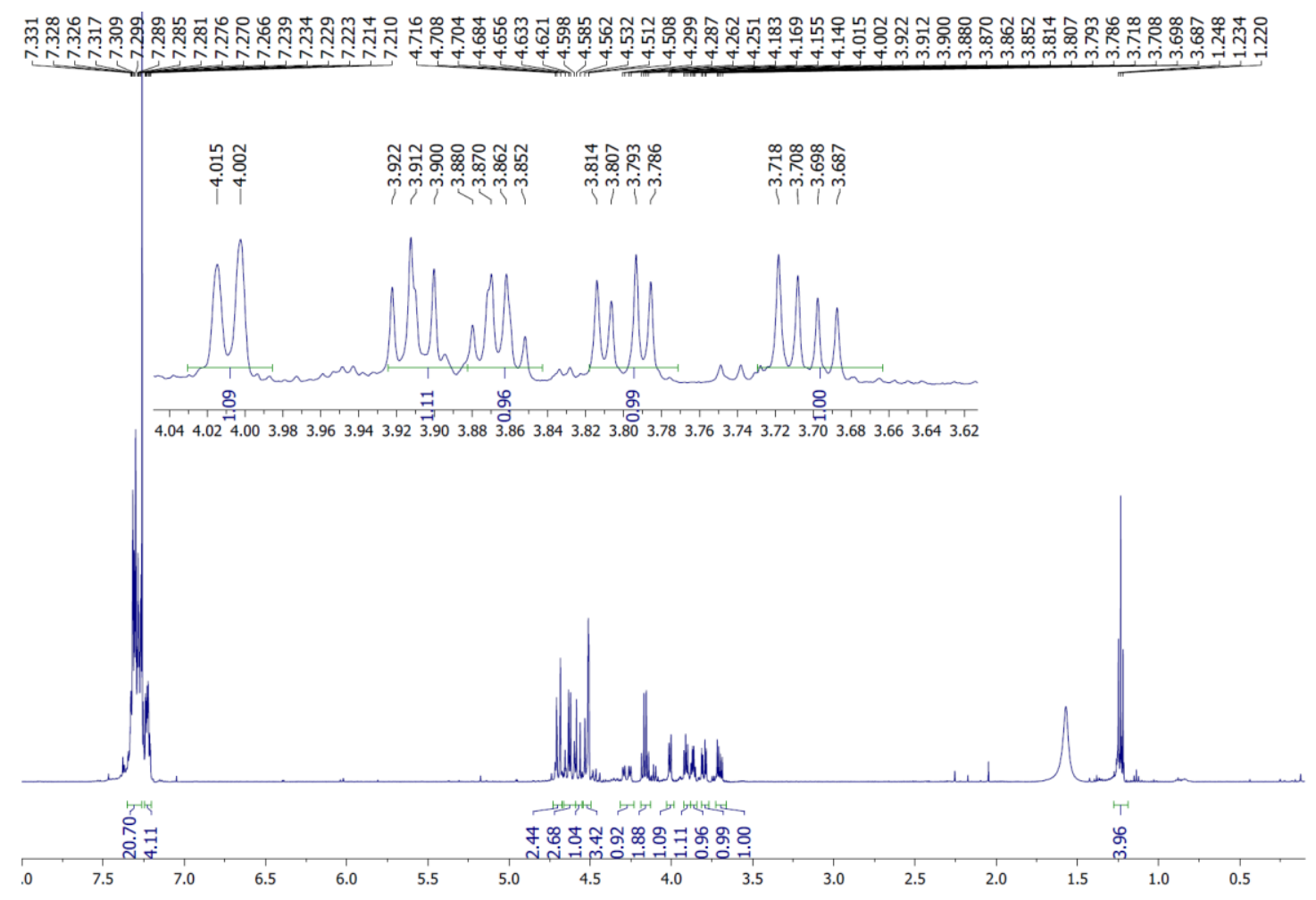

${ }^{19}$ F NMR

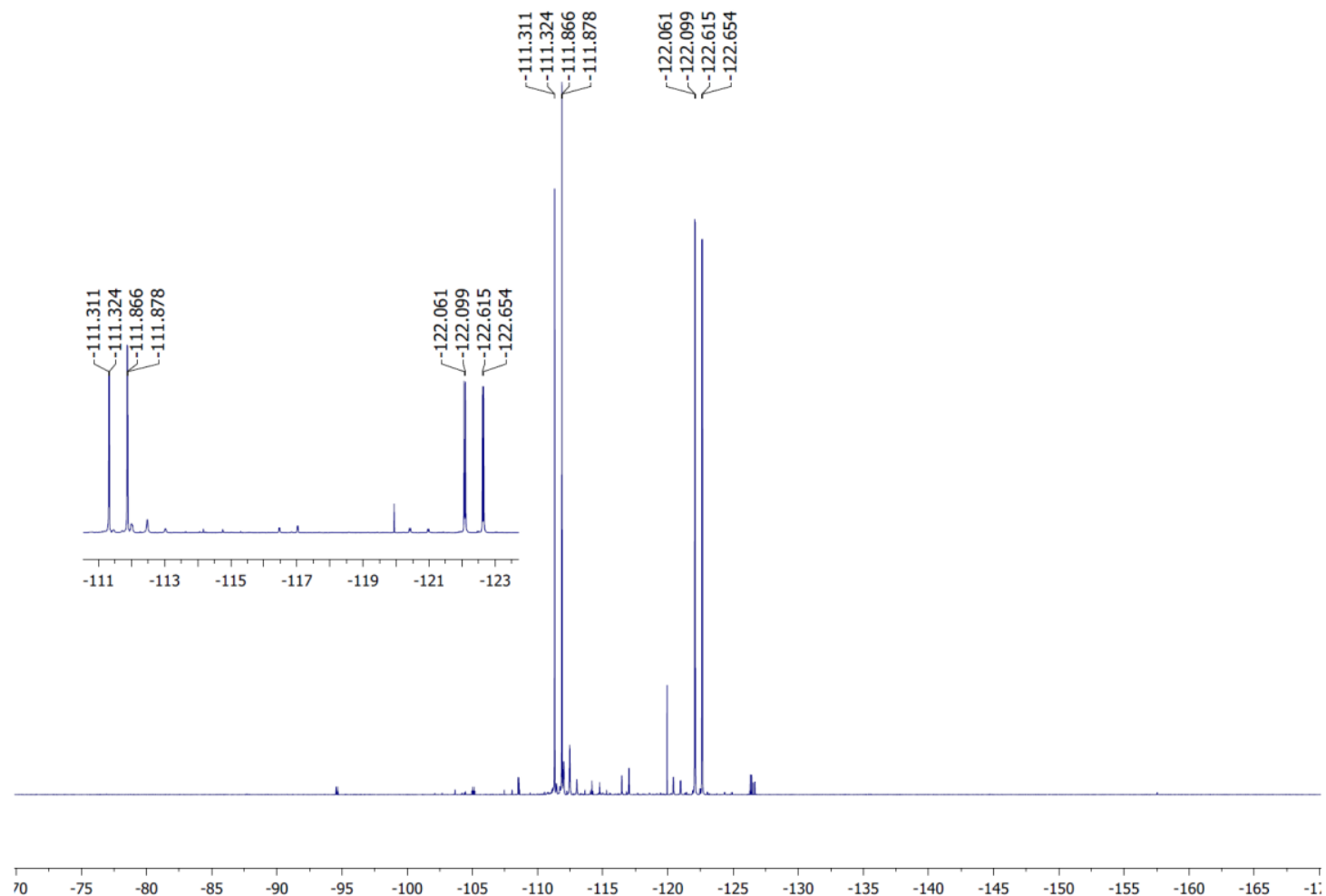


${ }^{13}$ C NMR

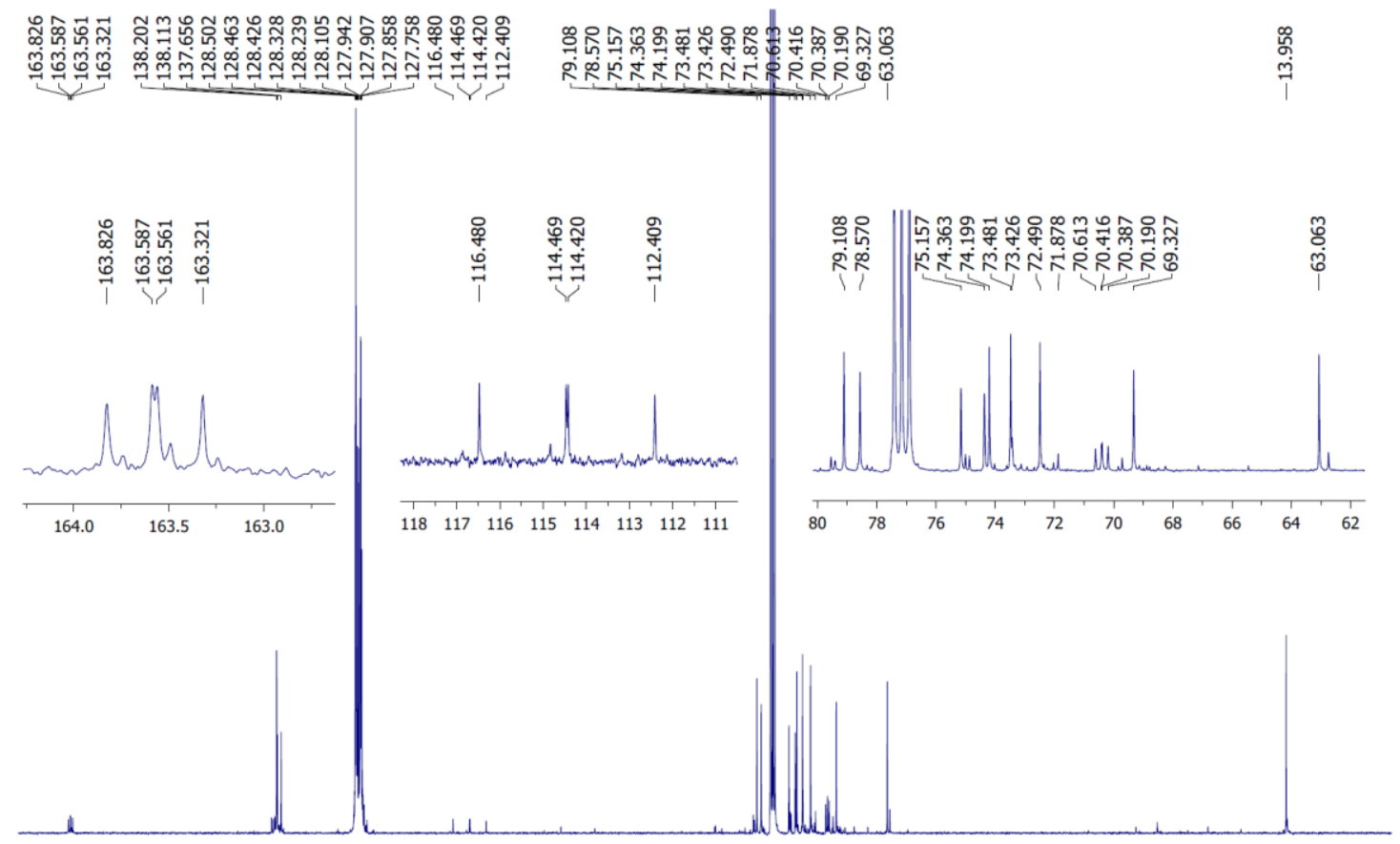

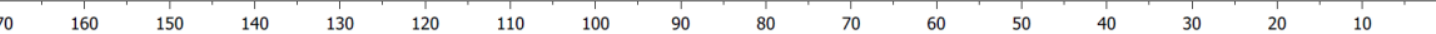




\section{D-Ribose}

Ethyl (1S/1R,2S,3R,4R)-2,3,4,5-tetra-O-benzyl-1',1'-difluoro-1-hydroxyheptanoate $((R / S)-3 b)$.<smiles>O=C[C@H](OCc1ccccc1)C(Br)(Br)Cc1ccccc1</smiles>

2b<smiles>CCOC(=O)C(F)(F)Br</smiles>

THF<smiles>CCOC(=O)C(F)(F)[C@H](O)[C@H](OCc1ccccc1)[C@H](Br)[C@@H](COc1ccccc1)Cc1ccccc1</smiles>

(R)-3b<smiles>CCOC[C@@H](Br)[C@@H](Br)[C@@H](OCc1ccccc1)[C@@H](O)C(F)(F)C(=O)OCC</smiles>

(S)-3b

$\mathrm{R}: \mathrm{S} d r 75: 25$

To a dry two-necked round bottomed flask was added "activated zinc" powder (753 $\mathrm{mg}$, $11.52 \mathrm{mmol}, 5.7$ equiv.). The flask was heated up at $40^{\circ} \mathrm{C}$ and the zinc powder suspended in dry THF ( $15 \mathrm{ml})$ under argon atmosphere. Then, TMSCl (90 $\mu \mathrm{l}, 0.69 \mathrm{mmol}, 0.4$ equiv.) was added dropwise and the reaction was stirred at $60^{\circ} \mathrm{C}$ for 15 mins. Next, a solution of aldehyde $2 \mathbf{b}$ ( $1.030 \mathrm{~g}, 2.02 \mathrm{mmol}, 1.0$ equiv.) and ethyl bromo-difluoroacetate $(0.310 \mathrm{ml}$, 2.42 mmol, 1.2 equiv.) dissolved homogenously in dry THF (15 ml) under argon atmosphere was added dropwise at $60^{\circ} \mathrm{C}$. The reaction was stirred vigorously for $2 \mathrm{~h}$ at $60^{\circ} \mathrm{C}$ and then cooled at room temperature. Ice water $(10 \mathrm{ml})$ and $\mathrm{HCl}(1 \mathrm{M}, 10 \mathrm{ml})$ were added and the mixture was diluted with EtOAc. The organic phase was washed with $\mathrm{NaHCO}_{3}$ (sat) (2 times), brine, dried over $\mathrm{MgSO}_{4}$ and concentrated under reduced pressure. Crude NMR analysis showed the formation of two diastereoisomer $(R)-3 \mathbf{b}$ and (S)-3b in 75:25 $d r$ ratio. Purification by silica gel chromatography (Cy/EtOAc 9:1) afforded pure stereoisomer $(R)-\mathbf{3 b}$ and $(S)$-3b in $64 \%$ overall yield. 
Ethyl (1S/1R,2S,3R,4R)-2,3,4,5-tetra-O-benzyl-1',1'-difluoro-1-hydroxyheptanoate (( $R)$ $3 b)$.<smiles>CCOC(F)(F)C(O)C(Br)C(Br)C(Br)C(Br)COCc1ccccc1</smiles>

(R)-3b

$[\alpha]^{20}{ }_{\mathrm{D}}:-5.3\left(\mathrm{c} 1.0, \mathrm{CHCl}_{3}\right) .{ }^{1} \mathrm{H} \mathrm{NMR}\left(500 \mathrm{MHz}, \mathrm{CDCl}_{3}\right) \delta=7.34-7.27\left(\mathrm{~m}, 18 \mathrm{H}, \mathrm{H}^{\text {arom }}\right)$, 7.26-7.23 (m, $\left.2 \mathrm{H}, \mathrm{H}^{\text {arom }}\right), 4.73\left(\mathrm{AB}, 1 \mathrm{H}, J_{\mathrm{A}-\mathrm{B}}=10.9 \mathrm{~Hz}, \mathrm{CH}_{2}{ }^{\mathrm{Bn}}\right), 4.71\left(\mathrm{AB}, 1 \mathrm{H}, J_{\mathrm{A}-\mathrm{B}}=\right.$ $\left.11.5 \mathrm{~Hz}, \mathrm{CH}_{2}{ }^{\mathrm{Bn}}\right), 4.67\left(\mathrm{AB}, 1 \mathrm{H}, J_{\mathrm{A}-\mathrm{B}}=11.5 \mathrm{~Hz}, \mathrm{CH}_{2}{ }^{\mathrm{Bn}}\right), 4.61\left(\mathrm{AB}, 1 \mathrm{H}, J_{\mathrm{A}-\mathrm{B}}=11.5 \mathrm{~Hz}\right.$, $\left.\mathrm{CH}_{2}{ }^{\mathrm{Bn}}\right), 4.59-4.49\left(\mathrm{~m}, 4 \mathrm{H}, \mathrm{H}-1, \mathrm{CH}_{2}{ }^{\mathrm{Bn}}\right), 4.46\left(\mathrm{AB}, 1 \mathrm{H}, J_{\mathrm{A}-\mathrm{B}}=10.9 \mathrm{~Hz}, \mathrm{CH}_{2}{ }^{\mathrm{Bn}}\right), 4.05(\mathrm{t}$, $2 \mathrm{H}, J=7.5 \mathrm{~Hz}, \mathrm{H}-2, \mathrm{H}-3), 4.02-3.94\left(\mathrm{~m}, 3 \mathrm{H}, \mathrm{H}-4, \mathrm{CH}_{2} \mathrm{CH}_{3}\right), 3.71\left(\mathrm{ABX}, 1 \mathrm{H}, J_{4-5 \mathrm{a}}=3.4\right.$ $\left.\mathrm{Hz}, J_{5 \mathrm{a}-5 \mathrm{~b}}=10.3 \mathrm{~Hz}, \mathrm{H}-5 \mathrm{a}\right), 3.64\left(\mathrm{ABX}, 1 \mathrm{H}, J_{4-5 \mathrm{~b}}=4.6 \mathrm{~Hz}, J_{5 \mathrm{a}-5 \mathrm{~b}}=10.3 \mathrm{~Hz}, \mathrm{H}-5 \mathrm{~b}\right), 3.44$ (bs, $1 \mathrm{H}, \mathrm{OH}), 1.11\left(\mathrm{t}, 3 \mathrm{H}, J_{\mathrm{CH} 2 \mathrm{CH} 3}=7.5 \mathrm{~Hz}, \mathrm{CH}_{2} \mathrm{CH}_{3}\right) \mathrm{ppm} .{ }^{13} \mathrm{C} \mathrm{NMR}\left(125 \mathrm{MHz}, \mathrm{CDCl}_{3}\right)$ $\delta=163.4\left(\mathrm{t}, J_{\mathrm{C}-\mathrm{F}}=31.4 \mathrm{~Hz}, \mathrm{C}_{\mathrm{q}} \mathrm{O}_{2} \mathrm{Et}\right), 138.3\left(\mathrm{C}_{\mathrm{q}}{ }^{\text {arom }}\right), 138.1\left(\mathrm{C}_{\mathrm{q}}{ }^{\text {arom }}\right), 137.8\left(2 \mathrm{C}_{\mathrm{q}}{ }^{\text {arom }}\right)$, 128.6-127.7 $\left(\mathrm{CH}^{\text {arom }}\right), 114.8\left(\mathrm{dd}, J_{\mathrm{C}-\mathrm{F}}=250.5 \mathrm{~Hz}, J_{\mathrm{C}-\mathrm{F}}=256.5 \mathrm{~Hz}, \mathrm{CF}_{2}\right), 78.7(\mathrm{C}-4), 77.9$ $(\mathrm{C}-2), 77.7(\mathrm{C}-3), 73.7\left(\mathrm{CH}_{2}{ }^{\mathrm{Bn}}\right), 73.6\left(\mathrm{CH}_{2}{ }^{\mathrm{Bn}}\right), 73.2\left(\mathrm{CH}_{2}{ }^{\mathrm{Bn}}\right), 73.1\left(\mathrm{CH}_{2}{ }^{\mathrm{Bn}}\right), 71.2\left(\mathrm{t}, J_{\mathrm{C}-\mathrm{F}}=\right.$ $22.9 \mathrm{~Hz}, \mathrm{C}-1), 69.3(\mathrm{C}-5), 62.6\left(\mathrm{CH}_{2} \mathrm{CH}_{3}\right), 13.8\left(\mathrm{CH}_{2} \mathrm{CH}_{3}\right) \mathrm{ppm} .{ }^{19} \mathrm{~F}$ NMR (471 MHz, $\left.\mathrm{CDCl}_{3}\right) \delta=-116.5\left(\mathrm{dd}, 1 \mathrm{~F}, J_{\mathrm{H}-\mathrm{Fa}}=9.0 \mathrm{~Hz}, J_{\mathrm{Fa}-\mathrm{Fb}}=260.9 \mathrm{~Hz}, \mathrm{Fa}\right),-122.0\left(\mathrm{dd}, 1 \mathrm{~F}, J_{\mathrm{H}-\mathrm{Fb}}=\right.$ $\left.15.9 \mathrm{~Hz}, J_{\mathrm{Fa}-\mathrm{Fb}}=260.9 \mathrm{~Hz}, \mathrm{Fb}\right) \mathrm{ppm}$. HRMS (ESI+): m/z calculated for $\mathrm{C}_{37} \mathrm{H}_{40} \mathrm{~F}_{2} \mathrm{NaO}_{7}$ $[\mathrm{M}+\mathrm{Na}]^{+}$: calc. 657.2634 ; found: 657.2625. 


\section{${ }^{1}$ H NMR}

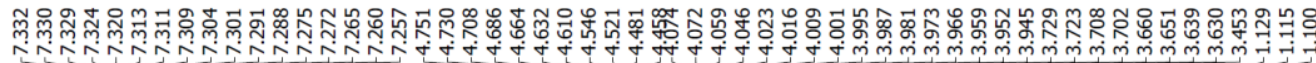

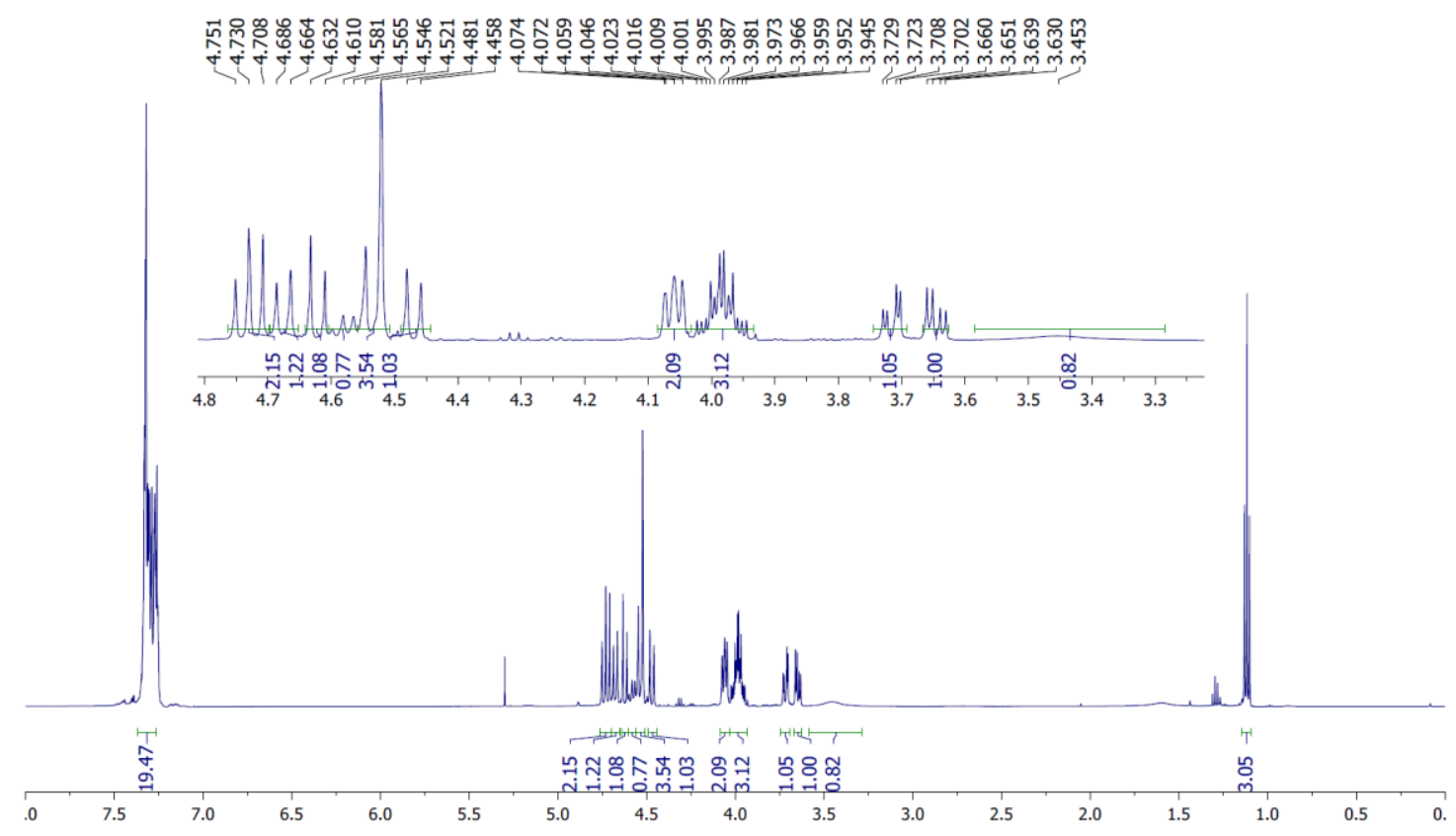

${ }^{19}$ F NMR

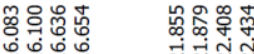

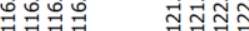

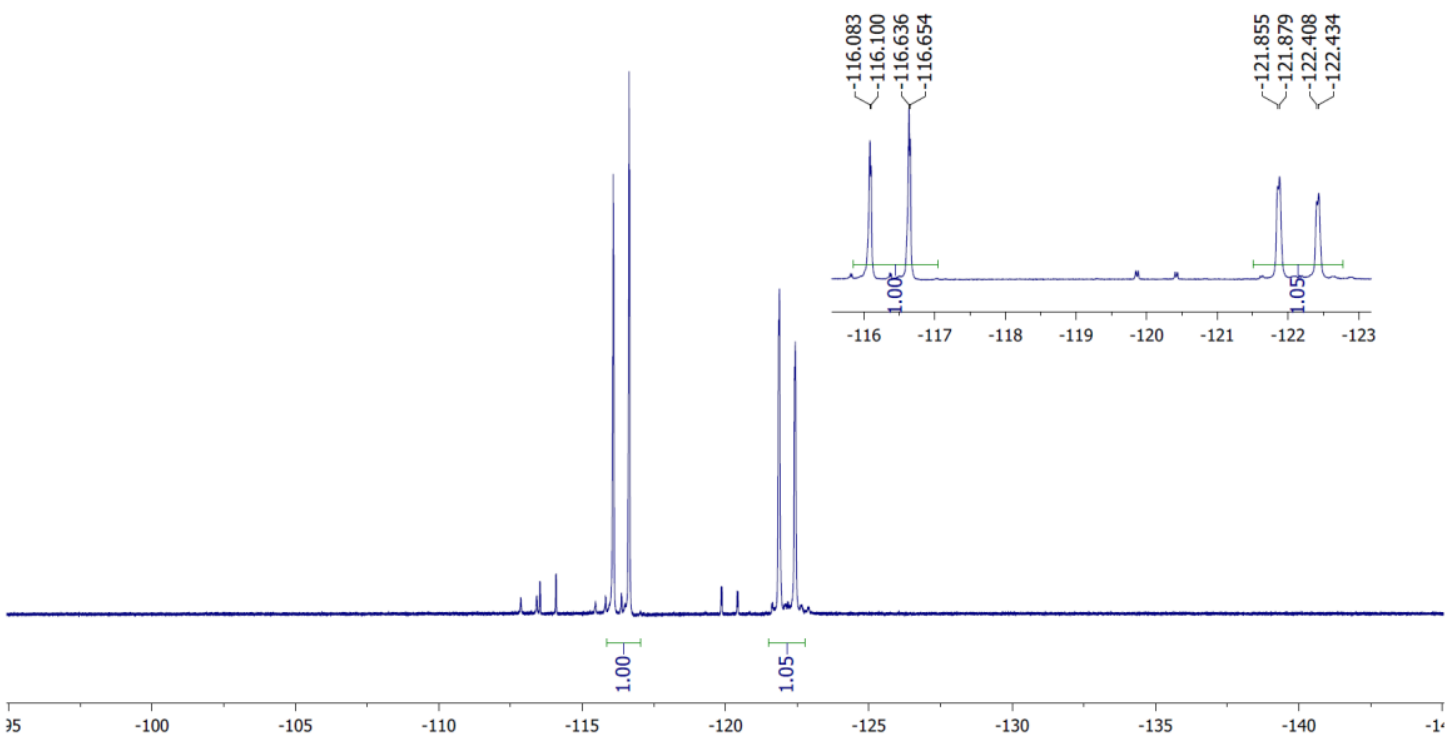


${ }^{13}$ C NMR

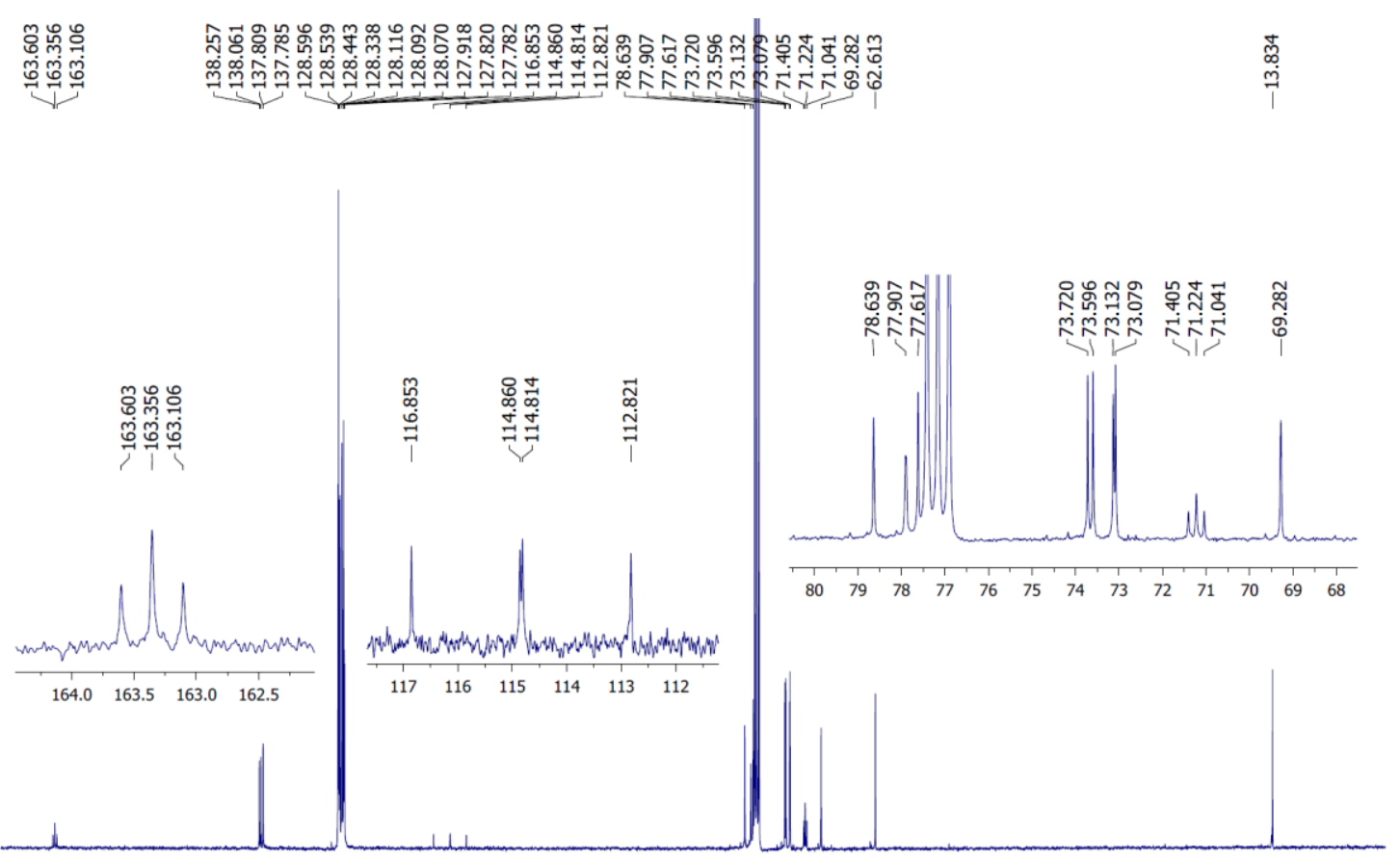

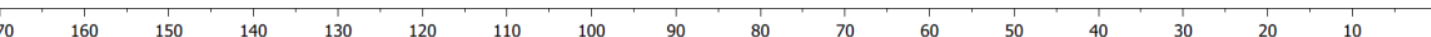


Ethyl (1S/1R,2S,3R,4R)-2,3,4,5-tetra-O-benzyl-1',1'-difluoro-1-hydroxyheptanoate ((S)$3 b)$.

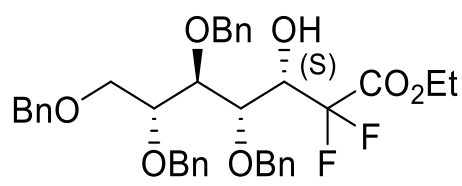

(S)-3b

$[\alpha]_{20}{ }^{\mathrm{D}}:-2.7\left(\mathrm{c} 1.0, \mathrm{CHCl}_{3}\right) .{ }^{1} \mathrm{H}$ NMR $\left(500 \mathrm{MHz}, \mathrm{CDCl}_{3}\right) \delta=7.34-7.27\left(\mathrm{~m}, 18 \mathrm{H}, \mathrm{H}^{\text {arom }}\right)$, 7.25-7.22 (m, $\left.2 \mathrm{H}, \mathrm{H}^{\text {arom }}\right), 4.72\left(\mathrm{AB}, 1 \mathrm{H}, J_{\mathrm{A}-\mathrm{B}}=12.0 \mathrm{~Hz}, \mathrm{CH}_{2}{ }^{\mathrm{Bn}}\right), 4.71\left(\mathrm{AB}, 1 \mathrm{H}, J_{\mathrm{A}-\mathrm{B}}=\right.$ $\left.10.9 \mathrm{~Hz}, \mathrm{CH}_{2}{ }^{\mathrm{Bn}}\right), 4.66-4.62\left(\mathrm{~m}, 3 \mathrm{H}, \mathrm{CH}_{2}{ }^{\mathrm{Bn}}\right), 4.55\left(\mathrm{AB}, 1 \mathrm{H}, J_{\mathrm{A}-\mathrm{B}}=10.9 \mathrm{~Hz}, \mathrm{CH}_{2}{ }^{\mathrm{Bn}}\right), 4.52$ $\left(\mathrm{AB}, 1 \mathrm{H}, J_{\mathrm{A}-\mathrm{B}}=12.0 \mathrm{~Hz}, \mathrm{CH}_{2}{ }^{\mathrm{Bn}}\right), 4.49\left(\mathrm{AB}, 1 \mathrm{H}, J_{\mathrm{A}-\mathrm{B}}=12.0 \mathrm{~Hz}, \mathrm{CH}_{2}{ }^{\mathrm{Bn}}\right), 4.38\left(\mathrm{dd}, 1 \mathrm{H}, J_{1-}\right.$ $\left.2=6.3 \mathrm{~Hz}, J_{1-\mathrm{Fb}}=19.5 \mathrm{~Hz}, \mathrm{H}-1\right), 4.24\left(\mathrm{q}, 2 \mathrm{H}, J_{\mathrm{CH} 2 \mathrm{CH} 3}=7.5 \mathrm{~Hz}, \mathrm{CH}_{2} \mathrm{CH}_{3}\right), 4.12(\mathrm{~d}, 1 \mathrm{H}$, $\left.J_{1-2}=6.3 \mathrm{~Hz}, \mathrm{H}-2\right), 3.96(\mathrm{~m}, 1 \mathrm{H}, \mathrm{H}-3), 3.89\left(\mathrm{q}, 1 \mathrm{H}, J_{3-4} \sim J_{4-5 \mathrm{a}}=3.4 \mathrm{~Hz}, J_{4-5 \mathrm{~b}}=5.2 \mathrm{~Hz}\right.$, $\mathrm{H}-4), 3.77\left(\mathrm{ABX}, 1 \mathrm{H}, J_{4-5 \mathrm{a}}=3.4 \mathrm{~Hz}, J_{5 \mathrm{a}-5 \mathrm{~b}}=10.3 \mathrm{~Hz}, \mathrm{H}-5 \mathrm{a}\right), 3.69\left(\mathrm{ABX}, 1 \mathrm{H}, J_{4-5 \mathrm{~b}}=5.2\right.$ $\left.\mathrm{Hz}, J_{5 \mathrm{a}-5 \mathrm{~b}}=10.3 \mathrm{~Hz}, \mathrm{H}-5 \mathrm{~b}\right), 1.28\left(\mathrm{t}, 3 \mathrm{H}, J_{\mathrm{CH} 2 \mathrm{CH} 3}=7.5 \mathrm{~Hz}, \mathrm{CH}_{2} \mathrm{CH}_{3}\right) \mathrm{ppm} .{ }^{13} \mathrm{C} \mathrm{NMR}(125$ $\left.\mathrm{MHz}, \mathrm{CDCl}_{3}\right) \delta=163.6\left(\mathrm{dd}, J_{\mathrm{C}-\mathrm{F}}=30.2 \mathrm{~Hz}, J_{\mathrm{C}-\mathrm{F}}=33.8 \mathrm{~Hz}, \mathrm{C}_{\mathrm{q}} \mathrm{O}_{2} \mathrm{Et}\right), 138.3\left(2 \mathrm{C}_{\mathrm{q}}{ }^{\text {arom }}\right)$, $138.0\left(\mathrm{C}_{\mathrm{q}}{ }^{\text {arom }}\right), 137.4\left(\mathrm{C}_{\mathrm{q}}{ }^{\text {arom }}\right), 128.6-127.7\left(\mathrm{CH}^{\text {arom }}\right), 114.8\left(\mathrm{dd}, J_{\mathrm{C}-\mathrm{F}}=254.1 \mathrm{~Hz}, J_{\mathrm{C}-\mathrm{F}}=\right.$ $\left.256.5 \mathrm{~Hz}, \mathrm{CF}_{2}\right), 79.2(\mathrm{C}-3), 78.1(\mathrm{C}-4), 74.7(\mathrm{C}-2), 74.2\left(\mathrm{CH}_{2}{ }^{\mathrm{Bn}}\right), 73.6\left(\mathrm{CH}_{2}{ }^{\mathrm{Bn}}\right), 73.5$ $\left(\mathrm{CH}_{2}{ }^{\mathrm{Bn}}\right), 72.6\left(\mathrm{CH}_{2}{ }^{\mathrm{Bn}}\right), 69.6(\mathrm{~m}, \mathrm{C}-1, \mathrm{C}-5), 63.1\left(\mathrm{CH}_{2} \mathrm{CH}_{3}\right), 14.0\left(\mathrm{CH}_{2} \mathrm{CH}_{3}\right) \mathrm{ppm} .{ }^{19} \mathrm{~F}$ $\operatorname{NMR}\left(471 \mathrm{MHz}, \mathrm{CDCl}_{3}\right) \delta=-113.1\left(\mathrm{~d}, 1 \mathrm{~F}, J_{\mathrm{Fa}-\mathrm{Fb}}=260.5 \mathrm{~Hz}, \mathrm{Fa}\right),-122.4\left(\mathrm{dd}, 1 \mathrm{~F}, J_{\mathrm{H} 1-\mathrm{Fb}}\right.$ $\left.=19.5 \mathrm{~Hz}, J_{\mathrm{Fa}-\mathrm{Fb}}=260.5 \mathrm{~Hz}, \mathrm{Fb}\right)$ ppm. HRMS (ESI+): $\mathrm{m} / \mathrm{z}$ calculated for $\mathrm{C}_{37} \mathrm{H}_{40} \mathrm{~F}_{2} \mathrm{NaO}_{7}$ $[\mathrm{M}+\mathrm{Na}]^{+}$: calc. 657.2634; found: 657.2630. 


\section{${ }^{1}$ H NMR}

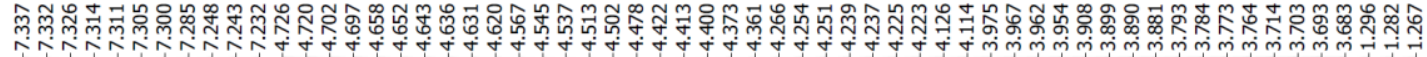

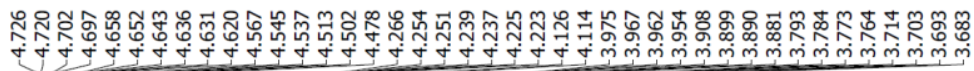

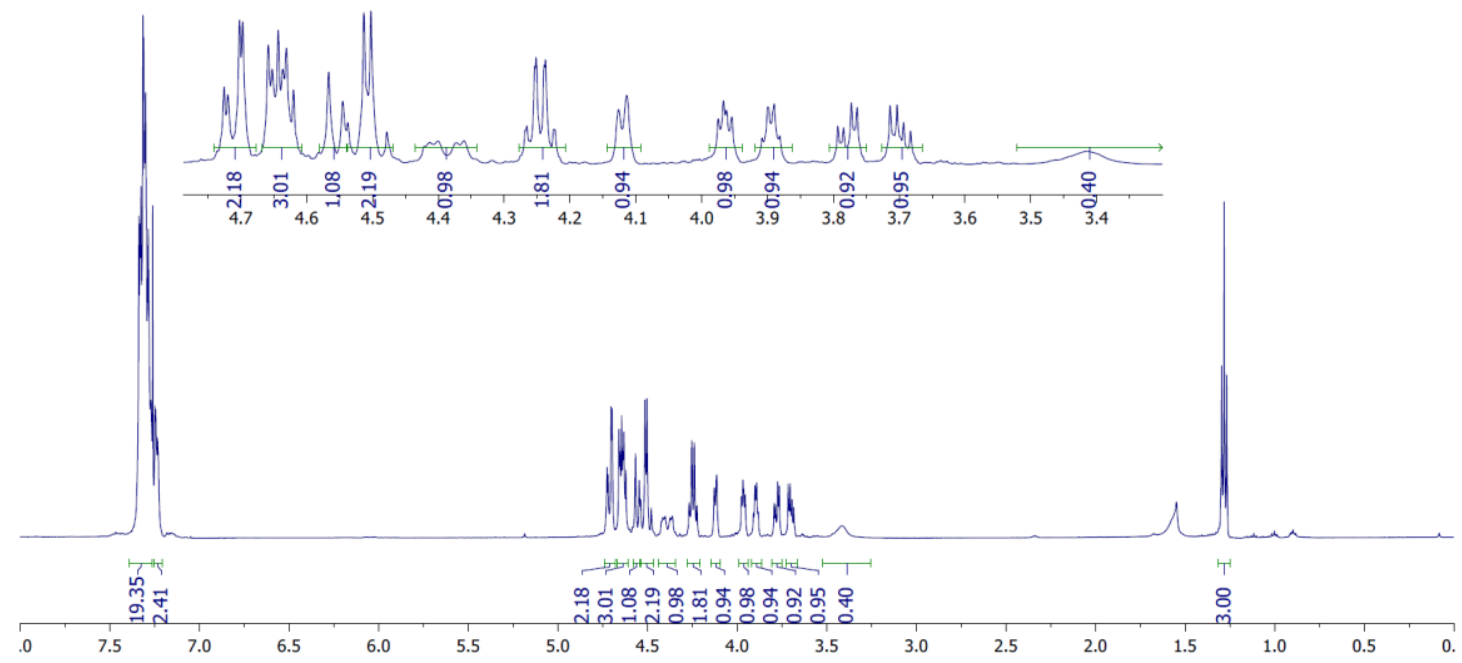

${ }^{19}$ F NMR
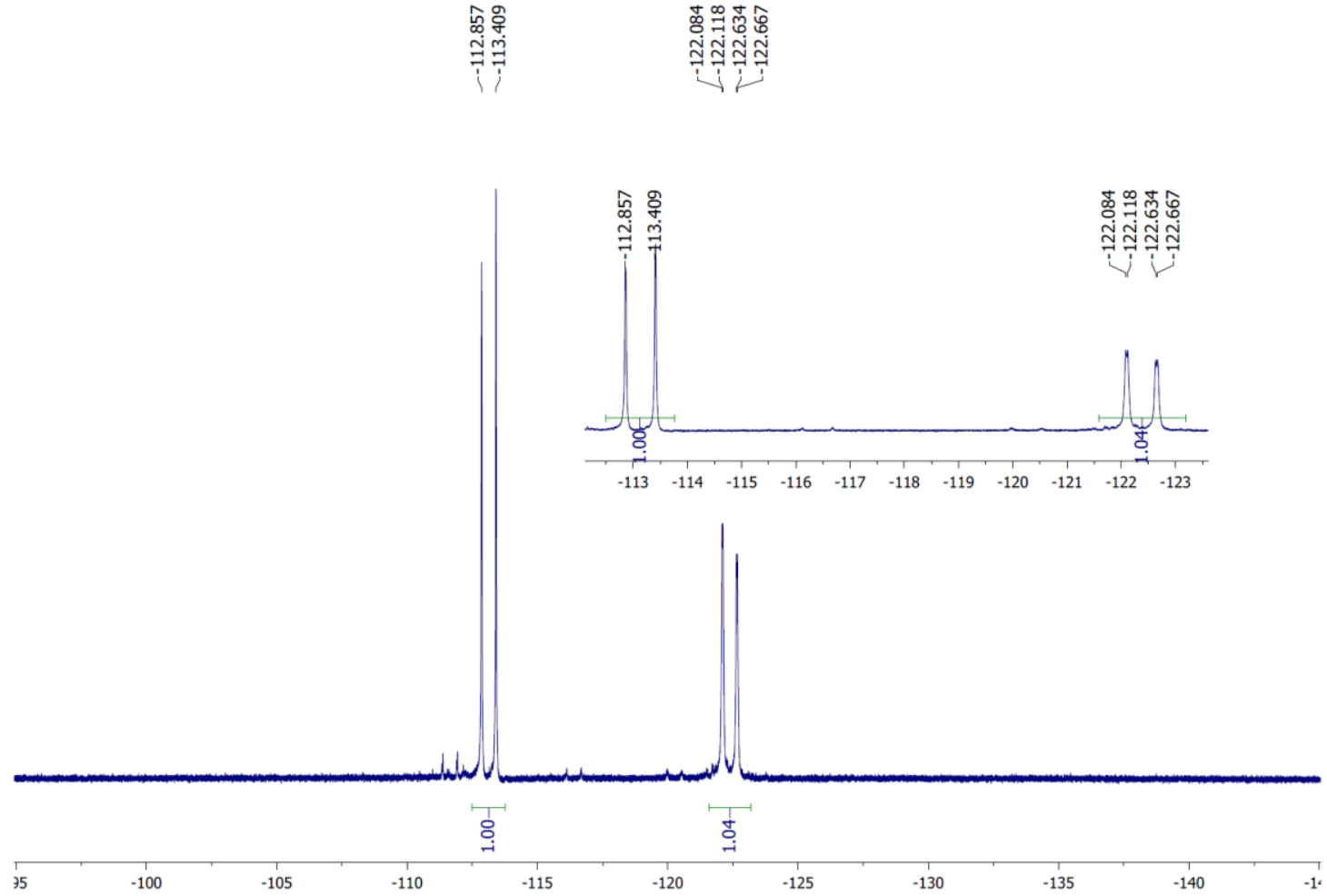
${ }^{13}$ C NMR
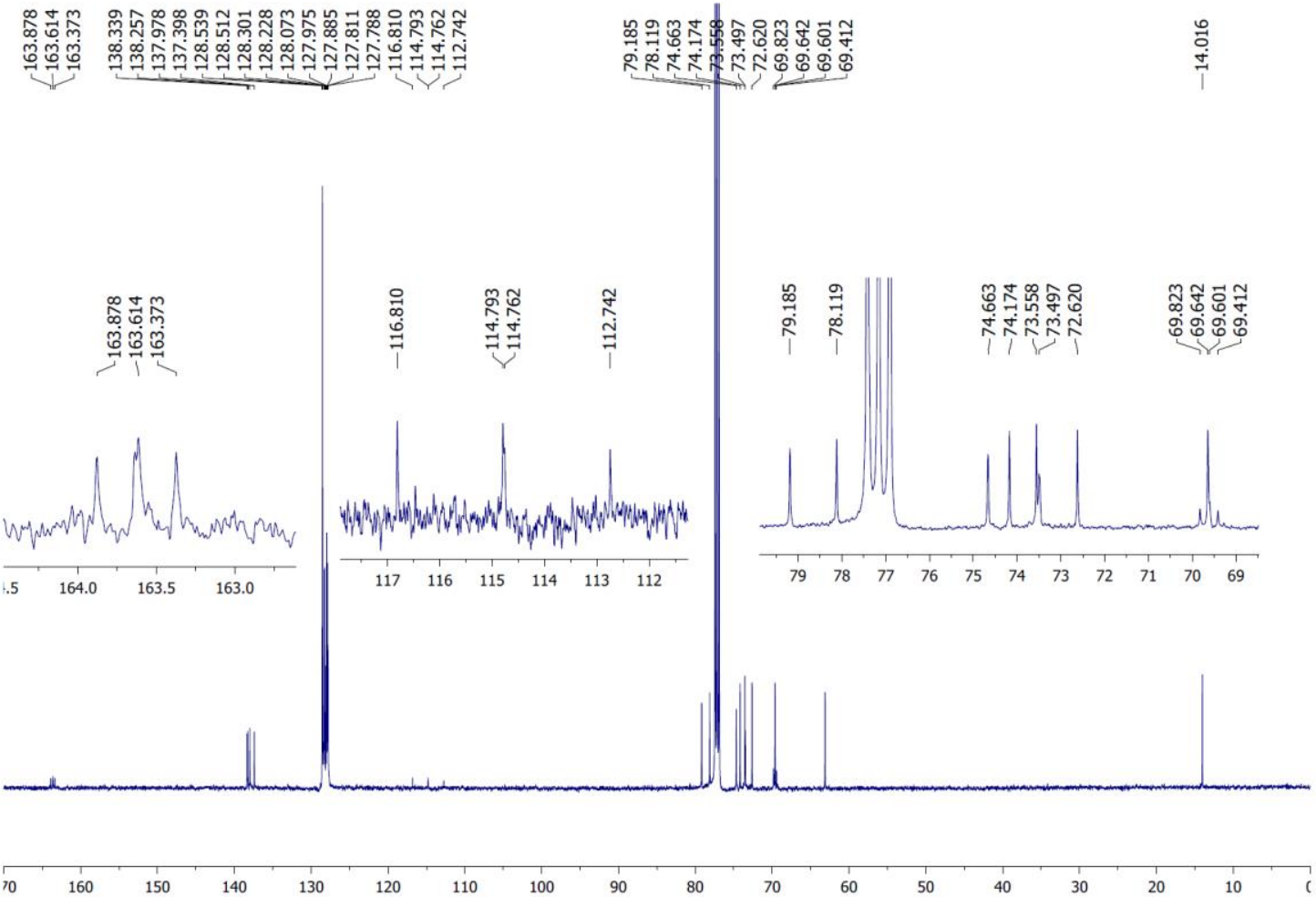


\section{D-Xylose}

Ethyl (1S/1R,2S,3S,4R)-2,3,4,5-tetra-O-benzyl-1',1'-difluoro-1-hydroxyheptanoate $((R / S)-3 c)$.

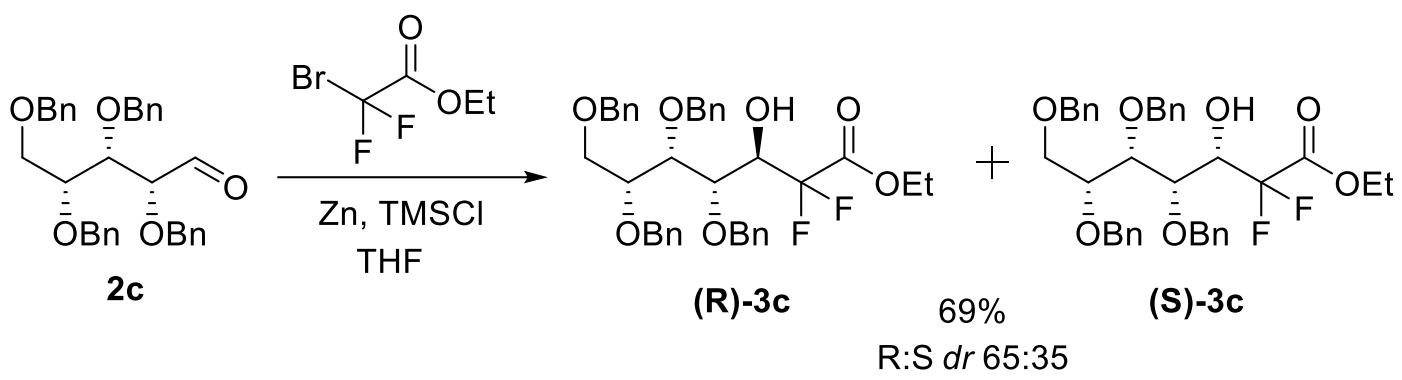

To a dry two-necked round bottomed flask was added "activated zinc" powder (298 $\mathrm{mg}$, $4.55 \mathrm{mmol}, 5.7$ equiv.). The flask was heated up at $40^{\circ} \mathrm{C}$ and the zinc powder suspended in dry THF ( $5 \mathrm{ml})$ under argon atmosphere. Then, TMSCl ( $44 \mu \mathrm{l}, 0.34 \mathrm{mmol}, 0.4$ equiv.) was added dropwise and the reaction was stirred at $60^{\circ} \mathrm{C}$ for 15 mins. Next, a solution of aldehyde 2 c (407 mg, $0.80 \mathrm{mmol}, 1.0$ equiv.) and ethyl bromo-difluoroacetate $(0.122 \mathrm{ml}$, $0.96 \mathrm{mmol}, 1.2$ equiv.) dissolved homogenously in dry THF $(5 \mathrm{ml})$ under argon atmosphere was added dropwise at $60^{\circ} \mathrm{C}$. The reaction was stirred vigorously for $1.5 \mathrm{~h}$ at $60^{\circ} \mathrm{C}$ and then cooled at room temperature. Ice water $(10 \mathrm{ml})$ and $\mathrm{HCl}(1 \mathrm{M}, 10 \mathrm{ml})$ were added and the mixture was diluted with EtOAc. The organic phase was washed with $\mathrm{NaHCO}_{3}$ (sat) (2 times), brine, dried over $\mathrm{MgSO}_{4}$ and concentrated under reduced pressure. Purification by silica gel chromatography (Cy/EtOAc 8:2) gave an inseparable mixture of two diastereoisomers $(R)-3 \mathbf{c a n d}(S)$-3c in 65:35 $d r$ ratio (326 $\mathrm{mg}, 69 \%$ ).

Noteworthy, few milligrams $(15 \mathrm{mg})$ of the major diastereoisomer $(R)-3 \mathrm{c}$ could be isolated and fully characterized. 
Ethyl (1S/1R,2S,3S,4R)-2,3,4,5-tetra-O-benzyl-1',1'-difluoro-1-hydroxyheptanoate ((R)$3 c$ ).<smiles>CCOC(F)(F)C(O)C(c1ccccc1)[C@@H](Br)[C@@H](Br)COCc1ccccc1</smiles>

(R)-3c

$[\alpha]_{20}{ }^{\mathrm{D}}:-2.1\left(\mathrm{c} 1.0, \mathrm{CHCl}_{3}\right) .{ }^{1} \mathrm{H}$ NMR $\left(500 \mathrm{MHz}, \mathrm{CDCl}_{3}\right) \delta=7.36-7.22\left(\mathrm{~m}, 20 \mathrm{H}, \mathrm{H}^{\text {arom }}\right)$, $4.76\left(\mathrm{AB}, 1 \mathrm{H}, J_{\mathrm{A}-\mathrm{B}}=10.9 \mathrm{~Hz}, \mathrm{CH}_{2}{ }^{\mathrm{Bn}}\right), 4.68\left(\mathrm{AB}, 2 \mathrm{H}, J_{\mathrm{A}-\mathrm{B}}=11.5 \mathrm{~Hz}, \mathrm{CH}_{2}{ }^{\mathrm{Bn}}\right), 4.61(\mathrm{AB}$, $\left.1 \mathrm{H}, J_{\mathrm{A}-\mathrm{B}}=11.5 \mathrm{~Hz}, \mathrm{CH}_{2}{ }^{\mathrm{Bn}}\right), 4.59\left(\mathrm{AB}, 1 \mathrm{H}, J_{\mathrm{A}-\mathrm{B}}=10.9 \mathrm{~Hz}, \mathrm{CH}_{2}{ }^{\mathrm{Bn}}\right), 4.56\left(\mathrm{AB}, 1 \mathrm{H}, J_{\mathrm{A}-\mathrm{B}}=\right.$ $\left.11.5 \mathrm{~Hz}, \mathrm{CH}_{2}{ }^{\mathrm{Bn}}\right), 4.44\left(\mathrm{AB}, 1 \mathrm{H}, J_{\mathrm{A}-\mathrm{B}}=12.0 \mathrm{~Hz}, \mathrm{CH}_{2}{ }^{\mathrm{Bn}}\right), 4.41\left(\mathrm{AB}, 1 \mathrm{H}, J_{\mathrm{A}-\mathrm{B}}=12.0 \mathrm{~Hz}\right.$, $\left.\mathrm{CH}_{2}{ }^{\mathrm{Bn}}\right), 4.30\left(\mathrm{dd}, 1 \mathrm{H}, J_{1-\mathrm{Fa}}=5.7 \mathrm{~Hz}, J_{1-\mathrm{Fb}}=18.9 \mathrm{~Hz}, \mathrm{H}-1\right), 4.20\left(\mathrm{q}, 2 \mathrm{H}, J_{\mathrm{CH} 2 \mathrm{CH} 3}=7.5 \mathrm{~Hz}\right.$, $\left.\mathrm{CH}_{2} \mathrm{CH}_{3}\right), 4.08\left(\mathrm{~d}, 1 \mathrm{H}, J_{2-3}=7.4 \mathrm{~Hz}, \mathrm{H}-2\right), 3.87\left(\mathrm{dd}, 1 \mathrm{H}, J_{2-3}=7.4 \mathrm{~Hz}, J_{3-4}=4.0 \mathrm{~Hz}, \mathrm{H}-\right.$ 3), $3.82\left(\mathrm{td}, 1 \mathrm{H}, J_{3-4}=4.0 \mathrm{~Hz}, J_{4-5 \mathrm{a}}=J_{4-5 \mathrm{~b}}=5.2 \mathrm{~Hz}, \mathrm{H}-4\right), 3.65\left(\mathrm{ABX}, 1 \mathrm{H}, J_{4-5 \mathrm{a}}=5.2 \mathrm{~Hz}\right.$, $\left.J_{5 \mathrm{a}-5 \mathrm{~b}}=9.7 \mathrm{~Hz}, \mathrm{H}-5 \mathrm{a}\right), 3.61\left(\mathrm{ABX}, 1 \mathrm{H}, J_{4-5 \mathrm{~b}}=5.2 \mathrm{~Hz}, J_{5 \mathrm{a}-5 \mathrm{~b}}=9.7 \mathrm{~Hz}, \mathrm{H}-5 \mathrm{~b}\right), 1.26(\mathrm{t}, 3 \mathrm{H}$, $\left.J_{\mathrm{CH} 2 \mathrm{CH} 3}=7.5 \mathrm{~Hz}, \mathrm{CH}_{2} \mathrm{CH}_{3}\right) \mathrm{ppm} .{ }^{13} \mathrm{C} \mathrm{NMR}\left(125 \mathrm{MHz}, \mathrm{CDCl}_{3}\right) \delta=163.6\left(\mathrm{dd}, J_{\mathrm{C}-\mathrm{F}}=29.9\right.$ $\left.\mathrm{Hz}, J_{\mathrm{C}-\mathrm{F}}=32.8 \mathrm{~Hz}, \mathrm{C}_{\mathrm{q}} \mathrm{O}_{2} \mathrm{Et}\right), 138.2\left(2 \mathrm{C}_{\mathrm{q}}{ }^{\text {arom }}\right), 138.1\left(\mathrm{C}_{\mathrm{q}}{ }^{\text {arom }}\right), 137.7\left(\mathrm{C}_{\mathrm{q}}{ }^{\text {arom }}\right), 128.6-127.7$ $\left(\mathrm{CH}^{\text {arom }}\right), 114.5\left(\mathrm{dd}, J_{\mathrm{C}-\mathrm{F}}=253.4 \mathrm{~Hz}, J_{\mathrm{C}-\mathrm{F}}=258.2 \mathrm{~Hz}, \mathrm{CF}_{2}\right), 78.5(\mathrm{C}-3), 77.3(\mathrm{C}-4), 74.8$ $\left(2 \mathrm{CH}_{2}{ }^{\mathrm{Bn}}\right), 74.5(\mathrm{C}-2), 73.4\left(\mathrm{CH}_{2}{ }^{\mathrm{Bn}}\right), 73.1\left(\mathrm{CH}_{2}{ }^{\mathrm{Bn}}\right), 69.9\left(\mathrm{dd}, J_{\mathrm{C}-\mathrm{F}}=24.5 \mathrm{~Hz}, J_{\mathrm{C}-\mathrm{F}}=28.5\right.$ $\mathrm{Hz}, \mathrm{C}-1), 69.6(\mathrm{C}-5), 63.1\left(\mathrm{CH}_{2} \mathrm{CH}_{3}\right), 14.0\left(\mathrm{CH}_{2} \mathrm{CH}_{3}\right)$ ppm. ${ }^{19} \mathrm{~F} \mathrm{NMR}\left(471 \mathrm{MHz}, \mathrm{CDCl}_{3}\right)$ $\delta=-111.5\left(\mathrm{dd}, 1 \mathrm{~F}, J_{\mathrm{H} 1-\mathrm{Fa}}=5.7 \mathrm{~Hz}, J_{\mathrm{Fa}-\mathrm{Fb}}=261.7 \mathrm{~Hz}, \mathrm{Fa}\right),-123.1\left(\mathrm{dd}, 1 \mathrm{~F}, J_{\mathrm{H} 1-\mathrm{Fb}}=18.9\right.$ $\left.\mathrm{Hz}, J_{\mathrm{Fa}-\mathrm{Fb}}=261.7 \mathrm{~Hz}, \mathrm{Fb}\right)$ ppm. HRMS $(\mathrm{ESI}+): \mathrm{m} / \mathrm{z}$ calculated for $\mathrm{C}_{37} \mathrm{H}_{40} \mathrm{~F}_{2} \mathrm{NaO}_{7}$ $[\mathrm{M}+\mathrm{Na}]^{+}$: calc. 657.2634 ; found: 657.2626 . 


\section{${ }^{1}$ H NMR}

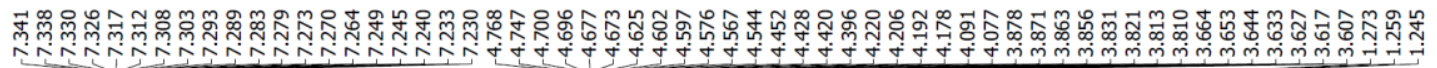

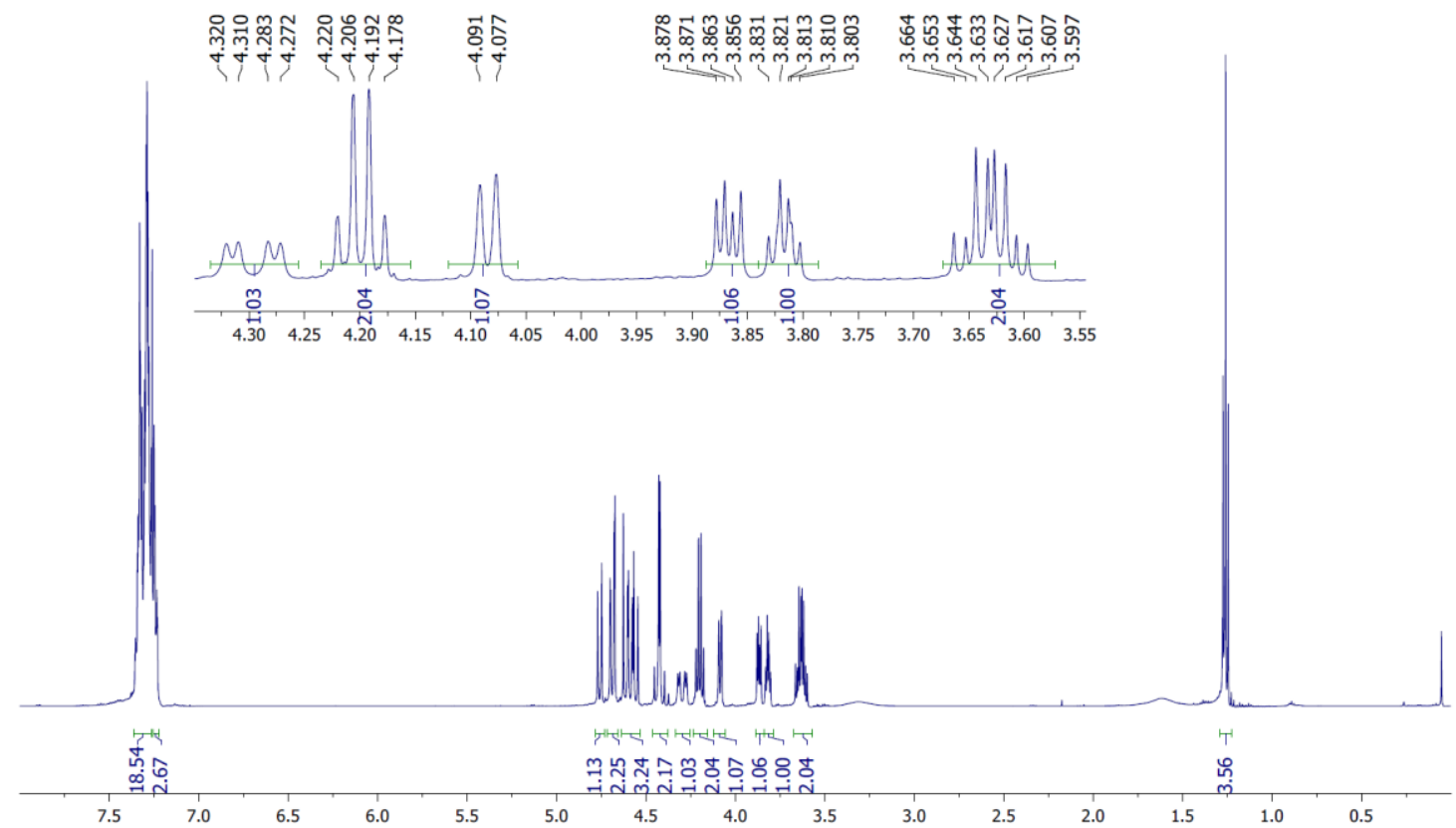

${ }^{19}$ F NMR

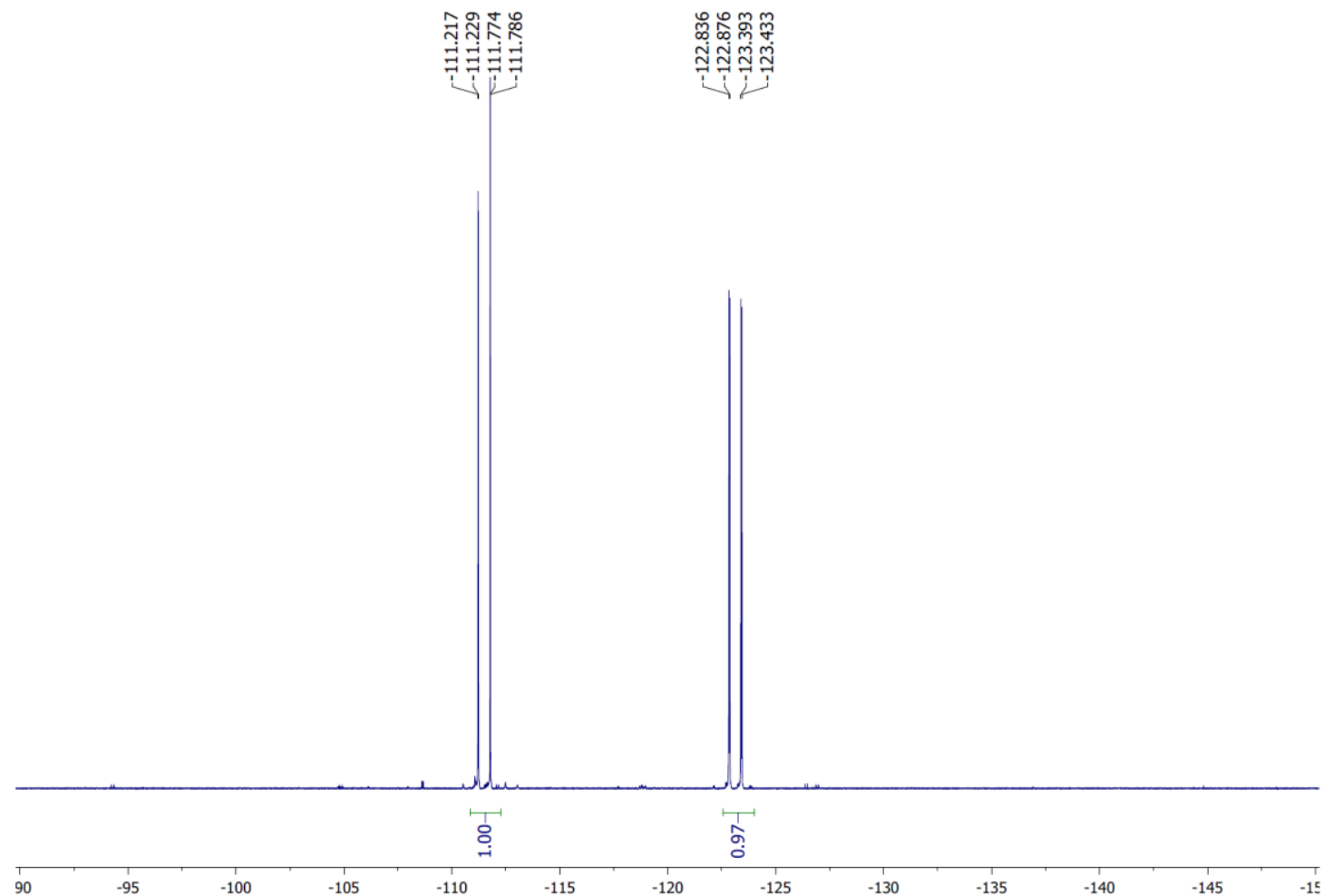


${ }^{13}$ C NMR
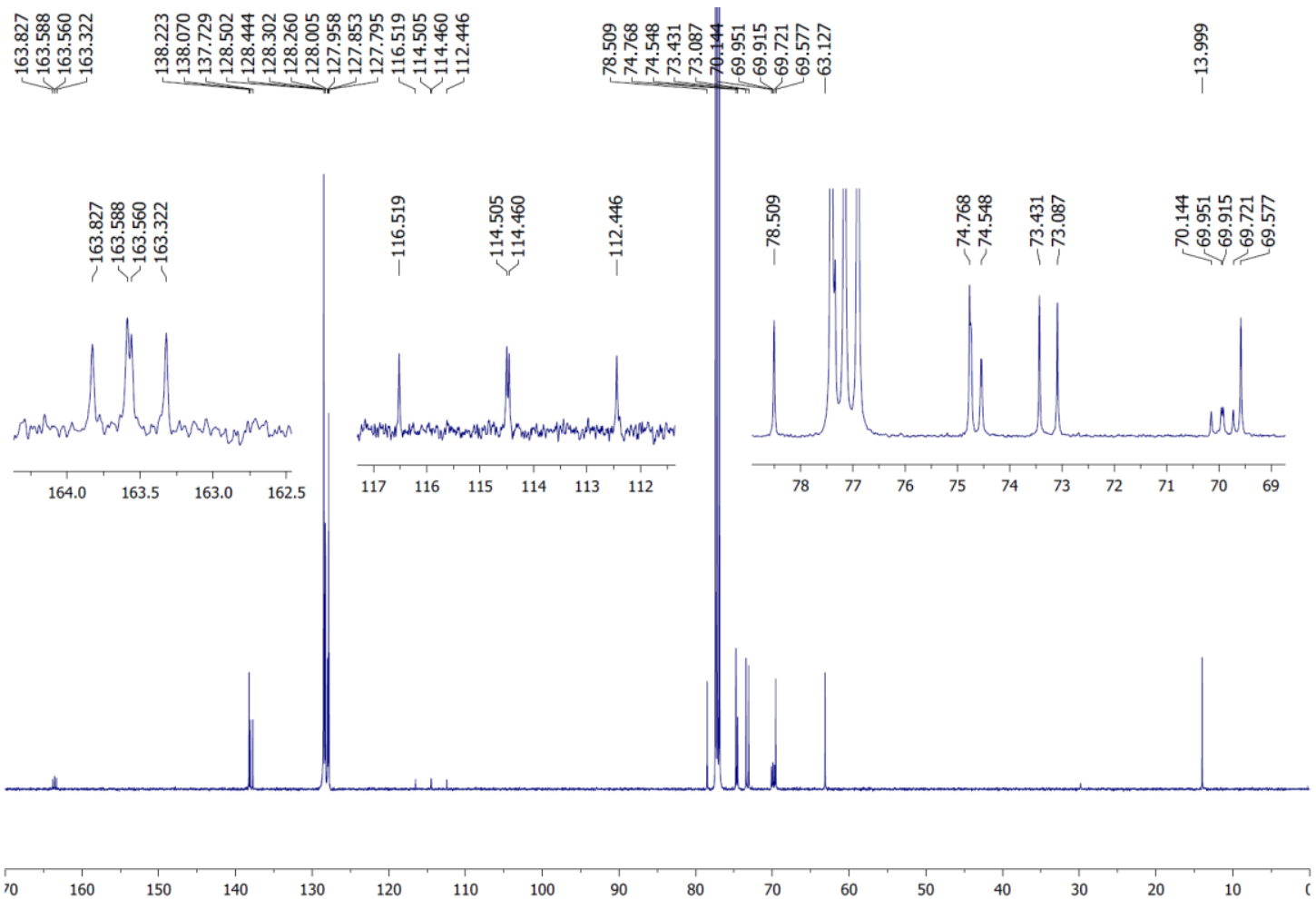

$((R / S)-3 c)$.

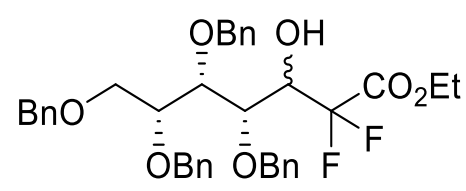

(R/S)-3c

${ }^{1} \mathrm{H}$ NMR (500 MHz, $\left.\mathrm{CDCl}_{3}\right) \delta=7.35-7.27\left(\mathrm{~m}, 32 \mathrm{H}, 16 \mathrm{HA}^{\text {arom }}, 16 \mathrm{HB}^{\text {arom }}\right), 7.25-7.17$ $\left(\mathrm{m}, 8 \mathrm{H}, 4 \mathrm{HA}^{\mathrm{arom}}, 4 \mathrm{HB}^{\mathrm{arom}}\right), 4.75\left(\mathrm{AB}, 1 \mathrm{H}, J_{\mathrm{A}-\mathrm{B}}=10.9 \mathrm{~Hz}, \mathrm{CH}_{2} \mathrm{~A}^{\mathrm{Bn}}\right), 4.73\left(\mathrm{AB}, 1 \mathrm{H}, J_{\mathrm{A}-}\right.$ в $\left.=12.0 \mathrm{~Hz}, \mathrm{CH}_{2} \mathrm{~B}^{\mathrm{Bn}}\right), 4.68\left(\mathrm{AB}, 2 \mathrm{H}, J_{\mathrm{A}-\mathrm{B}}=11.5 \mathrm{~Hz}, \mathrm{CH}_{2} \mathrm{~A}^{\mathrm{Bn}}\right), 4.65-4.53(\mathrm{~m}, 6 \mathrm{H}, 3$ $\left.\mathrm{CH}_{2} \mathrm{~A}^{\mathrm{Bn}}, 3 \mathrm{CH}_{2} \mathrm{~B}^{\mathrm{Bn}}\right), 4.49\left(\mathrm{AB}, 1 \mathrm{H}, J_{\mathrm{A}-\mathrm{B}}=11.5 \mathrm{~Hz}, \mathrm{CH}_{2} \mathrm{~B}^{\mathrm{Bn}}\right), 4.44-4.38(\mathrm{~m}, 6 \mathrm{H}, \mathrm{H}-1 \mathrm{~B}, 2$ $\mathrm{CH}_{2} \mathrm{~A}^{\mathrm{Bn}}, 3 \mathrm{CH}_{2} \mathrm{~B}^{\mathrm{Bn}}$ ), 4.29 (m, $\left.1 \mathrm{H}, \mathrm{H}-1 \mathrm{~A}\right), 4.19$ (q, 2H, $J_{\mathrm{CH} 2 \mathrm{CH} 3 \mathrm{~A}}=7.5 \mathrm{~Hz}, \mathrm{CH}_{2} \mathrm{CH}_{3} \mathrm{~A}$ ), 4.13-4.04 (m, 3H, H-2A, $\left.\mathrm{CH}_{2} \mathrm{CH}_{3} \mathrm{~B}\right), 4.01(\mathrm{t}, 1 \mathrm{H}, J=4.0 \mathrm{~Hz}, \mathrm{H}-4 \mathrm{~B}), 3.96(\mathrm{t}, 1 \mathrm{H}, J=4.6$ $\mathrm{Hz}, \mathrm{H}-3 \mathrm{~B}$ ), 3.93 (bs, 1H, OHB), 3.88 (d, $\left.1 \mathrm{H}, J_{2-3}=4.6 \mathrm{~Hz}, \mathrm{H}-2 \mathrm{~B}\right), 3.86$ (dd, $1 \mathrm{H}, J_{2-3}=7.4$ $\left.\mathrm{Hz}, J_{3-4}=4.0 \mathrm{~Hz}, \mathrm{H}-3 \mathrm{~A}\right), 3.81\left(\mathrm{td}, 1 \mathrm{H}, J_{3-4}=4.0 \mathrm{~Hz}, J_{4-5 \mathrm{a}}=J_{4-5 \mathrm{~b}}=5.2 \mathrm{~Hz}, \mathrm{H}-4 \mathrm{~A}\right), 3.66-$ 3.59 (m, 4 H, H-5aA, H-5bA, H-5aB, H-5bB), 1.25 (t, 3H, $J_{\mathrm{CH} 2 \mathrm{CH} 3 \mathrm{~A}}=7.5 \mathrm{~Hz}, \mathrm{CH}_{2} \mathrm{CH}_{3} \mathrm{~A}$ ), $1.18\left(\mathrm{t}, 3 \mathrm{H}, J_{\mathrm{CH} 2 \mathrm{CH} 3 \mathrm{~B}}=7.5 \mathrm{~Hz}, \mathrm{CH}_{2} \mathrm{CH}_{3} \mathrm{~B}\right) \mathrm{ppm} .{ }^{13} \mathrm{C} \mathrm{NMR}\left(125 \mathrm{MHz}, \mathrm{CDCl}_{3}\right) \delta=163.6$ $\left(\mathrm{dd}, J_{\mathrm{C}-\mathrm{F}}=30.2 \mathrm{~Hz}, J_{\mathrm{C}-\mathrm{F}}=33.8 \mathrm{~Hz}, \mathrm{C}_{\mathrm{q}} \mathrm{O}_{2} \mathrm{EtA}\right), 163.3\left(\mathrm{t}, J_{\mathrm{C}-\mathrm{F}}=31.4 \mathrm{~Hz}, \mathrm{C}_{\mathrm{q}} \mathrm{O}_{2} \mathrm{EtB}\right), 138.2$ $\left(2 \mathrm{C}_{\mathrm{q}} \mathrm{A}^{\text {arom }}, \mathrm{C}_{\mathrm{q}} \mathrm{B}^{\text {arom }}\right), 138.1\left(\mathrm{C}_{\mathrm{q}} \mathrm{A}^{\text {arom }}\right), 137.9\left(\mathrm{C}_{\mathrm{q}} \mathrm{B}^{\text {arom }}\right), 137.7\left(\mathrm{C}_{\mathrm{q}} \mathrm{A}^{\text {arom }}\right), 137.4\left(2 \mathrm{C}_{\mathrm{q}} \mathrm{B}^{\text {arom }}\right)$, 128.7-127.7 $\left(\mathrm{C}_{\mathrm{q}} \mathrm{A}^{\text {arom }}, \mathrm{C}_{\mathrm{q}} \mathrm{B}^{\text {arom }}\right), 114.8\left(\mathrm{t}, J_{\mathrm{C}-\mathrm{F}}=255.5 \mathrm{~Hz}, \mathrm{CF}_{2} \mathrm{~B}\right), 114.5\left(\mathrm{dd}, J_{\mathrm{C}-\mathrm{F}}=252.8\right.$ $\left.\mathrm{Hz}, J_{\mathrm{C}-\mathrm{F}}=258.9 \mathrm{~Hz}, \mathrm{CF}_{2} \mathrm{~A}\right), 78.5(\mathrm{C}-3 \mathrm{~A}), 78.2(\mathrm{C}-3 \mathrm{~B}), 77.4(\mathrm{C}-4 \mathrm{~A}), 77.3(\mathrm{C}-4 \mathrm{~B}), 74.7(2$ $\left.\mathrm{CH}_{2} \mathrm{~A}^{\mathrm{Bn}}\right), 74.5(\mathrm{C}-2 \mathrm{~A}), 74.4(\mathrm{C}-2 \mathrm{~B}), 74.0\left(\mathrm{CH}_{2} \mathrm{~B}^{\mathrm{Bn}}\right), 73.4\left(\mathrm{CH}_{2} \mathrm{~A}^{\mathrm{Bn}}, \mathrm{CH}_{2} \mathrm{~B}^{\mathrm{Bn}}\right), 73.2$ $\left(\mathrm{CH}_{2} \mathrm{~B}^{\mathrm{Bn}}\right), 73.2\left(\mathrm{CH}_{2} \mathrm{~B}^{\mathrm{Bn}}\right), 73.1\left(\mathrm{CH}_{2} \mathrm{~A}^{\mathrm{Bn}}\right), 71.7\left(\mathrm{t}, J_{\mathrm{C}-\mathrm{F}}=23.5 \mathrm{~Hz}, \mathrm{C}-1 \mathrm{~B}\right), 69.9\left(\mathrm{dd}, J_{\mathrm{C}-\mathrm{F}}=\right.$ $\left.24.5 \mathrm{~Hz}, J_{\mathrm{C}-\mathrm{F}}=28.5 \mathrm{~Hz}, \mathrm{C}-1 \mathrm{~A}\right), 69.6(\mathrm{C}-5 \mathrm{~A}), 69.5(\mathrm{C}-5 \mathrm{~B}), 63.1\left(\mathrm{CH}_{2} \mathrm{CH}_{3} \mathrm{~A}\right), 62.8$ $\left(\mathrm{CH}_{2} \mathrm{CH}_{3} \mathrm{~B}\right), 14.0\left(\mathrm{CH}_{2} \mathrm{CH}_{3} \mathrm{~A}\right), 13.9\left(\mathrm{CH}_{2} \mathrm{CH}_{3} \mathrm{~B}\right) \mathrm{ppm} .{ }^{19} \mathrm{~F} \mathrm{NMR}\left(471 \mathrm{MHz}, \mathrm{CDCl}_{3}\right) \delta=$ $-111.5\left(\mathrm{dd}, 1 \mathrm{~F}, J_{\mathrm{H} 1-\mathrm{Fa}}=5.7 \mathrm{~Hz}, J_{\mathrm{Fa}-\mathrm{Fb}}=261.7 \mathrm{~Hz}, \mathrm{FaA}\right),-118.4\left(\mathrm{dd}, 1 \mathrm{~F}, J=12.0 \mathrm{~Hz}, J_{\mathrm{Fa}-}\right.$ $\mathrm{Fb}=259.7 \mathrm{~Hz}, \mathrm{FaB}),-119.0\left(\mathrm{dd}, 1 \mathrm{~F}, J=12.3 \mathrm{~Hz}, J_{\mathrm{Fa}-\mathrm{Fb}}=259.7 \mathrm{~Hz}, \mathrm{FbB}\right),-123.1(\mathrm{dd}, 1 \mathrm{~F}$, $\left.J_{\mathrm{H} 1-\mathrm{Fb}}=18.9 \mathrm{~Hz}, J_{\mathrm{Fa}-\mathrm{Fb}}=261.7 \mathrm{~Hz}, \mathrm{FbA}\right) \mathrm{ppm}$. HRMS (ESI+): $\mathrm{m} / \mathrm{z}$ calculated for $\mathrm{C}_{37} \mathrm{H}_{40} \mathrm{~F}_{2} \mathrm{NaO}_{7}[\mathrm{M}+\mathrm{Na}]^{+}$: calc. 657.2634; found: 657.2628 . 


\section{${ }^{1}$ H NMR}

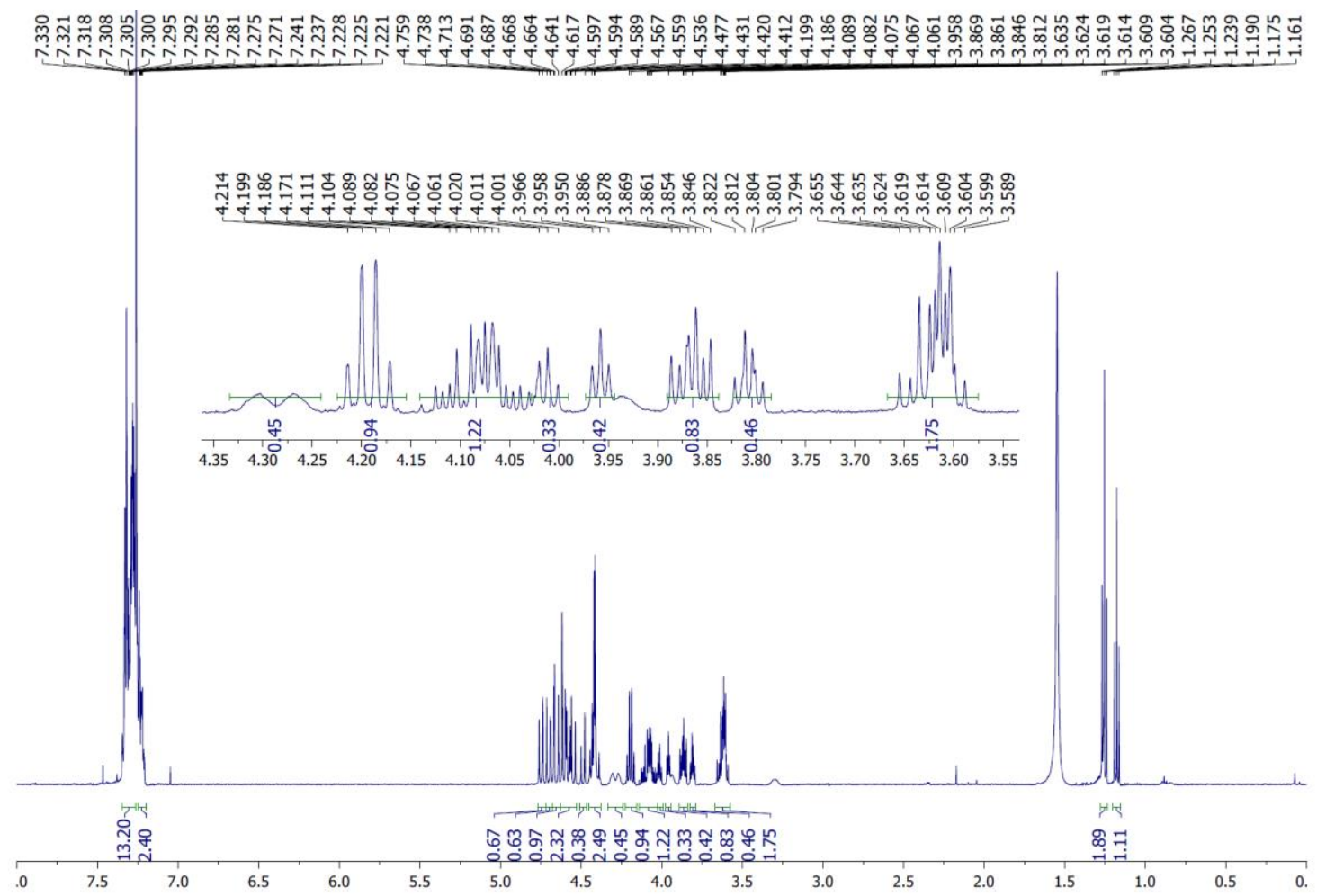

${ }^{19}$ F NMR

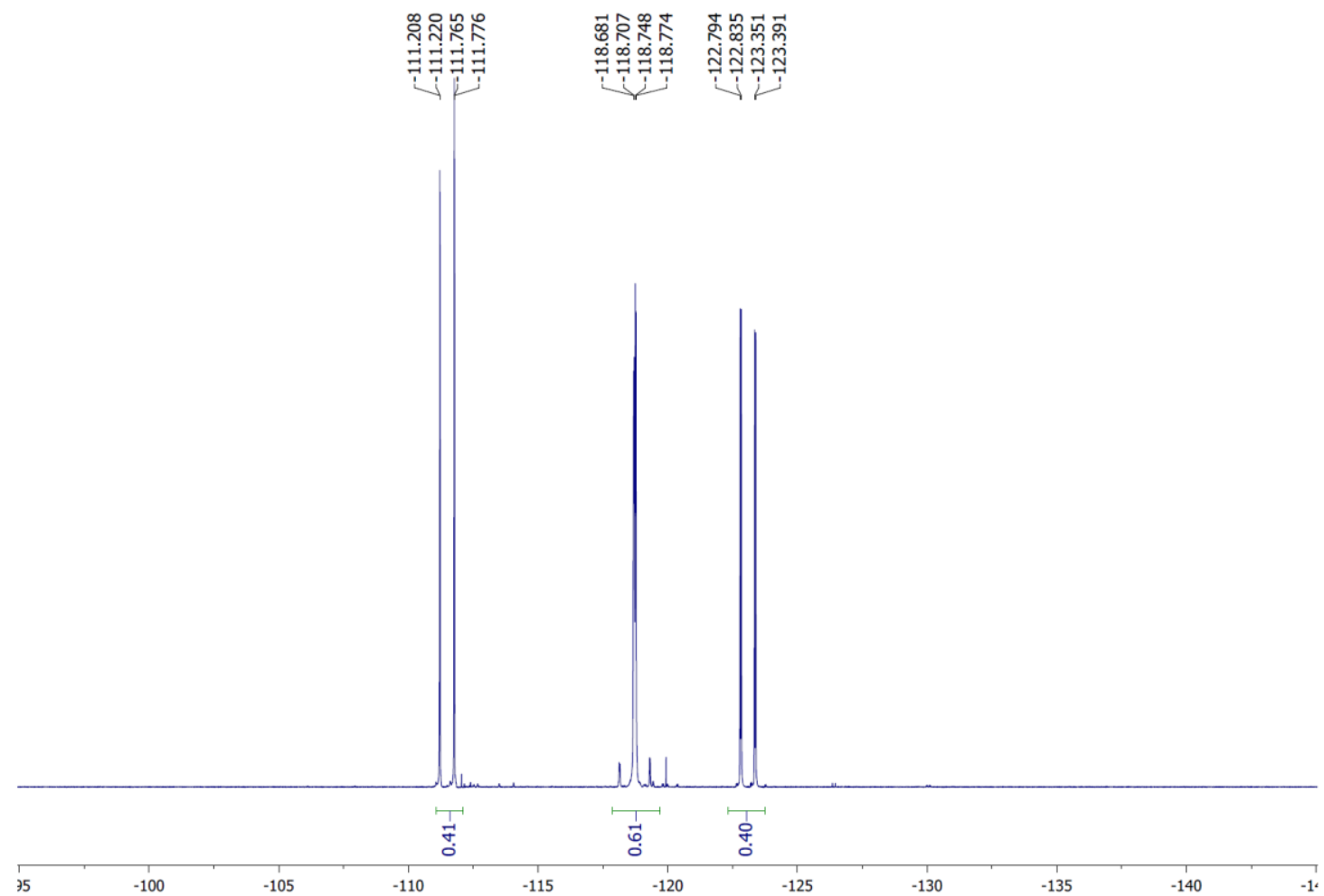


${ }^{13}$ C NMR

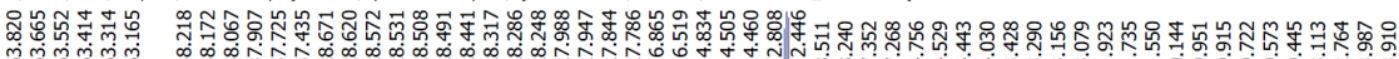

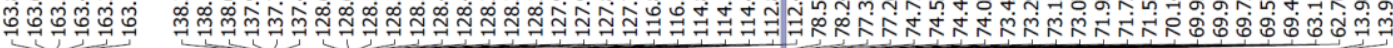

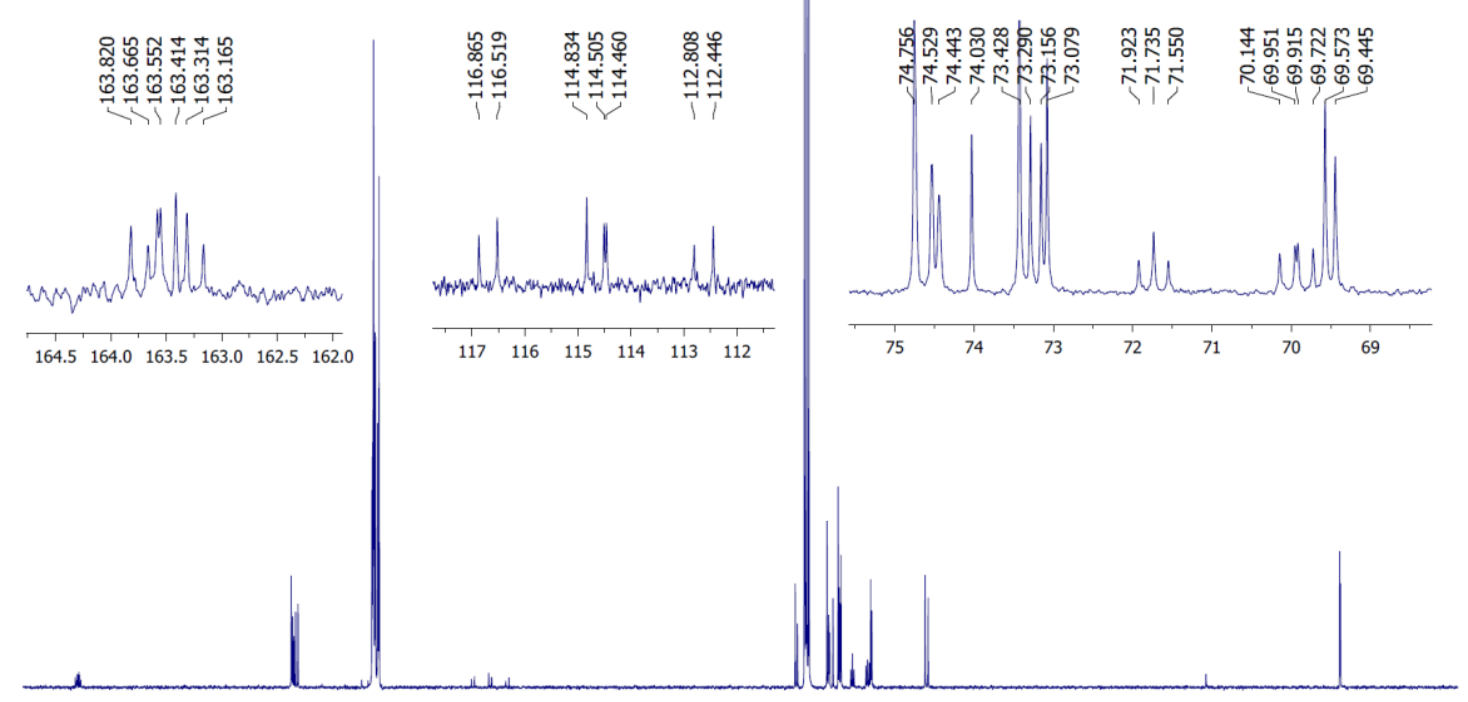

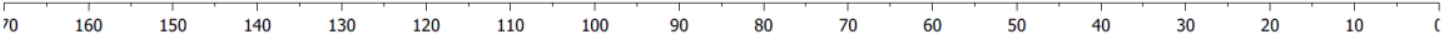




\section{D-Lyxose}

Ethyl 2,3,4,5-tetra-O-benzyl-1',1'-difluoro-1-hydroxyheptanoate ((R/S)-3d).<smiles>O=C[C@H](OCc1ccccc1)[C@H](Br)OCc1ccccc1</smiles>

2d

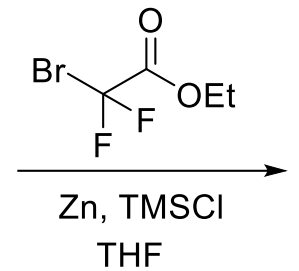

THF<smiles>CCOC(=O)C(F)(F)C(O)C(OCc1ccccc1)C(Cc1ccccc1)C(Cc1ccccc1)Cc1ccccc1</smiles>

(R)-3d

$73 \%$<smiles>CCOC(=O)C(F)(F)C(O)C(OCc1ccccc1)[C@H](OCc1ccccc1)[C@H](O)COC</smiles>

(S)-3d

$\mathrm{R}: \mathrm{S} d r 64: 36$

To a dry two-necked round bottomed flask was added "activated zinc" powder (1.472 g, $16.44 \mathrm{mmol}, 5.7$ equiv.). The flask was heated up at $40^{\circ} \mathrm{C}$ and the zinc powder suspended in dry THF $(20 \mathrm{ml})$ under argon atmosphere. Then, TMSCl $(120 \mu 1,0.98 \mathrm{mmol}, 0.4$ equiv.) was added dropwise and the reaction was stirred at $60^{\circ} \mathrm{C}$ for 15 mins. Next, a solution of aldehyde $2 \mathrm{c}(1.472 \mathrm{~g}, 2.88 \mathrm{mmol}, 1.0$ equiv.) and ethyl bromo-difluoroacetate ( $0.44 \mathrm{ml}, 3.46 \mathrm{mmol}, 1.2$ equiv.) dissolved homogenously in dry THF (20 ml) under argon atmosphere was added dropwise at $60^{\circ} \mathrm{C}$. The reaction was stirred vigorously for $1.5 \mathrm{~h}$ at $60^{\circ} \mathrm{C}$ and then cooled at room temperature. Ice water $(20 \mathrm{ml})$ and $\mathrm{HCl}(1 \mathrm{M}, 20 \mathrm{ml})$ were added and the mixture was diluted with EtOAc. The organic phase was washed with $\mathrm{NaHCO}_{3}$ (sat) (2 times), brine, dried over $\mathrm{MgSO}_{4}$ and concentrated under reduced pressure. Purification by silica gel chromatography (Cy/EtOAc 92:8) gave an inseparable mixture of two diastereoisomers $(R)-3 d a n d(S)-3 d$ in 64:36 $d r$ ratio $(1.337 \mathrm{~g}, 73 \%)$.

${ }^{1} \mathrm{H}$ NMR (500 MHz, $\left.\mathrm{CDCl}_{3}\right) \delta=7.38-7.24$ (m, 17H, $\left.\mathrm{H}^{\text {arom }}\right), 7.22-7.19\left(\mathrm{~m}, 3 \mathrm{H}, \mathrm{H}^{\text {arom }}\right)$, $4.79\left(\mathrm{AB}, 1 \mathrm{H}, J_{\mathrm{A}-\mathrm{B}}=11.3 \mathrm{~Hz}, \mathrm{CH}_{2} \mathrm{R}^{\mathrm{Bn}}\right), 4.76\left(\mathrm{AB}, 1 \mathrm{H}, J_{\mathrm{A}-\mathrm{B}}=11.9 \mathrm{~Hz}, \mathrm{CH}_{2} \mathrm{~S}^{\mathrm{Bn}}\right), 4.71$ $\left(\mathrm{AB}, 1 \mathrm{H}, J_{\mathrm{A}-\mathrm{B}}=11.3 \mathrm{~Hz}, \mathrm{CH}_{2} \mathrm{~S}^{\mathrm{Bn}}\right), 4.70\left(\mathrm{AB}, 1 \mathrm{H}, J_{\mathrm{A}-\mathrm{B}}=11.5 \mathrm{~Hz}, \mathrm{CH}_{2} \mathrm{R}^{\mathrm{Bn}}\right), 4.66(\mathrm{AB}$, $\left.1 \mathrm{H}, J_{\mathrm{A}-\mathrm{B}}=11.6 \mathrm{~Hz}, \mathrm{CH}_{2} \mathrm{R}^{\mathrm{Bn}}\right), 4.66\left(\mathrm{AB}, 1 \mathrm{H}, J_{\mathrm{A}-\mathrm{B}}=11.3 \mathrm{~Hz}, \mathrm{CH}_{2} \mathrm{~S}^{\mathrm{Bn}}\right), 4.62\left(\mathrm{AB}, 1 \mathrm{H}, J_{\mathrm{A}-}\right.$ В $\left.=11.3 \mathrm{~Hz}, \mathrm{CH}_{2} \mathrm{~S}^{\mathrm{Bn}}\right), 4.58\left(\mathrm{AB}, 1 \mathrm{H}, J_{\mathrm{A}-\mathrm{B}}=11.9 \mathrm{~Hz}, \mathrm{CH}_{2} \mathrm{~S}^{\mathrm{Bn}}\right), 4.55\left(\mathrm{AB}, 1 \mathrm{H}, J_{\mathrm{A}-\mathrm{B}}=11.0\right.$ $\left.\mathrm{Hz}, \mathrm{CH}_{2} \mathrm{~S}^{\mathrm{Bn}}\right), 4.53\left(\mathrm{AB}, 1 \mathrm{H}, J_{\mathrm{A}-\mathrm{B}}=11.1 \mathrm{~Hz}, \mathrm{CH}_{2} \mathrm{R}^{\mathrm{Bn}}\right), 4.51\left(\mathrm{~s}, 2 \mathrm{H}, \mathrm{CH}_{2} \mathrm{R}^{\mathrm{Bn}}\right), 4.48(\mathrm{~s}, 2 \mathrm{H}$, $\left.\mathrm{CH}_{2} \mathrm{~S}^{\mathrm{Bn}}\right), 4.40\left(\mathrm{AB}, 1 \mathrm{H}, J_{\mathrm{A}-\mathrm{B}}=10.8 \mathrm{~Hz}, \mathrm{CH}_{2} \mathrm{~S}^{\mathrm{Bn}}\right), 4.34\left(\mathrm{AB}, 1 \mathrm{H}, J_{\mathrm{A}-\mathrm{B}}=11.0 \mathrm{~Hz}, \mathrm{CH}_{2} \mathrm{R}^{\mathrm{Bn}}\right)$, 4.26 (q, $\left.2 \mathrm{H}, J_{\mathrm{CH} 2 \mathrm{CH} 3}=7.1 \mathrm{~Hz}, \mathrm{CH}_{2} \mathrm{CH}_{3} \mathrm{~S}\right), 4.13\left(\mathrm{~d}, 1 \mathrm{H}, J_{1-2}=6.5 \mathrm{~Hz}, \mathrm{H}-1 \mathrm{~S}\right), 4.02(\mathrm{~d}, 1 \mathrm{H}$, $\left.J_{1-2}=6.6 \mathrm{~Hz}, \mathrm{H}-1 \mathrm{R}\right), 3.95\left(\mathrm{qd}, 2 \mathrm{H}, J_{\mathrm{CH} 2 \mathrm{CH} 3}=7.2,2.8 \mathrm{~Hz}, \mathrm{CH}_{2} \mathrm{CH}_{3} \mathrm{R}\right), 3.91-3.85(\mathrm{~m}, 5 \mathrm{H}$, H-2R, H-2S, H-3R, H-3S, H-4R), 3.82 (d, 1H, $\left.J_{3-4}=J_{4-5}=9.3 \mathrm{~Hz}, \mathrm{H}-4 \mathrm{R}\right), 3.72$ (dd, 1H, $\left.J_{4-5}=9.3, J_{5 \mathrm{a}-5 \mathrm{~b}}=4.9 \mathrm{~Hz}, \mathrm{H}-5 \mathrm{~S}\right), 3.70\left(\mathrm{dd}, 1 \mathrm{H}, J_{4-5}=10.3, J_{5 \mathrm{a}-5 \mathrm{~b}}=4.3 \mathrm{~Hz}, \mathrm{H}-5 \mathrm{R}\right), 3.65$ (dd, 1H, J = 5.8, 9.8 Hz, H-5S), 3.62 (dd, 1H, J = 10.6, $4.9 \mathrm{~Hz}, \mathrm{H}-5 \mathrm{R}), 3.59$ (bs, 1H, OHR), 3.34 (bs, $1 \mathrm{H}, \mathrm{OHS}), 1.28\left(\mathrm{t}, 3 \mathrm{H}, J_{\mathrm{CH} 2 \mathrm{CH} 3}=7.1 \mathrm{~Hz}, \mathrm{CH}_{2} \mathrm{CH}_{3} \mathrm{~S}\right), 1.11\left(\mathrm{t}, 3 \mathrm{H}, J_{\mathrm{CH} 2 \mathrm{CH} 3}\right.$ $\left.=7.2 \mathrm{~Hz}, \mathrm{CH}_{2} \mathrm{CH}_{3} \mathrm{R}\right) \mathrm{ppm} .{ }^{13} \mathrm{C} \mathrm{NMR}\left(125 \mathrm{MHz}, \mathrm{CDCl}_{3}\right) \delta=163.6\left(\mathrm{t}, J_{\mathrm{C}-\mathrm{F}}=30.4 \mathrm{~Hz}\right.$, $\left.\mathrm{C}_{\mathrm{q}} \mathrm{O}_{2} \mathrm{EtR}\right), 163.3\left(\mathrm{t}, J_{\mathrm{C}-\mathrm{F}}=30.3 \mathrm{~Hz}, \mathrm{C}_{\mathrm{q}} \mathrm{O}_{2} \mathrm{EtS}\right), 138.43\left(\mathrm{C}_{\mathrm{q}}{ }^{\text {arom }}\right), 138.41\left(\mathrm{C}_{\mathrm{q}}{ }^{\text {arom }}\right), 138.0$ $\left(\mathrm{C}_{\mathrm{q}}{ }^{\text {arom }}\right), 137.9\left(\mathrm{C}_{\mathrm{q}}{ }^{\text {arom }}\right), 137.55\left(\mathrm{C}_{\mathrm{q}}{ }^{\text {arom }}\right), 137.49\left(\mathrm{C}_{\mathrm{q}}{ }^{\text {arom }}\right), 137.3\left(\mathrm{C}_{\mathrm{q}}{ }^{\text {arom }}\right), 128.6-127.8$ $\left(\mathrm{CH}^{\text {arom }}\right), 114.9\left(\mathrm{dd}, J_{\mathrm{C}-\mathrm{F}}=256.7 \mathrm{~Hz}, J_{\mathrm{C}-\mathrm{F}}=249.3 \mathrm{~Hz}, \mathrm{CF}_{2} \mathrm{R}\right), 114.7\left(\mathrm{dd}, J_{\mathrm{C}-\mathrm{F}}=258.3 \mathrm{~Hz}\right.$, $\left.J_{\mathrm{C}-\mathrm{F}}=253.8 \mathrm{~Hz}, \mathrm{CF}_{2} \mathrm{~S}\right), 80.8(\mathrm{C}-1 \mathrm{R}), 79.4(\mathrm{C}-2 \mathrm{R}, 2 \mathrm{~S}, 3 \mathrm{R}, 3 \mathrm{~S}$ or $4 \mathrm{~S}), 78.9(\mathrm{C}-2 \mathrm{R}, 2 \mathrm{~S}, 3 \mathrm{R}$, $3 \mathrm{~S}$ or $4 \mathrm{~S}), 78.49$ (C-2R, 2S, 3R, 3S or $4 \mathrm{~S}), 78.46$ (C-4R), 77.4 (C-2R, 2S, 3R, 3S or 4S), 74.94, 74.91, $74.5(\mathrm{C}-1 \mathrm{~S}), 73.7\left(\mathrm{CH}_{2}{ }^{\mathrm{Bn}}\right), 73.5\left(\mathrm{CH}_{2}{ }^{\mathrm{Bn}}\right), 73.4\left(\mathrm{CH}_{2}{ }^{\mathrm{Bn}}\right), 72.8\left(\mathrm{CH}_{2}{ }^{\mathrm{Bn}}\right), 72.4$ 
$\left(\mathrm{CH}_{2}{ }^{\mathrm{Bn}}\right), 70.2\left(\mathrm{t}, \mathrm{J}=22.3 \mathrm{~Hz}, \mathrm{CF}_{2} \mathrm{R}\right), 69.7(\mathrm{C}-5 \mathrm{R}), 69.6(\mathrm{C}-5 \mathrm{~S}), 69.4(\mathrm{t}, \mathrm{J}=25.9 \mathrm{~Hz}$, $\left.\mathrm{CF}_{2} \mathrm{R}\right), 63.1\left(\mathrm{CH}_{2} \mathrm{CH}_{3} \mathrm{~S}\right), 62.5\left(\mathrm{CH}_{2} \mathrm{CH}_{3} \mathrm{R}\right), 14.0\left(\mathrm{CH}_{2} \mathrm{CH}_{3} \mathrm{~S}\right), 13.8\left(\mathrm{CH}_{2} \mathrm{CH}_{3} \mathrm{R}\right) \mathrm{ppm} .{ }^{19} \mathrm{~F}$ $\operatorname{NMR}\left(471 \mathrm{MHz}, \mathrm{CDCl}_{3}\right) \delta=-112.1\left(\mathrm{dd}, 1 \mathrm{~F}, J_{\mathrm{H} 1-\mathrm{Fa}}=5.5 \mathrm{~Hz}, J_{\mathrm{Fa}-\mathrm{Fb}}=259.9 \mathrm{~Hz}, \mathrm{FaS}\right)$, $115.3\left(\mathrm{dd}, 1 \mathrm{~F}, J_{\mathrm{H} 1-\mathrm{Fa}}=6.6 \mathrm{~Hz}, J_{\mathrm{Fa}-\mathrm{Fb}}=260.1 \mathrm{~Hz}, \mathrm{FaR}\right),-123.0\left(\mathrm{dd}, 1 \mathrm{~F}, J_{\mathrm{H}-\mathrm{Fb}}=20.4 \mathrm{~Hz}\right.$, $\left.J_{\mathrm{Fa}-\mathrm{Fb}}=259.9 \mathrm{~Hz}, \mathrm{FbS}\right),-123.8\left(\mathrm{dd}, 1 \mathrm{~F}, J_{\mathrm{H}-\mathrm{Fb}}=18.7 \mathrm{~Hz}, J_{\mathrm{Fa}-\mathrm{Fb}}=260.1 \mathrm{~Hz}, \mathrm{FbR}\right) \mathrm{ppm}$. HRMS (ESI+): $\mathrm{m} / \mathrm{z}$ calculated for $\mathrm{C}_{37} \mathrm{H}_{41} \mathrm{~F}_{2} \mathrm{O}_{7}[\mathrm{M}+\mathrm{H}]^{+}$: calc. 635.2815; found: 635.2817. 


\section{${ }^{1}$ H NMR}

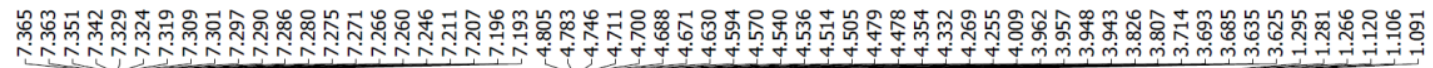

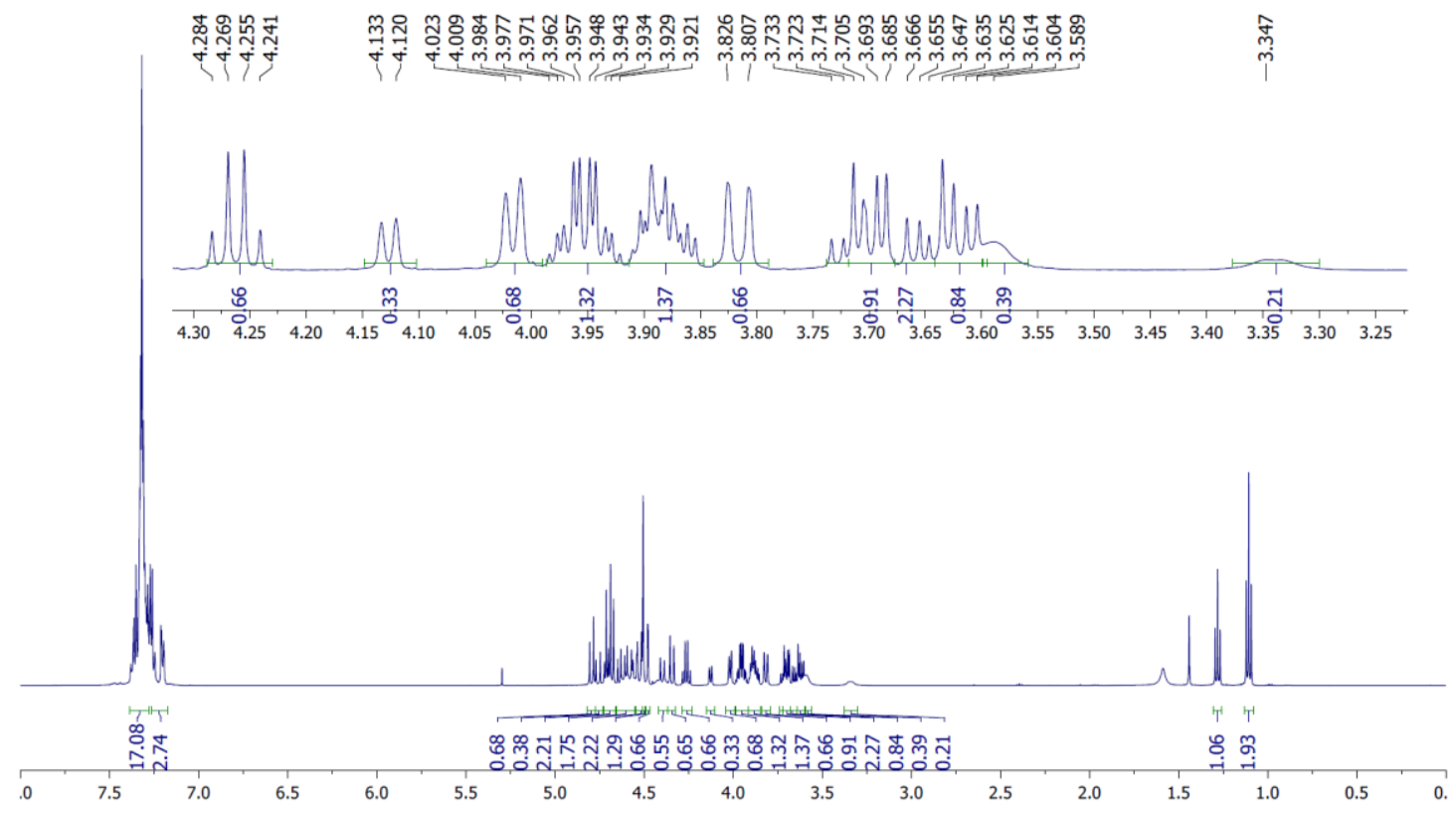

${ }^{19}$ F NMR

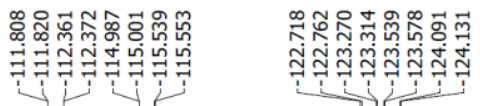

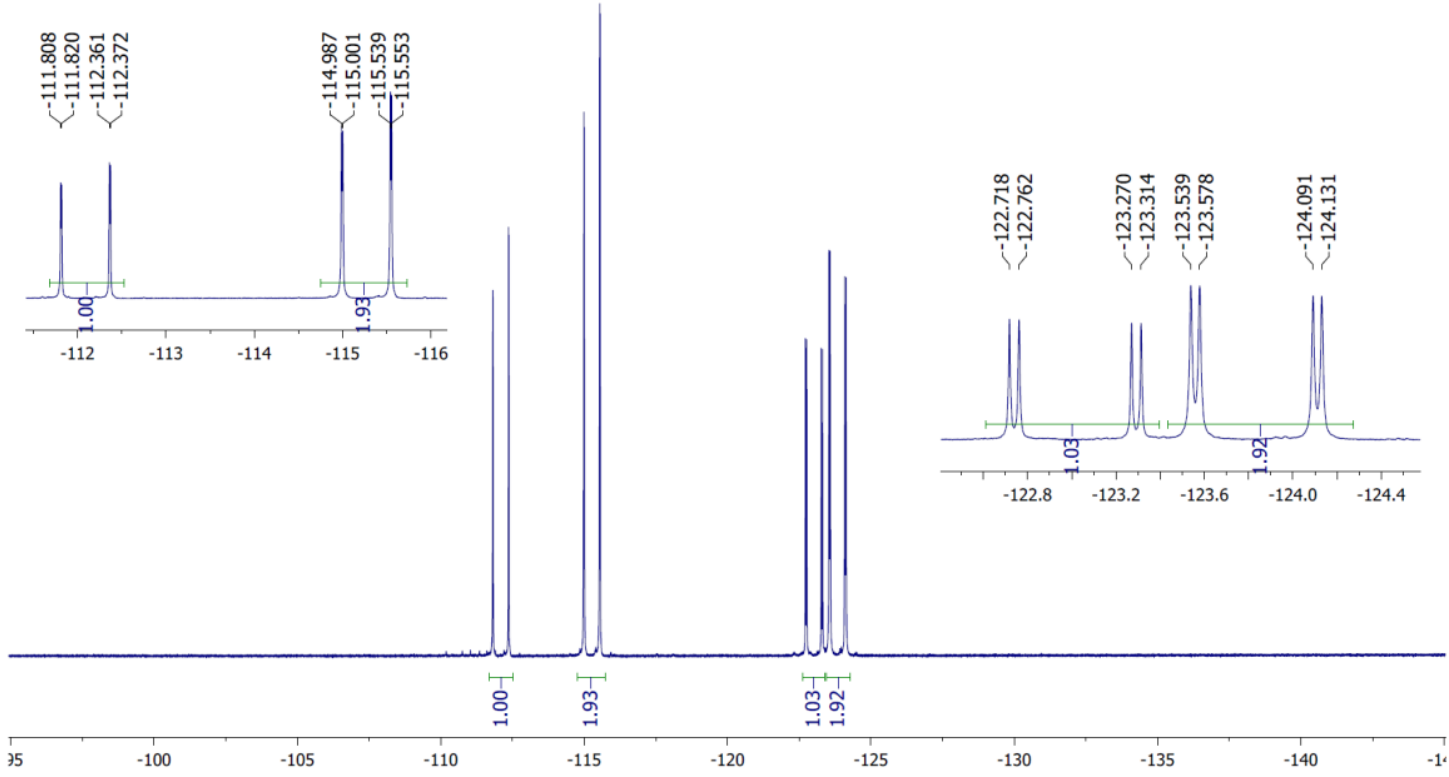


${ }^{13}$ C NMR

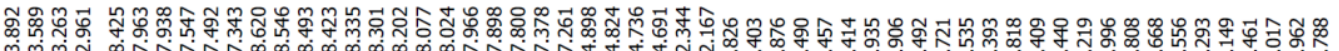

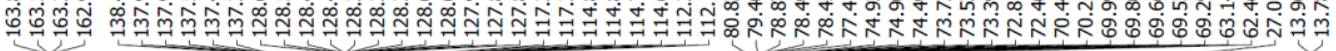

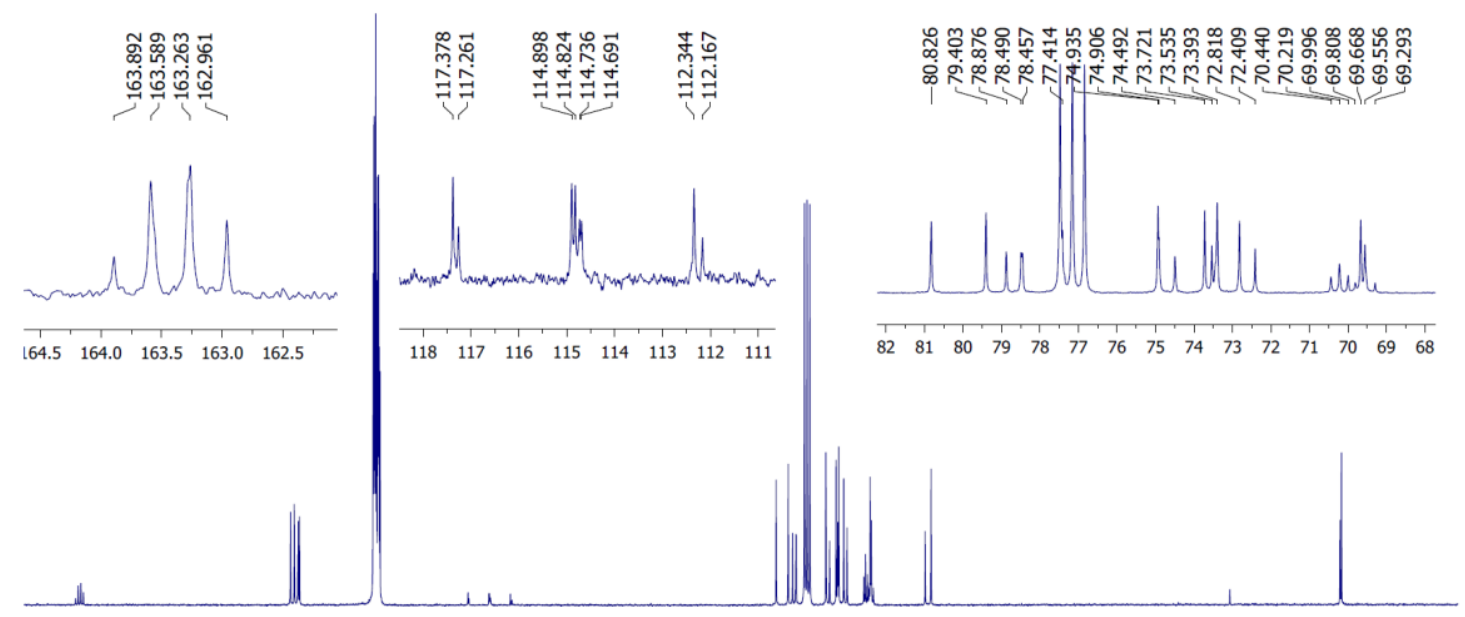

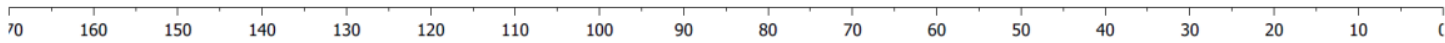




\section{L-Fucose}

Ethyl 2,3,4,5-tetra-O-benzyl-1',1'-difluoro-1-hydroxyoctanoate ((S/R)-3e).

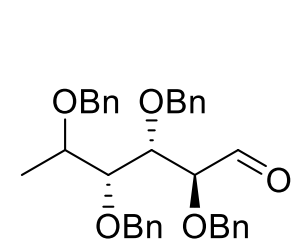

$2 e$<smiles>CCOC(=O)C(F)(Br)Br</smiles>

THF

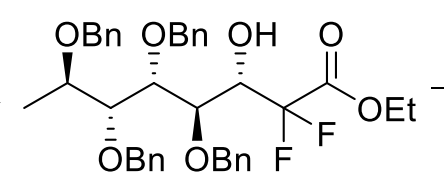

(S)-3e

$66 \%$

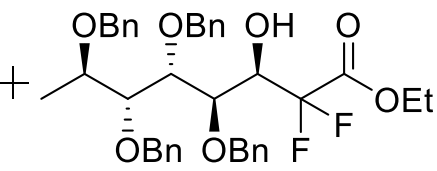

(R)-3e

S:R $d r 57: 43$

To a dry two-necked round bottomed flask was added "activated zinc" powder (660 mg, $10.1 \mathrm{mmol}, 5.7$ equiv.). The flask was heated up at $40^{\circ} \mathrm{C}$ and the zinc powder suspended in dry THF (13 ml) under argon atmosphere. Then, TMSCl ( $80 \mu 1,0.60 \mathrm{mmol}, 0.4$ equiv.) was added dropwise and the reaction was stirred at $60^{\circ} \mathrm{C}$ for 15 mins. Next, a solution of aldehyde $2 \mathrm{e}$ (929 mg, $1.77 \mathrm{mmol}, 1.0$ equiv.) and ethyl bromo-difluoroacetate $(0.27 \mathrm{ml}$, 2.12 mmol, 1.2 equiv.) dissolved homogenously in dry THF (13 ml) under argon atmosphere was added dropwise at $60^{\circ} \mathrm{C}$. The reaction was stirred vigorously for $2 \mathrm{~h}$ at $60^{\circ} \mathrm{C}$ and then cooled at room temperature. Ice water $(20 \mathrm{ml})$ and $\mathrm{HCl}(1 \mathrm{M}, 20 \mathrm{ml})$ were added and the mixture was diluted with EtOAc. The organic phase was washed with $\mathrm{NaHCO}_{3}$ (sat) (2 times), brine, dried over $\mathrm{MgSO}_{4}$ and concentrated under reduced pressure. Crude NMR analysis showed the formation of two diastereoisomer $(S)-3 \mathbf{e}$ and $(R)-3 \mathbf{e}$ in 57:43 $d r$ ratio. Purification by silica gel chromatography (Cy/EtOAc 93:7) afforded pure stereoisomer $(S)$-3e and $(R)-3 \mathbf{e}$ in $66 \%$ overall yield. 
Ethyl 4,5,6,7-tetrakis(benzyloxy)-2,2-difluoro-3-hydroxyoctanoate ((S)-3e).<smiles>CCOC(=O)C(F)(F)[C@H](O)[C@H](Br)[C@H](Br)c1ccccc1</smiles>

(S)-3e

$[\alpha]_{20}{ }^{\mathrm{D}}:-0.2\left(\mathrm{c} 1.0, \mathrm{CHCl}_{3}\right) .{ }^{1} \mathrm{H}$ NMR $\left(500 \mathrm{MHz}, \mathrm{CDCl}_{3}\right) \delta=7.33-7.27\left(\mathrm{~m}, 16 \mathrm{H}, \mathrm{H}^{\text {arom }}\right)$, 7.25-7.12 (m, $\left.4 \mathrm{H}, \mathrm{H}^{\mathrm{arom}}\right), 4.78\left(\mathrm{AB}, 1 \mathrm{H}, J_{\mathrm{A}-\mathrm{B}}=11.5 \mathrm{~Hz}, \mathrm{CH}_{2}{ }^{\mathrm{Bn}}\right), 4.74\left(\mathrm{AB}, 2 \mathrm{H}, J_{\mathrm{A}-\mathrm{B}}=\right.$ $\left.10.9 \mathrm{~Hz}, \mathrm{CH}_{2}{ }^{\mathrm{Bn}}\right), 4.62\left(\mathrm{AB}, 1 \mathrm{H}, J_{\mathrm{A}-\mathrm{B}}=11.5 \mathrm{~Hz}, \mathrm{CH}_{2}{ }^{\mathrm{Bn}}\right), 4.60\left(\mathrm{AB}, 1 \mathrm{H}, J_{\mathrm{A}-\mathrm{B}}=11.5 \mathrm{~Hz}\right.$, $\left.\mathrm{CH}_{2}{ }^{\mathrm{Bn}}\right), 4.57\left(\mathrm{AB}, 1 \mathrm{H}, J_{\mathrm{A}-\mathrm{B}}=10.9 \mathrm{~Hz}, \mathrm{CH}_{2}{ }^{\mathrm{Bn}}\right), 4.53\left(\mathrm{AB}, 1 \mathrm{H}, J_{\mathrm{A}-\mathrm{B}}=11.5 \mathrm{~Hz}, \mathrm{CH}_{2}{ }^{\mathrm{Bn}}\right)$, $4.50\left(\mathrm{AB}, 1 \mathrm{H}, J_{\mathrm{A}-\mathrm{B}}=10.9 \mathrm{~Hz}, \mathrm{CH}_{2}{ }^{\mathrm{Bn}}\right), 4.40\left(\mathrm{dd}, 1 \mathrm{H}, J_{1-\mathrm{Fa}}=5.7 \mathrm{~Hz}, J_{1-\mathrm{Fb}}=18.9 \mathrm{~Hz}, \mathrm{H}-1\right)$, $4.16\left(\mathrm{q}, 2 \mathrm{H}, J_{\mathrm{CH} 2 \mathrm{CH} 3}=7.5 \mathrm{~Hz}, \mathrm{CH}_{2} \mathrm{CH}_{3}\right), 4.09\left(\mathrm{~d}, 1 \mathrm{H}, J_{3-4}=6.3 \mathrm{~Hz}, \mathrm{H}-3\right), 3.92-3.87(\mathrm{~m}$, $2 \mathrm{H}, \mathrm{H}-2, \mathrm{H}-5), 3.71\left(\mathrm{dd}, 1 \mathrm{H}, J_{4-5}=4.0 \mathrm{~Hz}, J_{3-4}=6.3 \mathrm{~Hz}, \mathrm{H}-4\right), 1.23\left(\mathrm{t}, 3 \mathrm{H}, J_{\mathrm{CH} 2 \mathrm{CH} 3}=7.5\right.$ $\left.\mathrm{Hz}, \mathrm{CH}_{2} \mathrm{CH}_{3}\right), 1.21\left(\mathrm{~d}, 3 \mathrm{H}, J_{5-\mathrm{CH} 3}=6.3 \mathrm{~Hz}, \mathrm{CH}_{3}\right) \mathrm{ppm} .{ }^{13} \mathrm{C} \mathrm{NMR}\left(125 \mathrm{MHz}, \mathrm{CDCl}_{3}\right) \delta=$ $163.6\left(\mathrm{dd}, J_{\mathrm{C}-\mathrm{F}}=30.2 \mathrm{~Hz}, J_{\mathrm{C}-\mathrm{F}}=32.6 \mathrm{~Hz}, \mathrm{C}_{\mathrm{q}} \mathrm{O}_{2} \mathrm{Et}\right), 138.8\left(\mathrm{C}_{\mathrm{q}}{ }^{\text {arom }}\right), 138.2\left(\mathrm{C}_{\mathrm{q}}{ }^{\text {arom }}\right), 138.1$ $\left(\mathrm{C}_{\mathrm{q}}{ }^{\text {arom }}\right), 137.7\left(\mathrm{C}_{\mathrm{q}}{ }^{\text {arom }}\right), 128.5-127.5\left(\mathrm{CH}^{\text {arom }}\right), 114.6\left(\mathrm{dd}, J_{\mathrm{C}-\mathrm{F}}=252.3 \mathrm{~Hz}, J_{\mathrm{C}-\mathrm{F}}=259.5\right.$ $\left.\mathrm{Hz}, \mathrm{CF}_{2}\right), 84.3$ (C-4), 78.7 (C-2), $75.7(\mathrm{C}-5), 75.5(\mathrm{C}-3), 74.8\left(\mathrm{CH}_{2}{ }^{\mathrm{Bn}}\right), 74.4\left(\mathrm{CH}_{2}{ }^{\mathrm{Bn}}\right), 73.5$ $\left(\mathrm{CH}_{2}{ }^{\mathrm{Bn}}\right), 71.8\left(\mathrm{CH}_{2}{ }^{\mathrm{Bn}}\right), 70.0\left(\mathrm{dd}, J_{\mathrm{C}-\mathrm{F}}=24.1 \mathrm{~Hz}, J_{\mathrm{C}-\mathrm{F}}=27.8 \mathrm{~Hz}, \mathrm{C}-1\right), 63.0\left(\mathrm{CH}_{2} \mathrm{CH}_{3}\right)$, $17.0\left(\mathrm{CH}_{3}\right), 13.9\left(\mathrm{CH}_{2} \mathrm{CH}_{3}\right)$ ppm. ${ }^{19} \mathrm{~F}$ NMR $\left(471 \mathrm{MHz}, \mathrm{CDCl}_{3}\right) \delta=-111.0\left(\mathrm{dd}, 1 \mathrm{~F}, J_{\mathrm{H} 1-\mathrm{Fa}}\right.$ $\left.=5.7 \mathrm{~Hz}, J_{\mathrm{Fa}-\mathrm{Fb}}=260.5 \mathrm{~Hz}, \mathrm{Fa}\right),-122.6\left(\mathrm{dd}, 1 \mathrm{~F}, J_{\mathrm{H} 1-\mathrm{Fb}}=18.9 \mathrm{~Hz}, J_{\mathrm{Fa}-\mathrm{Fb}}=260.5 \mathrm{~Hz}, \mathrm{Fb}\right)$ ppm. HRMS (ESI+): $\mathrm{m} / \mathrm{z}$ calculated for $\mathrm{C}_{38} \mathrm{H}_{43} \mathrm{~F}_{2} \mathrm{O}_{7}[\mathrm{M}+\mathrm{H}]^{+}$: calc. 649.2971; found: 649.2971 . 


\section{${ }^{1}$ H NMR}

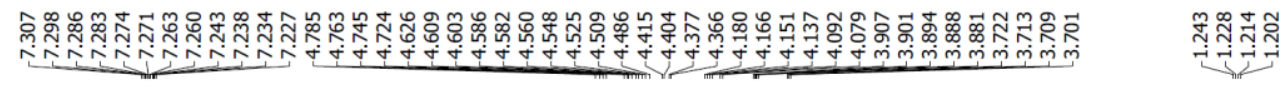

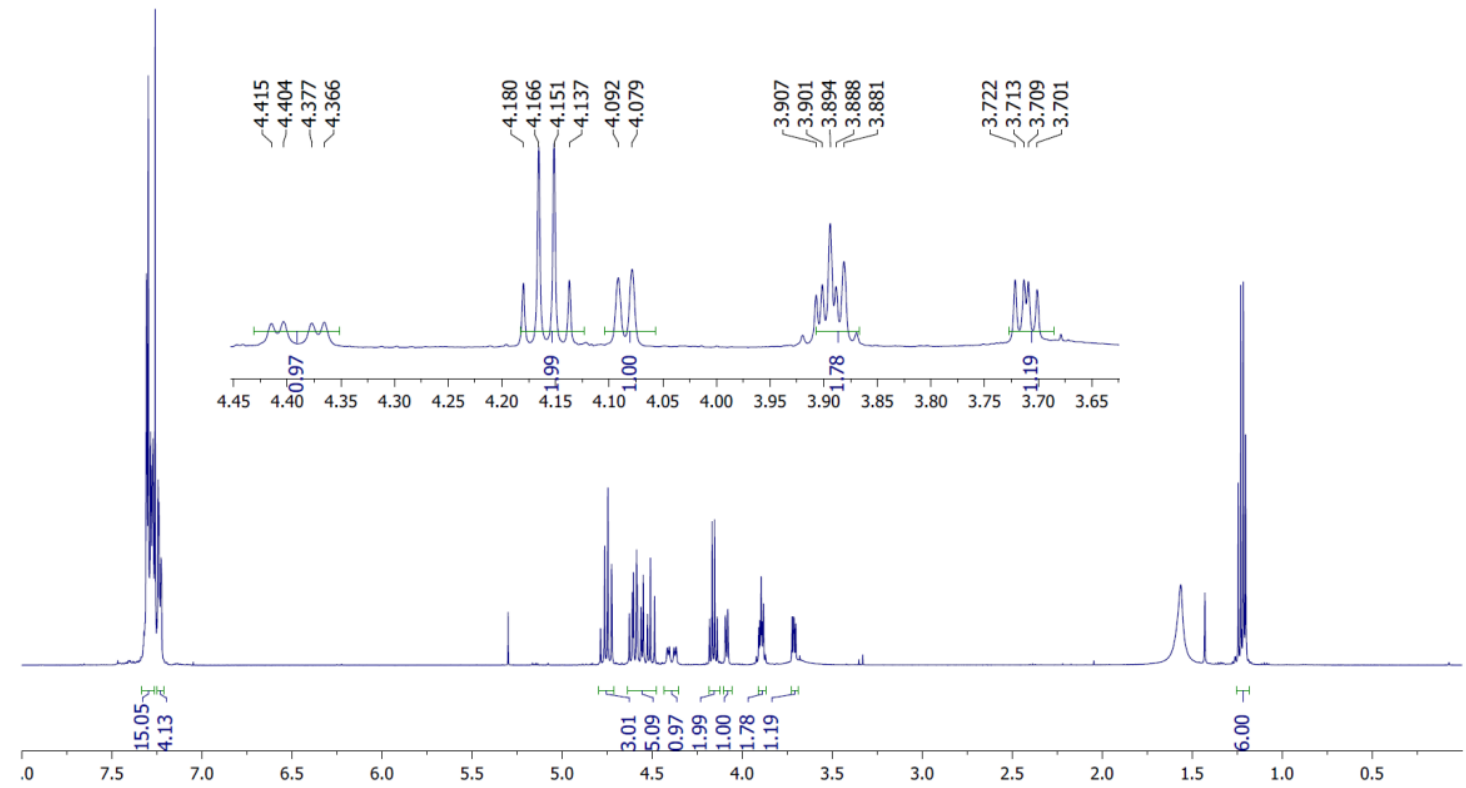

${ }^{19}$ F NMR

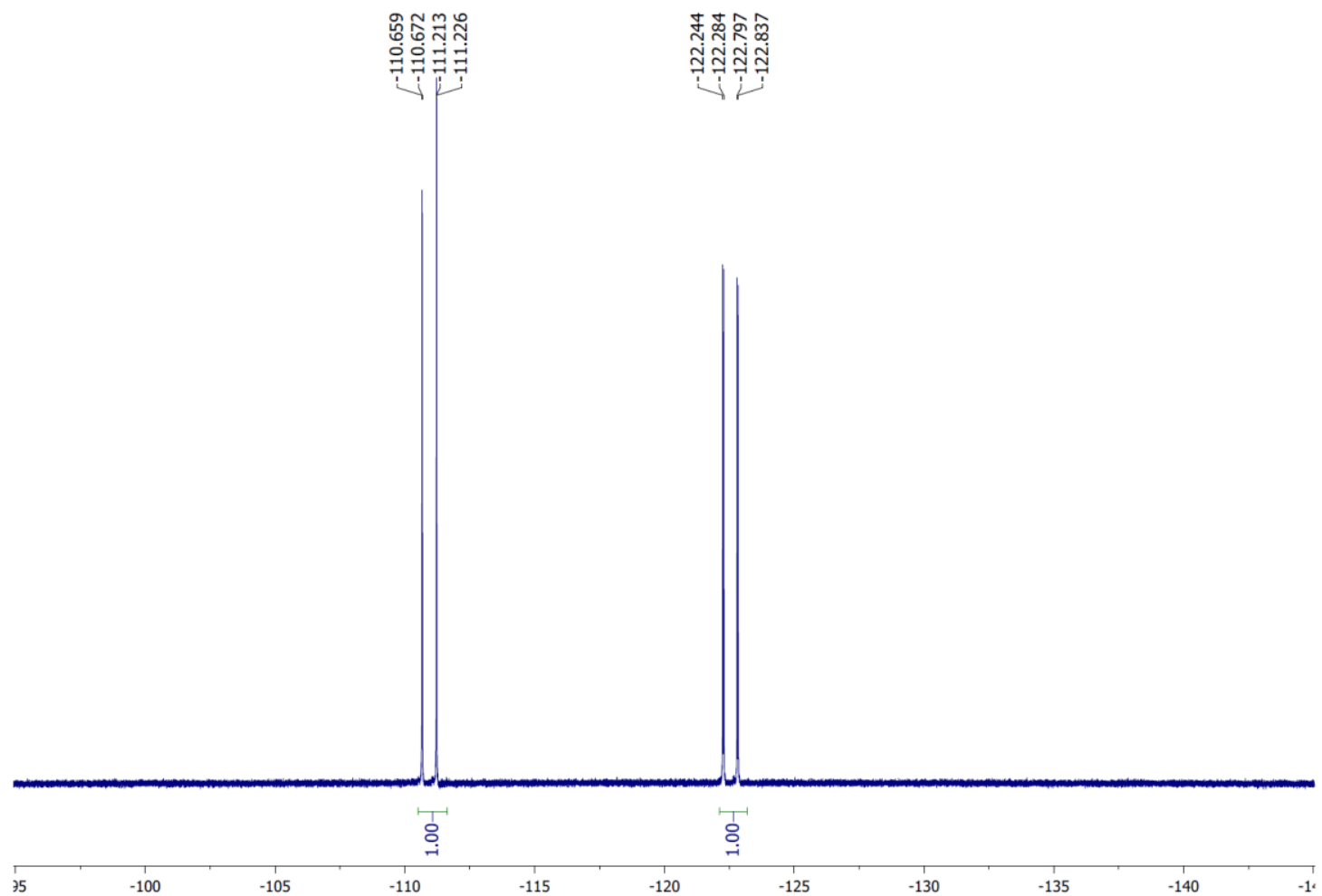


${ }^{13}$ C NMR
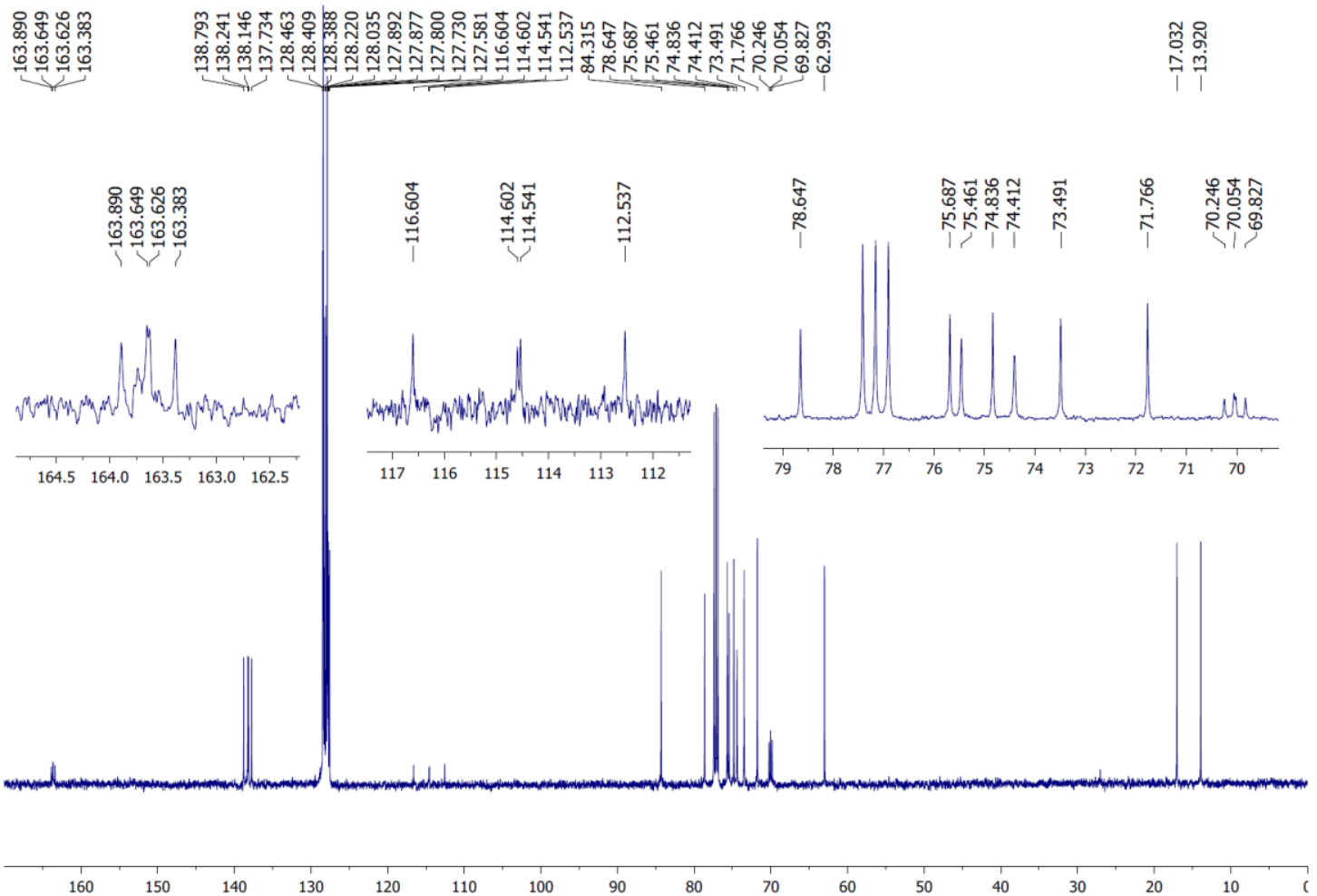
Ethyl 4,5,6,7-tetrakis(benzyloxy)-2,2-difluoro-3-hydroxyoctanoate ((R)-3e).<smiles>CCOC(=O)C(F)(F)[C@H](O)C(Cc1ccccc1)C(Cc1ccccc1)[C@H](Cc1ccccc1)OC(C)C</smiles>

(R)-3e

$[\alpha]_{20}{ }^{\mathrm{D}}:+26.3\left(\mathrm{c} 1.0, \mathrm{CHCl}_{3}\right) .{ }^{1} \mathrm{H} \mathrm{NMR}\left(500 \mathrm{MHz}, \mathrm{CDCl}_{3}\right) \delta=7.36-7.20\left(\mathrm{~m}, 20 \mathrm{H}, \mathrm{H}^{\text {arom }}\right)$, $4.79\left(\mathrm{AB}, 1 \mathrm{H}, J_{\mathrm{A}-\mathrm{B}}=10.9 \mathrm{~Hz}, \mathrm{CH}_{2}{ }^{\mathrm{Bn}}\right), 4.75\left(\mathrm{AB}, 1 \mathrm{H}, J_{\mathrm{A}-\mathrm{B}}=10.9 \mathrm{~Hz}, \mathrm{CH}_{2}{ }^{\mathrm{Bn}}\right), 4.67(\mathrm{AB}$, $\left.1 \mathrm{H}, J_{\mathrm{A}-\mathrm{B}}=10.9 \mathrm{~Hz}, \mathrm{CH}_{2}{ }^{\mathrm{Bn}}\right), 4.65\left(\mathrm{AB}, 1 \mathrm{H}, J_{\mathrm{A}-\mathrm{B}}=11.5 \mathrm{~Hz}, \mathrm{CH}_{2}{ }^{\mathrm{Bn}}\right), 4.59\left(\mathrm{AB}, 1 \mathrm{H}, J_{\mathrm{A}-\mathrm{B}}=\right.$ $\left.10.9 \mathrm{~Hz}, \mathrm{CH}_{2}{ }^{\mathrm{Bn}}\right), 4.55\left(\mathrm{AB}, 1 \mathrm{H}, J_{\mathrm{A}-\mathrm{B}}=10.9 \mathrm{~Hz}, \mathrm{CH}_{2}{ }^{\mathrm{Bn}}\right), 4.51\left(\mathrm{AB}, 1 \mathrm{H}, J_{\mathrm{A}-\mathrm{B}}=11.5 \mathrm{~Hz}\right.$, $\left.\mathrm{CH}_{2}{ }^{\mathrm{Bn}}\right), 4.51-4.44(\mathrm{~m}, 1 \mathrm{H}, \mathrm{H}-1), 4.44\left(\mathrm{AB}, 1 \mathrm{H}, J_{\mathrm{A}-\mathrm{B}}=10.9 \mathrm{~Hz}, \mathrm{CH}_{2}{ }^{\mathrm{Bn}}\right), 4.05\left(\mathrm{dd}, 1 \mathrm{H}, J_{1-}\right.$ $\left.\mathrm{Fa}=4.0 \mathrm{~Hz}, J_{3-4}=6.3 \mathrm{~Hz}, \mathrm{H}-3\right), 4.02-3.91\left(\mathrm{~m}, 4 \mathrm{H}, \mathrm{H}-2, \mathrm{H}-5, \mathrm{CH}_{2} \mathrm{CH}_{3}\right), 3.78\left(\mathrm{t}, 1 \mathrm{H}, J_{3-4}=\right.$ $6.3 \mathrm{~Hz}, \mathrm{H}-4), 3.69(\mathrm{bs}, 1 \mathrm{H}, \mathrm{OH}), 1.27\left(\mathrm{~d}, 3 \mathrm{H}, J_{5-\mathrm{CH} 3}=6.3 \mathrm{~Hz}, \mathrm{CH}_{3}\right), 1.12\left(\mathrm{t}, 3 \mathrm{H}, J_{\mathrm{CH} 2 \mathrm{CH}}\right.$ $\left.=7.5 \mathrm{~Hz}, \mathrm{CH}_{2} \mathrm{CH}_{3}\right) \mathrm{ppm} .{ }^{13} \mathrm{C} \mathrm{NMR}\left(125 \mathrm{MHz}, \mathrm{CDCl}_{3}\right) \delta=163.4\left(\mathrm{t}, J_{\mathrm{C}-\mathrm{F}}=31.4 \mathrm{~Hz}\right.$, $\left.\mathrm{C}_{\mathrm{q}} \mathrm{O}_{2} \mathrm{Et}\right), 138.7\left(\mathrm{C}_{\mathrm{q}}^{\text {arom }}\right), 138.0\left(\mathrm{C}_{\mathrm{q}}^{\text {arom }}\right), 137.9\left(\mathrm{C}_{\mathrm{q}}^{\text {arom }}\right), 137.6\left(\mathrm{C}_{\mathrm{q}}{ }^{\text {arom }}\right), 128.6-127.6$ $\left(\mathrm{CH}^{\mathrm{arom}}\right), 114.8\left(\mathrm{dd}, J_{\mathrm{C}-\mathrm{F}}=252.8 \mathrm{~Hz}, J_{\mathrm{C}-\mathrm{F}}=255.4 \mathrm{~Hz}, \mathrm{CF}_{2}\right), 83.0(\mathrm{C}-4), 77.7(\mathrm{C}-3), 76.7$ (C-2), $75.2(\mathrm{C}-5), 74.8\left(\mathrm{CH}_{2}{ }^{\mathrm{Bn}}\right), 73.4\left(2 \mathrm{CH}_{2}{ }^{\mathrm{Bn}}\right), 72.0\left(\mathrm{t}, J_{\mathrm{C}-\mathrm{F}}=23.9 \mathrm{~Hz}, \mathrm{C}-1\right), 71.4$ $\left(\mathrm{CH}_{2}{ }^{\mathrm{Bn}}\right), 62.7\left(\mathrm{CH}_{2} \mathrm{CH}_{3}\right), 17.0\left(\mathrm{CH}_{3}\right), 13.9\left(\mathrm{CH}_{2} \mathrm{CH}_{3}\right)$ ppm. ${ }^{19} \mathrm{~F} \mathrm{NMR}\left(471 \mathrm{MHz}, \mathrm{CDCl}_{3}\right)$ $\delta=-117.1\left(\mathrm{dd}, 1 \mathrm{~F}, J_{\mathrm{H} 1-\mathrm{Fa}}=9.9 \mathrm{~Hz}, J_{\mathrm{Fa}-\mathrm{Fb}}=260.0 \mathrm{~Hz}, \mathrm{Fa}\right),-120.5\left(\mathrm{dd}, 1 \mathrm{~F}, J_{\mathrm{H} 1-\mathrm{Fb}}=14.8\right.$ $\left.\mathrm{Hz}, J_{\mathrm{Fa}-\mathrm{Fb}}=260.0 \mathrm{~Hz}, \mathrm{Fb}\right) \mathrm{ppm}$. HRMS $(\mathrm{ESI}+)$ : $\mathrm{m} / \mathrm{z}$ calculated for $\mathrm{C}_{38} \mathrm{H}_{43} \mathrm{~F}_{2} \mathrm{O}_{7}[\mathrm{M}+\mathrm{H}]^{+}$: calc. 649.2971; found: 649.2964 . 


\section{${ }^{1}$ H NMR}

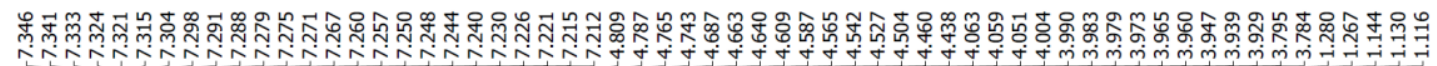

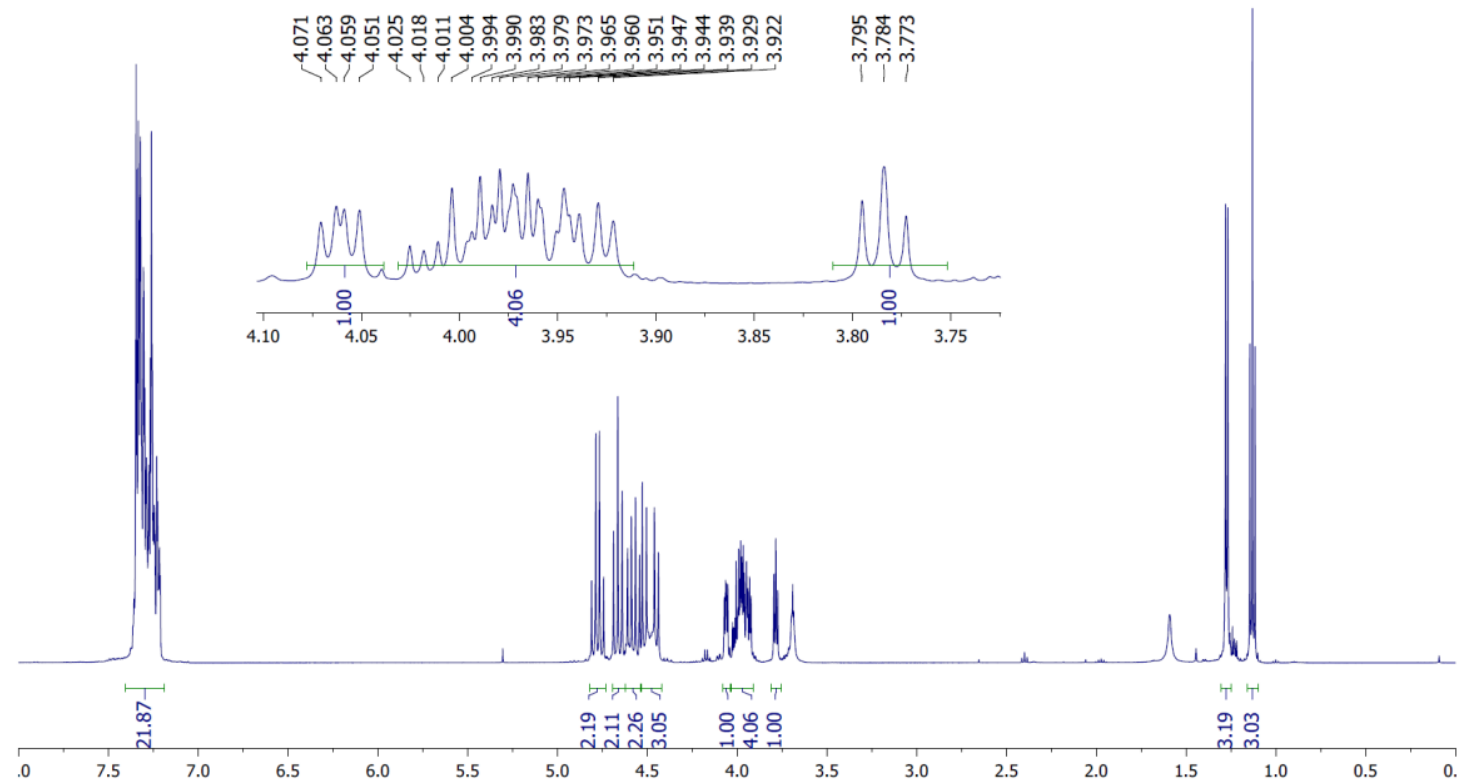

${ }^{19}$ F NMR

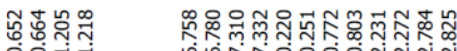

의큰

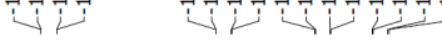

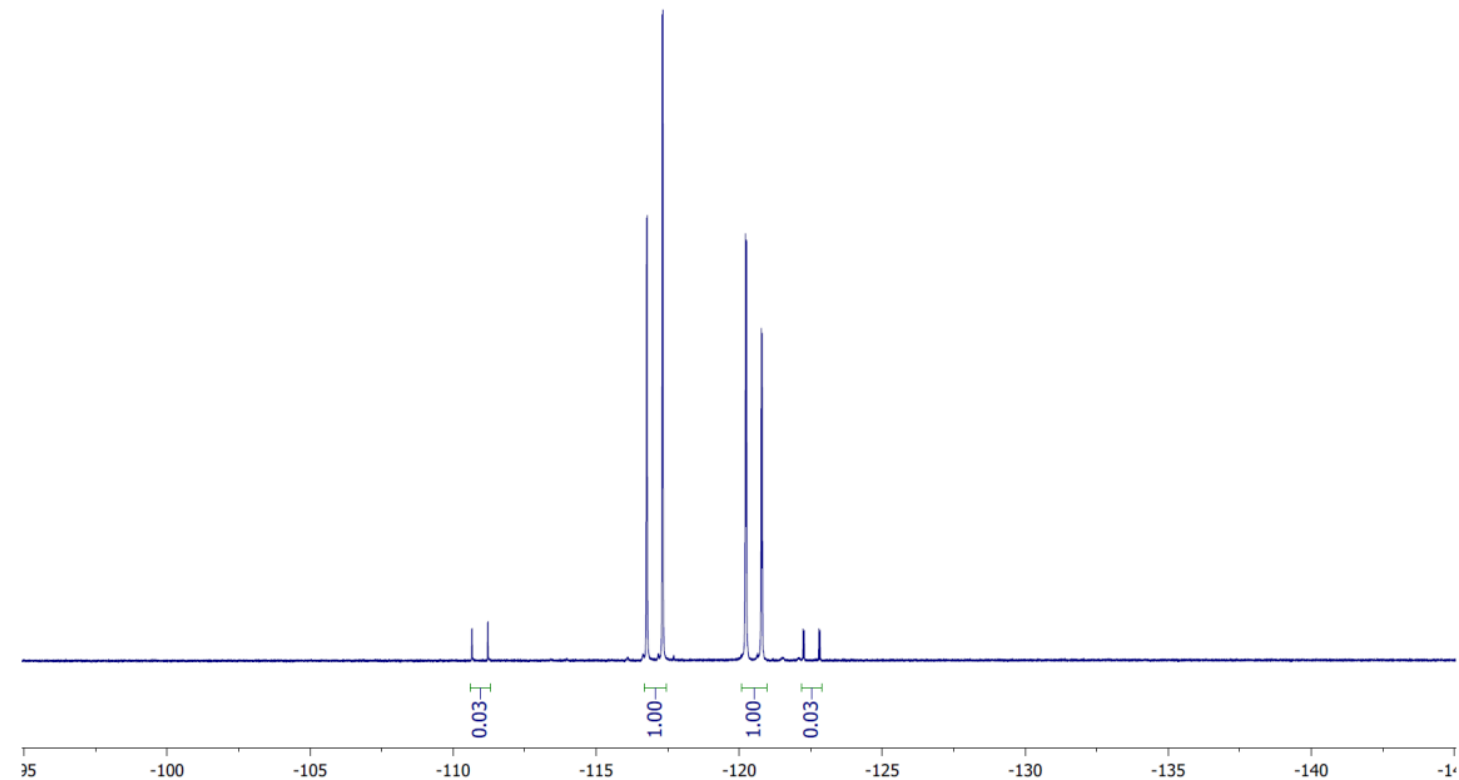


${ }^{13}$ C NMR

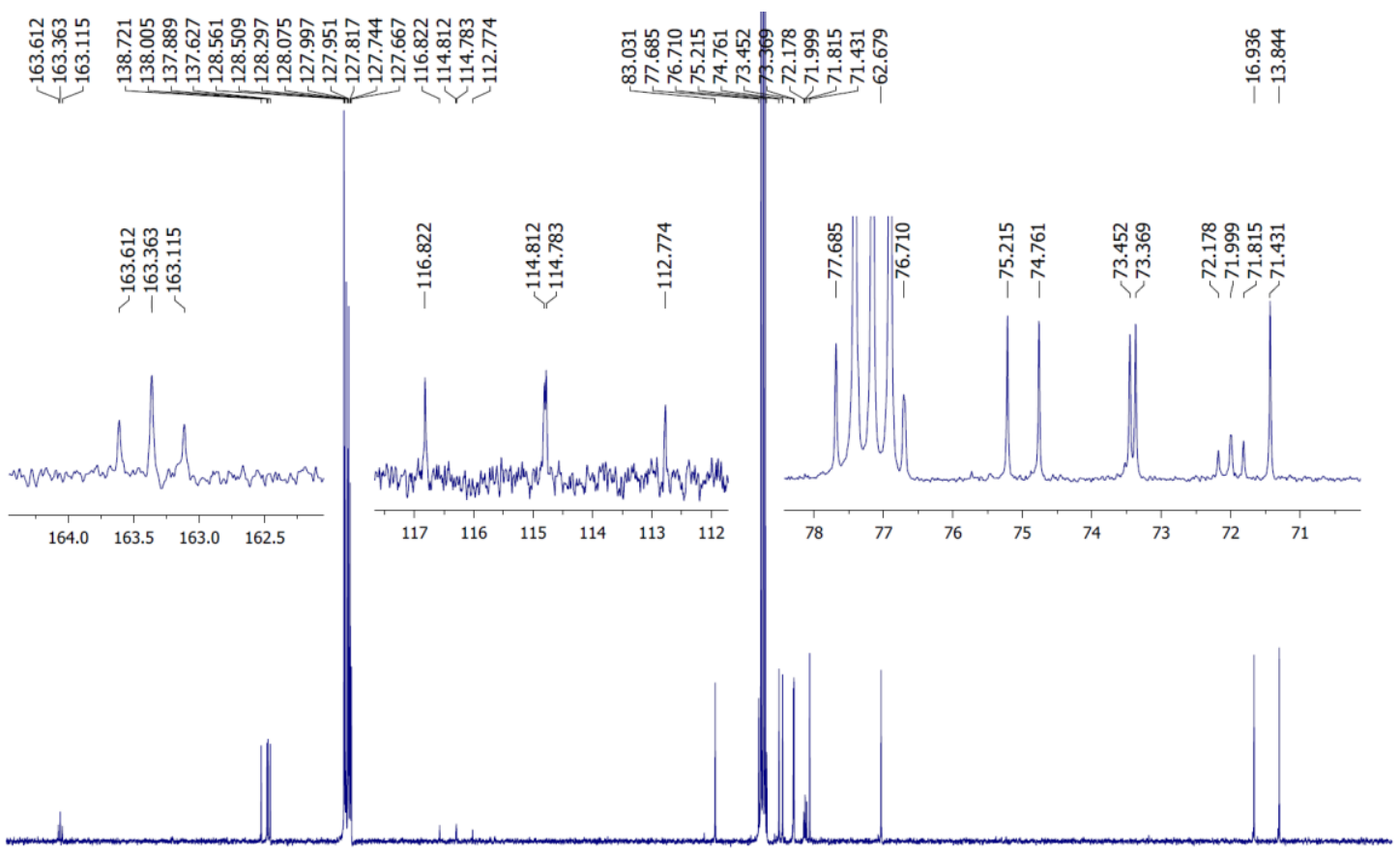

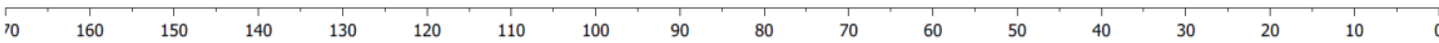




\section{D-Galactose}

Ethyl (3R,4S,5R,6S,7R)-4,5,6,7,8-pentakis(benzyloxy)-2,2-difluoro-3-hydroxyoctanoate $((R / S)-3 f)$.

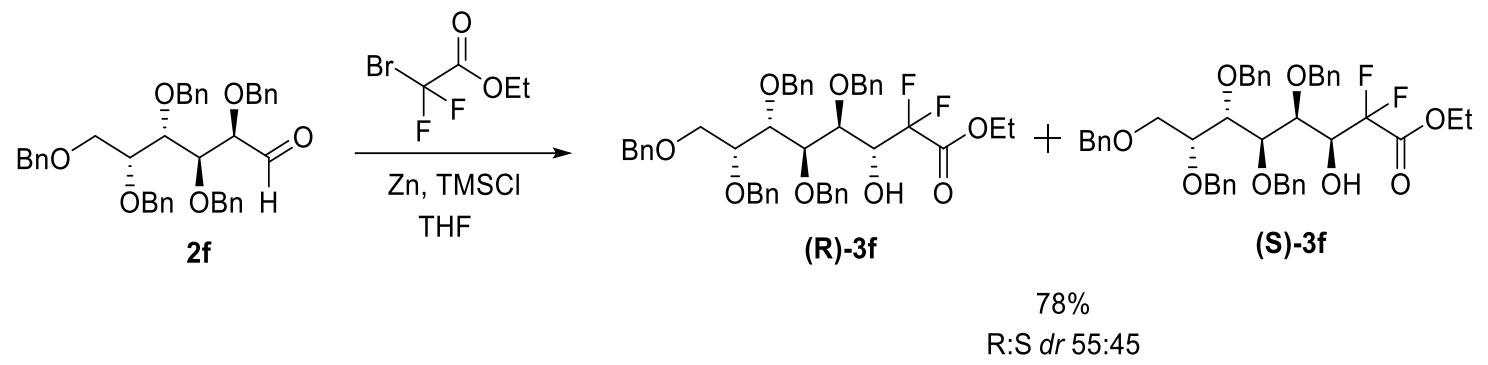

To a dry two-necked round bottomed flask was added "activated zinc" powder (309 mg, $4.73 \mathrm{mmol}, 5.7$ equiv.). The flask was heated up at $40^{\circ} \mathrm{C}$ and the zinc powder suspended in dry THF ( $6 \mathrm{ml})$ under argon atmosphere. Then, TMSCl ( $35 \mu \mathrm{l}, 0.28 \mathrm{mmol}, 0.4$ equiv.) was added dropwise and the reaction was stirred at $60^{\circ} \mathrm{C}$ for 15 mins. Next, a solution of aldehyde $2 \mathbf{f}$ (522 mg, $0.828 \mathrm{mmol}, 1.0$ equiv.) and ethyl bromo-difluoroacetate $(0.13 \mathrm{ml}$, $1.01 \mathrm{mmol}, 1.2$ equiv.) dissolved homogenously in dry THF (6 ml) under argon atmosphere was added dropwise at $60^{\circ} \mathrm{C}$. The reaction was stirred vigorously for $1.5 \mathrm{~h}$ at $60^{\circ} \mathrm{C}$ and then cooled at room temperature. Ice water $(5 \mathrm{ml})$ and $\mathrm{HCl}(1 \mathrm{M}, 10 \mathrm{ml})$ were added and the mixture was diluted with EtOAc. The organic phase was washed with $\mathrm{NaHCO}_{3}$ (sat) (2 times), brine, dried over $\mathrm{MgSO}_{4}$ and concentrated under reduced pressure. Crude NMR analysis showed the formation of two diastereoisomer $(R)-\mathbf{3 f}$ and $(S)$-3f in 55:45 $d r$ ratio. Purification by silica gel chromatography ( $\mathrm{Cy}_{2} / \mathrm{Et}_{2} \mathrm{O}$ 85:15) afforded pure diastereoisomers $(R)-\mathbf{3 f}$ and $(S)$-3f in $78 \%$ overall yield. 
Ethyl (3R,4S,5R,6S,7R)-4,5,6,7,8-pentakis(benzyloxy)-2,2-difluoro-3-hydroxyoctanoate ((S)-3f).

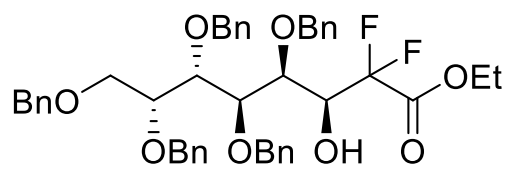

(S)-3f

$[\alpha]_{20}{ }^{\mathrm{D}}:-1.0\left(\mathrm{c} 1.0, \mathrm{CHCl}_{3}\right) .{ }^{1} \mathrm{H} \mathrm{NMR}\left(500 \mathrm{MHz}, \mathrm{CDCl}_{3}\right) \delta=7.34-7.25\left(\mathrm{~m}, 21 \mathrm{H}, \mathrm{H}^{\text {arom }}\right)$, 7.23-7.21 (m, $\left.2 \mathrm{H}, \mathrm{H}^{\text {arom }}\right), 7.19-7.17\left(\mathrm{~m}, 2 \mathrm{H}, \mathrm{H}^{\text {arom }}\right), 4.73\left(\mathrm{AB}, 1 \mathrm{H}, J_{\mathrm{A}-\mathrm{B}}=10.9 \mathrm{~Hz}, \mathrm{CH}_{2}{ }^{\mathrm{Bn}}\right)$, $4.70\left(\mathrm{AB}, 1 \mathrm{H}, J_{\mathrm{A}-\mathrm{B}}=10.9 \mathrm{~Hz}, \mathrm{CH}_{2}{ }^{\mathrm{Bn}}\right), 4.65\left(\mathrm{AB}, 1 \mathrm{H}, J_{\mathrm{A}-\mathrm{B}}=11.5 \mathrm{~Hz}, \mathrm{CH}_{2}{ }^{\mathrm{Bn}}\right), 4.64-4.58$ $\left(\mathrm{m}, 3 \mathrm{H}, \mathrm{CH}_{2}{ }^{\mathrm{Bn}}\right), 4.56\left(\mathrm{AB}, 1 \mathrm{H}, J_{\mathrm{A}-\mathrm{B}}=11.5 \mathrm{~Hz}, \mathrm{CH}_{2}{ }^{\mathrm{Bn}}\right), 4.51\left(\mathrm{AB}, 1 \mathrm{H}, J_{\mathrm{A}-\mathrm{B}}=10.9 \mathrm{~Hz}\right.$, $\left.\mathrm{CH}_{2}{ }^{\mathrm{Bn}}\right), 4.48\left(\mathrm{AB}, 1 \mathrm{H}, J_{\mathrm{A}-\mathrm{B}}=12.0 \mathrm{~Hz}, \mathrm{CH}_{2}{ }^{\mathrm{Bn}}\right), 4.45-4.36(\mathrm{~m}, 1 \mathrm{H}, \mathrm{H}-1), 4.42\left(\mathrm{AB}, 1 \mathrm{H}, J_{\mathrm{A}-}\right.$ в $\left.=12.0 \mathrm{~Hz}, \mathrm{CH}_{2}{ }^{\mathrm{Bn}}\right), 4.14\left(\mathrm{q}, 2 \mathrm{H}, J_{\mathrm{CH} 2 \mathrm{CH} 3}=7.5 \mathrm{~Hz}, \mathrm{CH}_{2} \mathrm{CH}_{3}\right), 4.05\left(\mathrm{~d}, 1 \mathrm{H}, J_{2-3}=6.3 \mathrm{~Hz}\right.$, H-2), 4.03 (dd, $\left.1 \mathrm{H}, J_{2-3}=6.3 \mathrm{~Hz}, J_{3-4}=4.0 \mathrm{~Hz}, \mathrm{H}-3\right), 3.97-3.93$ (m, 1H, H-5), 3.91 (dd, $\left.1 \mathrm{H}, J_{3-4}=4.0 \mathrm{~Hz}, J_{4-5}=6.9 \mathrm{~Hz}, \mathrm{H}-4\right), 3.71\left(\mathrm{~d}, 1 \mathrm{H}, J_{1-\mathrm{OH}}=8.6 \mathrm{~Hz}, \mathrm{OH}\right), 3.63(\mathrm{ABX}, 1 \mathrm{H}$, $\left.J_{5-6 \mathrm{a}}=4.0 \mathrm{~Hz}, J_{6 \mathrm{a}-6 \mathrm{~b}}=10.3 \mathrm{~Hz}, \mathrm{H}-6 \mathrm{a}\right), 3.59\left(\mathrm{ABX}, 1 \mathrm{H}, J_{5-6 \mathrm{a}}=4.6 \mathrm{~Hz}, J_{6 \mathrm{a}-6 \mathrm{~b}}=10.3 \mathrm{~Hz}, \mathrm{H}-\right.$ 6b), $1.22\left(\mathrm{t}, 3 \mathrm{H}, J_{\mathrm{CH} 2 \mathrm{CH} 3}=7.5 \mathrm{~Hz}, \mathrm{CH}_{2} \mathrm{CH}_{3}\right) \mathrm{ppm} .{ }^{13} \mathrm{C} \mathrm{NMR}\left(125 \mathrm{MHz}, \mathrm{CDCl}_{3}\right) \delta=163.7$ $\left(\mathrm{dd}, J_{\mathrm{C}-\mathrm{F}}=28.9 \mathrm{~Hz}, J_{\mathrm{C}-\mathrm{F}}=31.7 \mathrm{~Hz}, \mathrm{C}_{\mathrm{q}} \mathrm{O}_{2} \mathrm{Et}\right), 138.7\left(\mathrm{C}_{\mathrm{q}}{ }^{\text {arom }}\right), 138.3\left(\mathrm{C}_{\mathrm{q}}{ }^{\text {arom }}\right), 138.2(2$ $\left.\mathrm{C}_{\mathrm{q}}{ }^{\text {arom }}\right), 137.8\left(\mathrm{C}_{\mathrm{q}}{ }^{\text {arom }}\right), 128.6-127.6\left(\mathrm{CH}^{\text {arom }}\right), 114.6\left(\mathrm{dd}, J_{\mathrm{C}-\mathrm{F}}=252.5 \mathrm{~Hz}, J_{\mathrm{C}-\mathrm{F}}=254.9 \mathrm{~Hz}\right.$, $\left.\mathrm{CF}_{2}\right), 80.6(\mathrm{C}-3), 77.9(\mathrm{C}-4, \mathrm{C}-5), 75.7(\mathrm{C}-2), 74.8\left(\mathrm{CH}_{2}{ }^{\mathrm{Bn}}\right), 74.4\left(\mathrm{CH}_{2}{ }^{\mathrm{Bn}}\right), 73.6\left(\mathrm{CH}_{2}{ }^{\mathrm{Bn}}\right)$, $73.5\left(\mathrm{CH}_{2}{ }^{\mathrm{Bn}}\right), 73.3\left(\mathrm{CH}_{2}{ }^{\mathrm{Bn}}\right), 70.2(\mathrm{C}-6), 70.1\left(\mathrm{dd}, J_{\mathrm{C}-\mathrm{F}}=13.3 \mathrm{~Hz}, J_{\mathrm{C}-\mathrm{F}}=39.8 \mathrm{~Hz}, \mathrm{C}-1\right)$, $63.0\left(\mathrm{CH}_{2} \mathrm{CH}_{3}\right), 14.0\left(\mathrm{CH}_{2} \mathrm{CH}_{3}\right) \mathrm{ppm} .{ }^{19} \mathrm{~F} \mathrm{NMR}\left(471 \mathrm{MHz}, \mathrm{CDCl}_{3}\right) \delta=-110.8(\mathrm{dd}, 1 \mathrm{~F}$, $\left.J_{\mathrm{H} 1-\mathrm{Fa}}=6.3 \mathrm{~Hz}, J_{\mathrm{Fa}-\mathrm{Fb}}=261.4 \mathrm{~Hz}, \mathrm{Fa}\right),-122.4\left(\mathrm{dd}, 1 \mathrm{~F}, J_{\mathrm{H} 1-\mathrm{Fb}}=18.4 \mathrm{~Hz}, J_{\mathrm{Fa}-\mathrm{Fb}}=261.4 \mathrm{~Hz}\right.$, $\mathrm{Fb}$ ) ppm. HRMS (ESI+): m/z calculated for $\mathrm{C}_{45} \mathrm{H}_{49} \mathrm{~F}_{2} \mathrm{O}_{8}[\mathrm{M}+\mathrm{H}]^{+}$: calc. 755.3390; found: 755.3383 . 


\section{${ }^{1}$ H NMR}

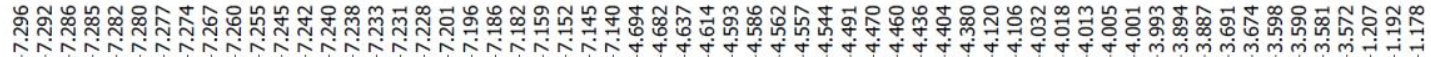

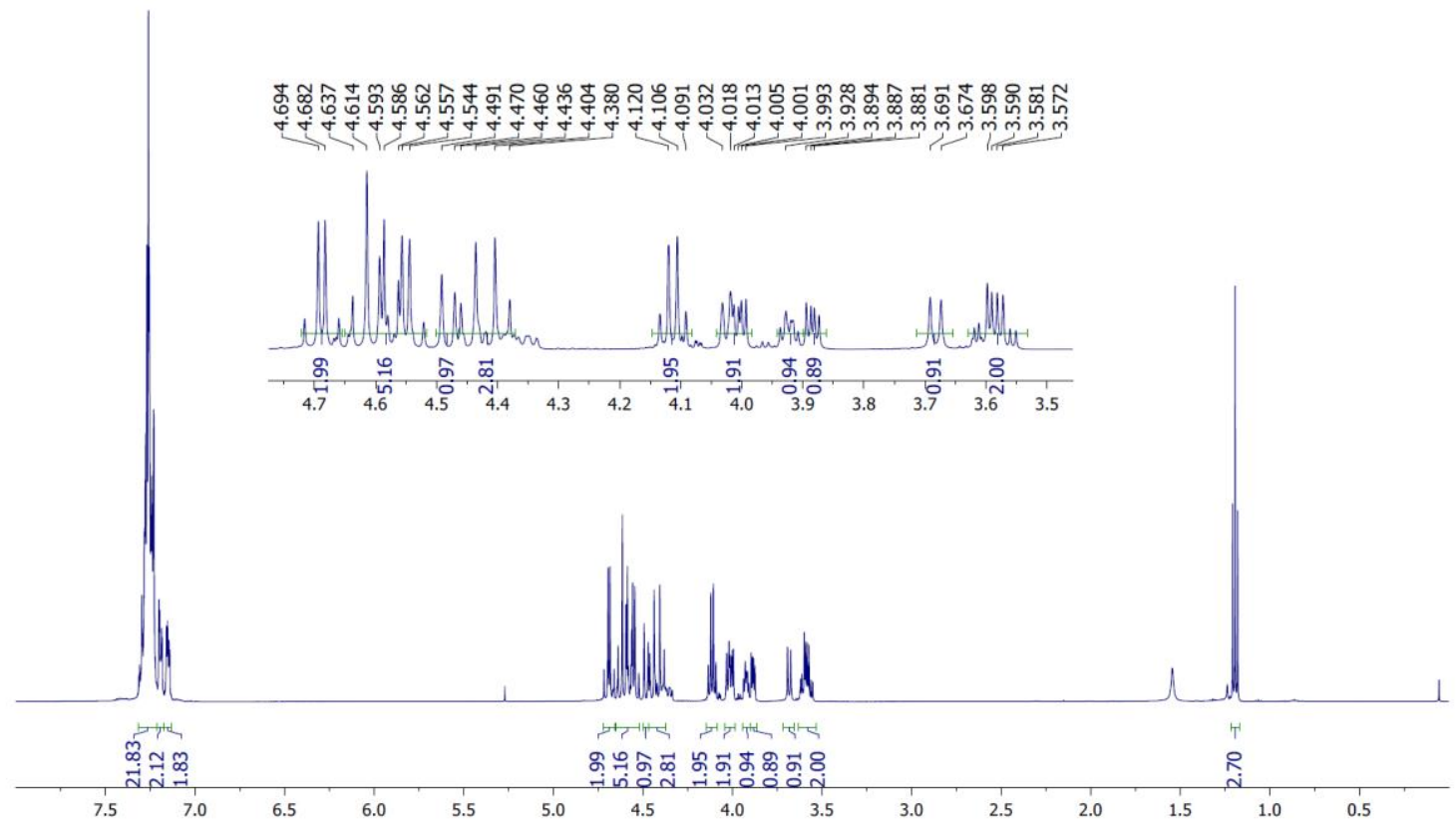

${ }^{19}$ F NMR

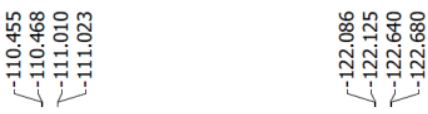

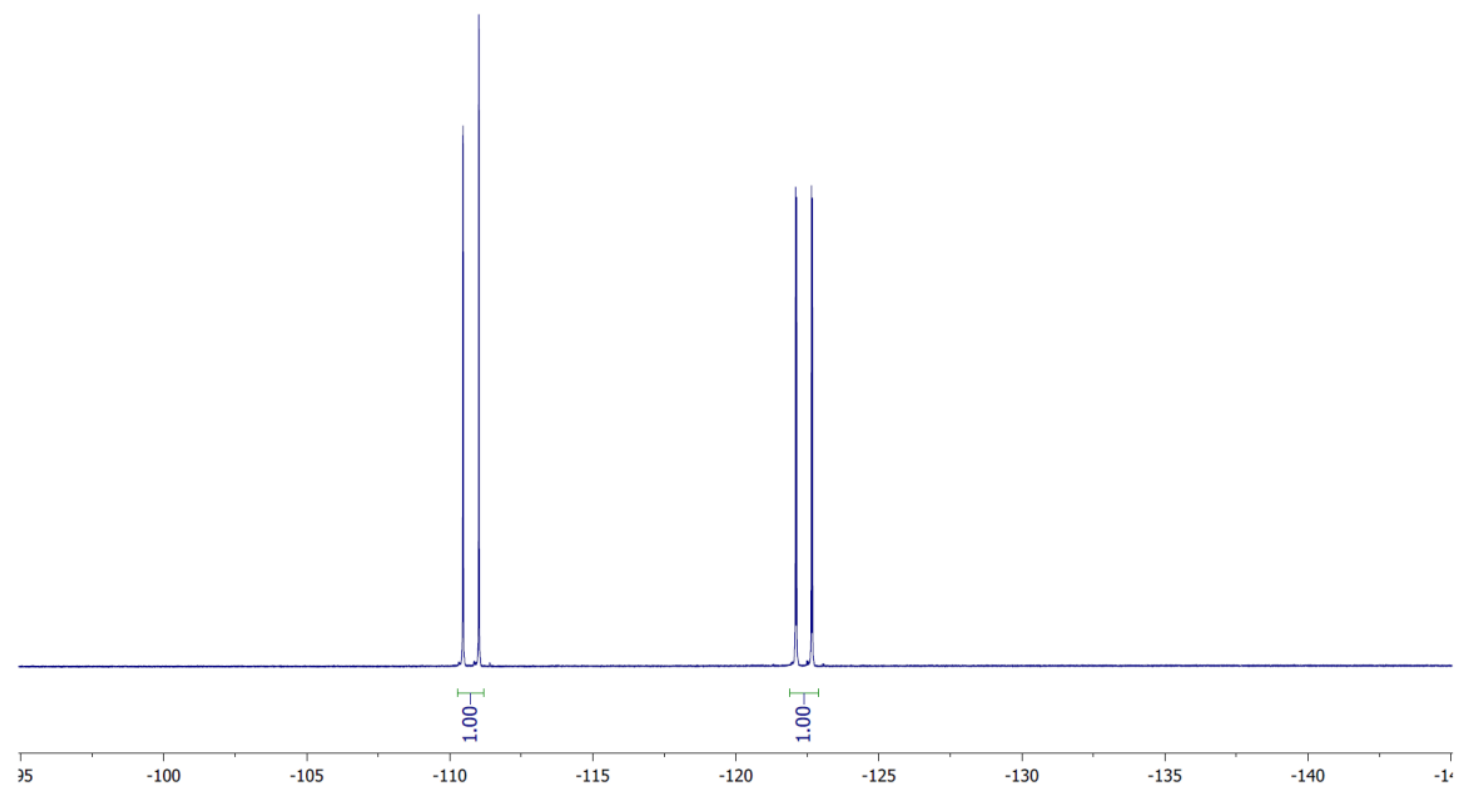


${ }^{13}$ C NMR

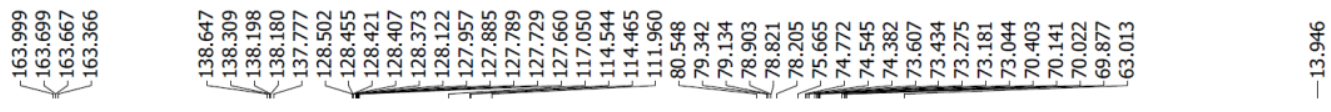

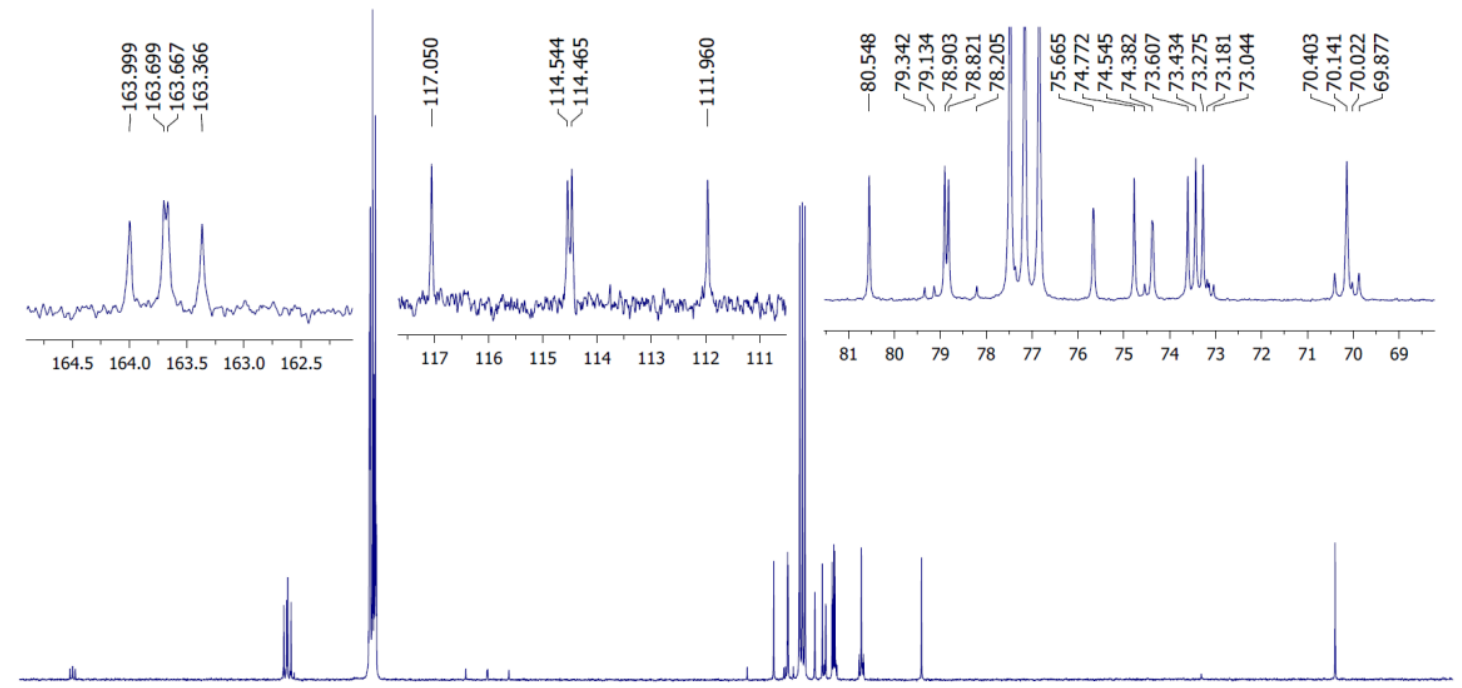

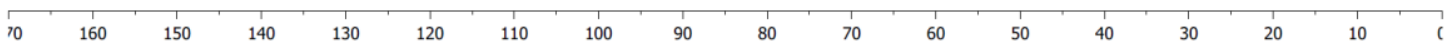


Ethyl 4,5,6,7,8-pentakis(benzyloxy)-2,2-difluoro-3-hydroxyoctanoate ((R)-3f).

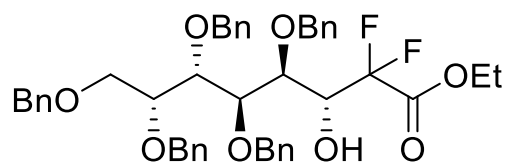

(R)-3f

$[\alpha]_{20} \mathrm{D}:+1.1\left(\mathrm{c} 1.0, \mathrm{CHCl}_{3}\right) .{ }^{1} \mathrm{H}$ NMR $\left(500 \mathrm{MHz}, \mathrm{CDCl}_{3}\right) \delta=7.32-7.27\left(\mathrm{~m}, 17 \mathrm{H}, \mathrm{H}^{\text {arom }}\right)$, 7.24-7.21 (m, $\left.6 \mathrm{H}, \mathrm{H}^{\text {arom }}\right), 7.16-7.13\left(\mathrm{~m}, 2 \mathrm{H}, \mathrm{H}^{\text {arom }}\right), 4.72\left(\mathrm{AB}, 1 \mathrm{H}, J_{\mathrm{A}-\mathrm{B}}=11.5 \mathrm{~Hz}, \mathrm{CH}_{2}{ }^{\mathrm{Bn}}\right)$, $4.71\left(\mathrm{AB}, 1 \mathrm{H}, J_{\mathrm{A}-\mathrm{B}}=11.5 \mathrm{~Hz}, \mathrm{CH}_{2}{ }^{\mathrm{Bn}}\right), 4.67\left(\mathrm{AB}, 1 \mathrm{H}, J_{\mathrm{A}-\mathrm{B}}=11.5 \mathrm{~Hz}, \mathrm{CH}_{2}{ }^{\mathrm{Bn}}\right), 4.62(\mathrm{AB}$, $\left.1 \mathrm{H}, J_{\mathrm{A}-\mathrm{B}}=10.9 \mathrm{~Hz}, \mathrm{CH}_{2}{ }^{\mathrm{Bn}}\right), 4.60\left(\mathrm{AB}, 1 \mathrm{H}, J_{\mathrm{A}-\mathrm{B}}=11.5 \mathrm{~Hz}, \mathrm{CH}_{2}{ }^{\mathrm{Bn}}\right), 4.53\left(\mathrm{AB}, 1 \mathrm{H}, J_{\mathrm{A}-\mathrm{B}}=\right.$ $\left.11.5 \mathrm{~Hz}, \mathrm{CH}_{2}{ }^{\mathrm{Bn}}\right), 4.50\left(\mathrm{AB}, 1 \mathrm{H}, J_{\mathrm{A}-\mathrm{B}}=10.9 \mathrm{~Hz}, \mathrm{CH}_{2}{ }^{\mathrm{Bn}}\right), 4.47\left(\mathrm{AB}, 1 \mathrm{H}, J_{\mathrm{A}-\mathrm{B}}=12.0 \mathrm{~Hz}\right.$, $\left.\mathrm{CH}_{2}{ }^{\mathrm{Bn}}\right), 4.46-4.37(\mathrm{~m}, 1 \mathrm{H}, \mathrm{H}-1), 4.41\left(\mathrm{AB}, 1 \mathrm{H}, J_{\mathrm{A}-\mathrm{B}}=12.0 \mathrm{~Hz}, \mathrm{CH}_{2}{ }^{\mathrm{Bn}}\right), 4.37\left(\mathrm{AB}, 1 \mathrm{H}, J_{\mathrm{A}-}\right.$ В $\left.=11.5 \mathrm{~Hz}, \mathrm{CH}_{2}{ }^{\mathrm{Bn}}\right), 4.08$ (t, $\left.1 \mathrm{H}, J=5.2 \mathrm{~Hz}, \mathrm{H}-4\right), 4.02-3.98$ (m, 2H, H-3, H-5), 3.98$3.89\left(\mathrm{~m}, 2 \mathrm{H}, \mathrm{CH}_{2} \mathrm{CH}_{3}\right), 3.86(\mathrm{dd}, 1 \mathrm{H}, J=4.6 \mathrm{~Hz}, J=8.6 \mathrm{~Hz}, \mathrm{H}-2), 3.67$ (m, $\left.1 \mathrm{H}, \mathrm{OH}\right)$, $3.64\left(\mathrm{ABX}, 1 \mathrm{H}, J_{5-6 \mathrm{a}}=4.6 \mathrm{~Hz}, J_{6 \mathrm{a}-6 \mathrm{~b}}=10.3 \mathrm{~Hz}, \mathrm{H}-6 \mathrm{a}\right), 3.60\left(\mathrm{ABX}, 1 \mathrm{H}, J_{5-6 \mathrm{~b}}=5.2 \mathrm{~Hz}, J_{6 \mathrm{a}-}\right.$ $\left.{ }_{6 \mathrm{~b}}=10.3 \mathrm{~Hz}, \mathrm{H}-6 \mathrm{~b}\right), 1.10\left(\mathrm{t}, 3 \mathrm{H}, J_{\mathrm{CH} 2-\mathrm{CH} 3}=7.5 \mathrm{~Hz}, \mathrm{CH}_{2} \mathrm{CH}_{3}\right) \mathrm{ppm} .{ }^{13} \mathrm{C} \mathrm{NMR}(125 \mathrm{MHz}$, $\left.\mathrm{CDCl}_{3}\right) \delta=163.3\left(\mathrm{t}, J_{\mathrm{C}-\mathrm{F}}=31.4 \mathrm{~Hz}, \mathrm{C}_{\mathrm{q}} \mathrm{O}_{2} \mathrm{Et}\right), 138.5\left(\mathrm{C}_{\mathrm{q}}{ }^{\text {arom }}\right), 138.1\left(\mathrm{C}_{\mathrm{q}}{ }^{\text {arom }}\right), 138.0\left(\mathrm{C}_{\mathrm{q}}{ }^{\text {arom }}\right)$, $137.9\left(\mathrm{C}_{\mathrm{q}}{ }^{\text {arom }}\right), 137.6\left(\mathrm{C}_{\mathrm{q}}^{\text {arom }}\right), 128.6-127.7\left(\mathrm{CH}^{\text {arom }}\right), 114.8\left(\mathrm{dd}, J_{\mathrm{C}-\mathrm{F}}=252.8 \mathrm{~Hz}, J_{\mathrm{C}-\mathrm{F}}=\right.$ $\left.256.5 \mathrm{~Hz}, \mathrm{CF}_{2}\right), 79.5(\mathrm{C}-4), 78.3\left(\mathrm{C}-3\right.$ or C-5), $78.2\left(\mathrm{C}-3\right.$ or C-5), $76.8(\mathrm{C}-2), 74.7\left(\mathrm{CH}_{2}{ }^{\mathrm{Bn}}\right)$, $73.6\left(\mathrm{CH}_{2}{ }^{\mathrm{Bn}}\right), 73.5\left(2 \mathrm{CH}_{2}{ }^{\mathrm{Bn}}\right), 73.1\left(\mathrm{CH}_{2}{ }^{\mathrm{Bn}}\right), 72.0\left(\mathrm{t}, J_{\mathrm{C}-\mathrm{F}}=22.9 \mathrm{~Hz}, \mathrm{C}-1\right), 70.0(\mathrm{C}-6), 62.7$ $\left(\mathrm{CH}_{2} \mathrm{CH}_{3}\right), 13.8\left(\mathrm{CH}_{2} \mathrm{CH}_{3}\right)$ ppm. ${ }^{19} \mathrm{~F} \mathrm{NMR}\left(471 \mathrm{MHz}, \mathrm{CDCl}_{3}\right) \delta=-117.0\left(\mathrm{dd}, 1 \mathrm{~F}, J_{\mathrm{H}-\mathrm{Fa}}=\right.$ $\left.9.9 \mathrm{~Hz}, J_{\mathrm{Fa}-\mathrm{Fb}}=260.0 \mathrm{~Hz}, \mathrm{Fa}\right),-120.3\left(\mathrm{dd}, 1 \mathrm{~F}, J_{\mathrm{H}-\mathrm{Fb}}=6.6 \mathrm{~Hz}, J_{\mathrm{H}-\mathrm{Fb}}=13.5 \mathrm{~Hz}, J_{\mathrm{Fa}-\mathrm{Fb}}=\right.$ $260.0 \mathrm{~Hz}, \mathrm{Fb}$ ) ppm. HRMS (ESI+): $\mathrm{m} / \mathrm{z}$ calculated for $\mathrm{C}_{45} \mathrm{H}_{49} \mathrm{~F}_{2} \mathrm{O}_{8}[\mathrm{M}+\mathrm{H}]^{+}$: calc. 755.3390; found: 755.3380 . 


\section{${ }^{1} \mathrm{H}$ NMR}

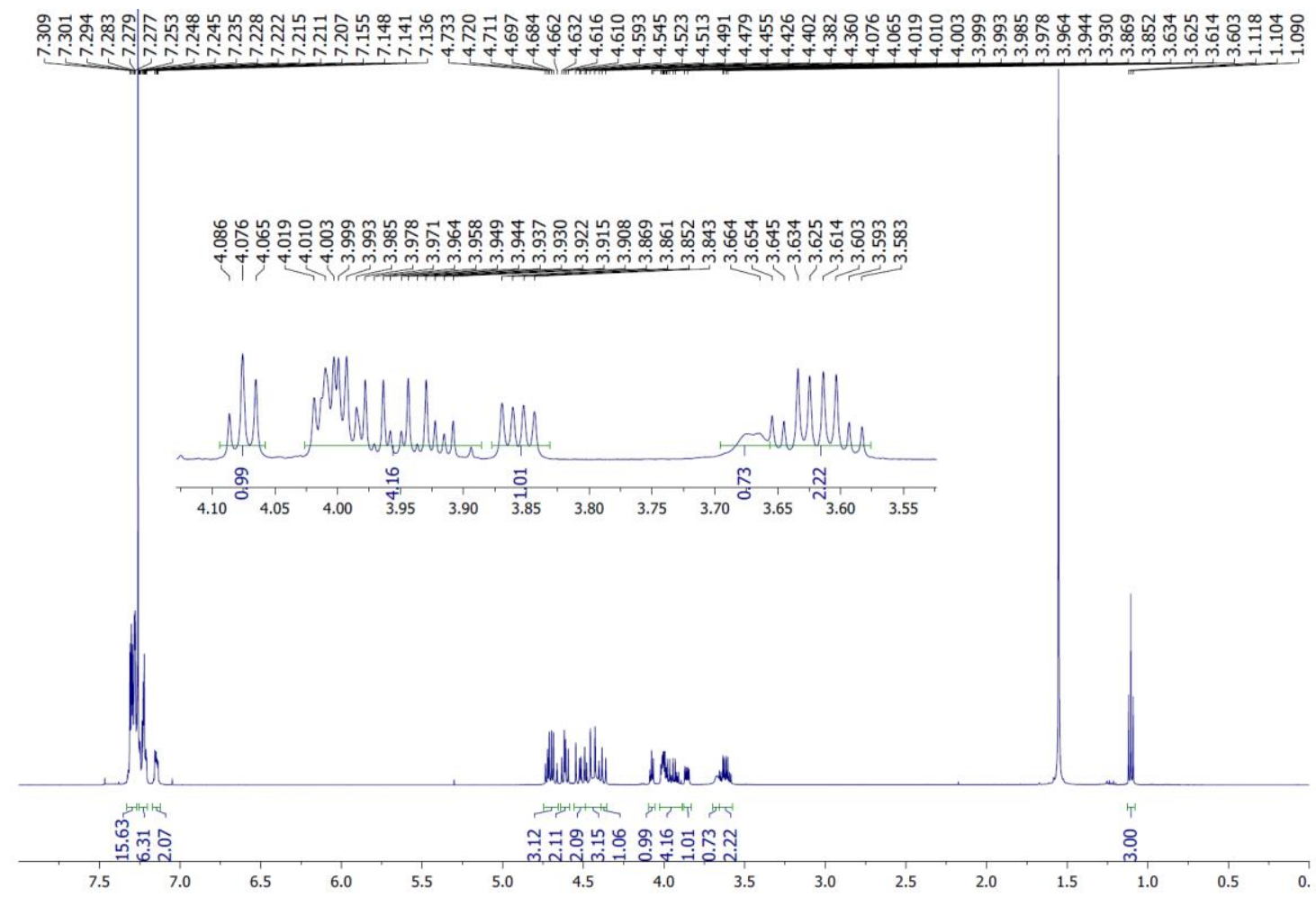

${ }^{19}$ F NMR

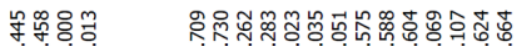

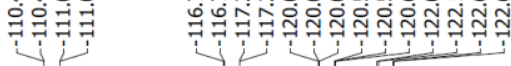

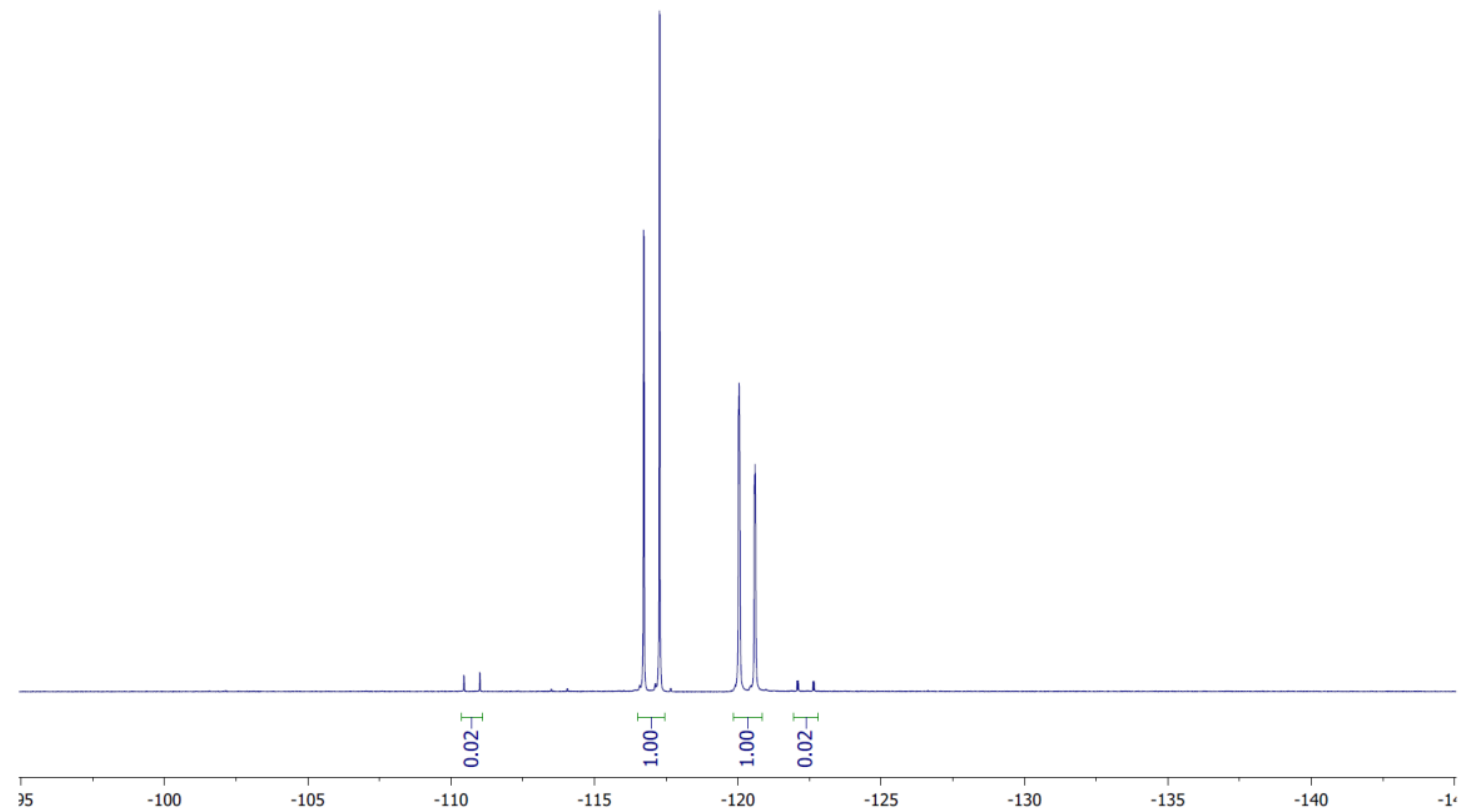

${ }^{13}$ C NMR 

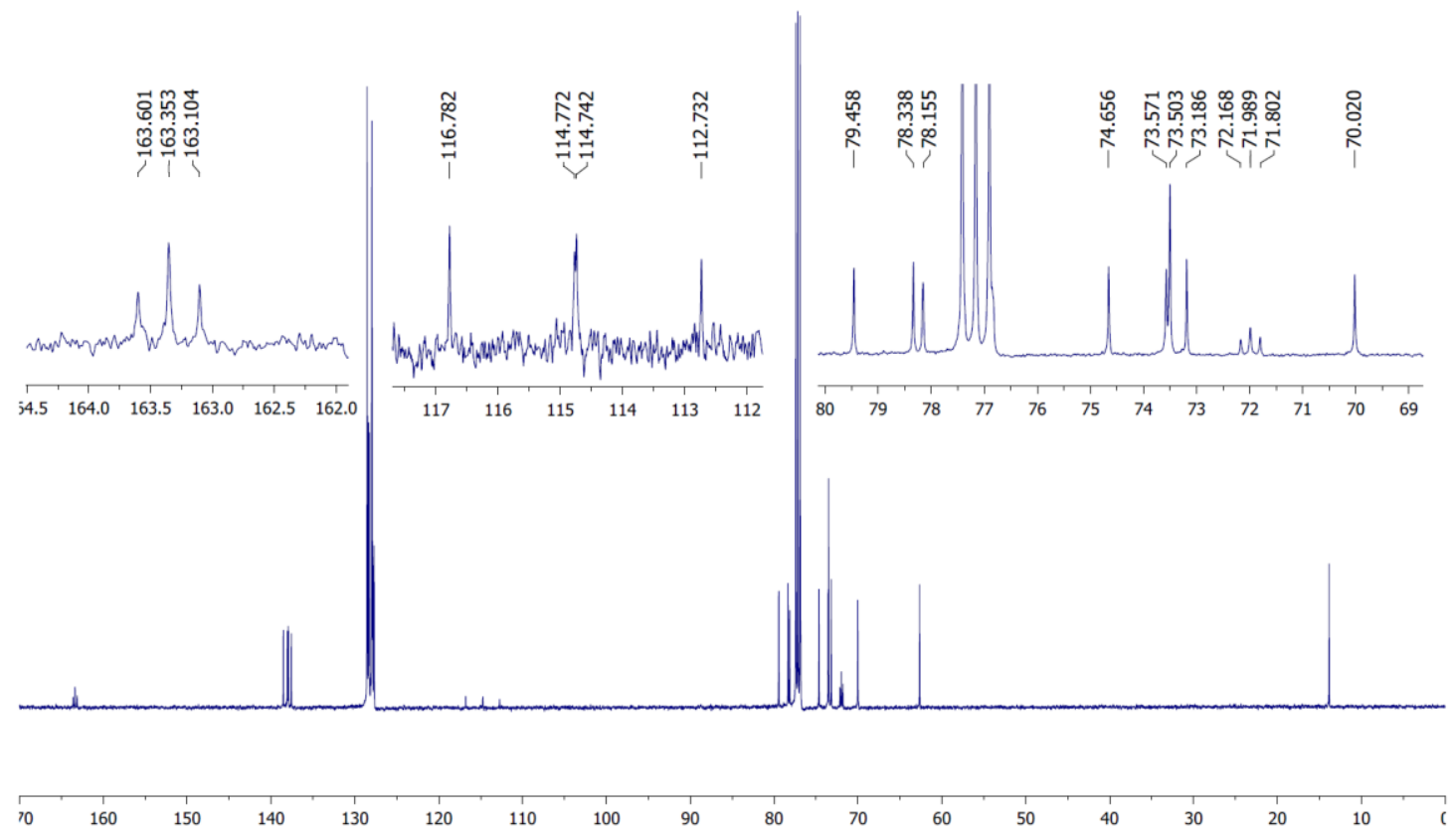
Ethyl 4,5,6,7,8-pentakis(benzyloxy)-2,2-difluoro-3-hydroxyoctanoate ((R/S)-3g).

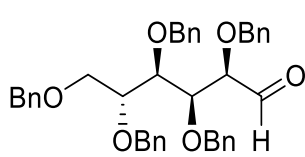

$2 g$<smiles>CCOC(=O)C(F)(F)Br</smiles>

$\mathrm{BnO}$<smiles>CCOC(=O)C(F)(F)C(O)C([18OH])C([18OH])C([18OH])C([18OH])CO</smiles>

(R)-3g<smiles>CCOC(=O)C(F)(F)C(O)C([18OH])C([18OH])C([18OH])C([18OH])COCc1ccccc1</smiles>

(S)-3g $75 \%$ R:S dr 69:31

To a dry two-necked round bottomed flask was added "activated zinc" powder (398 mg, $6.09 \mathrm{mmol}, 5.7$ equiv.). The flask was heated up at $40^{\circ} \mathrm{C}$ and the zinc powder suspended in dry THF ( $8 \mathrm{ml})$ under argon atmosphere. Then, TMSCl $(50 \mu 1,0.36 \mathrm{mmol}, 0.4$ equiv.) was added dropwise and the reaction was stirred at $60^{\circ} \mathrm{C}$ for 15 mins. Next, a solution of aldehyde $2 \mathrm{~g}$ (672 g, $1.065 \mathrm{mmol}, 1.0$ equiv.) and ethyl bromo-difluoroacetate $(0.17 \mathrm{ml}$, $1.28 \mathrm{mmol}, 1.2$ equiv.) dissolved homogenously in dry THF $(8 \mathrm{ml})$ under argon atmosphere was added dropwise at $60^{\circ} \mathrm{C}$. The reaction was stirred vigorously for $1.5 \mathrm{~h}$ at $60^{\circ} \mathrm{C}$ and then cooled at room temperature. Ice water $(5 \mathrm{ml})$ and $\mathrm{HCl}(1 \mathrm{M}, 10 \mathrm{ml})$ were added and the mixture was diluted with EtOAc. The organic phase was washed with $\mathrm{NaHCO}_{3}$ (sat) (2 times), brine, dried over $\mathrm{MgSO}_{4}$ and concentrated under reduced pressure. Crude NMR analysis showed the formation of two diastereoisomer $(R)-\mathbf{3 g}$ and $(S)-3 g$ in 69:31 $d r$ ratio. Purification by silica gel chromatography (Cy/EtOAc 92:8) afforded pure diastereoisomers $(R)-\mathbf{3 g}$ and $(S)-\mathbf{3 g}$ in $75 \%$ overall yield.

Ethyl 4,5,6,7,8-pentakis(benzyloxy)-2,2-difluoro-3-hydroxyoctanoate (( $R)$-3g). 


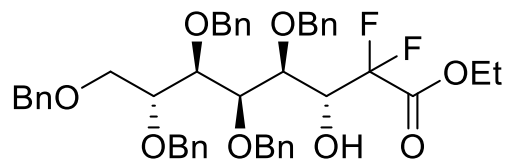

(R)-3g

$[\alpha]_{20}{ }^{\mathrm{D}}:+5.0\left(\mathrm{c} 1.0, \mathrm{CHCl}_{3}\right) .{ }^{1} \mathrm{H}$ NMR $\left(500 \mathrm{MHz}, \mathrm{CDCl}_{3}\right) \delta=7.33-7.24\left(\mathrm{~m}, 21 \mathrm{H}, \mathrm{H}^{\text {arom }}\right)$, 7.23-7.20 (m, $\left.4 \mathrm{H}, \mathrm{H}^{\text {arom }}\right), 4.73\left(\mathrm{AB}, 1 \mathrm{H}, J_{\mathrm{A}-\mathrm{B}}=10.3 \mathrm{~Hz}, \mathrm{CH}_{2}{ }^{\mathrm{Bn}}\right), 4.68\left(\mathrm{AB}, 1 \mathrm{H}, J_{\mathrm{A}-\mathrm{B}}=\right.$ $\left.11.5 \mathrm{~Hz}, \mathrm{CH}_{2}{ }^{\mathrm{Bn}}\right), 4.66\left(\mathrm{AB}, 1 \mathrm{H}, J_{\mathrm{A}-\mathrm{B}}=11.5 \mathrm{~Hz}, \mathrm{CH}_{2}{ }^{\mathrm{Bn}}\right), 4.64\left(\mathrm{AB}, 1 \mathrm{H}, J_{\mathrm{A}-\mathrm{B}}=12.0 \mathrm{~Hz}\right.$, $\left.\mathrm{CH}_{2}{ }^{\mathrm{Bn}}\right), 4.63\left(\mathrm{AB}, 1 \mathrm{H}, J_{\mathrm{A}-\mathrm{B}}=10.3 \mathrm{~Hz}, \mathrm{CH}_{2}{ }^{\mathrm{Bn}}\right), 4.53\left(\mathrm{AB}, 1 \mathrm{H}, J_{\mathrm{A}-\mathrm{B}}=11.5 \mathrm{~Hz}, \mathrm{CH}_{2}{ }^{\mathrm{Bn}}\right)$, $4.52\left(\mathrm{AB}, 1 \mathrm{H}, J_{\mathrm{A}-\mathrm{B}}=12.0 \mathrm{~Hz}, \mathrm{CH}_{2}{ }^{\mathrm{Bn}}\right), 4.50\left(\mathrm{AB}, 1 \mathrm{H}, J_{\mathrm{A}-\mathrm{B}}=11.5 \mathrm{~Hz}, \mathrm{CH}_{2}{ }^{\mathrm{Bn}}\right), 4.48(\mathrm{AB}$, $\left.1 \mathrm{H}, J_{\mathrm{A}-\mathrm{B}}=12.0 \mathrm{~Hz}, \mathrm{CH}_{2}{ }^{\mathrm{Bn}}\right), 4.42\left(\mathrm{AB}, 1 \mathrm{H}, J_{\mathrm{A}-\mathrm{B}}=12.0 \mathrm{~Hz}, \mathrm{CH}_{2}{ }^{\mathrm{Bn}}\right), 4.30\left(\mathrm{ddd}, 1 \mathrm{H}, J_{1-\mathrm{Fa}}=\right.$ $\left.4.6 \mathrm{~Hz}, J_{1-\mathrm{OH}}=9.7 \mathrm{~Hz}, J_{1-\mathrm{Fb}}=19.5 \mathrm{~Hz}, \mathrm{H}-1\right), 4.20\left(\mathrm{q}, 2 \mathrm{H}, J_{\mathrm{CH} 2-\mathrm{CH} 3}=7.5 \mathrm{~Hz}, C_{2} \mathrm{CH}_{3}\right)$, $4.10(\mathrm{~d}, 1 \mathrm{H}, J=6.9 \mathrm{~Hz}, \mathrm{H}-3), 3.94-3.88(\mathrm{~m}, 3 \mathrm{H}, \mathrm{H}-2, \mathrm{H}-4, \mathrm{H}-5), 3.86\left(\mathrm{ABX}, 1 \mathrm{H}, J_{5-6 \mathrm{a}}=\right.$ $\left.3.4 \mathrm{~Hz}, J_{6 \mathrm{a}-6 \mathrm{~b}}=10.3 \mathrm{~Hz}, \mathrm{H}-6 \mathrm{a}\right), 3.72\left(\mathrm{ABX}, 1 \mathrm{H}, J_{5-6 \mathrm{~b}}=5.2 \mathrm{~Hz}, J_{6 \mathrm{a}-6 \mathrm{~b}}=10.3 \mathrm{~Hz}, \mathrm{H}-6 \mathrm{~b}\right)$, $3.24\left(\mathrm{~d}, 1 \mathrm{H}, J_{1-\mathrm{OH}}=9.7 \mathrm{~Hz}, \mathrm{OH}\right), 1.26\left(\mathrm{t}, 3 \mathrm{H}, J_{\mathrm{CH} 2-\mathrm{CH} 3}=7.5 \mathrm{~Hz}, \mathrm{CH}_{2} \mathrm{CH}_{3}\right) \mathrm{ppm} .{ }^{13} \mathrm{C} \mathrm{NMR}$ $\left(125 \mathrm{MHz}, \mathrm{CDCl}_{3}\right) \delta=163.5\left(\mathrm{dd}, J_{\mathrm{C}-\mathrm{F}}=29.0 \mathrm{~Hz}, J_{\mathrm{C}-\mathrm{F}}=33.8 \mathrm{~Hz}, \mathrm{C}_{\mathrm{q}} \mathrm{O}_{2} \mathrm{Et}\right), 138.7\left(\mathrm{C}_{\mathrm{q}}{ }^{\text {arom }}\right)$, $138.4\left(\mathrm{C}_{\mathrm{q}}{ }^{\text {arom }}\right), 138.3\left(\mathrm{C}_{\mathrm{q}}{ }^{\text {arom }}\right), 138.2\left(\mathrm{C}_{\mathrm{q}}{ }^{\text {arom }}\right), 137.5\left(\mathrm{C}_{\mathrm{q}}{ }^{\text {arom }}\right), 128.5-127.6\left(\mathrm{CH}^{\text {arom }}\right), 114.6$ $\left(\mathrm{dd}, J_{\mathrm{C}-\mathrm{F}}=252.9 \mathrm{~Hz}, J_{\mathrm{C}-\mathrm{F}}=258.9 \mathrm{~Hz}, \mathrm{CF}_{2}\right), 78.7(\mathrm{C}-2, \mathrm{C}-4), 78.3(\mathrm{C}-5), 74.7\left(\mathrm{CH}_{2}{ }^{\mathrm{Bn}}\right)$, $74.6(\mathrm{C}-3), 74.5\left(\mathrm{CH}_{2}{ }^{\mathrm{Bn}}\right), 74.1\left(\mathrm{CH}_{2}{ }^{\mathrm{Bn}}\right), 73.5\left(\mathrm{CH}_{2}{ }^{\mathrm{Bn}}\right), 71.9\left(\mathrm{CH}_{2}{ }^{\mathrm{Bn}}\right), 69.8\left(\mathrm{dd}, J_{\mathrm{C}-\mathrm{F}}=24.1\right.$ $\left.\mathrm{Hz}, J_{\mathrm{C}-\mathrm{F}}=29.0 \mathrm{~Hz}, \mathrm{C}-1\right), 69.7(\mathrm{C}-6), 63.2\left(\mathrm{CH}_{2} \mathrm{CH}_{3}\right), 14.0\left(\mathrm{CH}_{2} \mathrm{CH}_{3}\right) \mathrm{ppm} .{ }^{19} \mathrm{~F}$ NMR $(471$ $\left.\mathrm{MHz}, \mathrm{CDCl}_{3}\right) \delta=-111.3\left(\mathrm{dd}, 1 \mathrm{~F}, J_{\mathrm{H} 1-\mathrm{Fa}}=4.6 \mathrm{~Hz}, J_{\mathrm{Fa}-\mathrm{Fb}}=261.4 \mathrm{~Hz}, \mathrm{Fa}\right),-123.7(\mathrm{dd}, 1 \mathrm{~F}$, $J_{\mathrm{H} 1-\mathrm{Fb}}=19.5 \mathrm{~Hz}, J_{\mathrm{Fa}-\mathrm{Fb}}=261.4 \mathrm{~Hz}, \mathrm{Fb}$ ) $\mathrm{ppm}$. HRMS (ESI+): m/z calculated for $\mathrm{C}_{45} \mathrm{H}_{49} \mathrm{~F}_{2} \mathrm{O}_{8}[\mathrm{M}+\mathrm{H}]^{+}$: calc. 755.3390; found: 755.3376 . 
${ }^{1}$ H NMR

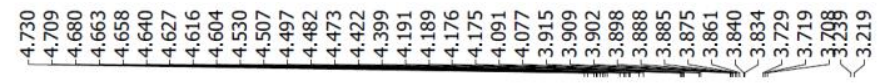

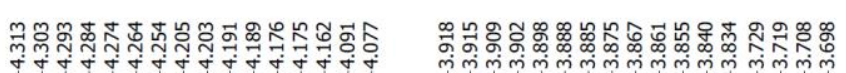

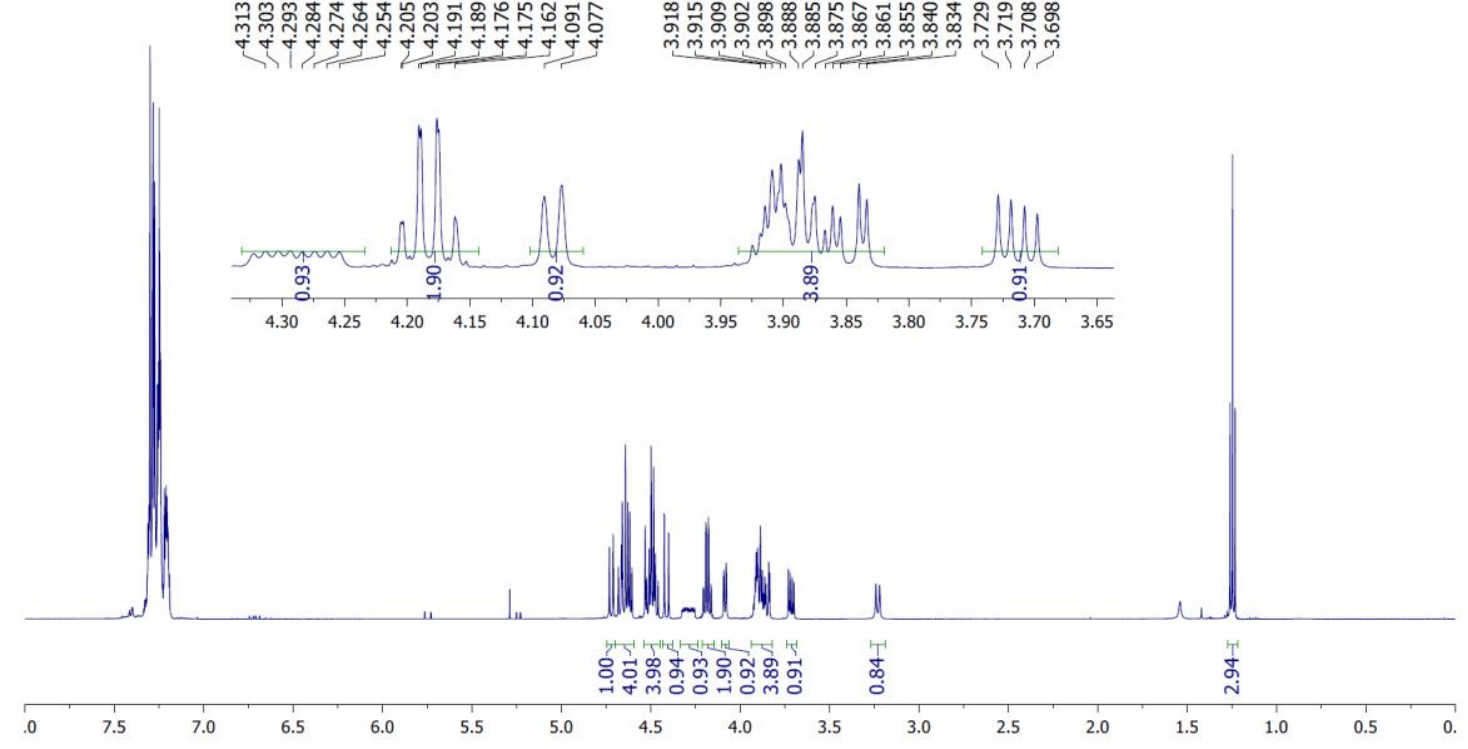

${ }^{19}$ F NMR

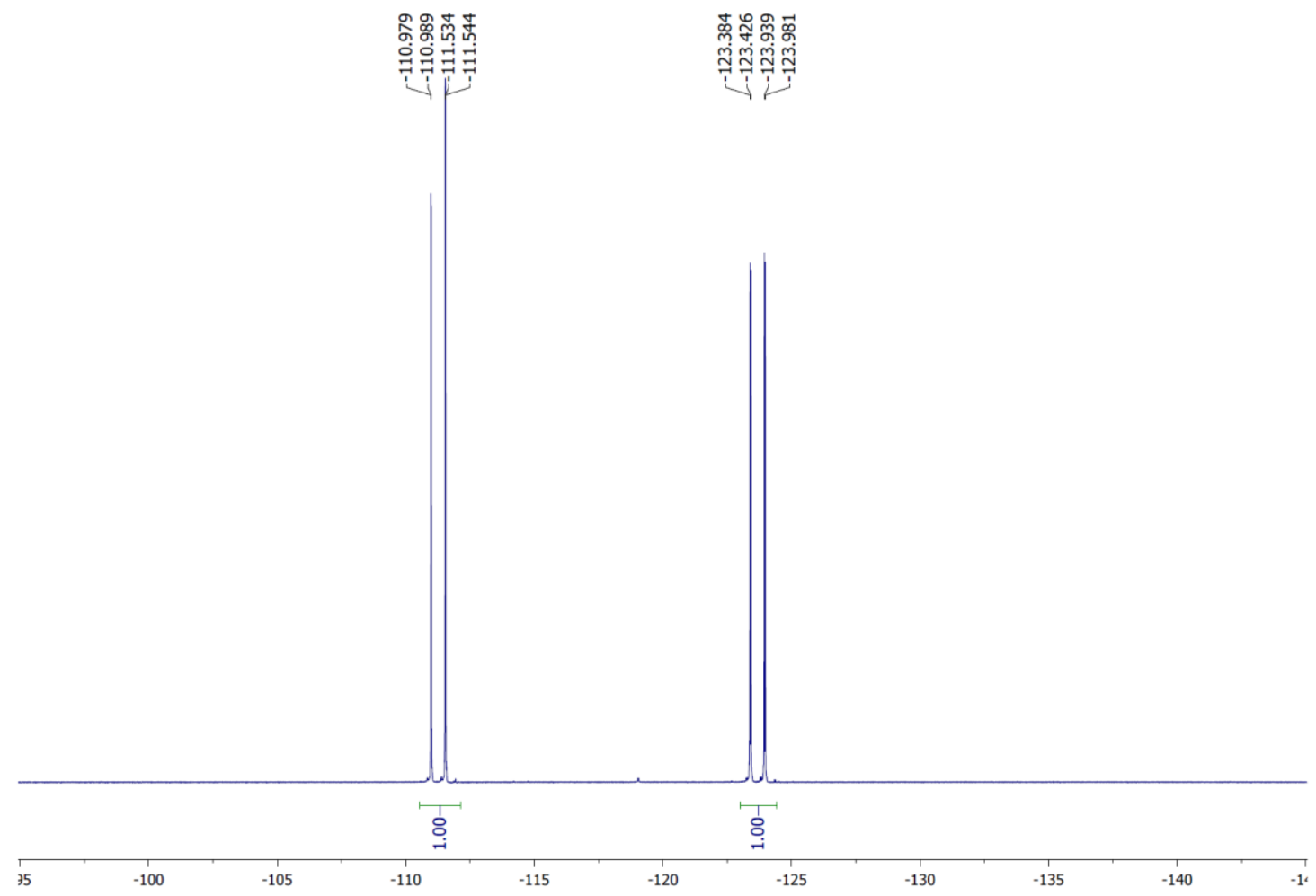


${ }^{13}$ C NMR

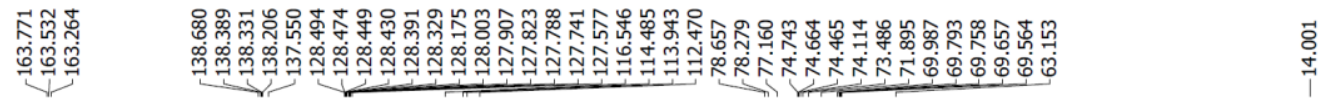

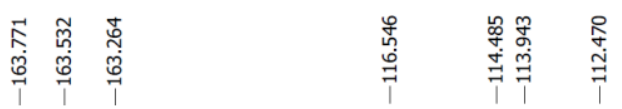
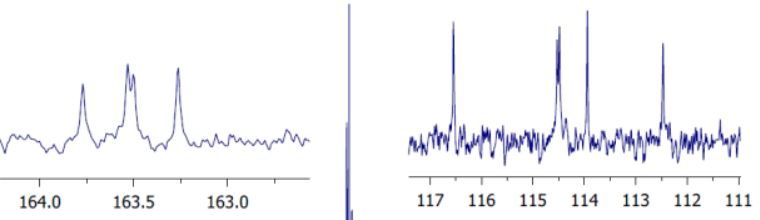

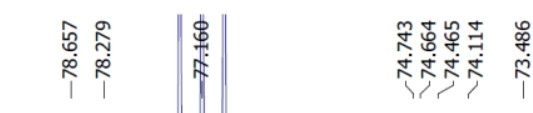

$\begin{array}{lllllllll} & 117 & 116 & 115 & 114 & 113 & 112 & 111\end{array}$

79

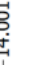

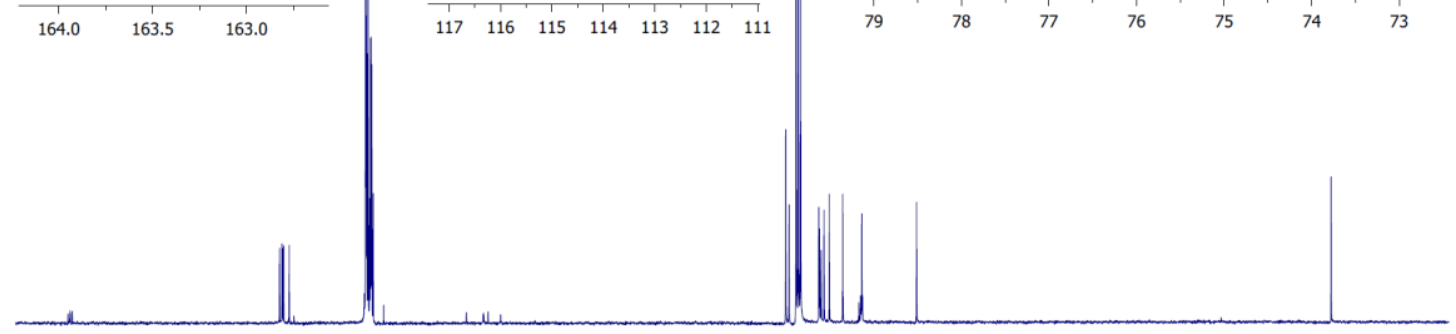

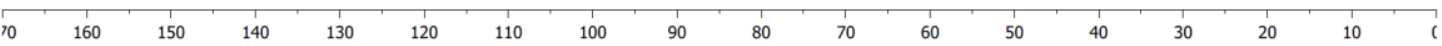


Ethyl 4,5,6,7,8-pentakis(benzyloxy)-2,2-difluoro-3-hydroxyoctanoate ((S)-3g).

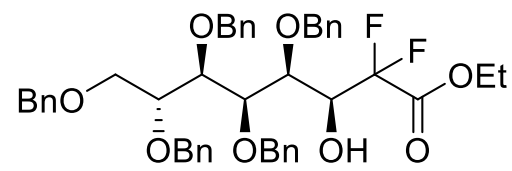

(S)-3g

$[\alpha]_{20} \mathrm{D}:-0.2\left(\mathrm{c} 0.5, \mathrm{CHCl}_{3}\right) .{ }^{1} \mathrm{H}$ NMR $\left(500 \mathrm{MHz}, \mathrm{CDCl}_{3}\right) \delta=7.34-7.27\left(\mathrm{~m}, 23 \mathrm{H}, \mathrm{H}^{\text {arom }}\right)$, 7.24-7.22 (m, $\left.2 \mathrm{H}, \mathrm{H}^{\mathrm{arom}}\right), 4.74\left(\mathrm{AB}, 1 \mathrm{H}, J_{\mathrm{A}-\mathrm{B}}=10.9 \mathrm{~Hz}, \mathrm{CH}_{2}{ }^{\mathrm{Bn}}\right), 4.71\left(\mathrm{AB}, 1 \mathrm{H}, J_{\mathrm{A}-\mathrm{B}}=\right.$ $\left.10.9 \mathrm{~Hz}, \mathrm{CH}_{2}{ }^{\mathrm{Bn}}\right), 4.67\left(\mathrm{AB}, 1 \mathrm{H}, J_{\mathrm{A}-\mathrm{B}}=12.0 \mathrm{~Hz}, \mathrm{CH}_{2}{ }^{\mathrm{Bn}}\right), 4.58\left(\mathrm{~s}, 2 \mathrm{H}, \mathrm{CH}_{2}{ }^{\mathrm{Bn}}\right), 4.53(\mathrm{AB}$, $\left.1 \mathrm{H}, J_{\mathrm{A}-\mathrm{B}}=12.6 \mathrm{~Hz}, \mathrm{CH}_{2}{ }^{\mathrm{Bn}}\right), 4.50\left(\mathrm{AB}, 1 \mathrm{H}, J_{\mathrm{A}-\mathrm{B}}=12.0 \mathrm{~Hz}, \mathrm{CH}_{2}{ }^{\mathrm{Bn}}\right), 4.48-4.44(\mathrm{~m}, 4 \mathrm{H}, 3$ $\left.\mathrm{CH}_{2}{ }^{\mathrm{Bn}}, \mathrm{H}-1\right), 4.16$ (t, $\left.1 \mathrm{H}, J_{3-4}=J_{4-5}=4.6 \mathrm{~Hz}, \mathrm{H}-4\right), 4.10-4.02\left(\mathrm{~m}, 3 \mathrm{H}, \mathrm{H}-3, \mathrm{CH}_{2} \mathrm{CH}_{3}\right), 3.91$ $(\mathrm{dd}, 1 \mathrm{H}, J=4.0 \mathrm{~Hz}, J=8.0 \mathrm{~Hz}, \mathrm{H}-2), 3.89\left(\mathrm{~d}, 1 \mathrm{H}, J_{1-\mathrm{OH}}=4.0 \mathrm{~Hz}, \mathrm{OH}\right), 3.86-3.81(\mathrm{~m}, 2 \mathrm{H}$, H-5, H-6a), $3.71\left(\mathrm{ABX}, 1 \mathrm{H}, J_{5-6 \mathrm{~b}}=4.6 \mathrm{~Hz}, J_{6 \mathrm{a}-6 \mathrm{~b}}=10.3 \mathrm{~Hz}, \mathrm{H}-6 \mathrm{~b}\right), 1.17$ (t, 3H, $J_{\mathrm{CH} 2-\mathrm{CH} 3}$ $\left.=7.5 \mathrm{~Hz}, \mathrm{CH}_{2} \mathrm{CH}_{3}\right) \mathrm{ppm} .{ }^{13} \mathrm{C} \mathrm{NMR}\left(125 \mathrm{MHz}, \mathrm{CDCl}_{3}\right) \delta=163.3\left(\mathrm{t}, J_{\mathrm{C}-\mathrm{F}}=31.4 \mathrm{~Hz}\right.$, $\left.\mathrm{C}_{\mathrm{q}} \mathrm{O}_{2} \mathrm{Et}\right), 138.5\left(\mathrm{C}_{\mathrm{q}}^{\text {arom }}\right), 138.3\left(\mathrm{C}_{\mathrm{q}}{ }^{\text {arom }}\right), 138.1\left(\mathrm{C}_{\mathrm{q}}{ }^{\text {arom }}\right), 137.5\left(\mathrm{C}_{\mathrm{q}}{ }^{\text {arom }}\right), 137.4\left(\mathrm{C}_{\mathrm{q}}^{\text {arom }}\right)$, 128.6- $127.7\left(\mathrm{CH}^{\text {arom }}\right), 114.8\left(\mathrm{t}, J_{\mathrm{C}-\mathrm{F}}=255.2 \mathrm{~Hz}, \mathrm{CF}_{2}\right), 79.1(\mathrm{C}-5), 78.7(\mathrm{C}-3), 77.9(\mathrm{C}-4)$, $74.4(\mathrm{C}-2), 74.3\left(\mathrm{CH}_{2}{ }^{\mathrm{Bn}}\right), 74.2\left(\mathrm{CH}_{2}{ }^{\mathrm{Bn}}\right), 73.5\left(\mathrm{CH}_{2}{ }^{\mathrm{Bn}}\right), 73.1\left(\mathrm{CH}_{2}{ }^{\mathrm{Bn}}\right), 72.1\left(\mathrm{CH}_{2}{ }^{\mathrm{Bn}}\right), 71.8$ $\left(\mathrm{t}, J_{\mathrm{C}-\mathrm{F}}=22.9 \mathrm{~Hz}, \mathrm{C}-1\right), 69.2(\mathrm{C}-6), 62.8\left(\mathrm{CH}_{2} \mathrm{CH}_{3}\right), 13.9\left(\mathrm{CH}_{2} \mathrm{CH}_{3}\right)$ ppm. ${ }^{19} \mathrm{~F}$ NMR $(471$ $\left.\mathrm{MHz}, \mathrm{CDCl}_{3}\right) \delta=-119.0$ (s, 1F, Fa), -119.1 (s, 1F, Fb) ppm. HRMS (ESI+): m/z calculated for $\mathrm{C}_{45} \mathrm{H}_{49} \mathrm{~F}_{2} \mathrm{O}_{8}[\mathrm{M}+\mathrm{H}]^{+}$: calc. 755.3390; found: 755.3381 . 


\section{${ }^{1}$ H NMR}

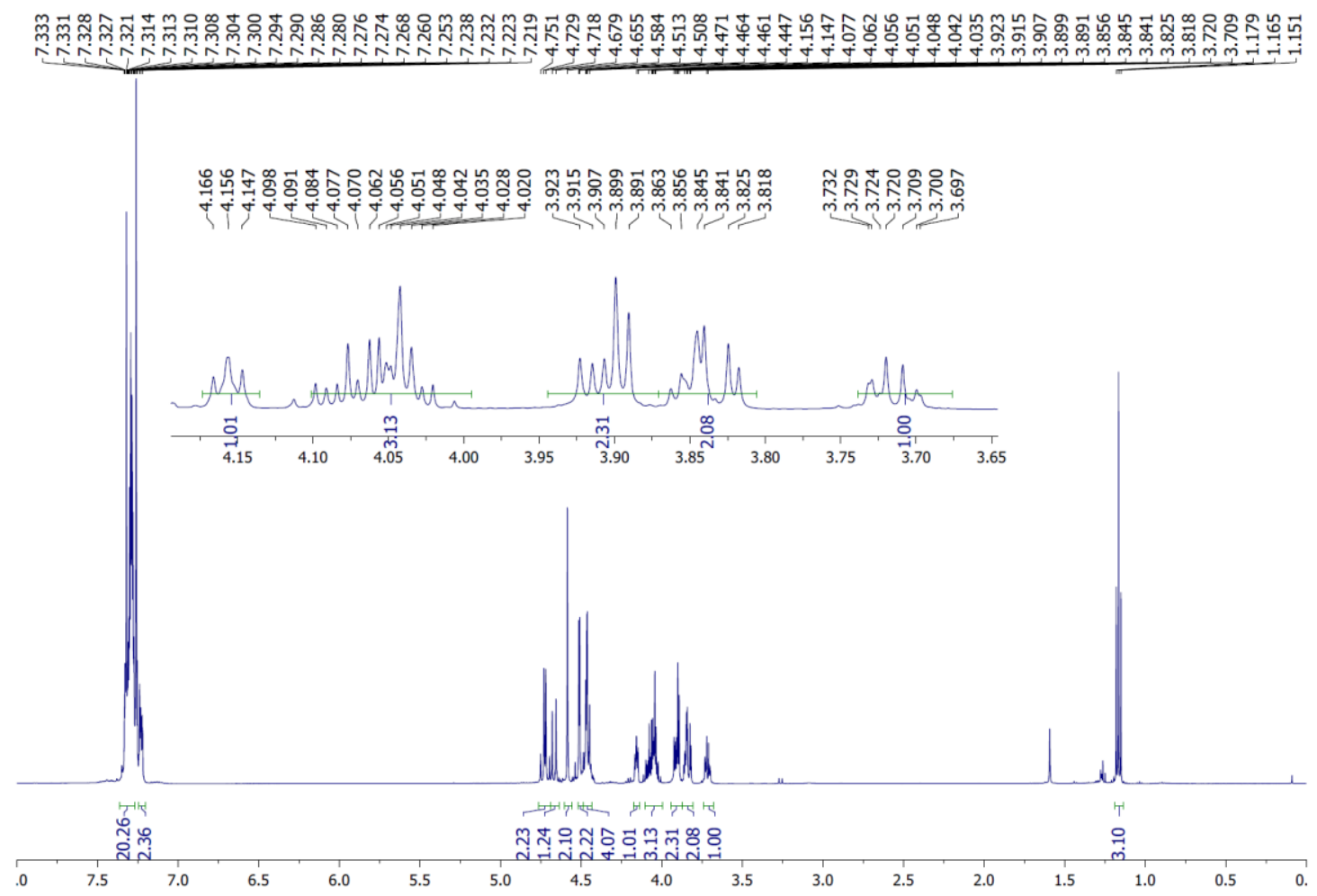

${ }^{19}$ F NMR

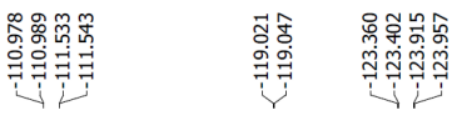

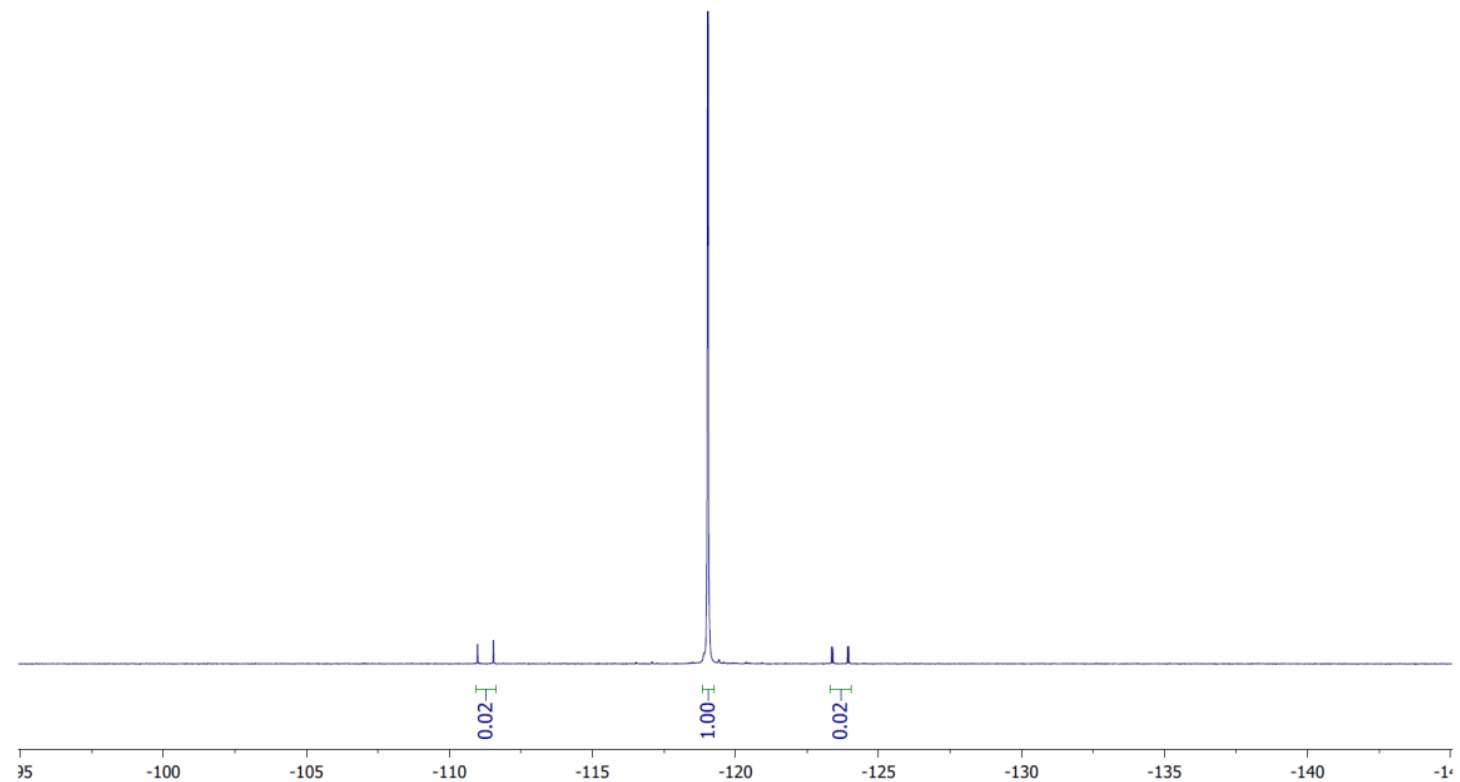


${ }^{13}$ C NMR
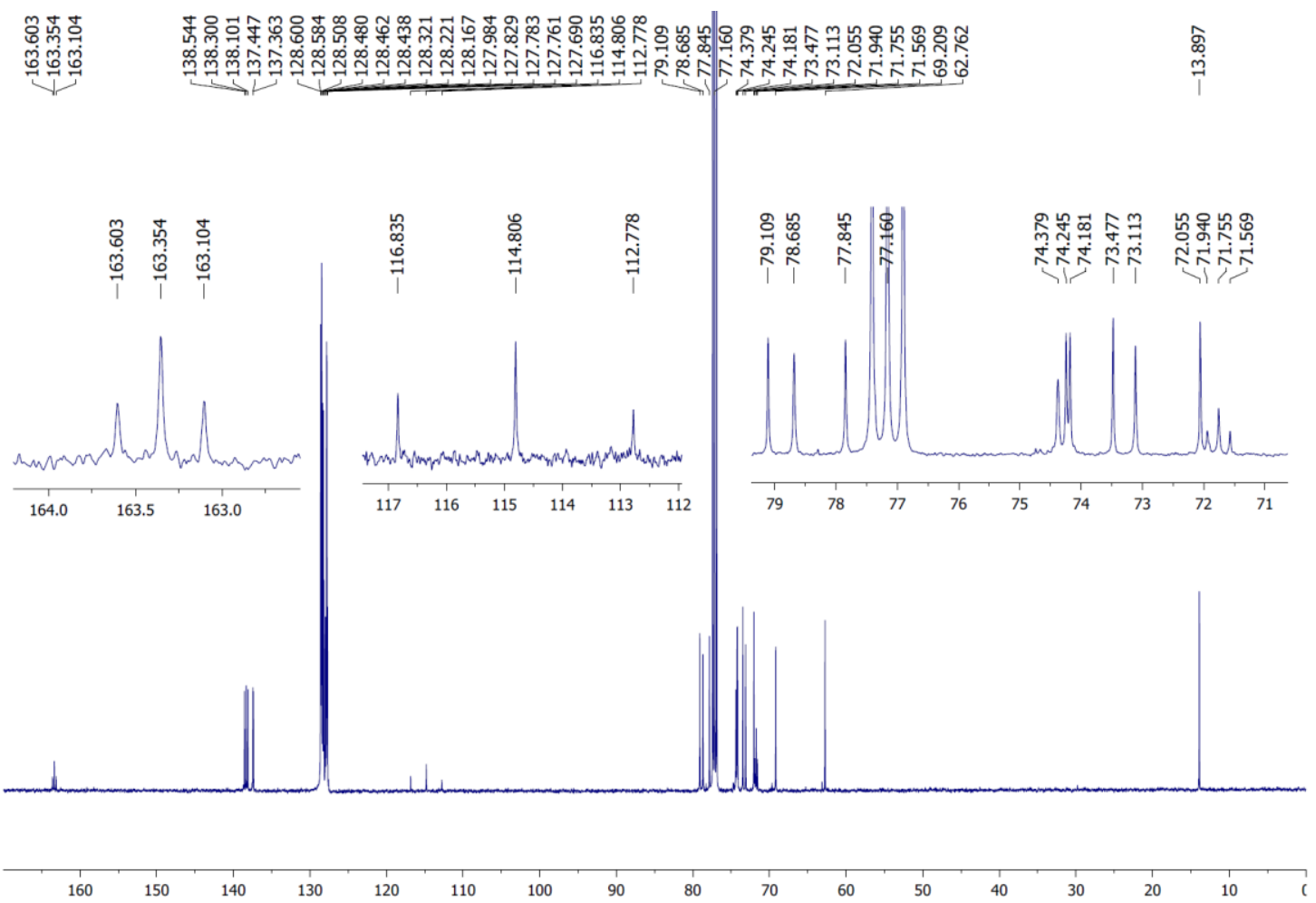


\section{D-Mannose}

Ethyl 4,5,6,7,8-pentakis(benzyloxy)-2,2-difluoro-3-hydroxyoctanoate ((A/B)-3h).

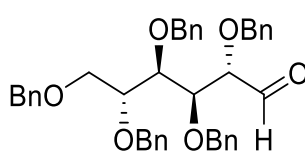

$2 \mathrm{~h}$

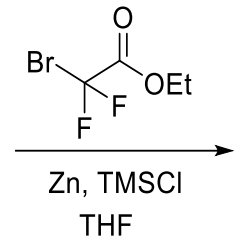

THF

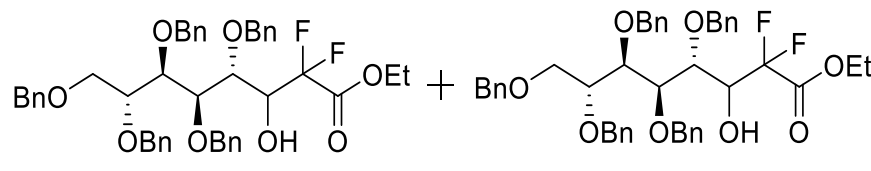

(A)-3h
$75 \%$

(B)-3h

$\mathrm{A}: \mathrm{B} d r 55: 45$

To a dry two-necked round bottomed flask was added "activated zinc" powder (293 mg, $4.46 \mathrm{mmol}, 5.7$ equiv.). The flask was heated up at $40^{\circ} \mathrm{C}$ and the zinc powder suspended in dry THF ( $6 \mathrm{ml})$ under argon atmosphere. Then, TMSCl $(40 \mu \mathrm{l}, 0.266 \mathrm{mmol}, 0.4$ equiv. $)$ was added dropwise and the reaction was stirred at $60^{\circ} \mathrm{C}$ for 15 mins. Next, a solution of aldehyde $\mathbf{2 h}$ (493 g, $0.782 \mathrm{mmol}, 1.0$ equiv.) and ethyl bromo-difluoroacetate $(0.12 \mathrm{ml}$, $0.938 \mathrm{mmol}, 1.2$ equiv.) dissolved homogenously in dry THF $(6 \mathrm{ml})$ under argon atmosphere was added dropwise at $60^{\circ} \mathrm{C}$. The reaction was stirred vigorously for $1.5 \mathrm{~h}$ at $60^{\circ} \mathrm{C}$ and then cooled at room temperature. Ice water $(5 \mathrm{ml})$ and $\mathrm{HCl}(1 \mathrm{M}, 10 \mathrm{ml})$ were added and the mixture was diluted with EtOAc. The organic phase was washed with $\mathrm{NaHCO}_{3}$ (sat) (2 times), brine, dried over $\mathrm{MgSO}_{4}$ and concentrated under reduced pressure. Crude NMR analysis showed the formation of two diastereoisomer $(A)-3 \mathbf{h}$ and $(B)-3 h$ in 55:45 $d r$ ratio. Purification by silica gel chromatography (Cy/EtOAc 92:8) afforded pure diastereoisomers $(A)-\mathbf{3 h}$ and $(B)-3 \mathbf{h}$ in $75 \%$ overall yield. 
Ethyl 4,5,6,7,8-pentakis(benzyloxy)-2,2-difluoro-3-hydroxyoctanoate ((A)-3h).

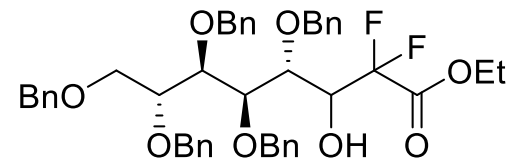

(A)-3h

$[\alpha]_{20} \mathrm{D}:+2.4\left(\mathrm{c} 1.0, \mathrm{CHCl}_{3}\right) .{ }^{1} \mathrm{H}$ NMR $\left(500 \mathrm{MHz}, \mathrm{CDCl}_{3}\right) \delta=7.35-7.26\left(\mathrm{~m}, 20 \mathrm{H}, \mathrm{H}^{\text {arom }}\right)$, 7.26-7.23 (m, $\left.3 \mathrm{H}, \mathrm{H}^{\mathrm{arom}}\right), 7.16-7.13\left(\mathrm{~m}, 2 \mathrm{H}, \mathrm{H}^{\text {arom }}\right), 4.78\left(\mathrm{AB}, 1 \mathrm{H}, J_{\mathrm{A}-\mathrm{B}}=11.5 \mathrm{~Hz}, \mathrm{CH}_{2}{ }^{\mathrm{Bn}}\right)$, $4.74\left(\mathrm{~s}, 2 \mathrm{H}, \mathrm{CH}_{2}{ }^{\mathrm{Bn}}\right), 4.66-4.57(\mathrm{~m}, 1 \mathrm{H}, \mathrm{H}-1), 4.66\left(\mathrm{AB}, 1 \mathrm{H}, J_{\mathrm{A}-\mathrm{B}}=10.9 \mathrm{~Hz}, \mathrm{CH}_{2}{ }^{\mathrm{Bn}}\right), 4.61$ $\left(\mathrm{AB}, 1 \mathrm{H}, J_{\mathrm{A}-\mathrm{B}}=12.0 \mathrm{~Hz}, \mathrm{CH}_{2}{ }^{\mathrm{Bn}}\right), 4.55\left(\mathrm{AB}, 1 \mathrm{H}, J_{\mathrm{A}-\mathrm{B}}=12.0 \mathrm{~Hz}, \mathrm{CH}_{2}{ }^{\mathrm{Bn}}\right), 4.49(\mathrm{AB}, 1 \mathrm{H}$, $\left.J_{\mathrm{A}-\mathrm{B}}=11.5 \mathrm{~Hz}, \mathrm{CH}_{2}{ }^{\mathrm{Bn}}\right), 4.48\left(\mathrm{AB}, 1 \mathrm{H}, J_{\mathrm{A}-\mathrm{B}}=11.5 \mathrm{~Hz}, \mathrm{CH}_{2}{ }^{\mathrm{Bn}}\right), 4.39\left(\mathrm{AB}, 1 \mathrm{H}, J_{\mathrm{A}-\mathrm{B}}=10.9\right.$ $\left.\mathrm{Hz}, \mathrm{CH}_{2}{ }^{\mathrm{Bn}}\right), 4.24\left(\mathrm{AB}, 1 \mathrm{H}, J_{\mathrm{A}-\mathrm{B}}=11.5 \mathrm{~Hz}, \mathrm{CH}_{2}{ }^{\mathrm{Bn}}\right), 4.06-4.02(\mathrm{~m}, 2 \mathrm{H}, \mathrm{H}-3, \mathrm{H}-4), 3.94-$ 3.86 (m, 3H, H-6a, $\mathrm{CH}_{2} \mathrm{CH}_{3}$ ), 3.85-3.81 (m, 2H, H-2, H-5), 3.71 (ABX, 1H, $J_{5-6 \mathrm{~b}}=4.6$ $\left.\mathrm{Hz}, J_{6 \mathrm{a}-6 \mathrm{~b}}=10.3 \mathrm{~Hz}, \mathrm{H}-6 \mathrm{~b}\right), 3.43\left(\mathrm{~d}, 1 \mathrm{H}, J_{1-\mathrm{OH}}=4.6 \mathrm{~Hz}, \mathrm{OH}\right), 1.08\left(\mathrm{t}, 3 \mathrm{H}, J_{\mathrm{CH} 2-\mathrm{CH} 3}=7.5\right.$ $\left.\mathrm{Hz}, \mathrm{CH}_{2} \mathrm{CH}_{3}\right) \mathrm{ppm} .{ }^{13} \mathrm{C} \mathrm{NMR}\left(125 \mathrm{MHz}, \mathrm{CDCl}_{3}\right) \delta=163.2\left(\mathrm{dd}, J_{\mathrm{C}-\mathrm{F}}=30.2 \mathrm{~Hz}, J_{\mathrm{C}-\mathrm{F}}=\right.$ $\left.32.6 \mathrm{~Hz}, \mathrm{C}_{\mathrm{q}} \mathrm{O}_{2} \mathrm{Et}\right), 138.5\left(\mathrm{C}_{\mathrm{q}}{ }^{\text {arom }}\right), 138.3\left(\mathrm{C}_{\mathrm{q}}{ }^{\text {arom }}\right), 138.2\left(\mathrm{C}_{\mathrm{q}}{ }^{\text {arom }}\right), 138.1\left(\mathrm{C}_{\mathrm{q}}{ }^{\text {arom }}\right), 137.4$ $\left(\mathrm{C}_{\mathrm{q}}{ }^{\text {arom }}\right), 128.6-127.7\left(\mathrm{CH}^{\text {arom }}\right), 114.9\left(\mathrm{dd}, J_{\mathrm{C}-\mathrm{F}}=247.8 \mathrm{~Hz}, J_{\mathrm{C}-\mathrm{F}}=258.4 \mathrm{~Hz}, \mathrm{CF}_{2}\right), 81.0$ (C-3 or C-4), 80.0 (C-3 or C-4), $79.0(\mathrm{C}-5), 78.9\left(\mathrm{~d}, J_{\mathrm{C}-\mathrm{F}}=3.6 \mathrm{~Hz}, \mathrm{C}-2\right), 75.2\left(\mathrm{CH}_{2}{ }^{\mathrm{Bn}}\right)$, $74.8\left(\mathrm{CH}_{2}{ }^{\mathrm{Bn}}\right), 73.6\left(\mathrm{CH}_{2}{ }^{\mathrm{Bn}}\right), 72.6\left(\mathrm{CH}_{2}{ }^{\mathrm{Bn}}\right), 72.1\left(\mathrm{CH}_{2}{ }^{\mathrm{Bn}}\right), 70.2\left(\mathrm{t}, J_{\mathrm{C}-\mathrm{F}}=22.2 \mathrm{~Hz}, \mathrm{C}-1\right), 69.1$ (C-6), $62.5\left(\mathrm{CH}_{2} \mathrm{CH}_{3}\right), 13.8\left(\mathrm{CH}_{2} \mathrm{CH}_{3}\right)$ ppm. ${ }^{19} \mathrm{~F} \mathrm{NMR}\left(471 \mathrm{MHz}, \mathrm{CDCl}_{3}\right) \delta=-114.5(\mathrm{dd}$, $\left.1 \mathrm{~F}, J_{\mathrm{H}-\mathrm{Fa}}=5.4 \mathrm{~Hz}, J_{\mathrm{Fa}-\mathrm{Fb}}=260.5 \mathrm{~Hz}, \mathrm{Fa}\right),-124.7\left(\mathrm{dd}, 1 \mathrm{~F}, J_{\mathrm{H}-\mathrm{Fb}}=19.8 \mathrm{~Hz}, J_{\mathrm{Fa}-\mathrm{Fb}}=260.5\right.$ $\mathrm{Hz}, \mathrm{Fb}$ ) ppm. HRMS (ESI+): m/z calculated for $\mathrm{C}_{45} \mathrm{H}_{49} \mathrm{~F}_{2} \mathrm{O}_{8}[\mathrm{M}+\mathrm{H}]^{+}$: calc. 755.3390; found: 755.3385 . 
${ }^{1}$ H NMR

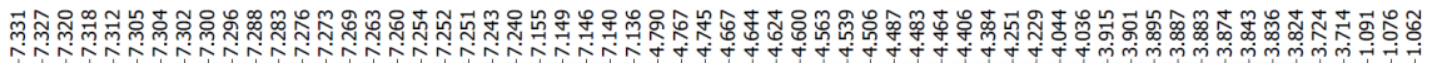

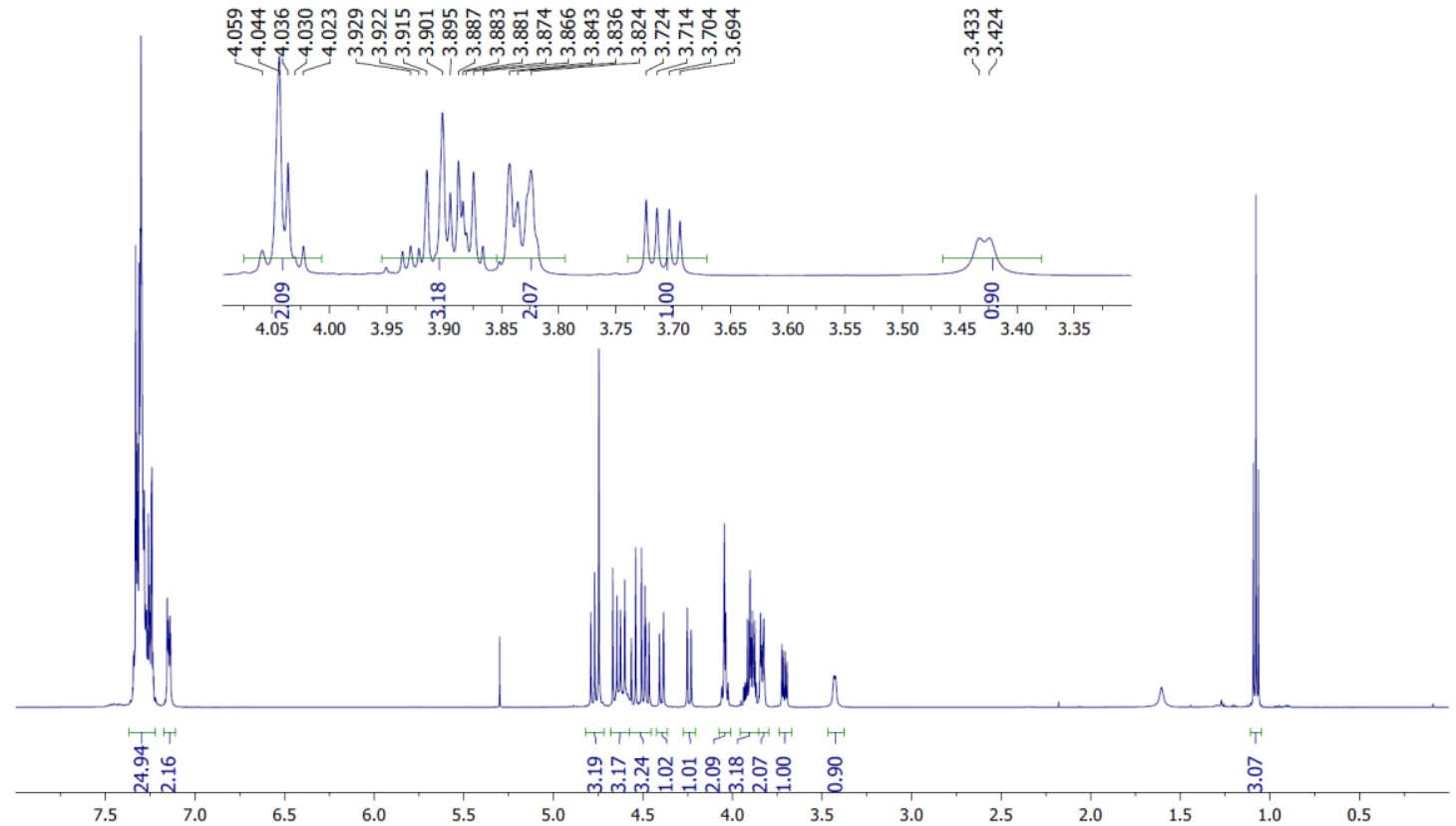

${ }^{19}$ F NMR

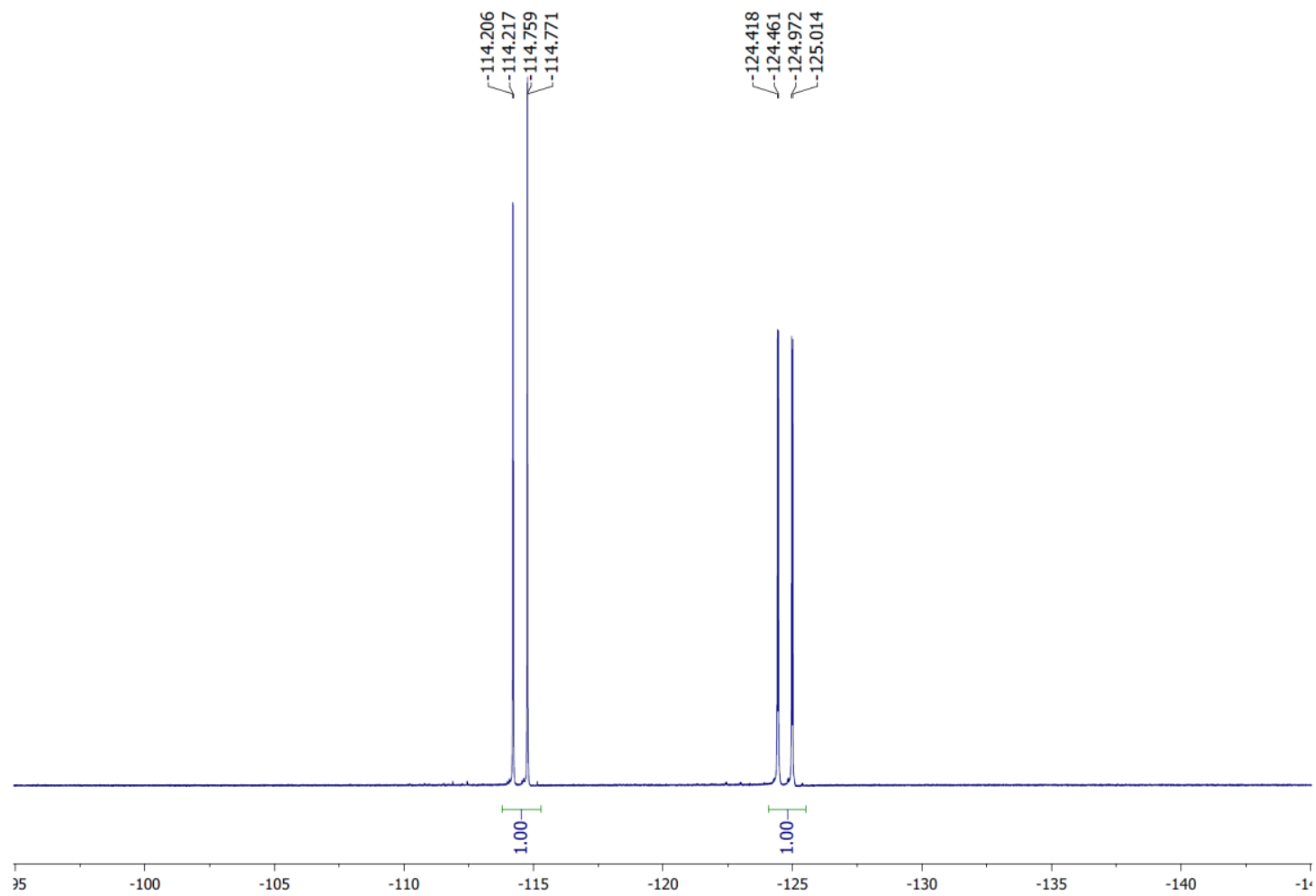


${ }^{13}$ C NMR

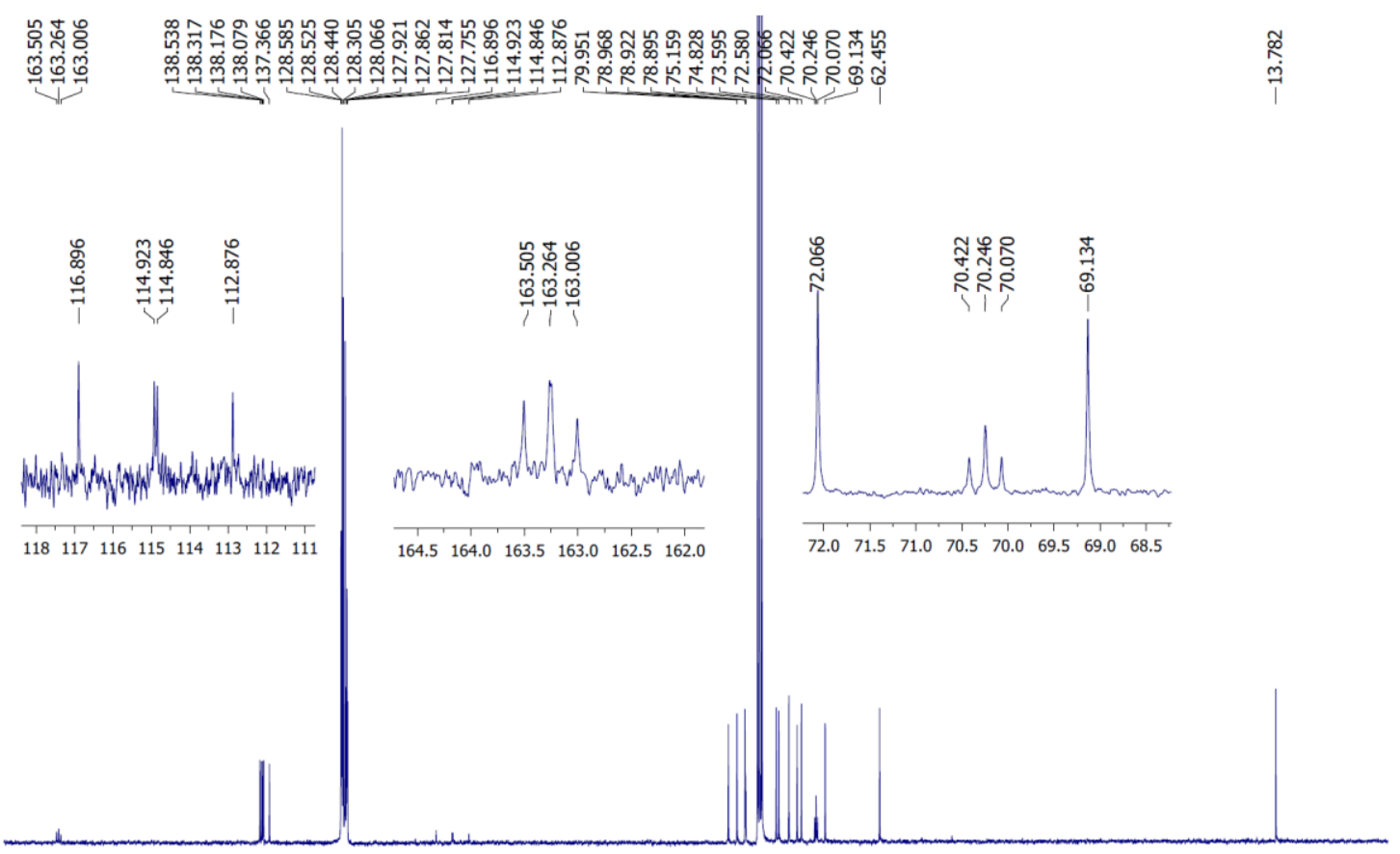

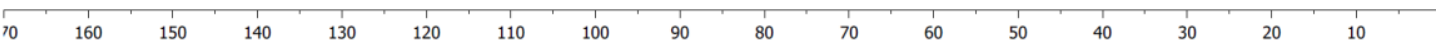


Ethyl 4,5,6,7,8-pentakis(benzyloxy)-2,2-difluoro-3-hydroxyoctanoate ((B)-3h).

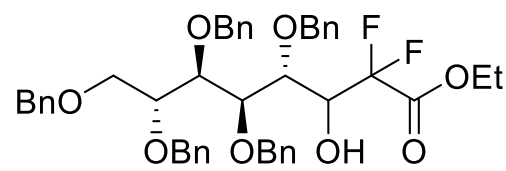

(B)-3h

$[\alpha]_{20}{ }^{\mathrm{D}}:+1.9\left(\mathrm{c} 1.0, \mathrm{CHCl}_{3}\right) .{ }^{1} \mathrm{H} \mathrm{NMR}\left(500 \mathrm{MHz}, \mathrm{CDCl}_{3}\right) \delta=7.32-7.26\left(\mathrm{~m}, 20 \mathrm{H}, \mathrm{H}^{\text {arom }}\right)$, 7.26-7.20 (m, $\left.5 \mathrm{H}, \mathrm{H}^{\mathrm{arom}}\right), 4.75\left(\mathrm{AB}, 1 \mathrm{H}, J_{\mathrm{A}-\mathrm{B}}=11.5 \mathrm{~Hz}, \mathrm{CH}_{2}{ }^{\mathrm{Bn}}\right), 4.71\left(\mathrm{AB}, 1 \mathrm{H}, J_{\mathrm{A}-\mathrm{B}}=\right.$ $\left.12.0 \mathrm{~Hz}, \mathrm{CH}_{2}{ }^{\mathrm{Bn}}\right), 4.67-4.61\left(\mathrm{~m}, 3 \mathrm{H}, \mathrm{CH}_{2}{ }^{\mathrm{Bn}}\right), 4.51\left(\mathrm{AB}, 1 \mathrm{H}, J_{\mathrm{A}-\mathrm{B}}=12.0 \mathrm{~Hz}, \mathrm{CH}_{2}{ }^{\mathrm{Bn}}\right), 4.50-$ $4.47\left(\mathrm{~m}, 2 \mathrm{H}, \mathrm{CH}_{2}{ }^{\mathrm{Bn}}\right), 4.44\left(\mathrm{AB}, 1 \mathrm{H}, J_{\mathrm{A}-\mathrm{B}}=10.9 \mathrm{~Hz}, \mathrm{CH}_{2}{ }^{\mathrm{Bn}}\right), 4.43-4.37(\mathrm{~m}, 1 \mathrm{H}, \mathrm{H}-1), 4.36$ $\left(\mathrm{AB}, 1 \mathrm{H}, J_{\mathrm{A}-\mathrm{B}}=10.9 \mathrm{~Hz}, \mathrm{CH}_{2}{ }^{\mathrm{Bn}}\right), 4.21\left(\mathrm{qd}, 2 \mathrm{H}, J=2.9 \mathrm{~Hz}, J_{\mathrm{CH} 2-\mathrm{CH} 3}=7.5 \mathrm{~Hz}, \mathrm{CH}_{2} \mathrm{CH}_{3}\right)$, $4.05(\mathrm{~d}, 1 \mathrm{H}, J=5.7 \mathrm{~Hz}, \mathrm{H}-2), 3.97$ (dd, $1 \mathrm{H}, J=4.0 \mathrm{~Hz}, J=5.7 \mathrm{~Hz}, \mathrm{H}-3), 3.95-3.88$ (m, $3 \mathrm{H}, \mathrm{H}-4, \mathrm{H}-5, \mathrm{H}-6 \mathrm{a}), 3.71$ (ABX, 1H, $\left.J_{5-6 \mathrm{~b}}=4.0 \mathrm{~Hz}, J_{6 \mathrm{a}-6 \mathrm{~b}}=9.7 \mathrm{~Hz}, \mathrm{H}-6 \mathrm{~b}\right), 3.42(\mathrm{~d}, 1 \mathrm{H}$, $\left.J_{1-\mathrm{OH}}=9.2 \mathrm{~Hz}, \mathrm{OH}\right), 1.23\left(\mathrm{t}, 3 \mathrm{H}, J_{\mathrm{CH} 2-\mathrm{CH} 3}=7.5 \mathrm{~Hz}, \mathrm{CH}_{2} \mathrm{CH}_{3}\right) \mathrm{ppm} .{ }^{13} \mathrm{C} \mathrm{NMR}(125 \mathrm{MHz}$, $\left.\mathrm{CDCl}_{3}\right) \delta=163.5\left(\mathrm{dd}, J_{\mathrm{C}-\mathrm{F}}=30.2 \mathrm{~Hz}, J_{\mathrm{C}-\mathrm{F}}=33.8 \mathrm{~Hz}, \mathrm{C}_{\mathrm{q}} \mathrm{O}_{2} \mathrm{Et}\right), 138.5\left(2 \mathrm{C}_{\mathrm{q}}{ }^{\text {arom }}\right), 138.2$ $\left(\mathrm{C}_{\mathrm{q}}{ }^{\text {arom }}\right), 138.1\left(\mathrm{C}_{\mathrm{q}}{ }^{\text {arom }}\right), 137.5\left(\mathrm{C}_{\mathrm{q}}{ }^{\text {arom }}\right), 128.6-127.7\left(\mathrm{CH}^{\text {arom }}\right), 114.6\left(\mathrm{dd}, J_{\mathrm{C}-\mathrm{F}}=254.2 \mathrm{~Hz}\right.$, $\left.J_{\mathrm{C}-\mathrm{F}}=257.8 \mathrm{~Hz}, \mathrm{CF}_{2}\right), 79.6(\mathrm{C}-3), 78.7(\mathrm{C}-4$ or C-5), $78.4(\mathrm{C}-4$ or C-5), $75.1(\mathrm{C}-2), 75.0$ $\left(\mathrm{CH}_{2}{ }^{\mathrm{Bn}}\right), 73.6\left(\mathrm{CH}_{2}{ }^{\mathrm{Bn}}\right), 73.2\left(2 \mathrm{CH}_{2}{ }^{\mathrm{Bn}}\right), 72.1\left(\mathrm{CH}_{2}{ }^{\mathrm{Bn}}\right), 69.7\left(\mathrm{dd}, J_{\mathrm{C}-\mathrm{F}}=24.1 \mathrm{~Hz}, J_{\mathrm{C}-\mathrm{F}}=29.0\right.$ $\mathrm{Hz}, \mathrm{C}-1), 69.6(\mathrm{C}-6), 63.1\left(\mathrm{CH}_{2} \mathrm{CH}_{3}\right), 14.0\left(\mathrm{CH}_{2} \mathrm{CH}_{3}\right) \mathrm{ppm} .{ }^{19} \mathrm{~F} \mathrm{NMR}\left(471 \mathrm{MHz}, \mathrm{CDCl}_{3}\right)$ $\delta=-112.2\left(\mathrm{dd}, 1 \mathrm{~F}, J_{\mathrm{H}-\mathrm{Fa}}=5.4 \mathrm{~Hz}, J_{\mathrm{Fa}-\mathrm{Fb}}=260.0 \mathrm{~Hz}, \mathrm{Fa}\right),-127.7\left(\mathrm{dd}, 1 \mathrm{~F}, J_{\mathrm{H}-\mathrm{Fb}}=20.2 \mathrm{~Hz}\right.$, $\left.J_{\mathrm{Fa}-\mathrm{Fb}}=260.0 \mathrm{~Hz}, \mathrm{Fb}\right) \mathrm{ppm}$. HRMS (ESI+): m/z calculated for $\mathrm{C}_{45} \mathrm{H}_{49} \mathrm{~F}_{2} \mathrm{O}_{8}[\mathrm{M}+\mathrm{H}]^{+}$: calc. 755.3390; found: 755.3379. 


\section{${ }^{1}$ H NMR}

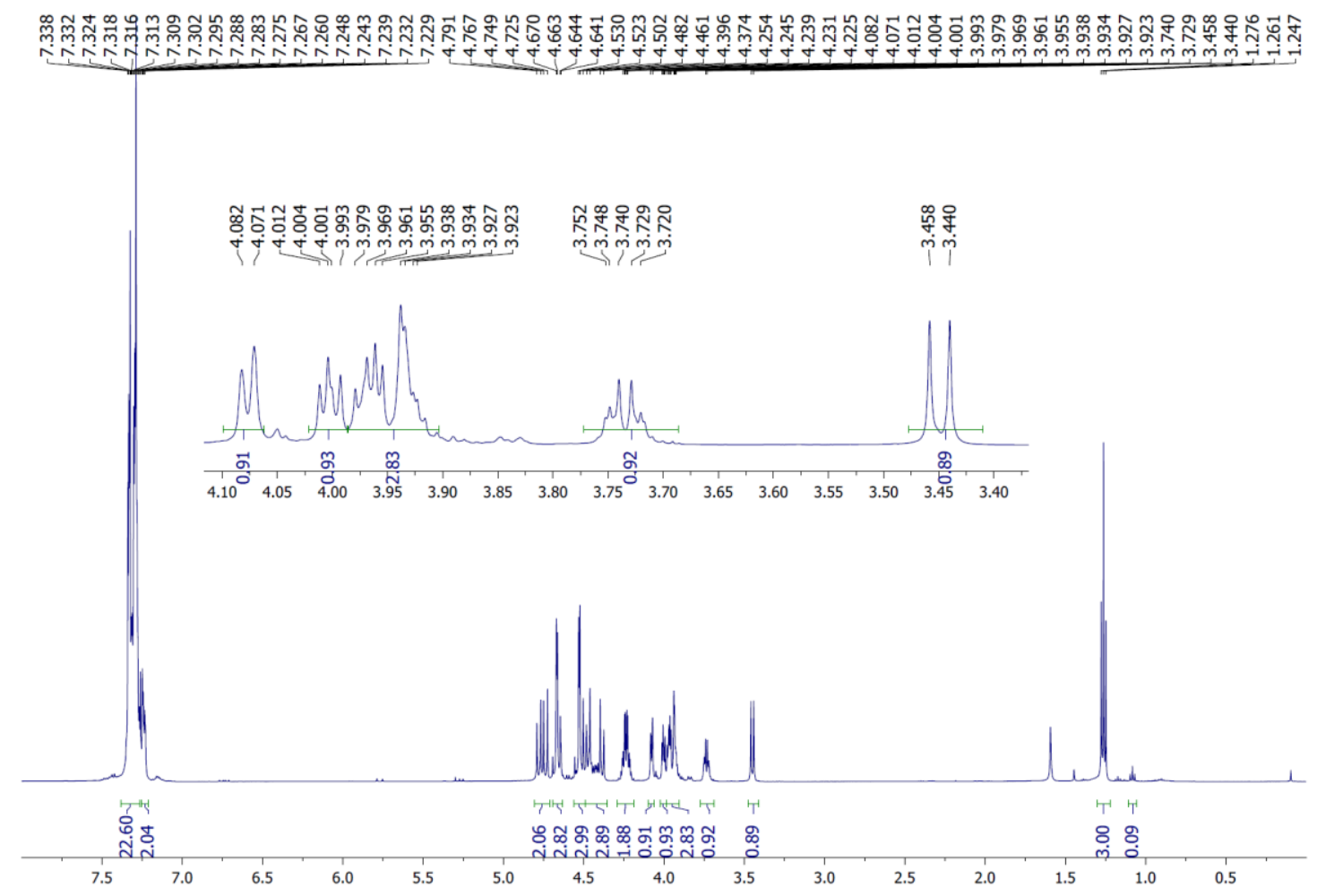

${ }^{19}$ F NMR
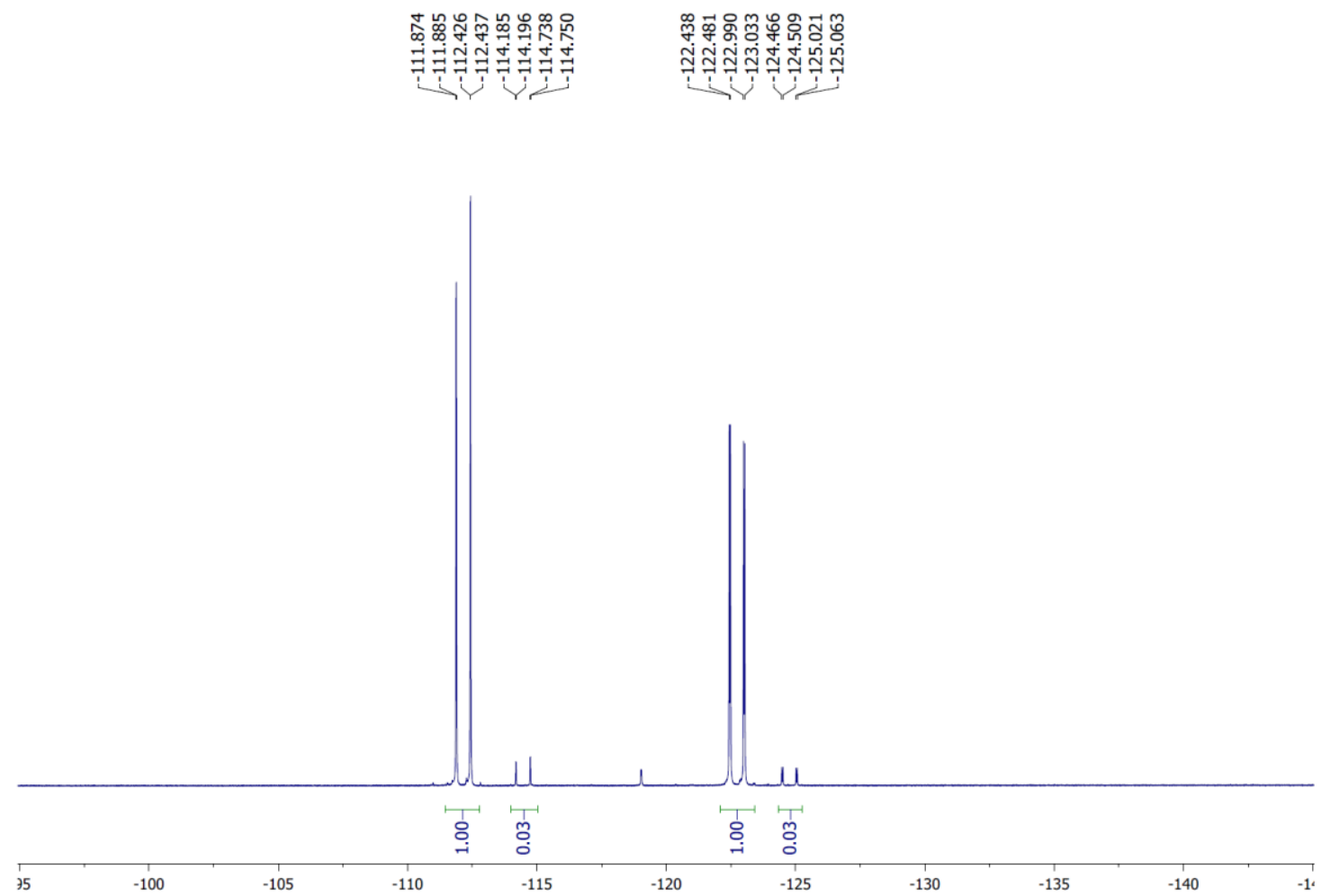
${ }^{13}$ C NMR
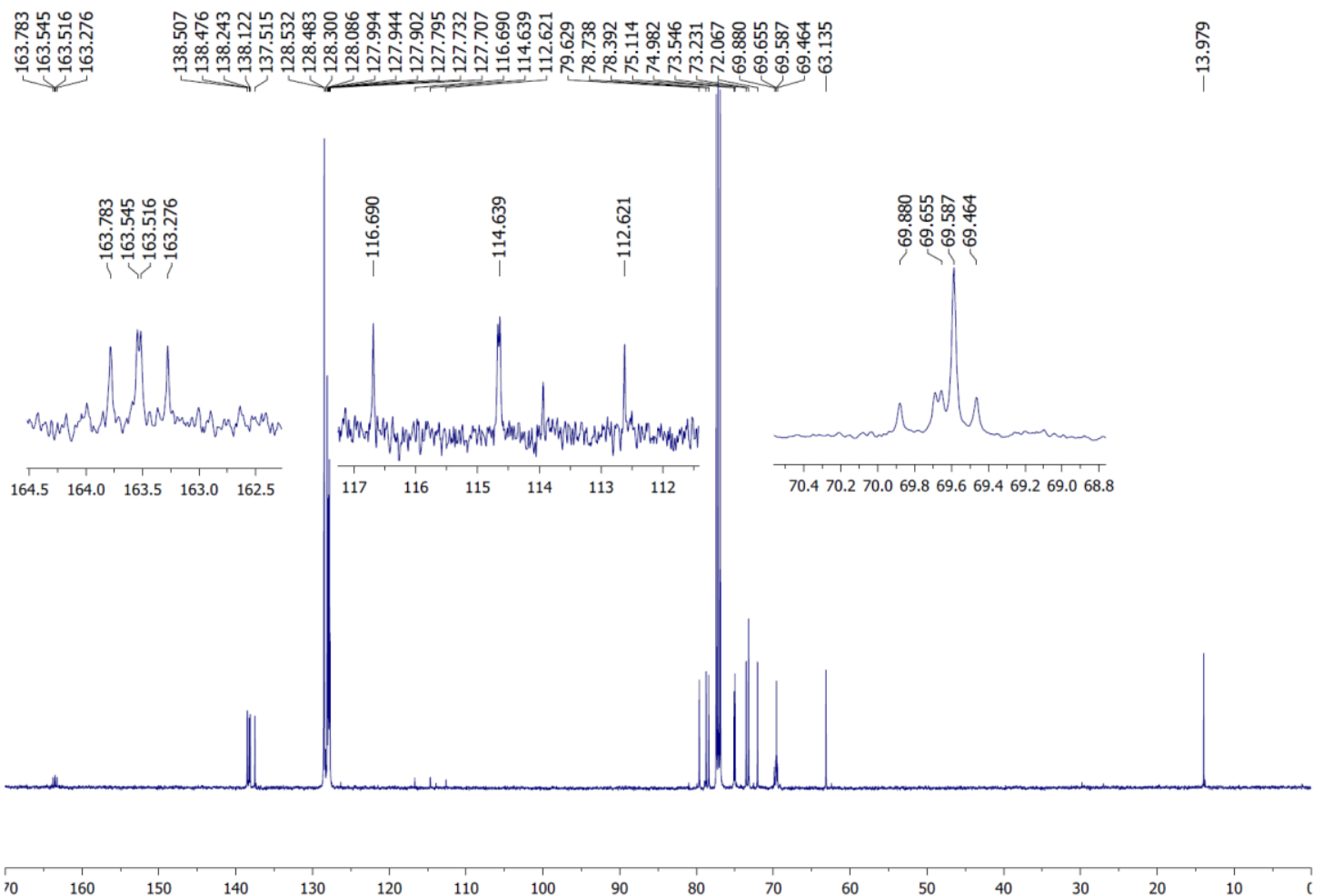


\section{$\mathrm{DBCE}$}

\section{D-Arabinose}

2,3,4,5-tetra-O-benzyl-1',1'-difluoro-1-hydroxy-2-((methylsulfonyl)oxy)heptanoate $((R / S)-4 c)$.

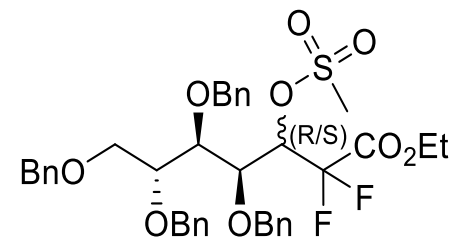

(R/S)-4c

To a solution of alcohol $(R / S)-3 \mathbf{a}(66 \mathrm{mg}, 0.105 \mathrm{mmol}, 93: 7 \mathrm{~S} / \mathrm{R}$ ratio) in dry DCM (2 ml) under argon atmosphere was added $\mathrm{Et}_{3} \mathrm{~N}(23 \mu \mathrm{L}, 0.165 \mathrm{mmol}, 1.5$ equiv.) and $\mathrm{MsCl}$ (13 $\mu \mathrm{l}, 0.165 \mathrm{mmol}, 1.5$ equiv.). The reaction was stirred for $17 \mathrm{~h}$ at r.t. before adding EtOAc $(10 \mathrm{~mL})$ and an aqueous sat. sol. of $\mathrm{NH}_{4} \mathrm{Cl}(10 \mathrm{~mL})$. The aqueous phase was extracted with EtOAc (3 times) and the combined organic phases were washed with brine, dried over $\mathrm{MgSO}_{4}$ and concentrated under reduced pressure. Purification by chromatography on silica gel (Cy/EtOAc 9:1) gave the mesylated product $(R / S)-\mathbf{4 c}$ as a colorless oil (53 $\mathrm{mg}, 0.0744 \mathrm{mmol}, 71 \%$, 93:7 $d r$ ratio).

${ }^{1} \mathrm{H}$ NMR $\left(500 \mathrm{MHz}, \mathrm{CDCl}_{3}\right) \delta=7.34-7.26\left(\mathrm{~m}, 16 \mathrm{H}, \mathrm{H}^{\mathrm{arom}}\right), 7.25-7.20\left(\mathrm{~m}, 4 \mathrm{H}, \mathrm{H}^{\mathrm{arom}}\right)$, $5.62\left(\mathrm{dt}, 1 \mathrm{H}, J_{1-\mathrm{F}}=16.2, J_{1-2}=7.0 \mathrm{~Hz}, \mathrm{H}-1\right), 5.62^{*}\left(\mathrm{ddd}, 1 \mathrm{H}, J_{1-\mathrm{Fa}}=11.0, J_{1-\mathrm{Fb}}=8.8, J_{1-2}\right.$ $=4.5 \mathrm{~Hz}, \mathrm{H}-1), 4.78\left(\mathrm{AB}, 1 \mathrm{H}, J_{\mathrm{A}-\mathrm{B}}=11.0 \mathrm{~Hz}, \mathrm{CH}_{2}{ }^{\mathrm{Bn}}\right), 4.72\left(\mathrm{AB}, 1 \mathrm{H}, J_{\mathrm{A}-\mathrm{B}}=11.7 \mathrm{~Hz}\right.$, $\left.\mathrm{CH}_{2}{ }^{\mathrm{Bn}}\right), 4.65\left(\mathrm{AB}, 1 \mathrm{H}, J_{\mathrm{A}-\mathrm{B}}=11.0 \mathrm{~Hz}, \mathrm{CH}_{2}{ }^{\mathrm{Bn}}\right), 4.56-4.51\left(\mathrm{~m}, 1 \mathrm{H}, \mathrm{CH}_{2}{ }^{\mathrm{Bn}}\right), 4.52(\mathrm{~s}, 2 \mathrm{H}$, $\left.\mathrm{CH}_{2}{ }^{\mathrm{Bn}}\right), 4.45\left(\mathrm{AB}, 1 \mathrm{H}, J_{\mathrm{A}-\mathrm{B}}=11.3 \mathrm{~Hz}, \mathrm{CH}_{2}{ }^{\mathrm{Bn}}\right), 4.43\left(\mathrm{AB}, 1 \mathrm{H}, J_{\mathrm{A}-\mathrm{B}}=11.9 \mathrm{~Hz}, \mathrm{CH}_{2}{ }^{\mathrm{Bn}}\right)$, $4.15\left(\mathrm{ddd}, 1 \mathrm{H}, J_{1-2}=6.6, J_{2-3}=2.1,1.0 \mathrm{~Hz}, \mathrm{H}-2\right), 4.06\left(\mathrm{dd}, 1 \mathrm{H}, J_{3-4}=7.5, J_{4-5}=2.3 \mathrm{~Hz}\right.$, $\mathrm{H}-4), 4.04-3.90\left(\mathrm{~m}, 2 \mathrm{H}, \mathrm{CH}_{2} \mathrm{CH}_{3}\right), 3.88\left(\mathrm{dd}, 1 \mathrm{H}, J_{5 \mathrm{a}-5 \mathrm{~b}}=10.6, J_{4-5}=2.8 \mathrm{~Hz}, \mathrm{H}-5\right), 3.84$ $\left(\mathrm{dt}, 1 \mathrm{H}, J_{3-4}=7.1, J_{2-3}=3.3 \mathrm{~Hz}, \mathrm{H}-3\right), 3.71\left(\mathrm{dd}, 1 \mathrm{H}, J_{5 \mathrm{a}-5 \mathrm{~b}}=10.5, J_{4-5}=3.9 \mathrm{~Hz}, \mathrm{H}-5\right), 2.98$ (s, $\left.3 \mathrm{H}, \mathrm{SO}_{2} \mathrm{CH}_{3}\right), 2.97 *\left(\mathrm{~s}, 3 \mathrm{H}, \mathrm{SO}_{2} \mathrm{CH}_{3}\right), 1.23^{*}\left(\mathrm{t}, 3 \mathrm{H}, J_{\mathrm{CH} 2 \mathrm{CH} 3}=7.2 \mathrm{~Hz}, \mathrm{CH}_{2} \mathrm{CH}_{3}\right), 1.08$ $\left(\mathrm{t}, 3 \mathrm{H}, J_{\mathrm{CH} 2 \mathrm{CH} 3}=7.2 \mathrm{~Hz}, \mathrm{CH}_{2} \mathrm{CH}_{3}\right) \mathrm{ppm} .{ }^{13} \mathrm{C} \mathrm{NMR}\left(125 \mathrm{MHz}, \mathrm{CDCl}_{3}\right) \delta=161.4$ (dd, JC$\left.\mathrm{F}=29.8 \mathrm{~Hz}, \mathrm{JC}-\mathrm{F}=32.0 \mathrm{~Hz}, \mathrm{C}_{\mathrm{q}} \mathrm{O}_{2} \mathrm{Et}\right), 138.4\left(\mathrm{C}_{\mathrm{q}}{ }^{\text {arom }}\right), 138.3\left(\mathrm{C}_{\mathrm{q}}{ }^{\text {arom }}\right), 138.1\left(\mathrm{C}_{\mathrm{q}}^{\text {arom }}\right)$, $137.3\left(\mathrm{C}_{\mathrm{q}}{ }^{\text {arom }}\right), 128.5-127.7\left(\mathrm{CH}^{\mathrm{arom}}\right), 112.8\left(\mathrm{dd}, J_{\mathrm{C}-\mathrm{F}}=253.2 \mathrm{~Hz}, J_{\mathrm{C}-\mathrm{F}}=257.4 \mathrm{~Hz}, \mathrm{CF}_{2}\right)$, $77.8(\mathrm{C}-3), 77.4\left(\mathrm{dd}, J_{\mathrm{C}-\mathrm{F}}=22.6 \mathrm{~Hz}, J_{\mathrm{C}-\mathrm{F}}=25.0 \mathrm{~Hz}, \mathrm{C}-1\right), 76.8(\mathrm{C}-4), 76.0(\mathrm{C}-2), 74.1$ $\left(\mathrm{CH}_{2}{ }^{\mathrm{Bn}}\right), 73.9\left(\mathrm{CH}_{2}{ }^{\mathrm{Bn}}\right), 73.6^{*}\left(\mathrm{CH}_{2}{ }^{\mathrm{Bn}}\right), 73.5\left(\mathrm{CH}_{2}{ }^{\mathrm{Bn}}\right), 72.0^{*}\left(\mathrm{CH}_{2}{ }^{\mathrm{Bn}}\right), 71.5\left(\mathrm{CH}_{2}{ }^{\mathrm{Bn}}\right), 68.7^{*}$ (C-5), $68.3(\mathrm{C}-5), 63.7 *\left(\mathrm{CH}_{2} \mathrm{CH}_{3}\right), 63.5\left(\mathrm{CH}_{2} \mathrm{CH}_{3}\right), 39.3\left(\mathrm{SO}_{2} \mathrm{CH}_{3}\right), 13.9 *\left(\mathrm{CH}_{2} \mathrm{CH}_{3}\right)$, $13.7\left(\mathrm{CH}_{2} \mathrm{CH}_{3}\right)$ ppm. ${ }^{19} \mathrm{~F}$ NMR $\left(471 \mathrm{MHz}, \mathrm{CDCl}_{3}\right) \delta=-109.8\left(\mathrm{dd}, 1 \mathrm{~F}, J_{\mathrm{H} 1-\mathrm{Fa}}=7.1 \mathrm{~Hz}\right.$, $\left.J_{\mathrm{Fa}-\mathrm{Fb}}=268.7 \mathrm{~Hz}, \mathrm{Fa}\right),-110.6^{*}\left(\mathrm{dd}, 1 \mathrm{~F}, J_{\mathrm{H} 1-\mathrm{Fa}}=8.3 \mathrm{~Hz}, J_{\mathrm{Fa}-\mathrm{Fb}}=269.3 \mathrm{~Hz}, \mathrm{Fa}\right),-114.6^{*}$ $\left(\mathrm{dd}, 1 \mathrm{~F}, J_{\mathrm{H}-\mathrm{Fb}}=11.0 \mathrm{~Hz}, J_{\mathrm{Fa}-\mathrm{Fb}}=268.6 \mathrm{~Hz}, \mathrm{Fb}\right),-118.7\left(\mathrm{dd}, 1 \mathrm{~F}, J_{\mathrm{H}-\mathrm{Fb}}=15.4 \mathrm{~Hz}, J_{\mathrm{Fa}-\mathrm{Fb}}=\right.$ $269.3 \mathrm{~Hz}, \mathrm{Fb}$ ) ppm. HRMS (ESI+): $\mathrm{m} / \mathrm{z}$ calculated for $\mathrm{C}_{38} \mathrm{H}_{43} \mathrm{~F}_{2} \mathrm{O}_{9} \mathrm{~S}[\mathrm{M}+\mathrm{H}]^{+}$: calc. 713.2590; found: 713.2584 . 


\section{${ }^{1} \mathbf{H}$ NMR}

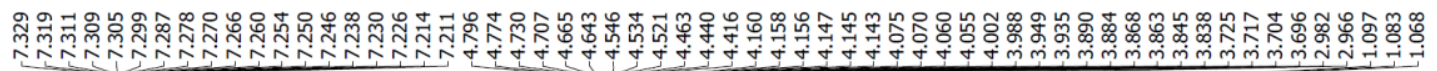

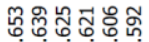

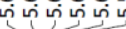

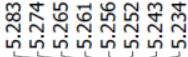

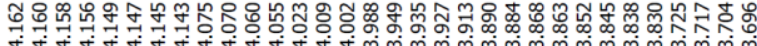

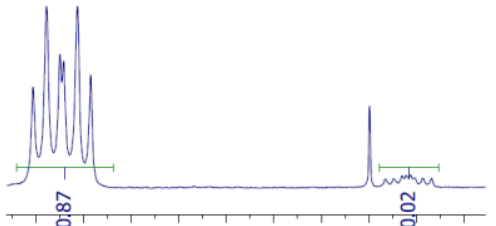

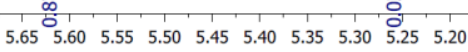
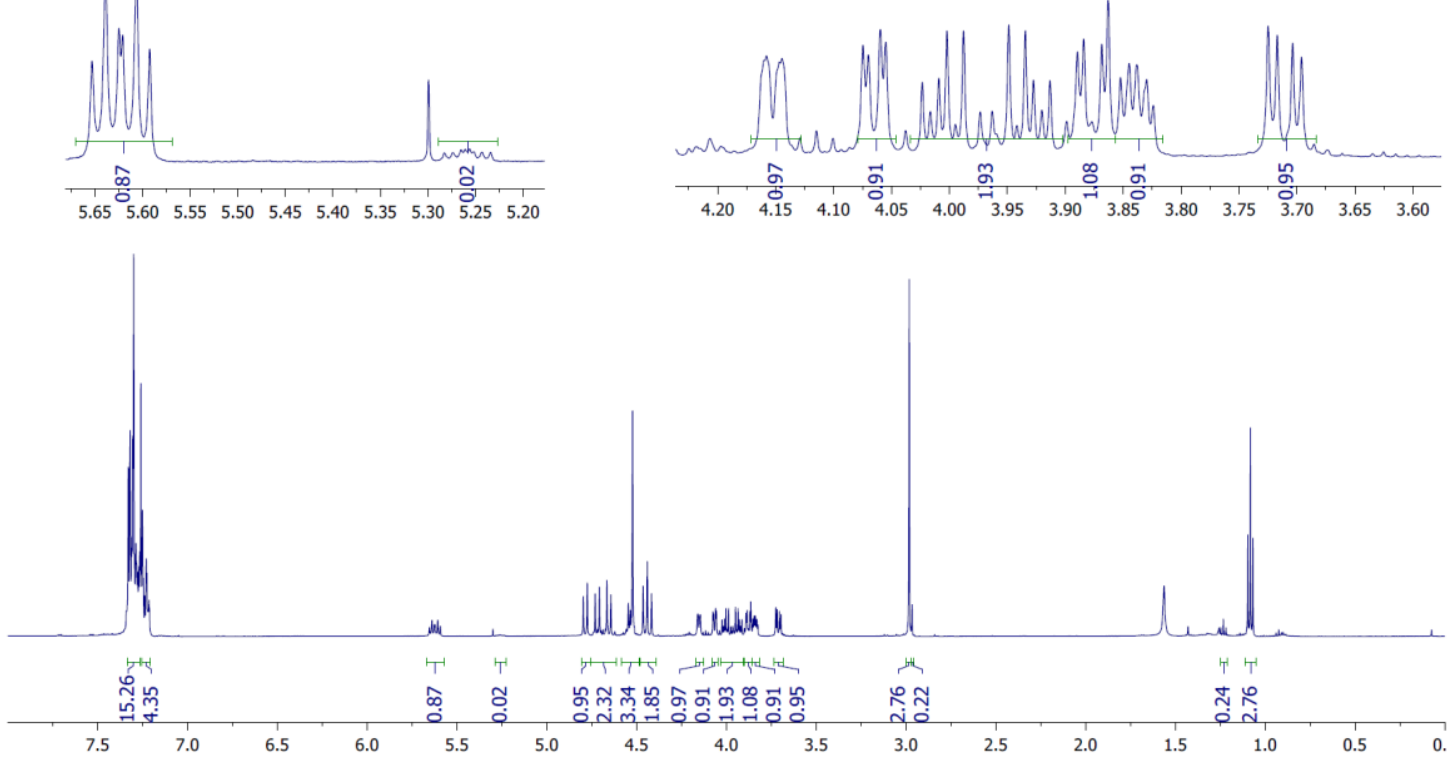

${ }^{19}$ F NMR

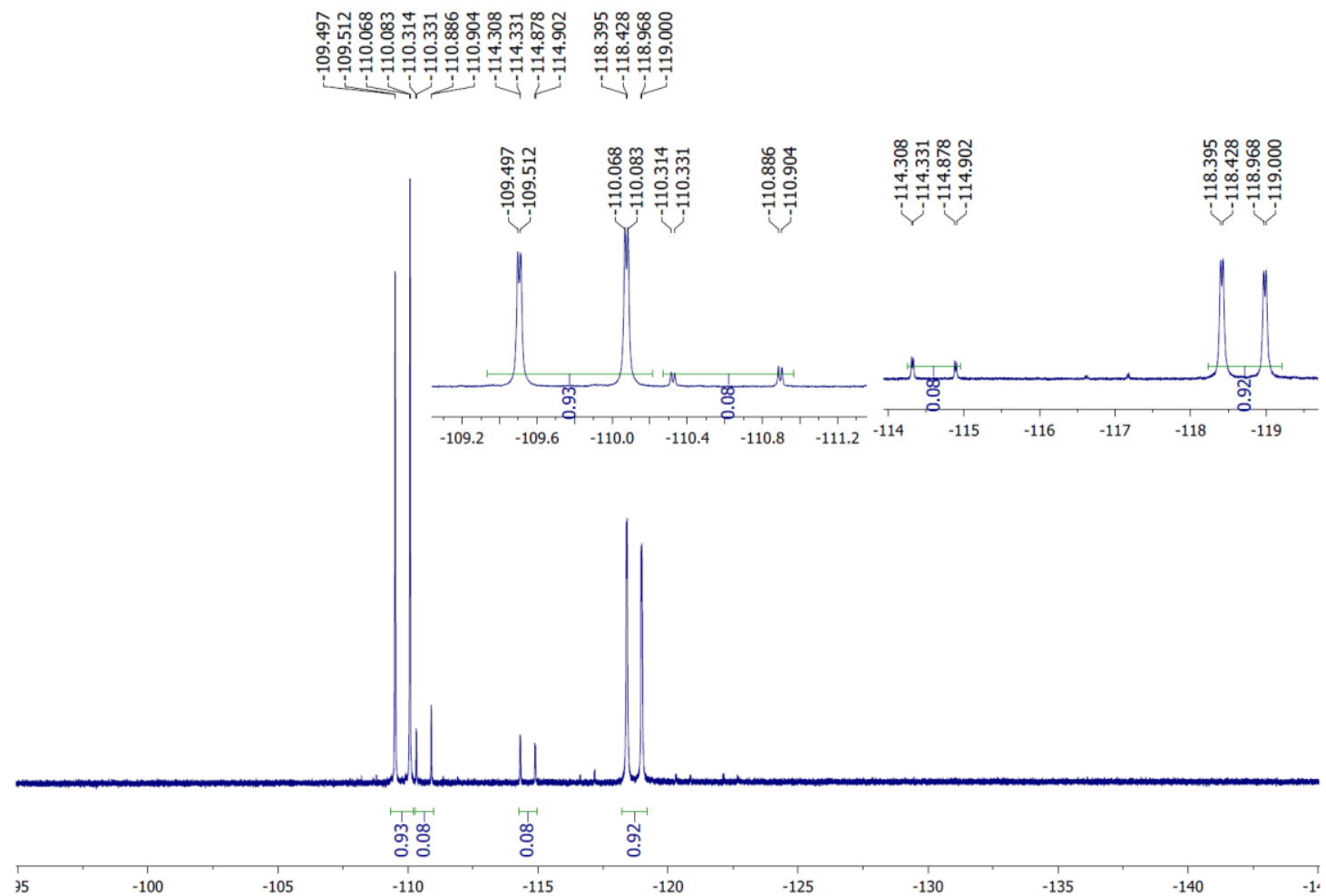


${ }^{13}$ C NMR

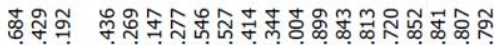

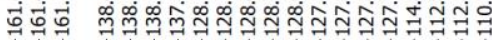

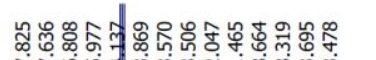

Fही

商

นूم

尚

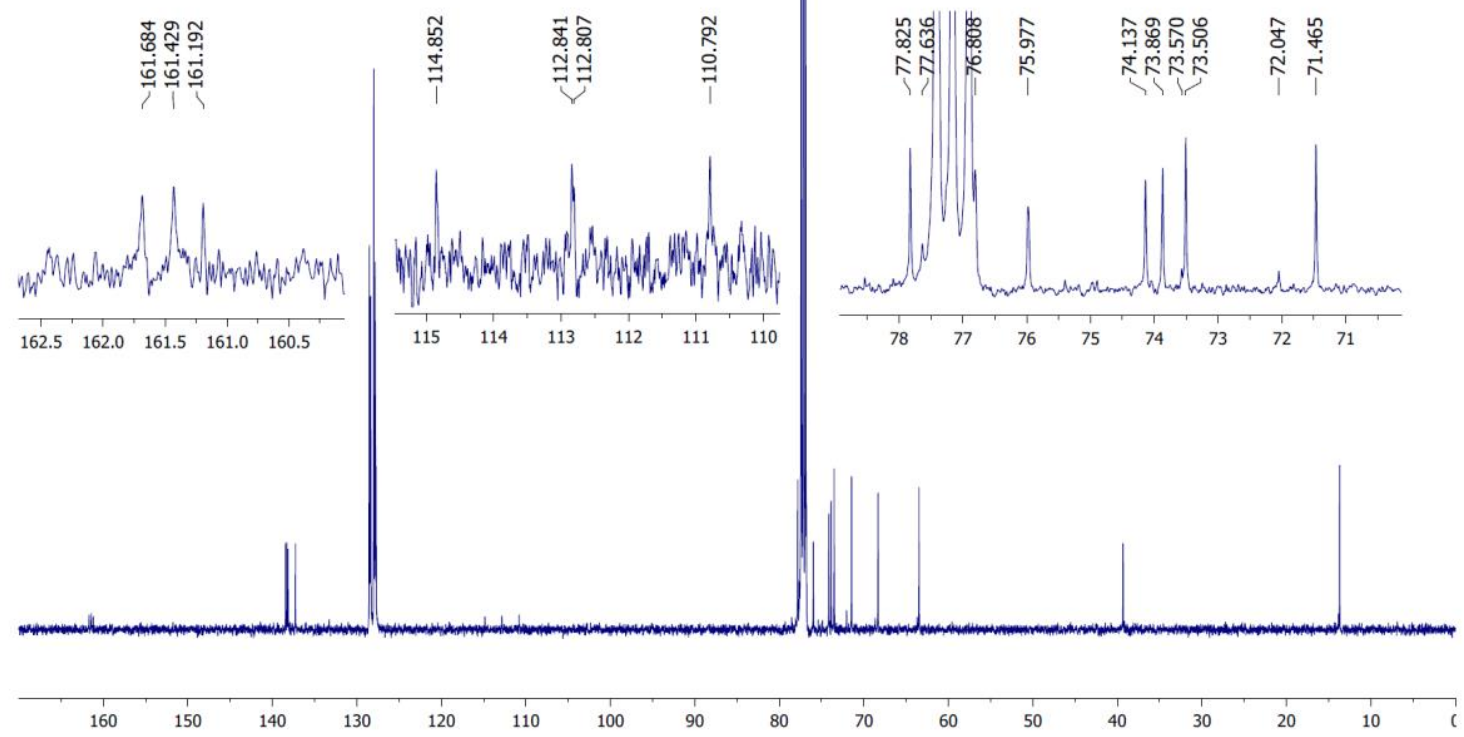


2,3,5-tri-O-benzyl-1-deoxy-1,1-difluoroethyl acetate- $\alpha$-D-arabinofuranose $(\alpha \beta)$-5a).<smiles></smiles>

To a solution of alcohols $(R / S)-3 \mathbf{a}(67 \mathrm{mg}, 0.12 \mathrm{mmol}, 89: 11 \mathrm{R}: \mathrm{S}$ ratio) in dry DCM $(1.5$ $\mathrm{ml})$ under argon atmosphere was added DAST (28 $\mu \mathrm{l}, 0.24 \mathrm{mmol}, 2.0$ equiv.) dropwise at $-78^{\circ} \mathrm{C}$. The reaction was allowed to warm from $-78^{\circ} \mathrm{C}$ to r.t for $2.5 \mathrm{~h}$ and stirred for $24 \mathrm{~h}$ at r.t. Then, the crude was diluted with dichloromethane. The organic phase was washed with $\mathrm{H}_{2} \mathrm{O}$ ( 3 times) and brine ( 3 times), dried over $\mathrm{MgSO}_{4}$ and concentrated under reduced pressure. Purification by chromatography on silica gel (Cy/EtOAc 9:1) gave the cyclized products $(\alpha)$-5a and ( $\beta$ )-5a as a colorless oil (44 mg, 74\%, 89:11 $\alpha: \beta$ ratio).

${ }^{1} \mathrm{H}$ NMR $\left(500 \mathrm{MHz}, \mathrm{CDCl}_{3}\right) \delta=7.35-7.26$ (m, 13H, $\left.\mathrm{H}^{\text {arom }}\right)$, 7.26-7.20 (m, $\left.2 \mathrm{H}, \mathrm{H}^{\text {arom }}\right)$, $4.68\left(\mathrm{ddd}, 1 \mathrm{H}, J_{1-2}=5.7 \mathrm{~Hz}, J_{1-\mathrm{Fa}}=10.8 \mathrm{~Hz}, J_{1-\mathrm{Fb}}=13.8 \mathrm{~Hz}, \mathrm{H}-1\right), 4.58\left(\mathrm{AB}, 1 \mathrm{H}, J_{\mathrm{A}-\mathrm{B}}=\right.$ $\left.12.6 \mathrm{~Hz}, \mathrm{CH}_{2}{ }^{\mathrm{Bn}}\right), 4.55\left(\mathrm{AB}, 1 \mathrm{H}, J_{\mathrm{A}-\mathrm{B}}=12.0 \mathrm{~Hz}, \mathrm{CH}_{2}{ }^{\mathrm{Bn}}\right), 4.51\left(\mathrm{AB}, 1 \mathrm{H}, J_{\mathrm{A}-\mathrm{B}}=12.6 \mathrm{~Hz}\right.$, $\left.\mathrm{CH}_{2}{ }^{\mathrm{Bn}}\right), 4.49\left(\mathrm{AB}, 1 \mathrm{H}, J_{\mathrm{A}-\mathrm{B}}=12.0 \mathrm{~Hz}, \mathrm{CH}_{2}{ }^{\mathrm{Bn}}\right), 4.47\left(\mathrm{AB}, 1 \mathrm{H}, J_{\mathrm{A}-\mathrm{B}}=12.0 \mathrm{~Hz}, \mathrm{CH}_{2}{ }^{\mathrm{Bn}}\right)$, $4.43\left(\mathrm{AB}, 1 \mathrm{H}, J_{\mathrm{A}-\mathrm{B}}=12.0 \mathrm{~Hz}, \mathrm{CH}_{2}{ }^{\mathrm{Bn}}\right), 4.28\left(\mathrm{dd}, 1 \mathrm{H}, J_{1-2}=5.7 \mathrm{~Hz}, J_{2-3}=3.4 \mathrm{~Hz}, \mathrm{H}-2\right)$, $4.24\left(\mathrm{td}, 1 \mathrm{H}, J_{3-4}=4.0 \mathrm{~Hz}, J_{4-5 \mathrm{a}}=J_{4-5 \mathrm{~b}}=6.3 \mathrm{~Hz}, \mathrm{H}-4\right), 4.12-3.99\left(\mathrm{~m}, 3 \mathrm{H}, \mathrm{H}-3, C_{2} \mathrm{CH}_{3}\right)$, $3.68\left(\mathrm{ABX}, 1 \mathrm{H}, J_{4-5 \mathrm{a}}=6.3 \mathrm{~Hz}, J_{5 \mathrm{a}-5 \mathrm{~b}}=10.3 \mathrm{~Hz}, \mathrm{H}-5 \mathrm{a}\right), 3.59\left(\mathrm{ABX}, 1 \mathrm{H}, J_{4-5 \mathrm{~b}}=6.3 \mathrm{~Hz}, J_{5 \mathrm{a}-}\right.$ $5 \mathrm{~b}=10.3 \mathrm{~Hz}, \mathrm{H}-5 \mathrm{~b}), 1.16$ (t, $\left.3 \mathrm{H}, J_{\mathrm{CH} 2-\mathrm{CH} 3}=7.5 \mathrm{~Hz}, \mathrm{CH}_{2} \mathrm{CH}_{3}\right) .{ }^{13} \mathrm{C} \mathrm{NMR}(125 \mathrm{MHz}$, $\left.\mathrm{CDCl}_{3}\right) \delta=163.0\left(\mathrm{t}, J_{\mathrm{C}-\mathrm{F}}=30.7 \mathrm{~Hz}, \mathrm{C}_{\mathrm{q}} \mathrm{O}_{2} \mathrm{Et}\right), 138.2\left(\mathrm{C}_{\mathrm{q}}{ }^{\text {arom }}\right), 137.6\left(\mathrm{C}_{\mathrm{q}}{ }^{\text {arom }}\right), 137.0\left(\mathrm{C}_{\mathrm{q}}{ }^{\text {arom }}\right)$, 128.7-126.7 $\left(\mathrm{CH}^{\text {arom }}\right), 113.1\left(\mathrm{dd}, J_{\mathrm{C}-\mathrm{F}}=249.9 \mathrm{~Hz}, J_{\mathrm{C}-\mathrm{F}}=258.3 \mathrm{~Hz}, \mathrm{CF}_{2}\right), 82.8(\mathrm{C}-2, \mathrm{C}-3$, C-4), $79.1\left(\mathrm{dd}, J_{\mathrm{C}-\mathrm{F}}=23.5 \mathrm{~Hz}, J_{\mathrm{C}-\mathrm{F}}=26.6 \mathrm{~Hz}, \mathrm{C}-1\right), 73.5\left(\mathrm{CH}_{2}{ }^{\mathrm{Bn}}\right), 72.8\left(\mathrm{CH}_{2}{ }^{\mathrm{Bn}}\right), 72.0$ $\left(\mathrm{CH}_{2}{ }^{\mathrm{Bn}}\right), 70.1(\mathrm{C}-5), 62.7\left(\mathrm{CH}_{2} \mathrm{CH}_{3}\right), 13.9\left(\mathrm{CH}_{2} \mathrm{CH}_{3}\right) .{ }^{19} \mathrm{~F}$ NMR $\left(471 \mathrm{MHz}, \mathrm{CDCl}_{3}\right) \delta=-$ $110.4\left(\mathrm{dd}, 1 \mathrm{~F}, J_{\mathrm{H} 1-\mathrm{Fa}}=10.8 \mathrm{~Hz}, J_{\mathrm{Fa}-\mathrm{Fb}}=274.4 \mathrm{~Hz}, \mathrm{Fa}\right),-114.5\left(\mathrm{dd}, 1 \mathrm{~F}, J_{\mathrm{H} 1-\mathrm{Fb}}=13.8 \mathrm{~Hz}\right.$, $\left.J_{\mathrm{Fa}-\mathrm{Fb}}=274.4 \mathrm{~Hz}, \mathrm{Fb}\right)$. HRMS (ESI+): $\mathrm{m} / \mathrm{z}$ calculated for $\mathrm{C}_{30} \mathrm{H}_{32} \mathrm{~F}_{2} \mathrm{NaO}_{6}[\mathrm{M}+\mathrm{Na}]^{+}$: calc. 549.2059; found: 549.2063. nOe: Correlation between $\mathrm{H}-1$ and $\mathrm{H}-4$ observed. 


\section{${ }^{1}$ H NMR}

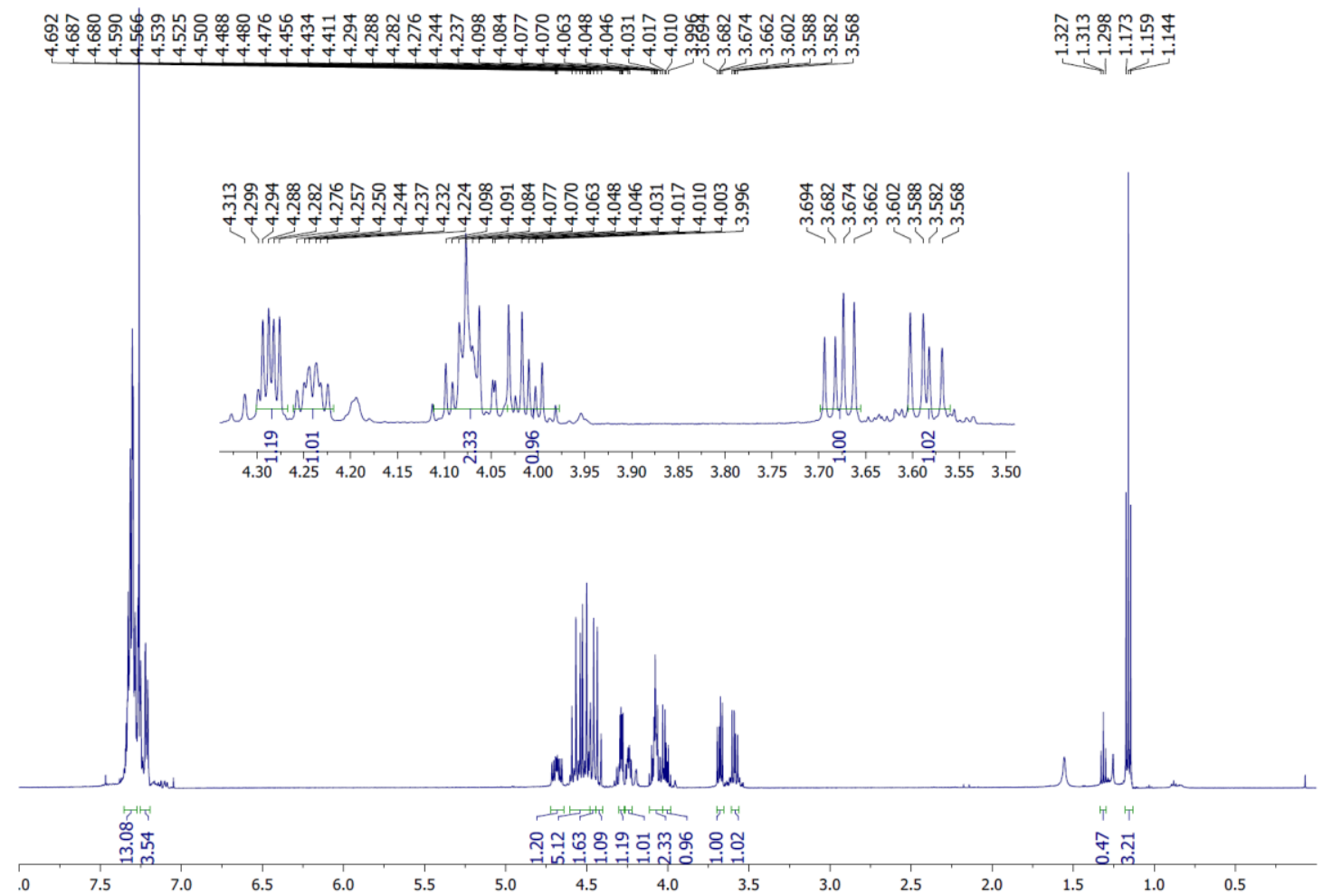

${ }^{19}$ F NMR

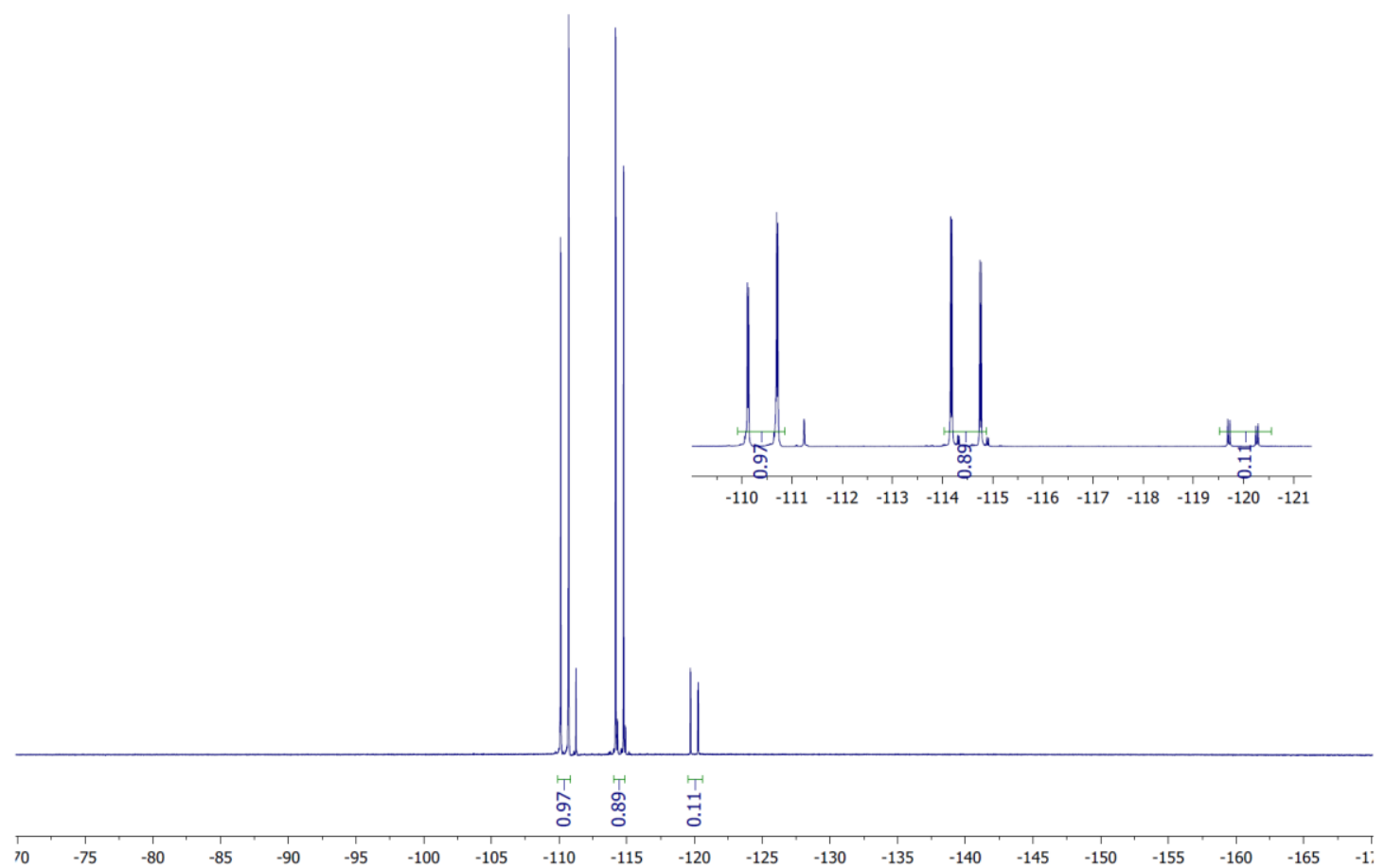


${ }^{13}$ C NMR
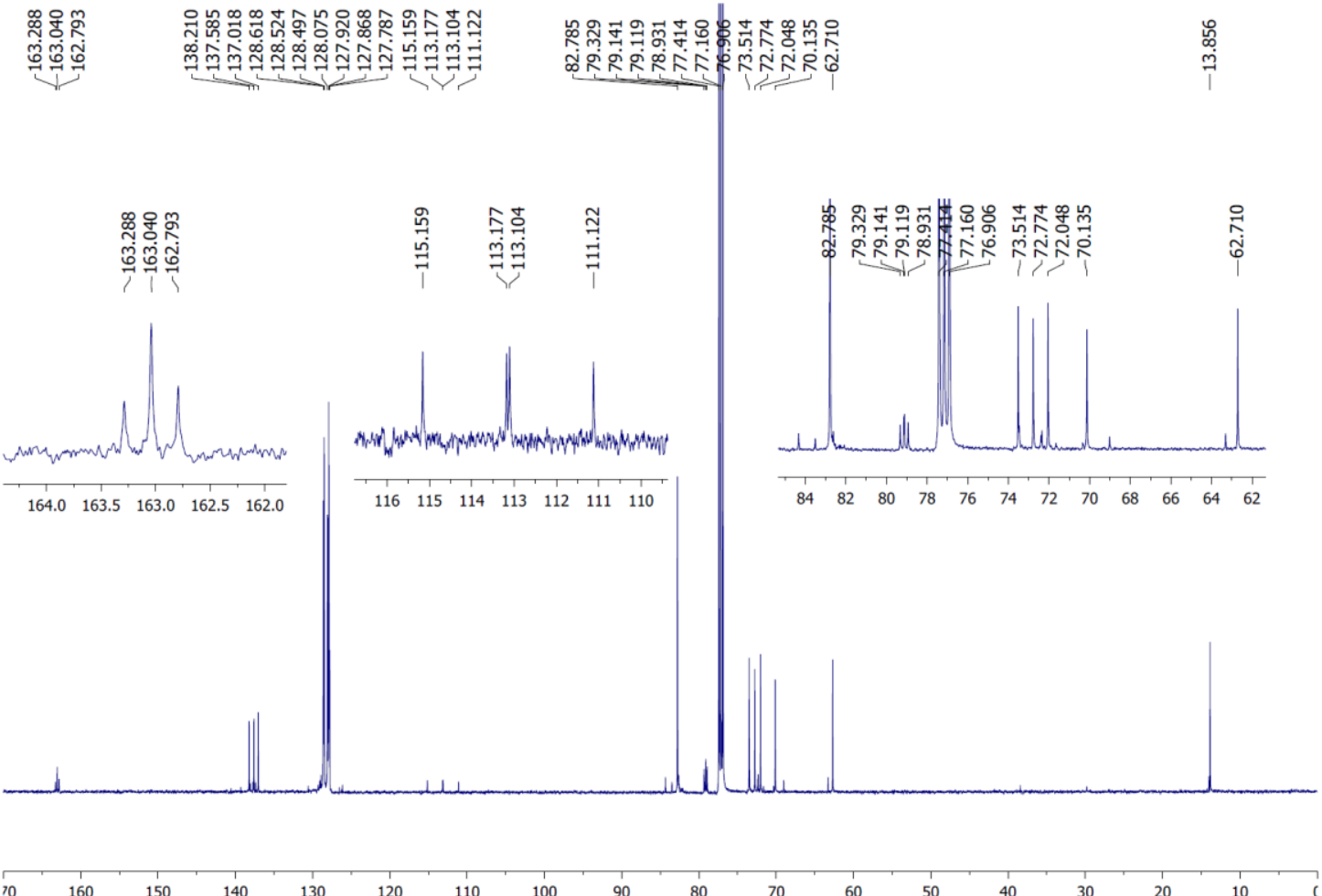
2,3,5-tri-O-benzyl-1-deoxy-1,1-difluoroethyl acetate- $\alpha$-D-arabinofuranose (( $\alpha)$-5a).<smiles>[B]OC[C@H]1O[C@H](C(F)(F)OCC)[C@H](O[Mg]Br)[C@@H]1Br</smiles>

To a solution of alcohol $(R)-3 \mathbf{a}(63 \mathrm{mg}, 0.11 \mathrm{mmol})$ in dry DCM $(1.5 \mathrm{ml})$ under argon atmosphere was added DAST ( $26 \mu 1,0.22 \mathrm{mmol}, 2.0$ equiv.) dropwise at $-78^{\circ} \mathrm{C}$. The reaction was allowed to warm from $-78^{\circ} \mathrm{C}$ to r.t for $2.5 \mathrm{~h}$ and stirred for $24 \mathrm{~h}$ at r.t. Then, the crude was diluted with dichloromethane. The organic phase was washed with $\mathrm{H}_{2} \mathrm{O}$ (3 times) and brine (3 times), dried over $\mathrm{MgSO}_{4}$ and concentrated under reduced pressure. Purification by chromatography on silica gel (Cy/EtOAc 9:1) gave the cyclized product $(\alpha)$-5a as a colorless oil (35 $\mathrm{mg}, 62 \%)$.

$[\alpha]_{20}$ D: $-0.3\left(\mathrm{c} 1.0, \mathrm{CHCl}_{3}\right) .{ }^{1} \mathrm{H}$ NMR $\left(500 \mathrm{MHz}, \mathrm{CDCl}_{3}\right) \delta=7.37-7.23\left(\mathrm{~m}, 15 \mathrm{H}, \mathrm{H}^{\text {arom }}\right)$, $4.59\left(\mathrm{AB}, 1 \mathrm{H}, J_{\mathrm{A}-\mathrm{B}}=11.7 \mathrm{~Hz}, \mathrm{CH}_{2}{ }^{\mathrm{Bn}}\right), 4.56\left(\mathrm{AB}, 1 \mathrm{H}, J_{\mathrm{A}-\mathrm{B}}=12.2 \mathrm{~Hz}, \mathrm{CH}_{2}{ }^{\mathrm{Bn}}\right), 4.54(\mathrm{AB}$, $\left.1 \mathrm{H}, J_{\mathrm{A}-\mathrm{B}}=12.2 \mathrm{~Hz}, \mathrm{CH}_{2}{ }^{\mathrm{Bn}}\right), 4.51\left(\mathrm{AB}, 1 \mathrm{H}, J_{\mathrm{A}-\mathrm{B}}=11.8 \mathrm{~Hz}, \mathrm{CH}_{2}{ }^{\mathrm{Bn}}\right), 4.50\left(\mathrm{AB}, 1 \mathrm{H}, J_{\mathrm{A}-\mathrm{B}}=\right.$ $\left.11.8 \mathrm{~Hz}, \mathrm{CH}_{2}{ }^{\mathrm{Bn}}\right), 4.48\left(\mathrm{AB}, 1 \mathrm{H}, J_{\mathrm{A}-\mathrm{B}}=11.7 \mathrm{~Hz}, \mathrm{CH}_{2}{ }^{\mathrm{Bn}}\right), 4.50-4.44(\mathrm{~m}, 2 \mathrm{H}, \mathrm{H}-1, \mathrm{H}-2)$, $4.31\left(\mathrm{q}, 2 \mathrm{H}, J_{\mathrm{CH} 2-\mathrm{CH} 3}=7.1 \mathrm{~Hz}, \mathrm{CH}_{2} \mathrm{CH}_{3}\right), 4.21-4.18(\mathrm{~m}, 2 \mathrm{H}, \mathrm{H}-3, \mathrm{H}-4), 3.63(\mathrm{ABX}, 1 \mathrm{H}$, $\left.J_{4-5 \mathrm{a}}=3.8 \mathrm{~Hz}, J_{5 \mathrm{a}-5 \mathrm{~b}}=10.8 \mathrm{~Hz}, \mathrm{H}-5 \mathrm{a}\right), 3.55\left(\mathrm{ABX}, 1 \mathrm{H}, J_{4-5 \mathrm{~b}}=4.2 \mathrm{~Hz}, J_{5 \mathrm{a}-5 \mathrm{~b}}=10.8 \mathrm{~Hz}, \mathrm{H}-\right.$ $5 b), 1.31\left(\mathrm{t}, 3 \mathrm{H}, J_{\mathrm{CH} 2-\mathrm{CH} 3}=7.1 \mathrm{~Hz}, \mathrm{CH}_{2} \mathrm{CH}_{3}\right) .{ }^{13} \mathrm{C} \mathrm{NMR}\left(125 \mathrm{MHz}, \mathrm{CDCl}_{3}\right) \delta=163.0(\mathrm{dd}$, $\left.J_{\mathrm{C}-\mathrm{F}}=30.2 \mathrm{~Hz}, J_{\mathrm{C}-\mathrm{F}}=32.6 \mathrm{~Hz}, \mathrm{C}_{\mathrm{q}} \mathrm{O}_{2} \mathrm{Et}\right), 138.1\left(\mathrm{C}_{\mathrm{q}}^{\text {arom }}\right), 137.7\left(\mathrm{C}_{\mathrm{q}}{ }^{\text {arom }}\right), 137.4\left(\mathrm{C}_{\mathrm{q}}^{\text {arom }}\right)$, 128.7-127.7 $\left(\mathrm{CH}^{\text {arom }}\right), 113.8\left(\mathrm{dd}, J_{\mathrm{C}-\mathrm{F}}=252.3 \mathrm{~Hz}, J_{\mathrm{C}-\mathrm{F}}=259.6 \mathrm{~Hz}, \mathrm{CF}_{2}\right), 84.3(\mathrm{C}-2), 83.5$ $(\mathrm{C}-4), 82.6(\mathrm{C}-3), 82.3\left(\mathrm{dd}, J_{\mathrm{C}-\mathrm{F}}=24.1 \mathrm{~Hz}, J_{\mathrm{C}-\mathrm{F}}=31.4 \mathrm{~Hz}, \mathrm{C}-1\right), 73.5\left(\mathrm{CH}_{2}{ }^{\mathrm{Bn}}\right), 72.4(2$ $\left.\mathrm{CH}_{2}{ }^{\mathrm{Bn}}\right), 69.0(\mathrm{C}-5), 63.3\left(\mathrm{CH}_{2} \mathrm{CH}_{3}\right), 14.0\left(\mathrm{CH}_{2} \mathrm{CH}_{3}\right) .{ }^{19} \mathrm{~F} \mathrm{NMR}\left(471 \mathrm{MHz}, \mathrm{CDCl}_{3}\right) \delta=-$ $111.0\left(\mathrm{dd}, 1 \mathrm{~F}, J_{\mathrm{H}-\mathrm{Fa}}=5.4 \mathrm{~Hz}, J_{\mathrm{Fa}-\mathrm{Fb}}=264.5 \mathrm{~Hz}, \mathrm{Fa}\right),-120.0\left(\mathrm{dd}, 1 \mathrm{~F}, J_{\mathrm{H}-\mathrm{Fb}}=20.7 \mathrm{~Hz}, J_{\mathrm{Fa}-}\right.$ $\mathrm{Fb}=264.5 \mathrm{~Hz}, \mathrm{Fb})$. HRMS (ESI+): $\mathrm{m} / \mathrm{z}$ calculated for $\mathrm{C}_{30} \mathrm{H}_{32} \mathrm{~F}_{2} \mathrm{NaO}_{6}[\mathrm{M}+\mathrm{Na}]^{+}$: calc. 549.2059; found: 549.2059. nOe: Correlation between H-1 and H-3 observed. 


\section{${ }^{1}$ H NMR}

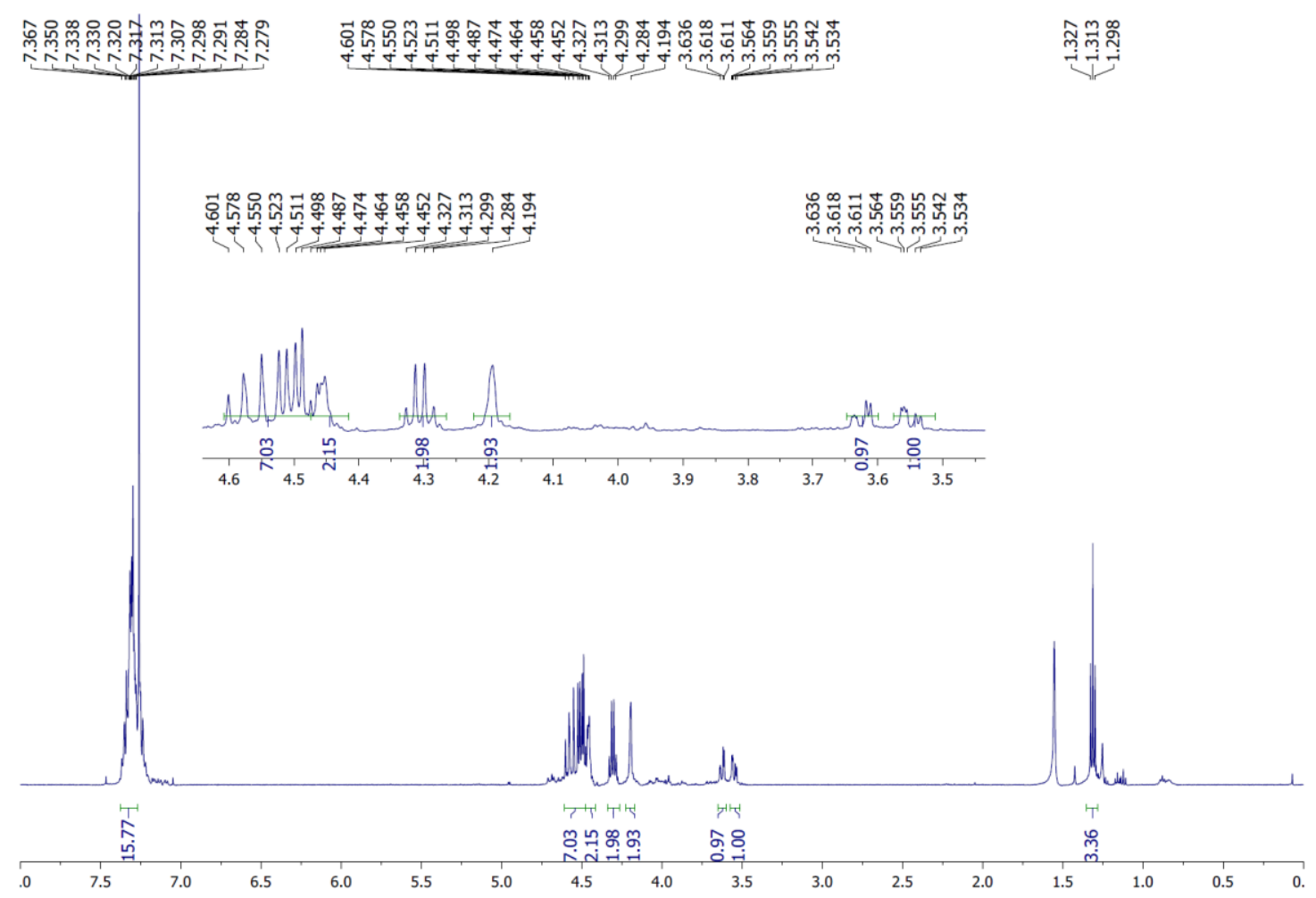

${ }^{19}$ F NMR
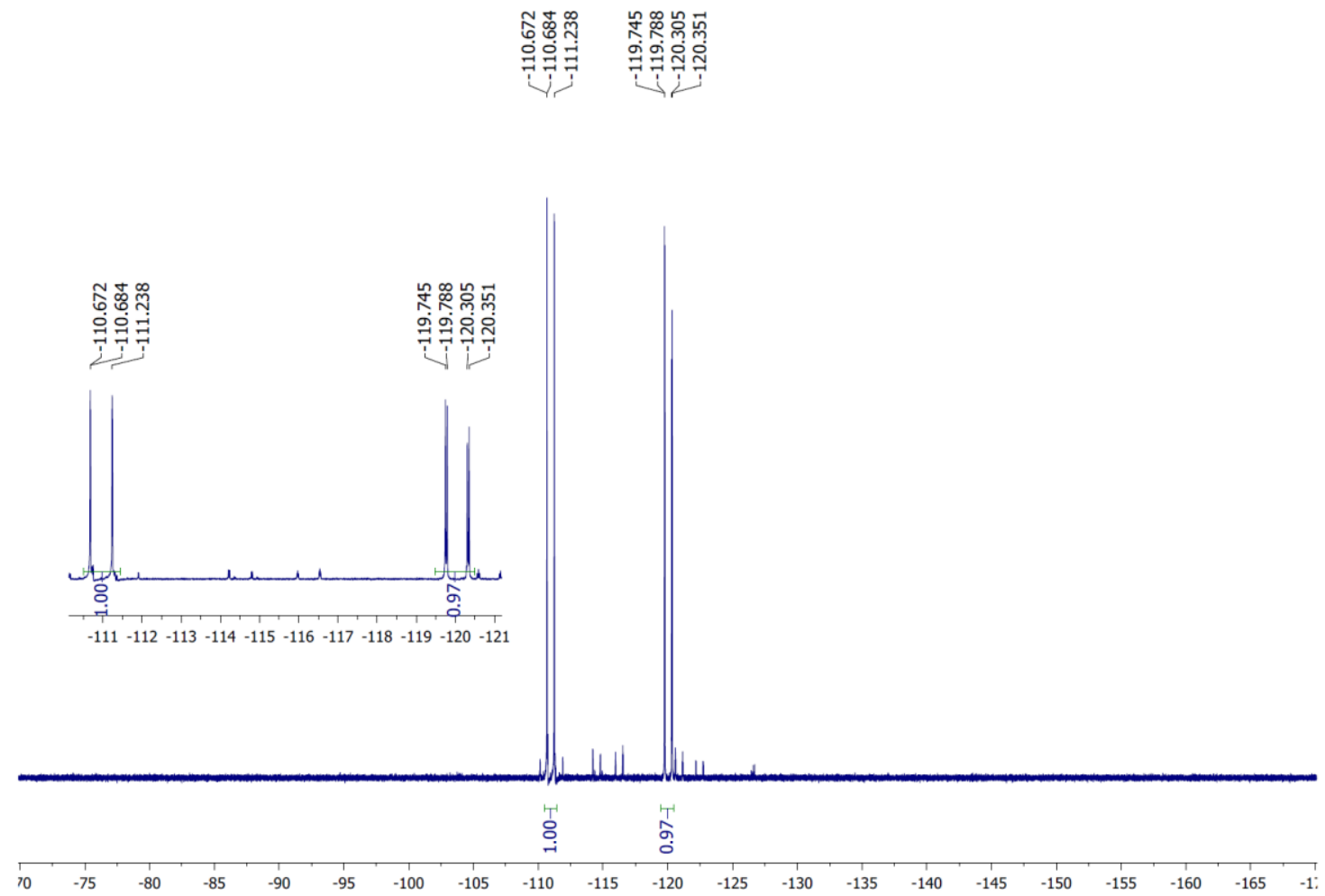
${ }^{13}$ C NMR

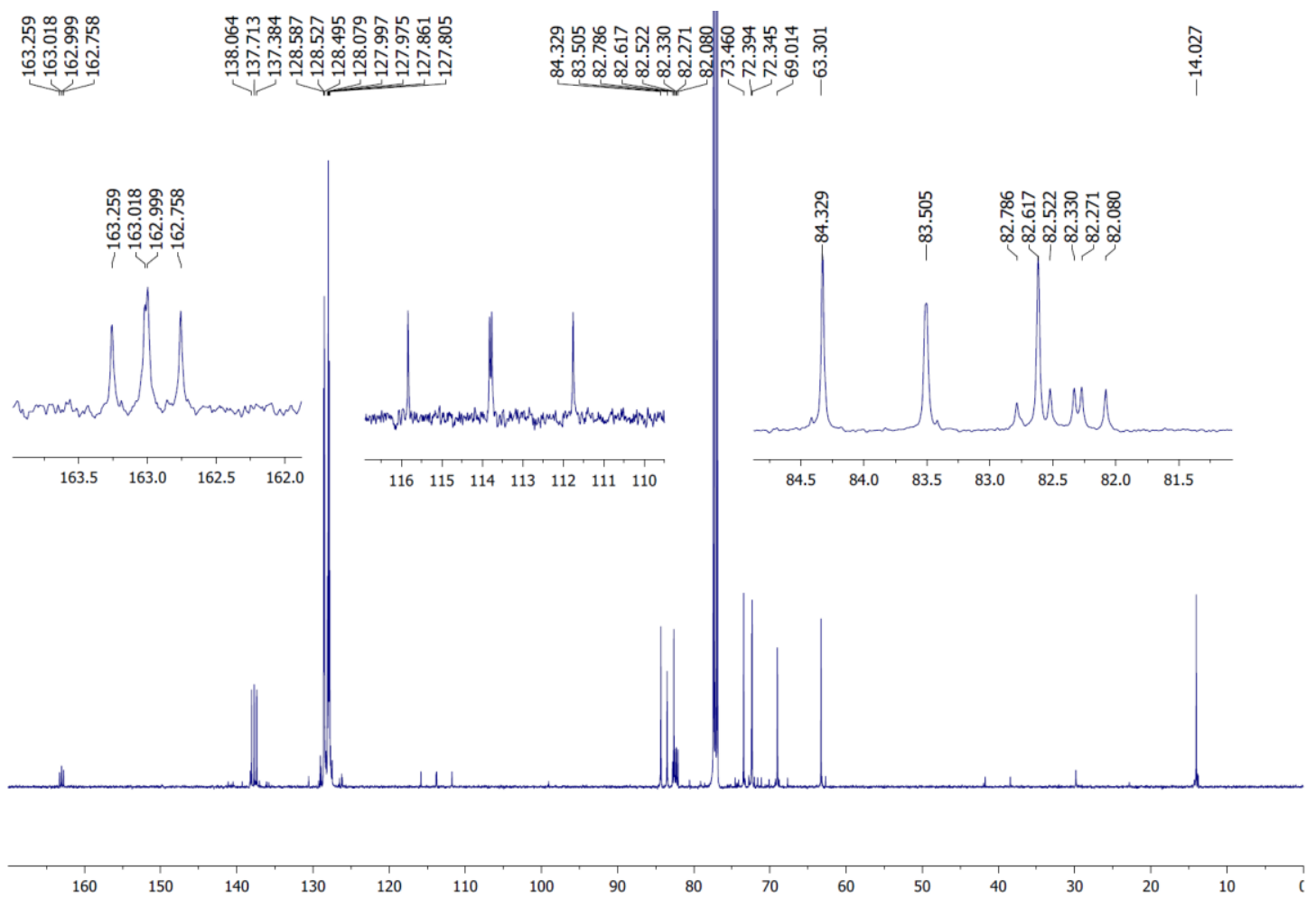




\section{D-Ribose}

2,3,5-tri-O-benzyl-1-deoxy-1,1-difluoroethyl acetate-D-ribofuranose (( $\alpha)$-5b).

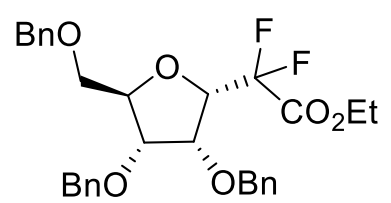

$(\alpha)-5 b$

To a solution of alcohol $(R)-3 \mathbf{b}(75 \mathrm{mg}, 0.118 \mathrm{mmol})$ in dry DCM $(2 \mathrm{ml})$ under argon atmosphere was added DAST ( $62 \mu \mathrm{l}, 0.473 \mathrm{mmol}, 4.0$ equiv.) dropwise at $0^{\circ} \mathrm{C}$. The reaction was allowed to warm from $0^{\circ} \mathrm{C}$ to r.t and stirred for $22 \mathrm{~h}$. DAST ( $62 \mu \mathrm{l}, 0.473$ mmol, 4.0 equiv.) was added sequentially at $0^{\circ} \mathrm{C}$ and the reaction was allowed to warm from $0^{\circ} \mathrm{C}$ to r.t and stirred for $23 \mathrm{~h}$. Then, the crude was diluted with dichloromethane. The organic phase was washed with $\mathrm{H}_{2} \mathrm{O}$ (2 times) and brine (1 times), dried over $\mathrm{MgSO}_{4}$ and concentrated under reduced pressure. Purification by chromatography on silica gel (Cy/EtOAc 9:1) gave the cyclized product $(\alpha)-5 \mathbf{b}$ as a colorless oil (4 $\mathrm{mg}, 6 \%)$.

$[\alpha]^{20} \mathrm{D}:+10.6\left(\mathrm{c} 0.5, \mathrm{CHCl}_{3}\right) .{ }^{1} \mathrm{H}$ NMR $\left(500 \mathrm{MHz}, \mathrm{CDCl}_{3}\right) \delta=7.35-7.23\left(\mathrm{~m}, 15 \mathrm{H}, \mathrm{H}^{\text {arom }}\right)$, $4.74\left(\mathrm{~d}, 1 \mathrm{H}, J_{\mathrm{A}-\mathrm{B}}=11.1 \mathrm{~Hz}, \mathrm{CH}_{2}{ }^{\mathrm{Bn}}\right), 4.67-4.59(\mathrm{~m}, 1 \mathrm{H}, \mathrm{H}-1), 4.59\left(\mathrm{dd}, 2 \mathrm{H}, J_{\mathrm{A}-\mathrm{B}}=11.9\right.$, $\left.4.5 \mathrm{~Hz}, \mathrm{H}-1, \mathrm{CH}_{2}{ }^{\mathrm{Bn}}\right), 4.51\left(\mathrm{~d}, 1 \mathrm{H}, J_{\mathrm{A}-\mathrm{B}}=11.9 \mathrm{~Hz}, \mathrm{CH}_{2}{ }^{\mathrm{Bn}}\right), 4.48\left(\mathrm{~d}, 2 \mathrm{H}, J_{\mathrm{A}-\mathrm{B}}=11.5 \mathrm{~Hz}\right.$, $\mathrm{CH}_{2}{ }^{\mathrm{Bn}}$ ), 4.33-4.27 (m, 2H, H-4 and H-3), $4.11\left(\mathrm{dd}, 1 \mathrm{H}, J_{1-2}\right.$ or $J_{2-3}=8.1 \mathrm{~Hz}, J_{1-2}$ or $J_{2-3}=$ $4.8 \mathrm{~Hz}, \mathrm{H}-2), 4.03-3.89\left(\mathrm{~m}, 2 \mathrm{H}, \mathrm{CH}_{2} \mathrm{CH}_{3}\right), 3.77\left(\mathrm{~d}, 1 \mathrm{H}, J_{5 \mathrm{a}-5 \mathrm{~b}}=11.2 \mathrm{~Hz}, J_{4-5 \mathrm{a}}=2.4 \mathrm{~Hz}\right.$, $\mathrm{H}-5 \mathrm{a}), 3.57\left(\mathrm{dd}, 1 \mathrm{H}, J_{5 \mathrm{a}-5 \mathrm{~b}}=11.2, J_{4-5 \mathrm{~b}}=3.1 \mathrm{~Hz}, \mathrm{H}-5 \mathrm{~b}\right), 1.11\left(\mathrm{t}, 3 \mathrm{H}, J_{\mathrm{CH} 2 \mathrm{CH} 3}=7.2 \mathrm{~Hz}\right.$, $\left.\mathrm{CH}_{2} \mathrm{CH}_{3}\right)$ ppm. ${ }^{13} \mathrm{C} \mathrm{NMR}\left(125 \mathrm{MHz}, \mathrm{CDCl}_{3}\right) \delta=163.1\left(\mathrm{t}, J_{\mathrm{C}-\mathrm{F}}=30.9 \mathrm{~Hz}, \mathrm{CqO}_{2} \mathrm{Et}\right), 138.1$ (Cq arom), $137.7(\mathrm{Cq}$ arom $), 137.5(\mathrm{Cq}$ arom $), 128.5-127.8\left(\mathrm{CH}^{\text {arom }}\right), 113.2\left(\mathrm{dd}, J_{\mathrm{C}-\mathrm{F}}=\right.$ $\left.247.4 \mathrm{~Hz}, J_{\mathrm{C}-\mathrm{F}}=259.3 \mathrm{~Hz}, \mathrm{CF}_{2}\right), 80.7(\mathrm{C}-3), 79.7\left(\mathrm{dd}, J_{\mathrm{C}-\mathrm{F}}=22.8 \mathrm{~Hz}, J_{\mathrm{C}-\mathrm{F}}=29.0 \mathrm{~Hz}, \mathrm{C}-\right.$ 1), $78.5(\mathrm{C}-4), 77.5(\mathrm{C}-2), 74.0\left(\mathrm{CH}_{2}{ }^{\mathrm{Bn}}\right), 73.6\left(\mathrm{CH}_{2}{ }^{\mathrm{Bn}}\right), 73.1\left(\mathrm{CH}_{2}{ }^{\mathrm{Bn}}\right), 68.6(\mathrm{C}-5), 62.5$ $\left(\mathrm{CH}_{2} \mathrm{CH}_{3}\right), 13.7\left(\mathrm{CH}_{2} \mathrm{CH}_{3}\right) \mathrm{ppm} .{ }^{19} \mathrm{~F} \mathrm{NMR}\left(471 \mathrm{MHz}, \mathrm{CDCl}_{3}\right) \delta=-109.5\left(\mathrm{dd}, 1 \mathrm{~F}, J_{\mathrm{H}-\mathrm{Fa}}=\right.$ $\left.9.5 \mathrm{~Hz}, J_{\mathrm{Fa}-\mathrm{Fb}}=277.9 \mathrm{~Hz}, \mathrm{Fa}\right),-114.4\left(\mathrm{dd}, 1 \mathrm{~F}, J_{\mathrm{H}-\mathrm{Fb}}=14.6 \mathrm{~Hz}, J_{\mathrm{Fa}-\mathrm{Fb}}=277.9 \mathrm{~Hz}, \mathrm{Fb}\right) \mathrm{ppm}$. HRMS (ESI+): $\mathrm{m} / \mathrm{z}$ calculated for $\mathrm{C}_{30} \mathrm{H}_{33} \mathrm{~F}_{2} \mathrm{O}_{6}$ [M+H] $]^{+}$: calc. 527.2240; found: 527.2241. nOe: Correlations were inconclusive. 


\section{${ }^{1}$ H NMR}

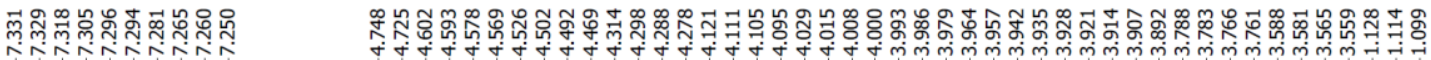

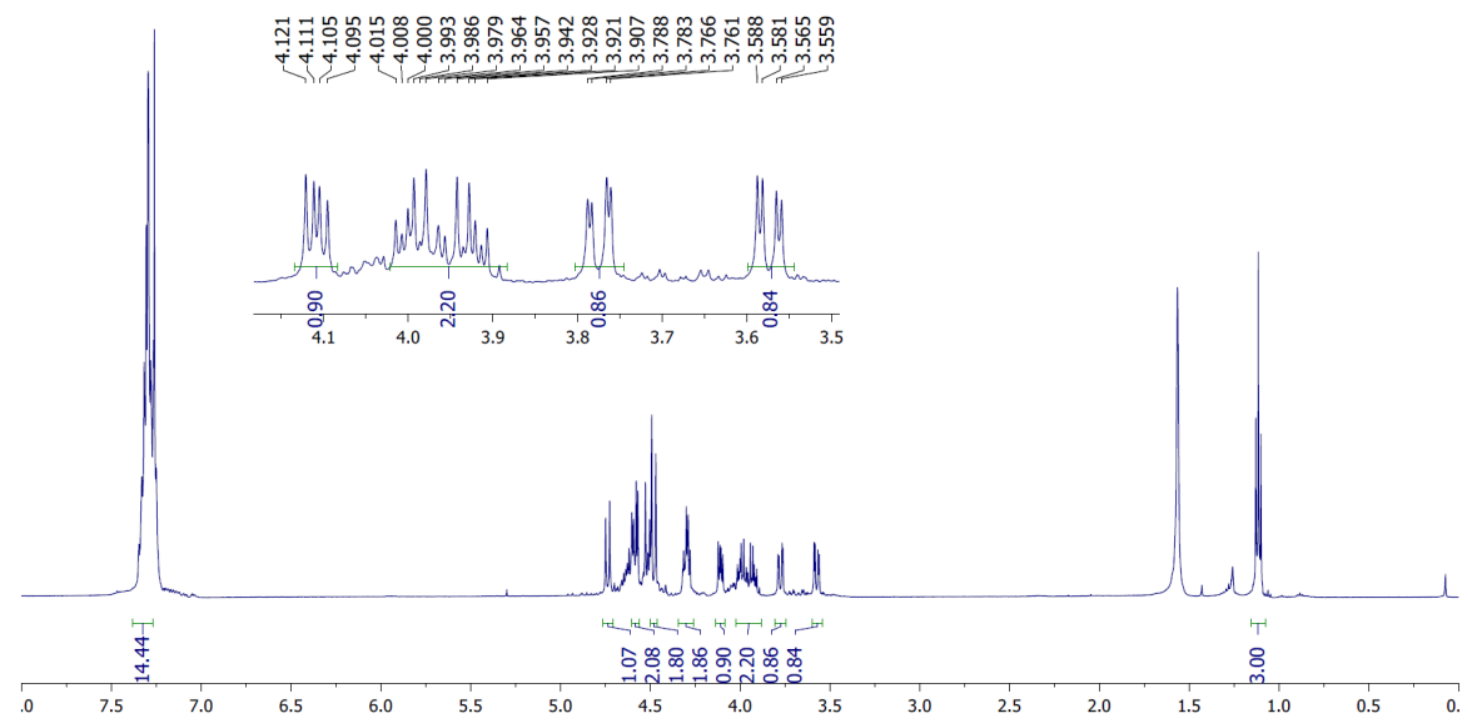

${ }^{19}$ F NMR
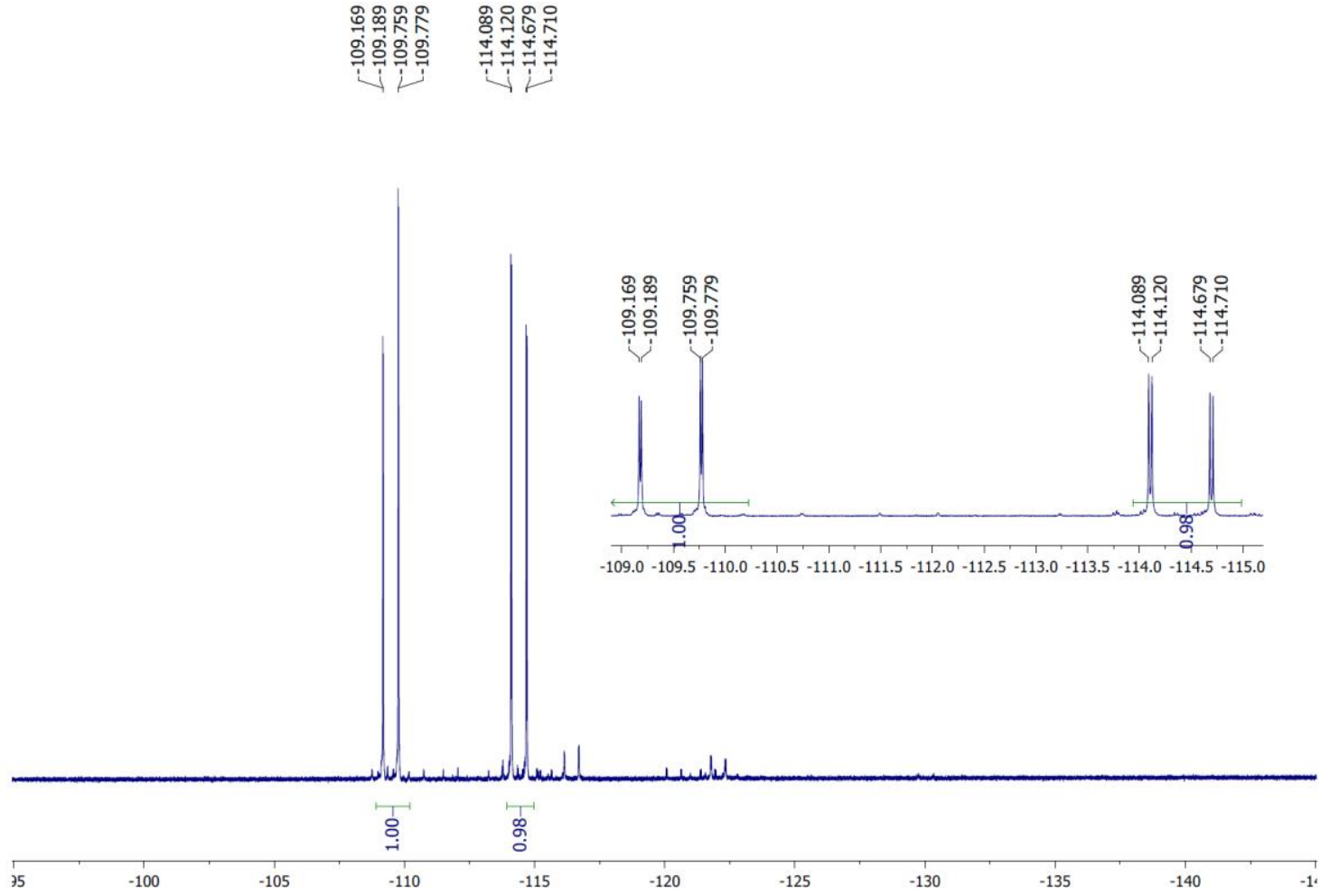
${ }^{13}$ C NMR
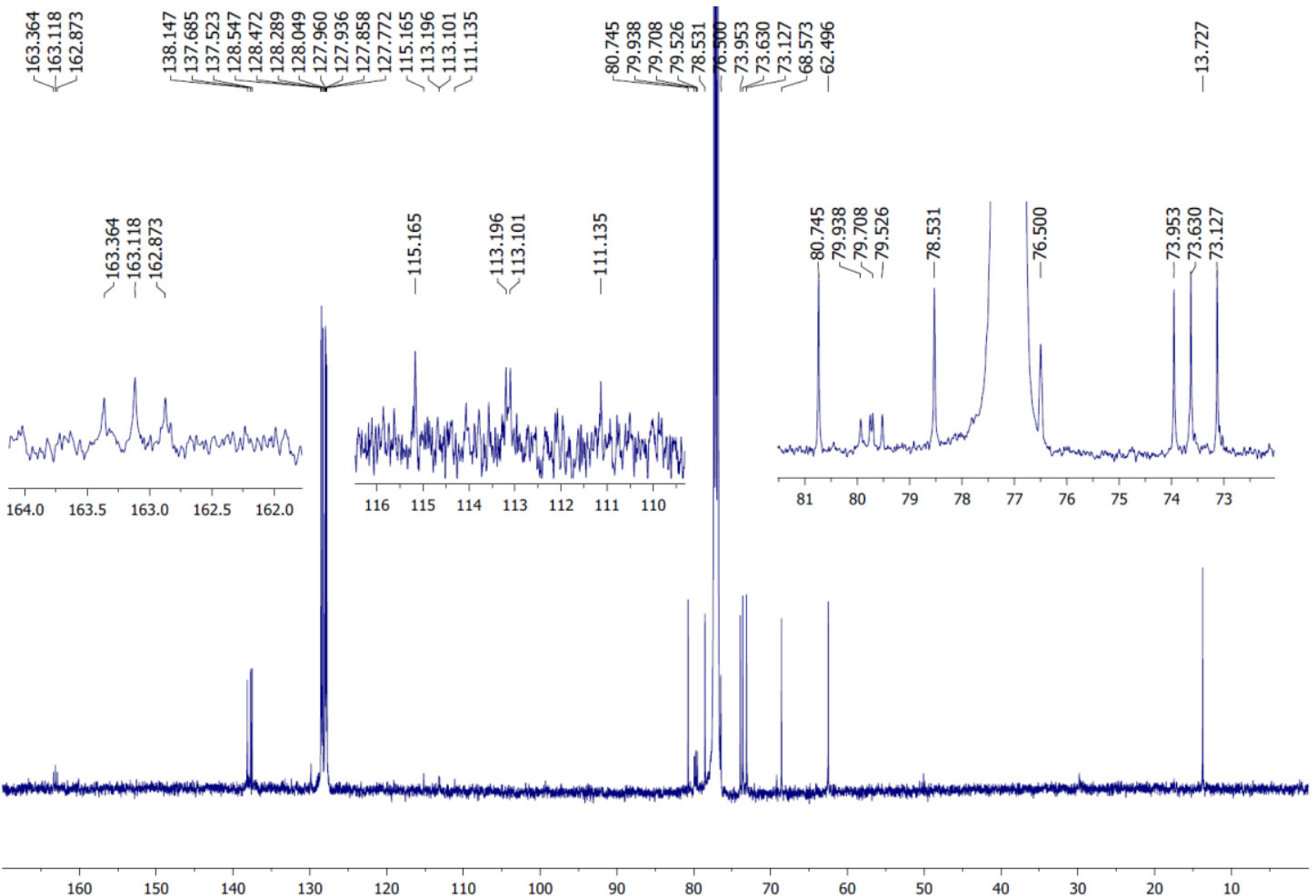


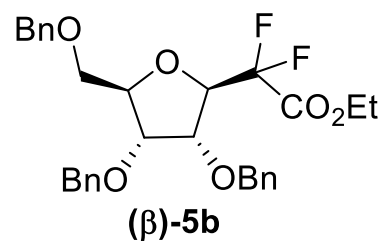

To a solution of alcohol $(S)-3 \mathbf{b}(33 \mathrm{mg}, 0.05 \mathrm{mmol})$ in dry DCM $(1.5 \mathrm{ml})$ under argon atmosphere was added DAST ( $27 \mu \mathrm{l}, 0.20 \mathrm{mmol}, 4.0$ equiv.) dropwise at $0^{\circ} \mathrm{C}$. The reaction was allowed to warm from $0^{\circ} \mathrm{C}$ to r.t and stirred for $19 \mathrm{~h}$. Then, the crude was diluted with dichloromethane. The organic phase was washed with $\mathrm{H}_{2} \mathrm{O}$ (3 times) and brine (3 times), dried over $\mathrm{MgSO}_{4}$ and concentrated under reduced pressure. Purification by chromatography on silica gel (Cy/EtOAc 9:1) gave the cyclized product $(\beta)-\mathbf{5 b}$ as a yellowish oil (17 mg, 62\%).

$[\alpha]^{20} \mathrm{D}:+32.4\left(\mathrm{c} 1.0, \mathrm{CHCl}_{3}\right) .{ }^{1} \mathrm{H} \mathrm{NMR}\left(500 \mathrm{MHz}, \mathrm{CDCl}_{3}\right) \delta=7.36-7.26\left(\mathrm{~m}, 15 \mathrm{H}, \mathrm{H}^{\text {arom }}\right)$, $4.63\left(\mathrm{AB}, 1 \mathrm{H}, J_{\mathrm{A}-\mathrm{B}}=12.0 \mathrm{~Hz}, \mathrm{CH}_{2}{ }^{\mathrm{Bn}}\right), 4.57-4.49\left(\mathrm{~m}, 5 \mathrm{H}, \mathrm{H}-1,2 \mathrm{x} \mathrm{CH}_{2}{ }^{\mathrm{Bn}}\right), 4.47(\mathrm{AB}, 1 \mathrm{H}$, $\left.J_{\mathrm{A}-\mathrm{B}}=11.5 \mathrm{~Hz}, \mathrm{CH}_{2}{ }^{\mathrm{Bn}}\right), 4.29-4.25(\mathrm{~m}, 1 \mathrm{H}, \mathrm{H}-4), 4.26\left(\mathrm{q}, 2 \mathrm{H}, J_{\mathrm{CH} 2-\mathrm{CH} 3}=7.1 \mathrm{~Hz}, \mathrm{CH}_{2} \mathrm{CH}_{3}\right)$, $4.20\left(\mathrm{dd}, 1 \mathrm{H}, J_{1-2}=3.4 \mathrm{~Hz}, J_{2-3}=5.2 \mathrm{~Hz}, \mathrm{H}-2\right), 3.87\left(\mathrm{td}, 1 \mathrm{H}, J_{3-4}=1.2 \mathrm{~Hz}, J_{2-3}=5.2 \mathrm{~Hz}\right.$, $\mathrm{H}-3), 3.59\left(\mathrm{ABX}, 1 \mathrm{H}, J_{4-5 \mathrm{a}}=4.0 \mathrm{~Hz}, J_{5 \mathrm{a}-5 \mathrm{~b}}=10.9 \mathrm{~Hz}, \mathrm{H}-5 \mathrm{a}\right), 3.53\left(\mathrm{ABX}, 1 \mathrm{H}, J_{4-5 \mathrm{~b}}=4.6\right.$ $\left.\mathrm{Hz}, J_{5 \mathrm{a}-5 \mathrm{~b}}=10.9 \mathrm{~Hz}, \mathrm{H}-5 \mathrm{~b}\right), 1.28\left(\mathrm{t}, 3 \mathrm{H}, J_{\mathrm{CH} 2-\mathrm{CH} 3}=7.5 \mathrm{~Hz}, \mathrm{CH}_{2} \mathrm{CH}_{3}\right)$ ppm. ${ }^{13} \mathrm{C} \mathrm{NMR}(125$ $\left.\mathrm{MHz}, \mathrm{CDCl}_{3}\right) \delta=162.8\left(\mathrm{dd}, J_{\mathrm{C}-\mathrm{F}}=30.2 \mathrm{~Hz}, J_{\mathrm{C}-\mathrm{F}}=32.6 \mathrm{~Hz}, \mathrm{CqO}_{2} \mathrm{Et}\right), 138.3\left(\mathrm{C}_{\mathrm{q}}{ }^{\text {arom }}\right)$, $137.6\left(\mathrm{C}_{\mathrm{q}}{ }^{\text {arom }}\right), 137.3\left(\mathrm{C}_{\mathrm{q}}{ }^{\text {arom }}\right), 128.6-127.7\left(\mathrm{CH}^{\text {arom }}\right), 113.3\left(\mathrm{dd}, J_{\mathrm{C}-\mathrm{F}}=251.6 \mathrm{~Hz}, J_{\mathrm{C}-\mathrm{F}}=\right.$ $\left.256.5 \mathrm{~Hz}, \mathrm{CF}_{2}\right), 81.9$ (dd, $\left.J_{\mathrm{C}-\mathrm{F}}=25.4 \mathrm{~Hz}, J_{\mathrm{C}-\mathrm{F}}=31.4 \mathrm{~Hz}, \mathrm{C}-1\right), 81.7(\mathrm{C}-4), 77.8(\mathrm{C}-3)$, $75.9(\mathrm{C}-2), 73.6\left(\mathrm{CH}_{2}{ }^{\mathrm{Bn}}\right), 72.4\left(\mathrm{CH}_{2}{ }^{\mathrm{Bn}}\right), 72.2\left(\mathrm{CH}_{2}{ }^{\mathrm{Bn}}\right) 69.7(\mathrm{C}-5), 62.3\left(\mathrm{CH}_{2} \mathrm{CH}_{3}\right), 14.0$ $\left(\mathrm{CH}_{2} \mathrm{CH}_{3}\right)$ ppm. ${ }^{19} \mathrm{~F} \mathrm{NMR}\left(471 \mathrm{MHz}, \mathrm{CDCl}_{3}\right) \delta=-113.5\left(\mathrm{dd}, 1 \mathrm{~F}, J_{\mathrm{H}-\mathrm{Fa}}=7.7 \mathrm{~Hz}, J_{\mathrm{Fa}-\mathrm{Fb}}=\right.$ $261.4 \mathrm{~Hz}, \mathrm{Fa}$ ), $-120.4\left(\mathrm{dd}, 1 \mathrm{~F}, J_{\mathrm{H}-\mathrm{Fb}}=18.0 \mathrm{~Hz}, J_{\mathrm{Fa}-\mathrm{Fb}}=261.4 \mathrm{~Hz}, \mathrm{Fb}\right.$ ) ppm. HRMS (ESI+): $\mathrm{m} / \mathrm{z}$ calculated for $\mathrm{C}_{30} \mathrm{H}_{32} \mathrm{~F}_{2} \mathrm{NaO}_{6}[\mathrm{M}+\mathrm{Na}]^{+}$: calc. 549.2059; found: 549.2057. nOe: No correlation between $\mathrm{H}-1$ and $\mathrm{H}-2$ or $\mathrm{H}-3$ observed. 
${ }^{1}$ H NMR

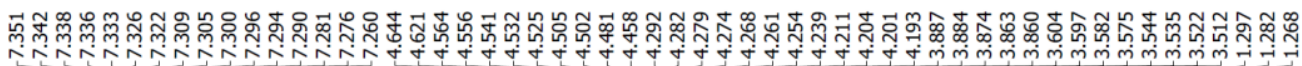

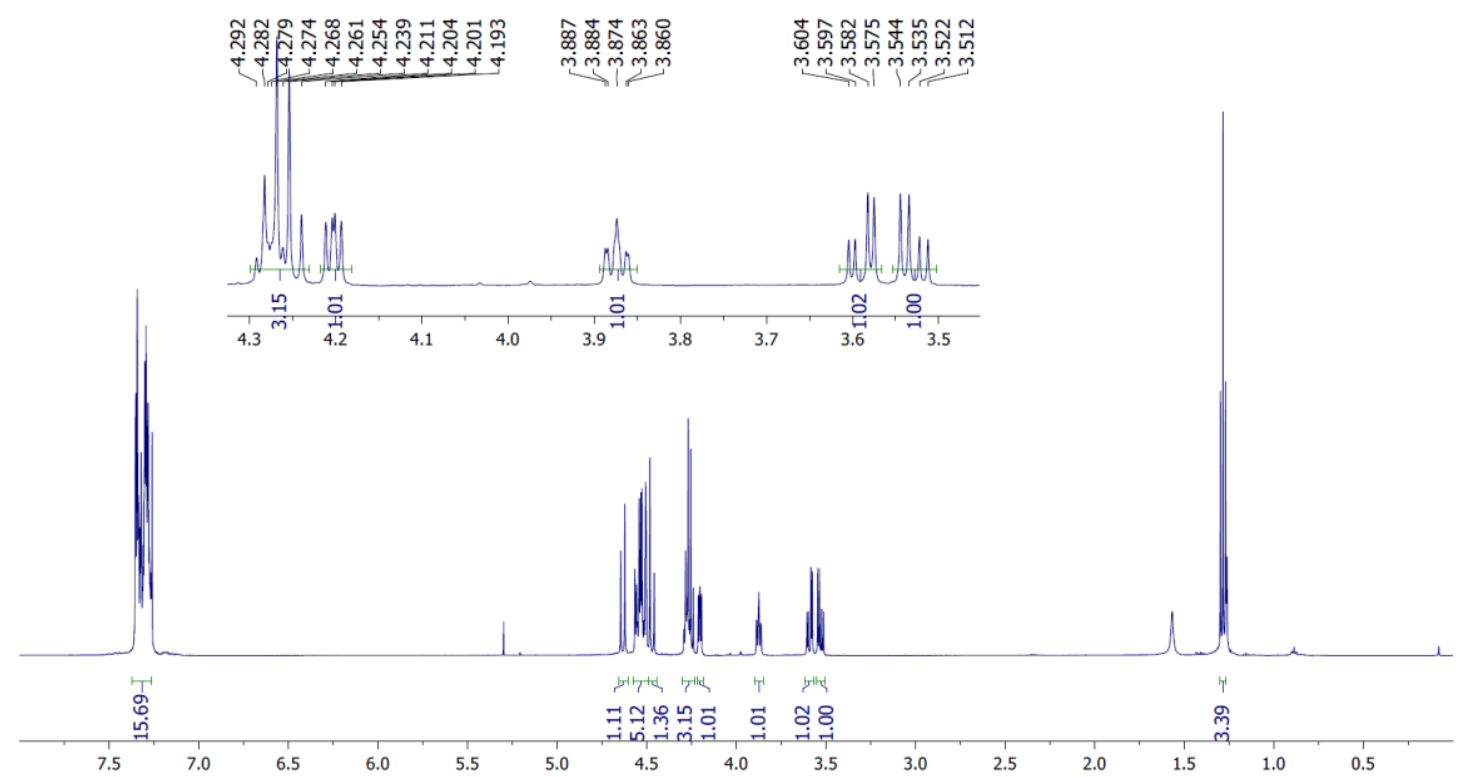

${ }^{19}$ F NMR

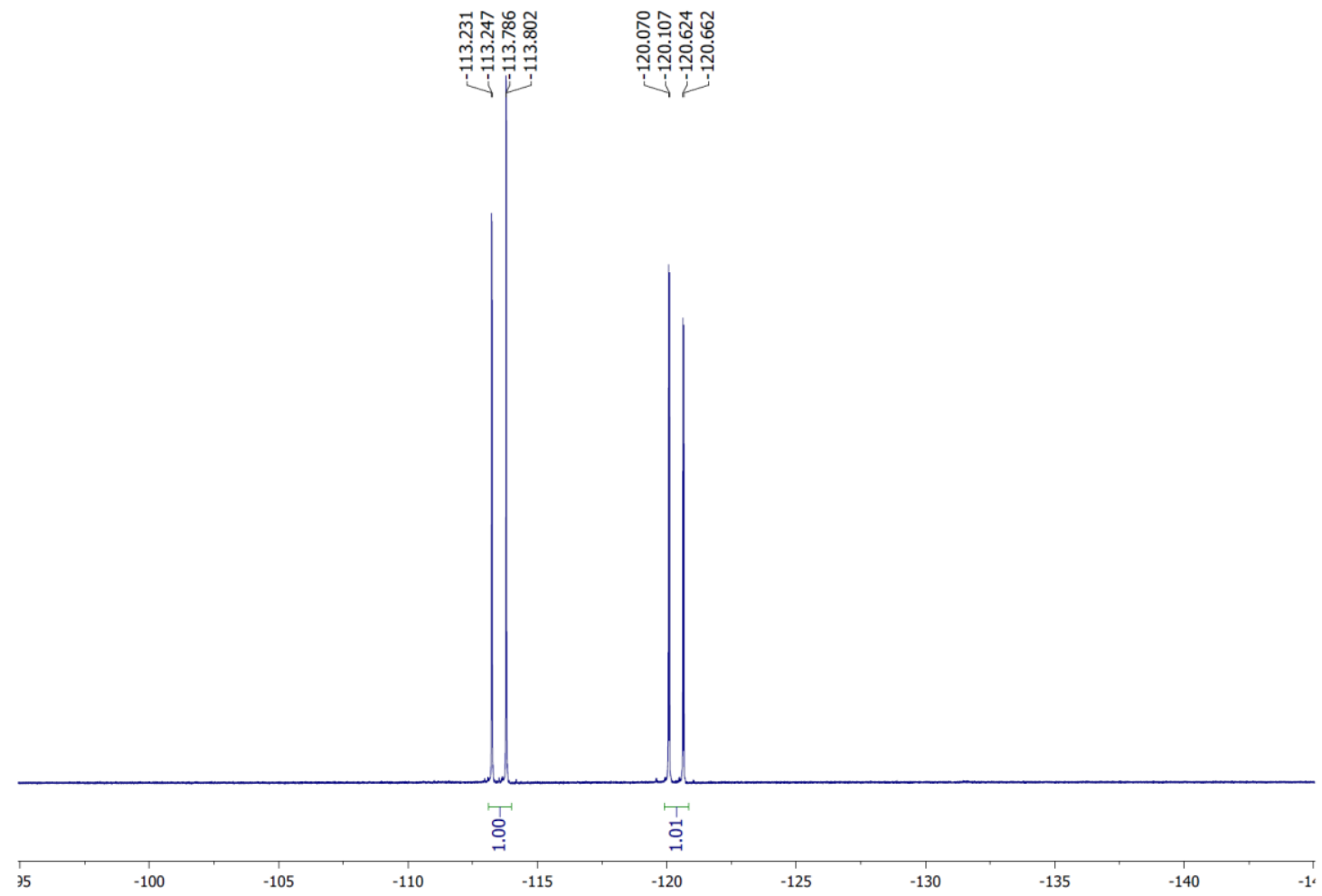


${ }^{13}$ C NMR

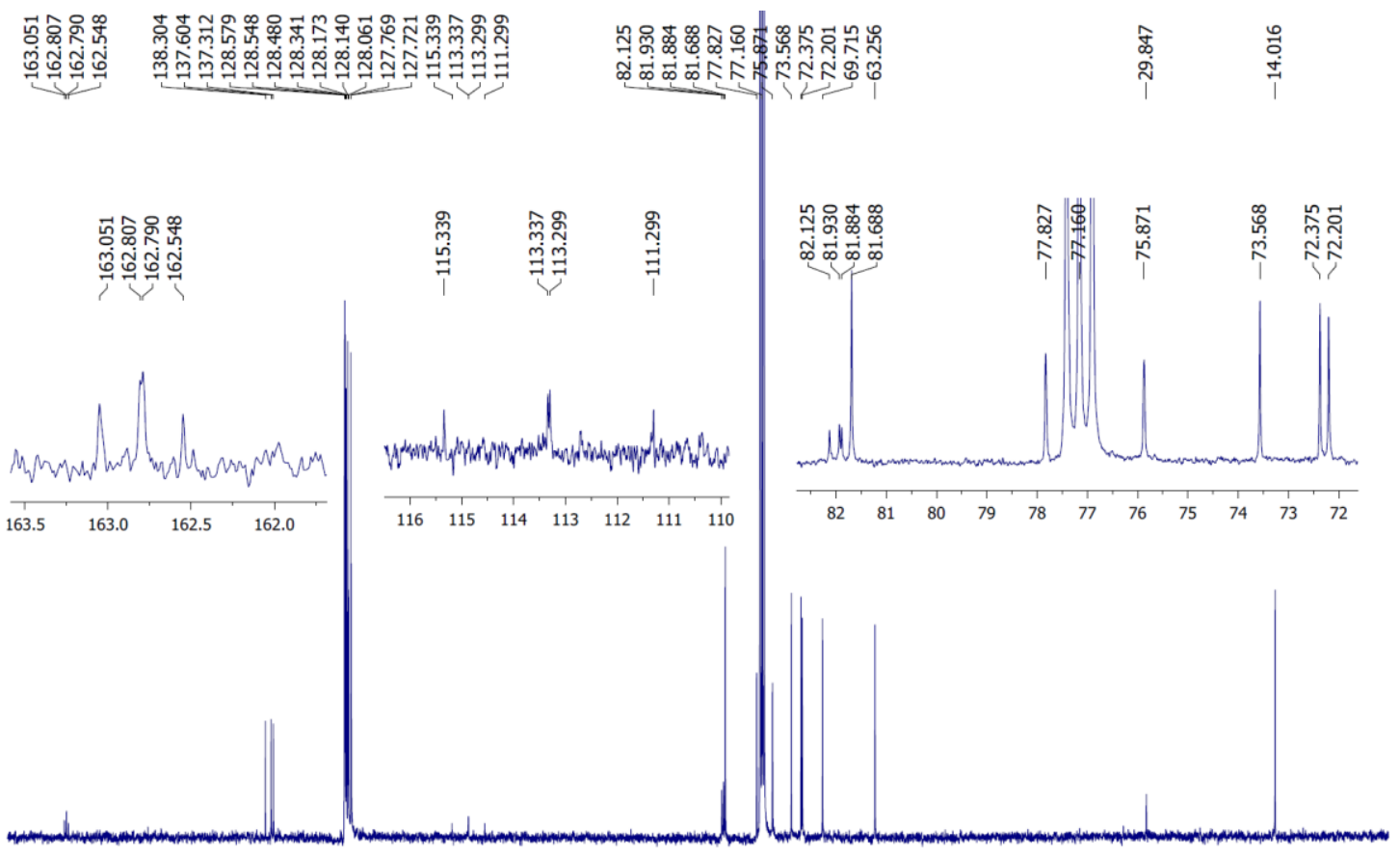

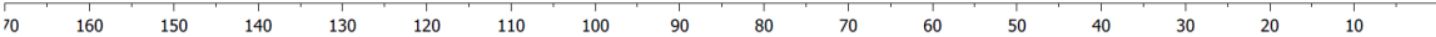




\section{D-Xylose}

2,3,5-tri-O-benzyl-1-deoxy-1,1-difluoroethyl acetate- $\alpha, \beta$-D-xylofuranose $((\alpha \beta)$-5c).

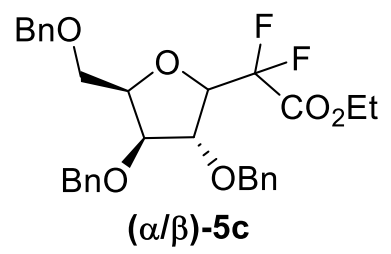

To a solution of alcohols $(R / S)-3 \mathrm{c}(84 \mathrm{mg}, 0.14 \mathrm{mmol})$ in dry DCM $(1.7 \mathrm{ml})$ under argon atmosphere was added DAST (35 $\mu 1,0.28 \mathrm{mmol}, 2.0$ equiv.) dropwise at $-78^{\circ} \mathrm{C}$. The reaction was allowed to warm from $0^{\circ} \mathrm{C}$ to r.t over $5 \mathrm{~h}$. TLC showed that starting material was still present. Thus, DAST ( $35 \mu 1,0.28 \mathrm{mmol}, 2.0$ equiv.) was added at $0^{\circ} \mathrm{C}$ and the reaction was stirred for $16 \mathrm{~h}$ at r.t. The crude was then diluted with dichloromethane and the organic phase was washed with $\mathrm{H}_{2} \mathrm{O}$ (3 times) and brine (3 times), dried over $\mathrm{MgSO}_{4}$ and concentrated under reduced pressure. ${ }^{1} \mathrm{H}$ and ${ }^{19} \mathrm{~F}$ crude NMR showed that the cyclized products $(\alpha / \beta)$-5c were formed in 65:35 $d r$ ratio. Purification by chromatography on silica gel (Cy/EtOAc 9:1) afforded pure compound $(\alpha)-5 \mathbf{c}(15 \mathrm{mg})$ and a mixture of $(\alpha / \beta)-5 \mathbf{c}$ in $54: 46 d r$ ratio $(47 \mathrm{mg})$ in $83 \%$ overall yield.

2,3,5-tri-O-benzyl-1-deoxy-1,1-difluoroethyl acetate- $\alpha, \beta$-D-xylofuranose (( $\alpha)$-5c).

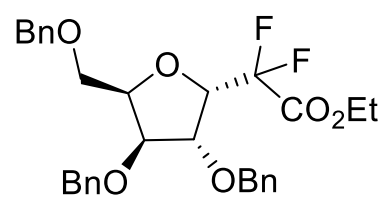

$(\alpha)-5 c$

$[\alpha]^{20} \mathrm{D}:-23.1\left(\mathrm{c} 1.0, \mathrm{CHCl}_{3}\right) .{ }^{1} \mathrm{H} \mathrm{NMR}\left(500 \mathrm{MHz}, \mathrm{CDCl}_{3}\right) \delta=7.38-7.26\left(\mathrm{~m}, 13 \mathrm{H}, \mathrm{H}^{\text {arom }}\right)$, 7.26-7.24 (m, $\left.2 \mathrm{H}, \mathrm{H}^{\mathrm{arom}}\right), 4.58\left(\mathrm{AB}, 1 \mathrm{H}, J_{\mathrm{A}-\mathrm{B}}=11.5 \mathrm{~Hz}, \mathrm{CH}_{2}{ }^{\mathrm{Bn}}\right), 4.55\left(\mathrm{AB}, 1 \mathrm{H}, J_{\mathrm{A}-\mathrm{B}}=\right.$ $\left.12.0 \mathrm{~Hz}, \mathrm{CH}_{2}{ }^{\mathrm{Bn}}\right), 4.53\left(\mathrm{AB}, 1 \mathrm{H}, J_{\mathrm{A}-\mathrm{B}}=12.0 \mathrm{~Hz}, \mathrm{CH}_{2}{ }^{\mathrm{Bn}}\right), 4.51\left(\mathrm{AB}, 1 \mathrm{H}, J_{\mathrm{A}-\mathrm{B}}=11.5 \mathrm{~Hz}\right.$, $\left.\mathrm{CH}_{2}{ }^{\mathrm{Bn}}\right), 4.48\left(\mathrm{AB}, 1 \mathrm{H}, J_{\mathrm{A}-\mathrm{B}}=11.5 \mathrm{~Hz}, \mathrm{CH}_{2}{ }^{\mathrm{Bn}}\right), 4.37-4.31\left(\mathrm{~m}, 3 \mathrm{H}, \mathrm{H}-1, \mathrm{H}-2, \mathrm{CH}_{2}{ }^{\mathrm{Bn}}\right), 4.28$ $\left(\mathrm{td}, 1 \mathrm{H}, J_{3-4}=4.0 \mathrm{~Hz}, J_{4-5 \mathrm{a}}=J_{4-5 \mathrm{~b}}=5.7 \mathrm{~Hz}, \mathrm{H}-4\right), 4.21\left(\mathrm{qd}, 2 \mathrm{H}, J=2.9 \mathrm{~Hz}, J_{\mathrm{CH} 2-\mathrm{CH} 3}=7.5\right.$ $\left.\mathrm{Hz}, \mathrm{CH}_{2} \mathrm{CH}_{3}\right), 4.00\left(\mathrm{~d}, 1 \mathrm{H}, J_{2-3} \sim J_{3-4}=4.0 \mathrm{~Hz}, \mathrm{H}-3\right), 3.79\left(\mathrm{ABX}, 1 \mathrm{H}, J_{4-5 \mathrm{a}}=5.7 \mathrm{~Hz}, J_{5 \mathrm{a}-}\right.$ $5 \mathrm{~b}=10.3 \mathrm{~Hz}, \mathrm{H}-5 \mathrm{a}), 3.70\left(\mathrm{ABX}, 1 \mathrm{H}, J_{4-5 \mathrm{~b}}=5.7 \mathrm{~Hz}, J_{5 \mathrm{a}-5 \mathrm{~b}}=10.3 \mathrm{~Hz}, \mathrm{H}-5 \mathrm{~b}\right), 1.26(\mathrm{t}, 3 \mathrm{H}$, $\left.J_{\mathrm{CH} 2-\mathrm{CH} 3}=7.5 \mathrm{~Hz}, \mathrm{CH}_{2} \mathrm{CH}_{3}\right)$ ppm. ${ }^{13} \mathrm{C} \mathrm{NMR}\left(125 \mathrm{MHz}, \mathrm{CDCl}_{3}\right) \delta=162.9\left(\mathrm{dd}, J_{\mathrm{C}-\mathrm{F}}=30.3\right.$ $\left.\mathrm{Hz}, J_{\mathrm{C}-\mathrm{F}}=32.3 \mathrm{~Hz}, \mathrm{CqO}_{2} \mathrm{Et}\right), 138.2\left(\mathrm{C}_{\mathrm{q}}{ }^{\text {arom }}\right), 137.6\left(\mathrm{C}_{\mathrm{q}}{ }^{\text {arom }}\right), 137.3\left(\mathrm{C}_{\mathrm{q}}{ }^{\text {arom }}\right), 128.7-127.8$ $\left(\mathrm{CH}^{\text {arom }}\right), 113.1\left(\mathrm{dd}, J_{\mathrm{C}-\mathrm{F}}=251.4 \mathrm{~Hz}, J_{\mathrm{C}-\mathrm{F}}=256.2 \mathrm{~Hz}, \mathrm{CF}_{2}\right), 84.0\left(\mathrm{dd}, J_{\mathrm{C}-\mathrm{F}}=27.5 \mathrm{~Hz}, J_{\mathrm{C}-}\right.$ $\mathrm{F}=30.3 \mathrm{~Hz}, \mathrm{C}-1), 82.2(\mathrm{C}-3), 81.7(\mathrm{C}-2, \mathrm{C}-4), 73.6\left(\mathrm{CH}_{2}{ }^{\mathrm{Bn}}\right), 72.1\left(\mathrm{CH}_{2}{ }^{\mathrm{Bn}}\right), 71.6\left(\mathrm{CH}_{2}{ }^{\mathrm{Bn}}\right)$, $67.8(\mathrm{C}-5), 63.2\left(\mathrm{CH}_{2} \mathrm{CH}_{3}\right), 14.0\left(\mathrm{CH}_{2} \mathrm{CH}_{3}\right) \mathrm{ppm} .{ }^{19} \mathrm{~F} \mathrm{NMR}\left(471 \mathrm{MHz}, \mathrm{CDCl}_{3}\right) \delta=-111.0$ $\left(\mathrm{dd}, 1 \mathrm{~F}, J_{\mathrm{H}-\mathrm{Fa}}=7.2 \mathrm{~Hz}, J_{\mathrm{Fa}-\mathrm{Fb}}=262.3 \mathrm{~Hz}, \mathrm{Fa}\right),-118.4\left(\mathrm{dd}, 1 \mathrm{~F}, J_{\mathrm{H}-\mathrm{Fb}}=15.7 \mathrm{~Hz}, J_{\mathrm{Fa}-\mathrm{Fb}}=\right.$ $262.3 \mathrm{~Hz}, \mathrm{Fb}$ ) ppm. HRMS (ESI+): $\mathrm{m} / \mathrm{z}$ calculated for $\mathrm{C}_{30} \mathrm{H}_{32} \mathrm{~F}_{2} \mathrm{NaO}_{6}[\mathrm{M}+\mathrm{Na}]^{+}$: calc. 549.2059; found: 549.2056. nOe: Correlations were inconclusive. 


\section{${ }^{1}$ H NMR}

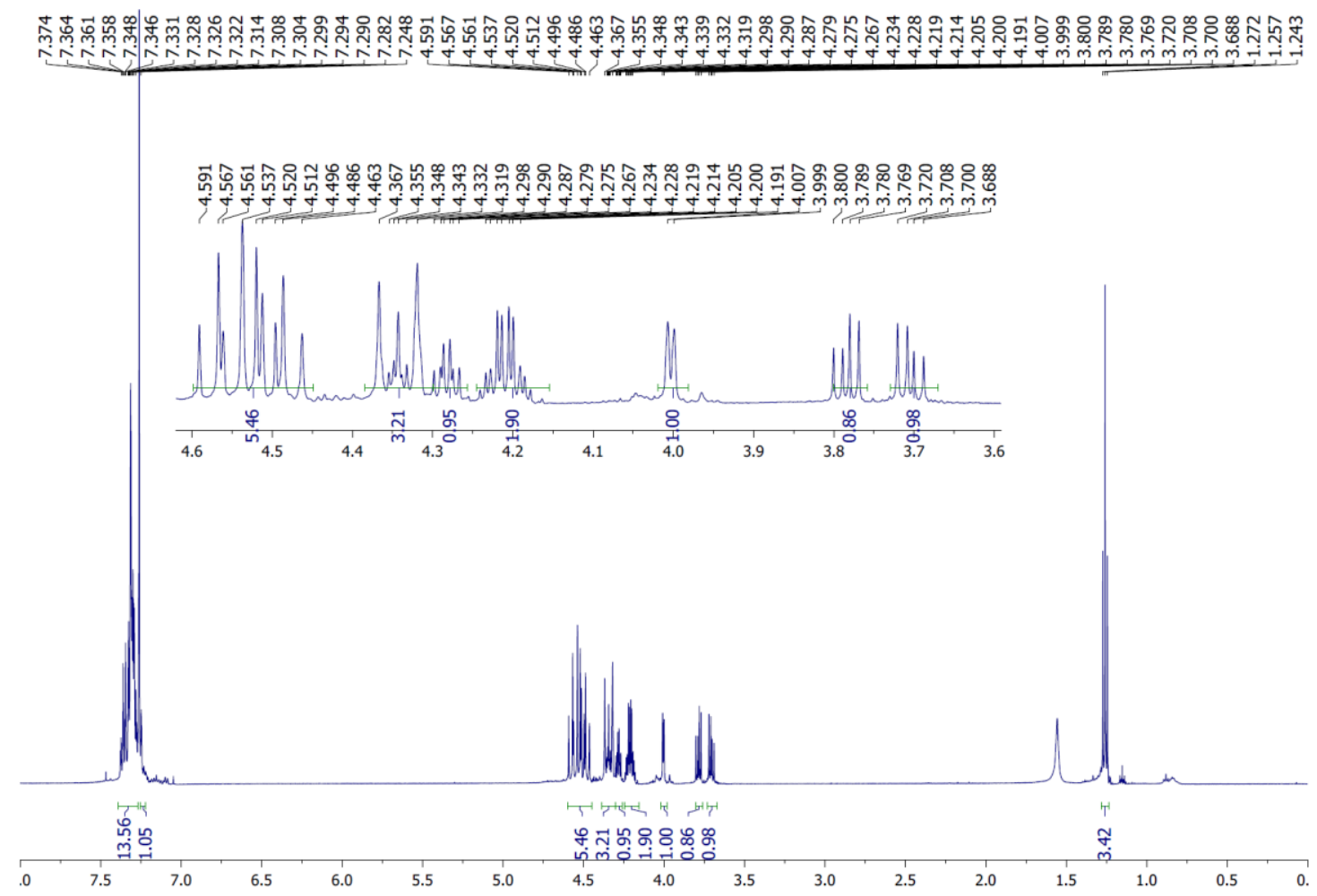

${ }^{19}$ F NMR

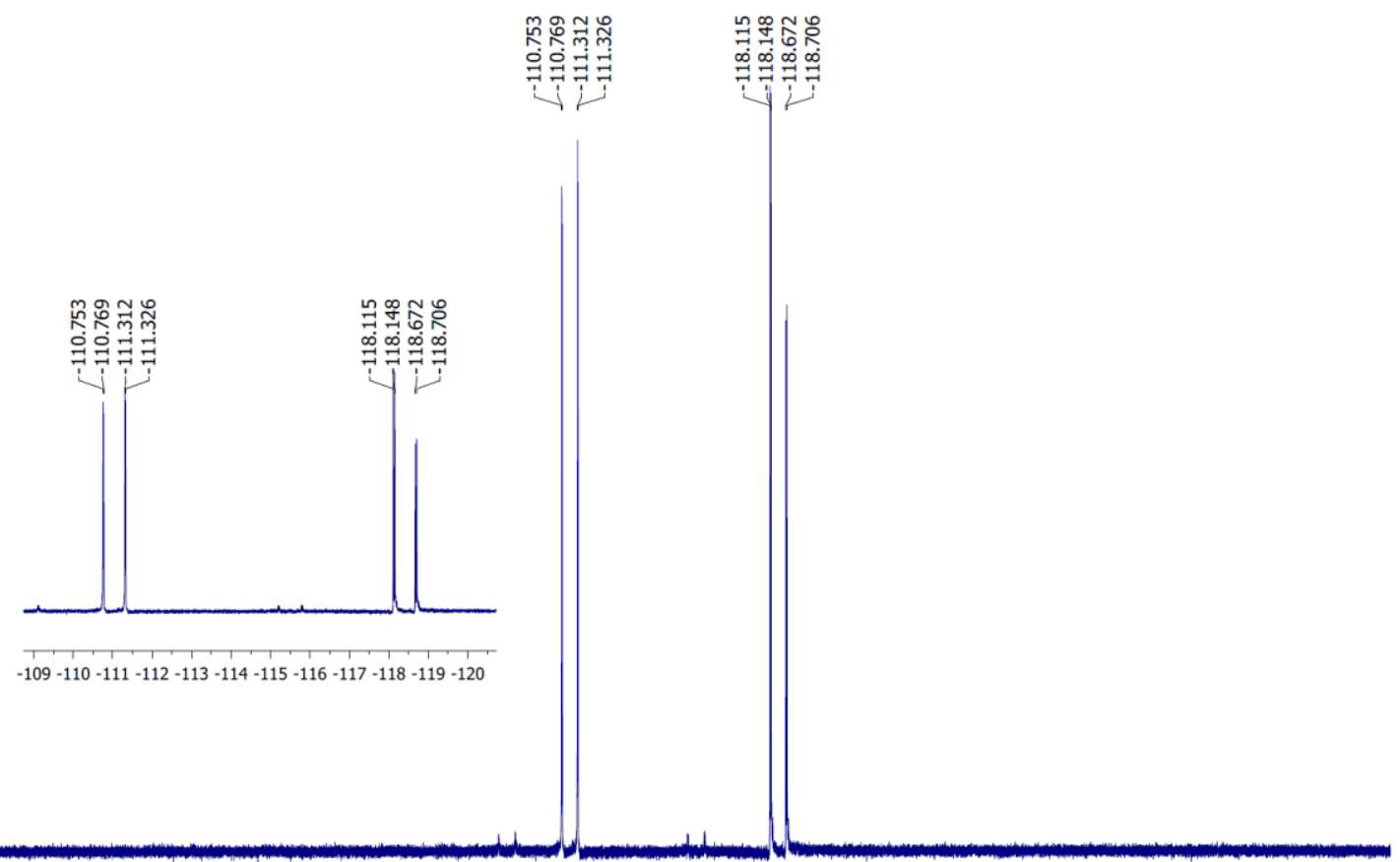

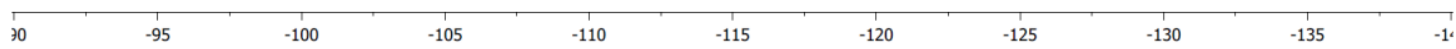


${ }^{13}$ C NMR

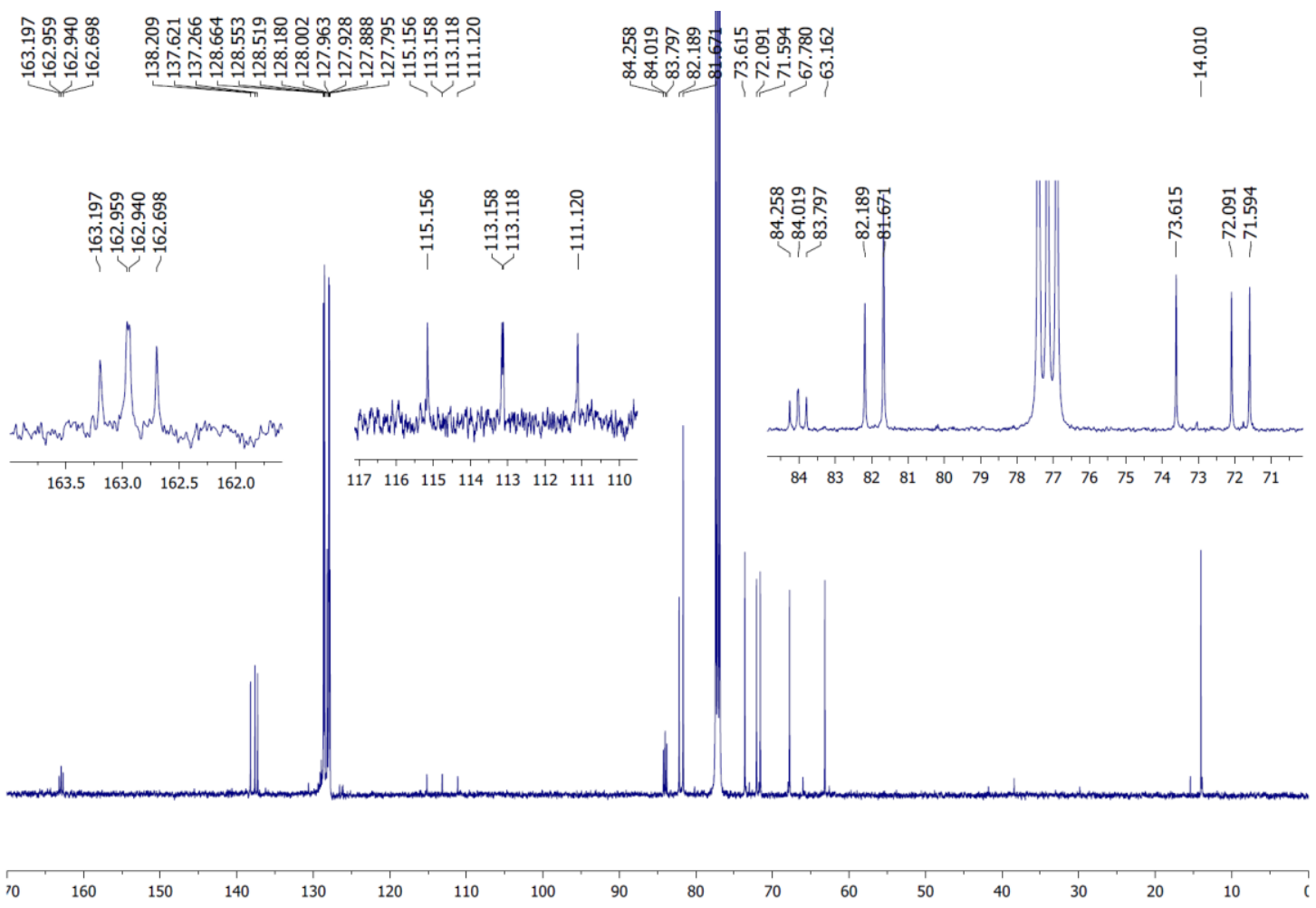


2,3,5-tri-O-benzyl-1-deoxy-1,1-difluoroethyl acetate- $\alpha, \beta$-D-xylofuranose (( $\alpha / \beta)$-5c).

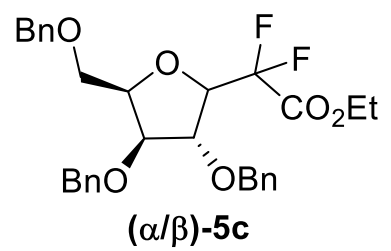

${ }^{1} \mathrm{H}$ NMR $\left(500 \mathrm{MHz}, \mathrm{CDCl}_{3}\right) \delta=7.38-7.26\left(\mathrm{~m}, 26 \mathrm{H}, 13 \mathrm{H \alpha}^{\text {arom }}, 13 \mathrm{H} \beta^{\text {arom }}\right), 7.26-7.22(\mathrm{~m}$, $\left.4 \mathrm{H}, 2 \mathrm{H \alpha}^{\text {arom }}, 2 \mathrm{H} \beta^{\text {arom }}\right), 4.73\left(\mathrm{ddd}, 1 \mathrm{H}, J_{1-2}=5.7 \mathrm{~Hz}, J_{1-\mathrm{Fa}}=9.7 \mathrm{~Hz}, J_{1-\mathrm{Fb}}=15.5 \mathrm{~Hz}, \mathrm{H}-\right.$ $1 \beta), 4.59\left(\mathrm{AB}, 1 \mathrm{H}, J_{\mathrm{A}-\mathrm{B}}=12.0 \mathrm{~Hz}, \mathrm{CH}_{2} \beta^{\mathrm{Bn}}\right), 4.58\left(\mathrm{AB}, 1 \mathrm{H}, J_{\mathrm{A}-\mathrm{B}}=12.0 \mathrm{~Hz}, \mathrm{CH}_{2} \alpha^{\mathrm{Bn}}\right)$, 4.55-4.52 (m, 4H, $\left.2 \mathrm{CH}_{2} \alpha^{\mathrm{Bn}}, 2 \mathrm{CH}_{2} \beta^{\mathrm{Bn}}\right), 4.51\left(\mathrm{AB}, 1 \mathrm{H}, J_{\mathrm{A}-\mathrm{B}}=12.0 \mathrm{~Hz}, \mathrm{CH}_{2} \alpha^{\mathrm{Bn}}\right), 4.50$ $\left(\mathrm{AB}, 1 \mathrm{H}, J_{\mathrm{A}-\mathrm{B}}=11.5 \mathrm{~Hz}, \mathrm{CH}_{2} \beta^{\mathrm{Bn}}\right), 4.47\left(\mathrm{AB}, 1 \mathrm{H}, J_{\mathrm{A}-\mathrm{B}}=12.0 \mathrm{~Hz}, \mathrm{CH}_{2} \alpha^{\mathrm{Bn}}\right), 4.44(\mathrm{AB}, 1 \mathrm{H}$, $\left.J_{\mathrm{A}-\mathrm{B}}=12.0 \mathrm{~Hz}, \mathrm{CH}_{2} \beta^{\mathrm{Bn}}\right), 4.40\left(\mathrm{AB}, 1 \mathrm{H}, J_{\mathrm{A}-\mathrm{B}}=11.5 \mathrm{~Hz}, \mathrm{CH}_{2} \beta^{\mathrm{Bn}}\right), 4.38-4.31(\mathrm{~m}, 3 \mathrm{H}, \mathrm{H}-$ $\left.1 \alpha, \mathrm{H}-2 \alpha, \mathrm{CH}_{2} \alpha^{\mathrm{Bn}}\right), 4.30-4.27(\mathrm{~m}, 2 \mathrm{H}, \mathrm{H}-4 \alpha, \mathrm{H}-2 \beta), 4.21\left(\mathrm{qd}, 2 \mathrm{H}, J=2.3 \mathrm{~Hz}, J_{\mathrm{CH} 2-\mathrm{CH} 3}=\right.$


$\left.J_{4-5 \mathrm{a}}=5.7 \mathrm{~Hz}, J_{5 \mathrm{a}-5 \mathrm{~b}}=10.3 \mathrm{~Hz}, \mathrm{H}-5 \mathrm{a} \alpha\right), 3.77-3.69$ (m, 3H, H-5b $\left.\alpha, \mathrm{H}-5 \mathrm{a} \beta, \mathrm{H}-5 \mathrm{~b} \beta\right), 1.26$ $\left(\mathrm{t}, 3 \mathrm{H}, J_{\mathrm{CH} 2-\mathrm{CH} 3 \alpha}=7.5 \mathrm{~Hz}, \mathrm{CH}_{2} \mathrm{CH}_{3} \alpha\right), 1.16\left(\mathrm{t}, 3 \mathrm{H}, J_{\mathrm{CH} 2-\mathrm{CH} 3 \beta}=7.5 \mathrm{~Hz}, \mathrm{CH}_{2} \mathrm{CH}_{3} \beta\right) \mathrm{ppm}$. ${ }^{13} \mathrm{C} \mathrm{NMR}\left(125 \mathrm{MHz}, \mathrm{CDCl}_{3}\right) \delta=163.2\left(\mathrm{t}, J_{\mathrm{C}-\mathrm{F}}=31.3 \mathrm{~Hz}, \mathrm{CqO}_{2} \mathrm{Et} \beta\right), 162.9\left(\mathrm{dd}, J_{\mathrm{C}-\mathrm{F}}=\right.$ $\left.30.8 \mathrm{~Hz}, J_{\mathrm{C}-\mathrm{F}}=32.8 \mathrm{~Hz}, \mathrm{CqO}_{2} \mathrm{Et} \alpha\right), 138.2\left(\mathrm{C}_{\mathrm{q}} \alpha^{\text {arom }}, \mathrm{C}_{\mathrm{q}} \beta^{\text {arom }}\right), 137.7\left(\mathrm{C}_{\mathrm{q}} \beta^{\text {arom }}\right), 137.6$ $\left(\mathrm{C}_{\mathrm{q}} \alpha^{\text {arom }}\right), 137.3\left(\mathrm{C}_{\mathrm{q}} \alpha^{\text {arom }}\right), 137.0\left(\mathrm{C}_{\mathrm{q}} \beta^{\text {arom }}\right), 128.7-127.7\left(\mathrm{CH}^{\text {arom }}, \mathrm{CH} \beta^{\text {arom }}\right), 113.5(\mathrm{dd}$, $\left.J_{\mathrm{C}-\mathrm{F}}=248.1 \mathrm{~Hz}, J_{\mathrm{C}-\mathrm{F}}=259.6 \mathrm{~Hz}, \mathrm{CF}_{2} \beta\right), 113.1\left(\mathrm{dd}, J_{\mathrm{C}-\mathrm{F}}=251.4 \mathrm{~Hz}, J_{\mathrm{C}-\mathrm{F}}=256.2 \mathrm{~Hz}\right.$, $\left.\mathrm{CF}_{2} \alpha\right), 84.0\left(\mathrm{dd}, J_{\mathrm{C}-\mathrm{F}}=27.9 \mathrm{~Hz}, J_{\mathrm{C}-\mathrm{F}}=29.9 \mathrm{~Hz}, \mathrm{C}-1 \alpha\right), 82.2(\mathrm{C}-3 \alpha), 82.0\left(\mathrm{~d}, J_{\mathrm{C}-\mathrm{F}}=2.9\right.$ $\mathrm{Hz}, \mathrm{C}-2 \beta), 81.7(\mathrm{C}-2 \alpha, \mathrm{C}-4 \alpha), 81.3(\mathrm{C}-3 \beta), 80.2(\mathrm{C}-4 \beta), 79.2\left(\mathrm{dd}, J_{\mathrm{C}-\mathrm{F}}=22.2 \mathrm{~Hz}, J_{\mathrm{C}-\mathrm{F}}=\right.$ $27.0 \mathrm{~Hz}, \mathrm{C}-1 \beta), 73.7\left(\mathrm{CH}_{2} \beta^{\mathrm{Bn}}\right), 73.6\left(\mathrm{CH}_{2} \alpha^{\mathrm{Bn}}\right), 73.1\left(\mathrm{CH}_{2} \beta^{\mathrm{Bn}}\right), 72.7\left(\mathrm{CH}_{2} \beta^{\mathrm{Bn}}\right), 72.1$ $\left(\mathrm{CH}_{2} \alpha^{\mathrm{Bn}}\right), 71.6\left(\mathrm{CH}_{2} \alpha^{\mathrm{Bn}}\right), 67.8(\mathrm{C}-5 \alpha, \mathrm{C}-5 \beta), 63.2\left(\mathrm{CH}_{2} \mathrm{CH}_{3} \alpha\right), 62.6\left(\mathrm{CH}_{2} \mathrm{CH}_{3} \beta\right), 14.0$ $\left(\mathrm{CH}_{2} \mathrm{CH}_{3} \alpha\right), 13.9\left(\mathrm{CH}_{2} \mathrm{CH}_{3} \beta\right)$ ppm. ${ }^{19} \mathrm{~F}$ NMR $\left(471 \mathrm{MHz}, \mathrm{CDCl}_{3}\right) \delta=-109.0\left(\mathrm{dd}, 1 \mathrm{~F}, J_{\mathrm{H}-}\right.$ $\left.\mathrm{Fa}=9.7 \mathrm{~Hz}, J_{\mathrm{Fa}-\mathrm{Fb}}=277.1 \mathrm{~Hz}, \mathrm{Fa} \beta\right),-111.0\left(\mathrm{dd}, 1 \mathrm{~F}, J_{\mathrm{H}-\mathrm{Fa}}=7.7 \mathrm{~Hz}, J_{\mathrm{Fa}-\mathrm{Fb}}=262.3 \mathrm{~Hz}\right.$, $\mathrm{Fa} \alpha),-115.3\left(\mathrm{dd}, 1 \mathrm{~F}, J_{\mathrm{H}-\mathrm{Fb}}=15.3 \mathrm{~Hz}, J_{\mathrm{Fa}-\mathrm{Fb}}=277.1 \mathrm{~Hz}, \mathrm{Fb} \beta\right),-118.4\left(\mathrm{dd}, 1 \mathrm{~F}, J_{\mathrm{H}-\mathrm{Fb}}=15.3\right.$ $\left.\mathrm{Hz}, J_{\mathrm{Fa}-\mathrm{Fb}}=262.3 \mathrm{~Hz}, \mathrm{Fb} \alpha\right)$ ppm. HRMS (ESI+): $\mathrm{m} / \mathrm{z}$ calculated for $\mathrm{C}_{30} \mathrm{H}_{32} \mathrm{~F}_{2} \mathrm{NaO}_{6}$ $[\mathrm{M}+\mathrm{Na}]^{+}$: calc. 549.2059; found: 549.2054. nOe ( $\beta$ anomer): Correlation between H-1 and $\mathrm{H}-2$ observed. No correlation between $\mathrm{H}-1$ and $\mathrm{H}-3$ or H-4 observed. 


\section{${ }^{1}$ H NMR}

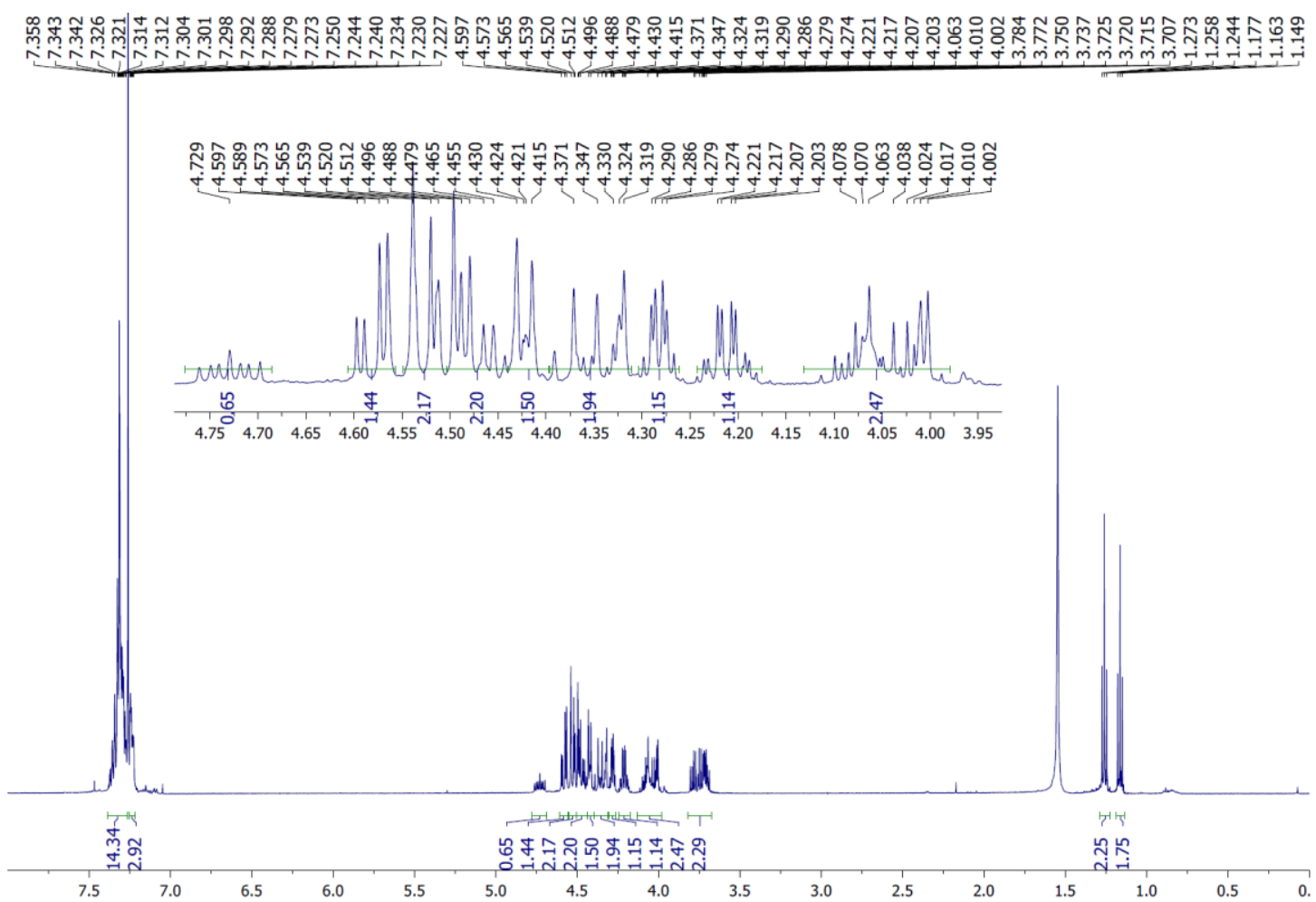

${ }^{19}$ F NMR

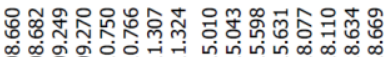

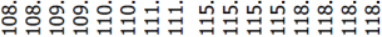

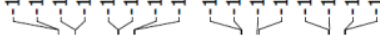

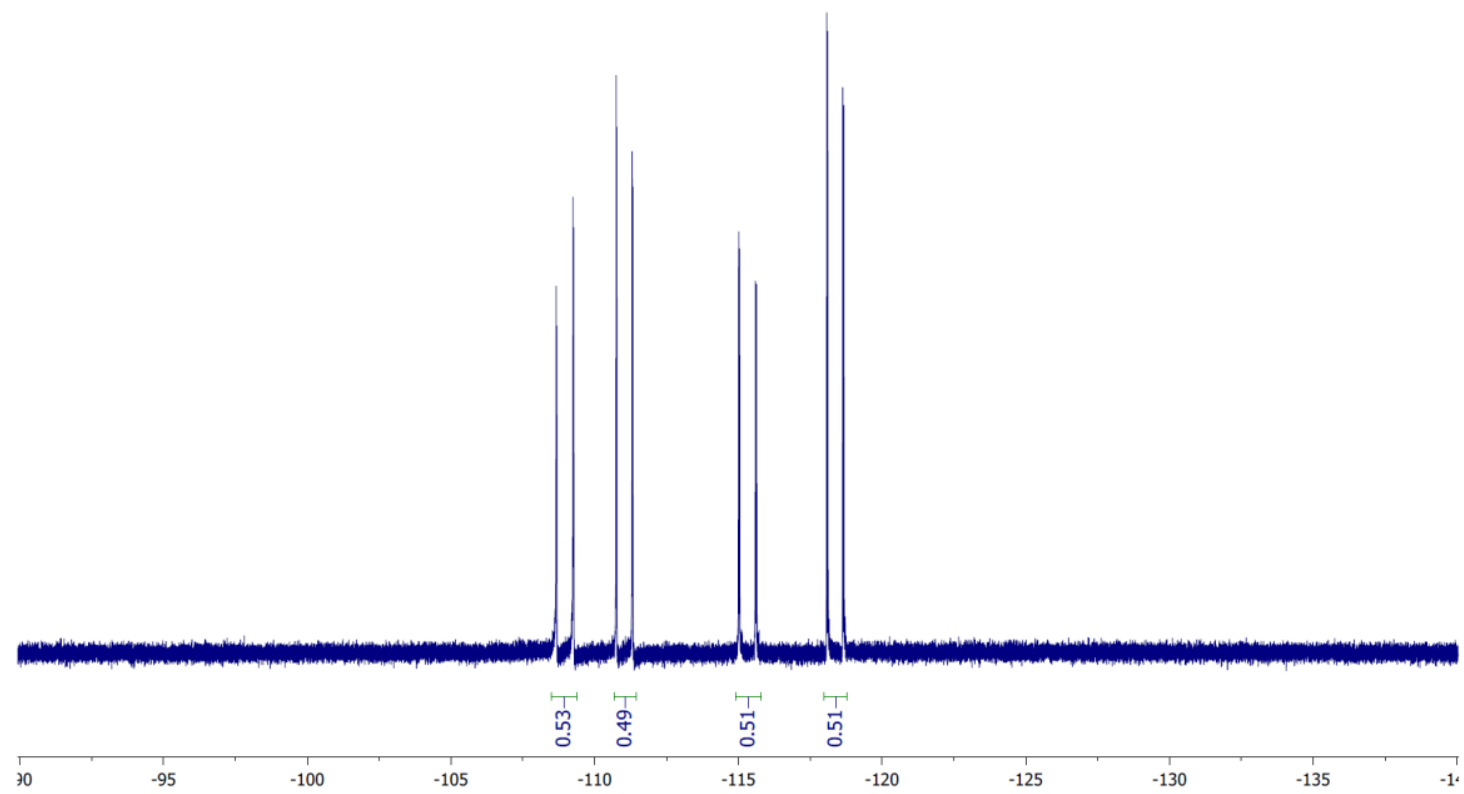


${ }^{13}$ C NMR

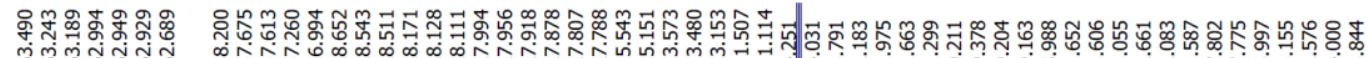

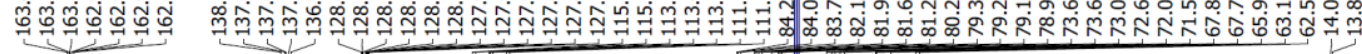
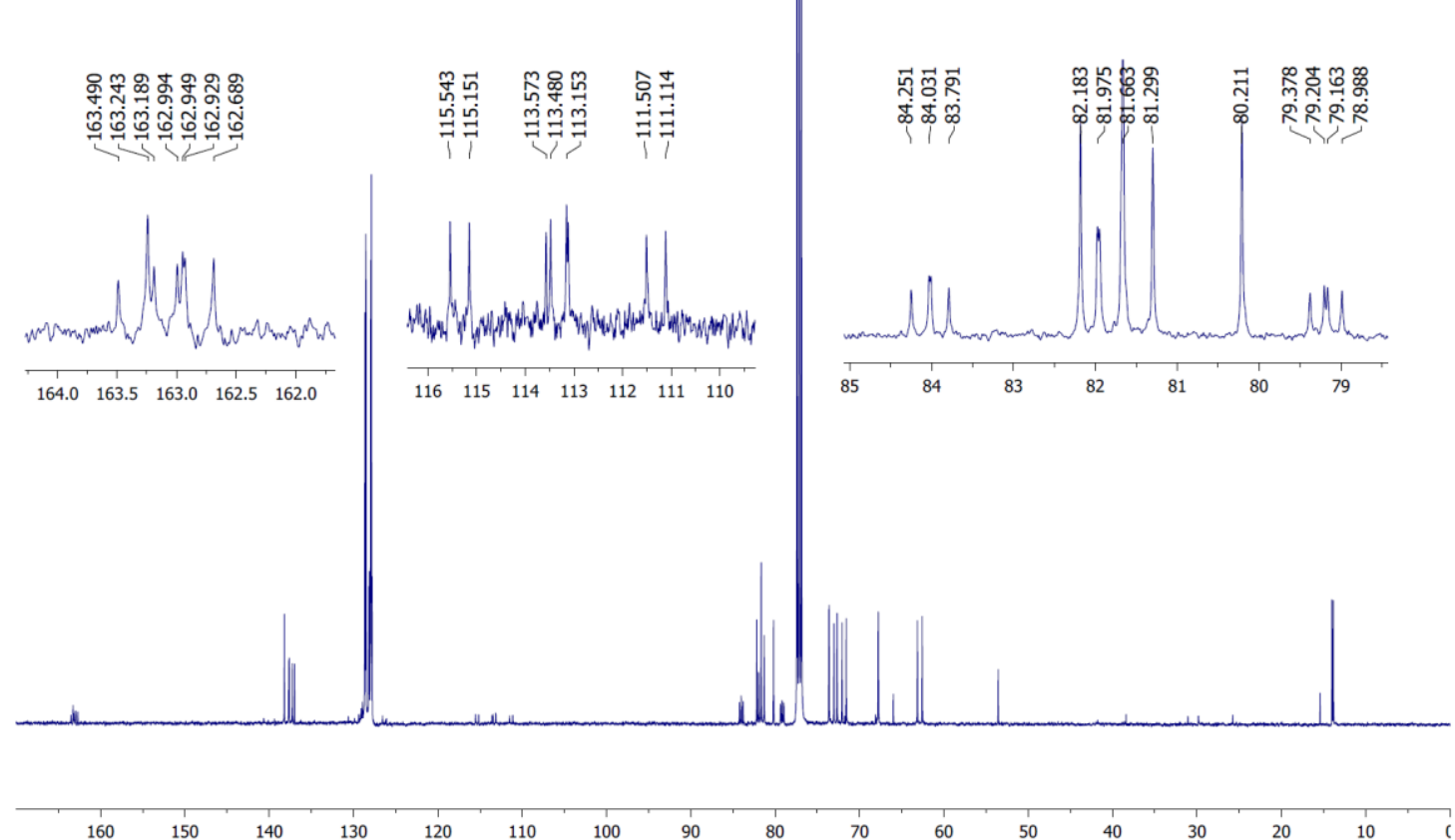


\section{D-Lyxose}

2,3,5-tri-O-benzyl-1-deoxy-1,1-difluoroethyl acetate- $\alpha, \beta$-D-lyxofuranose $((\alpha / \beta)$-5d).

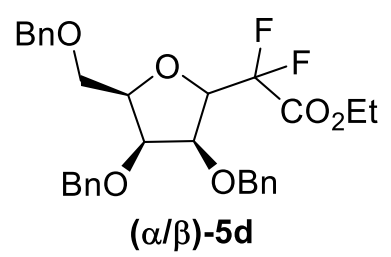

To a solution of alcohols $(R / S)-3 d(111 \mathrm{mg}, 0.175 \mathrm{mmol}, 64: 36 d r$ ratio) in dry DCM (3 $\mathrm{ml})$ under argon atmosphere was added DAST ( $92 \mu \mathrm{l}, 0.7 \mathrm{mmol}, 4.0$ equiv.) dropwise at $0^{\circ} \mathrm{C}$. The reaction was allowed to warm from $0^{\circ} \mathrm{C}$ to r.t. and the reaction was stirred for $18 \mathrm{~h}$ at r.t. The crude was then diluted with $\mathrm{CH}_{2} \mathrm{Cl}_{2}$ and the organic phase was washed with $\mathrm{H}_{2} \mathrm{O}$ ( 3 times) and brine ( 3 times), dried over $\mathrm{MgSO}_{4}$ and concentrated under reduced pressure. ${ }^{1} \mathrm{H}$ and ${ }^{19} \mathrm{~F}$ crude NMR showed that the cyclized products $(\alpha / \beta)-\mathbf{5 d}$ were formed in 51:49 $d r$ ratio with an overall conversion of $72 \%$. Purification by chromatography on silica gel (Cy/EtOAc 95:5 to 9:1) afforded pure compound $(\alpha)-5 d(16 \mathrm{mg})$ and $(\beta)-\mathbf{5 d}(22$ $\mathrm{mg}$ ) in $41 \%$ overall yield.

2,3,5-tri-O-benzyl-1-deoxy-1,1-difluoroethyl acetate- $\alpha, \beta$-D-lyxofuranose $((\alpha)$-5d).

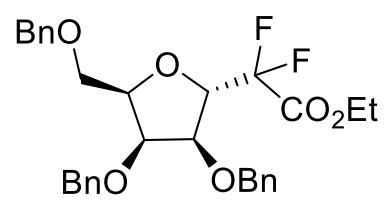

$(\alpha)-5 d$

$[\alpha]^{20} \mathrm{D}:+0.5\left(\mathrm{c} 1.0, \mathrm{CHCl}_{3}\right) .{ }^{1} \mathrm{H} \mathrm{NMR}\left(500 \mathrm{MHz}, \mathrm{CDCl}_{3}\right) \delta=7.36-7.26\left(\mathrm{~m}, 15 \mathrm{H}, \mathrm{H}^{\mathrm{arom}}\right)$, $4.69\left(\mathrm{AB}, 1 \mathrm{H}, J_{\mathrm{A}-\mathrm{B}}=11.6 \mathrm{~Hz}, \mathrm{CH}_{2}{ }^{\mathrm{Bn}}\right), 4.57\left(\mathrm{AB}, 1 \mathrm{H}, J_{\mathrm{A}-\mathrm{B}}=11.9 \mathrm{~Hz}, \mathrm{CH}_{2}{ }^{\mathrm{Bn}}\right), 4.56(\mathrm{AB}$, $\left.1 \mathrm{H}, J_{\mathrm{A}-\mathrm{B}}=11.6 \mathrm{~Hz}, \mathrm{CH}_{2}{ }^{\mathrm{Bn}}\right), 4.54-4.51\left(\mathrm{~m}, 3 \mathrm{H}, \mathrm{CH}_{2}{ }^{\mathrm{Bn}}\right), 4.50-4.47(\mathrm{~m}, 1 \mathrm{H}, \mathrm{H}-1), 4.31(\mathrm{dd}$, $\left.1 \mathrm{H}, J_{1-2}=6.2 \mathrm{~Hz}, J_{2-3}=4.6 \mathrm{~Hz}, \mathrm{H}-2\right), 4.28-4.25(\mathrm{~m}, 1 \mathrm{H}, \mathrm{H}-4), 4.27$ (ddq, $2 \mathrm{H}, J=10.1$ $\left.\mathrm{Hz}, J_{\mathrm{CH} 2 \mathrm{CH} 3}=7.1 \mathrm{~Hz}, J=3.2 \mathrm{~Hz}, \mathrm{CH}_{2} \mathrm{CH}_{3}\right), 4.11\left(\mathrm{t}, 1 \mathrm{H}, J_{2-3}=J_{3-4}=4.2 \mathrm{~Hz}, \mathrm{H}-3\right), 3.79$ $\left(\mathrm{dd}, 1 \mathrm{H}, J_{5 \mathrm{a}-5 \mathrm{~b}}=10.0 \mathrm{~Hz}, J_{4-5}=6.3 \mathrm{~Hz}, \mathrm{H}-5\right), 3.69\left(\mathrm{dd}, 1 \mathrm{H}, \mathrm{J}=J_{5 \mathrm{a}-5 \mathrm{~b}}=10.0, J_{4-5}=6.3 \mathrm{~Hz}\right.$, $\mathrm{H}-5), 1.29$ (t, 3H, $\left.J_{\mathrm{CH} 2 \mathrm{CH} 3}=7.2 \mathrm{~Hz}, \mathrm{CH}_{2} \mathrm{CH}_{3}\right) \mathrm{ppm} .{ }^{13} \mathrm{C} \mathrm{NMR}\left(125 \mathrm{MHz}, \mathrm{CDCl}_{3}\right) \delta=$ $163.0\left(\mathrm{t}, J_{\mathrm{C}-\mathrm{F}}=31.6 \mathrm{~Hz}, \mathrm{C}_{\mathrm{q}} \mathrm{O}_{2} \mathrm{Et}\right), 138.1\left(\mathrm{C}_{\mathrm{q}}{ }^{\text {arom }}\right), 138.0\left(\mathrm{C}_{\mathrm{q}}{ }^{\text {arom }}\right), 137.3\left(\mathrm{C}_{\mathrm{q}}{ }^{\text {arom }}\right), 128.6-$ $127.8\left(\mathrm{CH}^{\mathrm{arom}}\right), 114.0\left(\mathrm{dd}, J_{\mathrm{C}-\mathrm{F}}=252.6 \mathrm{~Hz}, J_{\mathrm{C}-\mathrm{F}}=256.7 \mathrm{~Hz}, \mathrm{CF}_{2}\right), 80.7(\mathrm{C}-4), 80.0(\mathrm{dd}$, $\left.J_{\mathrm{C}-\mathrm{F}}=23.7 \mathrm{~Hz}, J_{\mathrm{C}-\mathrm{F}}=29.4 \mathrm{~Hz}, \mathrm{C}-1\right), 78.66\left(\mathrm{~d}, J_{\mathrm{C}-\mathrm{F}}=2.7 \mathrm{~Hz}, \mathrm{C}-2\right), 77.3(\mathrm{C}-3), 73.64$ $\left(\mathrm{CH}_{2}{ }^{\mathrm{Bn}}\right), 73.56\left(\mathrm{CH}_{2}{ }^{\mathrm{Bn}}\right), 72.9\left(\mathrm{CH}_{2}{ }^{\mathrm{Bn}}\right), 68.1(\mathrm{C}-5), 63.2\left(\mathrm{CH}_{2} \mathrm{CH}_{3}\right), 14.0\left(\mathrm{CH}_{2} \mathrm{CH}_{3}\right) \mathrm{ppm}$. ${ }^{19} \mathrm{~F} \mathrm{NMR}\left(471 \mathrm{MHz}, \mathrm{CDCl}_{3}\right) \delta=-115.1\left(\mathrm{dd}, 1 \mathrm{~F}, J_{\mathrm{H}-\mathrm{Fa}}=8.1 \mathrm{~Hz}, J_{\mathrm{Fa}-\mathrm{Fb}}=259.9 \mathrm{~Hz}, \mathrm{Fa}\right)$, $120.5\left(\mathrm{dd}, 1 \mathrm{~F}, J_{\mathrm{H}-\mathrm{Fb}}=16.4 \mathrm{~Hz}, J_{\mathrm{Fa}-\mathrm{Fb}}=259.9 \mathrm{~Hz}, \mathrm{Fb}\right) \mathrm{ppm}$. HRMS (ESI+): $\mathrm{m} / \mathrm{z}$ calculated for $\mathrm{C}_{30} \mathrm{H}_{33} \mathrm{~F}_{2} \mathrm{O}_{6}[\mathrm{M}+\mathrm{H}]^{+}$: calc. 527.2240; found: 527.2235. nOe: Correlations were inconclusive. 


\section{${ }^{1} \mathrm{H}$ NMR}

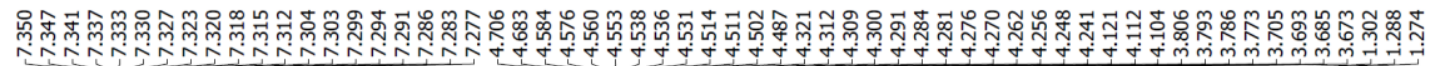

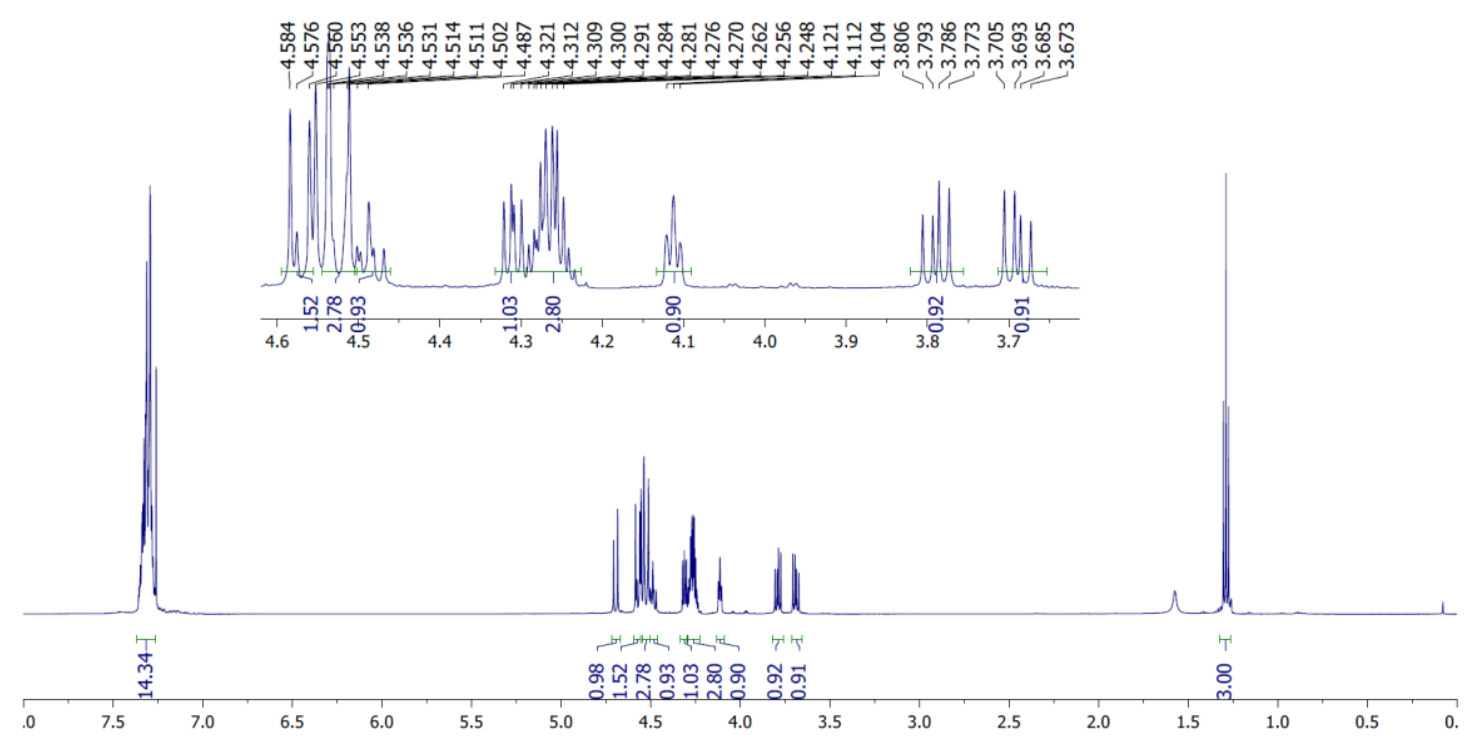

${ }^{19}$ F NMR

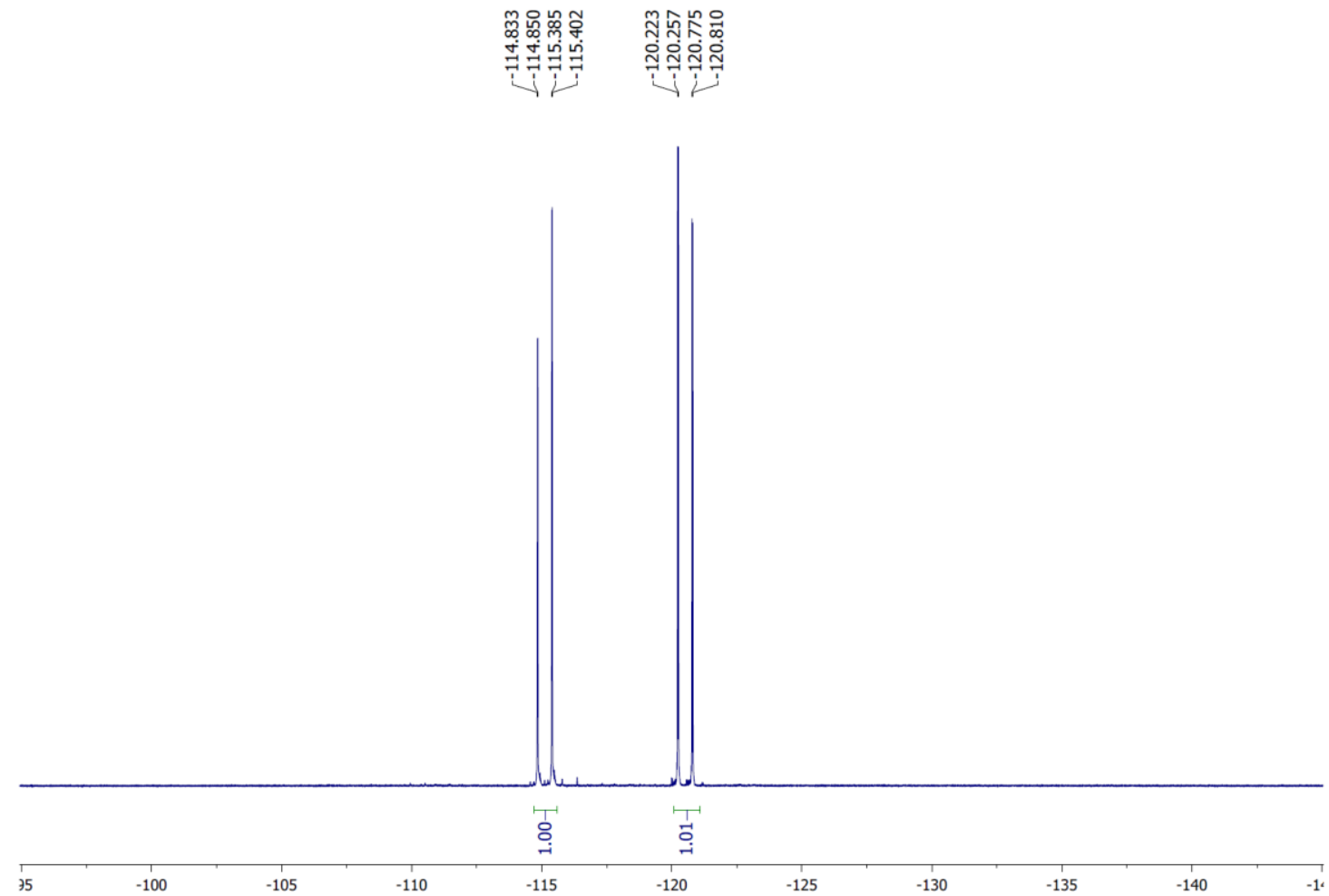


${ }^{13}$ C NMR

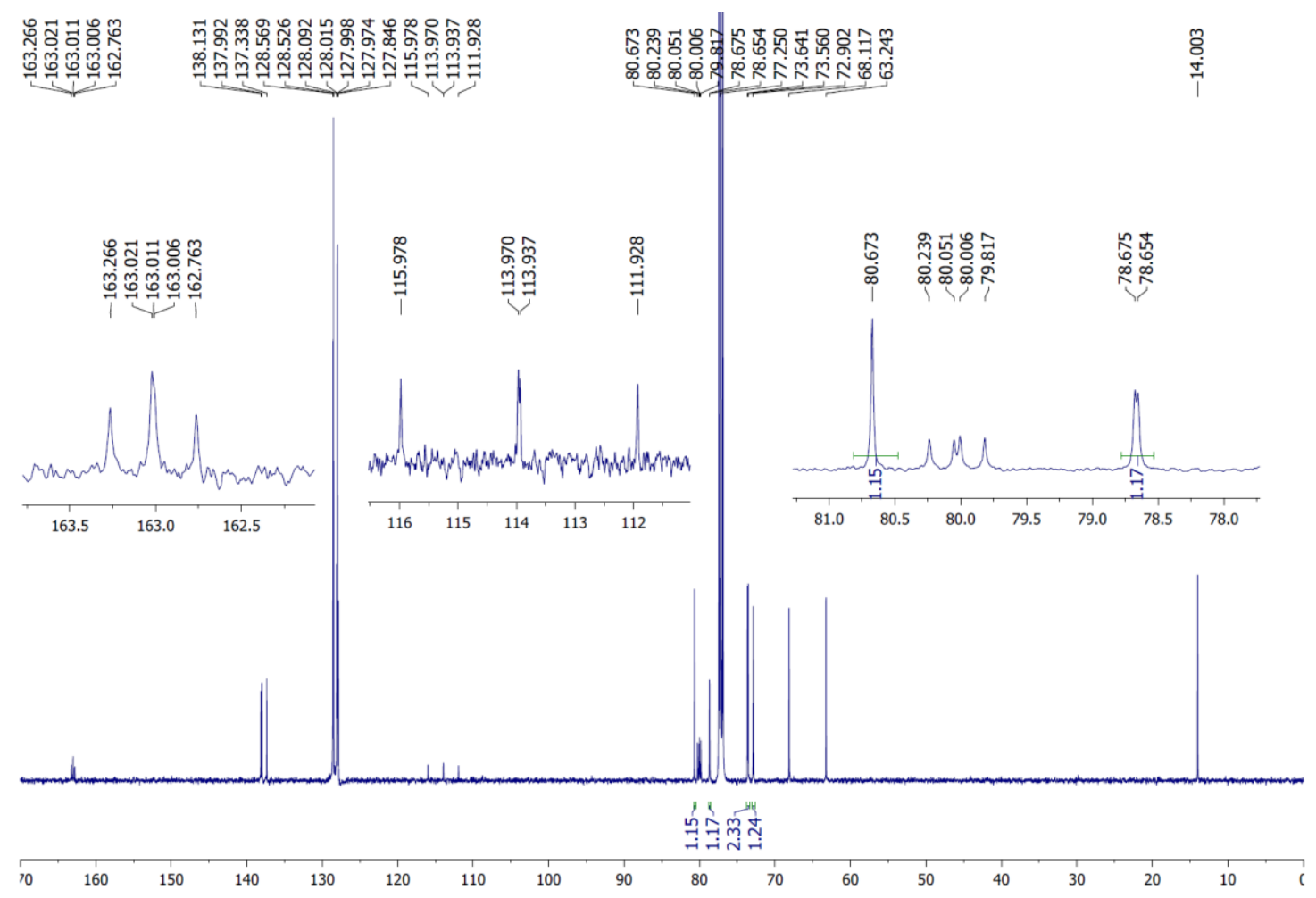


2,3,5-tri-O-benzyl-1-deoxy-1,1-difluoroethyl acetate- $\alpha, \beta$-D-lyxofuranose (( $\beta)$-5d).

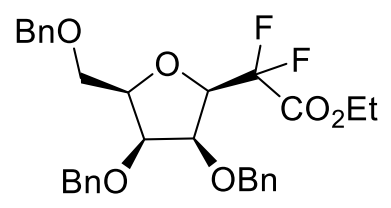

$(\beta)-5 d$

$[\alpha]^{20} \mathrm{D}:-0.2\left(\mathrm{c} 1.0, \mathrm{CHCl}_{3}\right) .{ }^{1} \mathrm{H} \mathrm{NMR}\left(500 \mathrm{MHz}, \mathrm{CDCl}_{3}\right) \delta=7.34-7.25\left(\mathrm{~m}, 15 \mathrm{H}, \mathrm{H}^{\text {arom }}\right)$, $4.75\left(\mathrm{AB}, 1 \mathrm{H}, J_{\mathrm{A}-\mathrm{B}}=11.4 \mathrm{~Hz}, \mathrm{CH}_{2}{ }^{\mathrm{Bn}}\right), 4.64\left(\mathrm{brs}, 2 \mathrm{H}, \mathrm{CH}_{2}{ }^{\mathrm{Bn}}\right), 4.61\left(\mathrm{AB}, 1 \mathrm{H}, J_{\mathrm{A}-\mathrm{B}}=11.9\right.$ $\left.\mathrm{Hz}, \mathrm{CH}_{2}{ }^{\mathrm{Bn}}\right), 4.63-4.56(\mathrm{~m}, 1 \mathrm{H}, \mathrm{H}-1), 4.51\left(\mathrm{AB}, 1 \mathrm{H}, J_{\mathrm{A}-\mathrm{B}}=11.9 \mathrm{~Hz}, \mathrm{CH}_{2}{ }^{\mathrm{Bn}}\right), 4.46(\mathrm{AB}$, $\left.1 \mathrm{H}, J_{\mathrm{A}-\mathrm{B}}=11.3 \mathrm{~Hz}, \mathrm{CH}_{2}{ }^{\mathrm{Bn}}\right), 4.34\left(\mathrm{q}, 1 \mathrm{H}, J_{3-4}=J_{4-5}=6.0 \mathrm{~Hz}, \mathrm{H}-4\right), 4.31\left(\mathrm{dd}, 1 \mathrm{H}, J_{1-2}=6.2\right.$ $\left.\mathrm{Hz}, J_{2-3}=4.6 \mathrm{~Hz}, \mathrm{H}-2\right), 4.16\left(\mathrm{dd}, 1 \mathrm{H}, J_{3-4}=5.9 \mathrm{~Hz}, J_{2-3}=4.7 \mathrm{~Hz}, \mathrm{H}-3\right), 4.06(\mathrm{dq}, 1 \mathrm{H}$, $\left.J_{\mathrm{CH} 2 \mathrm{CH} 3}=10.8 \mathrm{~Hz}, J_{\mathrm{CH} 2 \mathrm{CH} 3}=7.2 \mathrm{~Hz}, \mathrm{CH}_{2} \mathrm{CH}_{3}\right), 3.94-3.87\left(\mathrm{~m}, 2 \mathrm{H}, \mathrm{CH}_{2} \mathrm{CH}_{3}\right.$ and $\left.\mathrm{H}-5\right), 3.82$ $\left(\mathrm{dd}, 1 \mathrm{H}, J_{5 \mathrm{a}-5 \mathrm{~b}}=10.5 \mathrm{~Hz}, J_{4-5}=6.5 \mathrm{~Hz}, \mathrm{H}-5\right), 1.13\left(\mathrm{t}, 3 \mathrm{H}, J_{\mathrm{CH} 2 \mathrm{CH} 3}=7.2 \mathrm{~Hz}, \mathrm{CH}_{2} \mathrm{CH}_{3}\right)$ ppm. ${ }^{13} \mathrm{C} \mathrm{NMR}\left(125 \mathrm{MHz}, \mathrm{CDCl}_{3}\right) \delta=163.3\left(\mathrm{t}, J_{\mathrm{C}-\mathrm{F}}=30.9 \mathrm{~Hz}, \mathrm{C}_{\mathrm{q}} \mathrm{O}_{2} \mathrm{Et}\right), 138.4\left(\mathrm{C}_{\mathrm{q}}{ }^{\text {arom }}\right)$, $137.9\left(\mathrm{C}_{\mathrm{q}}{ }^{\text {arom }}\right), 137.5\left(\mathrm{C}_{\mathrm{q}}^{\text {arom }}\right), 128.5-127.6\left(\mathrm{CH}^{\text {arom }}\right), 112.9\left(\mathrm{dd}, J_{\mathrm{C}-\mathrm{F}}=247.5 \mathrm{~Hz}, J_{\mathrm{C}-\mathrm{F}}=\right.$ $\left.261.7 \mathrm{~Hz}, \mathrm{CF}_{2}\right), 79.7(\mathrm{C}-4), 78.44\left(\mathrm{dd}, J_{\mathrm{C}-\mathrm{F}}=23.1 \mathrm{~Hz}, J_{\mathrm{C}-\mathrm{F}}=26.3 \mathrm{~Hz}, \mathrm{C}-1\right), 78.36(\mathrm{C}-3)$, $78.3\left(\mathrm{~d}, J_{\mathrm{C}-\mathrm{F}}=3.5 \mathrm{~Hz}, \mathrm{C}-2\right), 73.9\left(\mathrm{CH}_{2}{ }^{\mathrm{Bn}}\right), 73.6\left(\mathrm{CH}_{2}{ }^{\mathrm{Bn}}\right), 73.4\left(\mathrm{CH}_{2}{ }^{\mathrm{Bn}}\right), 69.4(\mathrm{C}-5), 62.6$ $\left(\mathrm{CH}_{2} \mathrm{CH}_{3}\right), 13.8\left(\mathrm{CH}_{2} \mathrm{CH}_{3}\right)$ ppm. ${ }^{19} \mathrm{~F} \mathrm{NMR}\left(471 \mathrm{MHz}, \mathrm{CDCl}_{3}\right) \delta=-108.9\left(\mathrm{dd}, 1 \mathrm{~F}, J_{\mathrm{H}-\mathrm{Fa}}=\right.$ $\left.11.2 \mathrm{~Hz}, J_{\mathrm{Fa}-\mathrm{Fb}}=273.1 \mathrm{~Hz}, \mathrm{Fa}\right),-114.9\left(\mathrm{dd}, 1 \mathrm{~F}, J_{\mathrm{H}-\mathrm{Fb}}=14.4 \mathrm{~Hz}, J_{\mathrm{Fa}-\mathrm{Fb}}=273.2 \mathrm{~Hz}, \mathrm{Fb}\right)$ ppm. HRMS (ESI+): $\mathrm{m} / \mathrm{z}$ calculated for $\mathrm{C}_{30} \mathrm{H}_{33} \mathrm{~F}_{2} \mathrm{O}_{6}[\mathrm{M}+\mathrm{H}]^{+}$: calc. 527.2240; found: 527.2237. nOe: Correlation between $\mathrm{H}-1$ and $\mathrm{H}-3$ observed. 


\section{${ }^{1}$ H NMR}

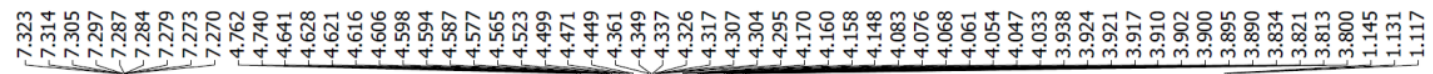

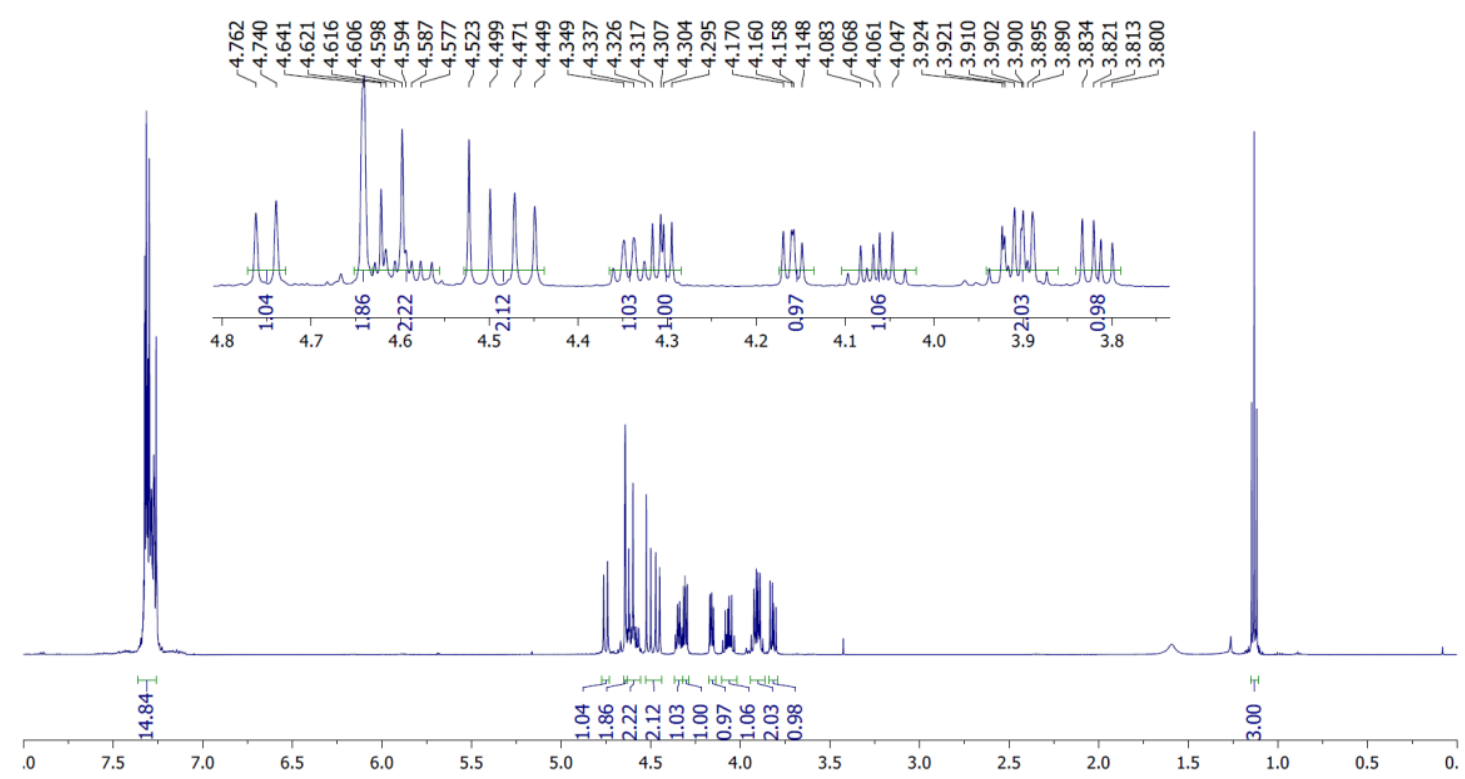

${ }^{19}$ F NMR

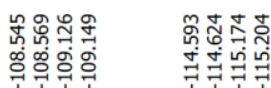

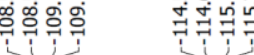

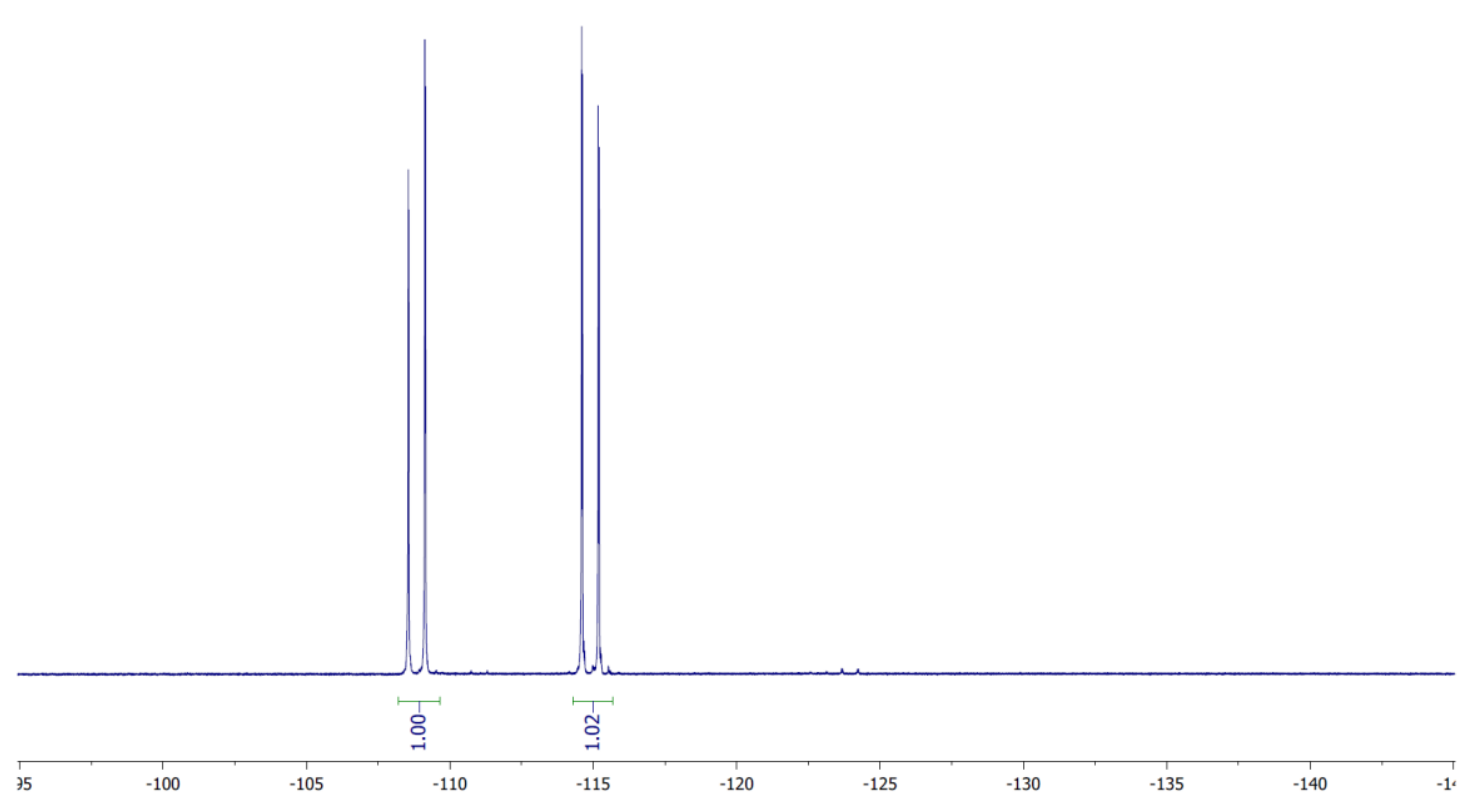


${ }^{13}$ C NMR
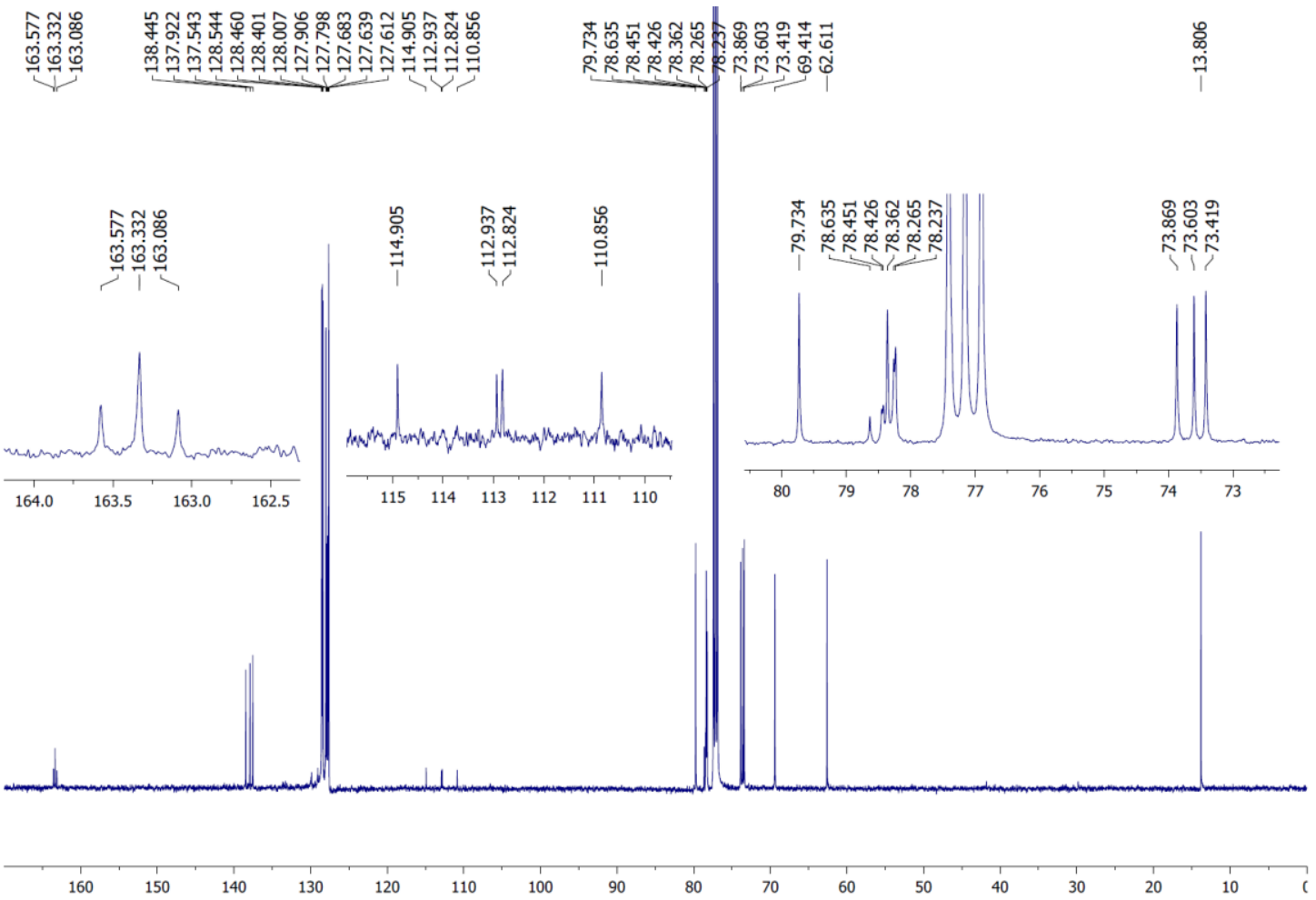


\section{L-Fucose}

5-methyl-2,3,5-tri-O-benzyl-1-deoxy-1,1-difluoroethyl acetate- $\alpha, \beta$-D-fucofuranose (( $\alpha)$ $5 e)$.<smiles>[B]OC(C)C1O[C@H](C(F)(F)OCC)[C@H](OCc2ccccc2)C1Oc1ccccc1</smiles>

$(\alpha)-5 e$

To a solution of alcohol $(R)-3 e(58.2 \mathrm{mg}, 0.09 \mathrm{mmol})$ in dry DCM $(2 \mathrm{ml})$ under argon atmosphere was added DAST ( $48 \mu 1,0.36 \mathrm{mmol}, 4.0$ equiv.) dropwise at $0^{\circ} \mathrm{C}$. The reaction was allowed to warm from $0^{\circ} \mathrm{C}$ to r.t. and the reaction was stirred for $18 \mathrm{~h}$ at r.t. The crude was then diluted with $\mathrm{CH}_{2} \mathrm{Cl}_{2}$ and the organic phase was washed with $\mathrm{H}_{2} \mathrm{O}$ (3 times) and brine (3 times), dried over $\mathrm{MgSO}_{4}$ and concentrated under reduced pressure. Purification by chromatography on silica gel (Cy/EtOAc 95:5) afforded pure compound $(\alpha)-5 e(26 \mathrm{mg}, 0.048 \mathrm{mmol}, 53 \%)$ as a colorless oil.

$[\alpha]_{20}{ }^{\mathrm{D}}:+2.9\left(\mathrm{c} 1.0, \mathrm{CHCl}_{3}\right) .{ }^{1} \mathrm{H} \mathrm{NMR}\left(500 \mathrm{MHz}, \mathrm{CDCl}_{3}\right) \delta=7.37-7.27\left(\mathrm{~m}, 13 \mathrm{H}, \mathrm{H}^{\text {arom }}\right)$, 7.22-7.20 (m, $\left.2 \mathrm{H}, \mathrm{H}^{\text {arom }}\right), 4.61\left(\mathrm{AB}, 1 \mathrm{H}, J_{\mathrm{A}-\mathrm{B}}=12.0 \mathrm{~Hz}, \mathrm{CH}_{2}{ }^{\mathrm{Bn}}\right), 4.60\left(\mathrm{AB}, 1 \mathrm{H}, J_{\mathrm{A}-\mathrm{B}}=\right.$ $\left.12.0 \mathrm{~Hz}, \mathrm{CH}_{2}{ }^{\mathrm{Bn}}\right), 4.54-4.41\left(\mathrm{~m}, 5 \mathrm{H}, \mathrm{H}-1, \mathrm{H}-2, \mathrm{CH}_{2}{ }^{\mathrm{Bn}}\right), 4.39\left(\mathrm{AB}, 1 \mathrm{H}, J_{\mathrm{A}-\mathrm{B}}=11.5 \mathrm{~Hz}\right.$, $\mathrm{CH}_{2}{ }^{\mathrm{Bn}}$ ), 4.31 (qd, $\left.2 \mathrm{H}, J=2.3 \mathrm{~Hz}, J_{\mathrm{CH} 2-\mathrm{CH} 3}=7.5 \mathrm{~Hz}, \mathrm{CH}_{2} \mathrm{CH}_{3}\right), 4.19$ (dd, $1 \mathrm{H}, J=4.0 \mathrm{~Hz}$, $\left.J_{3-4}=6.9 \mathrm{~Hz}, \mathrm{H}-3\right), 3.99\left(\mathrm{ddd}, 1 \mathrm{H}, J=2.3 \mathrm{~Hz}, J_{4-5}=4.0 \mathrm{~Hz}, J_{3-4}=6.9 \mathrm{~Hz}, \mathrm{H}-4\right), 3.62$ (qd, $\left.1 \mathrm{H}, J_{4-5}=4.0 \mathrm{~Hz}, J_{5-\mathrm{CH} 3}=6.3 \mathrm{~Hz}, \mathrm{H}-5\right), 1.32\left(\mathrm{t}, 3 \mathrm{H}, J_{\mathrm{CH} 2-\mathrm{CH} 3}=7.5 \mathrm{~Hz}, \mathrm{CH}_{2} \mathrm{CH}_{3}\right), 1.21(\mathrm{~d}$, $\left.3 \mathrm{H}, J_{5-\mathrm{CH} 3}=6.3 \mathrm{~Hz}, \mathrm{CH}_{3}\right) \mathrm{ppm} .{ }^{13} \mathrm{C} \mathrm{NMR}\left(125 \mathrm{MHz}, \mathrm{CDCl}_{3}\right) \delta=163.1\left(\mathrm{dd}, J_{\mathrm{C}-\mathrm{F}}=30.8\right.$ $\left.\mathrm{Hz}, J_{\mathrm{C}-\mathrm{F}}=33.2 \mathrm{~Hz}, \mathrm{C}_{\mathrm{q}} \mathrm{O}_{2} \mathrm{Et}\right), 138.5\left(\mathrm{C}_{\mathrm{q}}{ }^{\text {arom }}\right), 137.8\left(\mathrm{C}_{\mathrm{q}}{ }^{\text {arom }}\right), 137.5\left(\mathrm{C}_{\mathrm{q}}{ }^{\text {arom }}\right), 128.6-127.7$ $\left(\mathrm{CH}^{\text {arom }}\right), 114.0\left(\mathrm{dd}, J_{\mathrm{C}-\mathrm{F}}=252.8 \mathrm{~Hz}, J_{\mathrm{C}-\mathrm{F}}=260.2 \mathrm{~Hz}, \mathrm{CF}_{2}\right), 86.0(\mathrm{C}-4), 84.4(\mathrm{C}-3), 83.8$ $(\mathrm{C}-2), 82.1\left(\mathrm{dd}, J_{\mathrm{C}-\mathrm{F}}=24.1 \mathrm{~Hz}, J_{\mathrm{C}-\mathrm{F}}=32.6 \mathrm{~Hz}, \mathrm{C}-1\right), 73.1(\mathrm{C}-5), 72.3\left(2 \mathrm{CH}_{2}{ }^{\mathrm{Bn}}\right), 71.3$ $\left(\mathrm{CH}_{2}{ }^{\mathrm{Bn}}\right), 63.2\left(\mathrm{CH}_{2} \mathrm{CH}_{3}\right), 15.9\left(\mathrm{CH}_{3}\right), 14.1\left(\mathrm{CH}_{2} \mathrm{CH}_{3}\right) \mathrm{ppm} .{ }^{19} \mathrm{~F} \mathrm{NMR}\left(471 \mathrm{MHz}, \mathrm{CDCl}_{3}\right)$ $\delta=-110.6\left(\mathrm{dd}, 1 \mathrm{~F}, J_{\mathrm{H}-\mathrm{Fa}}=4.5 \mathrm{~Hz}, J_{\mathrm{Fa}-\mathrm{Fb}}=262.9 \mathrm{~Hz}, \mathrm{Fa}\right),-120.3\left(\mathrm{dd}, 1 \mathrm{~F}, J_{\mathrm{H}-\mathrm{Fb}}=22.3 \mathrm{~Hz}\right.$, $\left.J_{\mathrm{Fa}-\mathrm{Fb}}=262.9 \mathrm{~Hz}, \mathrm{Fb}\right)$ ppm. HRMS (ESI+): m/z calculated for $\mathrm{C}_{31} \mathrm{H}_{35} \mathrm{~F}_{2} \mathrm{O}_{6}[\mathrm{M}+\mathrm{H}]^{+}$: calc. 541.2396; found: 541.2395. nOe: Correlations were inconclusive. 


\section{${ }^{1}$ H NMR}

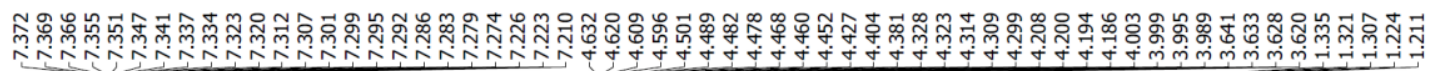

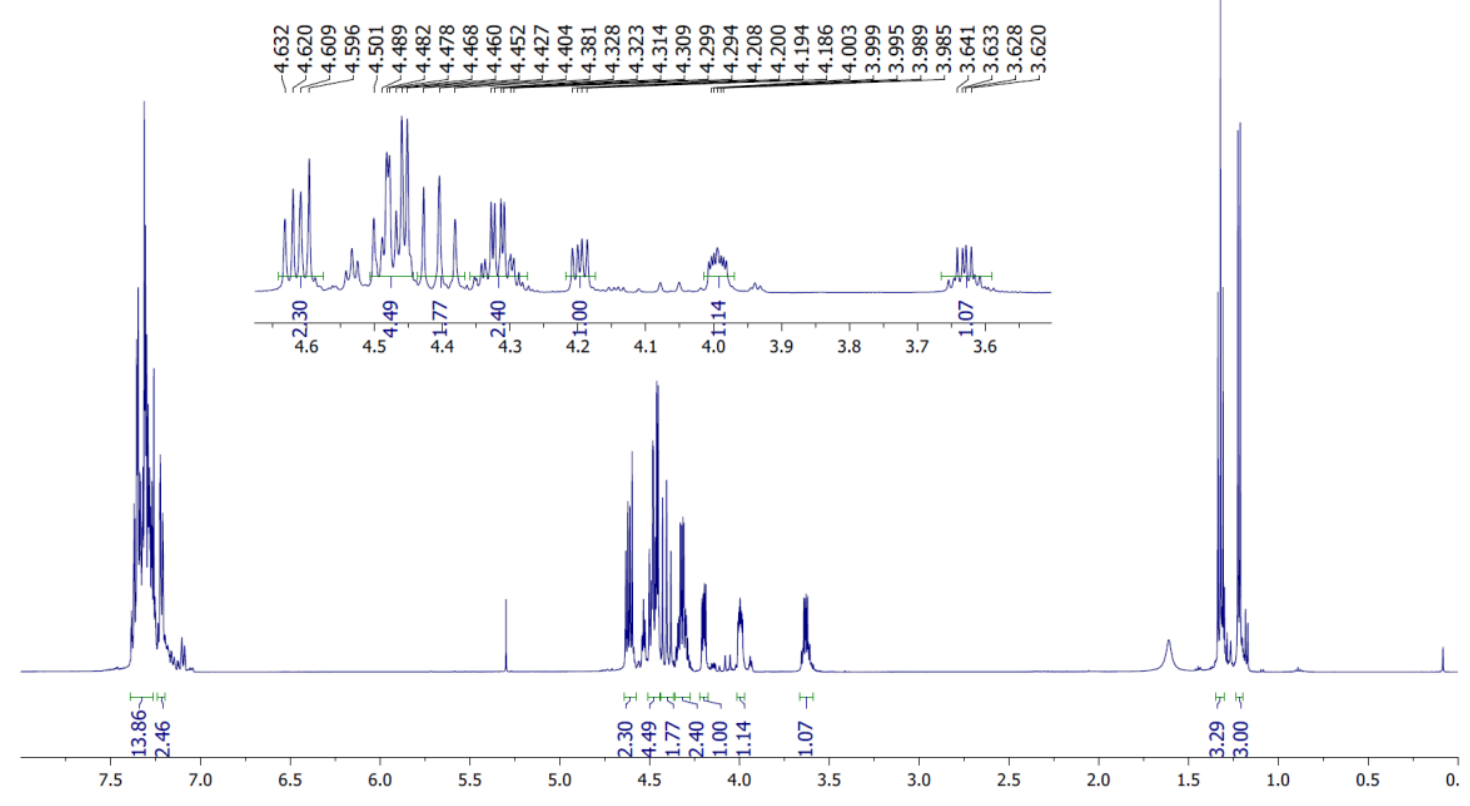

${ }^{19}$ F NMR
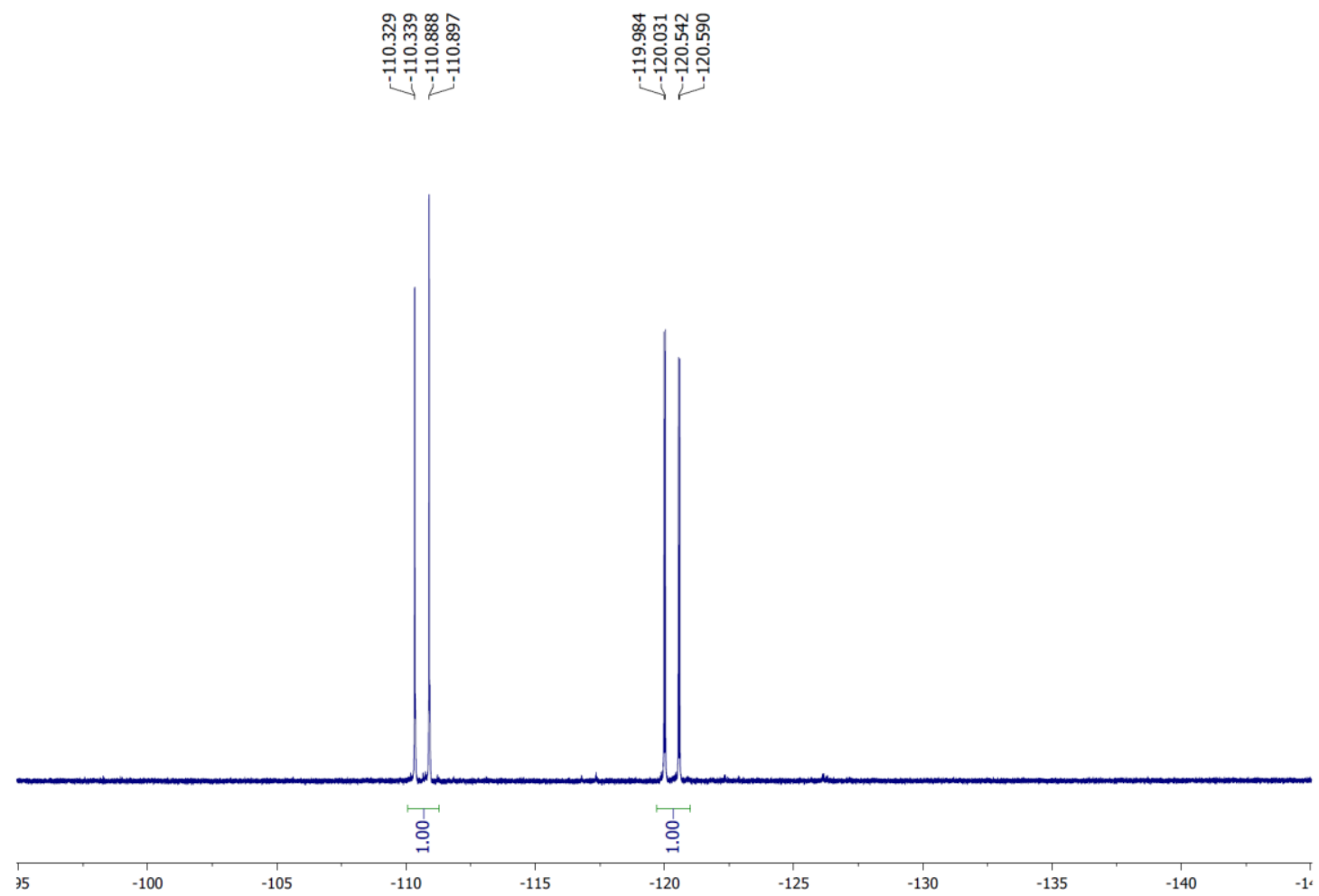
${ }^{13}$ C NMR

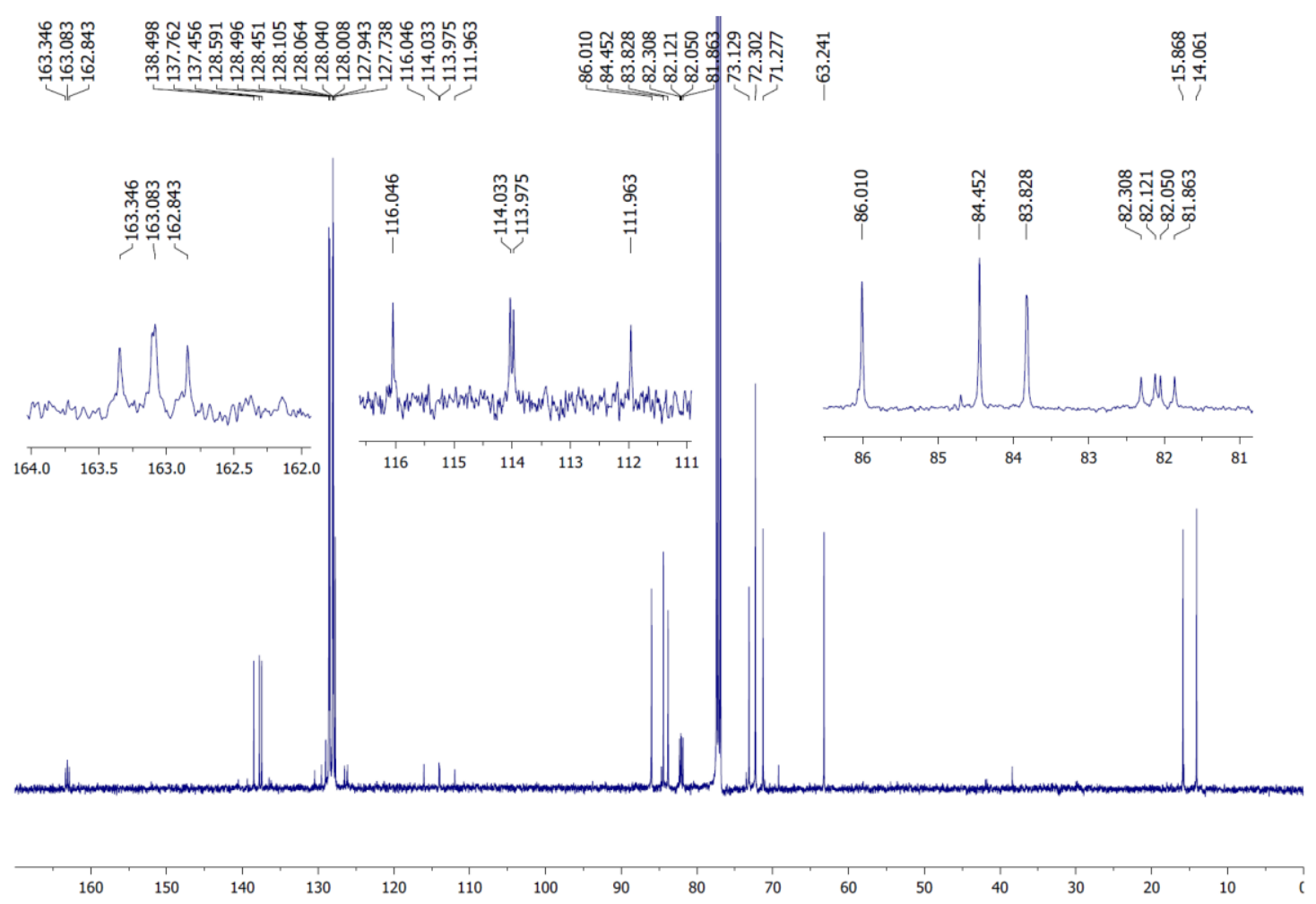



fucofuranose $((\beta)-6 \mathrm{e})$.<smiles>CCOC(F)(F)C1OC(C(C)O)C(O)C1OCc1ccccc1</smiles>

$(\beta)-6 e$

To a solution of alcohol $(S)-3 e(52.6 \mathrm{mg}, 0.081 \mathrm{mmol})$ in dry DCM $(2 \mathrm{ml})$ under argon atmosphere was added DAST ( $43 \mu \mathrm{l}, 0.32 \mathrm{mmol}, 4.0$ equiv.) dropwise at $0^{\circ} \mathrm{C}$. The reaction was allowed to warm from $0^{\circ} \mathrm{C}$ to r.t. and the reaction was stirred for $18 \mathrm{~h}$ at r.t. The crude was then diluted with $\mathrm{CH}_{2} \mathrm{Cl}_{2}$ and the organic phase was washed with $\mathrm{H}_{2} \mathrm{O}$ (3 times) and brine (3 times), dried over $\mathrm{MgSO}_{4}$ and concentrated under reduced pressure. Purification by chromatography on silica gel (Cy/EtOAc 95:5) afforded pure compound $(\beta)-5 e(11 \mathrm{mg}, 0.0203 \mathrm{mmol}, 25 \%)$ as a colorless oil and a side product $(\beta)-6 \mathbf{e}(13 \mathrm{mg}$, $0.029 \mathrm{mmol}, 36 \%)$ identified as similar to $(\beta)-5 \mathbf{e}$ with a free alcohol in C-5.

$[\alpha]_{20}{ }^{\mathrm{D}}:-4.5\left(\mathrm{c} 1.0, \mathrm{CHCl}_{3}\right) .{ }^{1} \mathrm{H}$ NMR $\left(500 \mathrm{MHz}, \mathrm{CDCl}_{3}\right) \delta=7.38-7.27\left(\mathrm{~m}, 10 \mathrm{H}, \mathrm{H}^{\text {arom }}\right)$, $4.64\left(\mathrm{td}, 1 \mathrm{H}, J_{1-2}=5.2 \mathrm{~Hz}, J_{1-\mathrm{Fa}}=J_{1-\mathrm{Fb}}=10.9 \mathrm{~Hz}, \mathrm{H}-1\right), 4.53\left(\mathrm{AB}, 1 \mathrm{H}, J_{\mathrm{A}-\mathrm{B}}=12.0 \mathrm{~Hz}\right.$, $\left.\mathrm{CH}_{2}{ }^{\mathrm{Bn}}\right), 4.50-4.47\left(\mathrm{~m}, 3 \mathrm{H}, \mathrm{CH}_{2}{ }^{\mathrm{Bn}}\right), 4.26\left(\mathrm{dd}, 1 \mathrm{H}, J_{2-3}=2.3 \mathrm{~Hz}, J_{1-2}=5.2 \mathrm{~Hz}, \mathrm{H}-2\right), 4.06$ $\left(\mathrm{qd}, 2 \mathrm{H}, J=2.9 \mathrm{~Hz}, J_{\mathrm{CH} 2-\mathrm{CH} 3}=7.5 \mathrm{~Hz}, \mathrm{CH}_{2} \mathrm{CH}_{3}\right), 3.98(\mathrm{~m}, 1 \mathrm{H}, \mathrm{H}-3), 3.88-3.81(\mathrm{~m}, 2 \mathrm{H}$, $\mathrm{H}-4, \mathrm{H}-5), 1.19\left(\mathrm{t}, 3 \mathrm{H}, J_{\mathrm{CH} 2-\mathrm{CH} 3}=7.5 \mathrm{~Hz}, \mathrm{CH}_{2} \mathrm{CH}_{3}\right), 1.16\left(\mathrm{~d}, 3 \mathrm{H}, J_{5-\mathrm{CH} 3}=6.3 \mathrm{~Hz}, \mathrm{CH}_{3}\right)$ ppm. ${ }^{13} \mathrm{C} \mathrm{NMR}\left(125 \mathrm{MHz}, \mathrm{CDCl}_{3}\right) \delta=163.1\left(\mathrm{t}, J_{\mathrm{C}-\mathrm{F}}=30.7 \mathrm{~Hz}, \mathrm{C}_{\mathrm{q}} \mathrm{O}_{2} \mathrm{Et}\right), 137.3\left(\mathrm{C}_{\mathrm{q}}^{\text {arom }}\right)$, $136.7\left(\mathrm{C}_{\mathrm{q}}{ }^{\text {arom }}\right), 128.8-127.8\left(\mathrm{CH}^{\mathrm{arom}}\right), 113.0\left(\mathrm{t}, J_{\mathrm{C}-\mathrm{F}}=253.5 \mathrm{~Hz}, \mathrm{CF}_{2}\right), 88.5(\mathrm{C}-4), 82.7(\mathrm{C}-$ 2), $82.3(\mathrm{C}-3), 79.8\left(\mathrm{t}, J_{\mathrm{C}-\mathrm{F}}=27.1 \mathrm{~Hz}, \mathrm{C}-1\right), 73.0\left(\mathrm{CH}_{2}{ }^{\mathrm{Bn}}\right), 72.2\left(\mathrm{CH}_{2}{ }^{\mathrm{Bn}}\right), 67.2(\mathrm{C}-5), 62.9$ $\left(\mathrm{CH}_{2} \mathrm{CH}_{3}\right), 19.2\left(\mathrm{CH}_{3}\right), 13.9\left(\mathrm{CH}_{2} \mathrm{CH}_{3}\right) \mathrm{ppm} .{ }^{19} \mathrm{~F} \mathrm{NMR}\left(471 \mathrm{MHz}, \mathrm{CDCl}_{3}\right) \delta=-111.1(\mathrm{dd}$, $\left.1 \mathrm{~F}, J_{\mathrm{H} 1-\mathrm{Fa}}=10.9 \mathrm{~Hz}, J_{\mathrm{Fa}-\mathrm{Fb}}=278.0 \mathrm{~Hz}, \mathrm{Fa}\right),-111.8\left(\mathrm{dd}, 1 \mathrm{~F}, J_{\mathrm{H} 1-\mathrm{Fb}}=10.9 \mathrm{~Hz}, J_{\mathrm{Fa}-\mathrm{Fb}}=278.0\right.$ $\mathrm{Hz}, \mathrm{Fb}$ ) ppm. HRMS (ESI+): m/z calculated for $\mathrm{C}_{24} \mathrm{H}_{29} \mathrm{~F}_{2} \mathrm{O}_{6}[\mathrm{M}+\mathrm{H}]^{+}$: calc. 451.1927; found: 451.1926. nOe: Correlation between $\mathrm{H}-1$ and $\mathrm{H}-4$ observed. 


\section{${ }^{1}$ H NMR}

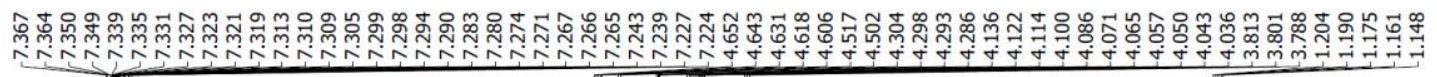

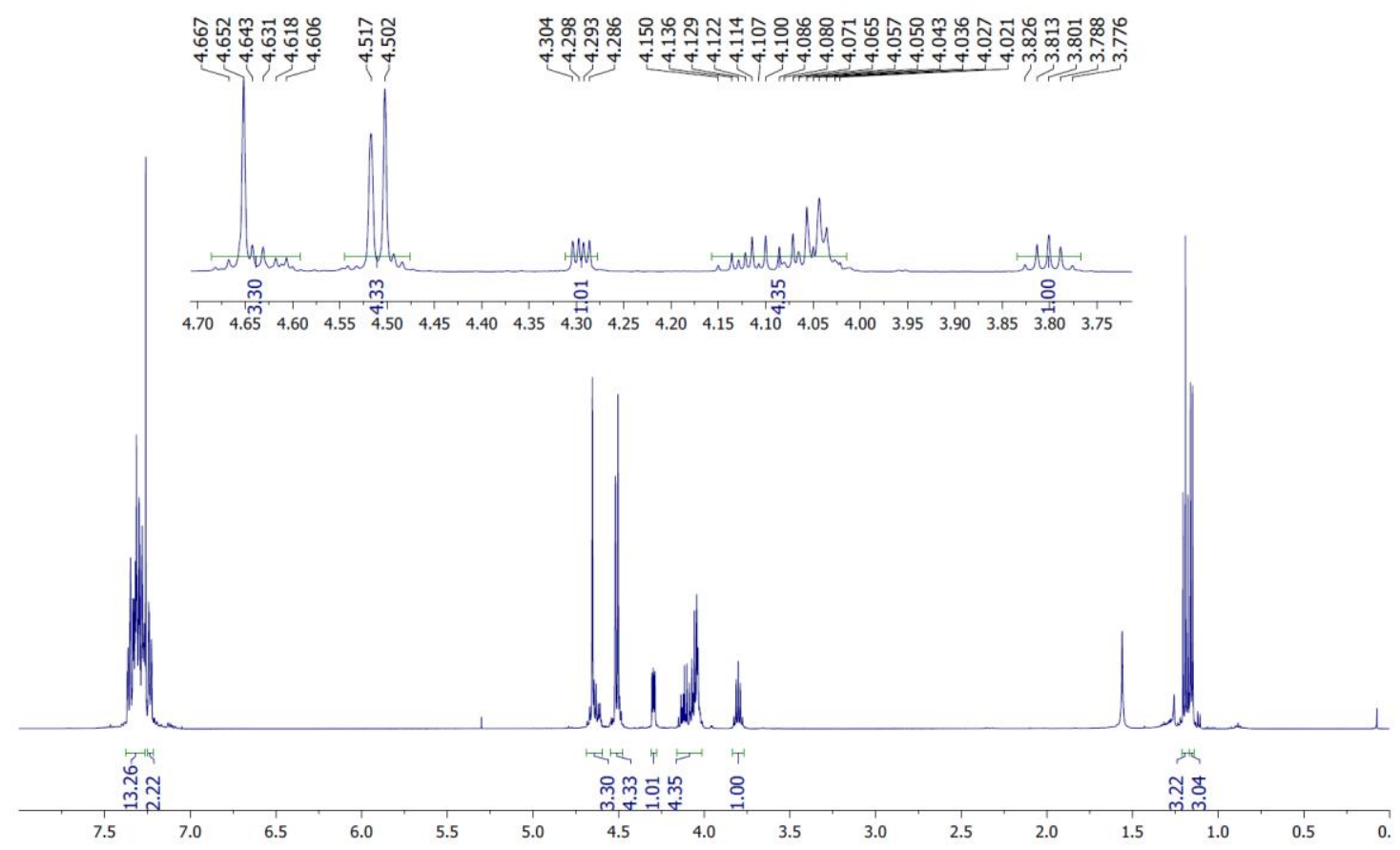

${ }^{19}$ F NMR
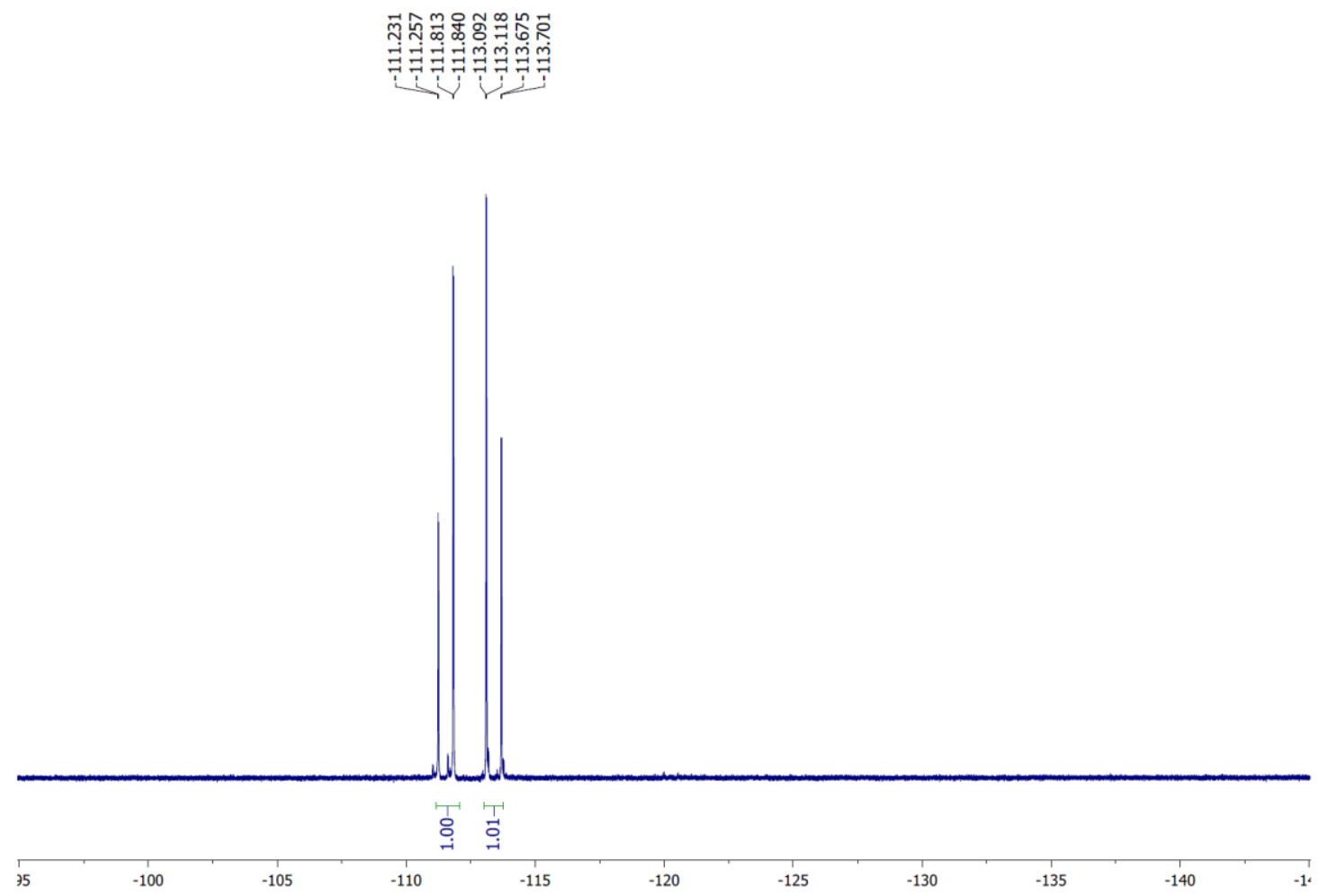
${ }^{13}$ C NMR

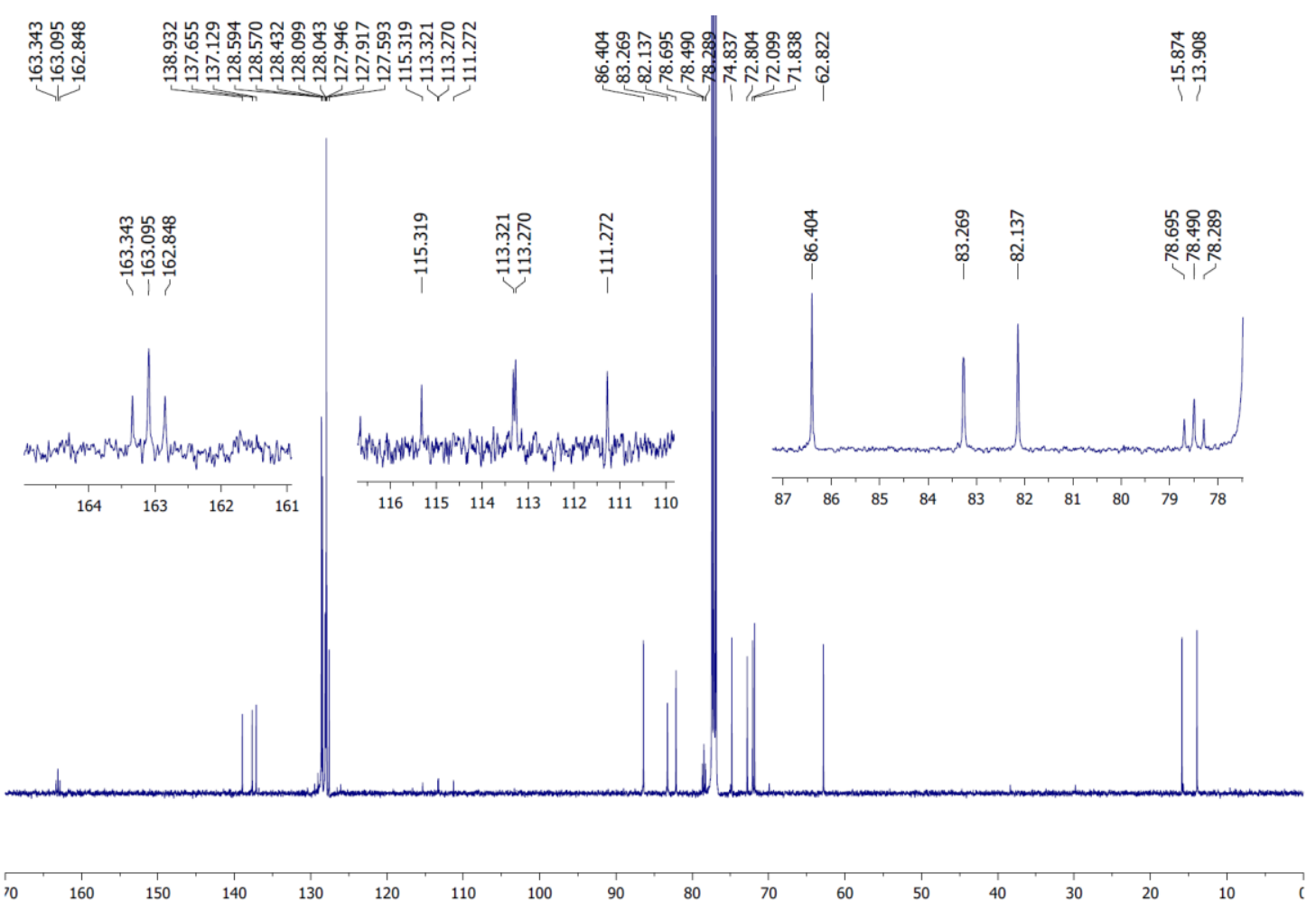


5-methyl-2,3,5-tri-O-benzyl-1-deoxy-1,1-difluoroethyl acetate- $\alpha, \beta$-D-fucofuranose $((\beta)$ $5 e)$.

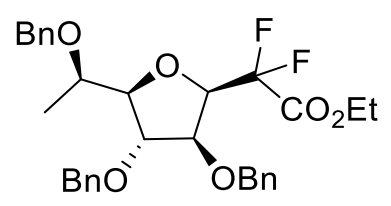

$(\beta)-5 e$

To a solution of alcohol $(S)-3 e(52.6 \mathrm{mg}, 0.081 \mathrm{mmol})$ in dry DCM $(2 \mathrm{ml})$ under argon atmosphere was added DAST ( $43 \mu \mathrm{l}, 0.32 \mathrm{mmol}, 4.0$ equiv.) dropwise at $0^{\circ} \mathrm{C}$. The reaction was allowed to warm from $0^{\circ} \mathrm{C}$ to r.t. and the reaction was stirred for $18 \mathrm{~h}$ at r.t. The crude was then diluted with $\mathrm{CH}_{2} \mathrm{Cl}_{2}$ and the organic phase was washed with $\mathrm{H}_{2} \mathrm{O}$ (3 times) and brine (3 times), dried over $\mathrm{MgSO}_{4}$ and concentrated under reduced pressure. Purification by chromatography on silica gel (Cy/EtOAc 95:5) afforded pure compound $(\beta)-5 e(11 \mathrm{mg}, 0.0203 \mathrm{mmol}, 25 \%)$ as a colorless oil and a side product $(\beta)-6 \mathbf{e}(13 \mathrm{mg}$, $0.029 \mathrm{mmol}, 36 \%)$ identified as similar to $(\beta)-5 \mathbf{e}$ with a free alcohol in C-5.

$[\alpha]_{20}$ D: -7.2 (c 1.0, $\left.\mathrm{CHCl}_{3}\right) .{ }^{1} \mathrm{H}$ NMR $\left(500 \mathrm{MHz}, \mathrm{CDCl}_{3}\right) \delta=7.37-7.27\left(\mathrm{~m}, 13 \mathrm{H}, \mathrm{H}^{\text {arom }}\right)$, 7.24-7.22 (m, $\left.2 \mathrm{H}, \mathrm{H}^{\mathrm{arom}}\right), 4.68-4.60\left(\mathrm{td}, 1 \mathrm{H}, J_{1-2}=5.7 \mathrm{~Hz}, J_{1-\mathrm{Fa}}=J_{1-\mathrm{Fb}}=12.6 \mathrm{~Hz}, \mathrm{H}-1\right)$, 4.65 (bs, $2 \mathrm{H}, \mathrm{CH}_{2}{ }^{\mathrm{Bn}}$ ), 4.54-4.48 (m, $4 \mathrm{H}, \mathrm{CH}_{2}{ }^{\mathrm{Bn}}$ ), 4.30 (dd, $1 \mathrm{H}, J_{2-3}=3.4 \mathrm{~Hz}, J_{1-2}=5.7 \mathrm{~Hz}$, $\mathrm{H}-2$ ), 4.15-4.02 (m, 4H, H-3, H-4, $\mathrm{CH}_{2} \mathrm{CH}_{3}$ ), 3.80 (quin, $1 \mathrm{H}, J=6.3 \mathrm{~Hz}, \mathrm{H}-5$ ), 1.19 (t, $\left.3 \mathrm{H}, J_{\mathrm{CH} 2-\mathrm{CH} 3}=7.5 \mathrm{~Hz}, \mathrm{CH}_{2} \mathrm{CH}_{3}\right), 1.15\left(\mathrm{~d}, 3 \mathrm{H}, J_{5-\mathrm{CH} 3}=6.3 \mathrm{~Hz}, \mathrm{CH}_{3}\right) \mathrm{ppm} .{ }^{13} \mathrm{C}$ NMR $(125$ $\left.\mathrm{MHz}, \mathrm{CDCl}_{3}\right) \delta=163.1\left(\mathrm{t}, J_{\mathrm{C}-\mathrm{F}}=30.8 \mathrm{~Hz}, \mathrm{C}_{\mathrm{q}} \mathrm{O}_{2} \mathrm{Et}\right), 138.9\left(\mathrm{C}_{\mathrm{q}}{ }^{\text {arom }}\right), 137.7\left(\mathrm{C}_{\mathrm{q}}{ }^{\text {arom }}\right), 137.1$ $\left(\mathrm{C}_{\mathrm{q}}{ }^{\text {arom }}\right), 128.6-127.6\left(\mathrm{CH}^{\text {arom }}\right), 113.3\left(\mathrm{dd}, J_{\mathrm{C}-\mathrm{F}}=251.1 \mathrm{~Hz}, J_{\mathrm{C}-\mathrm{F}}=258.3 \mathrm{~Hz}, \mathrm{CF}_{2}\right), 86.4$ $(\mathrm{C}-4), 83.3(\mathrm{C}-2), 82.1(\mathrm{C}-3), 78.5\left(\mathrm{t}, J_{\mathrm{C}-\mathrm{F}}=25.4 \mathrm{~Hz}, \mathrm{C}-1\right), 74.8(\mathrm{C}-5), 72.8\left(\mathrm{CH}_{2}{ }^{\mathrm{Bn}}\right), 72.1$ $\left(\mathrm{CH}_{2}{ }^{\mathrm{Bn}}\right), 71.8\left(\mathrm{CH}_{2}{ }^{\mathrm{Bn}}\right), 62.8\left(\mathrm{CH}_{2} \mathrm{CH}_{3}\right), 15.9\left(\mathrm{CH}_{3}\right), 13.9\left(\mathrm{CH}_{2} \mathrm{CH}_{3}\right)$ ppm. ${ }^{19} \mathrm{~F}$ NMR (471 $\left.\mathrm{MHz}, \mathrm{CDCl}_{3}\right) \delta=-111.6\left(\mathrm{dd}, 1 \mathrm{~F}, J_{\mathrm{H} 1-\mathrm{Fa}}=12.6 \mathrm{~Hz}, J_{\mathrm{Fa}-\mathrm{Fb}}=274.0 \mathrm{~Hz}, \mathrm{Fa}\right),-113.4(\mathrm{dd}, 1 \mathrm{~F}$, $\left.J_{\mathrm{H} 1-\mathrm{Fb}}=12.6 \mathrm{~Hz}, J_{\mathrm{Fa}-\mathrm{Fb}}=274.0 \mathrm{~Hz}, \mathrm{Fb}\right) \mathrm{ppm}$. HRMS (ESI+): m/z calculated for $\mathrm{C}_{31} \mathrm{H}_{35} \mathrm{~F}_{2} \mathrm{O}_{6}[\mathrm{M}+\mathrm{H}]^{+}$: calc. 541.2396; found: 541.2396. nOe: Correlations were inconclusive. 


\section{${ }^{1}$ H NMR}

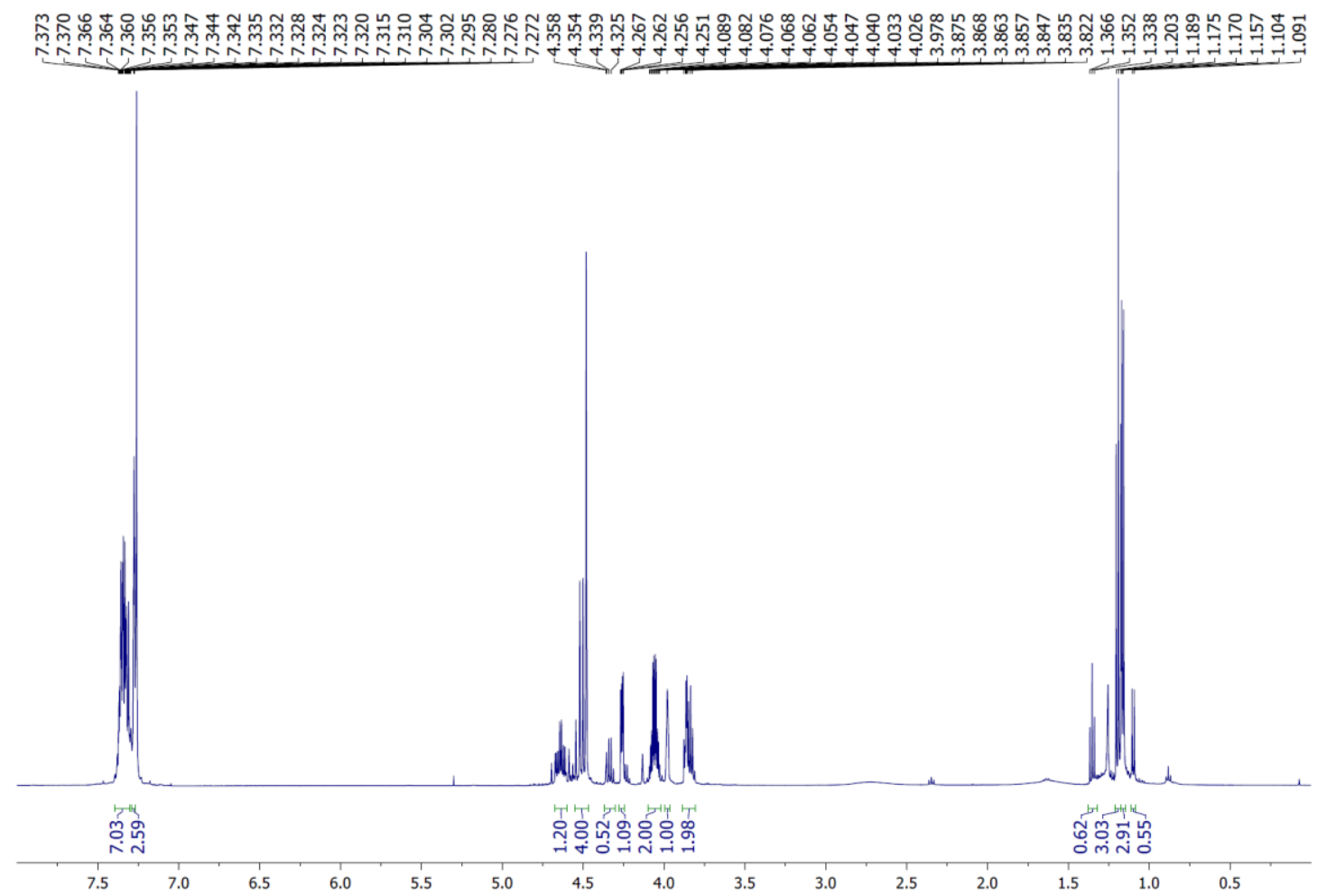

${ }^{19}$ F NMR

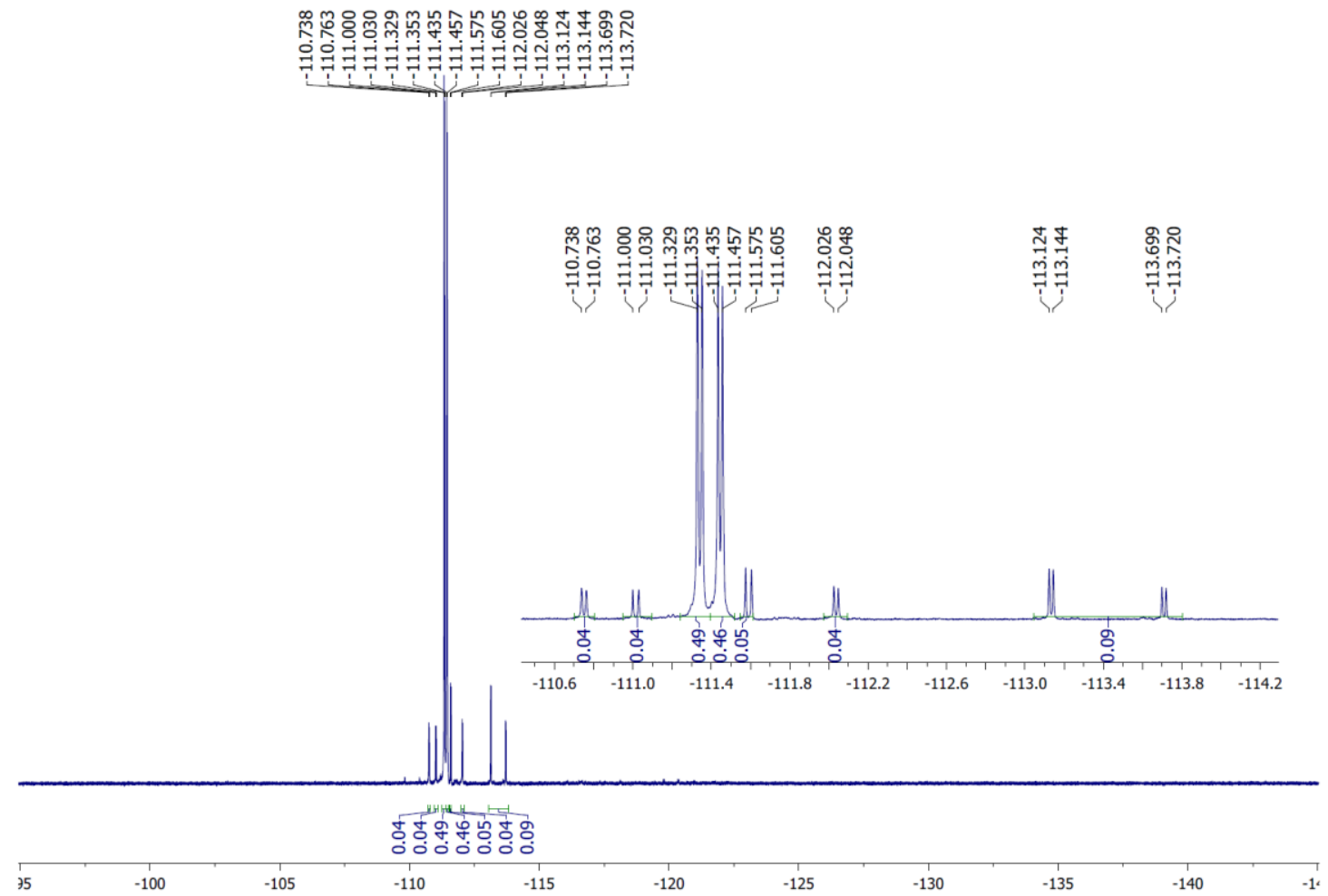

\section{${ }^{13}$ C NMR}




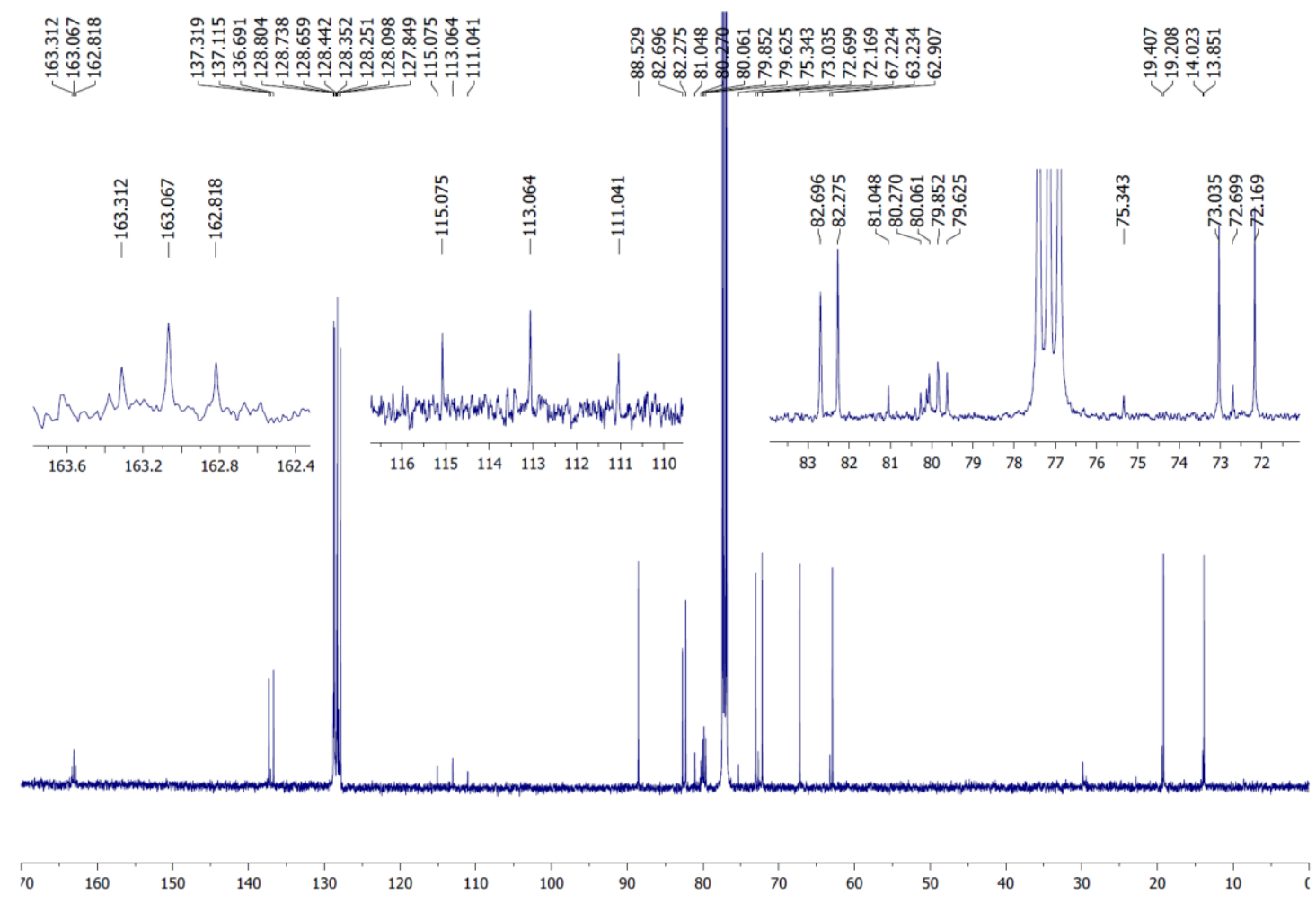




\section{D-Galactose}

2,3,5,6-tetra-O-benzyl-1-deoxy-1,1-difluoroethyl acetate- $\alpha, \beta$-D-galactofuranose $((\alpha)$ $5 f)$.

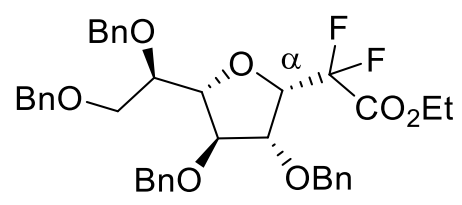

$(\alpha)-5 f$

To a solution of alcohol $(R)-\mathbf{3 f}(38 \mathrm{mg}, 0.0503 \mathrm{mmol})$ in dry DCM $(1.5 \mathrm{ml})$ under argon atmosphere was added DAST ( $26 \mu \mathrm{l}, 0.20 \mathrm{mmol}, 4.0$ equiv.) dropwise at $0^{\circ} \mathrm{C}$. The reaction was allowed to warm from $0^{\circ} \mathrm{C}$ to r.t. and the reaction was stirred for $18 \mathrm{~h}$ at r.t. The crude was then diluted with $\mathrm{CH}_{2} \mathrm{Cl}_{2}$ and the organic phase was washed with $\mathrm{H}_{2} \mathrm{O}$ (3 times) and brine (3 times), dried over $\mathrm{MgSO}_{4}$ and concentrated under reduced pressure. Purification by chromatography on silica gel (Cy/EtOAc 95:5) afforded pure compound ( $\alpha)-5 f(19 \mathrm{mg}, 0.0294 \mathrm{mmol}, 58 \%)$ as a colorless oil.

$[\alpha]_{20}{ }^{\mathrm{D}}:+2.9\left(\mathrm{c} 1.0, \mathrm{CHCl}_{3}\right) .{ }^{1} \mathrm{H}$ NMR $\left(500 \mathrm{MHz}, \mathrm{CDCl}_{3}\right) \delta=7.38-7.36\left(\mathrm{~m}, 2 \mathrm{H}, \mathrm{H}^{\text {arom}}\right)$, 7.33-7.26 (m, $\left.12 \mathrm{H}, \mathrm{H}^{\text {arom }}\right), 7.26-7.19\left(\mathrm{~m}, 6 \mathrm{H}, \mathrm{H}^{\text {arom }}\right), 4.77\left(\mathrm{AB}, 1 \mathrm{H}, J_{\mathrm{A}-\mathrm{B}}=12.0 \mathrm{~Hz}\right.$, $\left.\mathrm{CH}_{2}{ }^{\mathrm{Bn}}\right), 4.74\left(\mathrm{AB}, 1 \mathrm{H}, J_{\mathrm{A}-\mathrm{B}}=12.0 \mathrm{~Hz}, \mathrm{CH}_{2}{ }^{\mathrm{Bn}}\right), 4.64\left(\mathrm{td}, 1 \mathrm{H}, J_{1-2}=5.2 \mathrm{~Hz}, J_{1-\mathrm{Fa}}=J_{1-\mathrm{Fb}}=\right.$ $12.6 \mathrm{~Hz}, \mathrm{H}-1), 4.46-4.41\left(\mathrm{~m}, 6 \mathrm{H}, \mathrm{CH}_{2}{ }^{\mathrm{Bn}}\right), 4.25$ (dd, $\left.1 \mathrm{H}, J_{1-2}=5.2 \mathrm{~Hz}, J_{2-3}=3.4 \mathrm{~Hz}, \mathrm{H}-2\right)$, $4.20(\mathrm{~m}, 1 \mathrm{H}, \mathrm{H}-3), 4.17\left(\mathrm{dd}, 1 \mathrm{H}, J_{3-4}=4.0 \mathrm{~Hz}, J_{4-5}=5.7 \mathrm{~Hz}, \mathrm{H}-2\right), 4.15-4.01(\mathrm{~m}, 2 \mathrm{H}$, $\left.\mathrm{CH}_{2} \mathrm{CH}_{3}\right), 3.87\left(\mathrm{td}, 1 \mathrm{H}, J_{5-6 \mathrm{a}}=4.0 \mathrm{~Hz}, J_{4-5}=J_{5-6 \mathrm{~b}}=5.7 \mathrm{~Hz}, \mathrm{H}-5\right), 3.64\left(\mathrm{ABX}, 1 \mathrm{H}, J_{5-6 \mathrm{a}}=\right.$ $\left.4.0 \mathrm{~Hz}, J_{6 \mathrm{a}-6 \mathrm{~b}}=10.3 \mathrm{~Hz}, \mathrm{H}-6 \mathrm{a}\right), 3.54\left(\mathrm{ABX}, 1 \mathrm{H}, J_{5-6 \mathrm{~b}}=5.7 \mathrm{~Hz}, J_{6 \mathrm{a}-6 \mathrm{~b}}=10.3 \mathrm{~Hz}, \mathrm{H}-6 \mathrm{~b}\right)$, $1.19\left(\mathrm{t}, 3 \mathrm{H}, J_{\mathrm{CH} 2-\mathrm{CH} 3}=7.5 \mathrm{~Hz}, \mathrm{CH}_{2} \mathrm{CH}_{3}\right) \mathrm{ppm} .{ }^{13} \mathrm{C} \mathrm{NMR}\left(125 \mathrm{MHz}, \mathrm{CDCl}_{3}\right) \delta=163.1(\mathrm{t}$, $\left.J_{\mathrm{C}-\mathrm{F}}=31.4 \mathrm{~Hz}, \mathrm{C}_{\mathrm{q}} \mathrm{O}_{2} \mathrm{Et}\right), 138.9\left(\mathrm{C}_{\mathrm{q}}{ }^{\text {arom }}\right), 138.4\left(\mathrm{C}_{\mathrm{q}}{ }^{\text {arom }}\right), 137.8\left(\mathrm{C}_{\mathrm{q}}{ }^{\text {arom }}\right), 137.2\left(\mathrm{C}_{\mathrm{q}}^{\text {arom }}\right)$, 128.6-127.6 $\left(\mathrm{CH}^{\mathrm{arom}}\right), 113.2\left(\mathrm{dd}, J_{\mathrm{C}-\mathrm{F}}=251.1 \mathrm{~Hz}, J_{\mathrm{C}-\mathrm{F}}=258.3 \mathrm{~Hz}, \mathrm{CF}_{2}\right), 85.0(\mathrm{C}-4), 83.0$ $(\mathrm{C}-2), 81.6(\mathrm{C}-3), 78.8\left(\mathrm{t}, J_{\mathrm{C}-\mathrm{F}}=25.4 \mathrm{~Hz}, \mathrm{C}-1\right), 77.9(\mathrm{C}-5), 73.5\left(\mathrm{CH}_{2}{ }^{\mathrm{Bn}}\right), 73.4\left(\mathrm{CH}_{2}{ }^{\mathrm{Bn}}\right)$, $72.6\left(\mathrm{CH}_{2}{ }^{\mathrm{Bn}}\right), 72.0\left(\mathrm{CH}_{2}{ }^{\mathrm{Bn}}\right), 70.7(\mathrm{C}-6), 62.8\left(\mathrm{CH}_{2} \mathrm{CH}_{3}\right), 13.9\left(\mathrm{CH}_{2} \mathrm{CH}_{3}\right)$ ppm. ${ }^{19} \mathrm{~F} \mathrm{NMR}$ $\left(471 \mathrm{MHz}, \mathrm{CDCl}_{3}\right) \delta=-110.8\left(\mathrm{dd}, 1 \mathrm{~F}, J_{\mathrm{H} 1-\mathrm{Fa}}=12.6 \mathrm{~Hz}, J_{\mathrm{Fa}-\mathrm{Fb}}=274.0 \mathrm{~Hz}, \mathrm{Fa}\right),-113.5(\mathrm{dd}$, $\left.1 \mathrm{~F}, J_{\mathrm{H} 1-\mathrm{Fb}}=12.6 \mathrm{~Hz}, J_{\mathrm{Fa}-\mathrm{Fb}}=274.0 \mathrm{~Hz}, \mathrm{Fb}\right) \mathrm{ppm}$. HRMS (ESI+): $\mathrm{m} / \mathrm{z}$ calculated for $\mathrm{C}_{38} \mathrm{H}_{41} \mathrm{~F}_{2} \mathrm{O}_{7}[\mathrm{M}+\mathrm{H}]^{+}$: calc. 647.2815; found: 647.2814. nOe: Correlation H-1 - H-2 and $\mathrm{H}-1$ - H-4 observed. 


\section{${ }^{1}$ H NMR}

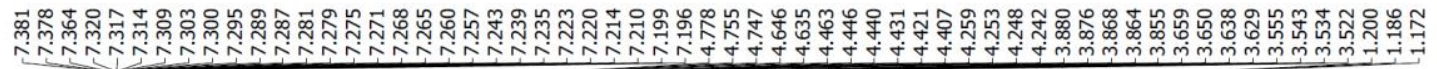
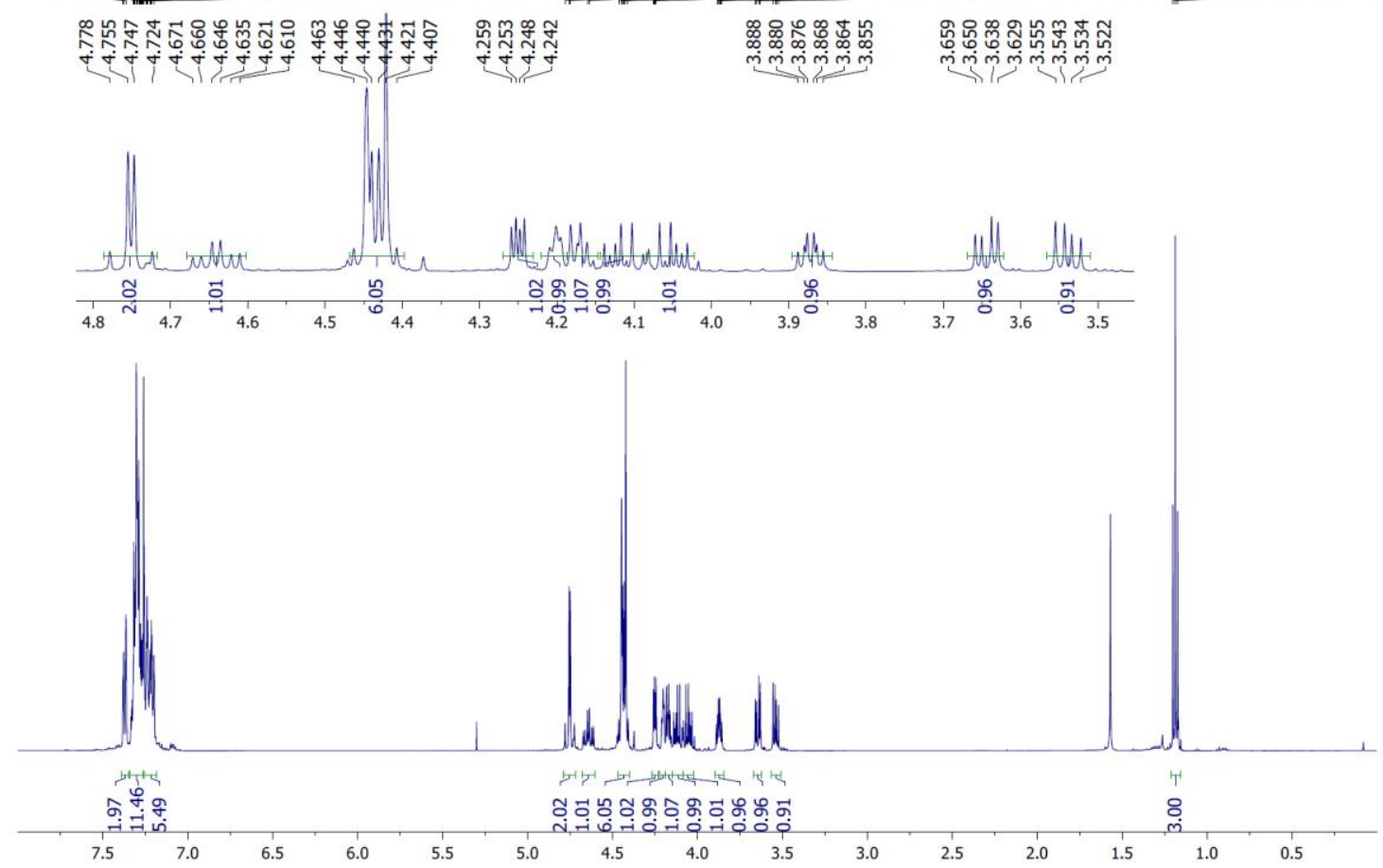

${ }^{19}$ F NMR

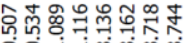

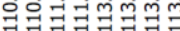

पij

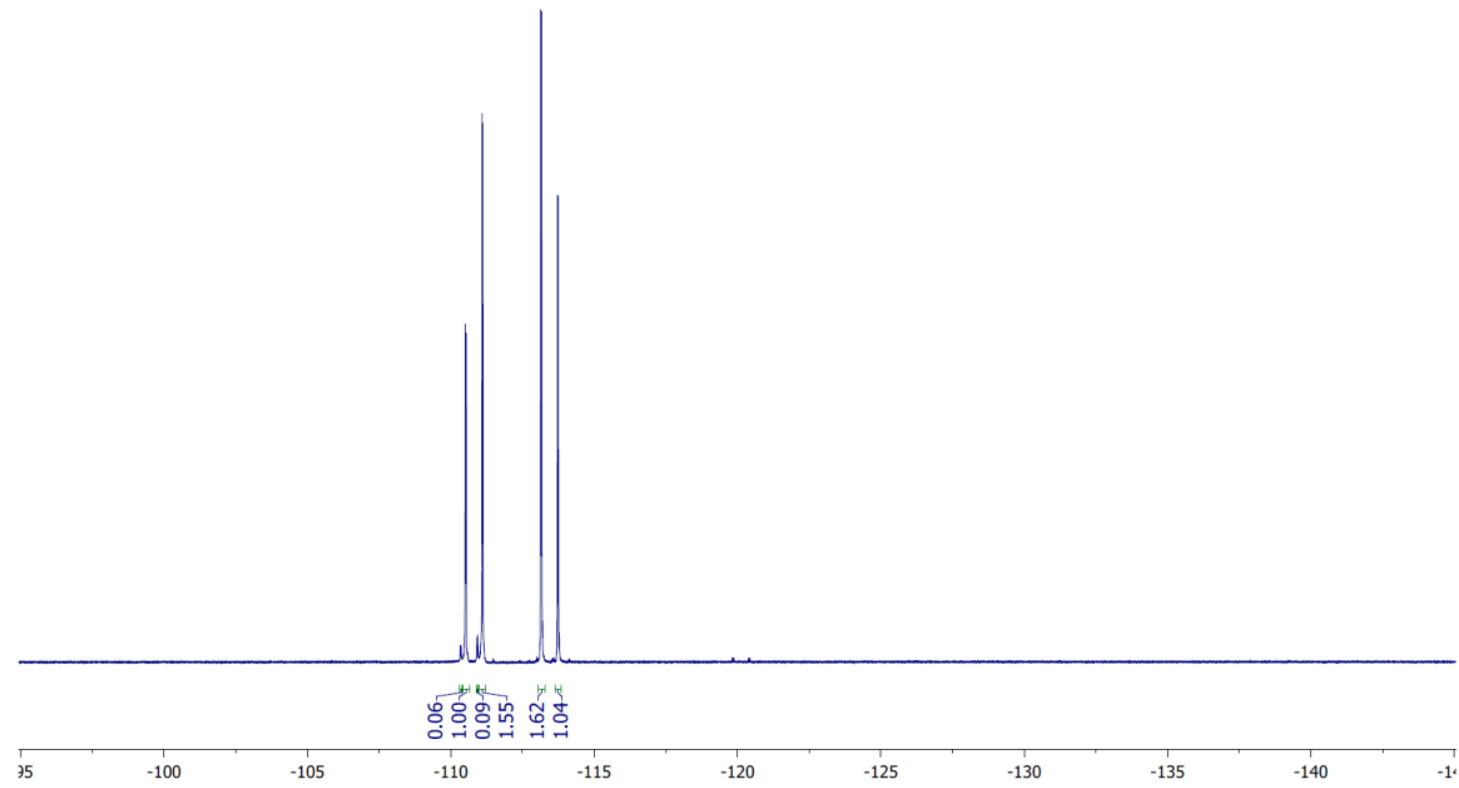


${ }^{13}$ C NMR

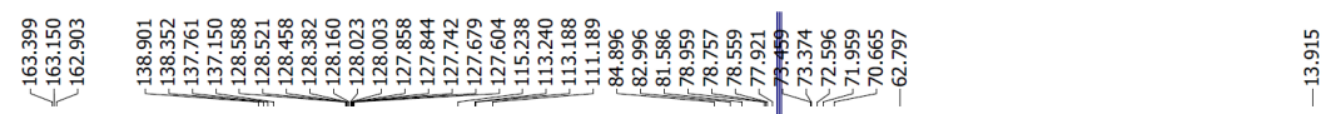

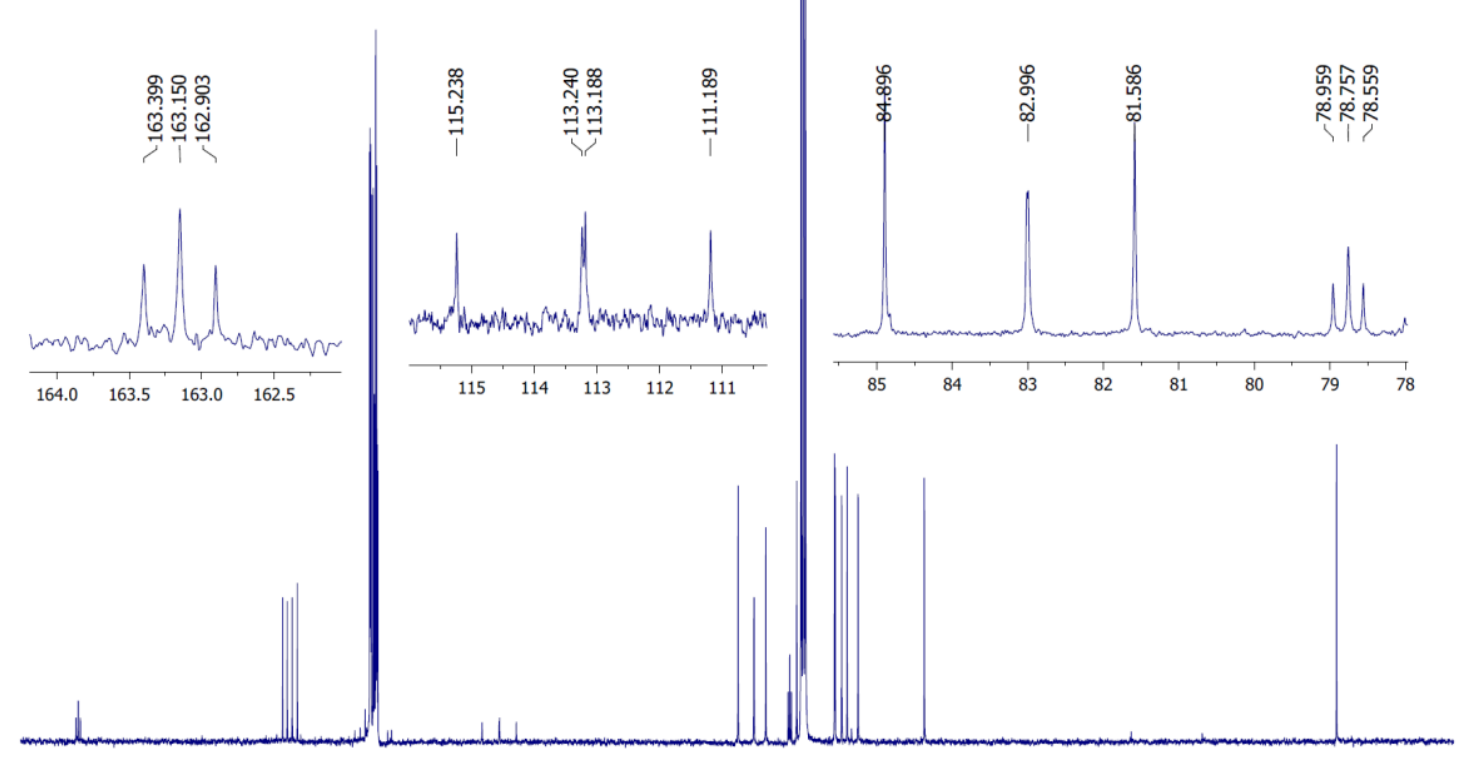

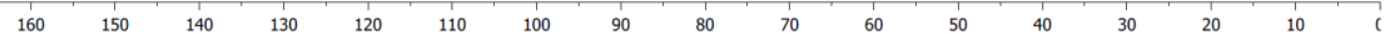


<smiles>CCOC(F)(F)[C@H]1O[C@H]([C@H](O)COc2ccccc2)[C@@H](O)[C@H]1OCc1ccccc1</smiles>

$(\beta)-5 f$

To a solution of alcohol $(S)$-3f $(86 \mathrm{mg}, 0.1139 \mathrm{mmol})$ in dry DCM (2 ml) under argon atmosphere was added DAST (60 $\mu \mathrm{l}, 0.456 \mathrm{mmol}, 4.0$ equiv.) dropwise at $0^{\circ} \mathrm{C}$. The reaction was allowed to warm from $0^{\circ} \mathrm{C}$ to r.t. and the reaction was stirred for $18 \mathrm{~h}$ at r.t. The crude was then diluted with $\mathrm{CH}_{2} \mathrm{Cl}_{2}$ and the organic phase was washed with $\mathrm{H}_{2} \mathrm{O}$ (3 times) and brine (3 times), dried over $\mathrm{MgSO}_{4}$ and concentrated under reduced pressure. Purification by chromatography on silica gel (Cy/EtOAc 95:5) afforded pure compound $(\beta)-5 f(40 \mathrm{mg}, 0.062 \mathrm{mmol}, 54 \%)$ as a colorless oil.

$[\alpha]_{20} \mathrm{D}:-13.3\left(\mathrm{c} 1.0, \mathrm{CHCl}_{3}\right) .{ }^{1} \mathrm{H} \mathrm{NMR}\left(500 \mathrm{MHz}, \mathrm{CDCl}_{3}\right) \delta=7.38-7.26\left(\mathrm{~m}, 18 \mathrm{H}, \mathrm{H}^{\mathrm{arom}}\right)$, 7.21-7.18 (m, $\left.2 \mathrm{H}, \mathrm{H}^{\text {arom }}\right), 4.71\left(\mathrm{AB}, 1 \mathrm{H}, J_{\mathrm{A}-\mathrm{B}}=11.5 \mathrm{~Hz}, \mathrm{CH}_{2}{ }^{\mathrm{Bn}}\right), 4.60\left(\mathrm{AB}, 1 \mathrm{H}, J_{\mathrm{A}-\mathrm{B}}=\right.$ $\left.11.5 \mathrm{~Hz}, \mathrm{CH}_{2}{ }^{\mathrm{Bn}}\right), 4.52-4.44\left(\mathrm{~m}, 6 \mathrm{H}, \mathrm{CH}_{2}{ }^{\mathrm{Bn}}, \mathrm{H}-1, \mathrm{H}-2\right), 4.43\left(\mathrm{AB}, 1 \mathrm{H}, J_{\mathrm{A}-\mathrm{B}}=11.5 \mathrm{~Hz}\right.$, $\left.\mathrm{CH}_{2}{ }^{\mathrm{Bn}}\right), 4.31\left(\mathrm{AB}, 1 \mathrm{H}, J_{\mathrm{A}-\mathrm{B}}=12.0 \mathrm{~Hz}, \mathrm{CH}_{2}{ }^{\mathrm{Bn}}\right), 4.30-4.25\left(\mathrm{~m}, 2 \mathrm{H}, \mathrm{CH}_{2} \mathrm{CH}_{3}\right), 4.25-4.22$ (dd, $1 \mathrm{H}, J=4.0 \mathrm{~Hz}, J=6.9 \mathrm{~Hz}, \mathrm{H}-3), 4.15$ (m, 1H, H-4), 3.72-3.68 (m, 2H, H-5, H-6a), 3.64-3.61 (m, $1 \mathrm{H}, \mathrm{H}-6 \mathrm{~b}), 1.28\left(\mathrm{t}, 3 \mathrm{H}, J_{\mathrm{CH} 2-\mathrm{CH} 3}=7.5 \mathrm{~Hz}, \mathrm{CH}_{2} \mathrm{CH}_{3}\right) \mathrm{ppm} .{ }^{13} \mathrm{C} \mathrm{NMR}(125$ $\left.\mathrm{MHz}, \mathrm{CDCl}_{3}\right) \delta=163.0\left(\mathrm{dd}, J_{\mathrm{C}-\mathrm{F}}=30.8 \mathrm{~Hz}, J_{\mathrm{C}-\mathrm{F}}=32.0 \mathrm{~Hz}, \mathrm{C}_{\mathrm{q}} \mathrm{O}_{2} \mathrm{Et}\right), 138.3\left(2 \mathrm{C}_{\mathrm{q}}{ }^{\text {arom }}\right)$, $137.8\left(\mathrm{C}_{\mathrm{q}}{ }^{\text {arom }}\right), 137.5\left(\mathrm{C}_{\mathrm{q}}{ }^{\text {arom }}\right), 128.6-127.7\left(\mathrm{CH}^{\text {arom }}\right), 113.9\left(\mathrm{dd}, J_{\mathrm{C}-\mathrm{F}}=252.9 \mathrm{~Hz}, J_{\mathrm{C}-\mathrm{F}}=\right.$ $\left.260.2 \mathrm{~Hz}, \mathrm{CF}_{2}\right), 84.1(\mathrm{C}-3), 83.8(\mathrm{C}-2), 83.2(\mathrm{C}-4), 82.2\left(\mathrm{dd}, J_{\mathrm{C}-\mathrm{F}}=23.5 \mathrm{~Hz}, J_{\mathrm{C}-\mathrm{F}}=32.0\right.$ $\mathrm{Hz}, \mathrm{C}-1), 76.4(\mathrm{C}-5), 73.7\left(\mathrm{CH}_{2}{ }^{\mathrm{Bn}}\right), 73.3\left(\mathrm{CH}_{2}{ }^{\mathrm{Bn}}\right), 72.3\left(2 \mathrm{CH}_{2}{ }^{\mathrm{Bn}}\right), 70.8(\mathrm{C}-6), 63.2$ $\left(\mathrm{CH}_{2} \mathrm{CH}_{3}\right), 14.0\left(\mathrm{CH}_{2} \mathrm{CH}_{3}\right)$ ppm. ${ }^{19} \mathrm{~F} \mathrm{NMR}\left(471 \mathrm{MHz}, \mathrm{CDCl}_{3}\right) \delta=-110.9\left(\mathrm{dd}, 1 \mathrm{~F}, J_{\mathrm{H}-\mathrm{Fa}}=\right.$ $\left.4.5 \mathrm{~Hz}, J_{\mathrm{Fa}-\mathrm{Fb}}=263.1 \mathrm{~Hz}, \mathrm{Fa}\right),-120.1\left(\mathrm{dd}, 1 \mathrm{~F}, J_{\mathrm{H}-\mathrm{Fb}}=22.0 \mathrm{~Hz}, J_{\mathrm{Fa}-\mathrm{Fb}}=263.1 \mathrm{~Hz}, \mathrm{Fb}\right) \mathrm{ppm}$. HRMS (ESI+): $\mathrm{m} / \mathrm{z}$ calculated for $\mathrm{C}_{38} \mathrm{H}_{40} \mathrm{~F}_{2} \mathrm{O}_{7} \mathrm{Na}[\mathrm{M}+\mathrm{Na}]^{+}$: calc. 669.2634; found: 669.2623. nOe: Correlations were inconclusive. 


\section{${ }^{1}$ H NMR}

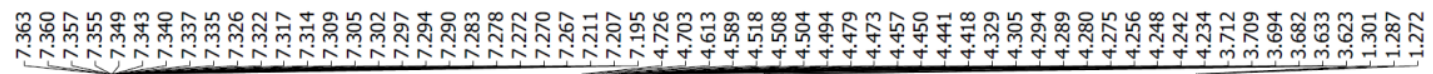

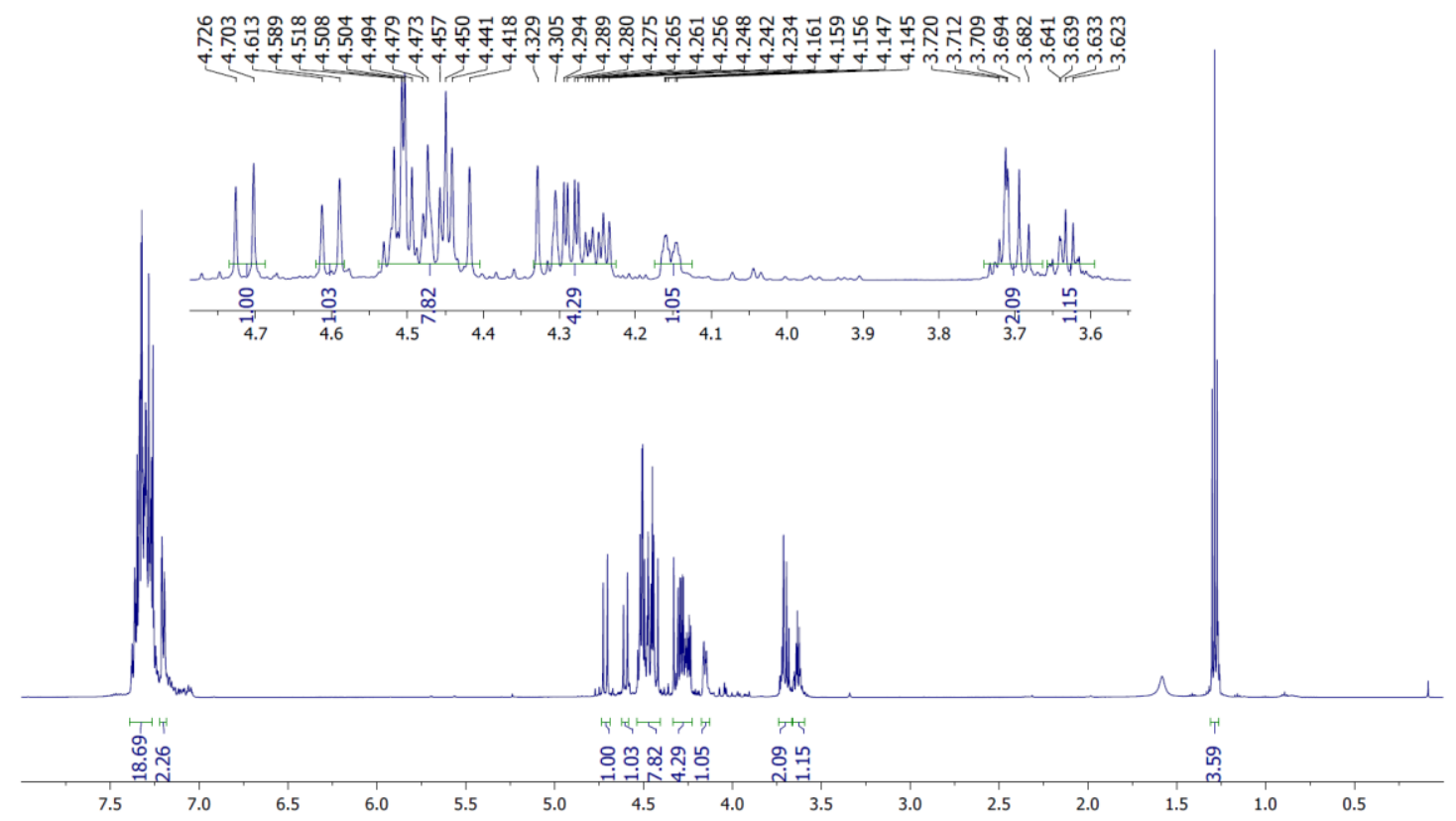

${ }^{19}$ F NMR

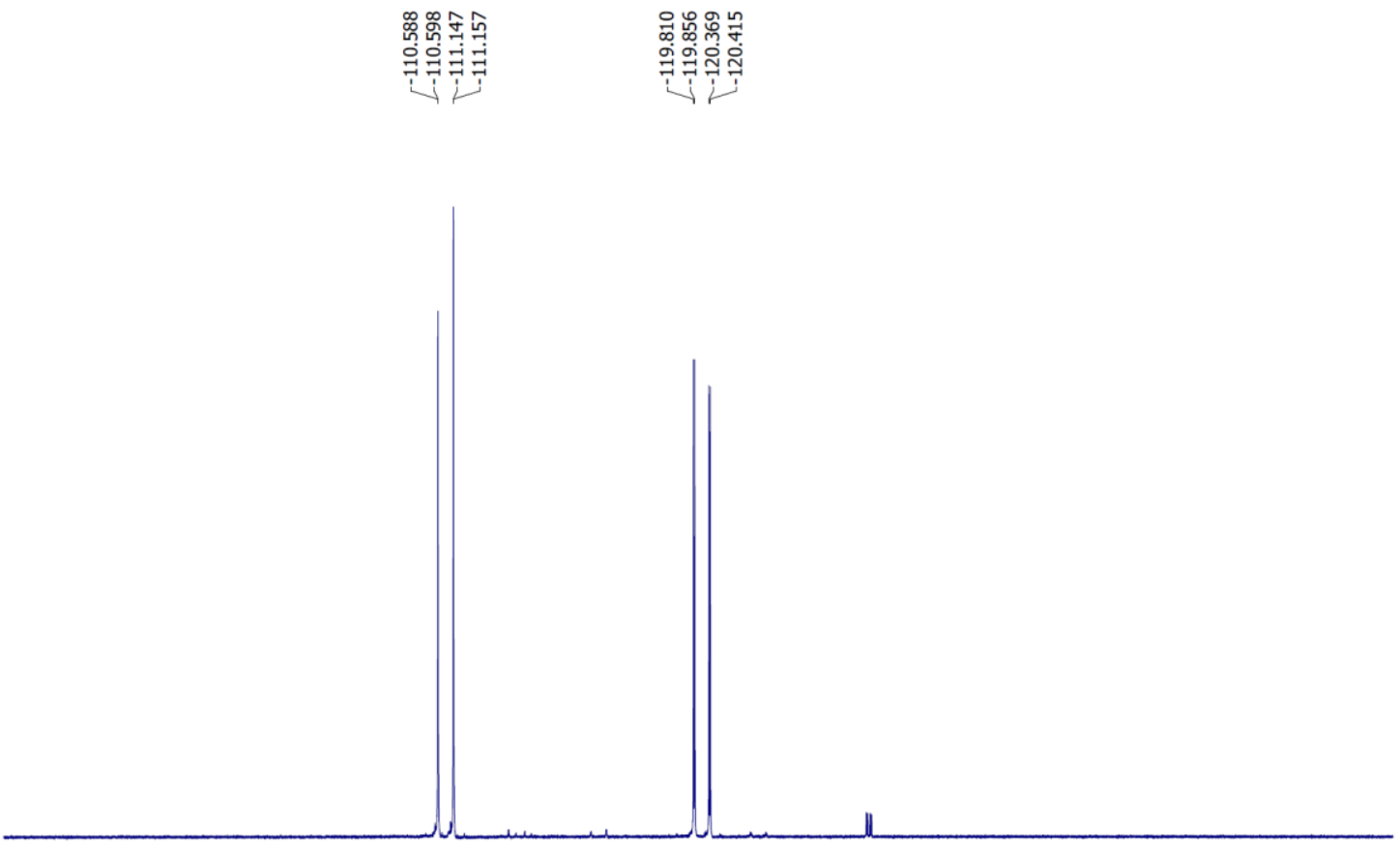

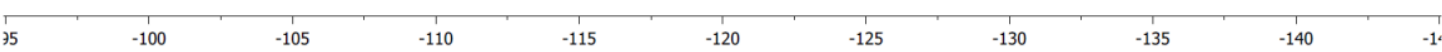


${ }^{13}$ C NMR

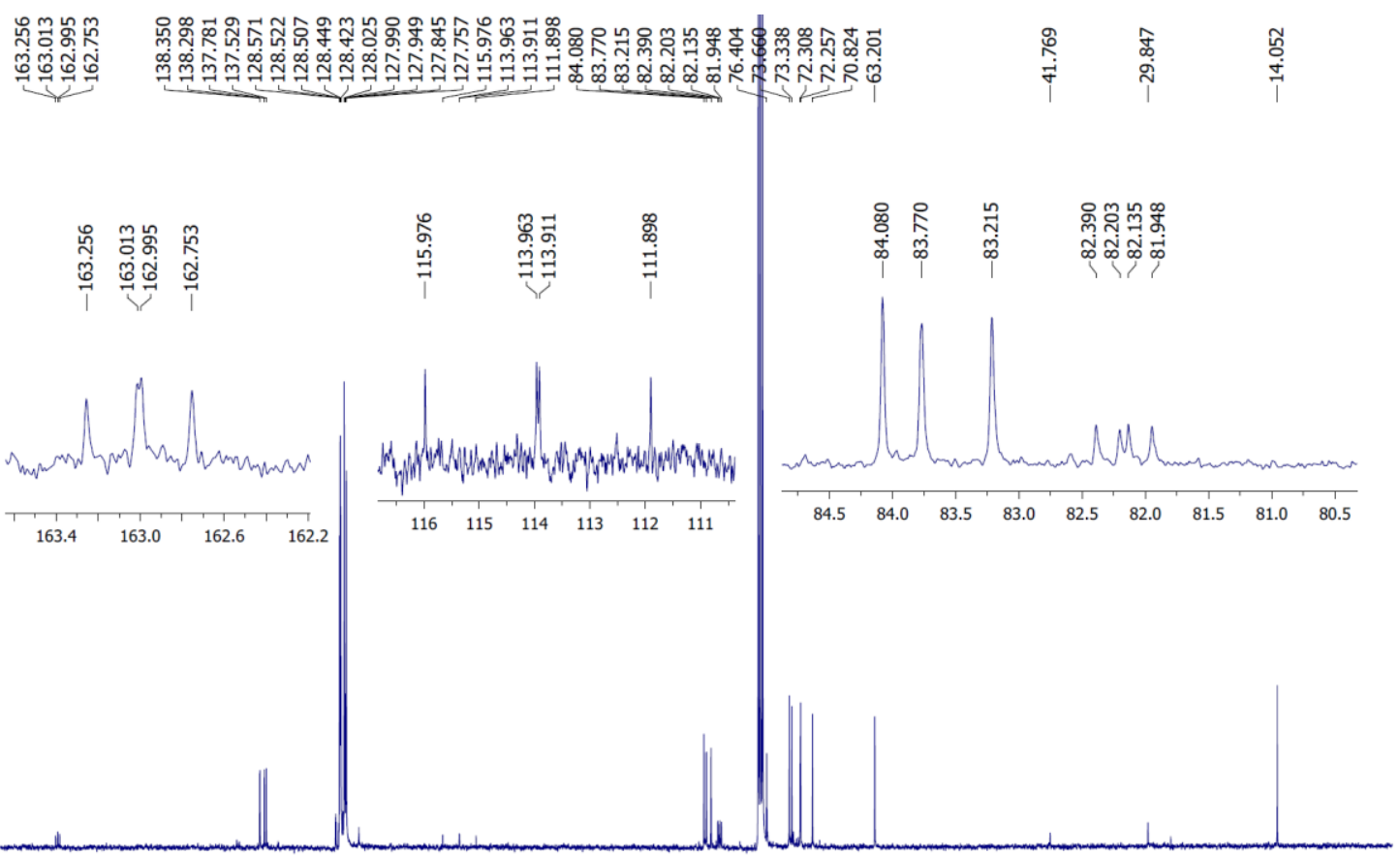

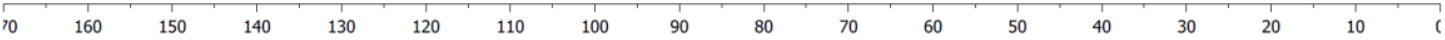




\section{D-Glucose}

2,3,5,6-tetra-O-benzyl-1-deoxy-1,1-difluoroethyl acetate- $\alpha, \beta$-D-glucofuranose (( $\beta)$-5g).<smiles>CCOC(F)(F)[C@H]1OC([C@@H](COc2ccccc2)OCc2ccccc2)[C@@H](O)[C@H]1OCc1ccccc1</smiles>

$(\beta)-5 g$

To a solution of alcohol $(S)-3 \mathrm{~g}(60.5 \mathrm{mg}, 0.0801 \mathrm{mmol})$ in dry DCM $(1.5 \mathrm{ml})$ under argon atmosphere was added DAST ( $42 \mu 1,0.32 \mathrm{mmol}, 4.0$ equiv.) dropwise at $0^{\circ} \mathrm{C}$. The reaction was allowed to warm from $0^{\circ} \mathrm{C}$ to r.t. and the reaction was stirred for $18 \mathrm{~h}$ at r.t. The crude was then diluted with $\mathrm{CH}_{2} \mathrm{Cl}_{2}$ and the organic phase was washed with $\mathrm{H}_{2} \mathrm{O}$ (3 times) and brine (3 times), dried over $\mathrm{MgSO}_{4}$ and concentrated under reduced pressure. Purification by chromatography on silica gel (Cy/EtOAc 96:4) afforded pure compound ( $\beta$ )-5g (45 mg, $0.0696 \mathrm{mmol}, 87 \%)$ as a colorless oil.

$[\alpha]_{20} \mathrm{D}:-10.9\left(\mathrm{c} 0.5, \mathrm{CHCl}_{3}\right) .{ }^{1} \mathrm{H} \mathrm{NMR}\left(500 \mathrm{MHz}, \mathrm{CDCl}_{3}\right) \delta=7.37-7.23\left(\mathrm{~m}, 20 \mathrm{H}, \mathrm{H}^{\text {arom }}\right)$, $4.75\left(\mathrm{AB}, 1 \mathrm{H}, J_{\mathrm{A}-\mathrm{B}}=11.5 \mathrm{~Hz}, \mathrm{CH}_{2}{ }^{\mathrm{Bn}}\right), 4.56-4.53\left(\mathrm{~m}, 3 \mathrm{H}, \mathrm{CH}_{2}{ }^{\mathrm{Bn}}\right), 4.52-4.46(\mathrm{~m}, 3 \mathrm{H}$, $\left.\mathrm{CH}_{2}{ }^{\mathrm{Bn}}\right), 4.38-4.31\left(\mathrm{~m}, 3 \mathrm{H}, \mathrm{H}-1, \mathrm{H}-3, \mathrm{CH}_{2}{ }^{\mathrm{Bn}}\right), 4.20\left(\mathrm{q}, 2 \mathrm{H}, J_{\mathrm{CH} 2-\mathrm{CH} 3}=7.5 \mathrm{~Hz}, \mathrm{CH}_{2} \mathrm{CH}_{3}\right)$, 4.22-4.17 (m, $1 \mathrm{H}, \mathrm{H}-4), 4.12(\mathrm{~d}, 1 \mathrm{H}, J=3.4 \mathrm{~Hz}, \mathrm{H}-2), 4.00$ (ddd, $1 \mathrm{H}, J_{5-6 \mathrm{a}}=1.7 \mathrm{~Hz}, J_{5-}$ $\left.6 \mathrm{~b}=5.2 \mathrm{~Hz}, J_{4-5}=8.6 \mathrm{~Hz}, \mathrm{H}-5\right), 3.81\left(\mathrm{ABX}, 1 \mathrm{H}, J_{5-6 \mathrm{a}}=1.7 \mathrm{~Hz}, J_{6 \mathrm{a}-6 \mathrm{~b}}=10.9 \mathrm{~Hz}, \mathrm{H}-6 \mathrm{a}\right)$, $3.62\left(\mathrm{ABX}, 1 \mathrm{H}, J_{5-6 \mathrm{~b}}=5.2 \mathrm{~Hz}, J_{6 \mathrm{a}-6 \mathrm{~b}}=10.9 \mathrm{~Hz}, \mathrm{H}-6 \mathrm{~b}\right), 1.24\left(\mathrm{t}, 3 \mathrm{H}, J_{\mathrm{CH} 2-\mathrm{CH} 3}=7.5 \mathrm{~Hz}\right.$, $\left.\mathrm{CH}_{2} \mathrm{CH}_{3}\right) \mathrm{ppm} .{ }^{13} \mathrm{C} \mathrm{NMR}\left(125 \mathrm{MHz}, \mathrm{CDCl}_{3}\right) \delta=163.0\left(\mathrm{dd}, J_{\mathrm{C}-\mathrm{F}}=30.2 \mathrm{~Hz}, J_{\mathrm{C}-\mathrm{F}}=32.6\right.$ $\left.\mathrm{Hz}, \mathrm{C}_{\mathrm{q}} \mathrm{O}_{2} \mathrm{Et}\right), 138.9\left(\mathrm{C}_{\mathrm{q}}{ }^{\text {arom }}\right), 138.7\left(\mathrm{C}_{\mathrm{q}}{ }^{\text {arom }}\right), 137.6\left(\mathrm{C}_{\mathrm{q}}{ }^{\text {arom }}\right), 137.3\left(\mathrm{C}_{\mathrm{q}}{ }^{\text {arom }}\right), 128.7-127.5$ $\left(\mathrm{CH}^{\text {arom }}\right), 113.1\left(\mathrm{dd}, J_{\mathrm{C}-\mathrm{F}}=249.9 \mathrm{~Hz}, J_{\mathrm{C}-\mathrm{F}}=257.1 \mathrm{~Hz}, \mathrm{CF}_{2}\right), 84.5\left(\mathrm{dd}, J_{\mathrm{C}-\mathrm{F}}=27.0 \mathrm{~Hz}, J_{\mathrm{C}-}\right.$ $\mathrm{F}=31.7 \mathrm{~Hz}, \mathrm{C}-1), 82.1(\mathrm{C}-2), 81.4(\mathrm{C}-4), 81.3(\mathrm{C}-3), 75.6(\mathrm{C}-5), 73.5\left(\mathrm{CH}_{2}{ }^{\mathrm{Bn}}\right), 72.7$ $\left(\mathrm{CH}_{2}{ }^{\mathrm{Bn}}\right), 72.0\left(\mathrm{CH}_{2}{ }^{\mathrm{Bn}}\right), 71.6\left(\mathrm{CH}_{2}{ }^{\mathrm{Bn}}\right), 70.6(\mathrm{C}-6), 63.0\left(\mathrm{CH}_{2} \mathrm{CH}_{3}\right), 14.1\left(\mathrm{CH}_{2} \mathrm{CH}_{3}\right) \mathrm{ppm}$. ${ }^{19} \mathrm{~F} \mathrm{NMR}\left(471 \mathrm{MHz}, \mathrm{CDCl}_{3}\right) \delta=-110.8\left(\mathrm{dd}, 1 \mathrm{~F}, J_{\mathrm{H}-\mathrm{Fa}}=7.2 \mathrm{~Hz}, J_{\mathrm{Fa}-\mathrm{Fb}}=262.0 \mathrm{~Hz}, \mathrm{Fa}\right),-$ $119.1\left(\mathrm{dd}, 1 \mathrm{~F}, J_{\mathrm{H}-\mathrm{Fb}}=17.1 \mathrm{~Hz}, J_{\mathrm{Fa}-\mathrm{Fb}}=262.0 \mathrm{~Hz}, \mathrm{Fb}\right) \mathrm{ppm}$. HRMS (ESI+): $\mathrm{m} / \mathrm{z}$ calculated for $\mathrm{C}_{38} \mathrm{H}_{41} \mathrm{~F}_{2} \mathrm{O}_{7}[\mathrm{M}+\mathrm{H}]^{+}$: calc. 647.2815; found: 647.2809. nOe: Correlation between $\mathrm{H}-$ 1 and $\mathrm{H}-3$ observed. 


\section{${ }^{1}$ H NMR}

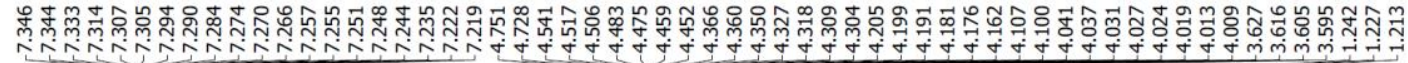

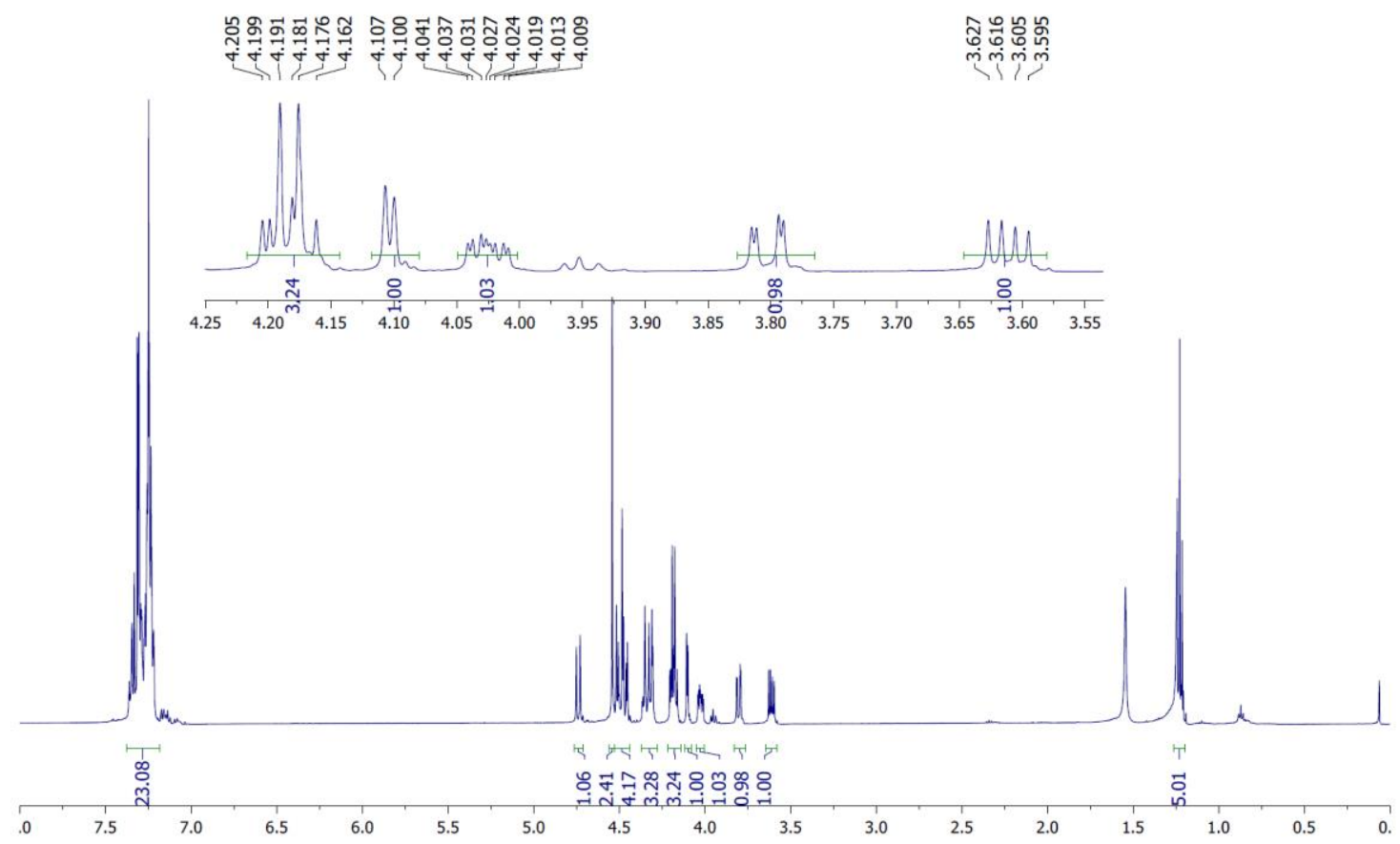

${ }^{19}$ F NMR

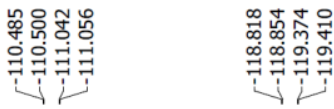

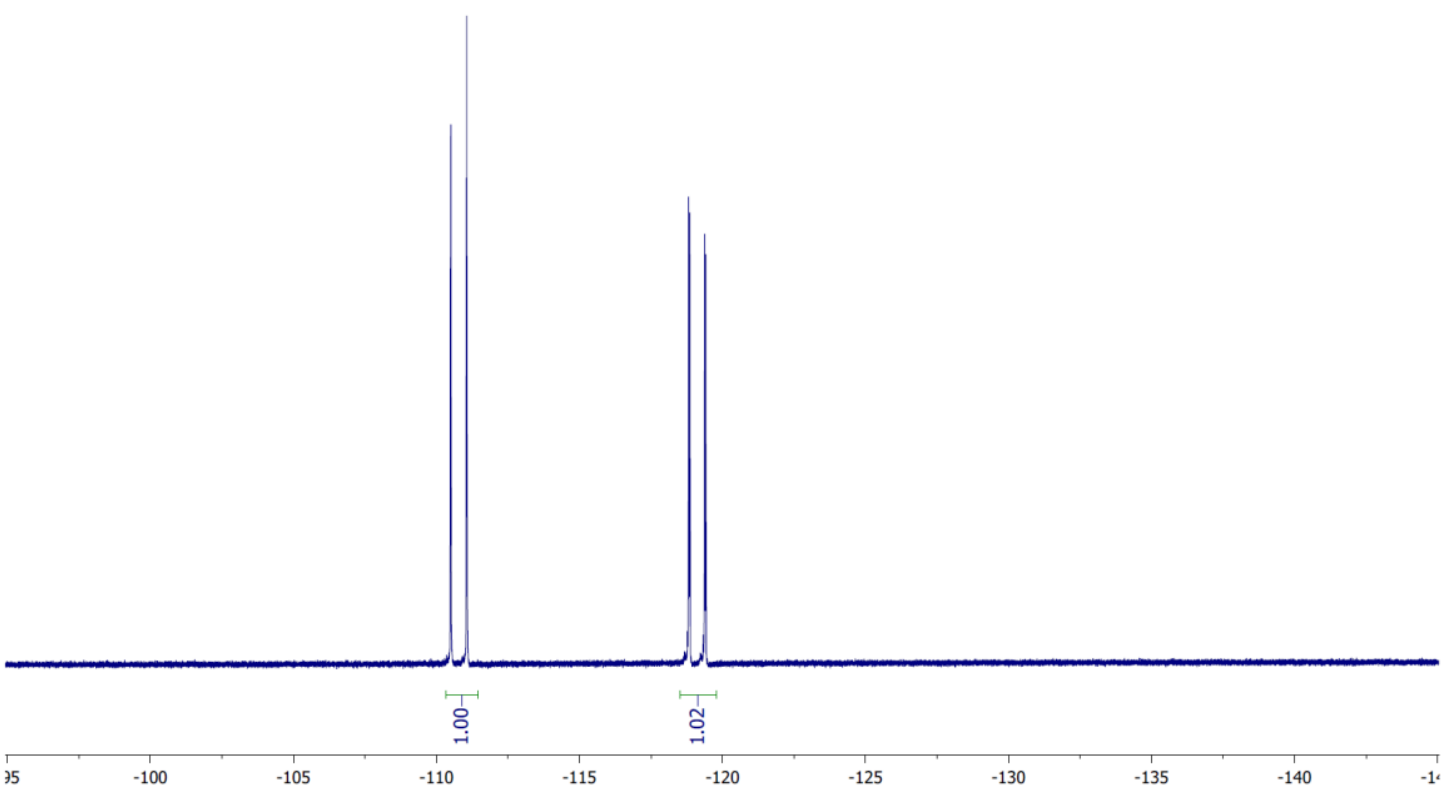




\section{${ }^{13}$ C NMR}

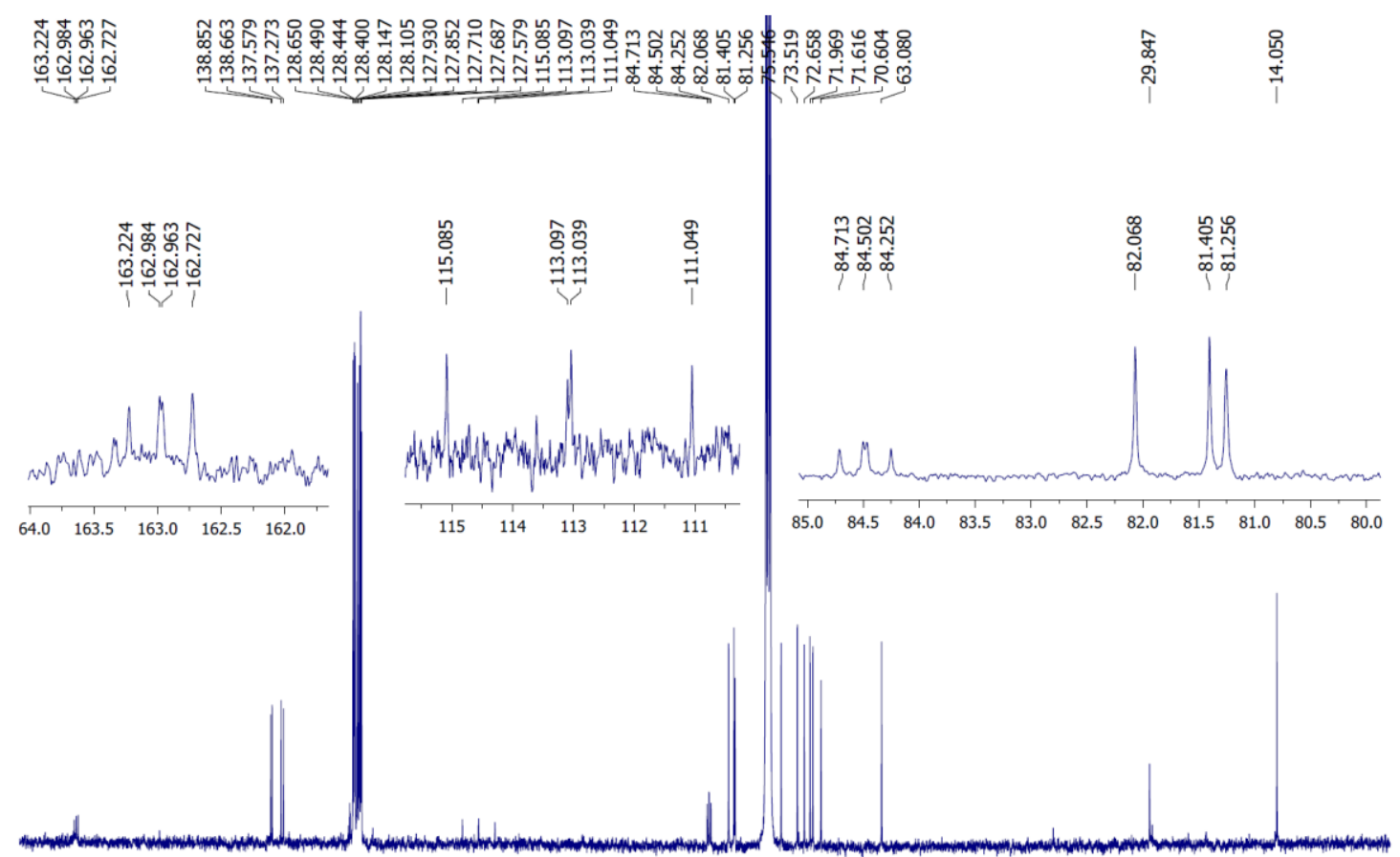

